\title{
Design of P-Chirogenic Aminophosphine-Phosphinite Ligands at both Phosphorus Centers: Origin of Enantioselectivities in Pd-Catalyzed Allylic Reactions
}

Antonin Jaillet, ${ }^{a}$ Christophe Darcel, ${ }^{a, b}$ Jérôme Bayardon, ${ }^{a}$ Adrien Schlachter, ${ }^{c}$ Christine Salomon, ${ }^{a \delta}$ Yoann Rousselin, ${ }^{a}$ Pierre Harvey, ${ }^{c}$ and Sylvain Jugé ${ }^{* a}$

${ }^{a}$ Université de Bourgogne-Franche-Comté, Institut de Chimie Moléculaire (ICMUB UMRCNRS 6302),

9 avenue A. Savary, BP 47870, 21078 Dijon Cedex, France

${ }^{\text {b } U n i v e r s i t e ́ ~ d e ~ R e n n e s, ~ I n s t i t u t ~ d e s ~ S c i e n c e s ~ C h i m i q u e s ~ d e ~ R e n n e s ~(I S C R ~ U M R-C N R S ~ 6226), ~}$ 35000 Rennes, France

${ }^{\mathrm{c}}$ Département de Chimie, Université de Sherbrooke, Sherbrooke, Québec, Canada J1K2R1

*E-mail: Sylvain.Juge@u-bourgogne.fr

\section{Part A:}

Table of contents: ${ }^{1} \mathrm{H},{ }^{13} \mathrm{C},{ }^{31} \mathrm{P}$ NMR spectra of compounds

${ }^{1} \mathrm{H},{ }^{31} \mathrm{P}$ NMR spectra of compound (-)-10b $\quad$ S4

${ }^{1} \mathrm{H},{ }^{13} \mathrm{C},{ }^{31} \mathrm{P}$ NMR spectra of compound $\left(R_{\mathrm{p}}\right)-\mathbf{1 1 e} \quad$ S5

${ }^{1} \mathrm{H},{ }^{31} \mathrm{P}$ NMR spectra of compound $\left(S_{\mathrm{p}}\right)-\mathbf{1 1 i} \quad$ S6

${ }^{1} \mathrm{H},{ }^{13} \mathrm{C},{ }^{31} \mathrm{P}$ NMR spectra of compound $\left(R_{\mathrm{p}}\right)-\mathbf{1 1} \mathbf{r} \quad \mathrm{S} 7$

${ }^{1} \mathrm{H},{ }^{13} \mathrm{C},{ }^{31} \mathrm{P}$ NMR spectra of compound $\left(M, S_{\mathrm{p}}\right)-\mathbf{1 1} \mathbf{v} \quad$ S9

${ }^{1} \mathrm{H},{ }^{13} \mathrm{C},{ }^{31} \mathrm{P}$ NMR spectra of compound $\left(S_{\mathrm{p}}\right)-\mathbf{1 2} \mathbf{i} \quad \mathrm{S} 10$

${ }^{1} \mathrm{H},{ }^{13} \mathrm{C},{ }^{31} \mathrm{P}$ NMR spectra of compound $\left(R_{\mathrm{p}}\right)-\mathbf{1 2 m} \quad \mathrm{S} 12$

${ }^{1} \mathrm{H},{ }^{13} \mathrm{C},{ }^{31} \mathrm{P}$ NMR spectra of compound $\left(R_{\mathrm{p}}\right)-\mathbf{1 2 n} \quad \mathrm{S} 13$

${ }^{1} \mathrm{H},{ }^{13} \mathrm{C},{ }^{31} \mathrm{P}$ NMR spectra of compound $\left(R_{\mathrm{p}}\right)-\mathbf{1 2 0} \quad \mathrm{S} 15$

${ }^{1} \mathrm{H},{ }^{13} \mathrm{C},{ }^{31} \mathrm{P}$ NMR spectra of compound $\left(R_{\mathrm{p}}\right)-\mathbf{1 2 p} \quad \mathrm{S} 16$

${ }^{1} \mathrm{H},{ }^{13} \mathrm{C},{ }^{31} \mathrm{P}$ NMR spectra of compound $\left(R_{\mathrm{p}}\right)-\mathbf{1 2 q} \quad \mathrm{S} 18$

${ }^{1} \mathrm{H},{ }^{13} \mathrm{C},{ }^{31} \mathrm{P}$ NMR spectra of compound $\left(R_{\mathrm{p}}\right)-\mathbf{1 2} \mathrm{r} \quad \mathrm{S} 19$

${ }^{1} \mathrm{H},{ }^{13} \mathrm{C},{ }^{31} \mathrm{P}$ NMR spectra of compound $\left(R_{\mathrm{p}}\right)-\mathbf{1 2 s} \quad \mathrm{S} 21$

${ }^{1} \mathrm{H},{ }^{13} \mathrm{C},{ }^{31} \mathrm{P}$ NMR spectra of compound $\left(R_{\mathrm{p}}\right)-\mathbf{1 2 t} \quad$ S22

${ }^{1} \mathrm{H},{ }^{13} \mathrm{C},{ }^{31} \mathrm{P}$ NMR spectra of compound $\left(M, S_{\mathrm{p}}\right)-\mathbf{1 2 v} \quad$ S24

${ }^{1} \mathrm{H},{ }^{13} \mathrm{C},{ }^{31} \mathrm{P}$ NMR spectra of compound $\left(S_{\mathrm{p}}\right)-\mathbf{1 2 w} \quad$ S25 
${ }^{31} \mathrm{P}$ NMR spectra of compound $\left(S_{\mathrm{p}}\right)-\mathbf{1 3 i}$

${ }^{31} \mathrm{P}$ NMR spectra of compound $\left(R_{\mathrm{p}}\right)-\mathbf{1 3 m}$

${ }^{31} \mathrm{P}$ NMR spectra of compound $\left(R_{\mathrm{p}}\right)-\mathbf{1 3 n}$ S28

${ }^{31} \mathrm{P}$ NMR spectra of compound $\left(R_{\mathrm{p}}\right)-\mathbf{1 3 0}$ S28

${ }^{31} \mathrm{P}$ NMR spectra of compound $\left(R_{\mathrm{p}}\right)-\mathbf{1 3 p}$

${ }^{31} \mathrm{P}$ NMR spectra of compound $\left(R_{\mathrm{p}}\right)-\mathbf{1 3 q}$

${ }^{31} \mathrm{P}$ NMR spectra of compound $\left(R_{\mathrm{p}}\right)-\mathbf{1 3 r}$ S30

${ }^{31} \mathrm{P}$ NMR spectra of compound $\left(R_{\mathrm{p}}\right)-\mathbf{1 3 s}$ S30

${ }^{31} \mathrm{P}$ NMR spectra of compound $\left(R_{\mathrm{p}}\right)-\mathbf{1 3 t}$ S31

${ }^{31} \mathrm{P}$ NMR spectra of compound $\left(M, S_{\mathrm{p}}\right)-\mathbf{1 3} \mathbf{v}$

${ }^{31} \mathrm{P}$ NMR spectra of compound $\left(S_{\mathrm{p}}\right)-\mathbf{1 3 w}$

${ }^{1} \mathrm{H},{ }^{13} \mathrm{C},{ }^{31} \mathrm{P}$ NMR spectra of compound $\left(S_{\mathrm{p}}\right)$-21a

${ }^{1} \mathrm{H},{ }^{13} \mathrm{C},{ }^{31} \mathrm{P}$ NMR spectra of compound $\left(S_{\mathrm{p}}\right)-\mathbf{2 1 b}$

${ }^{1} \mathrm{H},{ }^{13} \mathrm{C},{ }^{31} \mathrm{P}$ NMR spectra of compound $\left(R_{\mathrm{p}}\right)-21 \mathrm{c}$

${ }^{1} \mathrm{H},{ }^{13} \mathrm{C},{ }^{31} \mathrm{P}$ NMR spectra of compound $\left(R_{\mathrm{p}}\right)-\mathbf{2 1 d}$

${ }^{1} \mathrm{H},{ }^{13} \mathrm{C},{ }^{31} \mathrm{P}$ NMR spectra of compound $\left(R_{\mathrm{p}}\right)-\mathbf{2 1 e}$ S38

${ }^{1} \mathrm{H},{ }^{13} \mathrm{C},{ }^{31} \mathrm{P}$ NMR spectra of compound $\left(R_{\mathrm{p}}\right)$-21f S40

${ }^{1} \mathrm{H},{ }^{13} \mathrm{C},{ }^{31} \mathrm{P}$ NMR spectra of compound $\left(R_{\mathrm{p}}\right)-\mathbf{2 1 g}$

${ }^{1} \mathrm{H},{ }^{13} \mathrm{C},{ }^{31} \mathrm{P}$ NMR spectra of compound $\left(S_{\mathrm{p}}\right)-\mathbf{2 1 h}$

${ }^{1} \mathrm{H},{ }^{13} \mathrm{C},{ }^{31} \mathrm{P}$ NMR spectra of compound $\left(S_{\mathrm{p}}\right)-\mathbf{2 1 i}$

${ }^{1} \mathrm{H},{ }^{31} \mathrm{P}$ NMR spectra of compound $\left(R_{\mathrm{p}}\right)-\mathbf{2 0 a}$

${ }^{1} \mathrm{H},{ }^{31} \mathrm{P}$ NMR spectra of compound $\left(S_{\mathrm{p}}\right)-\mathbf{2 0 a}$

${ }^{1} \mathrm{H},{ }^{31} \mathrm{P}$ NMR spectra of compound $\left(R_{\mathrm{p}}\right)-\mathbf{2 0 b}$

${ }^{1} \mathrm{H},{ }^{31} \mathrm{P}$ NMR spectra of compound $\left(R_{\mathrm{p}}\right)-20 \mathrm{c}$

${ }^{1} \mathrm{H},{ }^{31} \mathrm{P}$ NMR spectra of compound $\left(R_{\mathrm{p}}\right)$-20d

${ }^{1} \mathrm{H},{ }^{31} \mathrm{P}$ NMR spectra of compound $\left(R_{\mathrm{p}}\right)$-20f

${ }^{1} \mathrm{H},{ }^{31} \mathrm{P}$ NMR spectra of compound $\left(R_{\mathrm{p}}\right)-\mathbf{2 0 g}$

${ }^{1} \mathrm{H},{ }^{31} \mathrm{P}$ NMR spectra of compound $\left(S_{\mathrm{p}}\right)-\mathbf{2 0 i}$

${ }^{1} \mathrm{H},{ }^{13} \mathrm{C},{ }^{31} \mathrm{P}$ NMR spectra of complex 30

${ }^{1} \mathrm{H},{ }^{13} \mathrm{C},{ }^{31} \mathrm{P}$ NMR spectra of complex 31

${ }^{1} \mathrm{H},{ }^{13} \mathrm{C},{ }^{31} \mathrm{P}$ NMR spectra of complex 32

\section{Part B:}

Table of contents:

Crystallographic data of compounds: 
Compound 21b

Compound 21c

Compound 21d $\left(\boldsymbol{R}_{\mathrm{p}}\right)$

Compound 21d $\left(S_{\mathrm{p}}\right)$

Compound 21e

\section{Part C:}

Table of contents:

HPLC chromatograms on chiral column

Allylic alkylation of ( \pm )-1,3-diphenylpropenyl acetate 24 with dimethylmalonate into compound $(R)-\mathbf{2 8}$

Allylic alkylation of ( \pm )-1,3-diphenylpropenyl acetate $\mathbf{2 4}$ with benzylamine into compound $(S)$-29

Complementary results in allylic alkylation of ( \pm )-1,3-diphenylpropenyl acetate 24, catalyzed by Pd-complexes with AMPP*

Computational details

Number of Imaginary Frequences and Cartesian Coordinates

Investigated structures

Fukui index calculations

Structures, Representations of frontier MOs HOMO/LUMO, atomic contributions at the $\mathrm{C} 1$ and $\mathrm{C} 3$ positions of the complexed $\pi$-allyl group

33M(boat)

33M(half-chair)

33W(half-chair) 
(2S,4R,5S)-3,4-Dimethyl-2-biphenyl-5-diphenyl-1,3,2-oxazaphospholidine-2-borane (-)-10b.

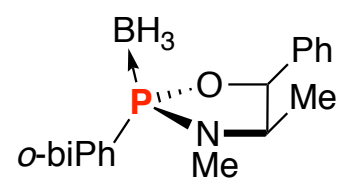

${ }^{1} \mathrm{H}$ NMR $\left(300 \mathrm{MHz}, \mathrm{CD}_{2} \mathrm{Cl}_{2}\right)$

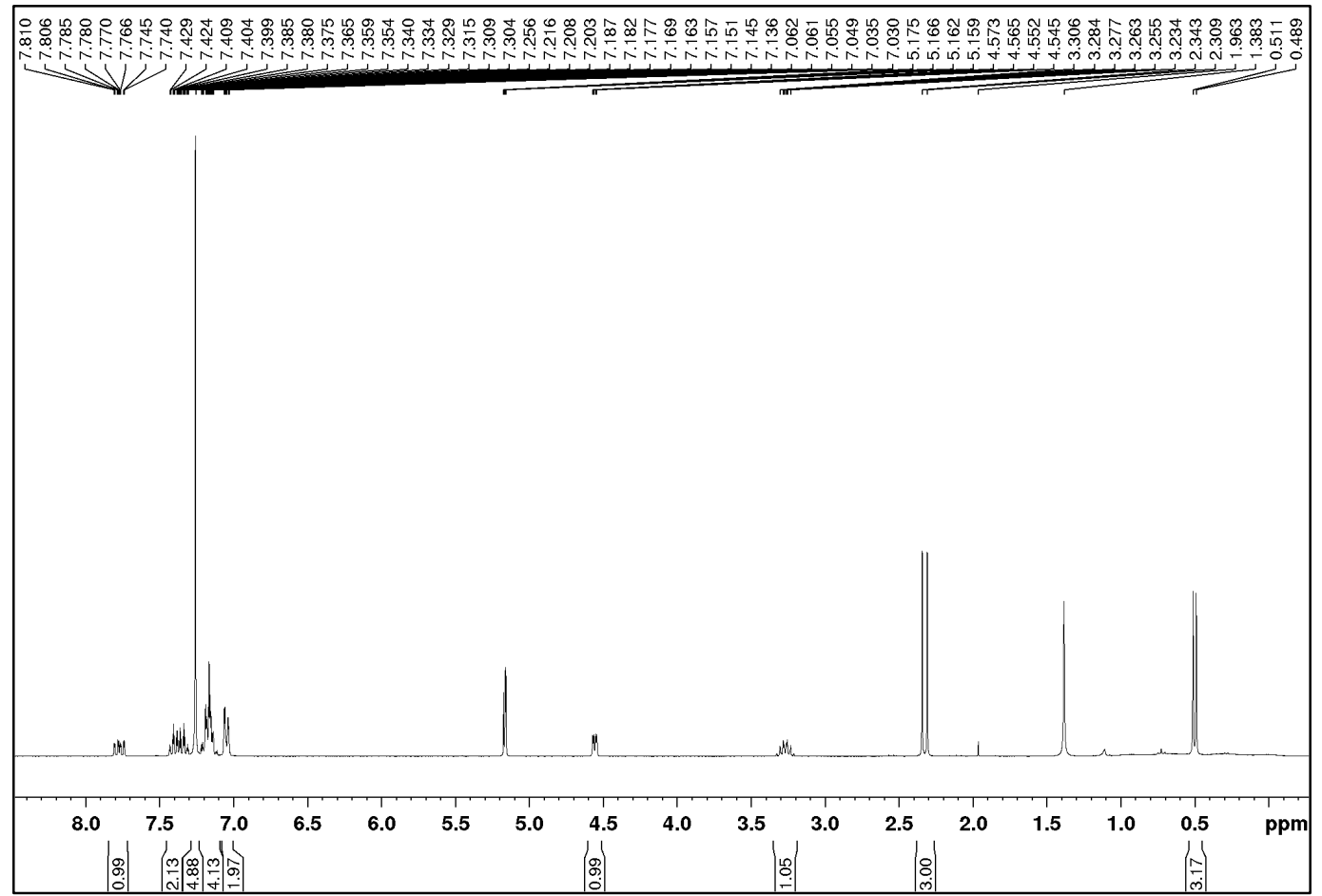

${ }^{31} \mathrm{P}\left\{{ }^{1} \mathrm{H}\right\}$ NMR (121.5 MHz, $\left.\mathrm{CD}_{2} \mathrm{Cl}_{2}\right)$

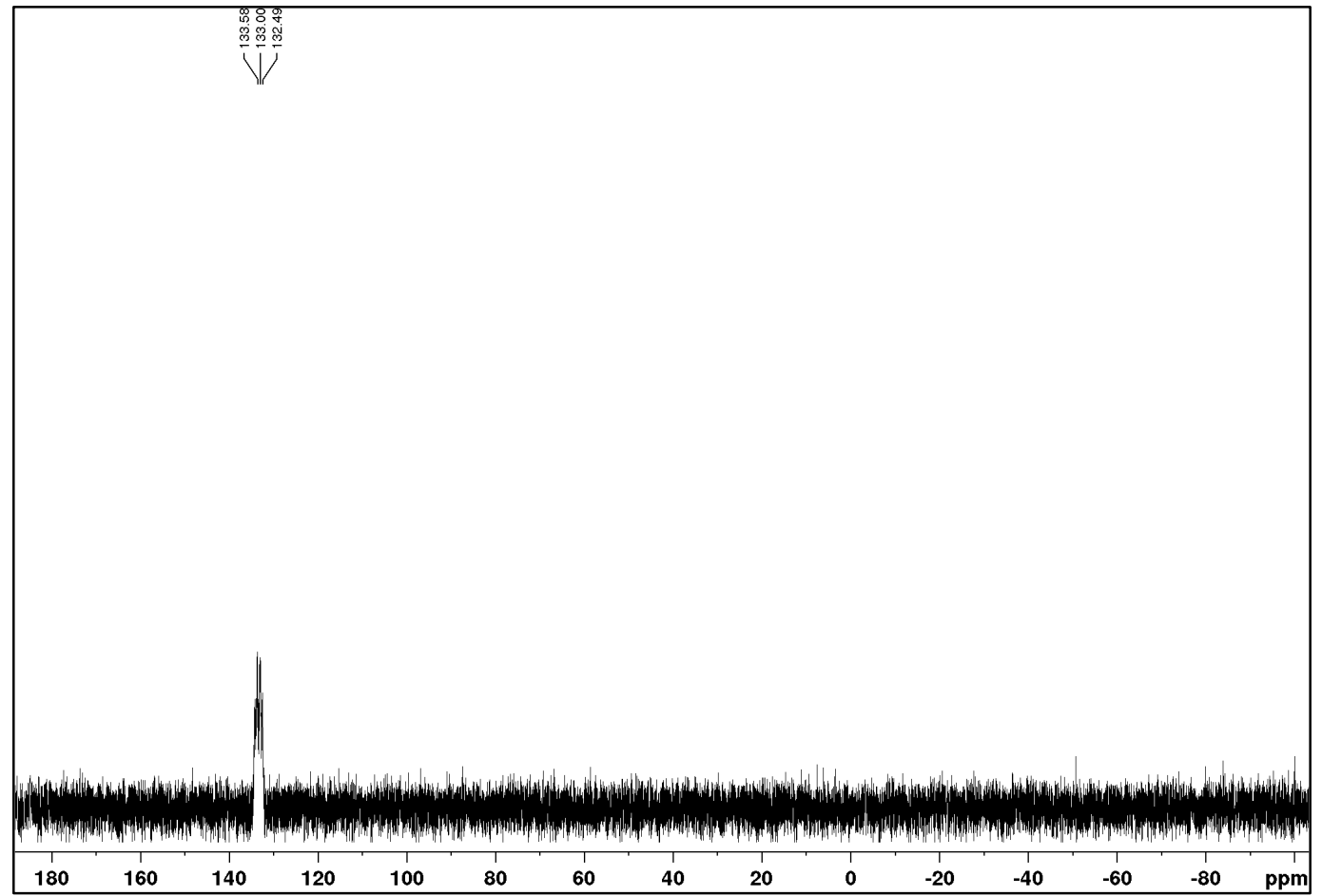


P-chirogenic aminophosphine-boranes 11.

$\left(R_{\mathrm{p}}\right)-(-)-\mathrm{N}-[(1 R, 2 S)-2-\mathrm{Hydroxy}-1-\mathrm{methyl}-2$-phenylethyl], $\quad$ N-methylamino(phenyl-p-tolyl) phosphine-borane 11e.

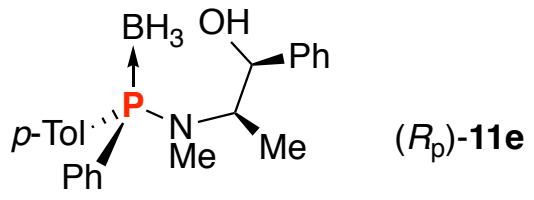

${ }^{1} \mathrm{H}$ NMR (300 MHz, $\left.\mathrm{CDCl}_{3}\right)$

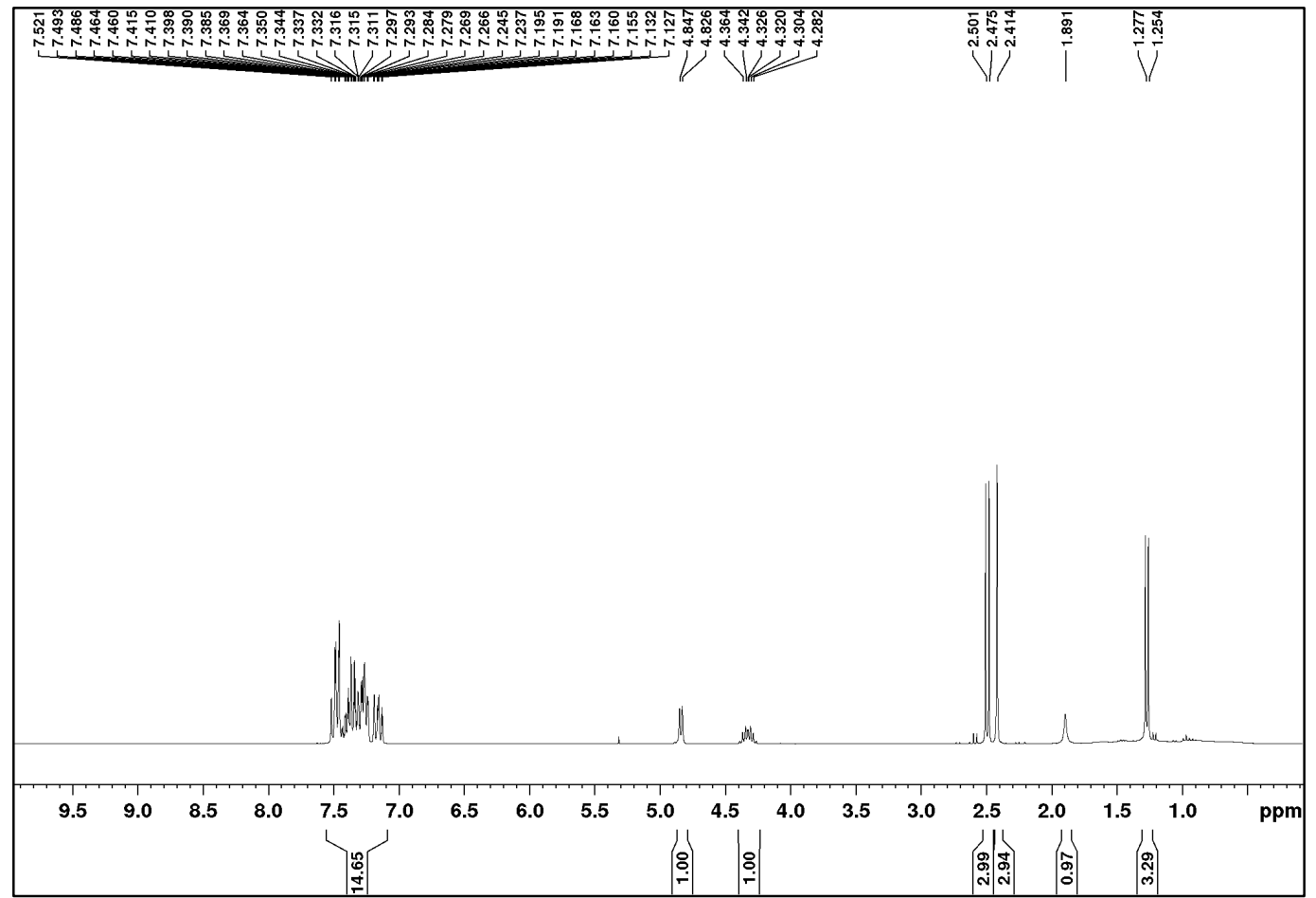

${ }^{13} \mathrm{C}\left\{{ }^{1} \mathrm{H}\right\}$ J-mod NMR $\left(75.5 \mathrm{MHz}, \mathrm{CDCl}_{3}\right)$

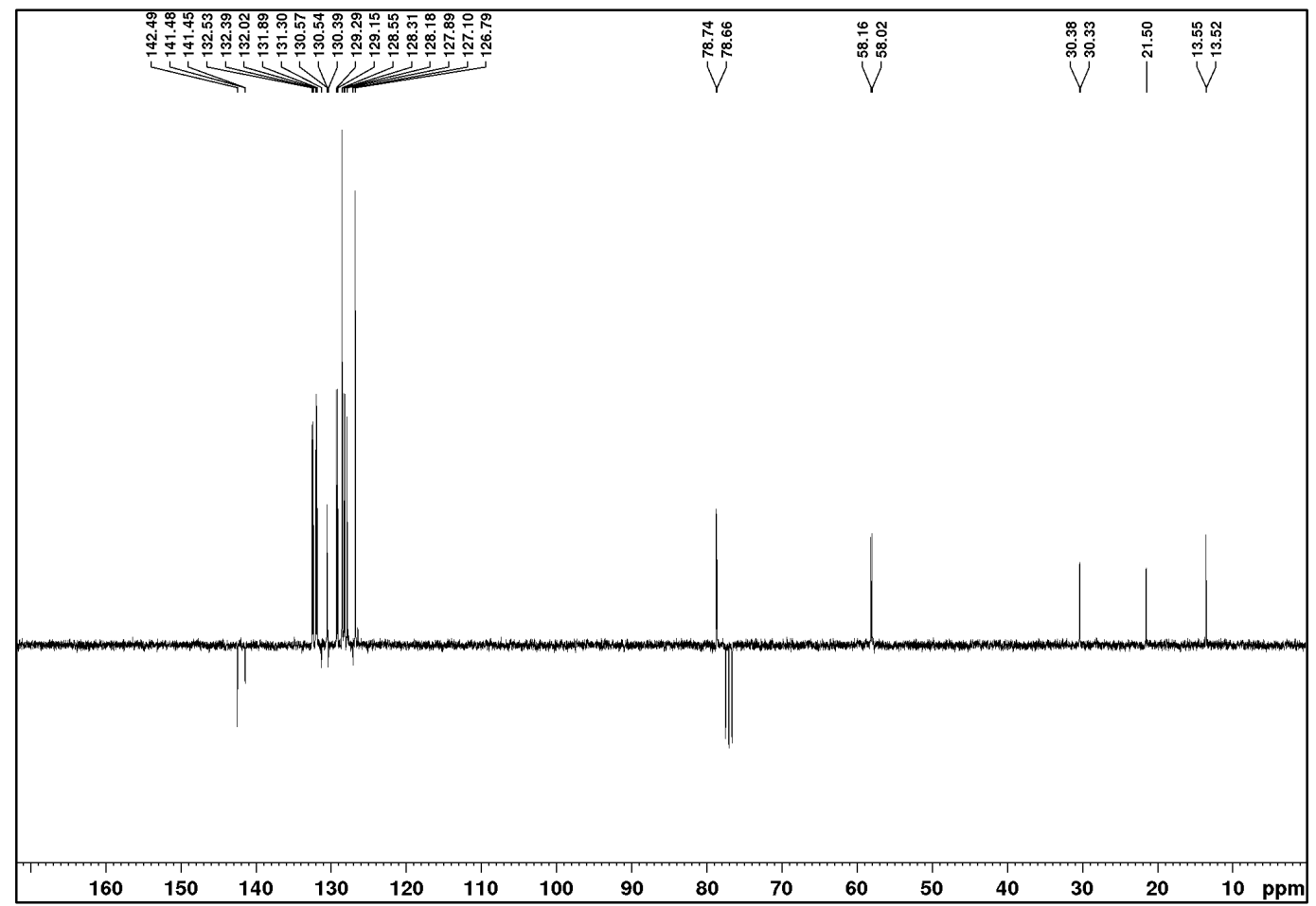


${ }^{31} \mathrm{P}\left\{{ }^{1} \mathrm{H}\right\}$ NMR (121.5 MHz, $\left.\mathrm{CDCl}_{3}\right)$

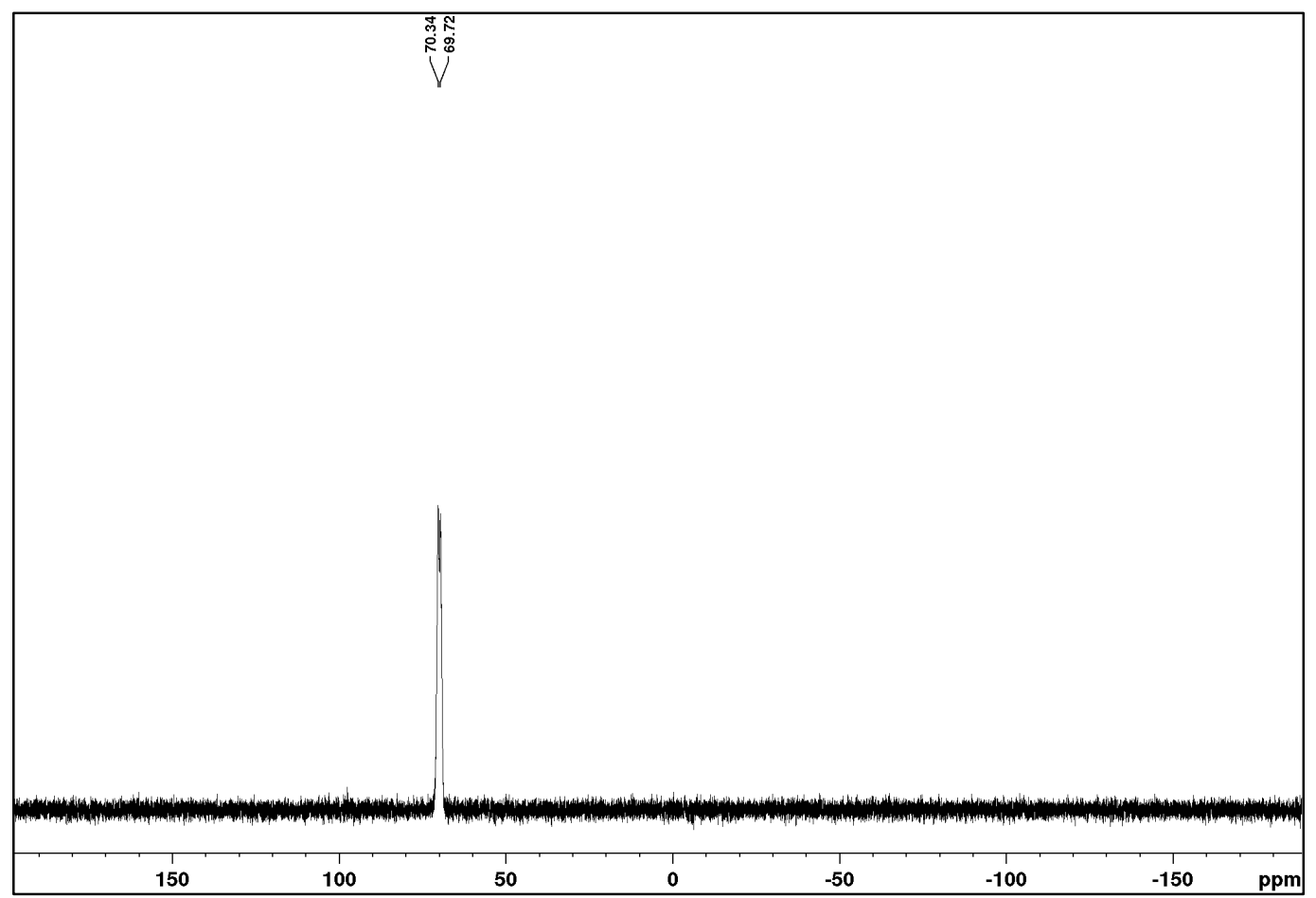

$\left(S_{\mathrm{p}}\right)-(+)-\mathrm{N}-[(1 R, 2 S)-2-H y d r o x y-1-m e t h y l-2-p h e n y l e t h y l], \quad \mathrm{N}$-methylamino(o-biphenyl)phenyl phosphine-borane 11i.

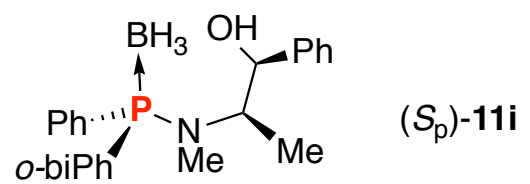

${ }^{1} \mathrm{H} \mathrm{NMR}\left(300 \mathrm{MHz}, \mathrm{CD}_{2} \mathrm{Cl}_{2}\right)$

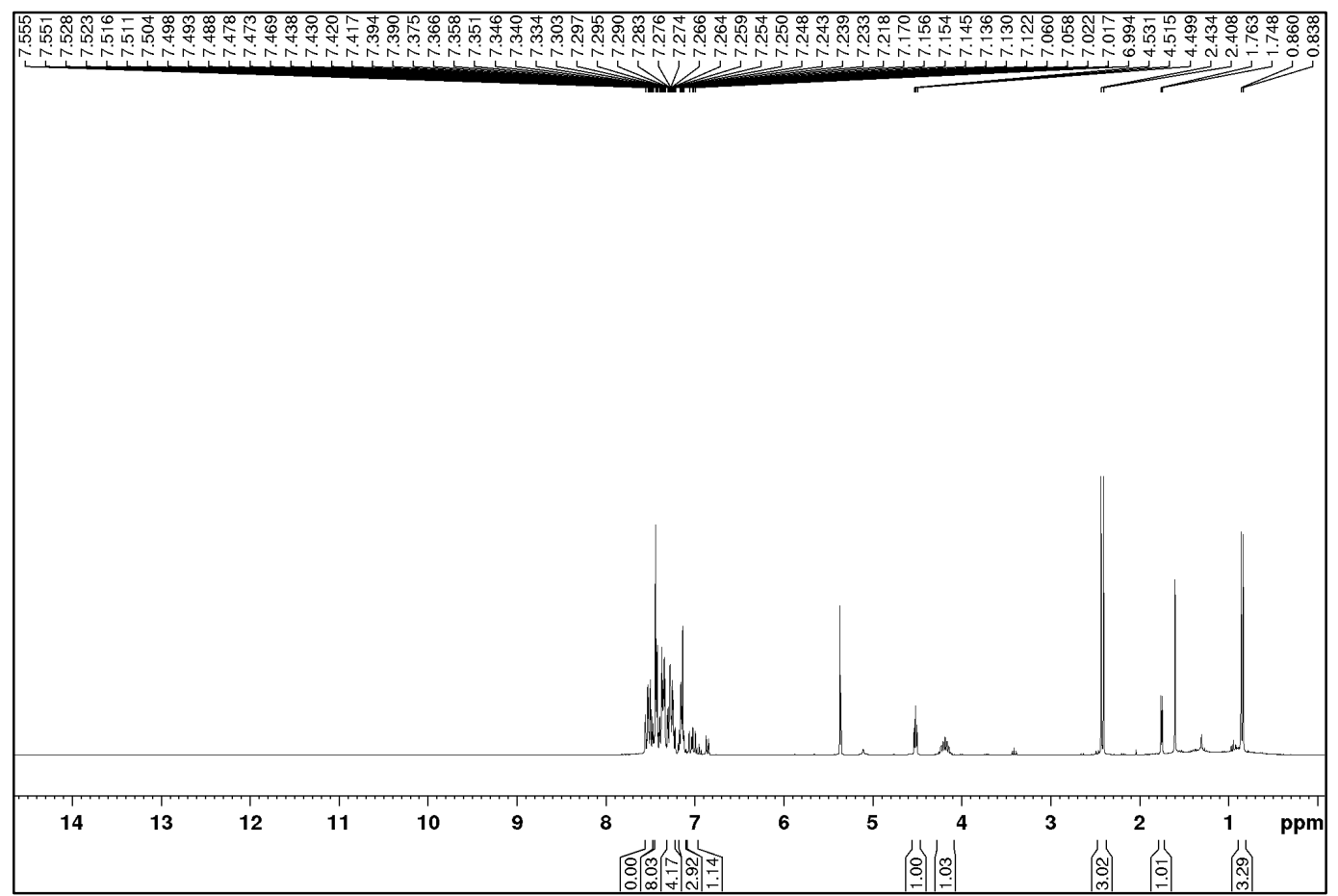


${ }^{31} \mathrm{P}\left\{{ }^{1} \mathrm{H}\right\}$ NMR (121.5 MHz, $\left.\mathrm{CD}_{2} \mathrm{Cl}_{2}\right)$

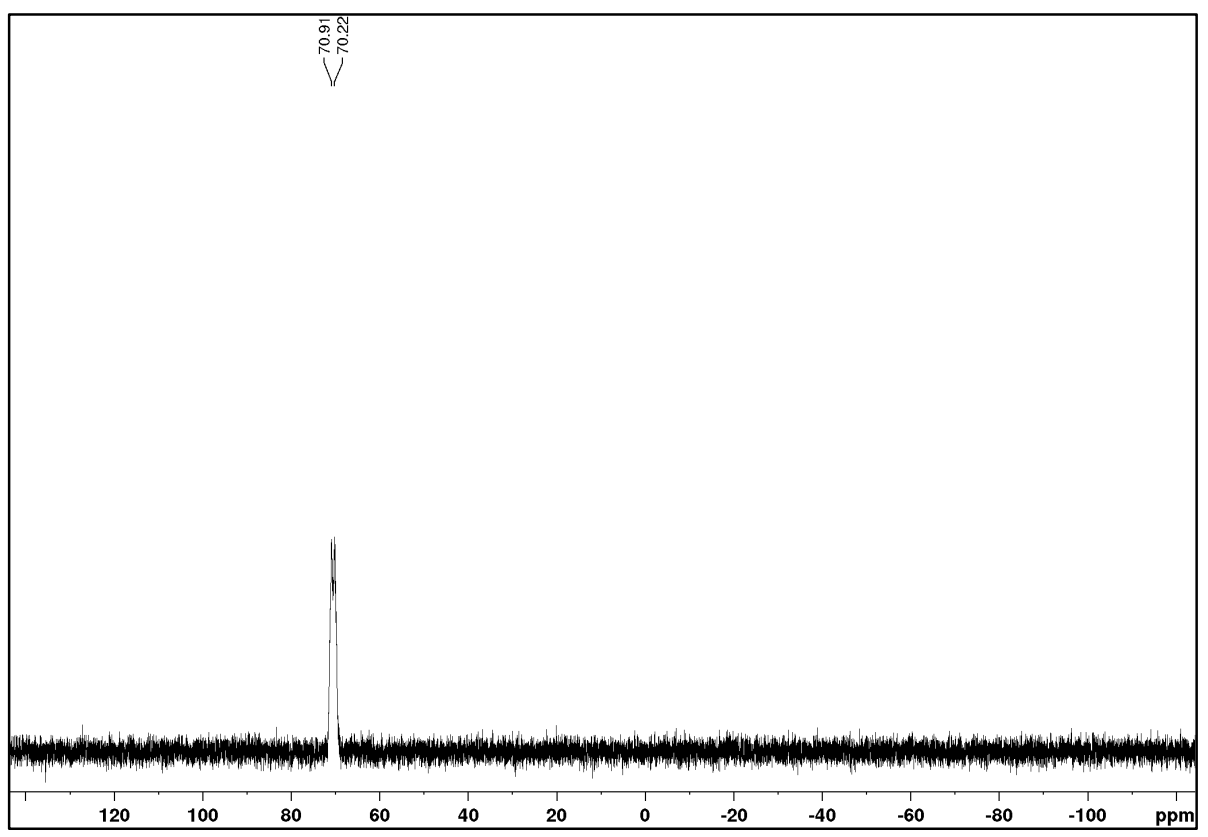

$\left(R_{\mathrm{p}}\right)-\mathrm{N}$-[(1R,2S)-2-Hydroxy-1-methyl-2-phenylethyl],

$\mathrm{N}$-(methylamino)phenyl(2-pyridyl) phosphine-borane 11r.

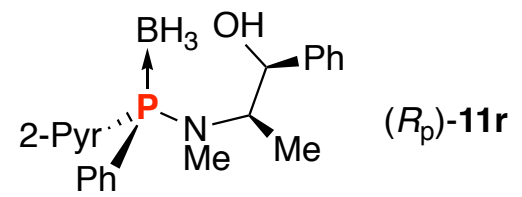

${ }^{1} \mathrm{H}$ NMR $\left(300 \mathrm{MHz}, \mathrm{CDCl}_{3}\right)$

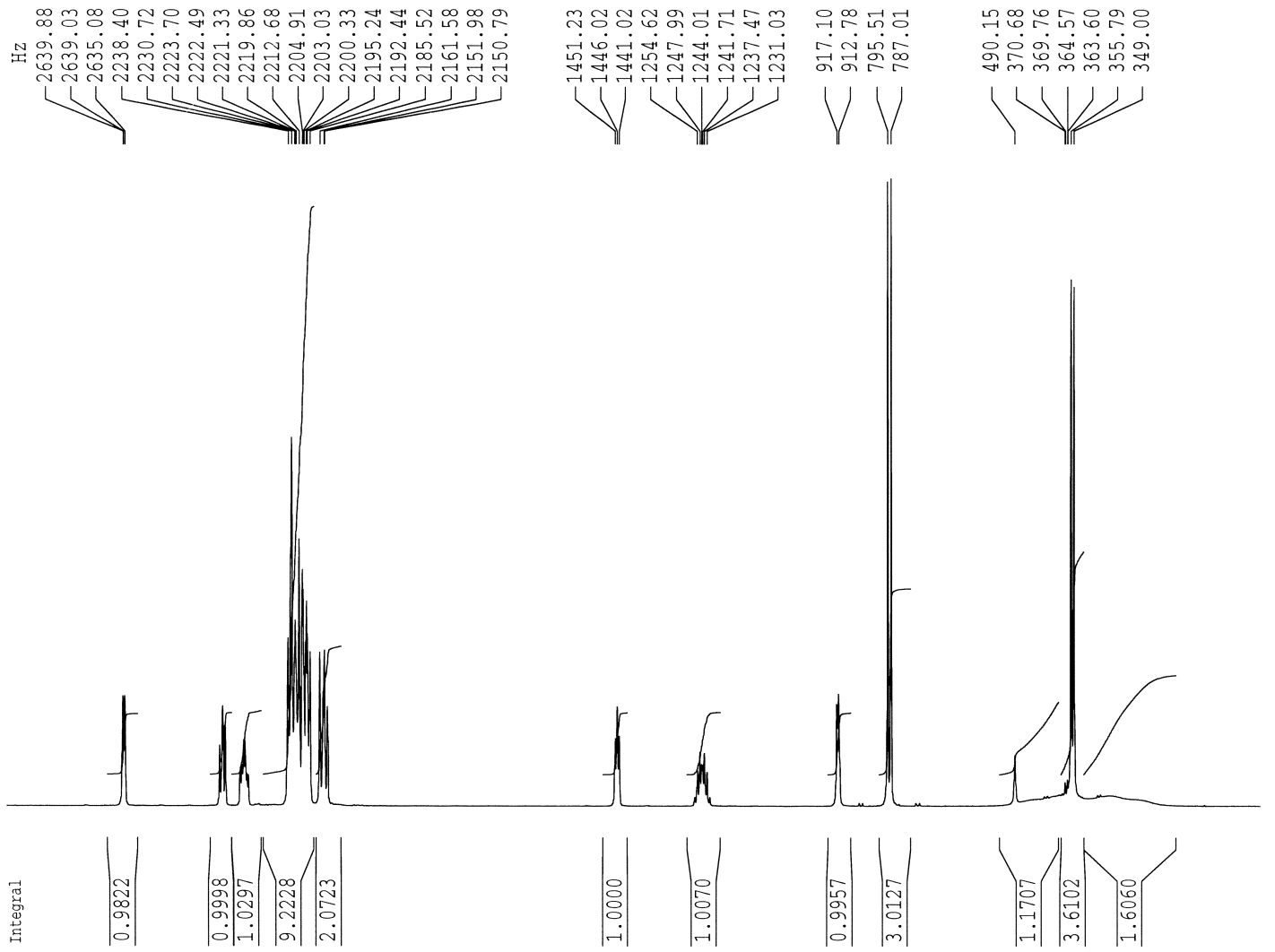

ppm 
${ }^{13} \mathrm{C}\left\{{ }^{1} \mathrm{H}\right\}$ NMR $\left(75.5 \mathrm{MHz}, \mathrm{CDCl}_{3}\right)$

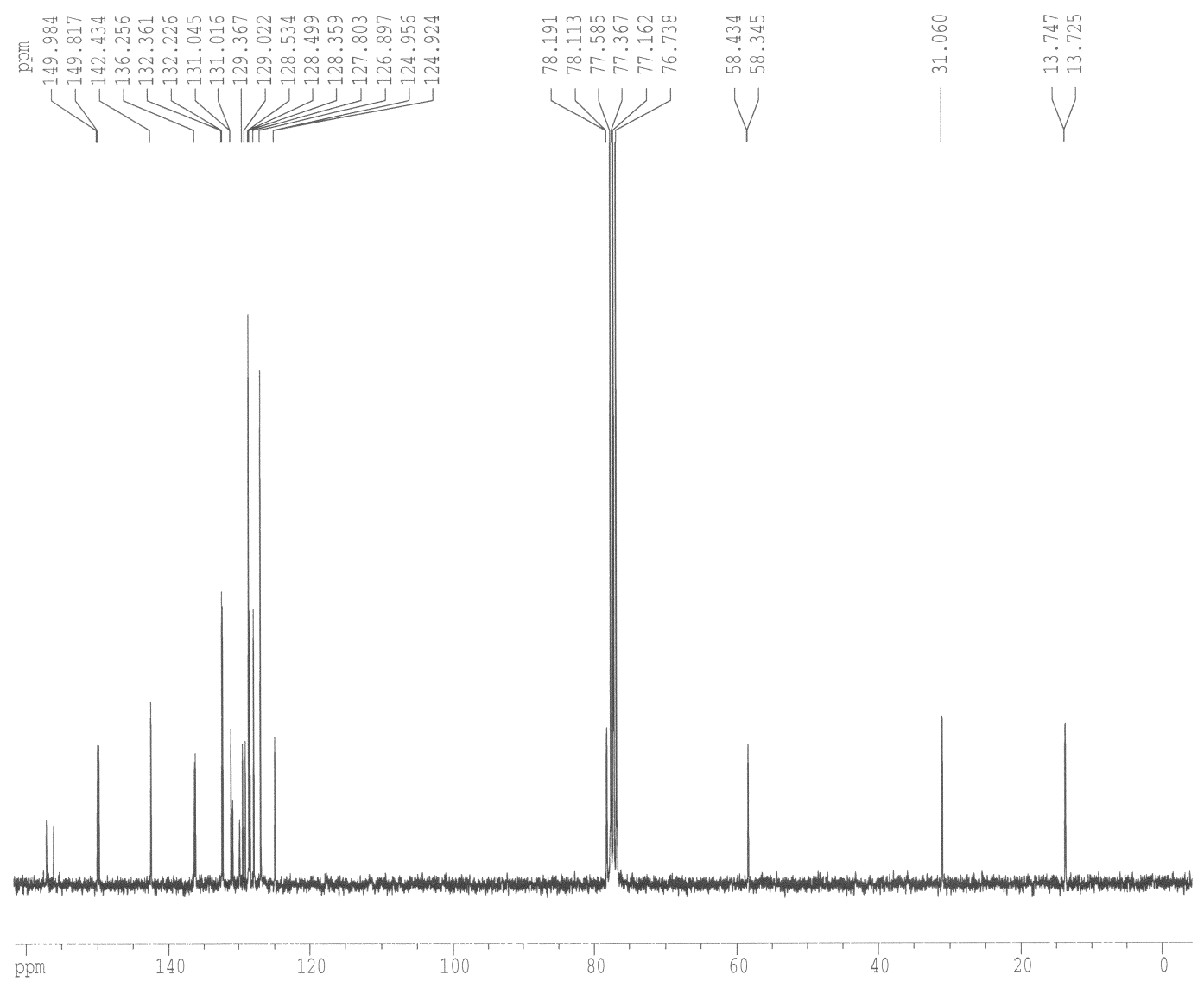

${ }^{31} \mathrm{P}\left\{{ }^{1} \mathrm{H}\right\}$ NMR (121.5 MHz, $\left.\mathrm{CDCl}_{3}\right)$

言

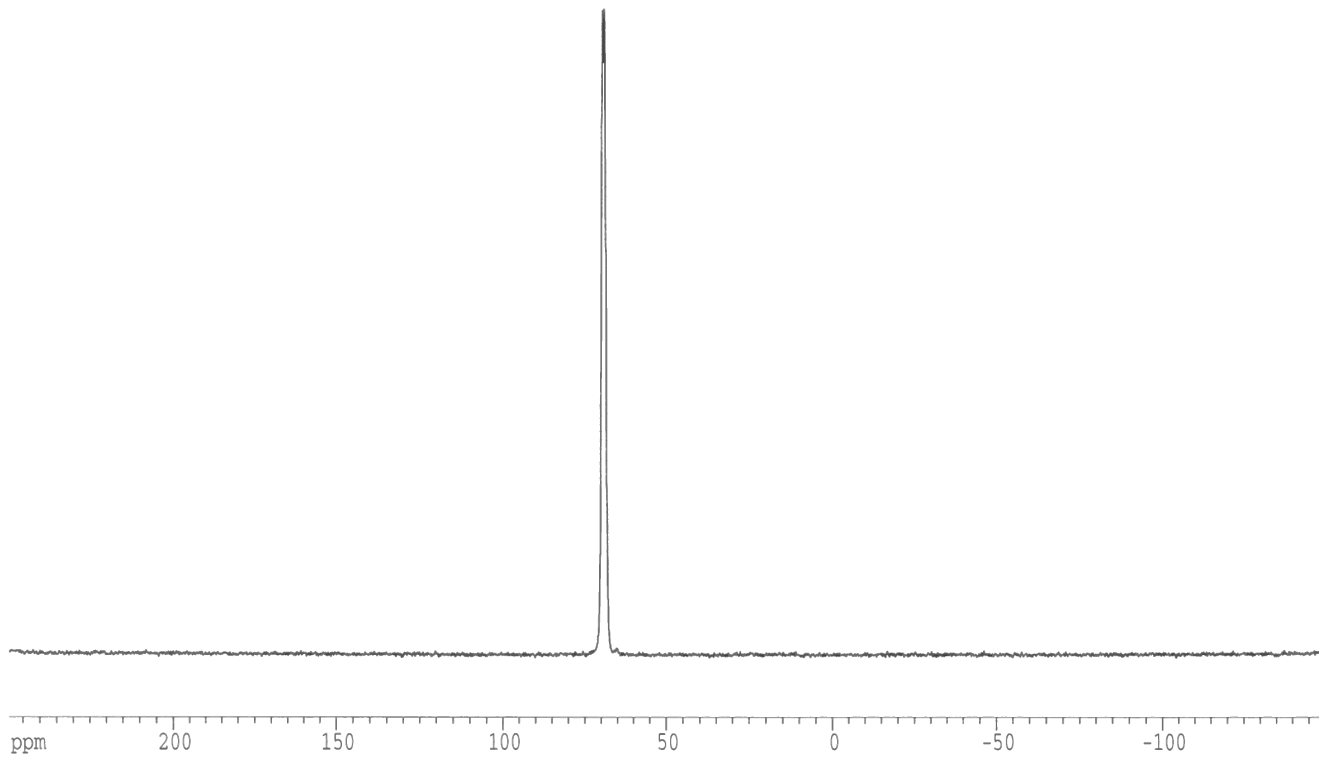


$\left(M, S_{\mathrm{p}}\right)-(+)-\mathrm{N}-[(1 S, 2 R)-2-(H y d r o x y-1-m e t h y l-2-p h e n y l e t h y l)], \mathrm{N}-m e t h y l a m i n o-2-\left(1,1^{\prime}\right.$-binaphtyl) phenylphosphine-borane 11v.

${ }^{1} \mathrm{H}$ NMR $\left(500 \mathrm{MHz}, \mathrm{CDCl}_{3}\right)$<smiles>[B][PH](c1ccccc1)(c1ccc2ccccc2c1-c1ccccc1)N(C)C(C)C(O)c1ccccc1</smiles>

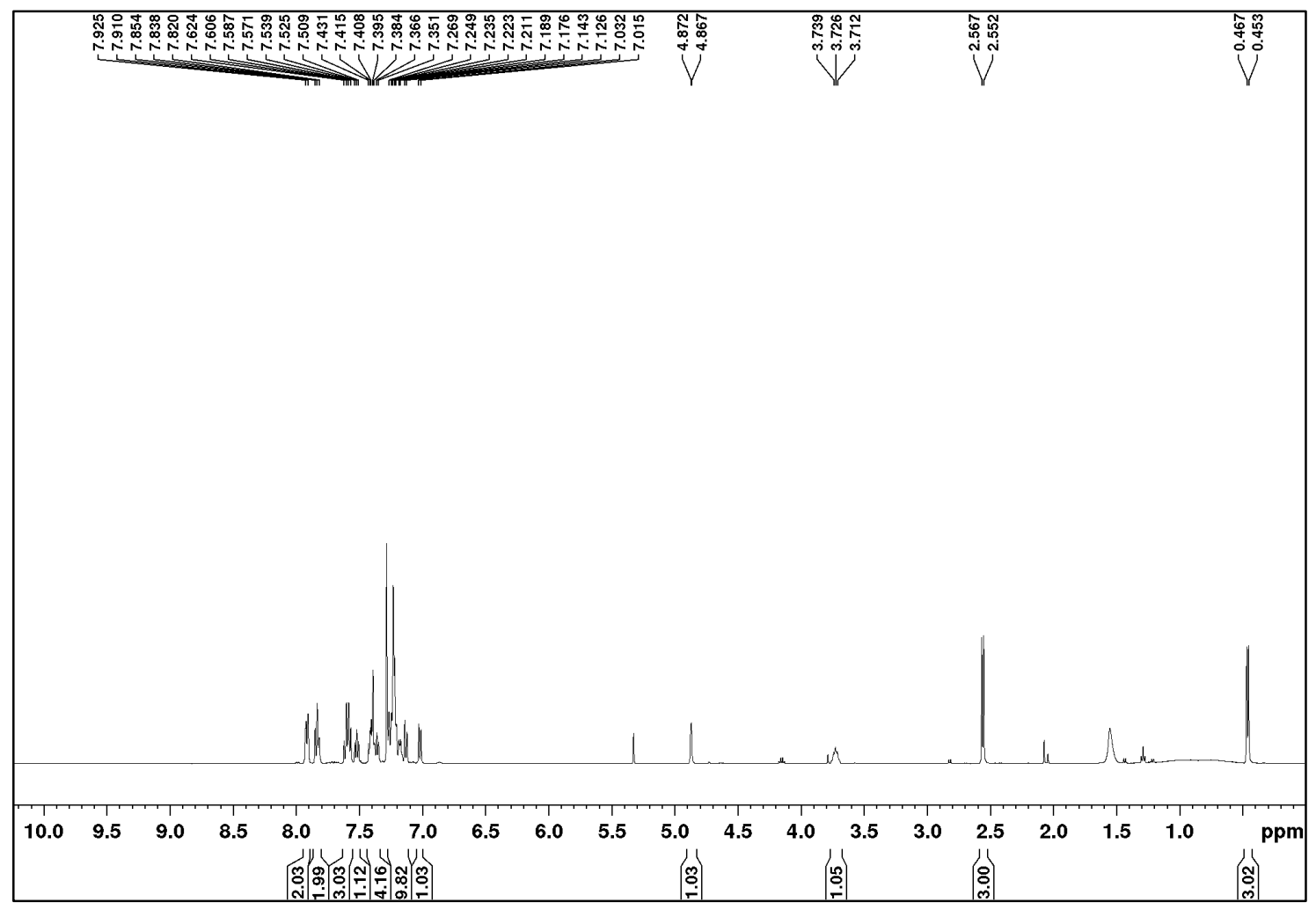

${ }^{13} \mathrm{C}\left\{{ }^{1} \mathrm{H}\right\} \mathrm{J}-\bmod \mathrm{NMR}\left(125.8 \mathrm{MHz}, \mathrm{CDCl}_{3}\right)$

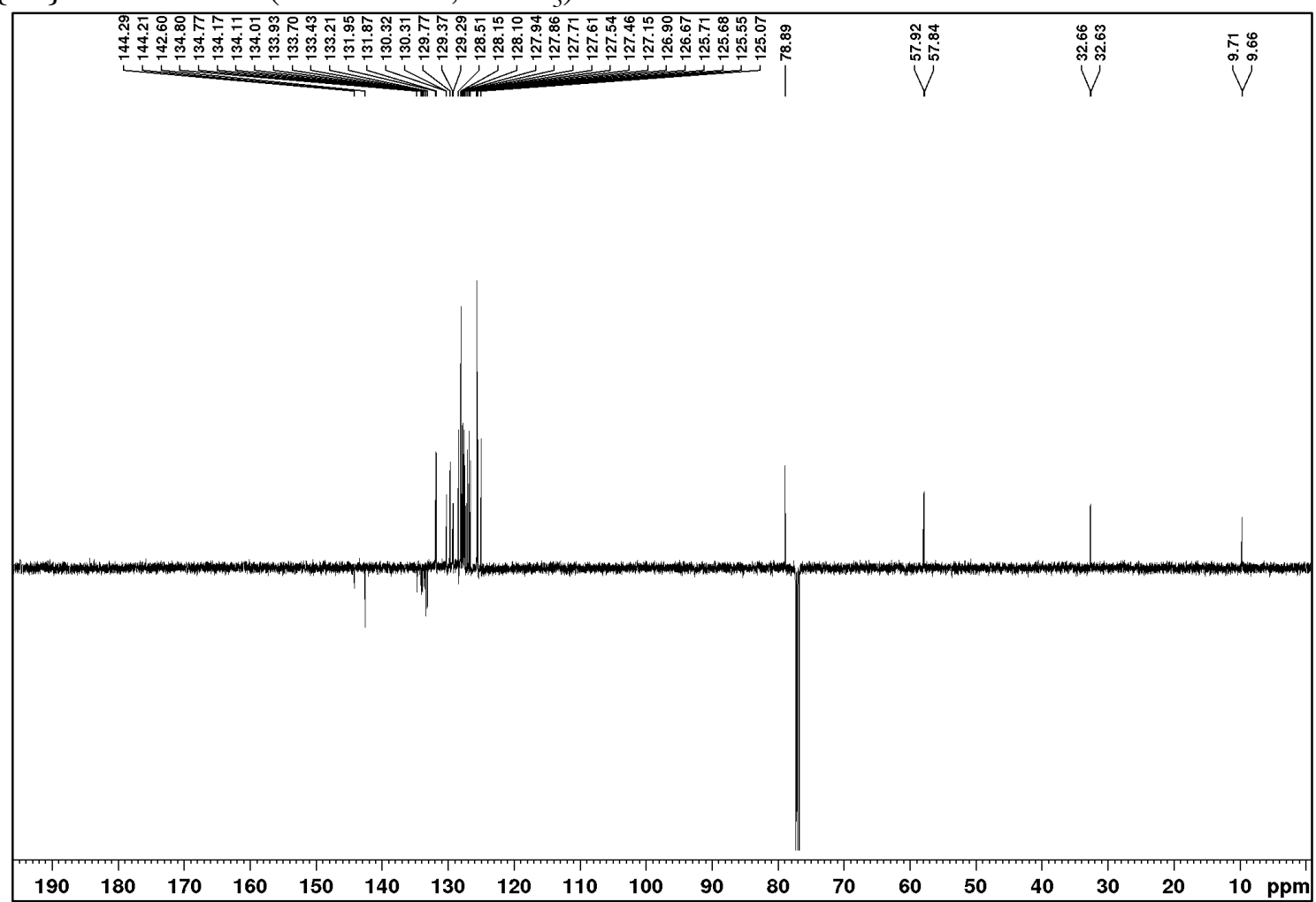


${ }^{31} \mathrm{P}\left\{{ }^{1} \mathrm{H}\right\}$ NMR (202.5 MHz, $\left.\mathrm{CDCl}_{3}\right)$

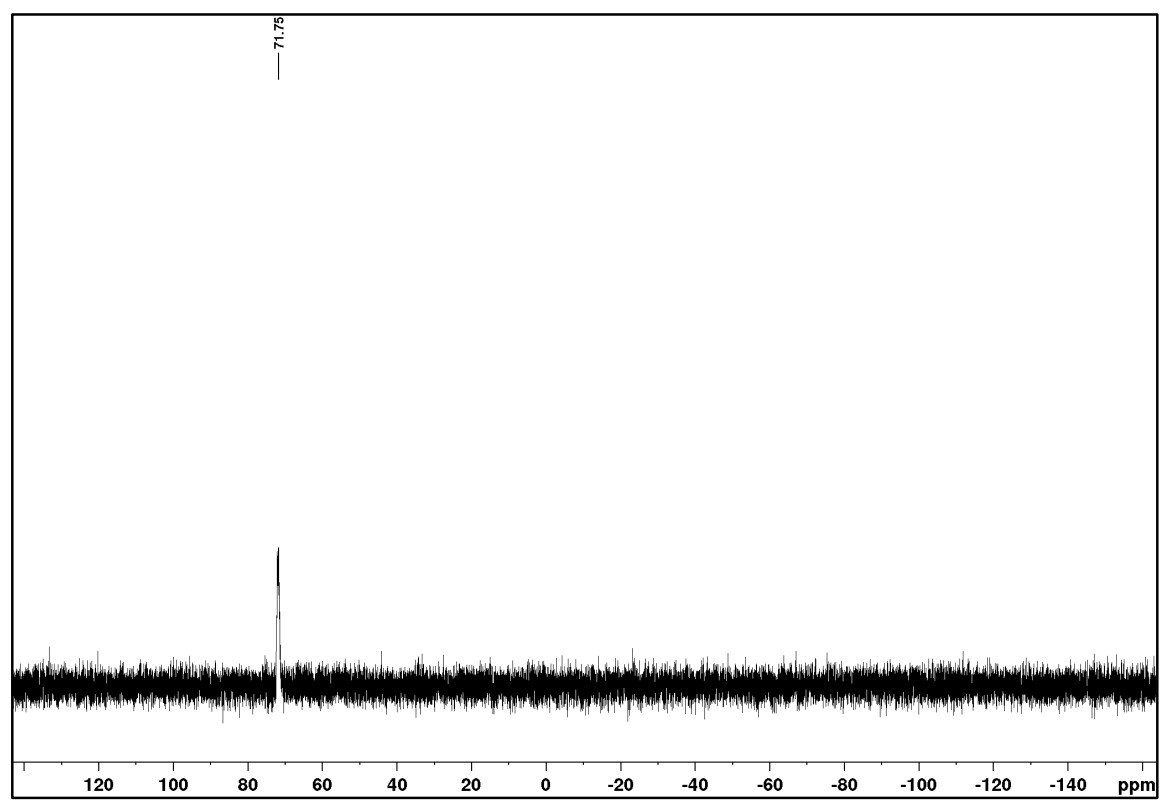

\section{P-chirogenic aminophosphine-phosphinite-diborane 12.}

$\left(S_{p}\right)-(-)-\mathrm{N}-[(1 R, 2 S)-2-($ Diphenylphosphinito-borane)-1-methyl-2-phenylethyl], N-methylamino (o-biphenyl)phenylphosphine-borane 12i.

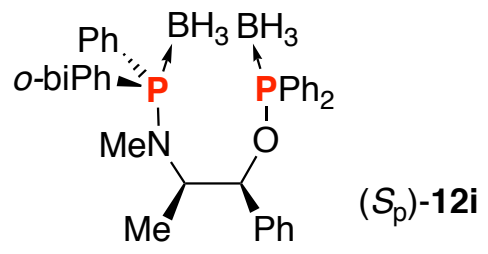

${ }^{1} \mathrm{H}$ NMR (500 MHz, $\mathrm{CD}_{2} \mathrm{Cl}_{2}$ )

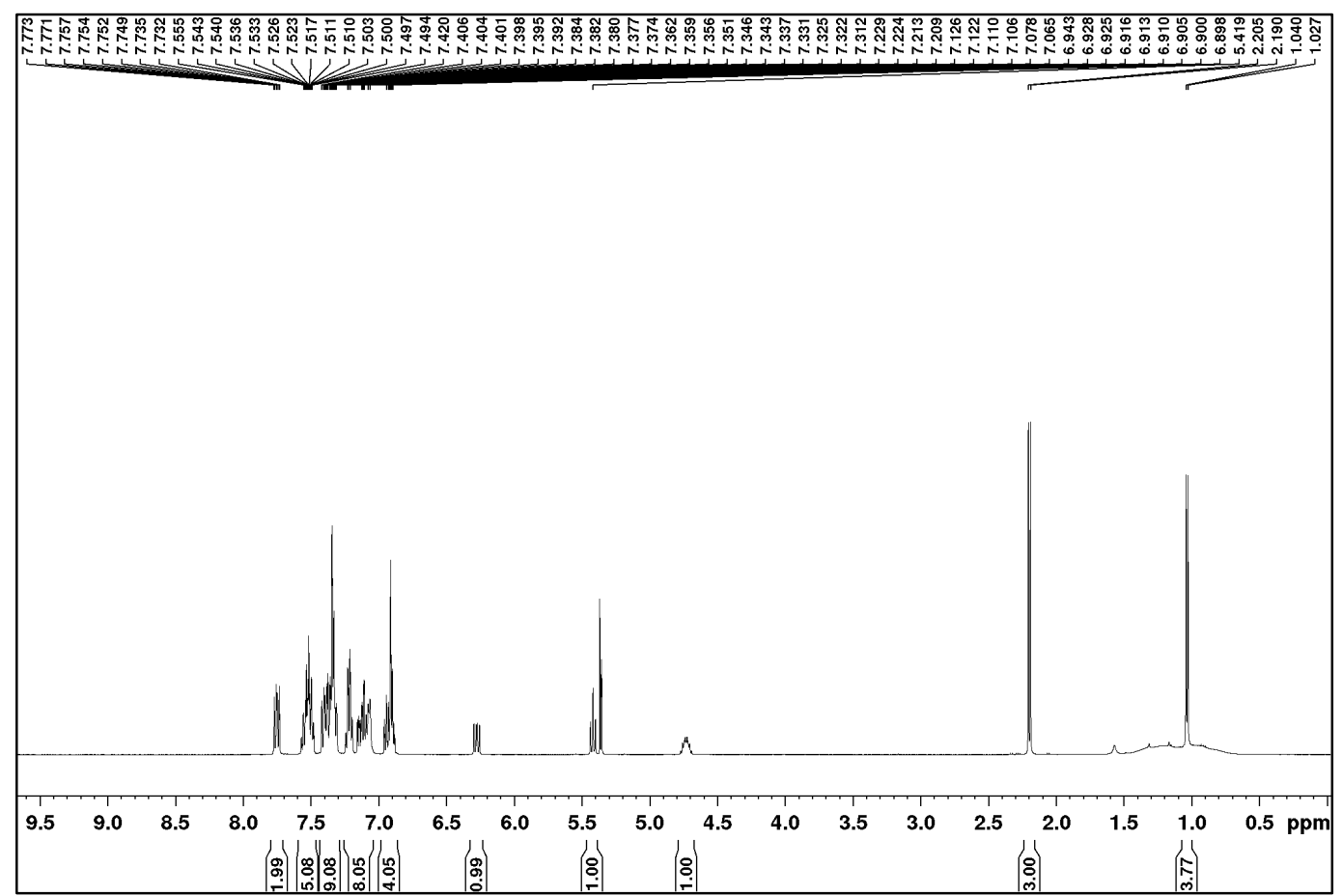


${ }^{13} \mathrm{C}\left\{{ }^{1} \mathrm{H}\right\} \mathrm{J}$-mod NMR (125.8 $\left.\mathrm{MHz}, \mathrm{CD}_{2} \mathrm{Cl}_{2}\right)$

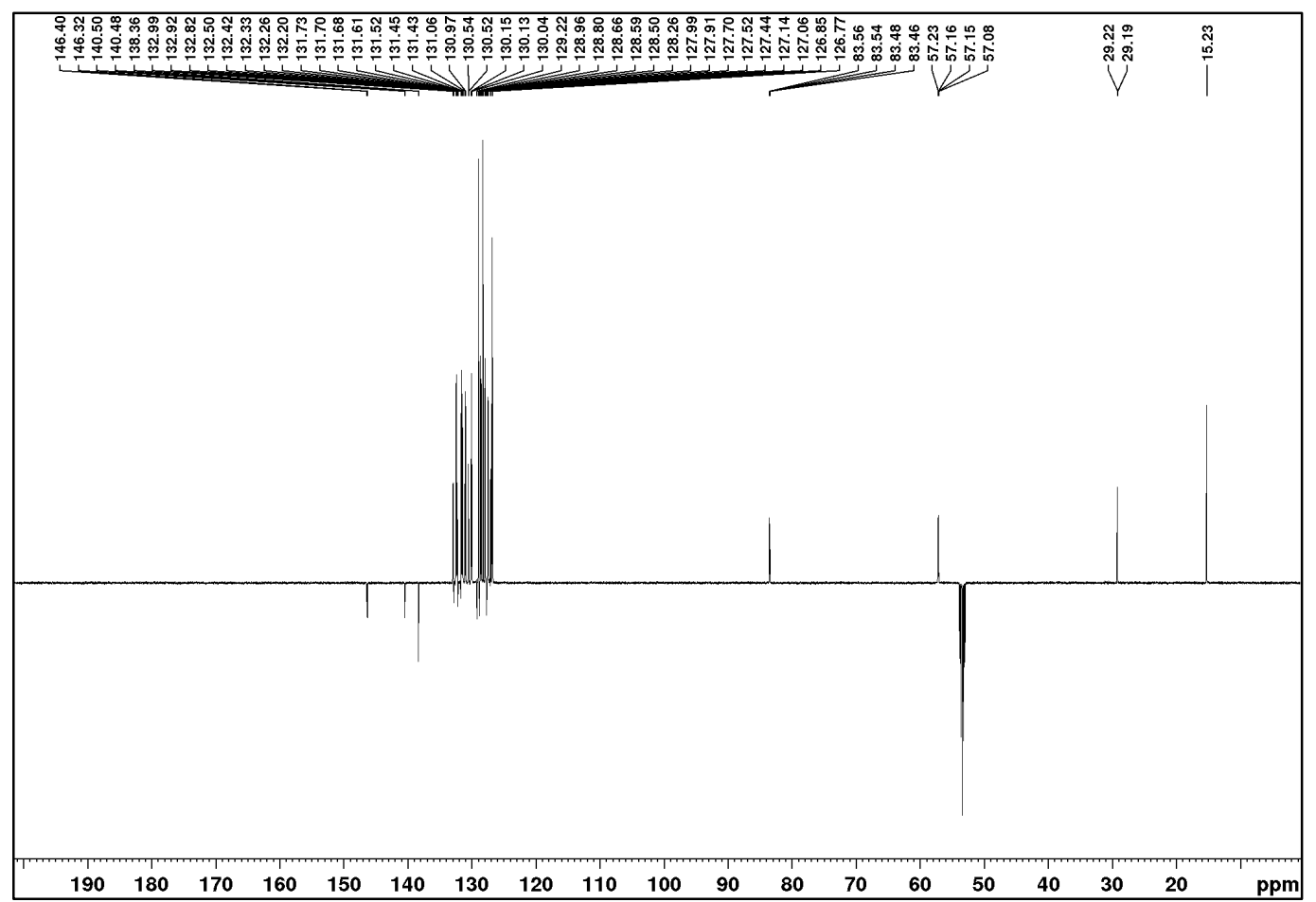

${ }^{31} \mathrm{P}\left\{{ }^{1} \mathrm{H}\right\} \mathrm{NMR}\left(202.5 \mathrm{MHz}, \mathrm{CD}_{2} \mathrm{Cl}_{2}\right)$

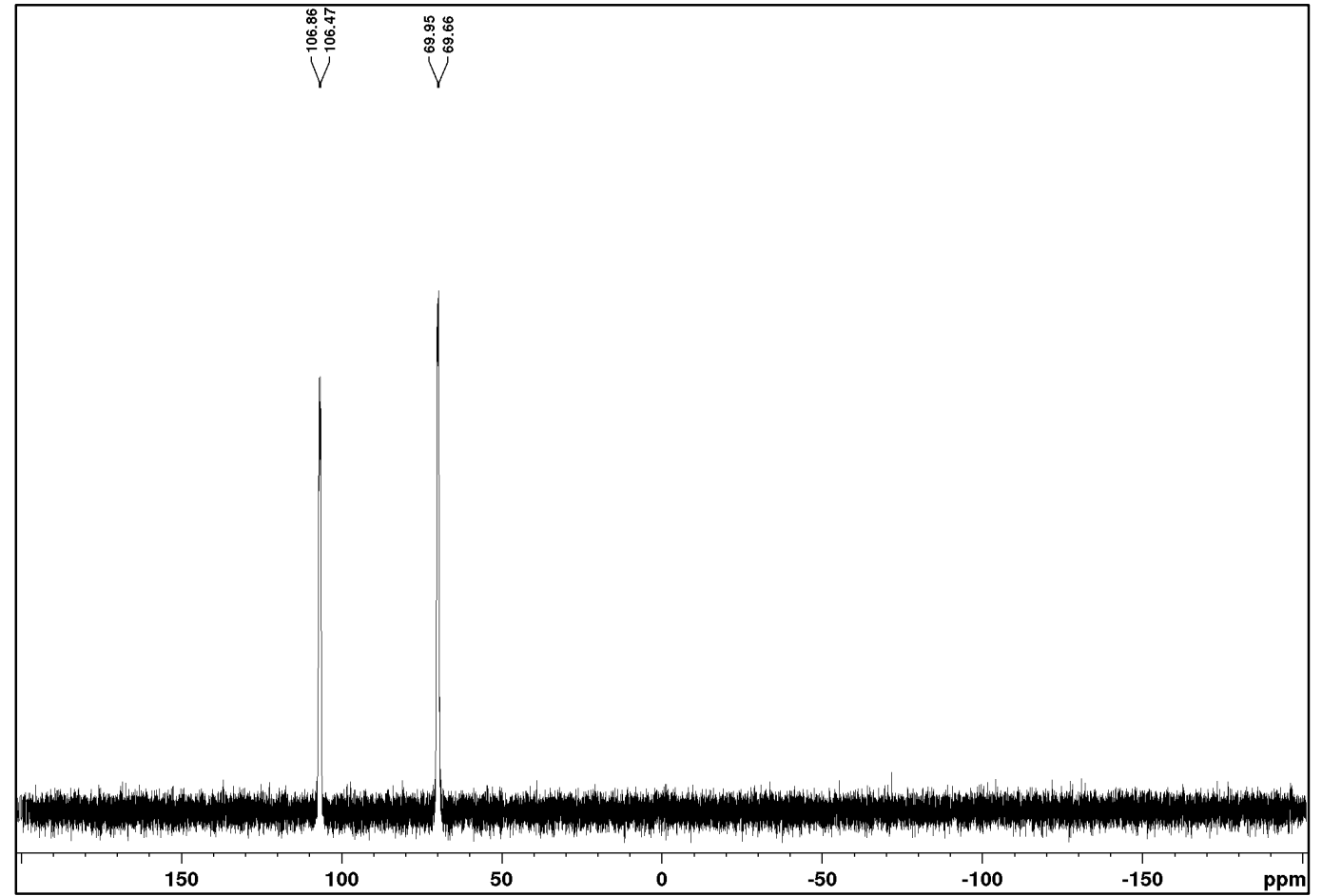


$\left(R_{\mathrm{p}}\right)-(-)-\mathrm{N}-[(1 R, 2 S)-2-($ Diphenylphosphinito-borane)-1-methyl-2-phenylethyl], N-methylamino (2-isopropoxyphenyl)phenylphosphine-borane $\mathbf{1 2} \mathbf{m}$.

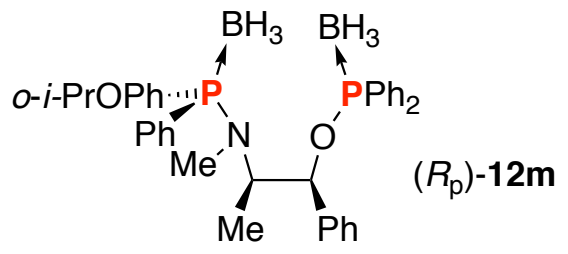

${ }^{1} \mathrm{H}$ NMR $\left(400 \mathrm{MHz}, \mathrm{CDCl}_{3}\right)$

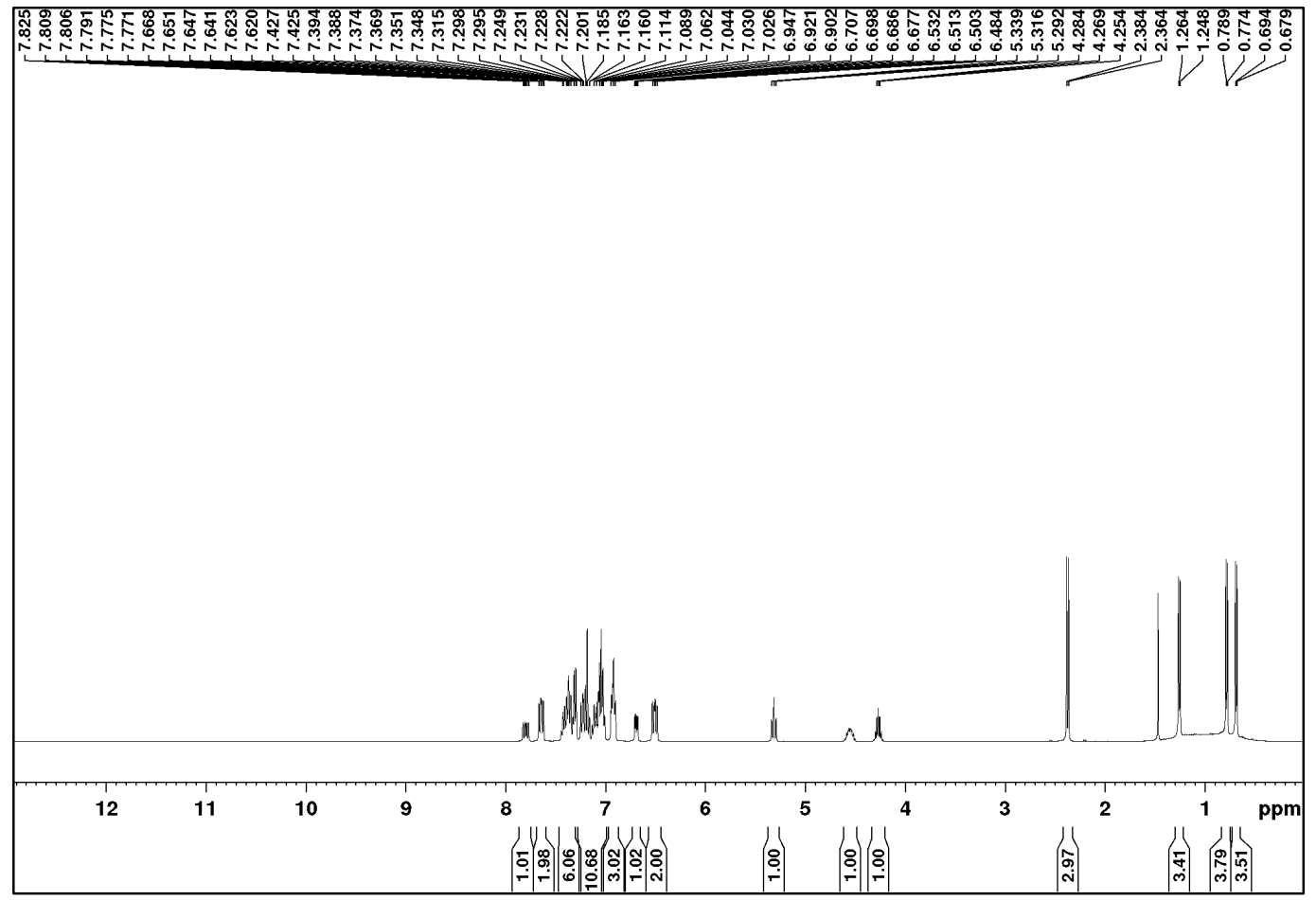

${ }^{13} \mathrm{C}\left\{{ }^{1} \mathrm{H}\right\} \mathrm{J}-\bmod \mathrm{NMR}\left(100.6 \mathrm{MHz}, \mathrm{CDCl}_{3}\right)$

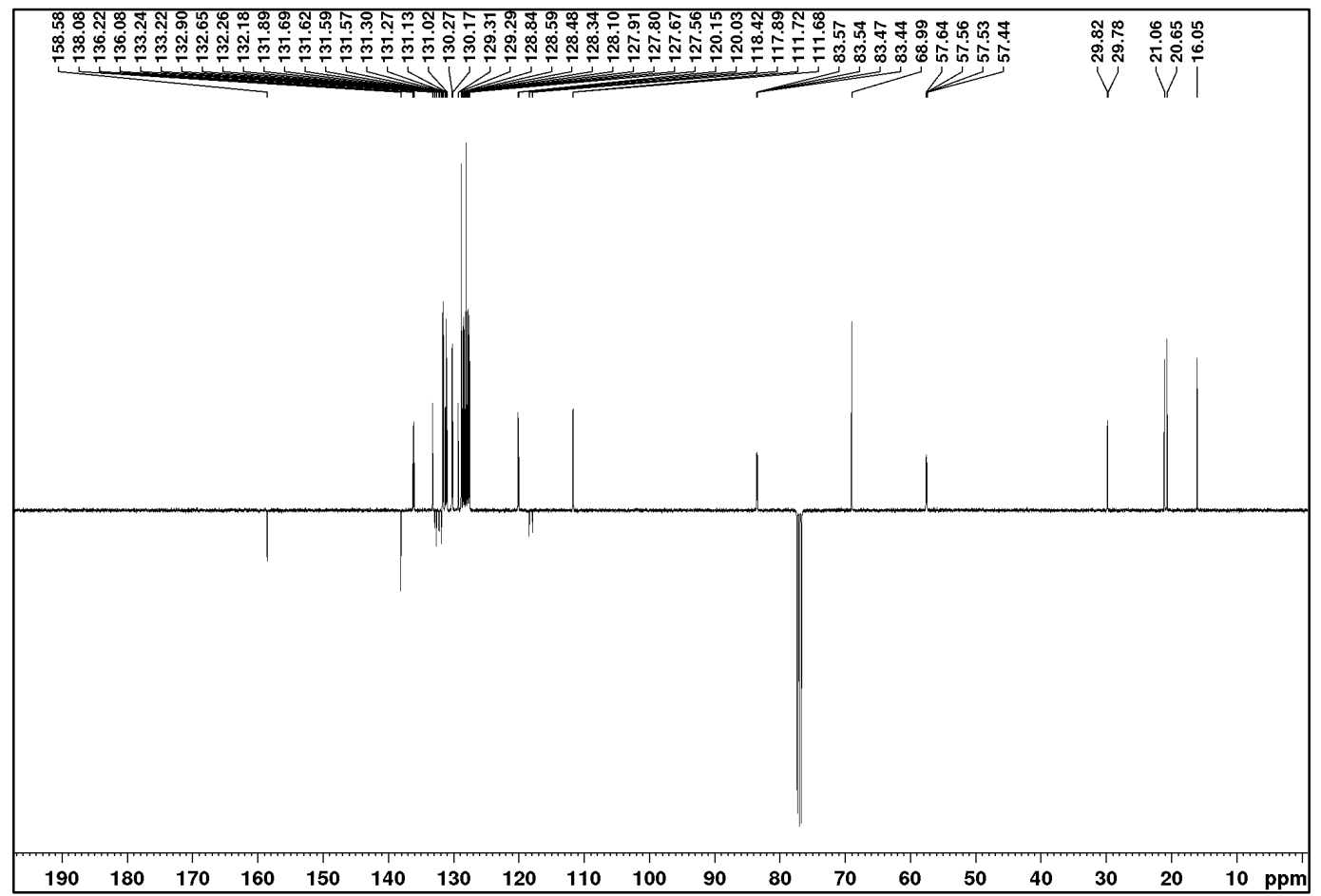


${ }^{31} \mathrm{P}\left\{{ }^{1} \mathrm{H}\right\}$ NMR $\left(161.9 \mathrm{MHz}, \mathrm{CDCl}_{3}\right)$

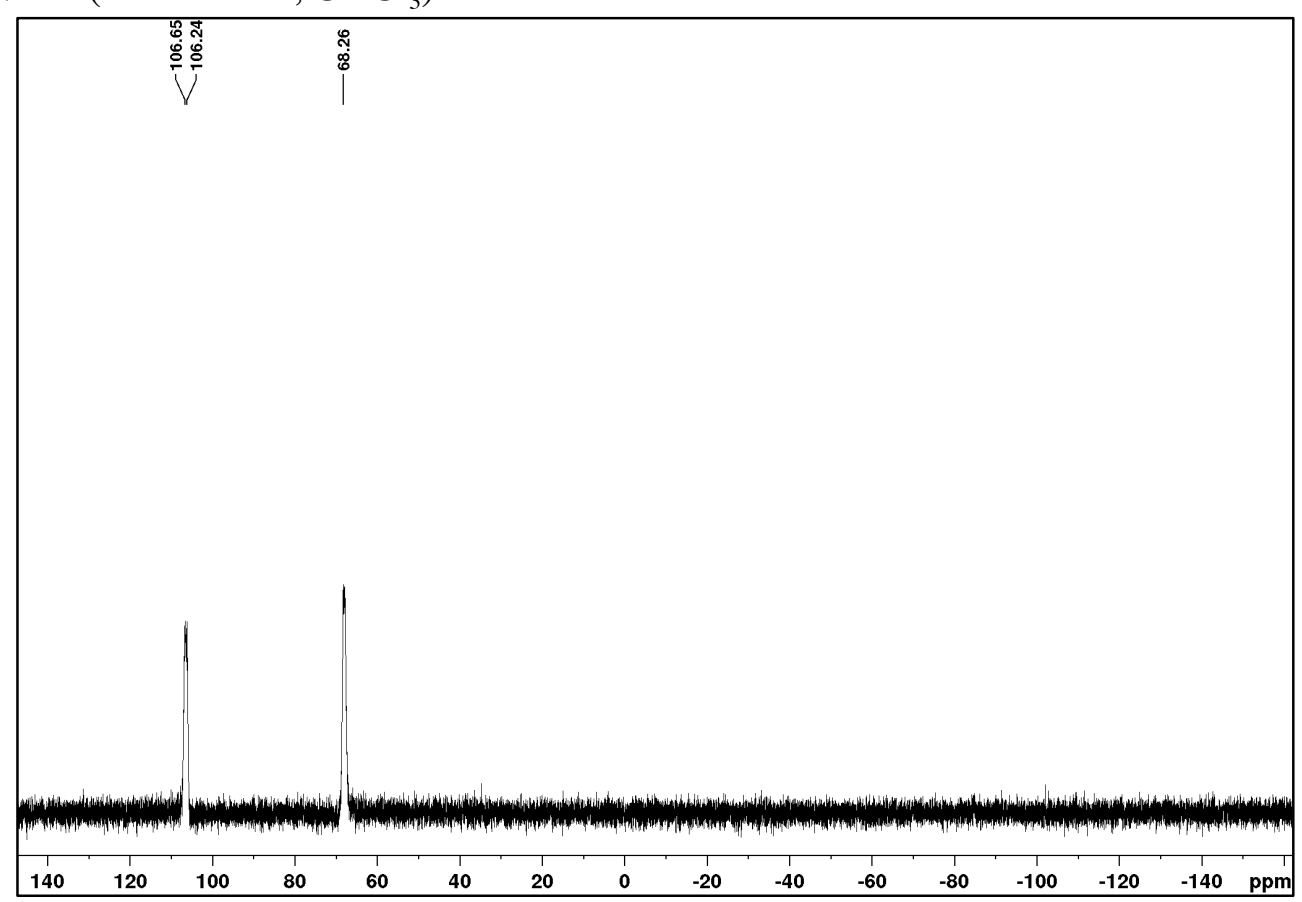

$\left(R_{\mathrm{p}}\right)-(-)-\mathrm{N}-[(1 R, 2 S)-2-($ Diphenylphosphinito-borane)-1-methyl-2-phenylethyl], $\quad \mathrm{N}$-methylamino (2-benzyloxyphenyl)phenylphosphine-borane 12n.

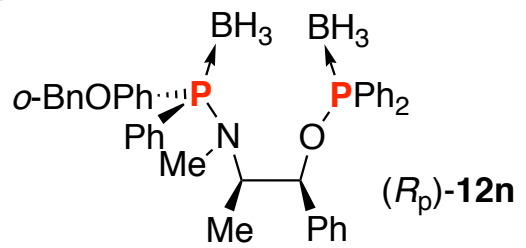

${ }^{1} \mathrm{H} \mathrm{NMR}\left(300 \mathrm{MHz}, \mathrm{CDCl}_{3}\right)$

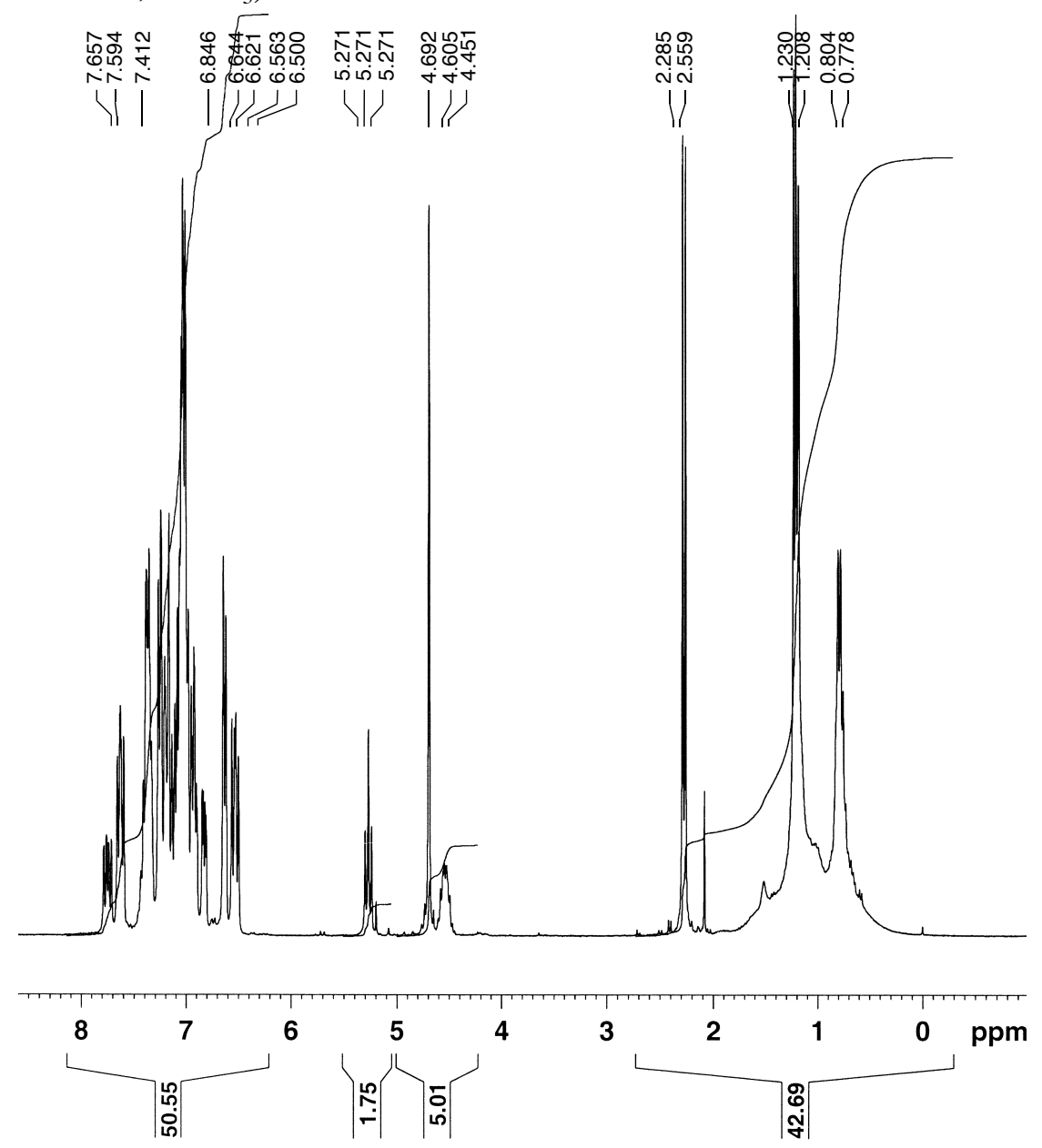


${ }^{13} \mathrm{C}\left\{{ }^{1} \mathrm{H}\right\}$ DEPT NMR (75.5 MHz, $\left.\mathrm{CDCl}_{3}\right)$
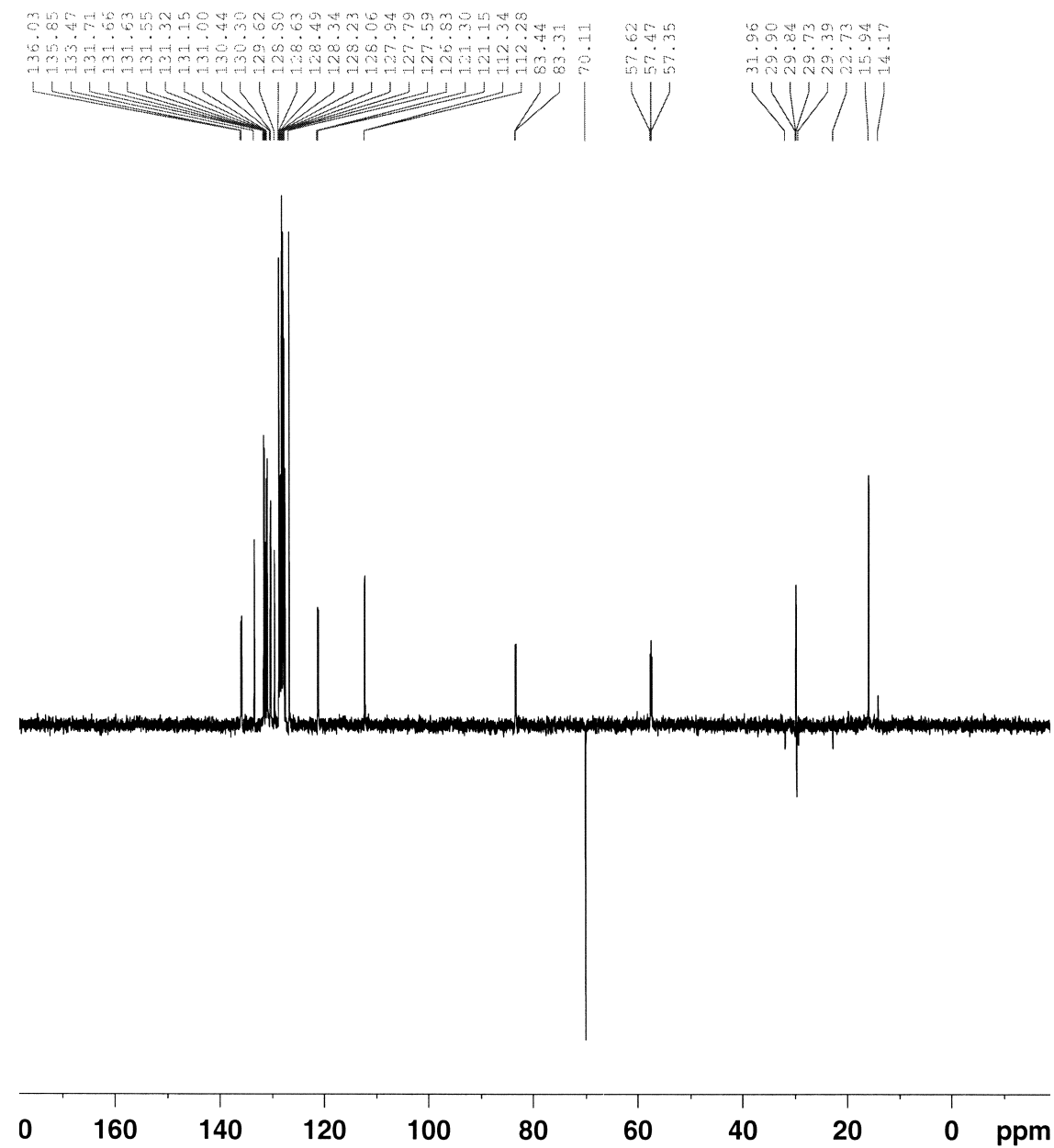

${ }^{31} \mathrm{P}\left\{{ }^{1} \mathrm{H}\right\}$ NMR $\left(121.5 \mathrm{MHz}, \mathrm{CDCl}_{3}\right)$

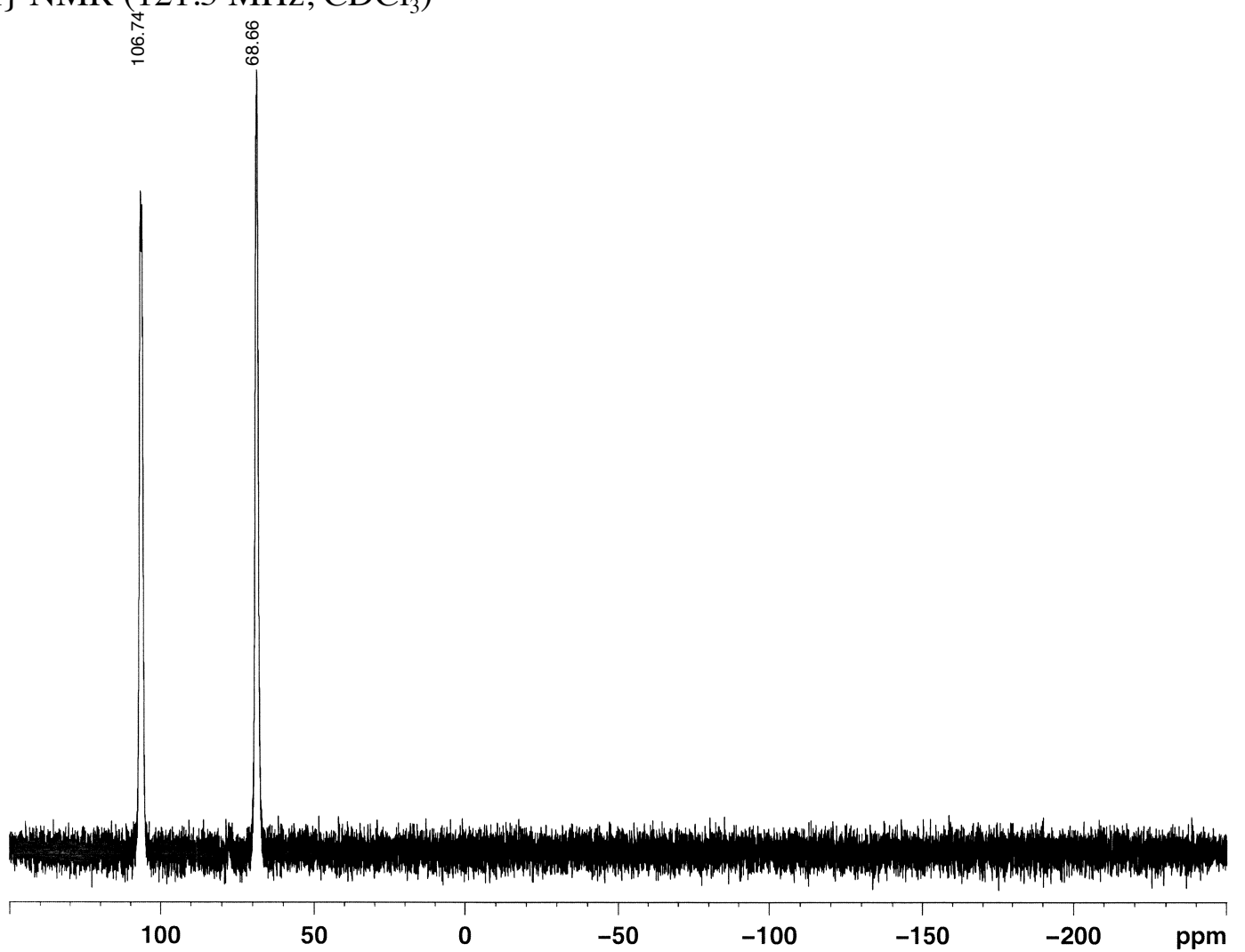


$\left(R_{\mathrm{p}}\right)-(-)-\mathrm{N}-[(1 R, 2 S)-2-($ Diphenylphosphinito-borane)-1-methyl-2-phenylethyl], N-methylamino (3,5-dimethylphenyl)phenylphosphine-borane 120.

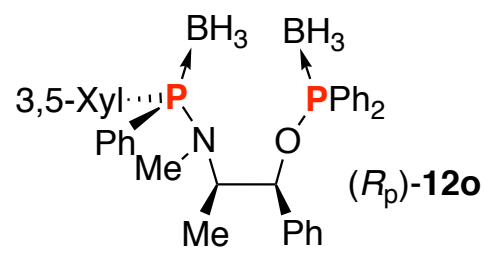

${ }^{1} \mathrm{H} \mathrm{NMR}\left(500 \mathrm{MHz}, \mathrm{CDCl}_{3}\right)$

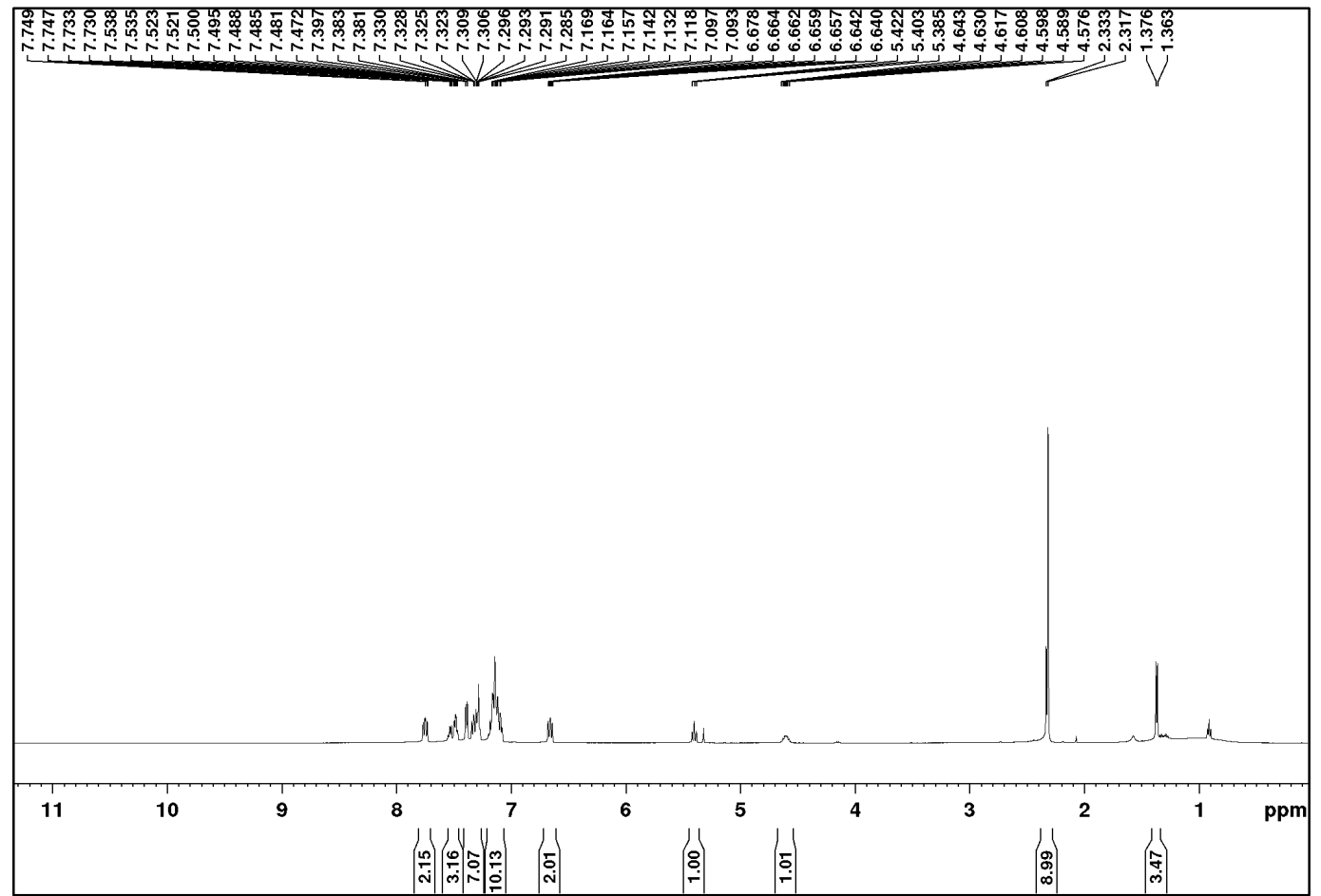

${ }^{13} \mathrm{C}\left\{{ }^{1} \mathrm{H}\right\} \mathrm{J}-\bmod \mathrm{NMR}\left(125.8 \mathrm{MHz}, \mathrm{CDCl}_{3}\right)$

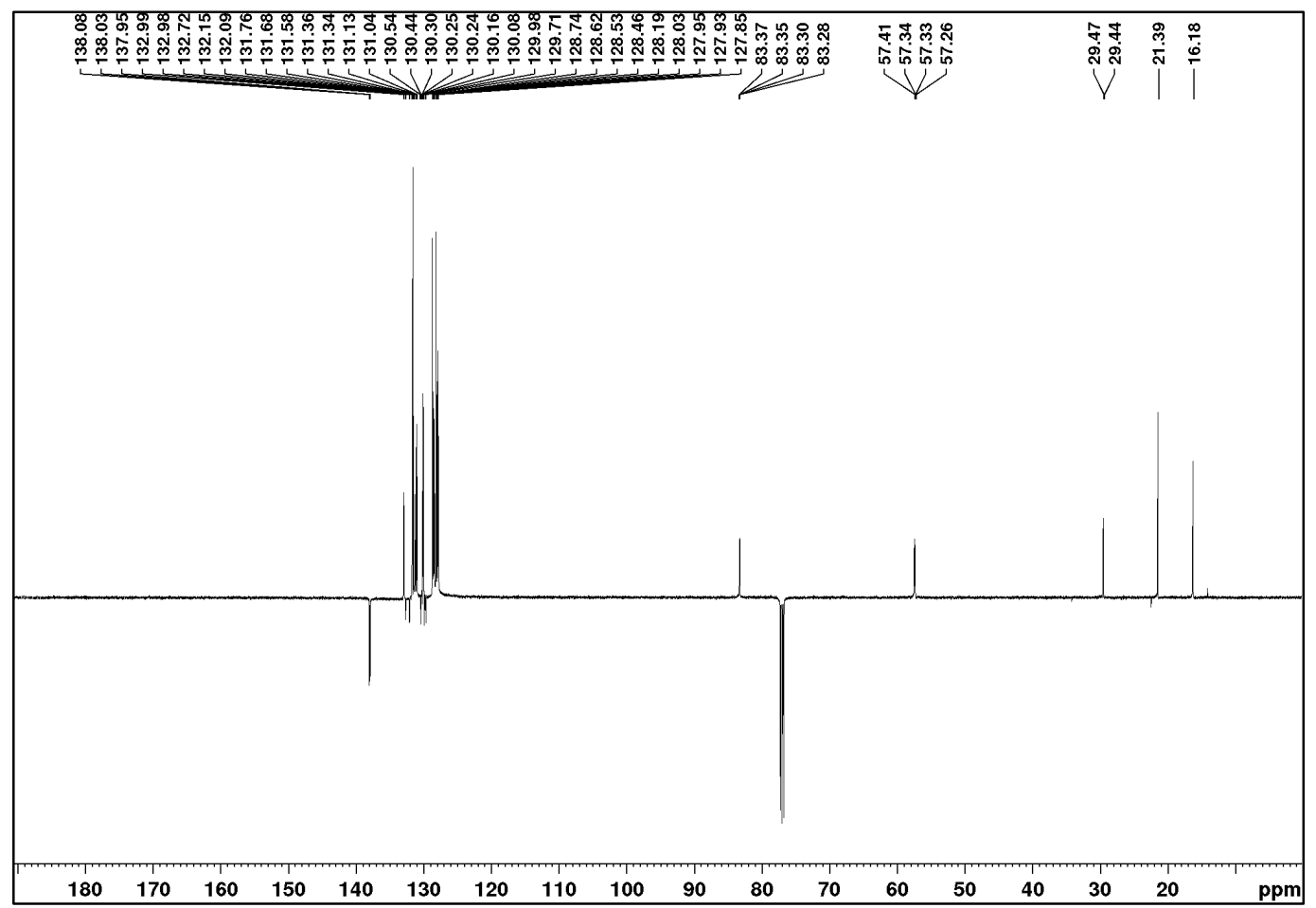


${ }^{31} \mathrm{P}\left\{{ }^{1} \mathrm{H}\right\} \mathrm{NMR}\left(202.5 \mathrm{MHz}, \mathrm{CDCl}_{3}\right)$

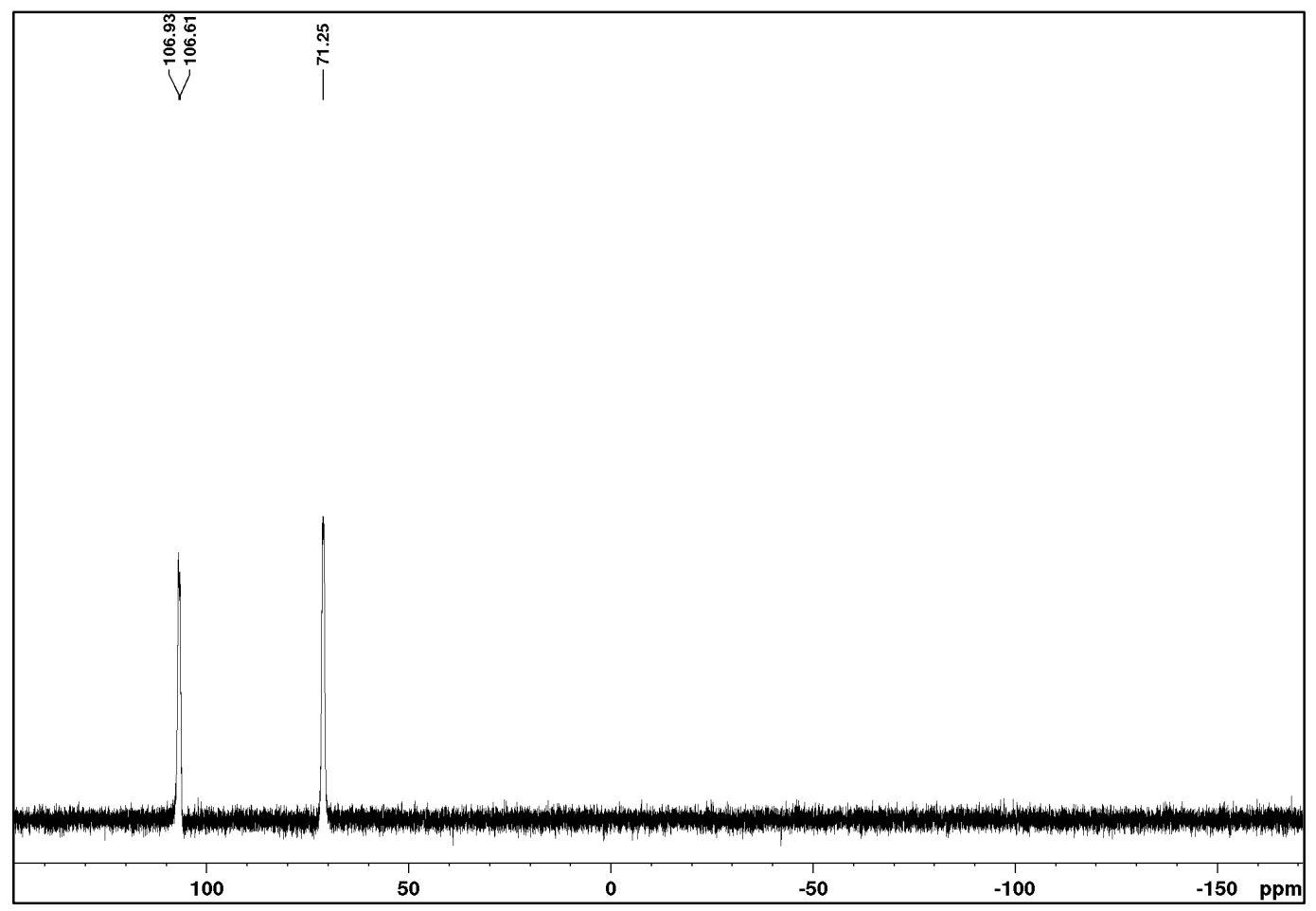

$\left(R_{\mathrm{p}}\right)-(-)-\mathrm{N}-[(1 R, 2 S)-2-($ Diphenylphosphinito-borane)-1-methyl-2-phenylethyl], $\quad \mathrm{N}$-methylamino (3,5-di-t-butylphenyl)phenylphosphine-borane 12p.

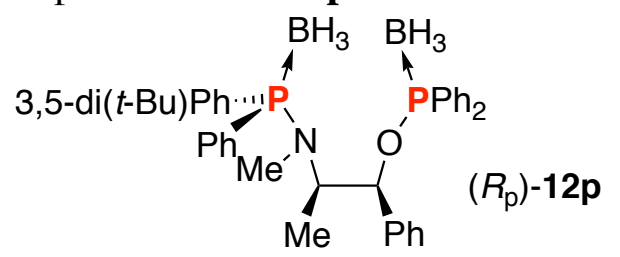

${ }^{1} \mathrm{H} \mathrm{NMR}\left(500 \mathrm{MHz}, \mathrm{CDCl}_{3}\right)$

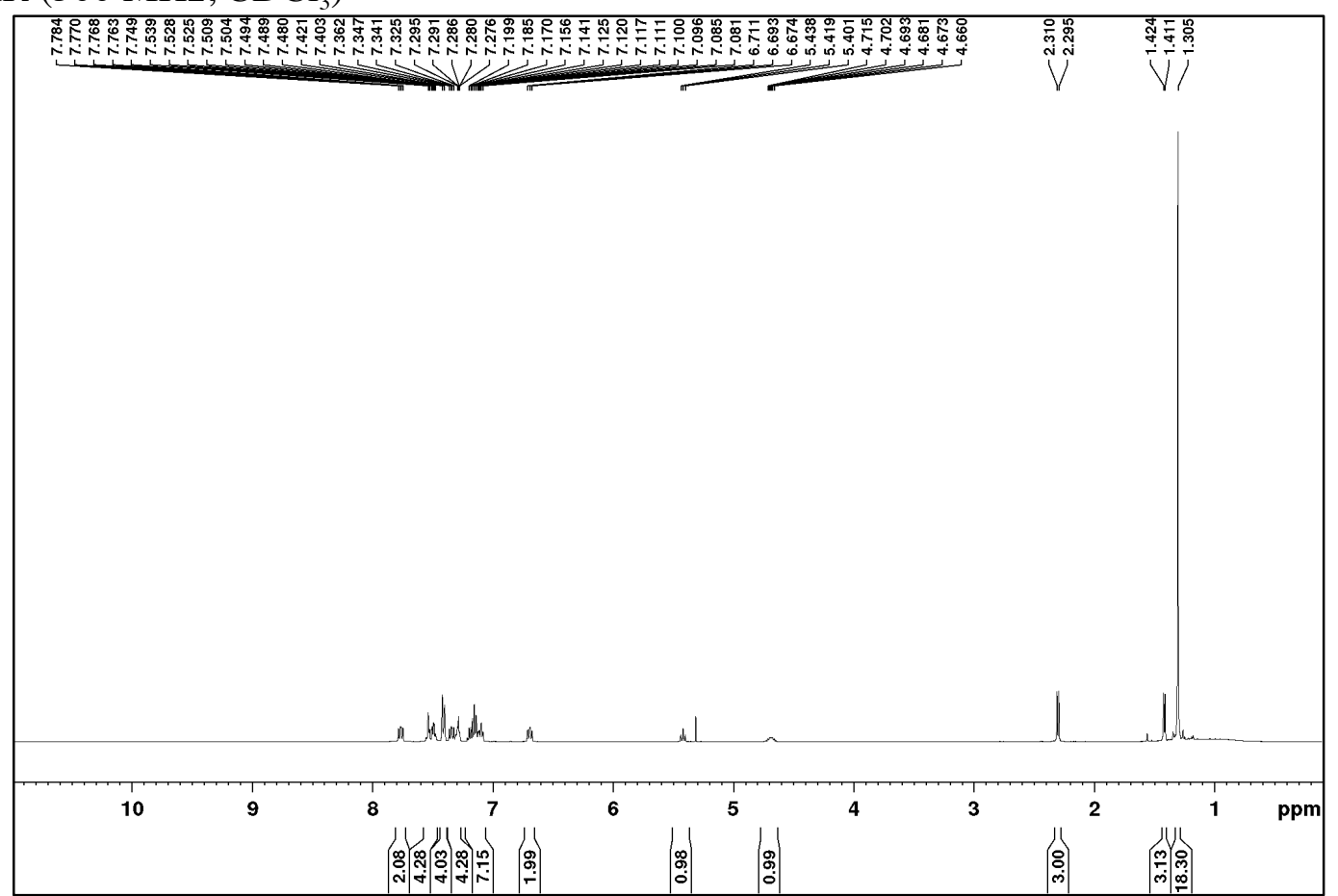


${ }^{13} \mathrm{C}\left\{{ }^{1} \mathrm{H}\right\} \mathrm{J}-\bmod$ NMR $\left(125.8 \mathrm{MHz}, \mathrm{CDCl}_{3}\right)$

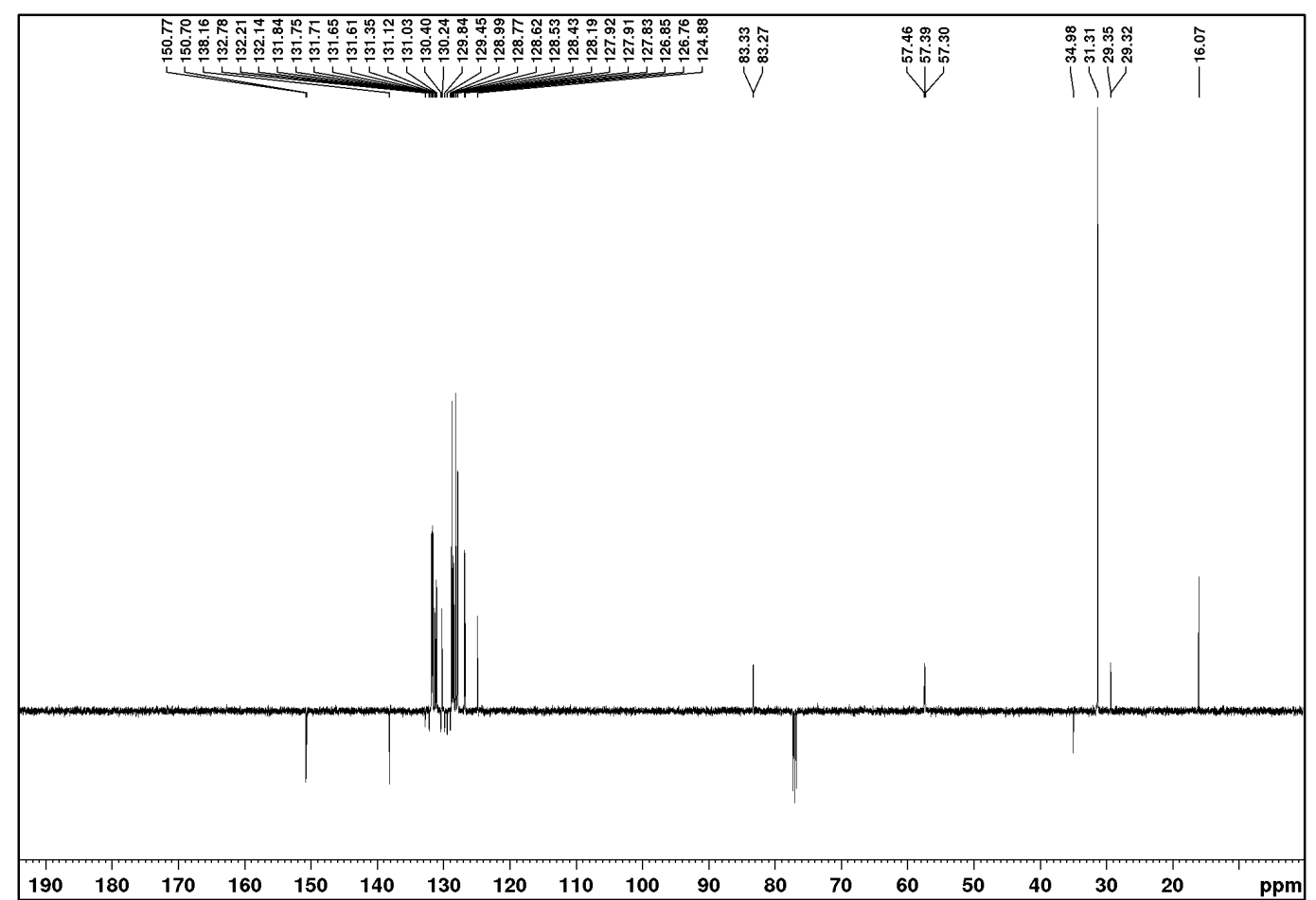

${ }^{31} \mathrm{P}\left\{{ }^{1} \mathrm{H}\right\}$ NMR $\left(202.5 \mathrm{MHz}, \mathrm{CDCl}_{3}\right)$

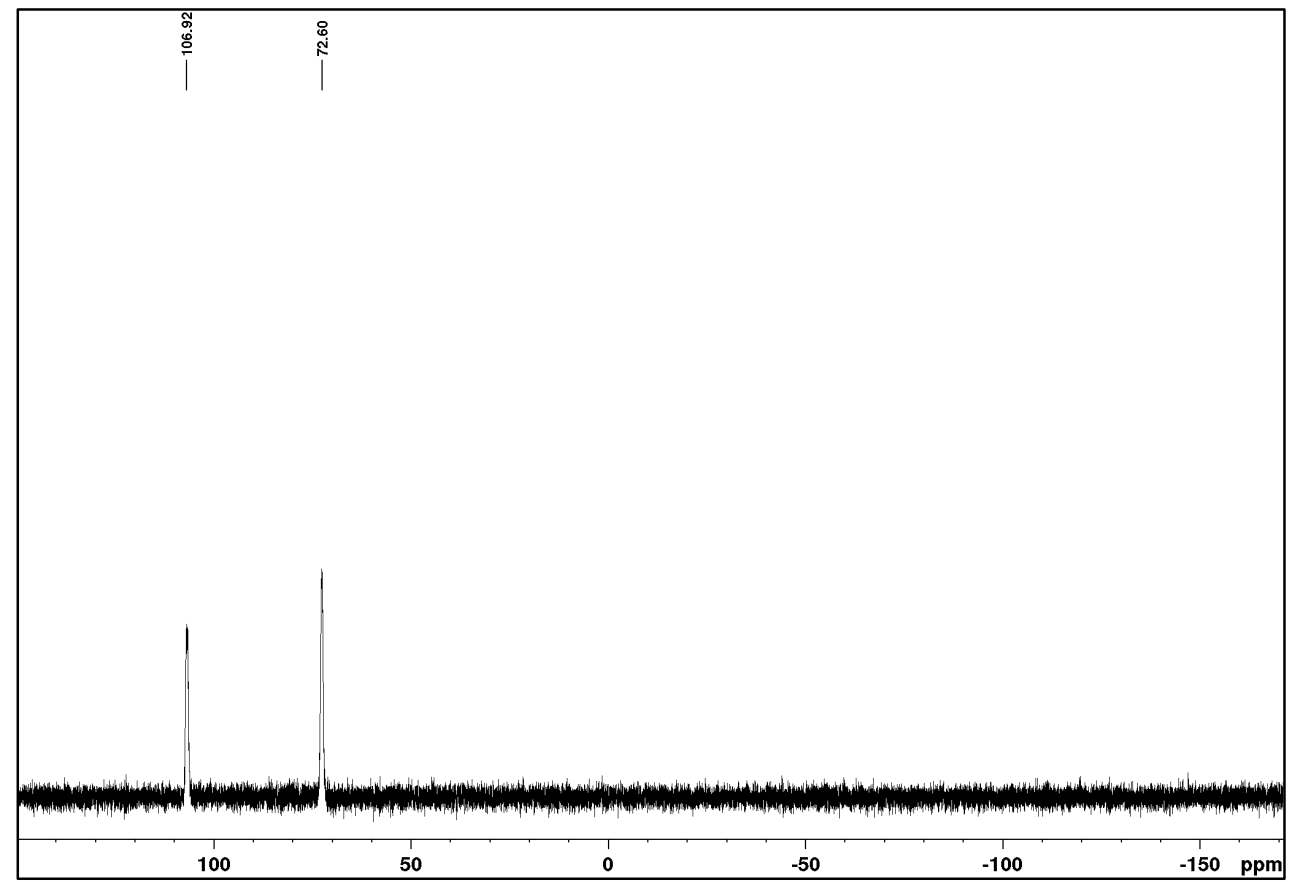


$\left(R_{\mathrm{p}}\right)-(-)-\mathrm{N}-[(1 R, 2 S)-2-($ Diphenylphosphinito-borane)-1-methyl-2-phenylethyl], $\quad \mathrm{N}$-methylamino (3,5-di-t-butyl-4-methoxyphenyl)phenylphosphine-borane 12q.

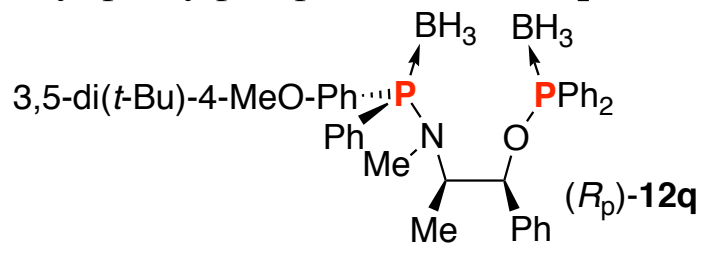

${ }^{1} \mathrm{H} \mathrm{NMR}\left(500 \mathrm{MHz}, \mathrm{CDCl}_{3}\right)$

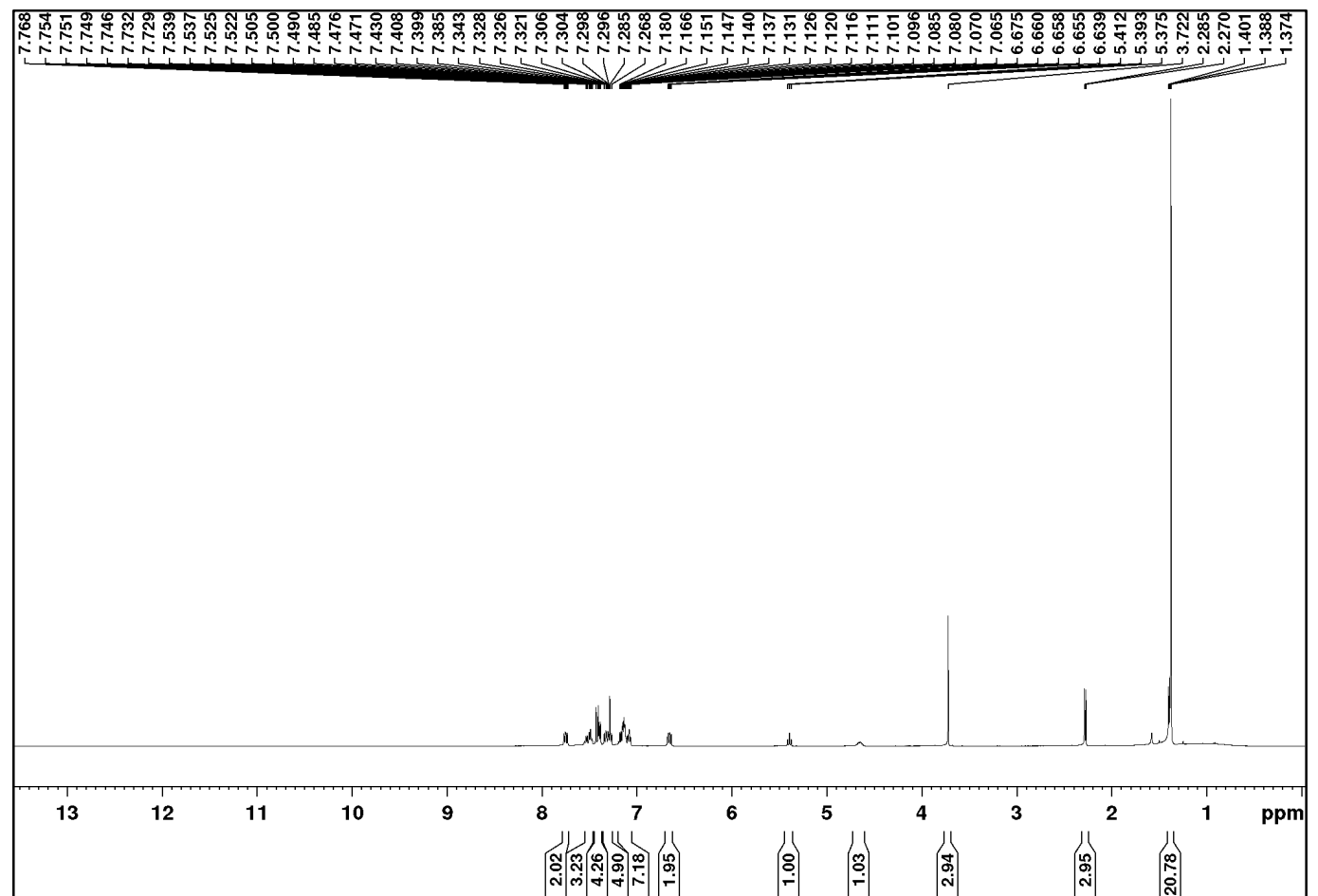

${ }^{13} \mathrm{C}\left\{{ }^{1} \mathrm{H}\right\}$ NMR $\left(125.8 \mathrm{MHz}, \mathrm{CDCl}_{3}\right)$

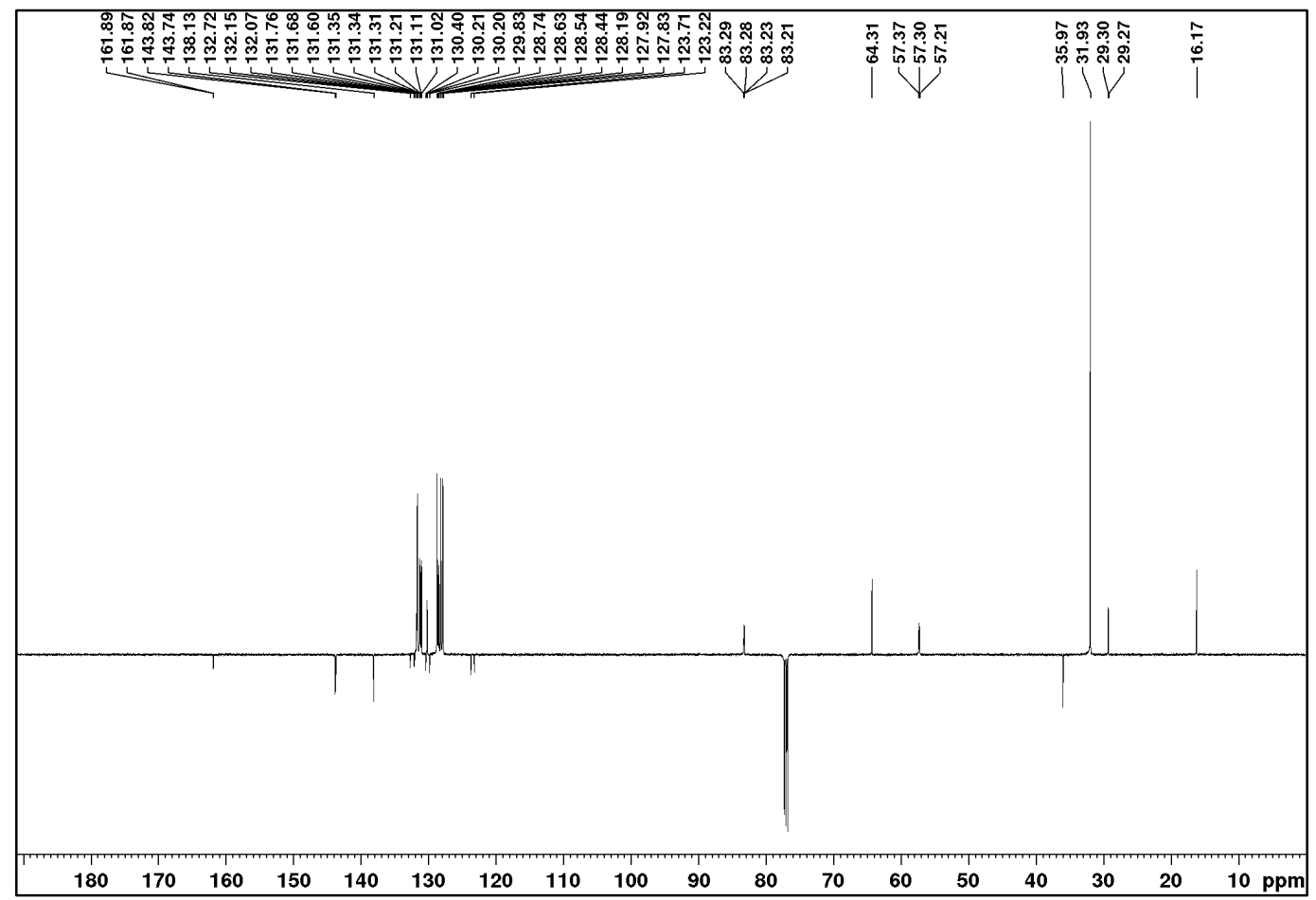


${ }^{31} \mathrm{P}\left\{{ }^{1} \mathrm{H}\right\}$ NMR (202.5 MHz, $\left.\mathrm{CDCl}_{3}\right)$

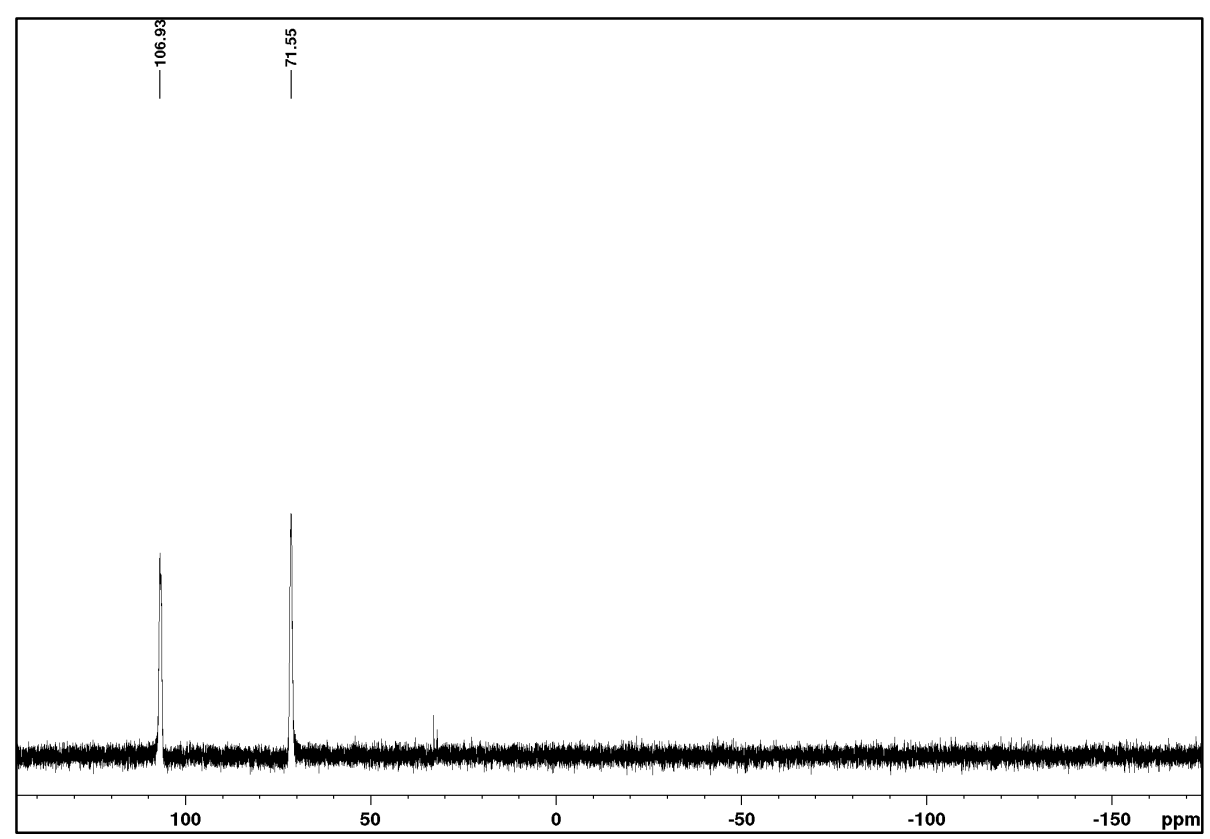

$\left(R_{\mathrm{p}}\right)-(-)-\mathrm{N}-[(1 R, 2 S)-2-($ Diphenylphosphinito-borane)-1-methyl-2-phenylethyl], $\quad \mathrm{N}$-methylamino (phenyl(2-pyridyl)phosphine-borane 12r.

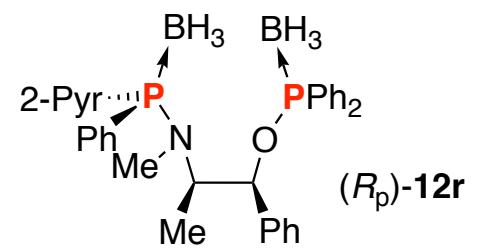

${ }^{1} \mathrm{H}$ NMR (300 MHz, $\left.\mathrm{CDCl}_{3}\right)$

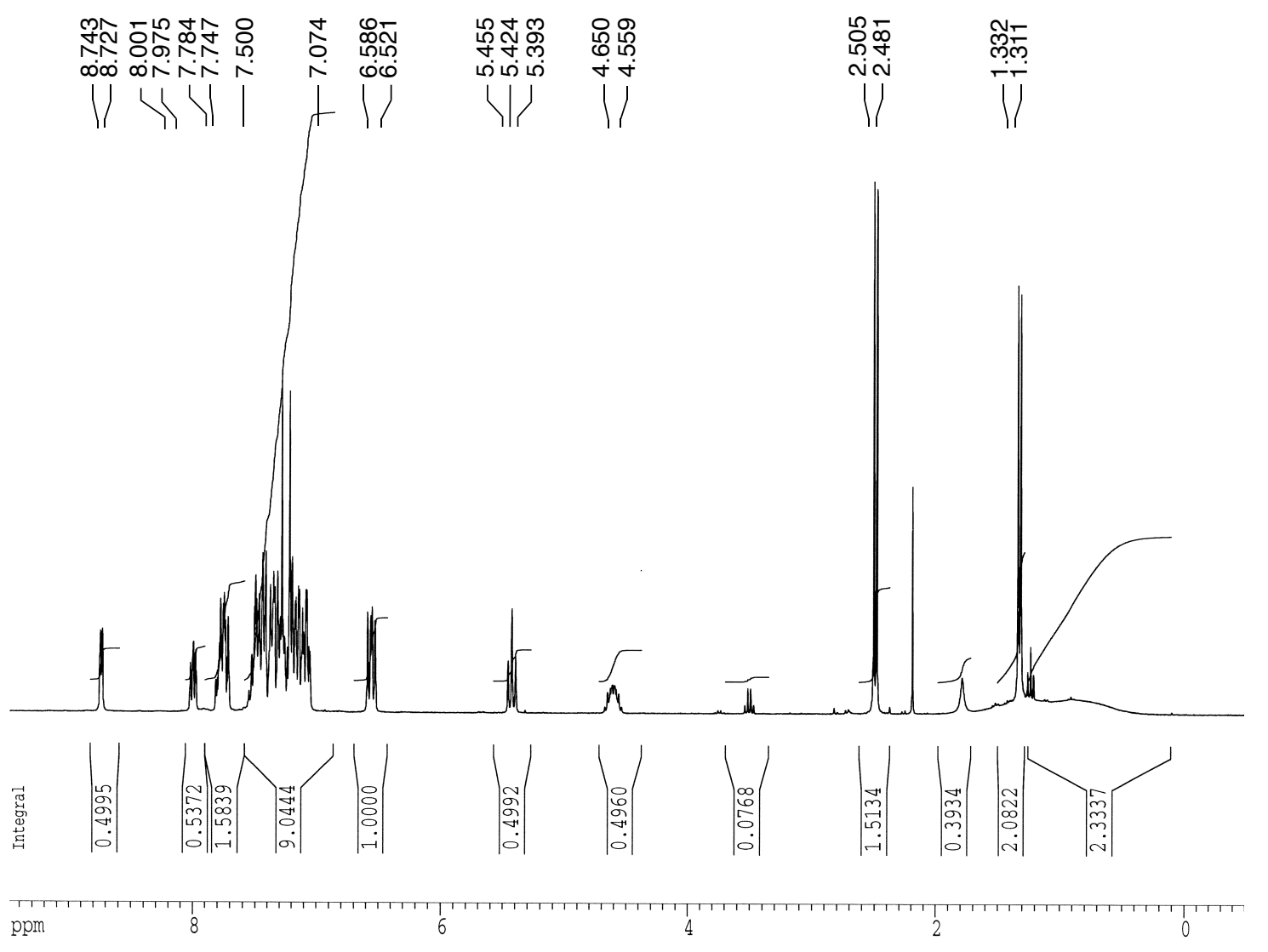


${ }^{13} \mathrm{C}\left\{{ }^{1} \mathrm{H}\right\}$ NMR $\left(75.5 \mathrm{MHz}, \mathrm{CDCl}_{3}\right)$

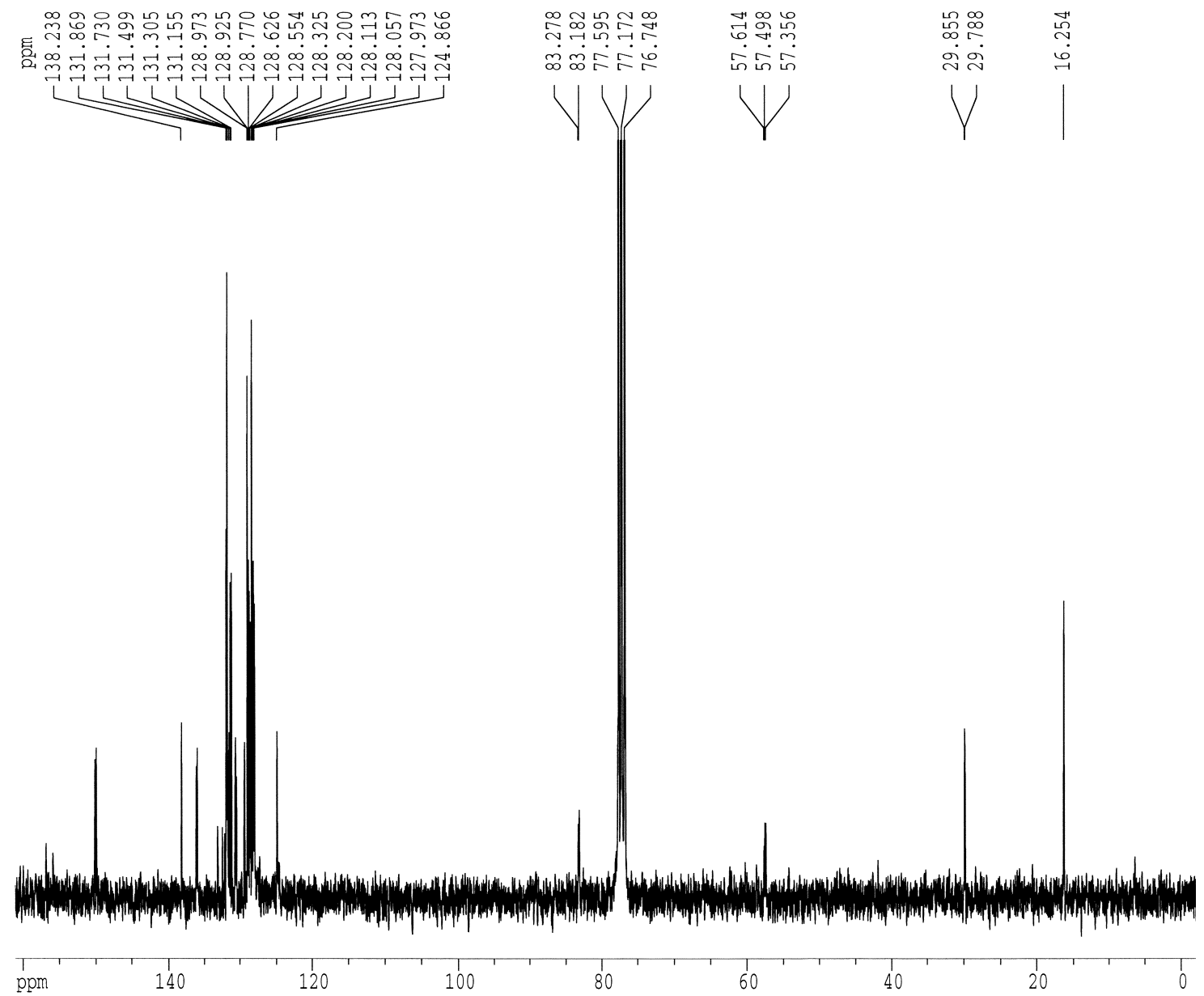

${ }^{31} \mathrm{P}\left\{{ }^{1} \mathrm{H}\right\}$ NMR $\left(121.5 \mathrm{MHz}, \mathrm{CDCl}_{3}\right)$

镸

|

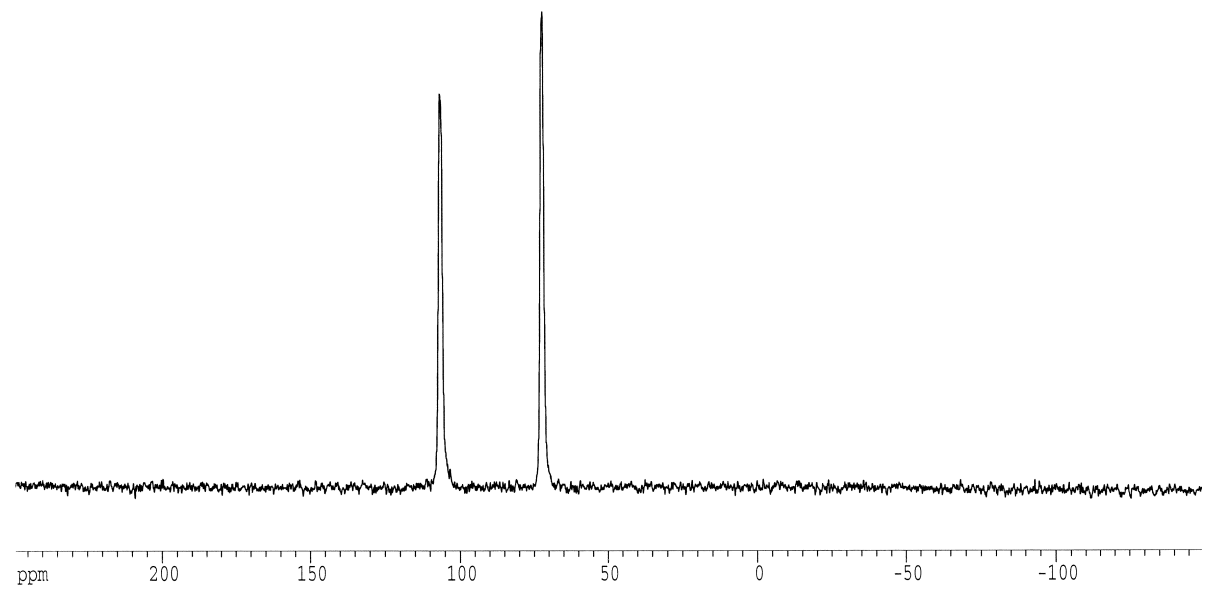


$\left(R_{\mathrm{p}}\right)-(-)-\mathrm{N}-[(1 R, 2 S)-2-($ Diphenylphosphinito-borane)-1-methyl-2-phenylethyl], $\quad \mathrm{N}$-methyl amino-2-(2', 6'-dimethoxy-1,1'-biphenyl)phenylphosphine-borane 12s.

${ }^{1} \mathrm{H} \mathrm{NMR}\left(500 \mathrm{MHz}, \mathrm{CDCl}_{3}\right)$
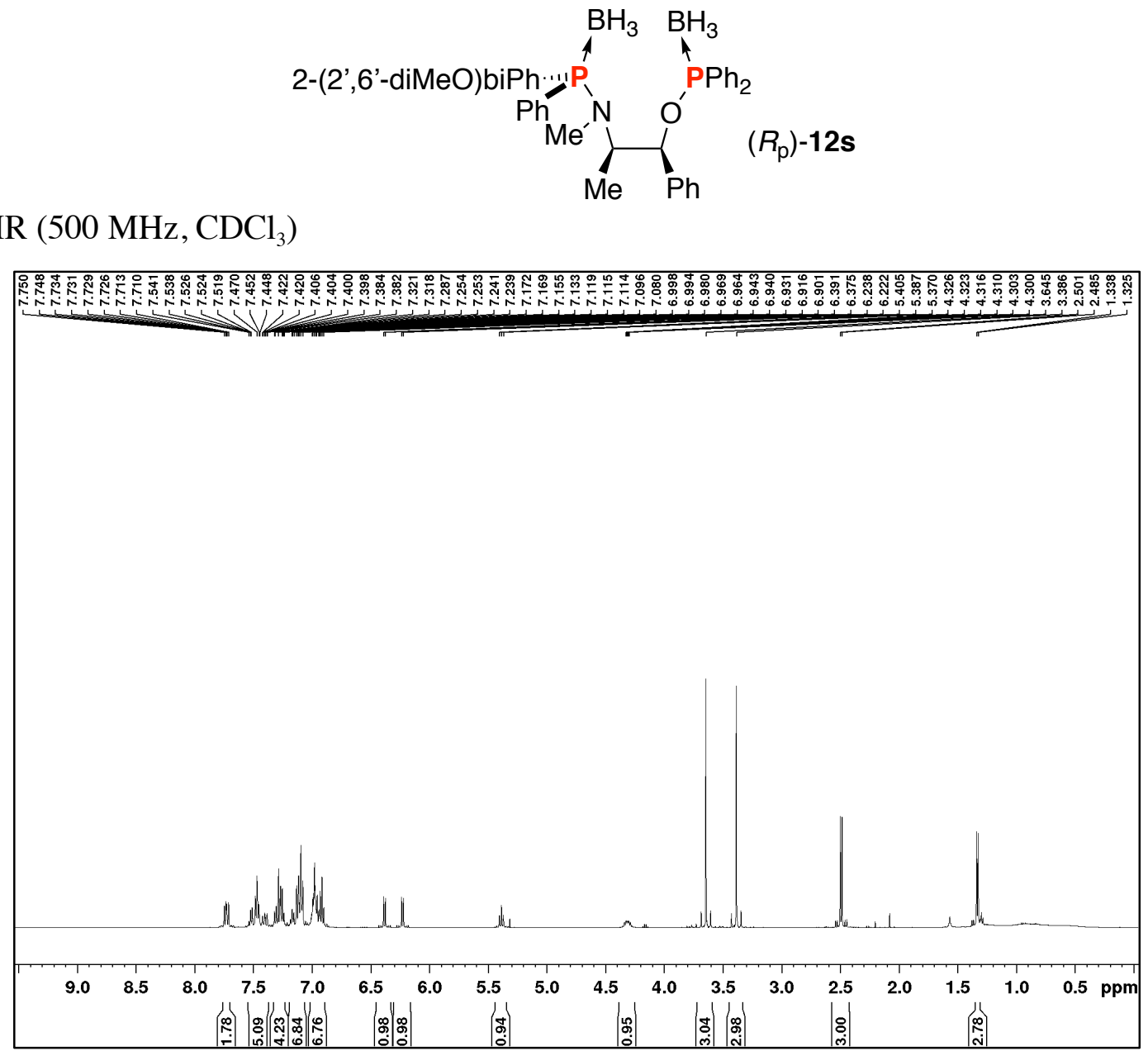

${ }^{13} \mathrm{C}\left\{{ }^{1} \mathrm{H}\right\} \mathrm{J}-\bmod \mathrm{NMR}\left(125.8 \mathrm{MHz}, \mathrm{CDCl}_{3}\right)$

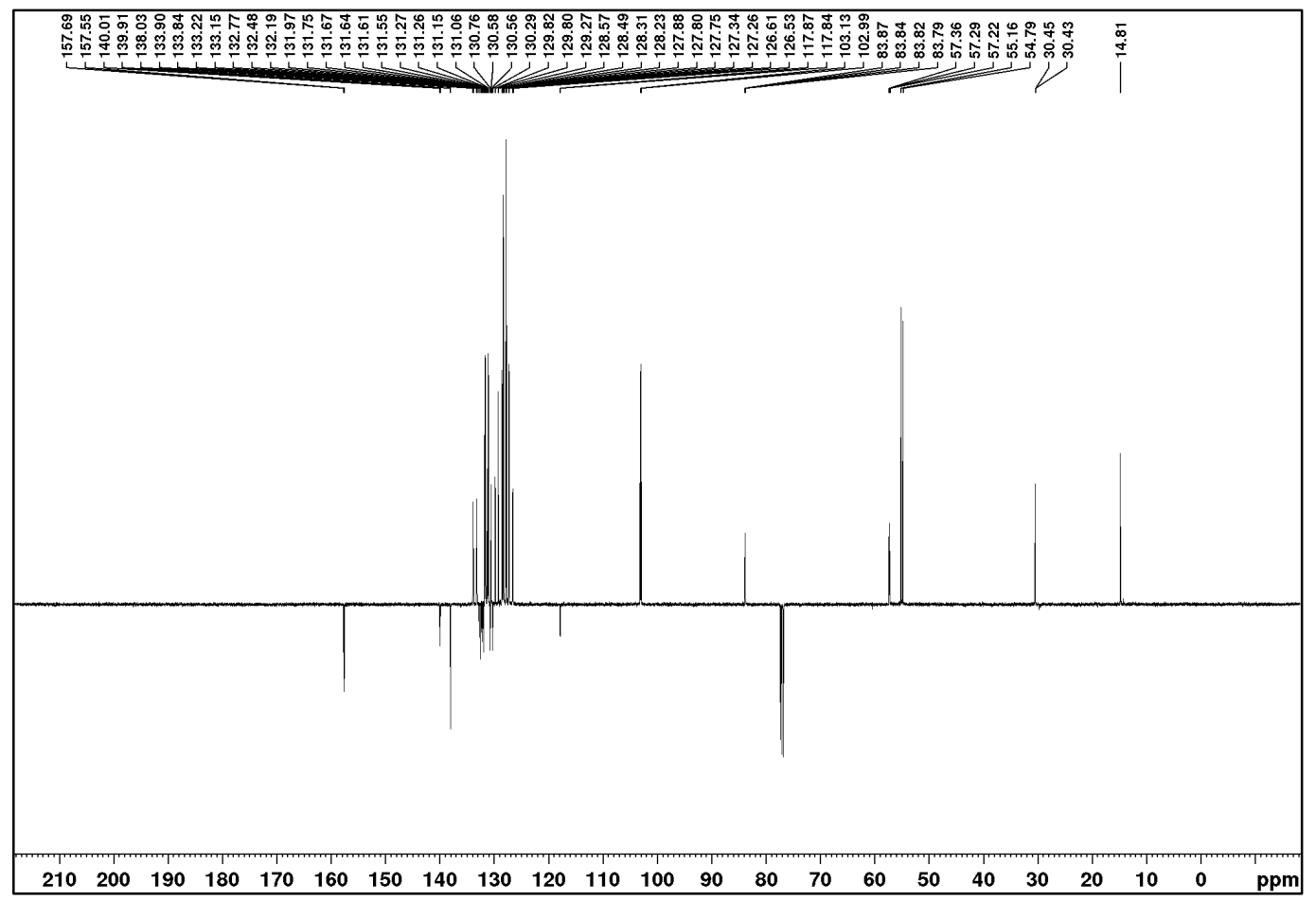


${ }^{31} \mathrm{P}\left\{{ }^{1} \mathrm{H}\right\} \mathrm{NMR}\left(202.5 \mathrm{MHz}, \mathrm{CDCl}_{3}\right)$

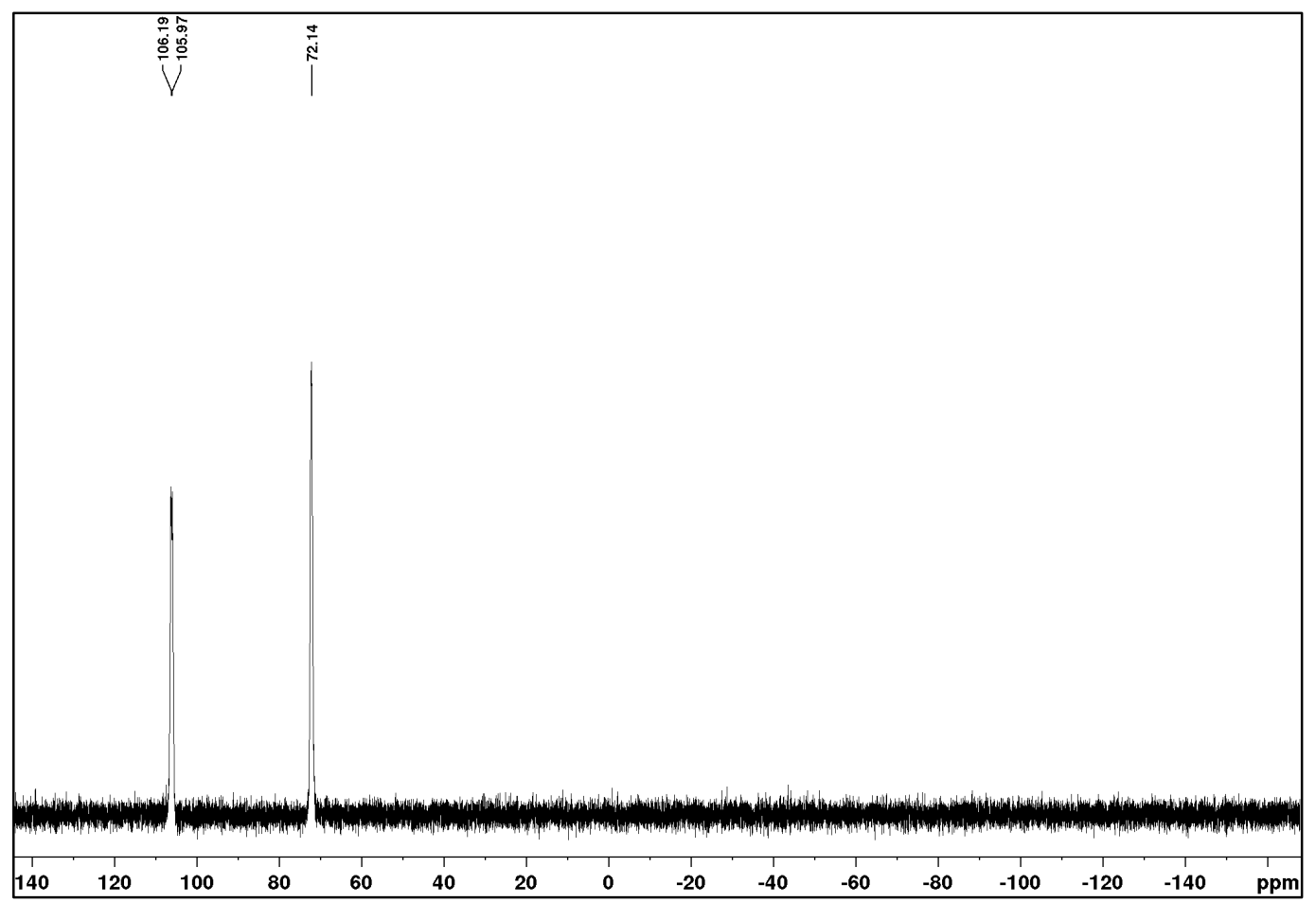

$\left(R_{\mathrm{p}}\right)-(-)-\mathrm{N}-[(1 R, 2 S)-(2-(\mathrm{Di}-\alpha-n a p h t y l p h o s p h i n i t o-b o r a n e)-1-m e t h y l-2-p h e n y l e t h y l], \quad \mathrm{N}$-methyl amino(o-biphenyl)phenylphosphine-borane $\mathbf{1 2 t}$.

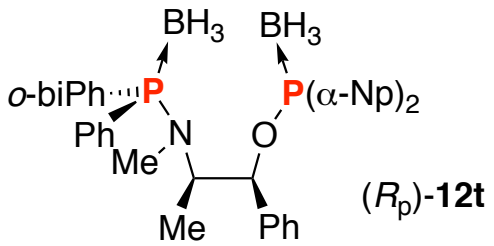

${ }^{1} \mathrm{H}$ NMR $\left(500 \mathrm{MHz}, \mathrm{CDCl}_{3}\right)$

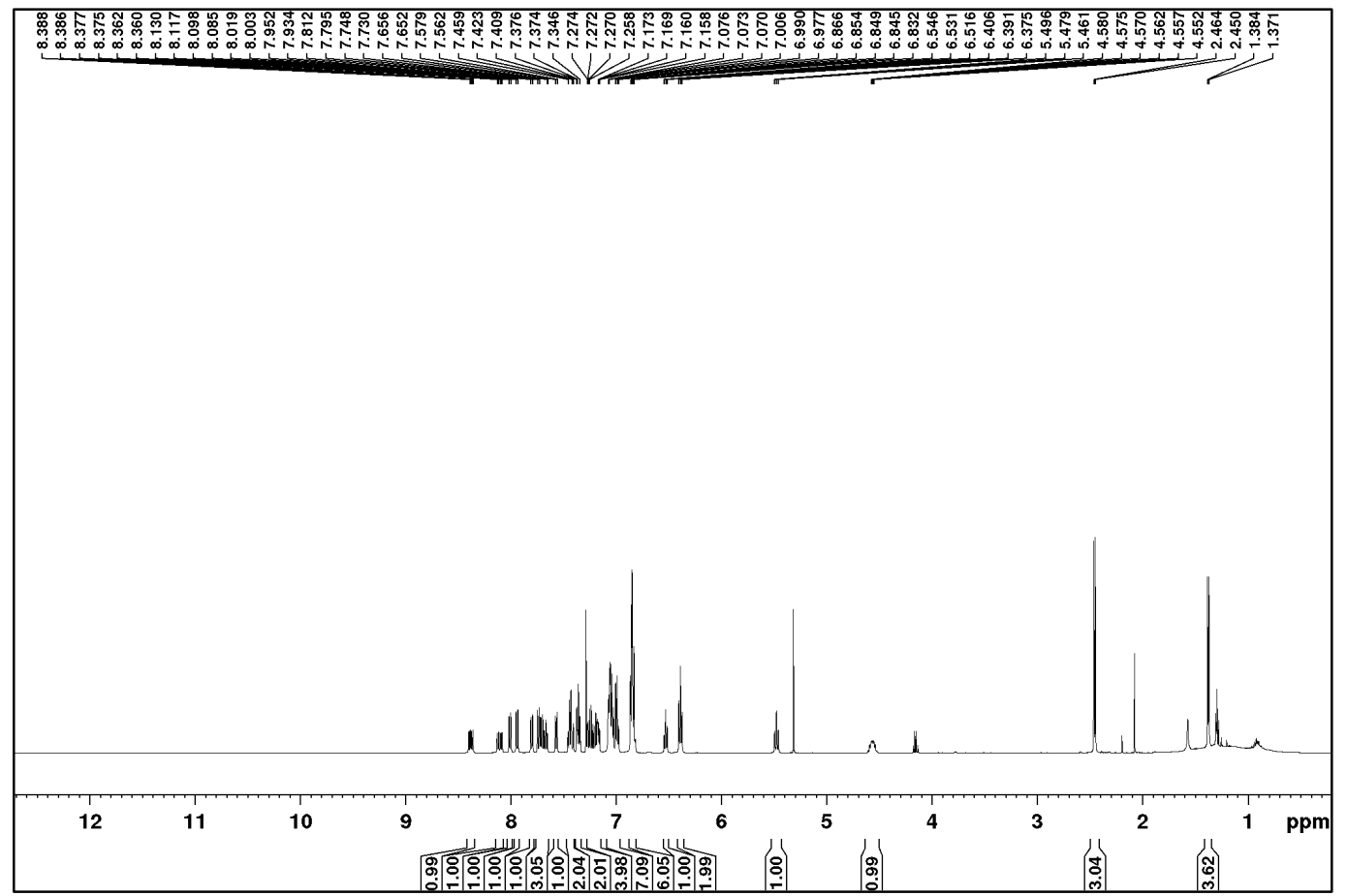


${ }^{13} \mathrm{C}\left\{{ }^{1} \mathrm{H}\right\} \mathrm{J}-\bmod$ NMR $\left(125.8 \mathrm{MHz}, \mathrm{CDCl}_{3}\right)$

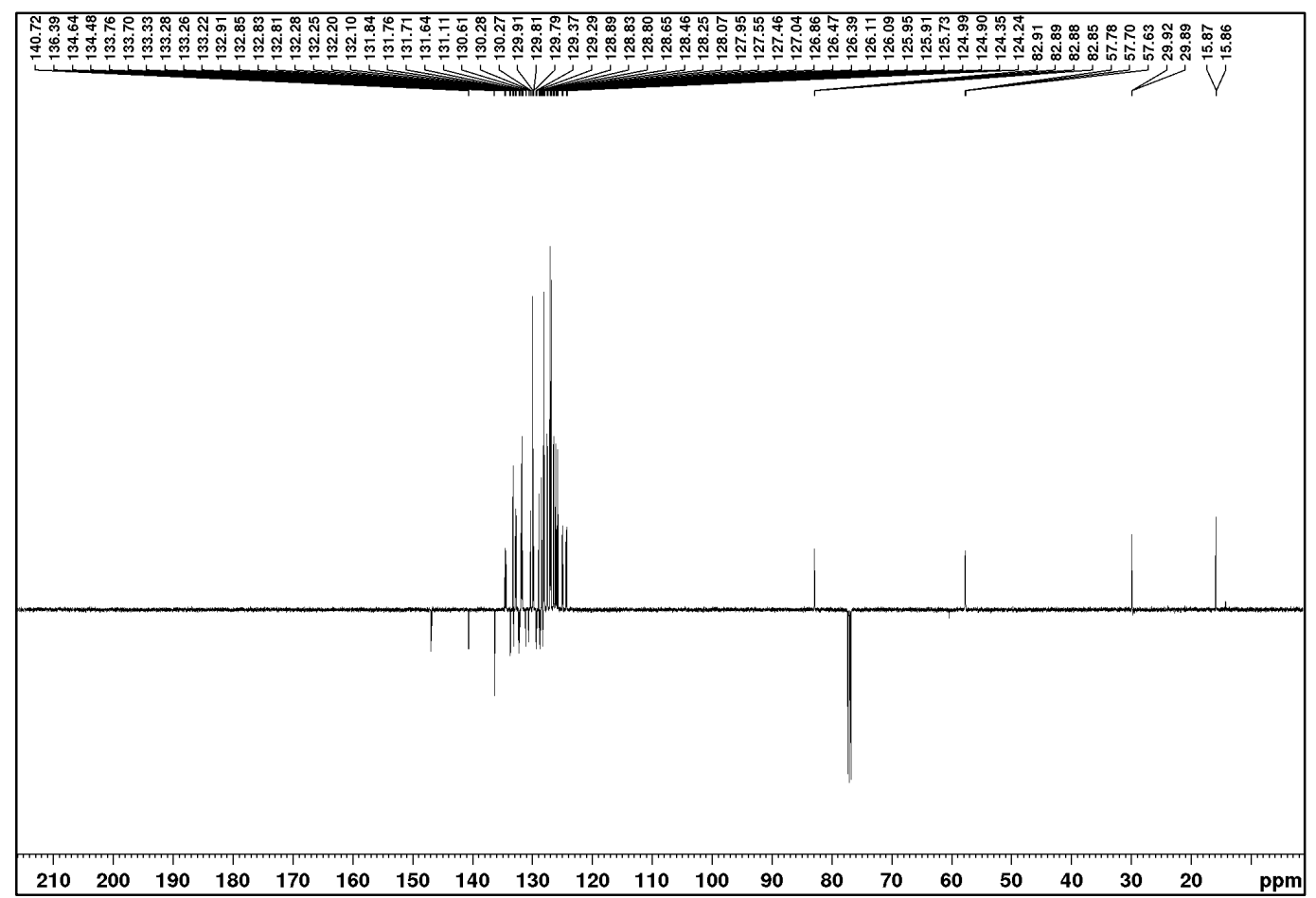

${ }^{31} \mathrm{P}\left\{{ }^{1} \mathrm{H}\right\}$ NMR $\left(202.5 \mathrm{MHz}, \mathrm{CDCl}_{3}\right)$

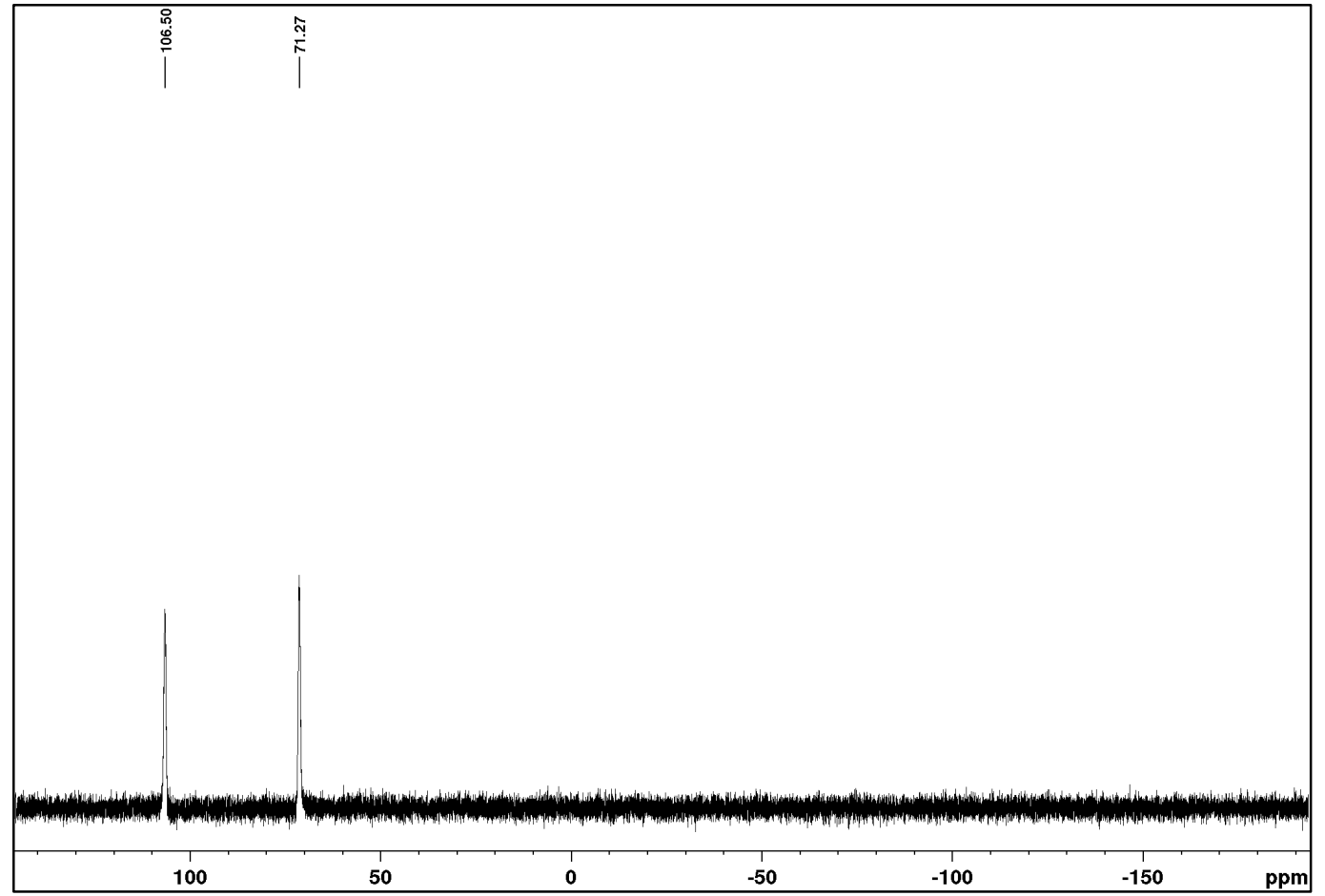


$\left(M, S_{\mathrm{p}}\right)-(+)-\mathrm{N}-[(1 S, 2 R)-2-($ Diphenylphosphinito-borane)-1-methyl-2-phenylethyl], $\quad \mathrm{N}$-methyl amino-2-(1,1'-binaphtyl)phenylphosphine-borane $\mathbf{1 2 v}$.<smiles>[B][P+](c1ccccc1)(c1ccc2ccccc2c1-c1cccc2ccccc12)N(C)C(C)C(c1ccccc1)[PH](C)(C)C</smiles>

${ }^{1} \mathrm{H} \mathrm{NMR}\left(500 \mathrm{MHz}, \mathrm{CDCl}_{3}\right)$

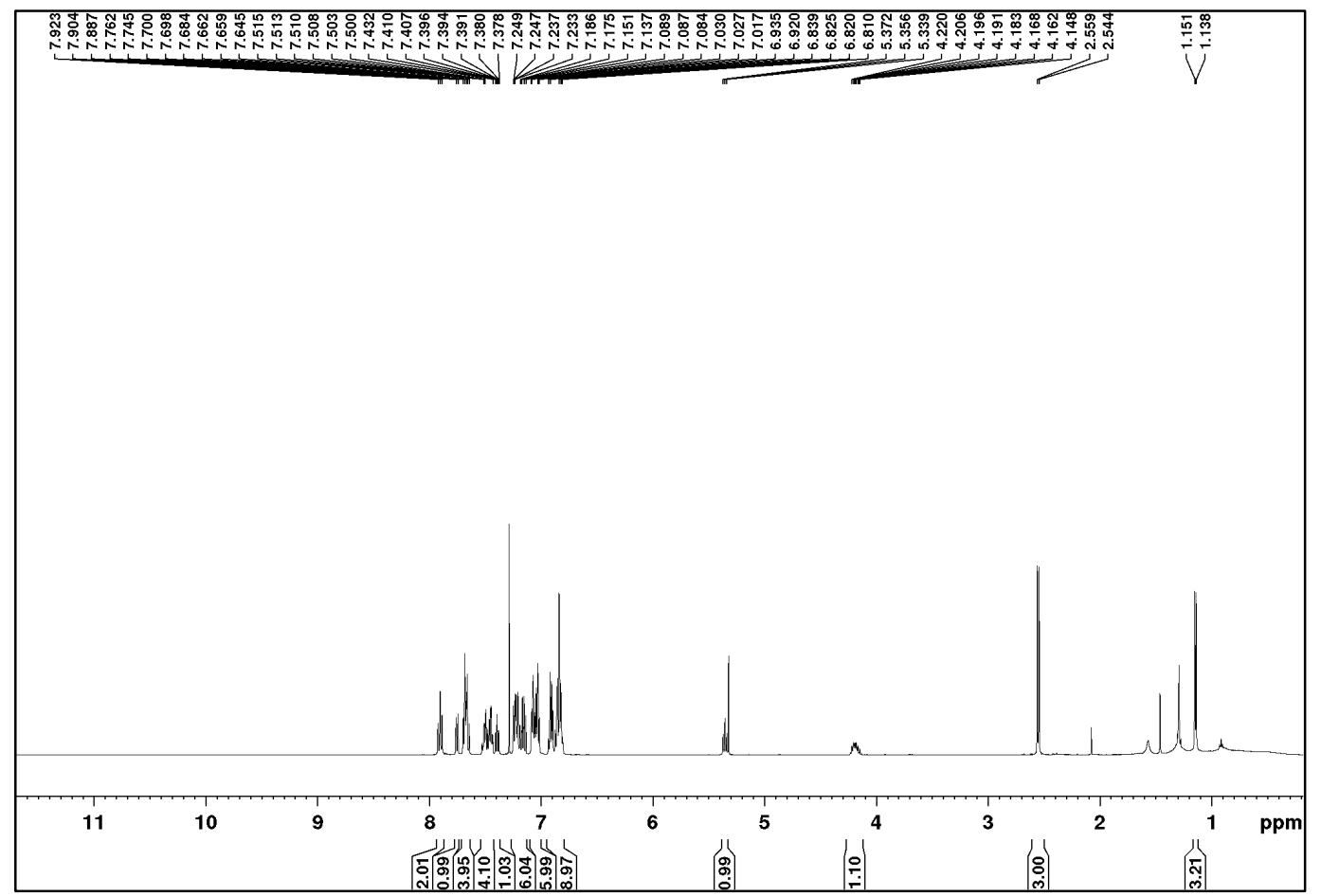

${ }^{13} \mathrm{C}\left\{{ }^{1} \mathrm{H}\right\} \mathrm{J}-\bmod \mathrm{NMR}\left(125.8 \mathrm{MHz}, \mathrm{CDCl}_{3}\right)$

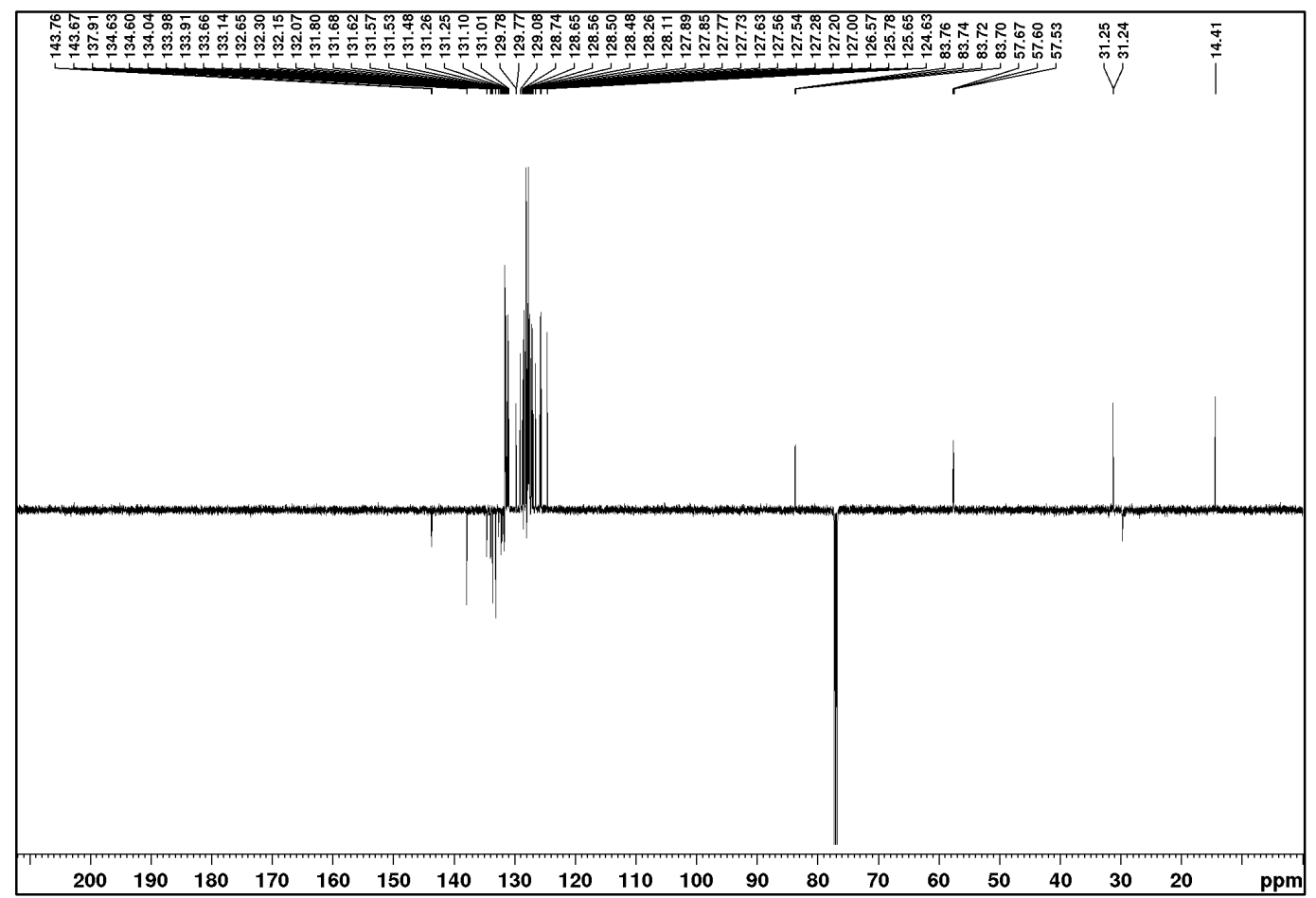


${ }^{31} \mathrm{P}\left\{{ }^{1} \mathrm{H}\right\}$ NMR (202.5 MHz, $\left.\mathrm{CDCl}_{3}\right)$

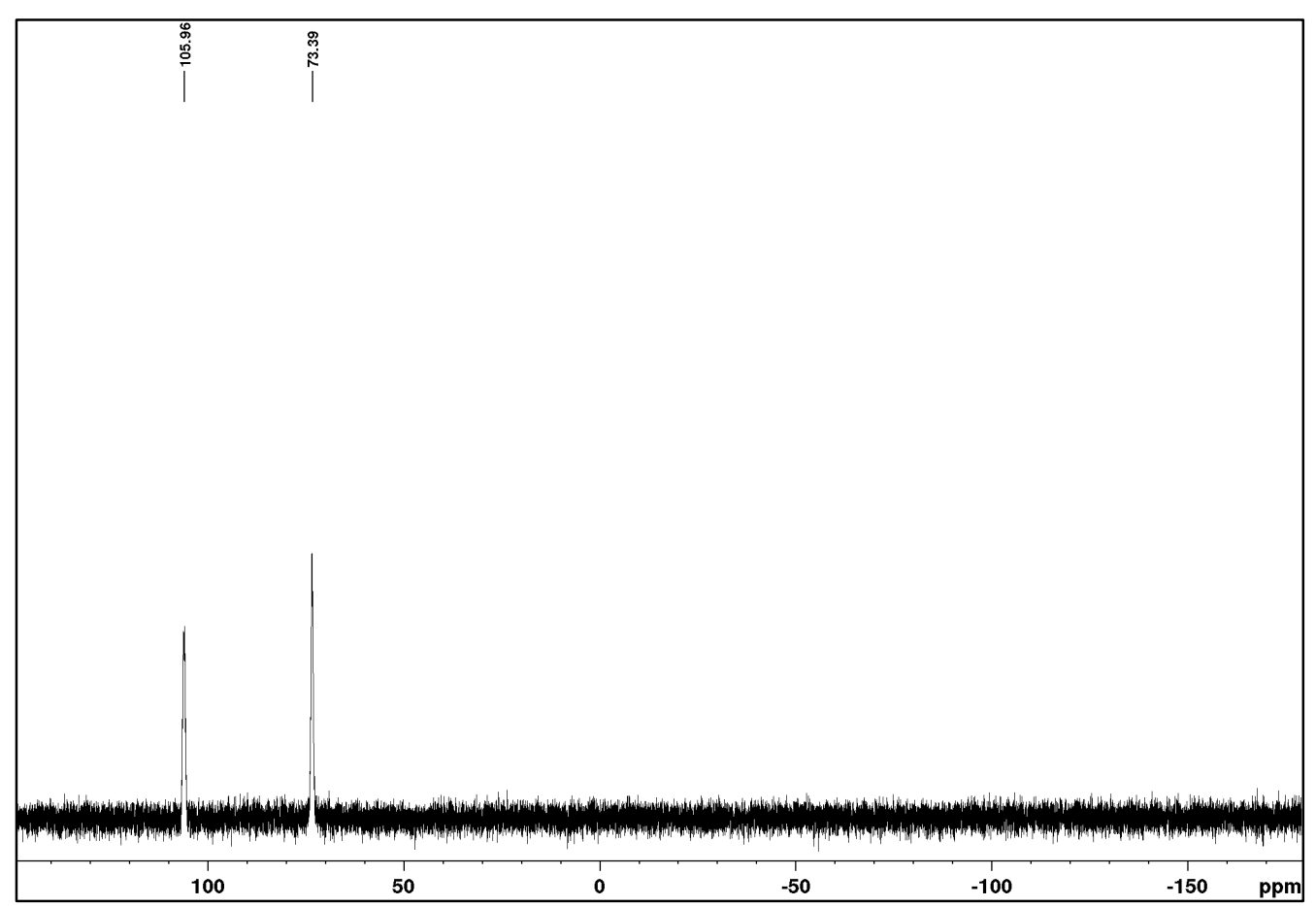

$\left(S_{\mathrm{p}}\right)-(+)-\mathrm{N}-[(1 S, 2 R)-2-[\mathrm{Bis}(3,5-d i m e t h y l p h e n y l) p h o s p h i n i t o-b o r a n e]-1-m e t h y l-2-p h e n y l e t h y l], \mathrm{N}-$ methyl amino(o-biphenyl)phenylphosphine-borane $\mathbf{1 2} \mathbf{w}$.

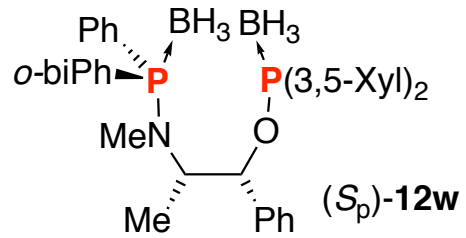

${ }^{1} \mathrm{H} \mathrm{NMR}\left(500 \mathrm{MHz}, \mathrm{CDCl}_{3}\right)$

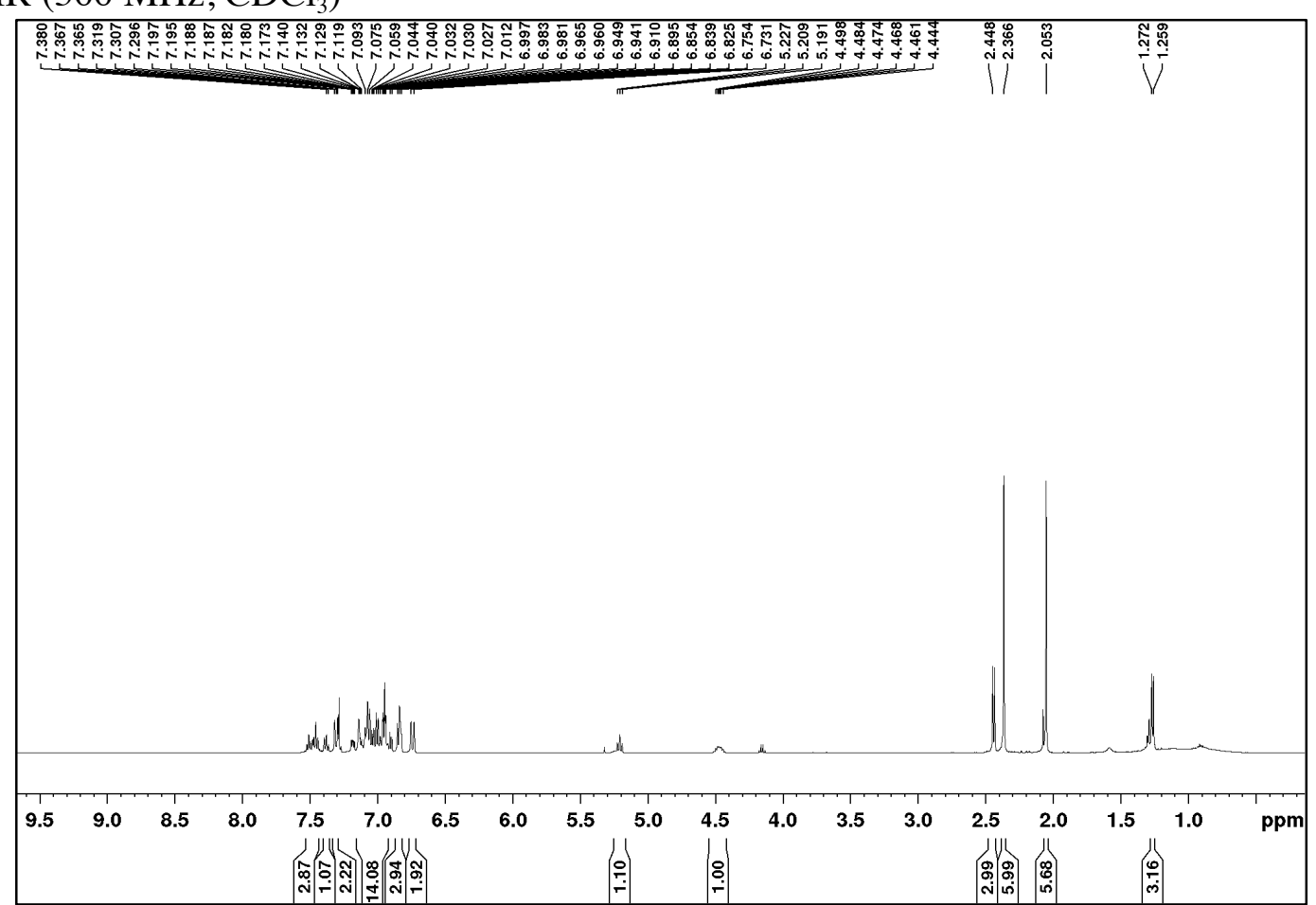


${ }^{13} \mathrm{C}\left\{{ }^{1} \mathrm{H}\right\} \mathrm{J}-\bmod$ NMR $\left(125.8 \mathrm{MHz}, \mathrm{CDCl}_{3}\right)$

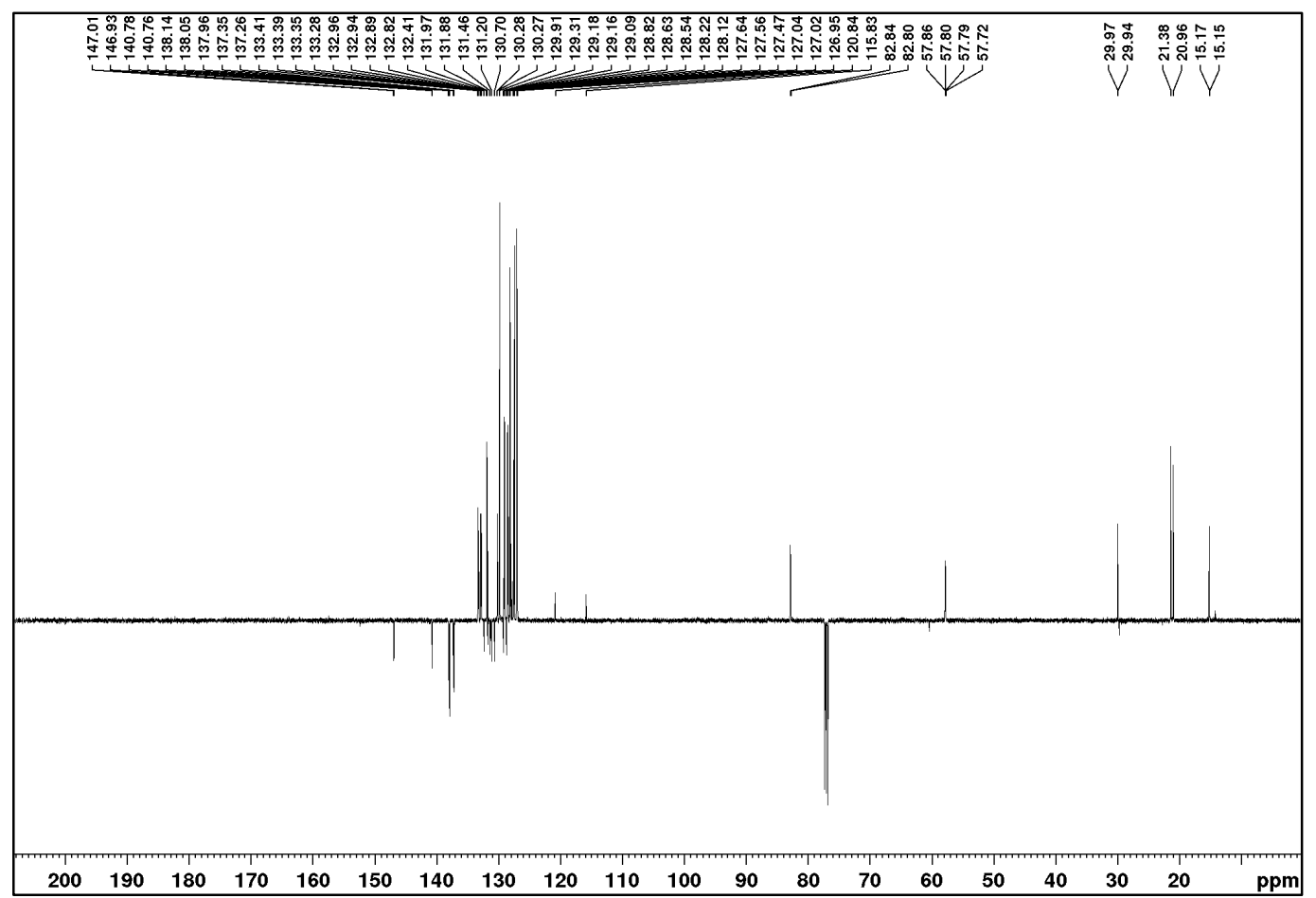

${ }^{31} \mathrm{P}\left\{{ }^{1} \mathrm{H}\right\}$ NMR $\left(202.5 \mathrm{MHz}, \mathrm{CDCl}_{3}\right)$

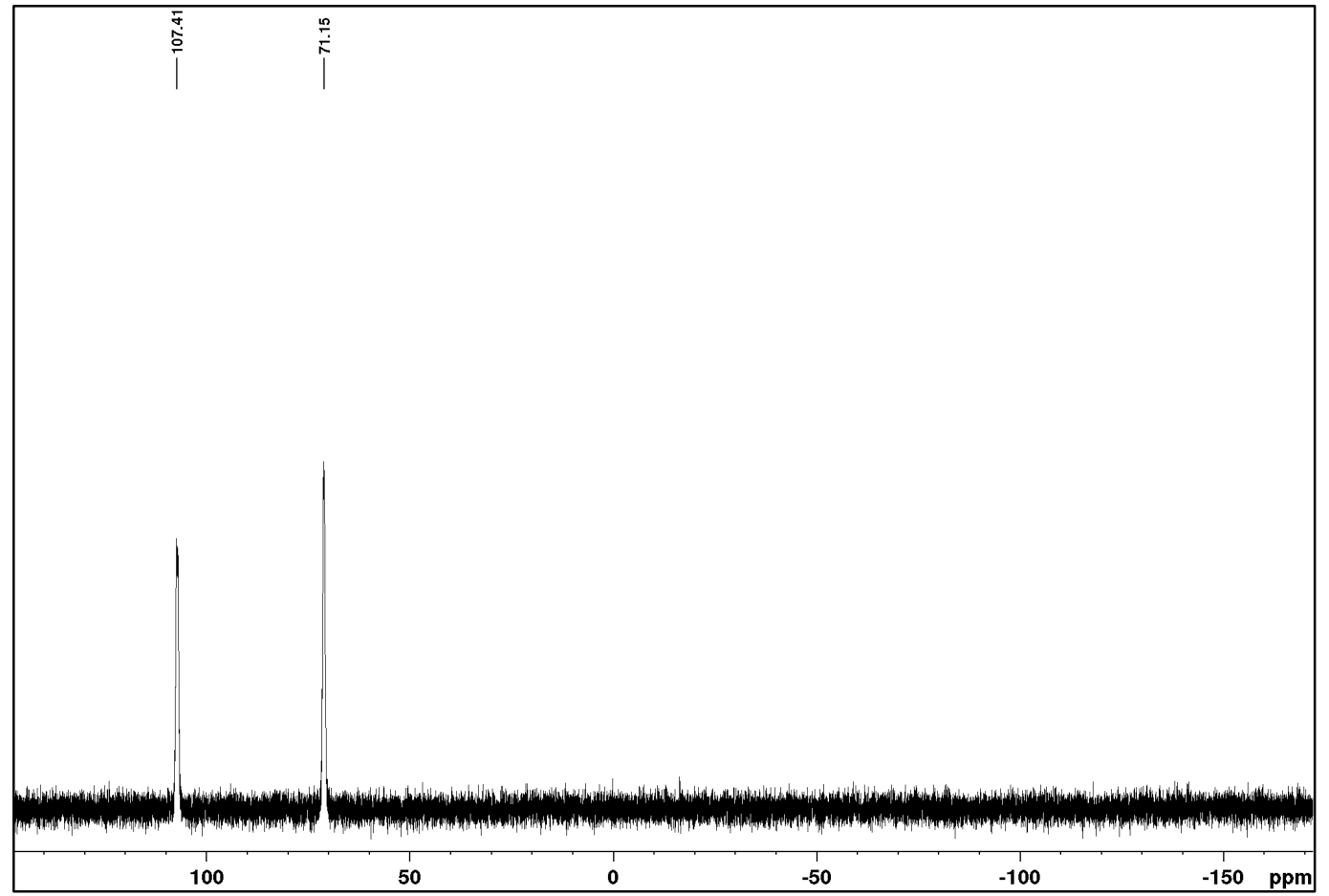


P-chirogenic aminophosphine-phosphinites 13.

$\left(S_{\mathrm{p}}\right)-(-)-\mathrm{N}-[(1 R, 2 S)-2-($ Diphenylphosphinito)-1-methyl-2-phenyl]ethyl], N-methylamino(obiphenyl)phenylphosphine $\mathbf{1 3 i}$.

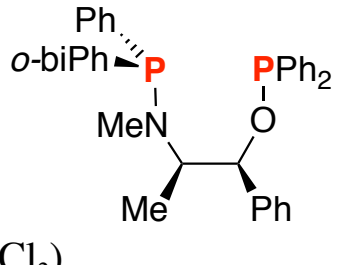

${ }^{31} \mathrm{P}\left\{{ }^{1} \mathrm{H}\right\}$ NMR $\left(121.5 \mathrm{MHz}, \mathrm{CD}_{2} \mathrm{Cl}_{2}\right)$

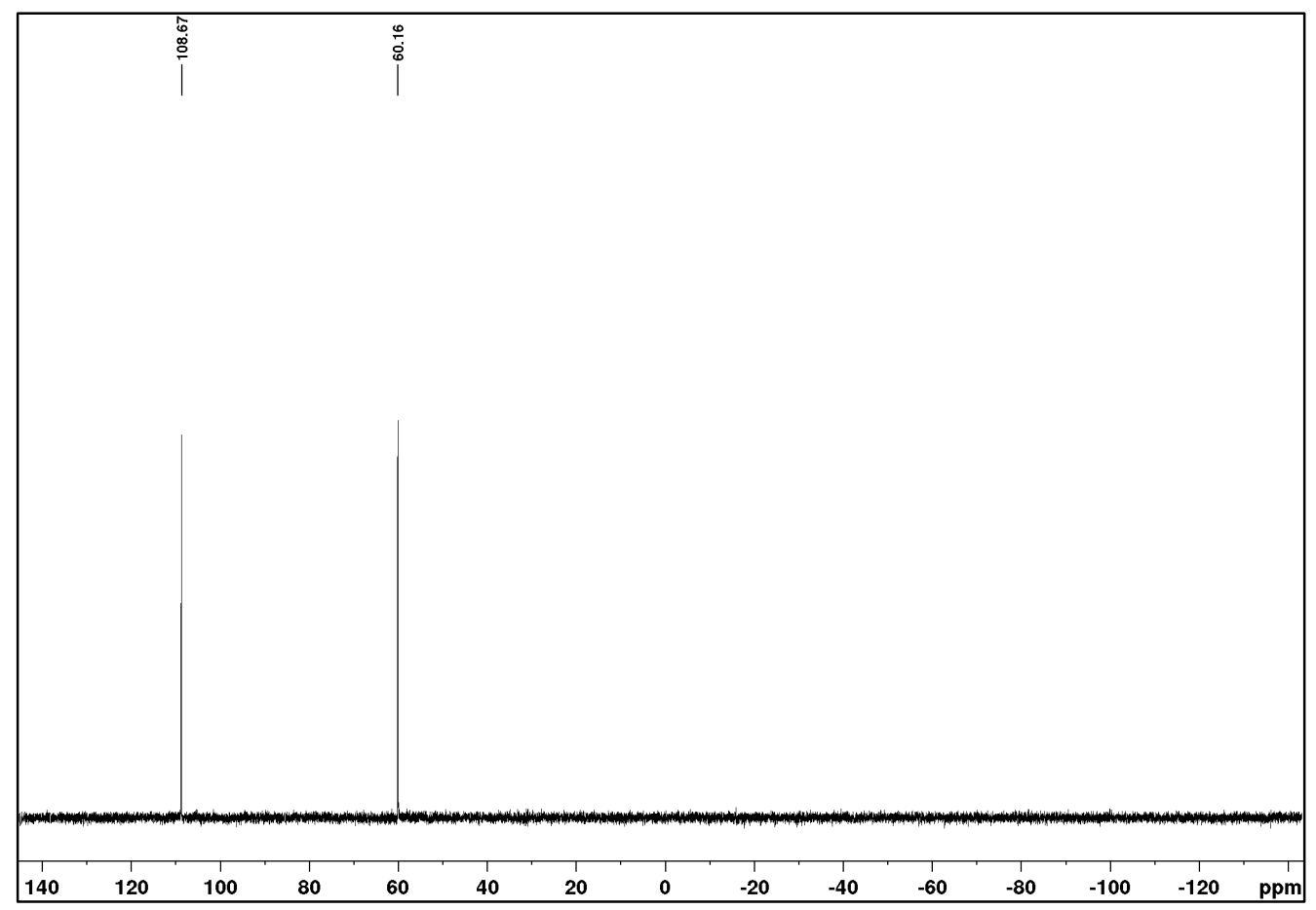

$\left(R_{\mathrm{p}}\right)$-N-[(1R,2S)-2-(Diphenylphosphinito)-1-methyl-2-phenylethyl],

N-methylamino(2isopropoxyphenyl)phenylphosphine $\mathbf{1 3 m}$.

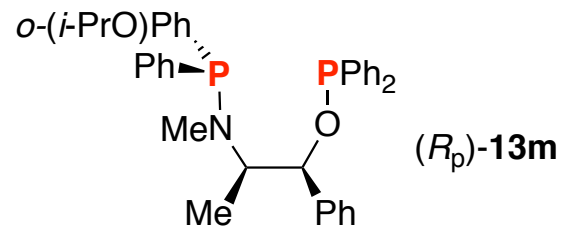

${ }^{31} \mathrm{P}\left\{{ }^{1} \mathrm{H}\right\}$ NMR $\left(121.5 \mathrm{MHz}, \mathrm{CDCl}_{3}\right)$

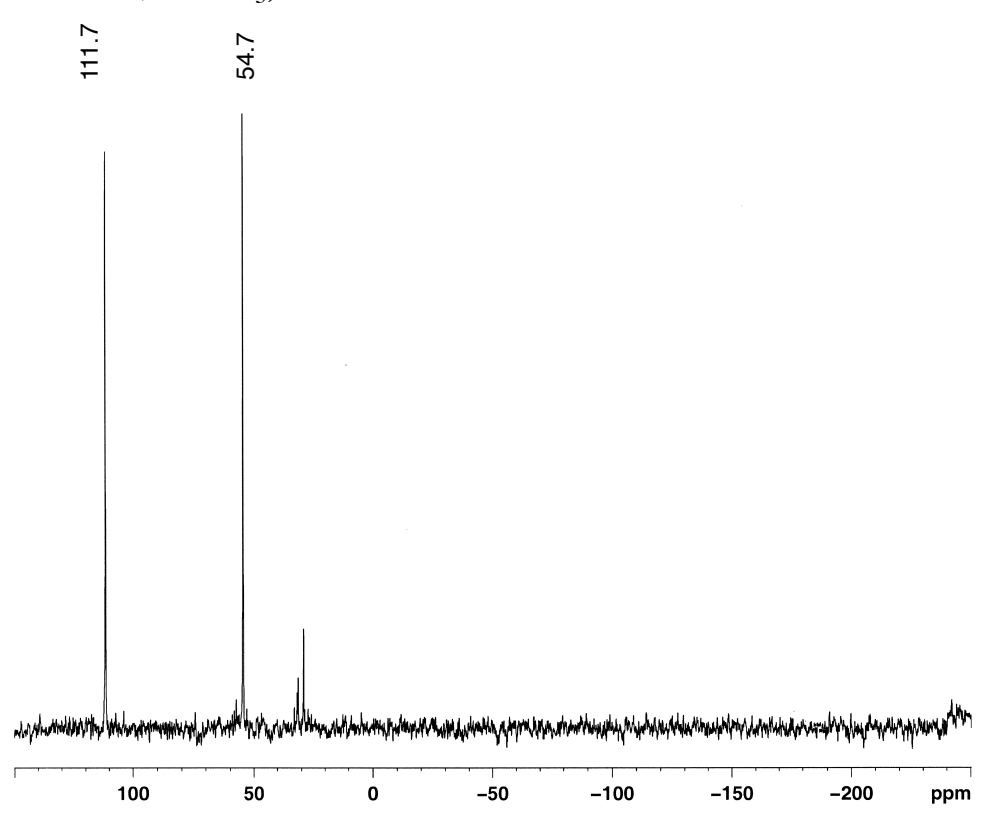


$\left(R_{\mathrm{p}}\right)-\mathrm{N}-[(1 R, 2 S)$-2-(Diphenylphosphinito)-1-methyl-2-phenylethyl], $\quad \mathrm{N}$-methylamino(2-benzyl oxyphenyl)phenylphosphine 13n.

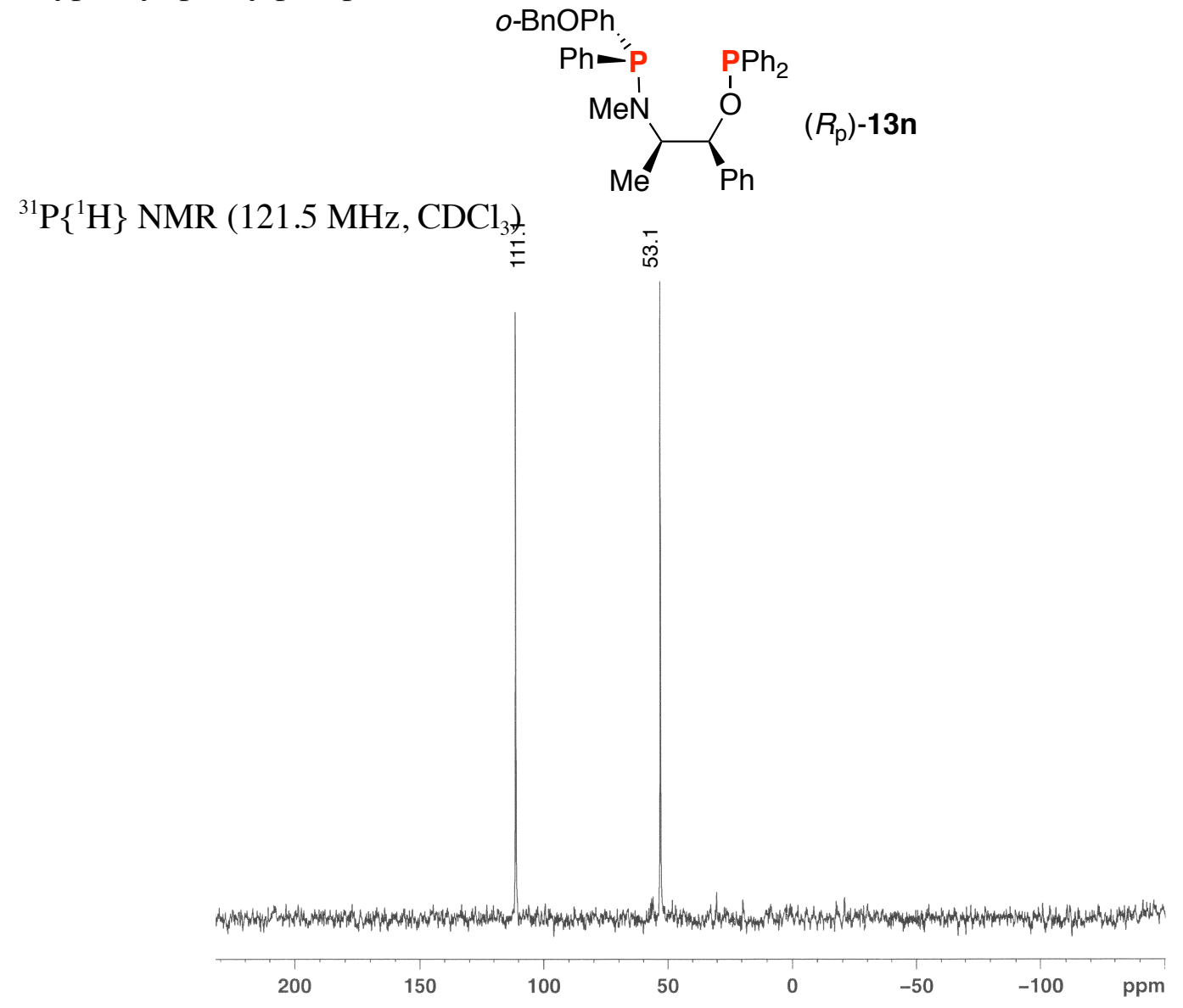

$\left(R_{\mathrm{p}}\right)-\mathrm{N}-[(1 R, 2 S)$-2-(Diphenylphosphinito)-1-methyl-2-phenylethyl],

$\mathrm{N}$-methylamino(3,5dimethylphenyl)phenylphosphine $\mathbf{1 3 0}$.

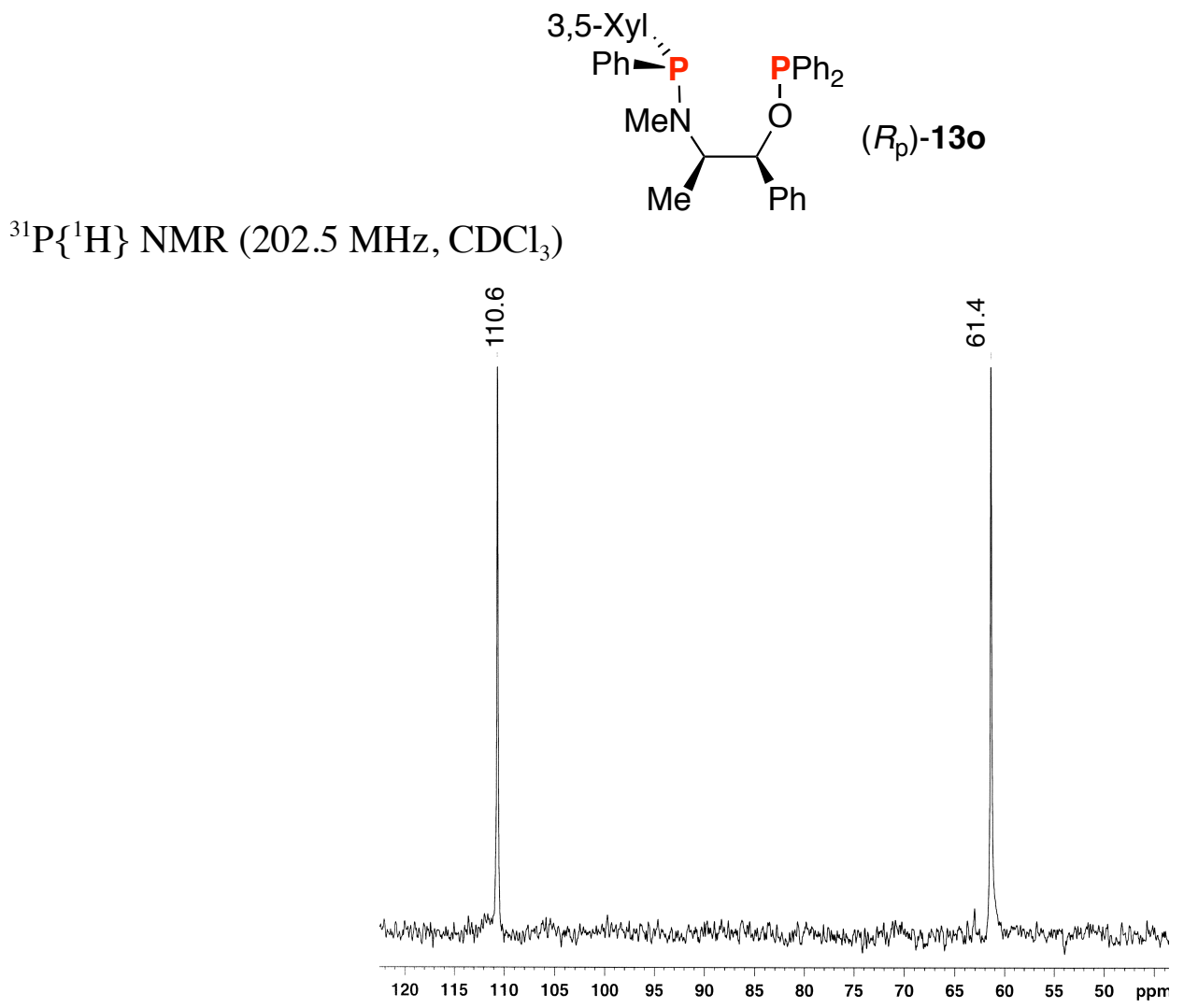


$\left(R_{\mathrm{p}}\right)-\mathrm{N}-[(1 R, 2 S)$-2-(Diphenylphosphinito)-1-methyl-2-phenylethyl], $\quad \mathrm{N}$-methylamino(3,5-di-tbutylphenyl)phenylphosphine 13p.

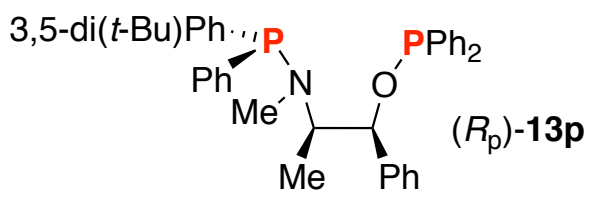

${ }^{31} \mathrm{P}\left\{{ }^{1} \mathrm{H}\right\} \mathrm{NMR}\left(121.5 \mathrm{MHz}, \mathrm{CDCl}_{3}\right)$ :

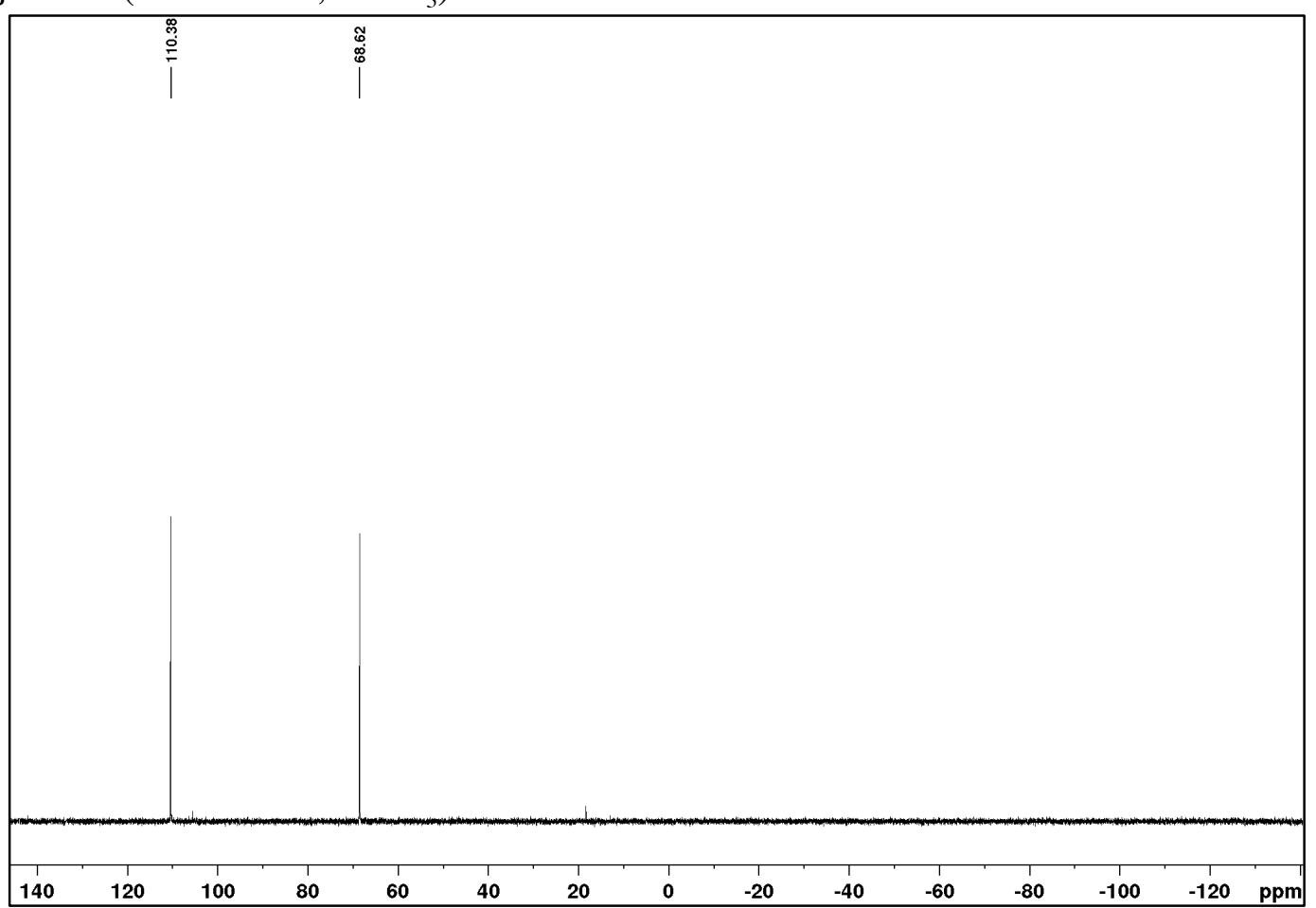

$\left(R_{\mathrm{p}}\right)$-N-[(1R,2S)-2-(Diphenylphosphinito)-1-methyl-2-phenylethyl], $\quad \mathrm{N}$-methylamino(3,5-di-tbutyl-4-methoxyphenyl)phenylphosphine 13q.

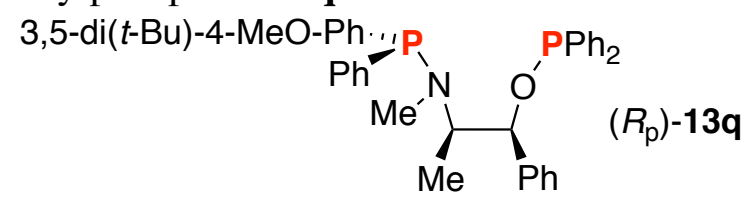

${ }^{31} \mathrm{P}\left\{{ }^{1} \mathrm{H}\right\}$ NMR $\left.121.5, \mathrm{CDCl}_{3}\right)$

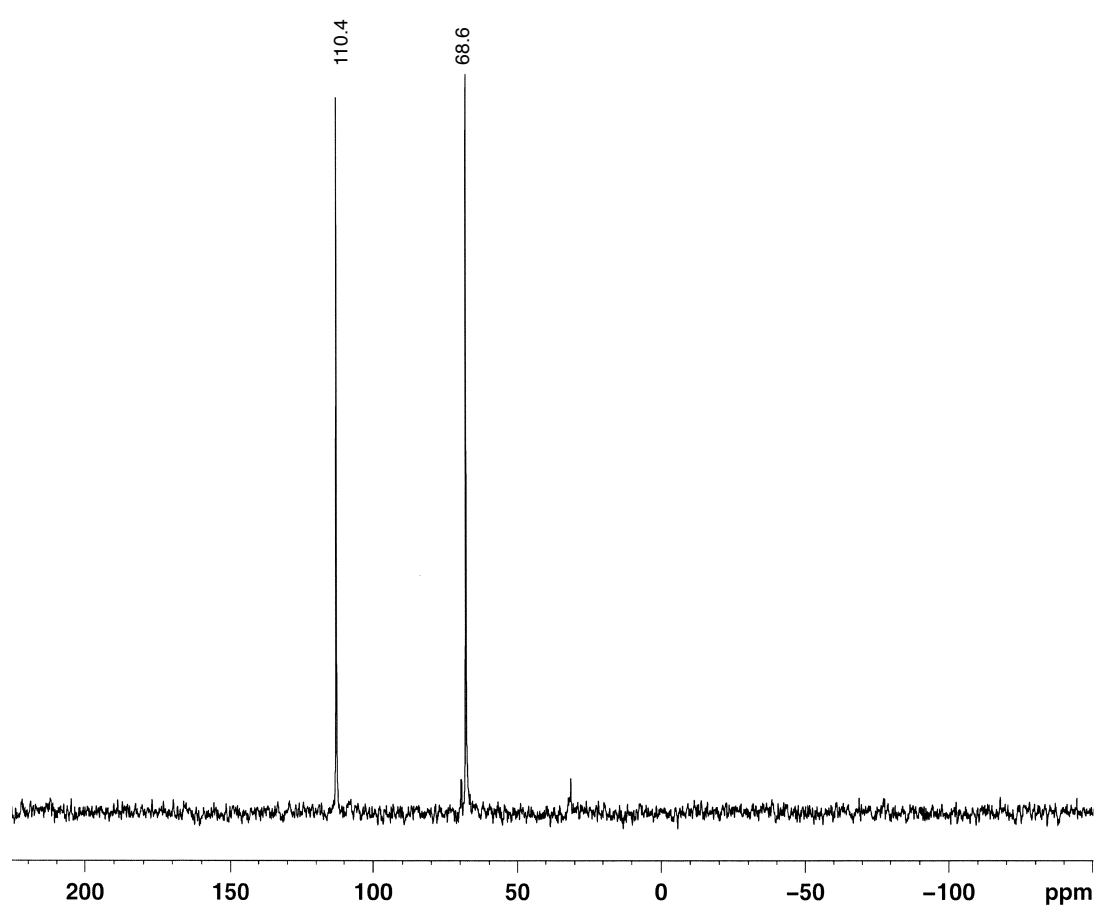


$\left(R_{\mathrm{p}}\right)-\mathrm{N}-[(1 R, 2 S)$-2-(Diphenylphosphinito)-1-methyl-2-phenylethyl], $\mathrm{N}$-(methylamino)phenyl(2pyridyl)phosphine 13r.

${ }^{31} \mathrm{P}\left\{{ }^{1} \mathrm{H}\right\}$ NMR $\left(202.5 \mathrm{MHz}, \mathrm{CDCl}_{3}\right)$
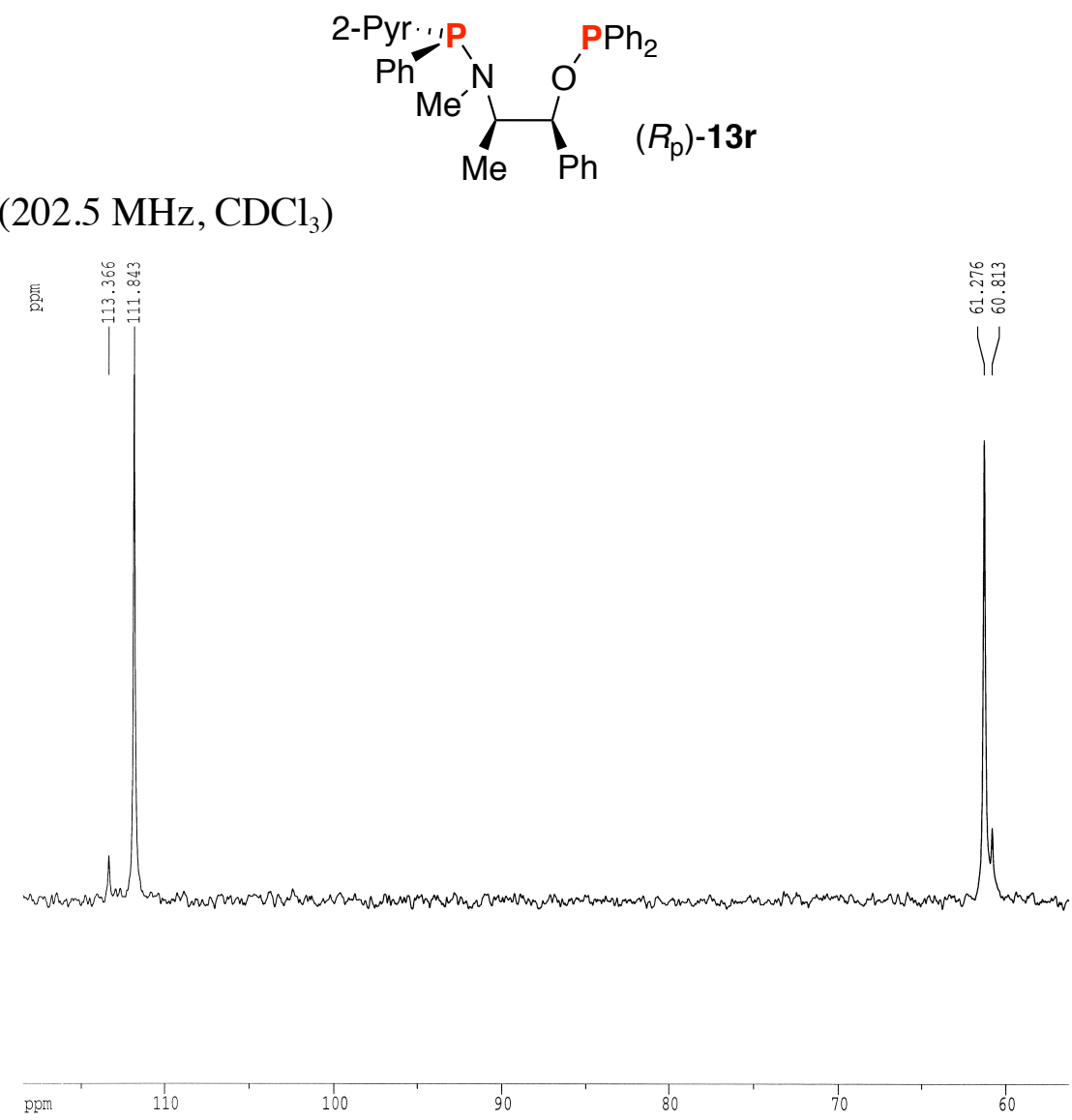

$\left(R_{\mathrm{p}}\right)-\mathrm{N}-[(1 R, 2 S)$-2-(Diphenylphosphinito)-1-methyl-2-phenylethyl], $\quad$ N-methylamino-2-(2',6'dimethoxy-1,1'-biphenyl)phenylphosphine 13s.

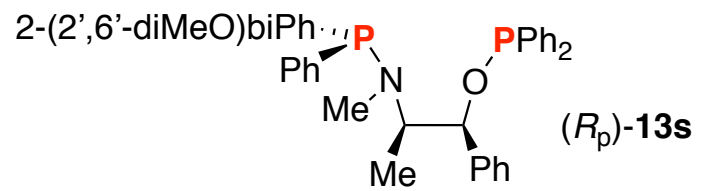

${ }^{31} \mathrm{P}\left\{{ }^{1} \mathrm{H}\right\} \mathrm{NMR}\left(121.5 \mathrm{MHz}, \mathrm{CDCl}_{3}\right)$ :

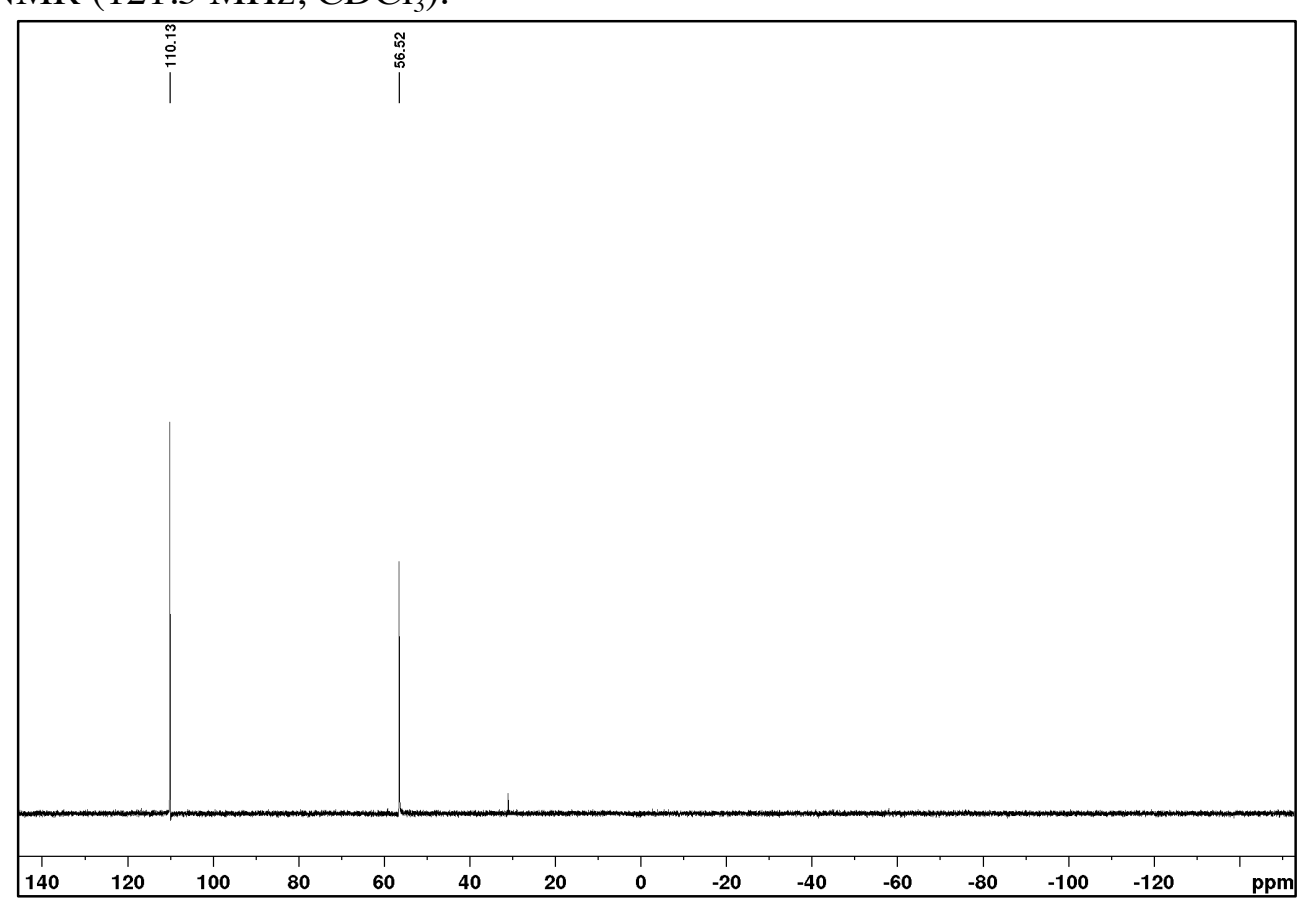




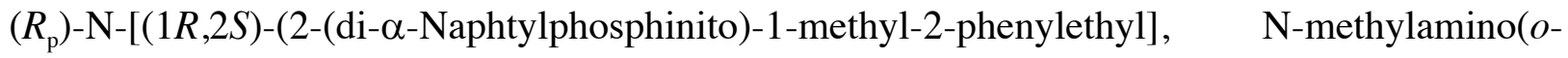
biphenyl)phenylphosphine 13t.

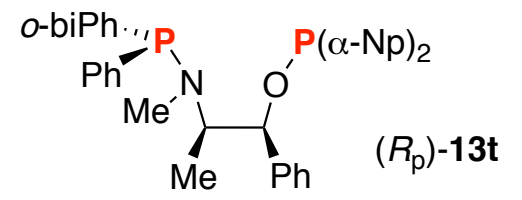

${ }^{31} \mathrm{P}\left\{{ }^{1} \mathrm{H}\right\}$ NMR $\left(121.5 \mathrm{MHz}, \mathrm{CDCl}_{3}\right)$

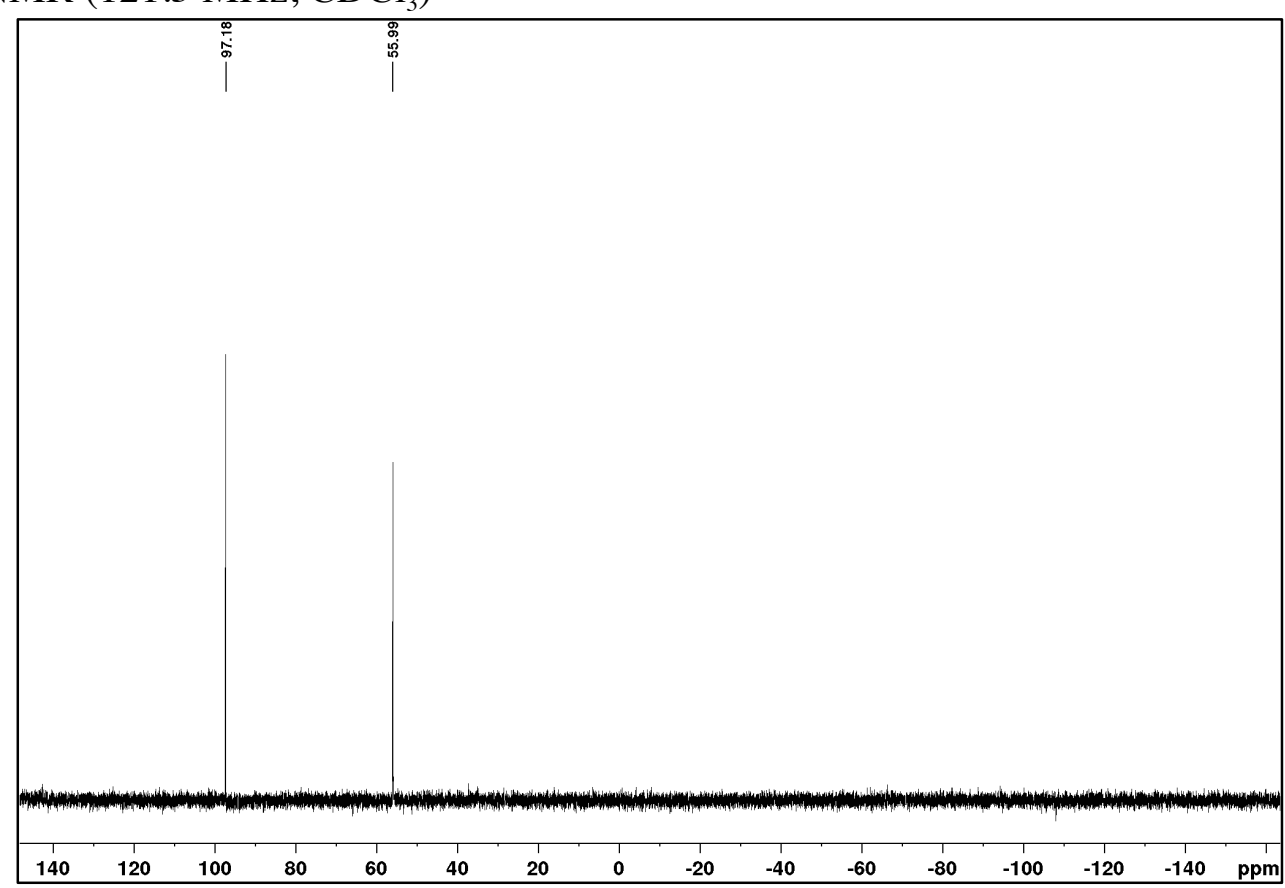

$\left(M, S_{\mathrm{p}}\right)-(+)-\mathrm{N}-[(1 S, 2 R)-2-($ Diphenylphosphinito)-1-methyl-2-phenylethyl], $\quad \mathrm{N}$-methylamino-2(1,1'-binaphtyl)phenylphosphine 13v.

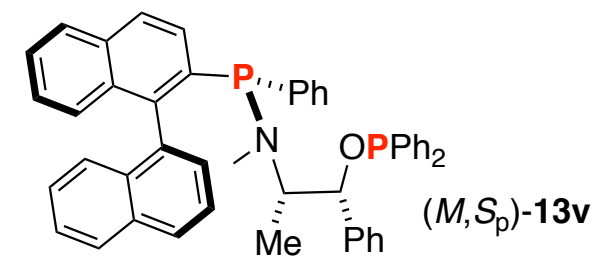

${ }^{31} \mathrm{P}\left\{{ }^{1} \mathrm{H}\right\} \mathrm{NMR}\left(202.5 \mathrm{MHz}, \mathrm{CDCl}_{3}\right)$

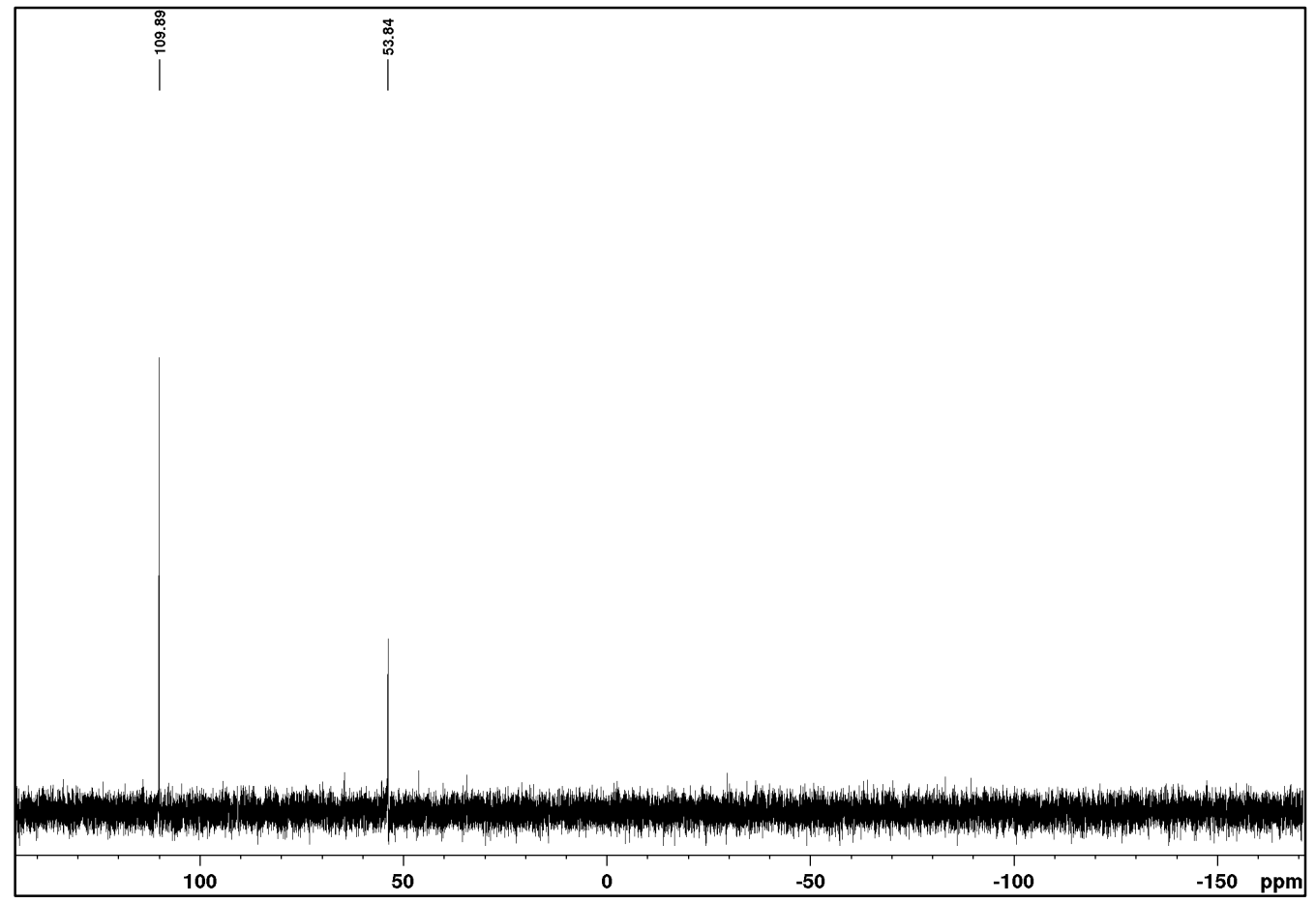


$\left(S_{\mathrm{p}}\right)-\mathrm{N}-[(1 S, 2 R)$-2-Bis(3,5-dimethylphenyl)phosphinito]-1-methyl-2-phenylethyl], $\quad \mathrm{N}$-methyl amino(o-biphenyl)phenylphosphine-borane 13w.

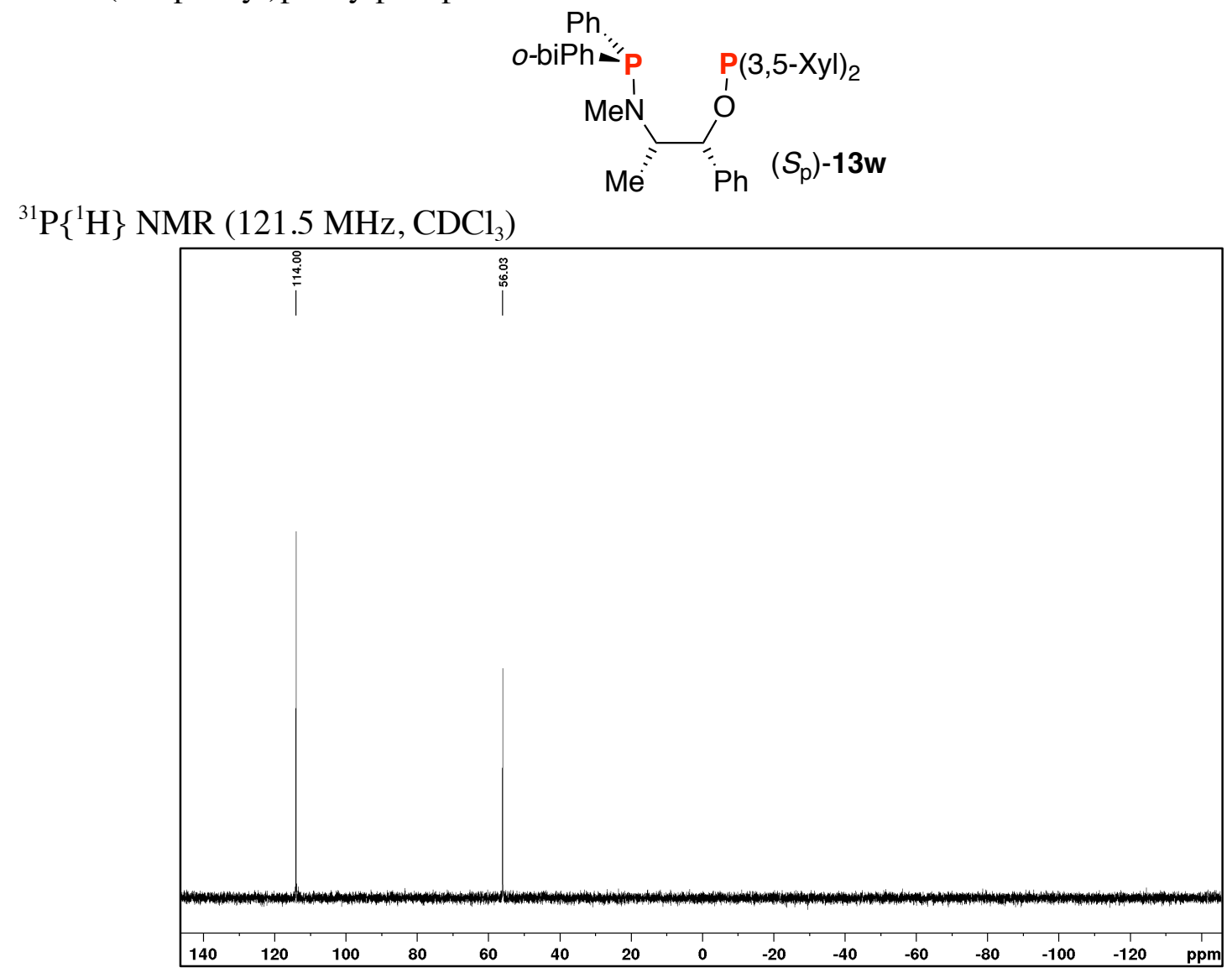

P-Chirogenic Aminophosphine-Phosphinite-Diborane 21.

$\left(S_{\mathrm{p}}\right)-(+)-\mathrm{N}-[(1 S, 2 R)-2-(o-A n i s y l p h e n y l p h o s p h i n i t o-b o r a n e)-1-m e t h y l-2-p h e n y l e t h y l], \quad \mathrm{N}$-methyl aminodiphenylphosphine-borane $\mathbf{2 1 a}$.

${ }^{1} \mathrm{H} \mathrm{NMR}\left(500 \mathrm{MHz}, \mathrm{CDCl}_{3}\right)$
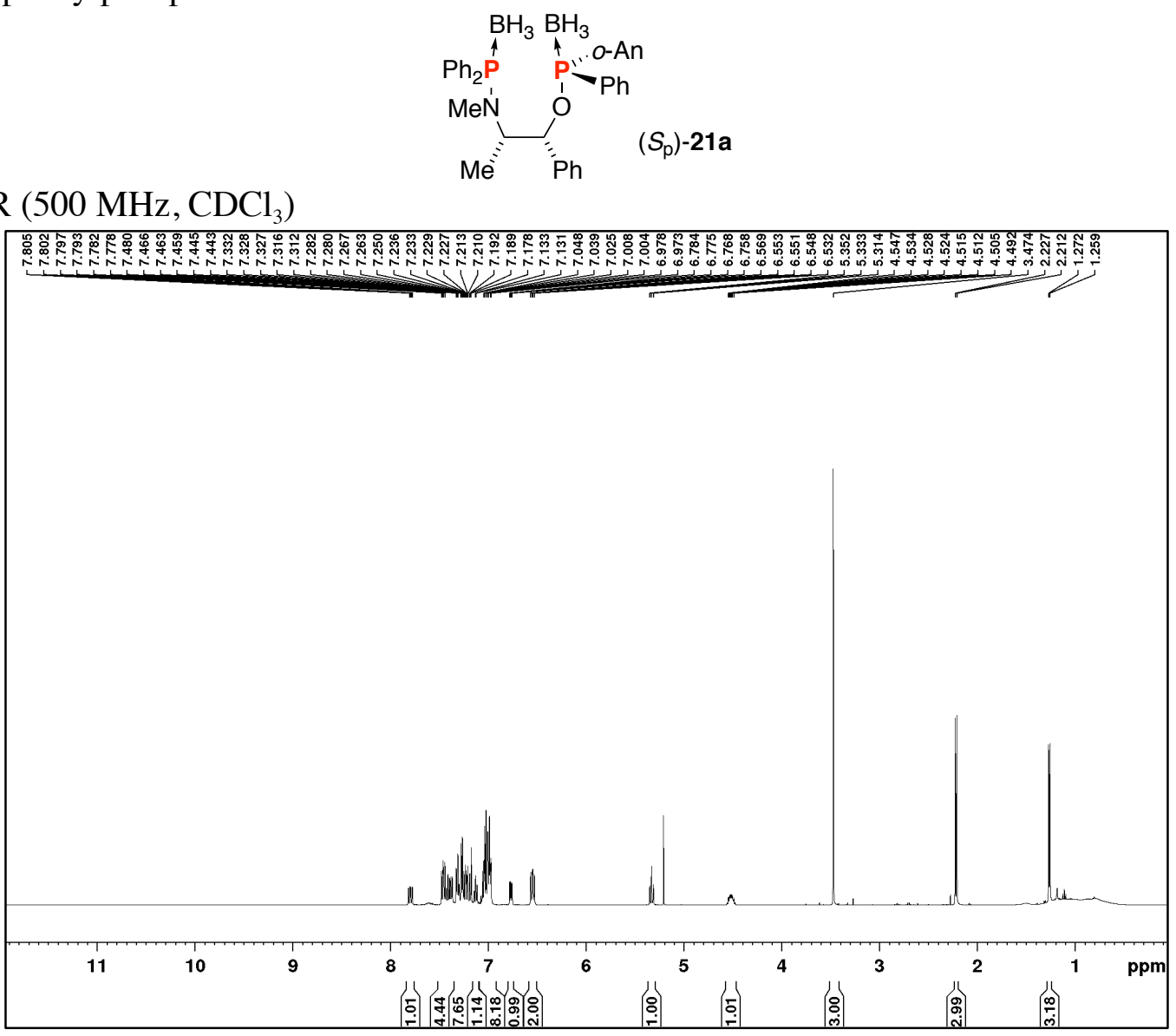
${ }^{13} \mathrm{C}\left\{{ }^{1} \mathrm{H}\right\} \mathrm{J}-\bmod$ NMR $\left(125.8 \mathrm{MHz}, \mathrm{CDCl}_{3}\right)$

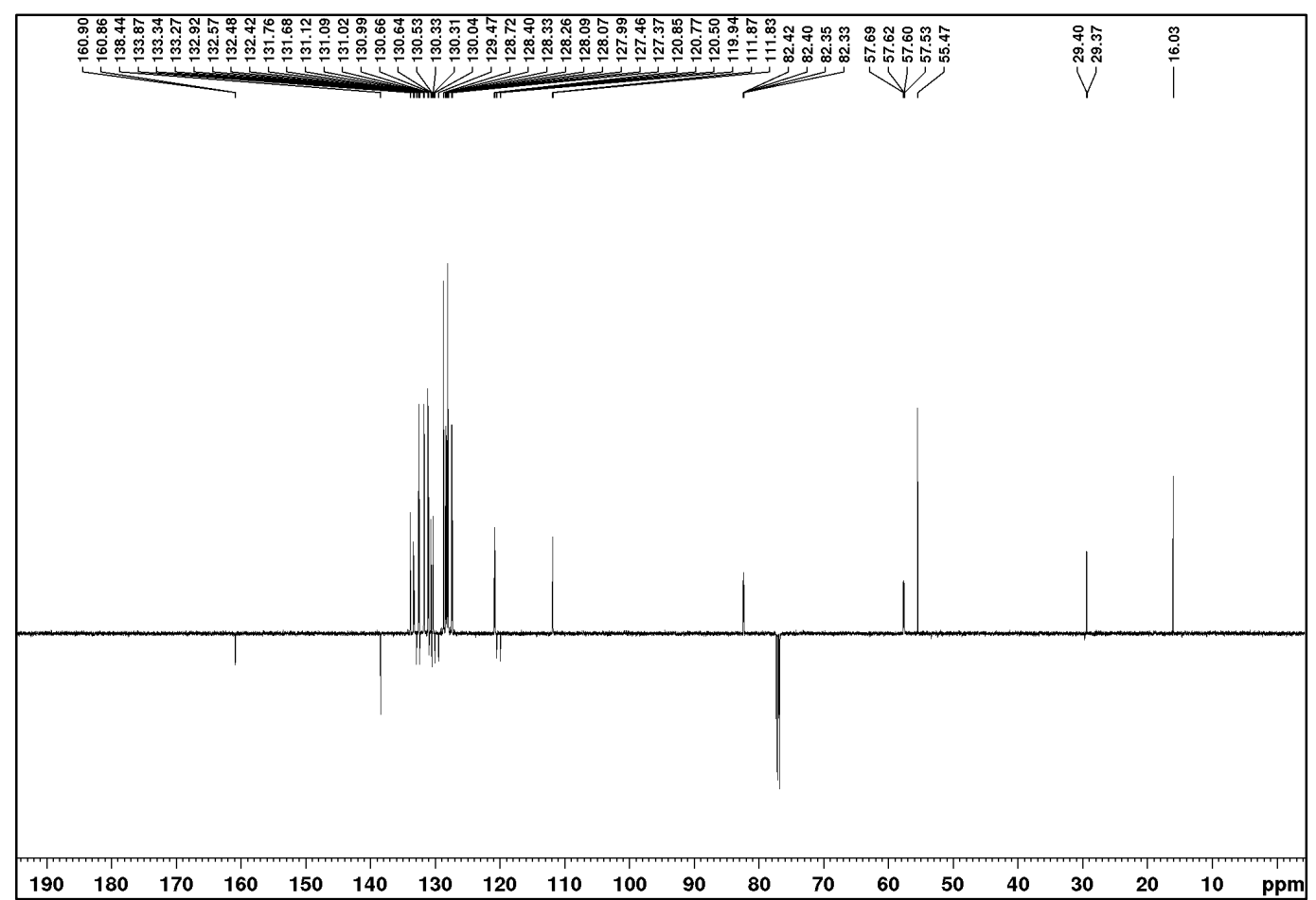

${ }^{31} \mathrm{P}\left\{{ }^{1} \mathrm{H}\right\} \mathrm{NMR}\left(202.5 \mathrm{MHz}, \mathrm{CDCl}_{3}\right)$

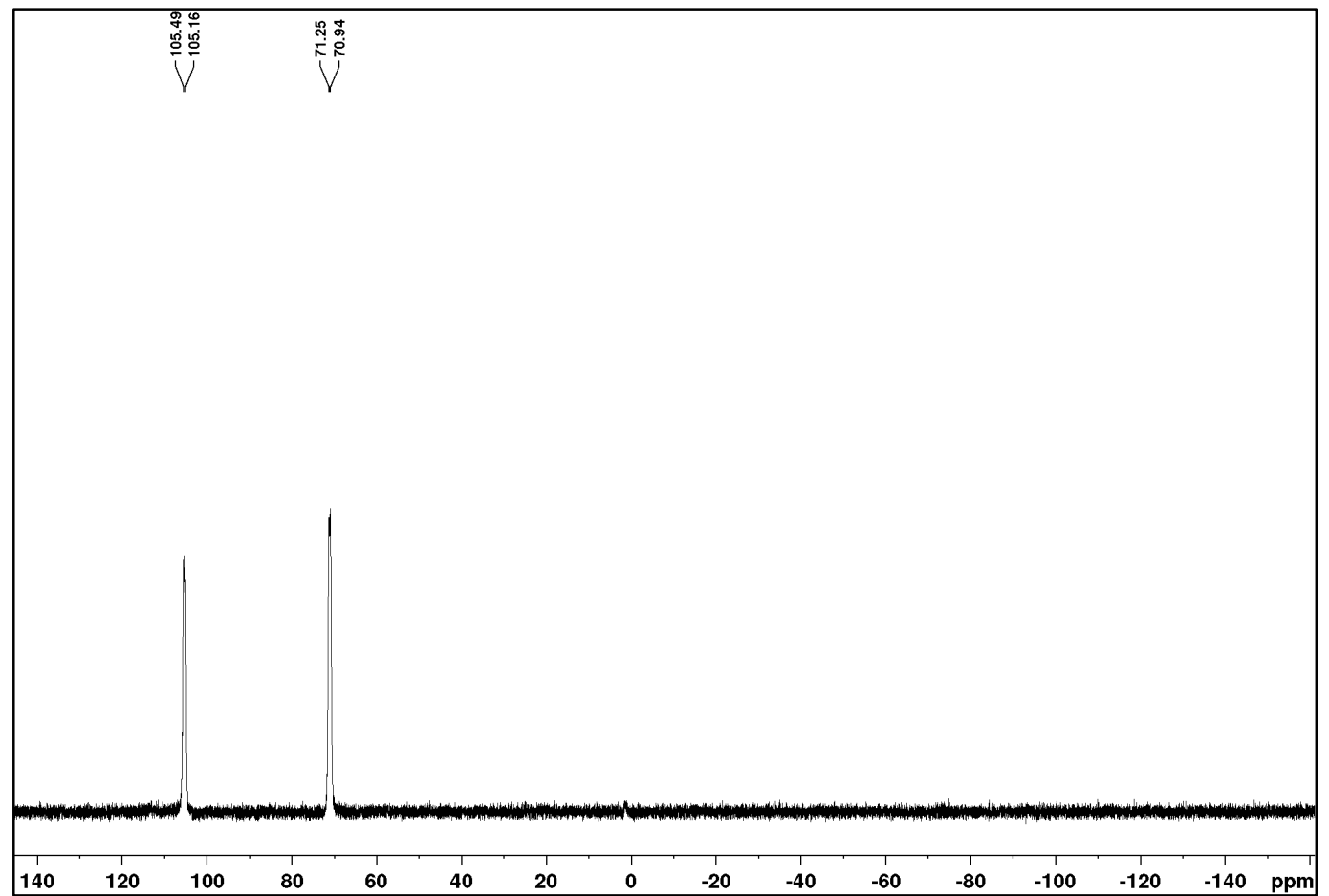


$\left(S_{\mathrm{p}}\right)-(+)-\mathrm{N}-[(1 S, 2 R)-2-(\alpha-N a p h t y l p h e n y l p h o s p h i n i t o-b o r a n e)-1-m e t h y l-2-p h e n y l e t h y l], \mathrm{N}-m e t h y l$ aminodiphenylphosphine-borane $\mathbf{2 1 b}$.

${ }^{1} \mathrm{H} \mathrm{NMR}\left(500 \mathrm{MHz}, \mathrm{CDCl}_{3}\right)$
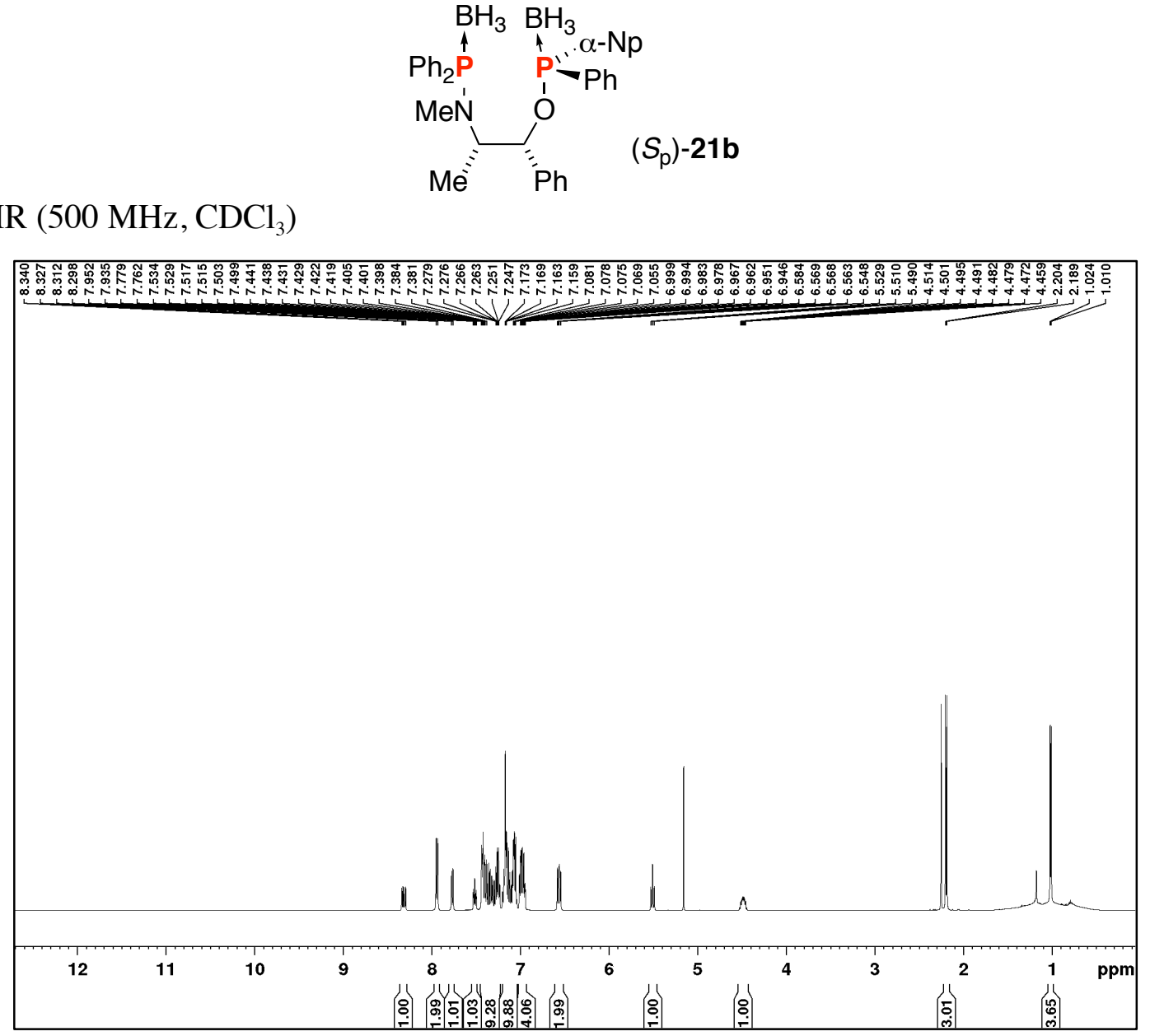

${ }^{13} \mathrm{C}\left\{{ }^{1} \mathrm{H}\right\} \mathrm{J}-\bmod \mathrm{NMR}\left(125.8 \mathrm{MHz}, \mathrm{CDCl}_{3}\right)$

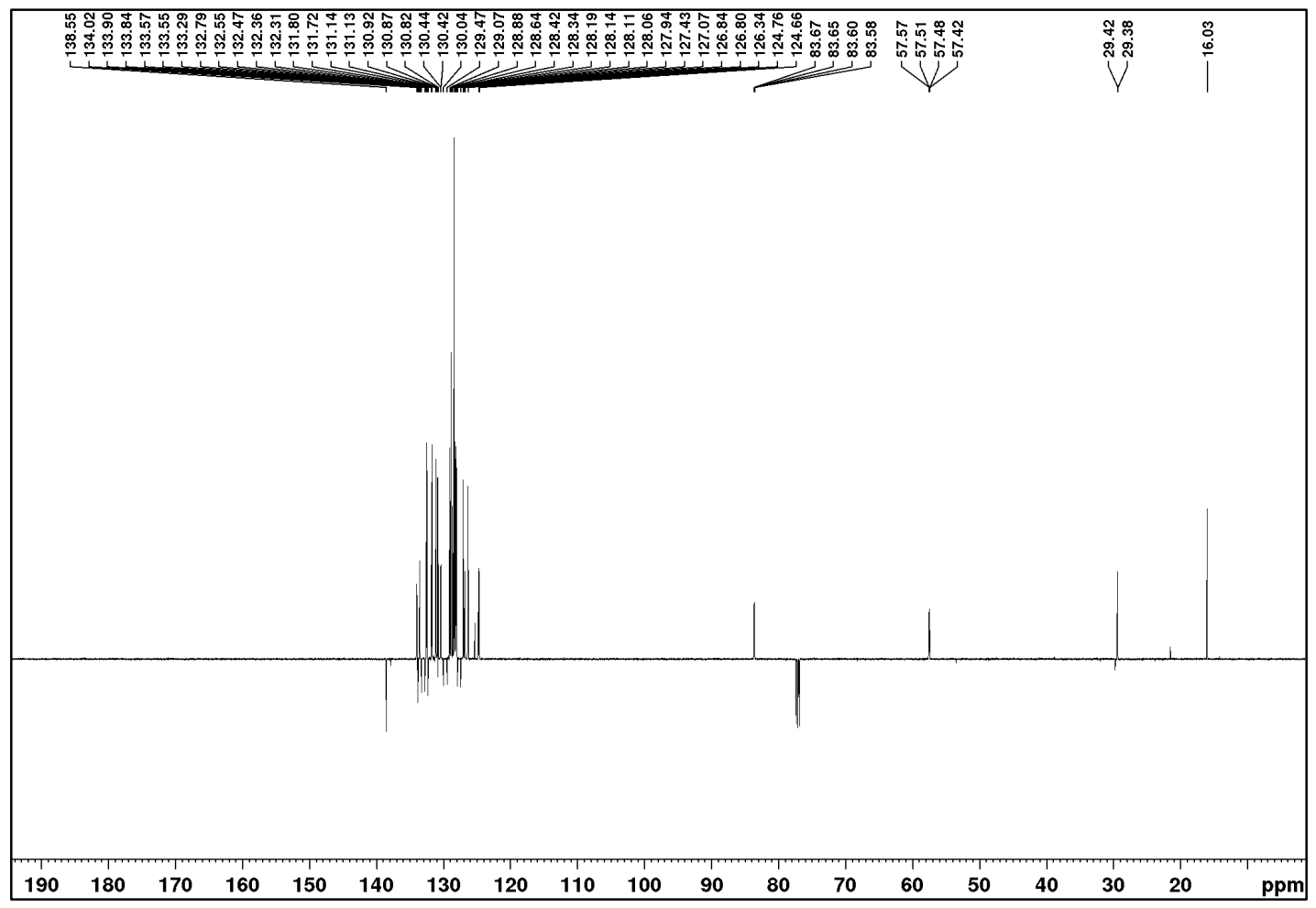


${ }^{31} \mathrm{P}\left\{{ }^{1} \mathrm{H}\right\}$ NMR (202.5 MHz, $\left.\mathrm{CDCl}_{3}\right)$

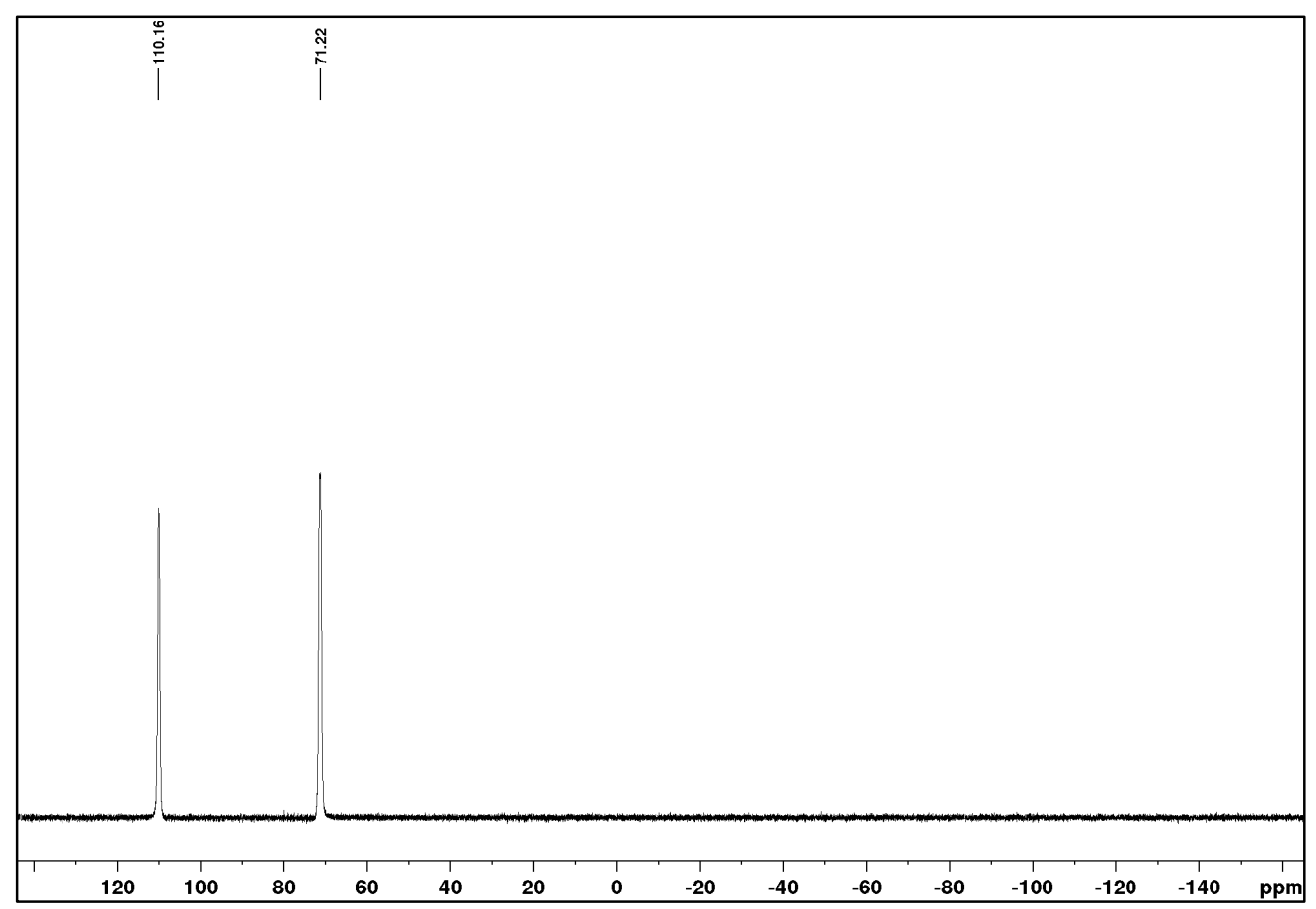

$\left(R_{\mathrm{p}}\right)-(-)-\mathrm{N}-[(1 R, 2 S)-2-(\beta-\mathrm{Naphtylphenylphosphinito-borane)-1-methyl-2-phenylethyl],} \mathrm{N-methyl}$ aminodiphenylphosphine-borane 21c.

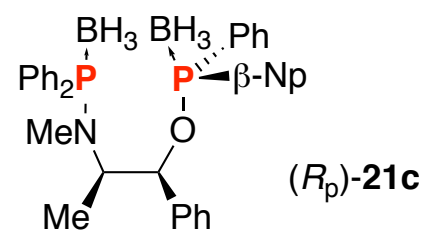

${ }^{1} \mathrm{H}$ NMR (300 MHz, $\mathrm{CDCl}_{3}$ )

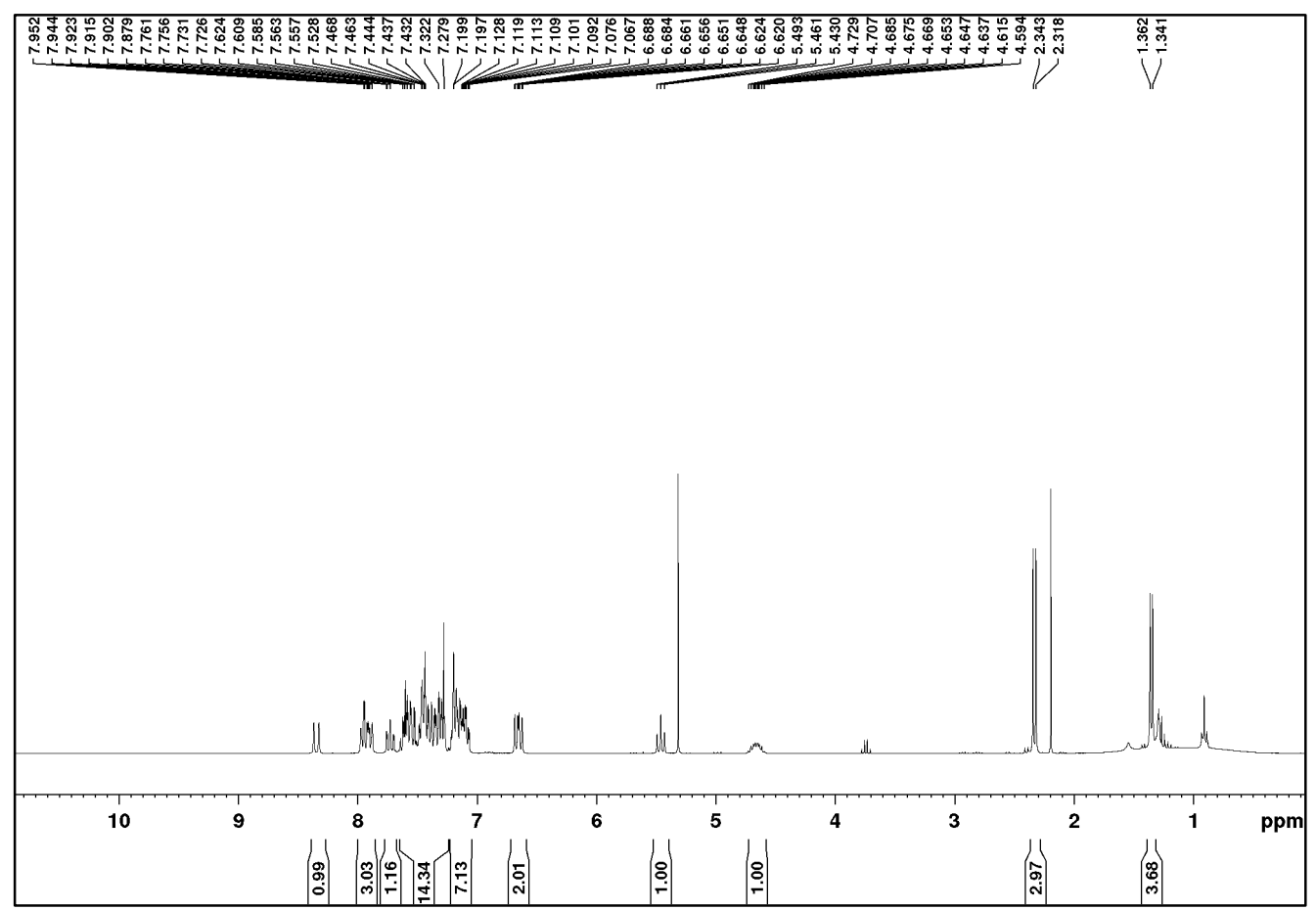


${ }^{13} \mathrm{C}\left\{{ }^{1} \mathrm{H}\right\} \mathrm{J}-\bmod \mathrm{NMR}\left(75.5 \mathrm{MHz}, \mathrm{CDCl}_{3}\right)$

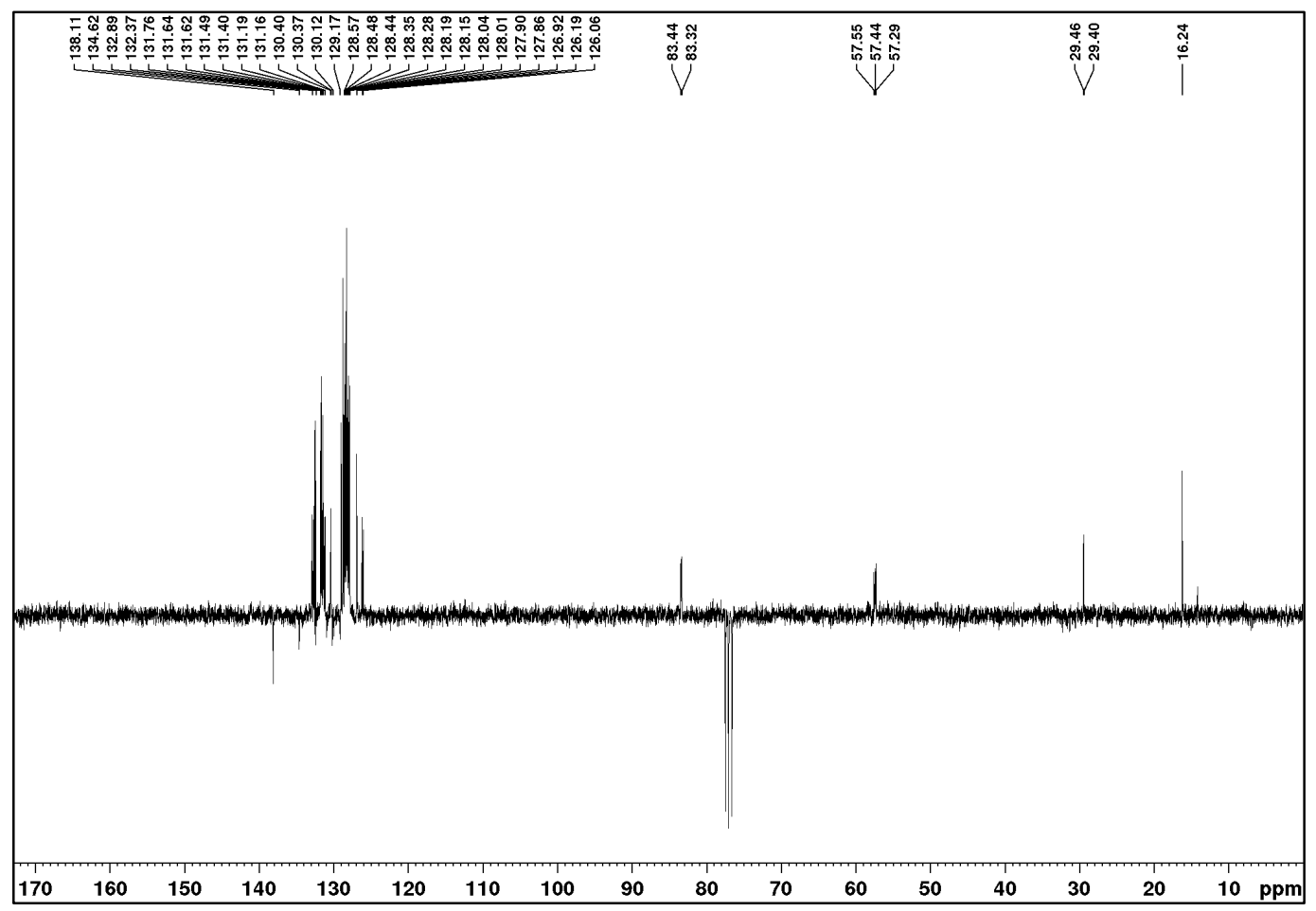

${ }^{31} \mathrm{P}\left\{{ }^{1} \mathrm{H}\right\} \mathrm{NMR}\left(121.5 \mathrm{MHz}, \mathrm{CDCl}_{3}\right)$

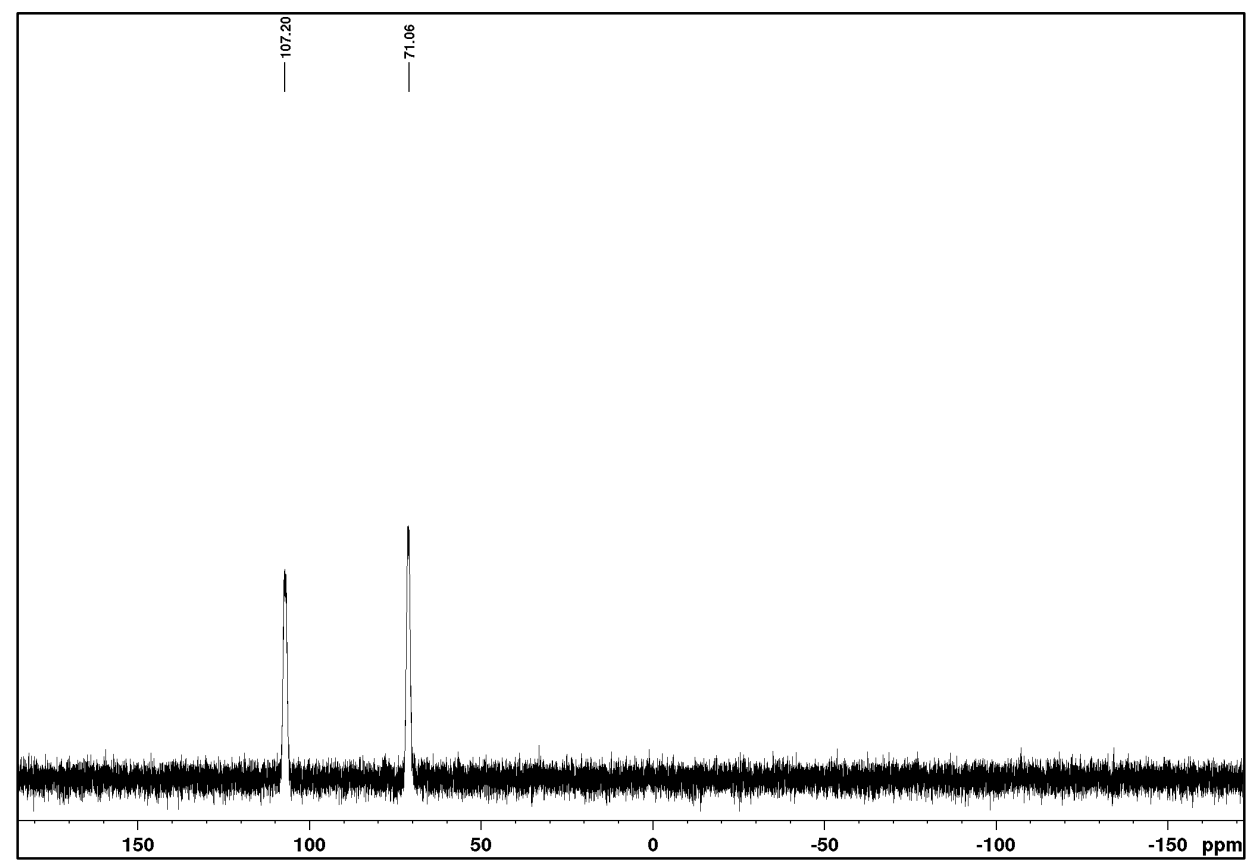


$\left(R_{\mathrm{p}}\right)-(-)-\mathrm{N}-[(1 R, 2 S), \quad$ 2-(Phenyl-o-tolylphosphinito-borane)-1-methyl-2-phenylethyl], N-methyl aminodiphenylphosphine-borane 21d.

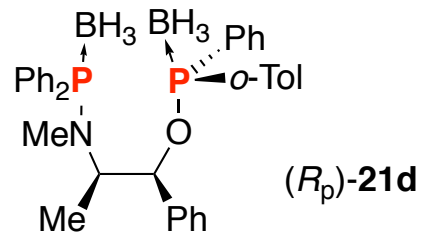

${ }^{1} \mathrm{H}$ NMR $\left(300 \mathrm{MHz}, \mathrm{CDCl}_{3}\right)$

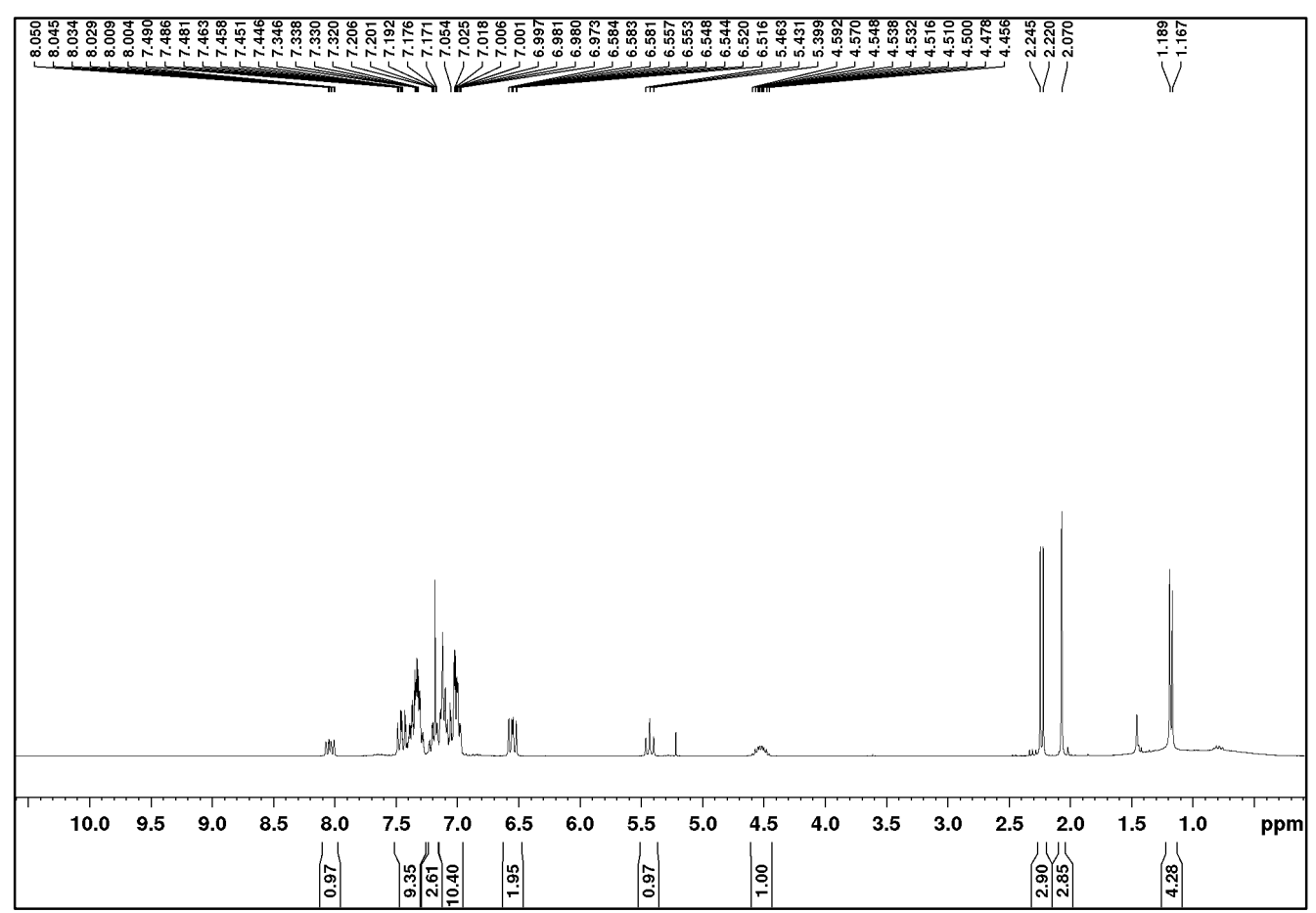

${ }^{13} \mathrm{C}\left\{{ }^{1} \mathrm{H}\right\}$ J-mod NMR $\left(75.5 \mathrm{MHz}, \mathrm{CDCl}_{3}\right)$

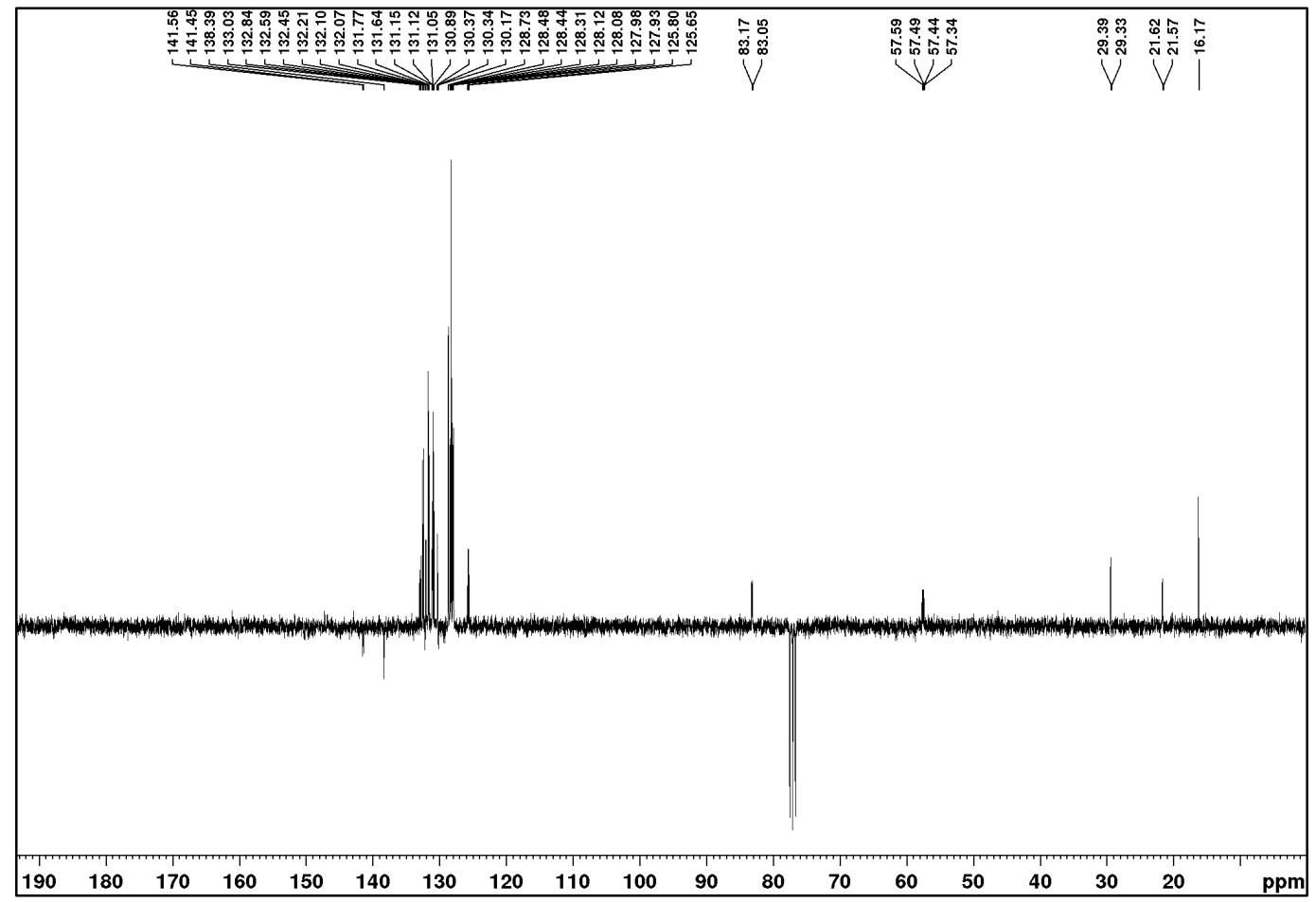


${ }^{31} \mathrm{P}\left\{{ }^{1} \mathrm{H}\right\}$ NMR (121.5 MHz, $\left.\mathrm{CDCl}_{3}\right)$

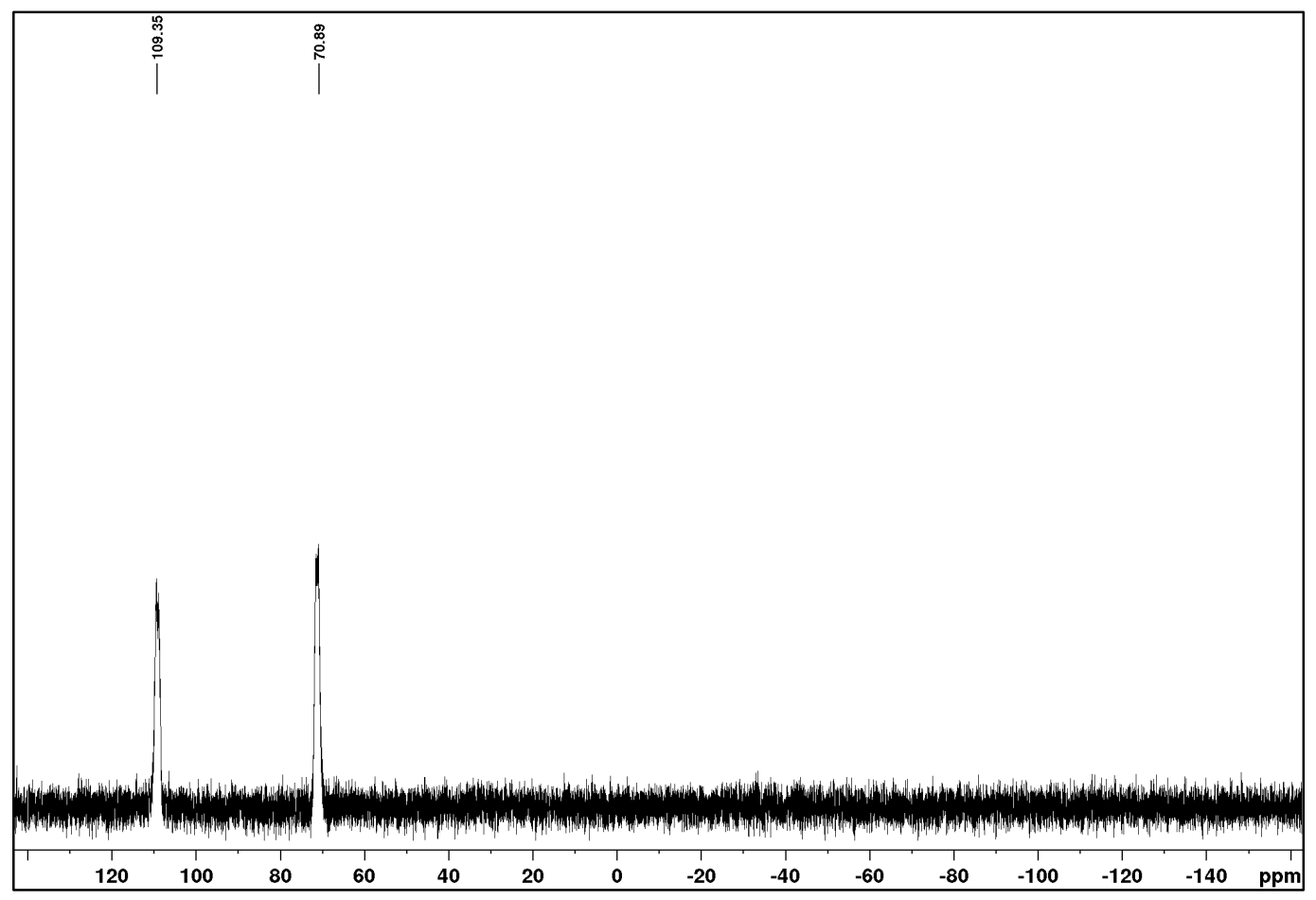

$\left(R_{\mathrm{p}}\right)-(-)-\mathrm{N}-[(1 R, 2 S), \quad 2-($ Phenyl-p-tolylphosphinito-borane)-1-methyl-2-phenylethyl], N-methyl aminodiphenylphosphine-borane 21e.

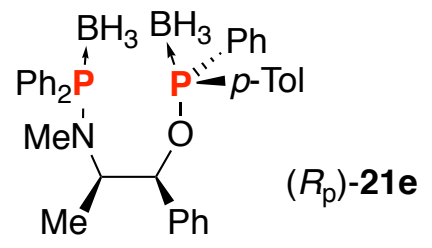

${ }^{1} \mathrm{H} \mathrm{NMR}\left(500 \mathrm{MHz}, \mathrm{CDCl}_{3}\right.$ )

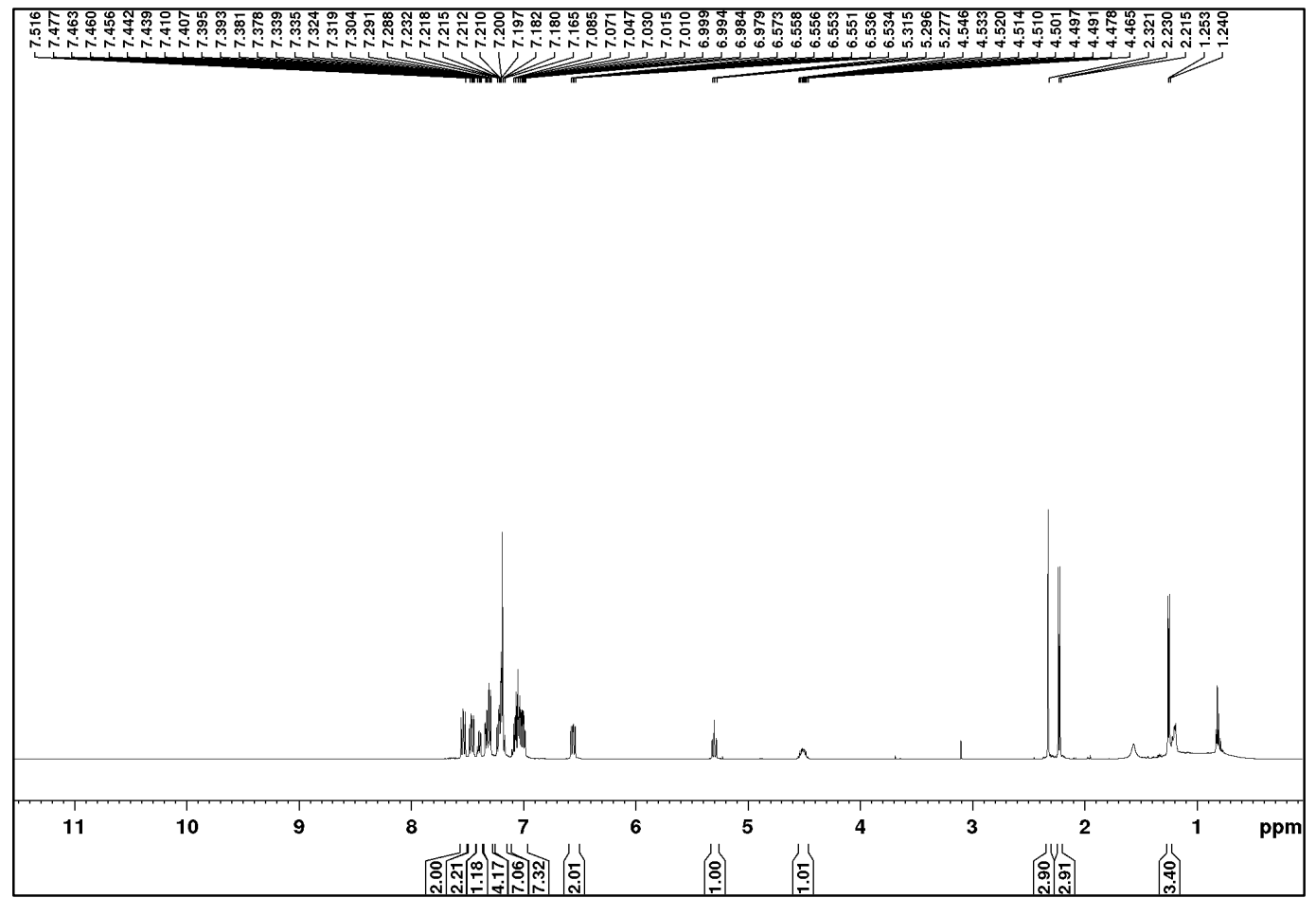


${ }^{13} \mathrm{C}\left\{{ }^{1} \mathrm{H}\right\} \mathrm{J}-\bmod$ NMR $\left(125.8 \mathrm{MHz}, \mathrm{CDCl}_{3}\right)$

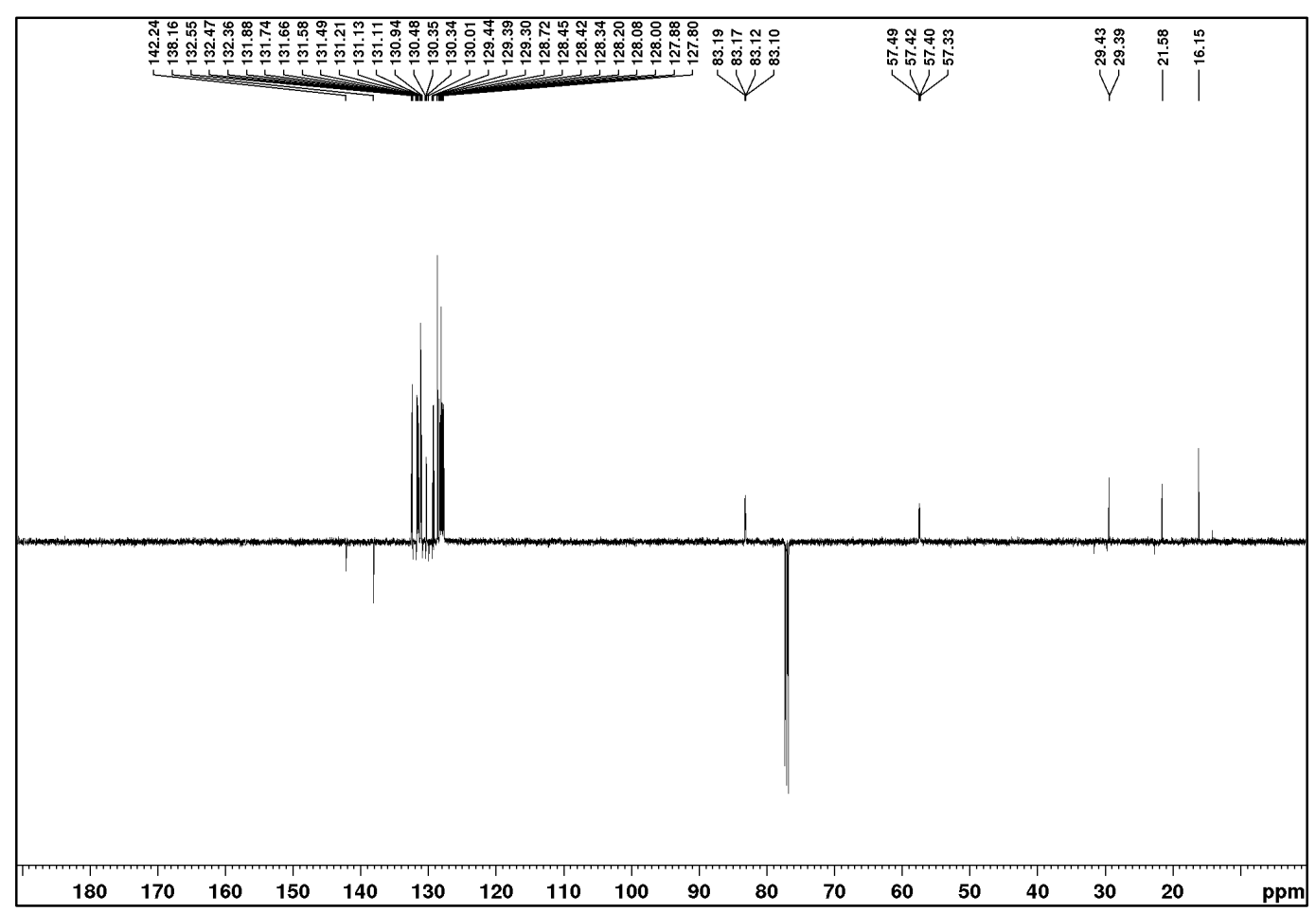

${ }^{31} \mathrm{P}\left\{{ }^{1} \mathrm{H}\right\}$ NMR $\left(202.5 \mathrm{MHz}, \mathrm{CDCl}_{3}\right)$

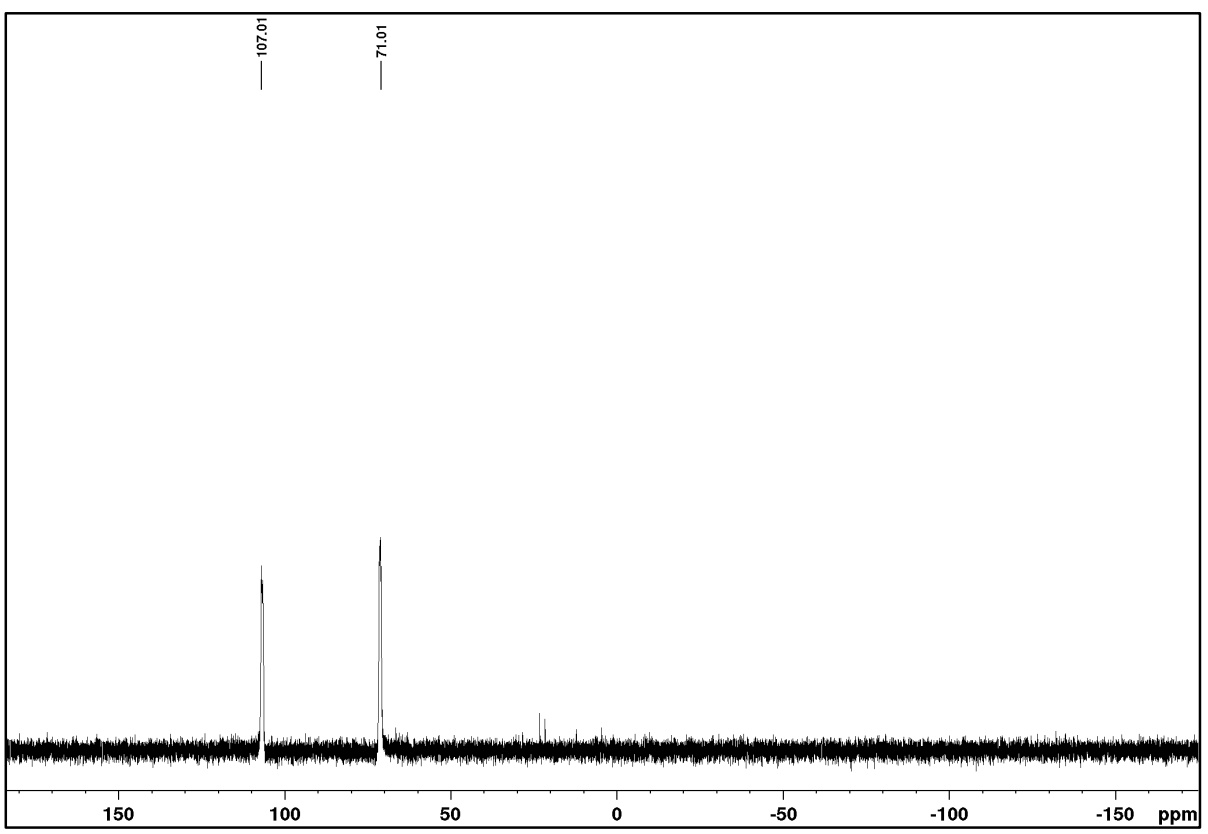


$\left(R_{\mathrm{p}}\right)-(-)-\mathrm{N}-[(1 R, 2 S), \quad$ 2-(Phenyl-o-biphenylphosphinito-borane)-1-methyl-2-phenylethyl], methylaminodiphenylphosphine-borane $21 f$.

${ }^{1} \mathrm{H}$ NMR (500 MHz, $\left.\mathrm{CD}_{2} \mathrm{Cl}_{2}\right)$<smiles>[B][PH]([B])(OC(Pc1ccccc1)[C@@H](C)P(c1ccccc1)c1ccccc1)c1ccccc1</smiles>

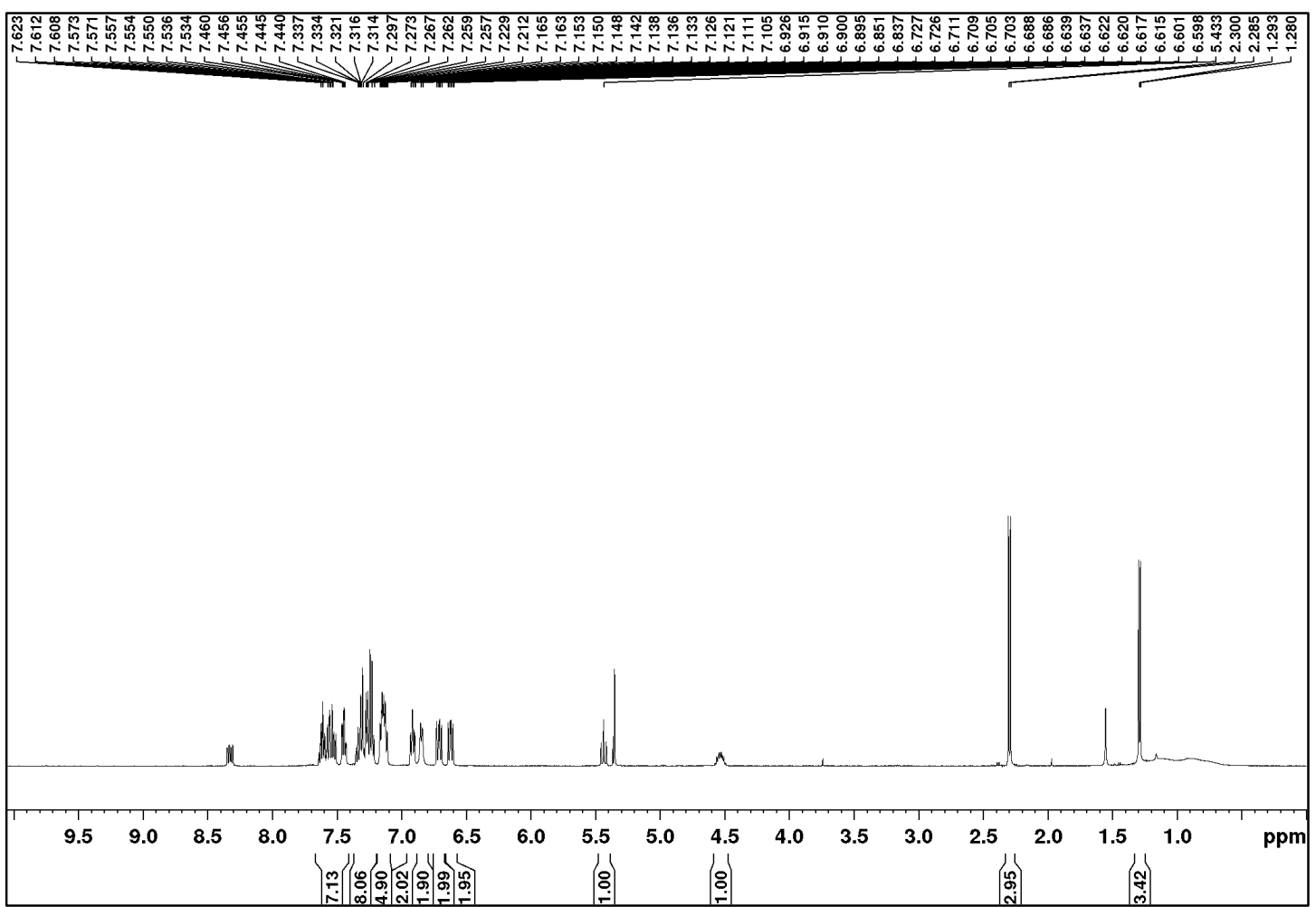

${ }^{13} \mathrm{C}\left\{{ }^{1} \mathrm{H}\right\}$ J-mod NMR (125.8 $\left.\mathrm{MHz}, \mathrm{CD}_{2} \mathrm{Cl}_{2}\right)$

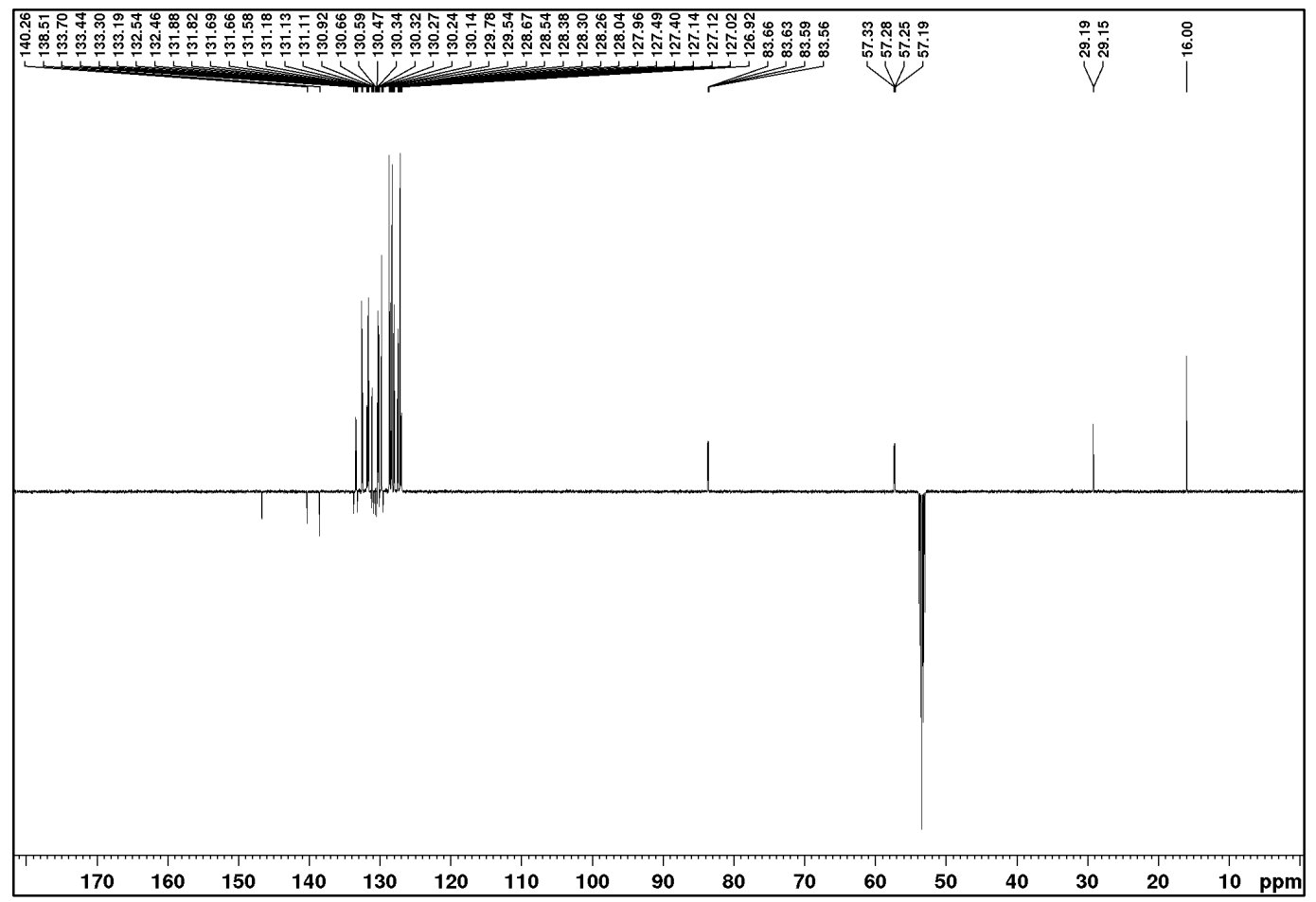


${ }^{31} \mathrm{P}\left\{{ }^{1} \mathrm{H}\right\} \mathrm{NMR}\left(202.5 \mathrm{MHz}, \mathrm{CD}_{2} \mathrm{Cl}_{2}\right)$

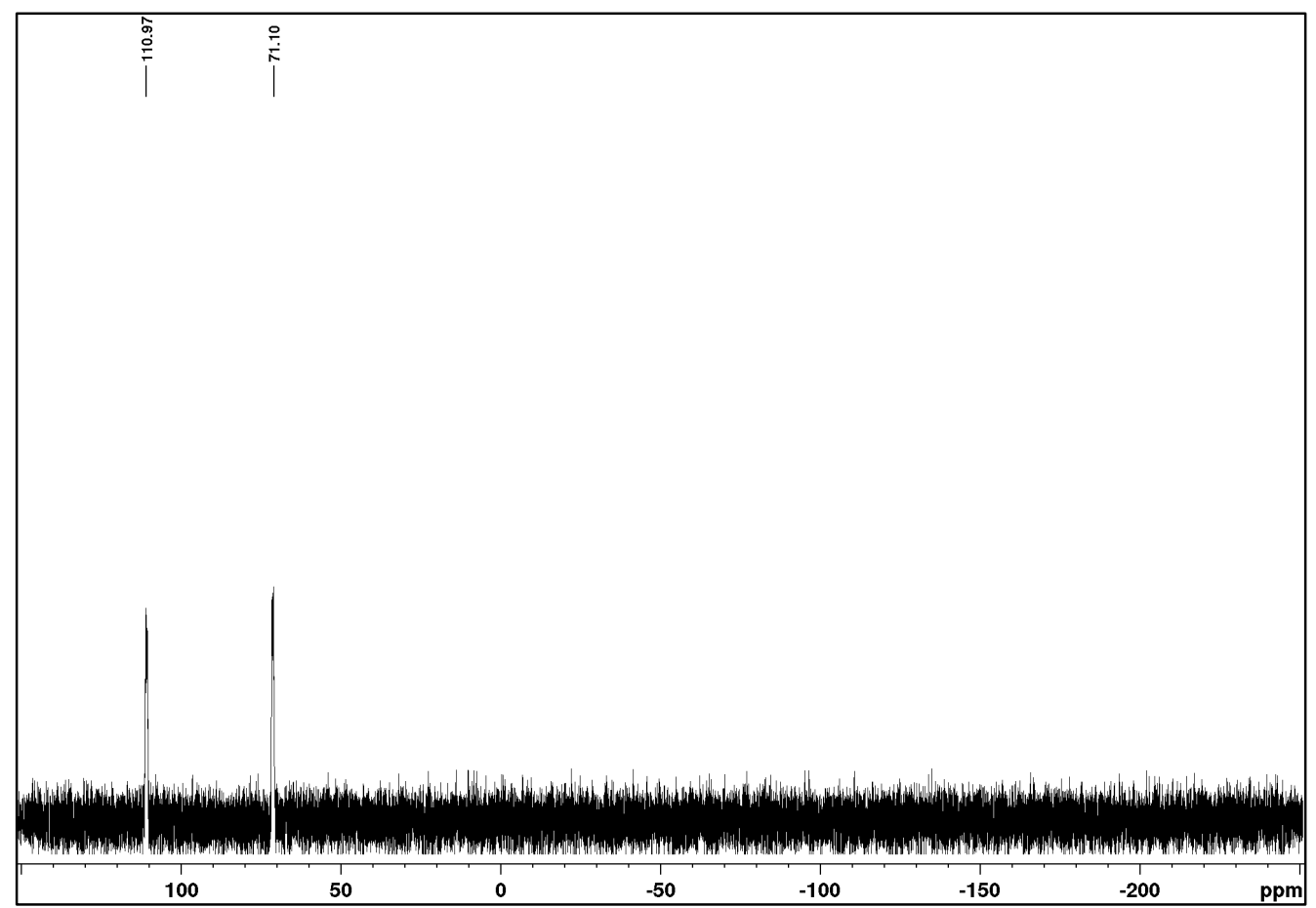

$\left(R_{\mathrm{p}}\right)-(+)-\mathrm{N}-[(1 R, 2 S), 2-$ Ferrocenylphenylphosphinito-borane)-1-methyl-2-phenylethyl], N-methyl aminodiphenylphosphine-borane $\mathbf{2 1 g}$.

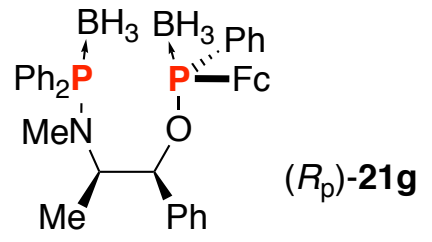

${ }^{1} \mathrm{H} \mathrm{NMR}\left(500 \mathrm{MHz}, \mathrm{CDCl}_{3}\right)$

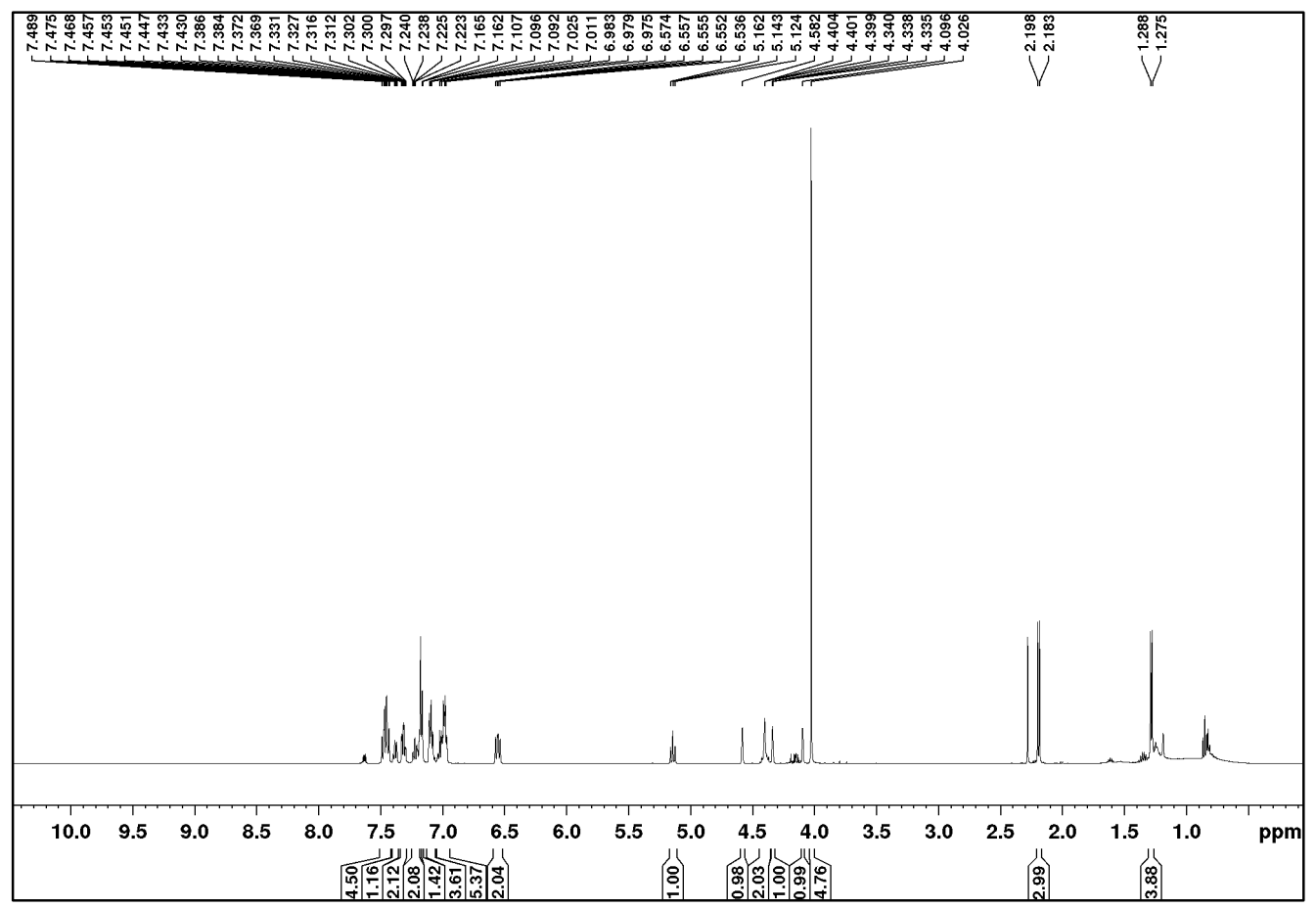


${ }^{13} \mathrm{C}\left\{{ }^{1} \mathrm{H}\right\} \mathrm{J}-\bmod$ NMR $\left(125.8 \mathrm{MHz}, \mathrm{CDCl}_{3}\right)$

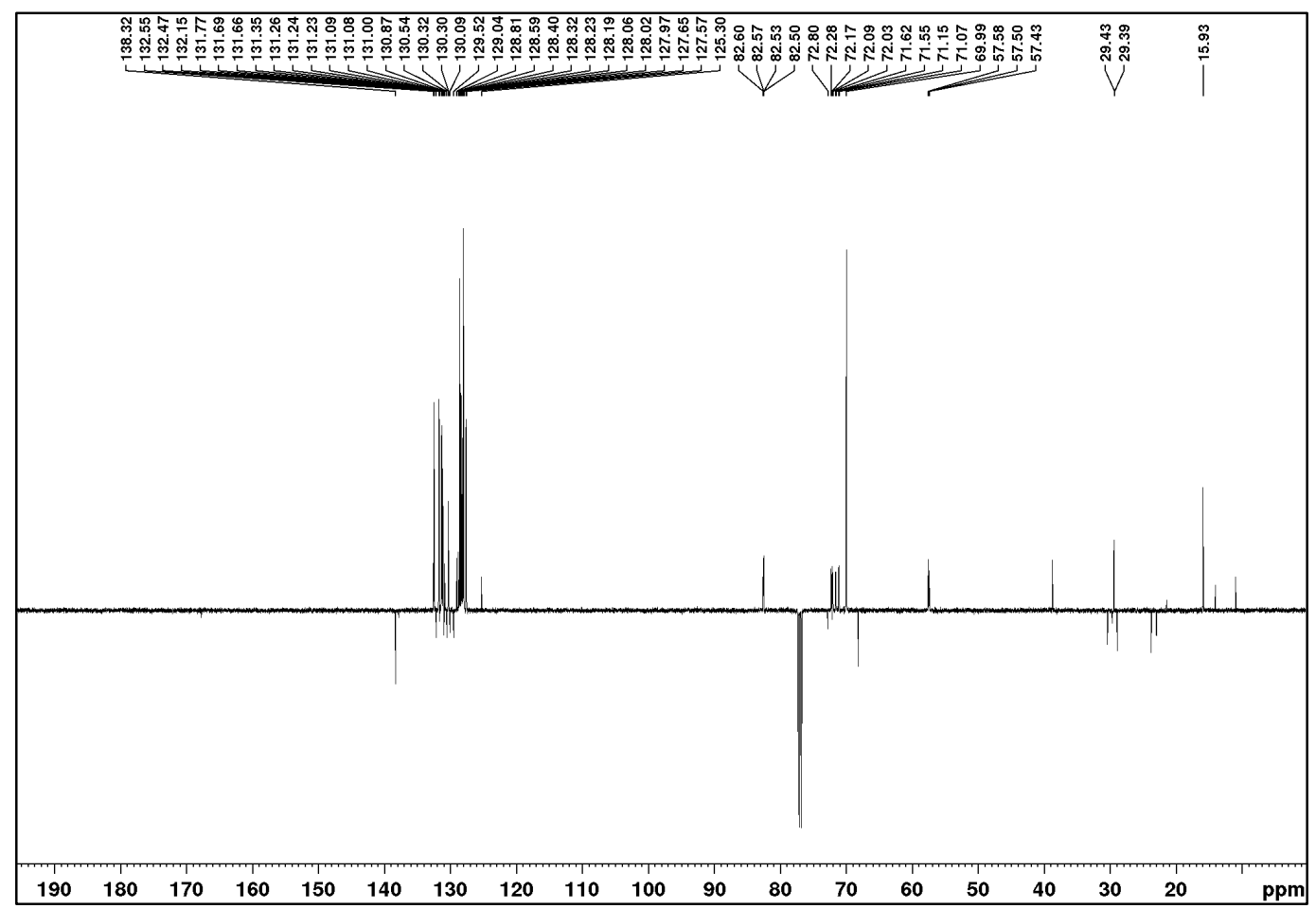

${ }^{31} \mathrm{P}\left\{{ }^{1} \mathrm{H}\right\}$ NMR $\left(202.5 \mathrm{MHz}, \mathrm{CDCl}_{3}\right)$

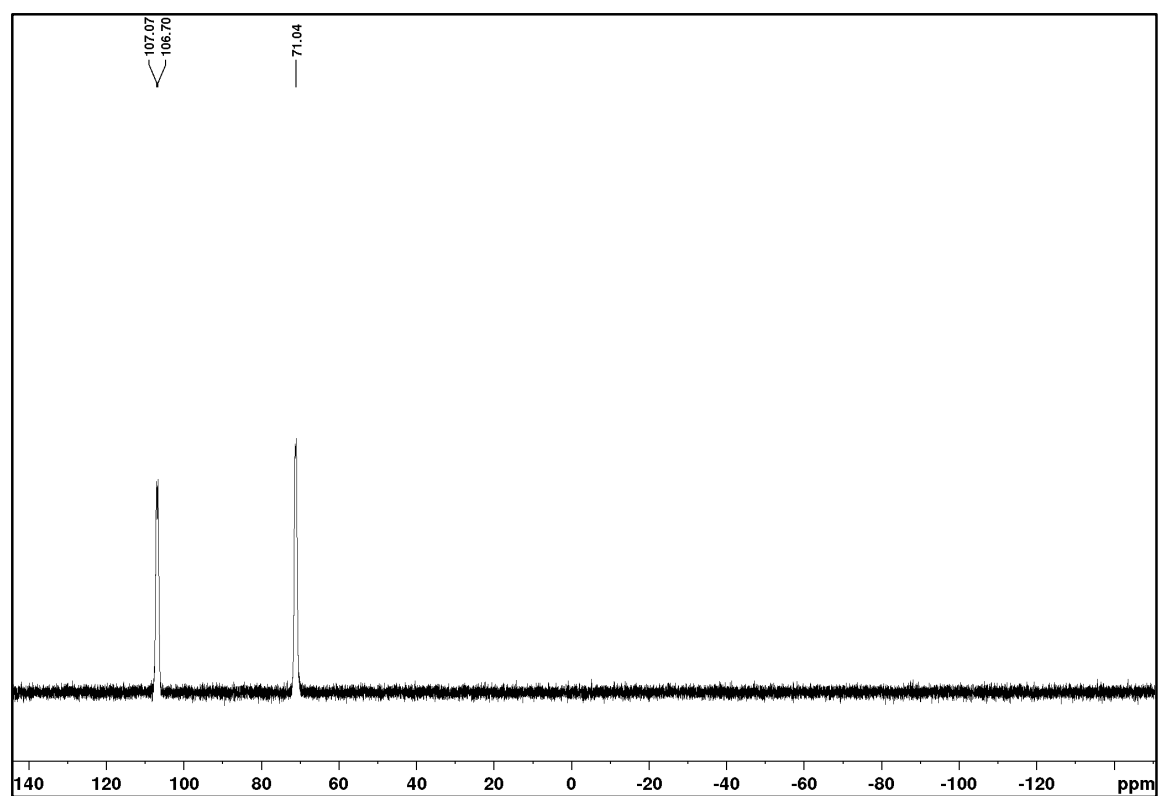


$\left(S_{\mathrm{p}}\right)-(-)-\mathrm{N}-[(1 R, 2 S), \quad$ 2-t-Butylphenylphosphinito-borane)-1-methyl-2-phenylethyl], $\quad \mathrm{N}$-methyl amino-diphenylphosphine-borane $\mathbf{2 1 h}$.

${ }^{1} \mathrm{H} \mathrm{NMR}\left(300 \mathrm{MHz}, \mathrm{CDCl}_{3}\right)$
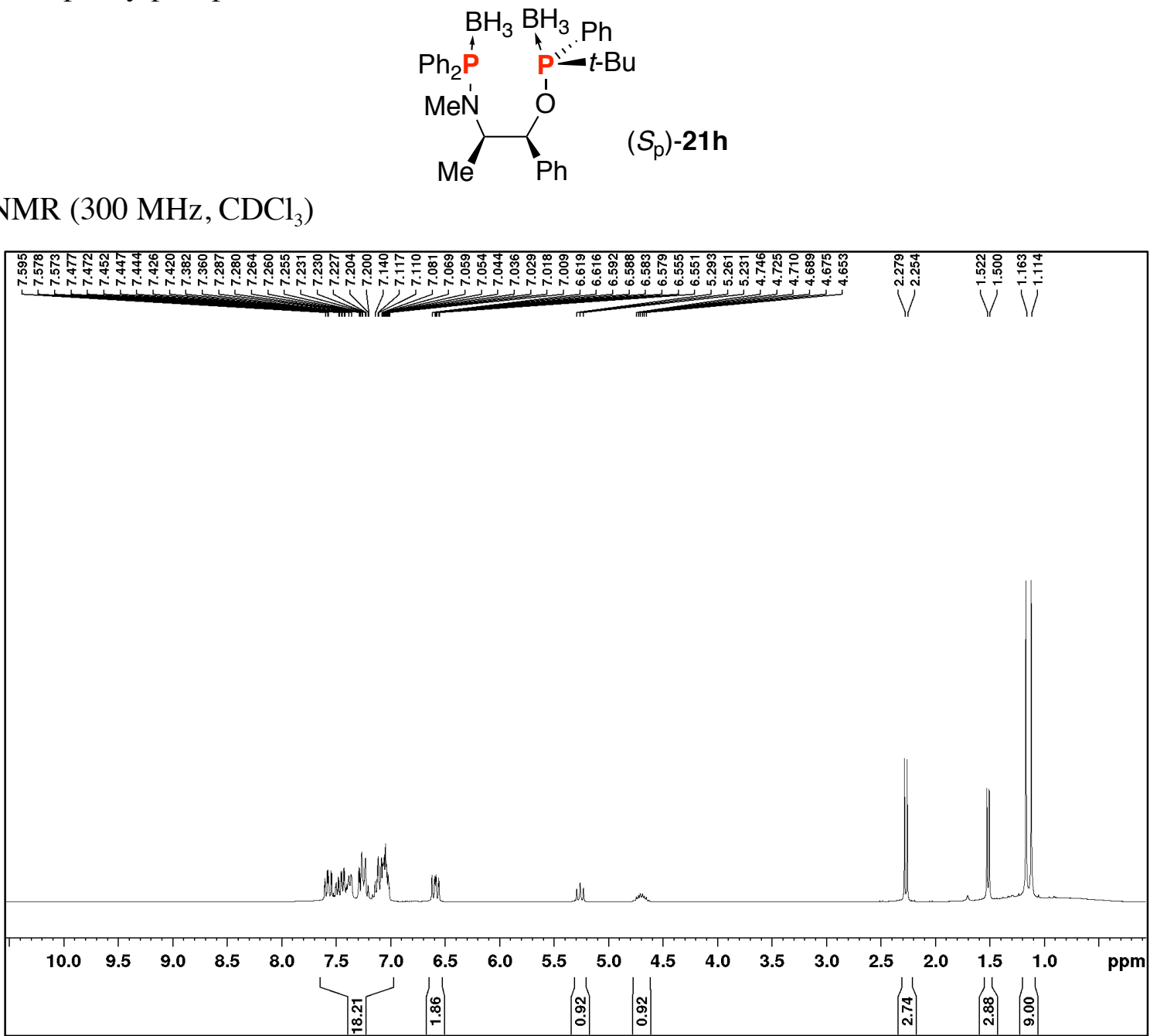

${ }^{13} \mathrm{C}\left\{{ }^{1} \mathrm{H}\right\} \mathrm{NMR}\left(75.5 \mathrm{MHz}, \mathrm{CDCl}_{3}\right)$

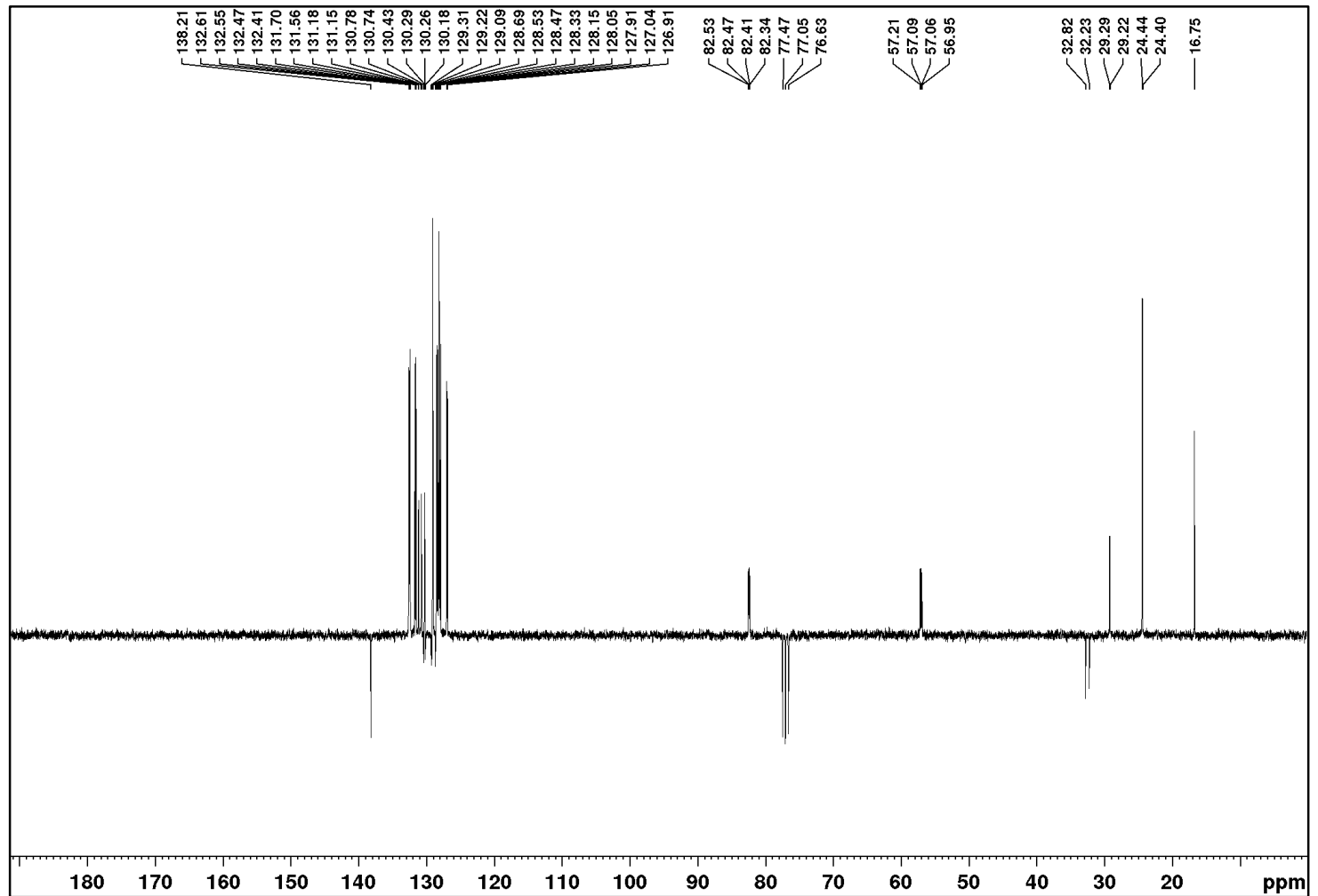


${ }^{31} \mathrm{P}\left\{{ }^{1} \mathrm{H}\right\}$ NMR (121.5 MHz, $\left.\mathrm{CDCl}_{3}\right)$

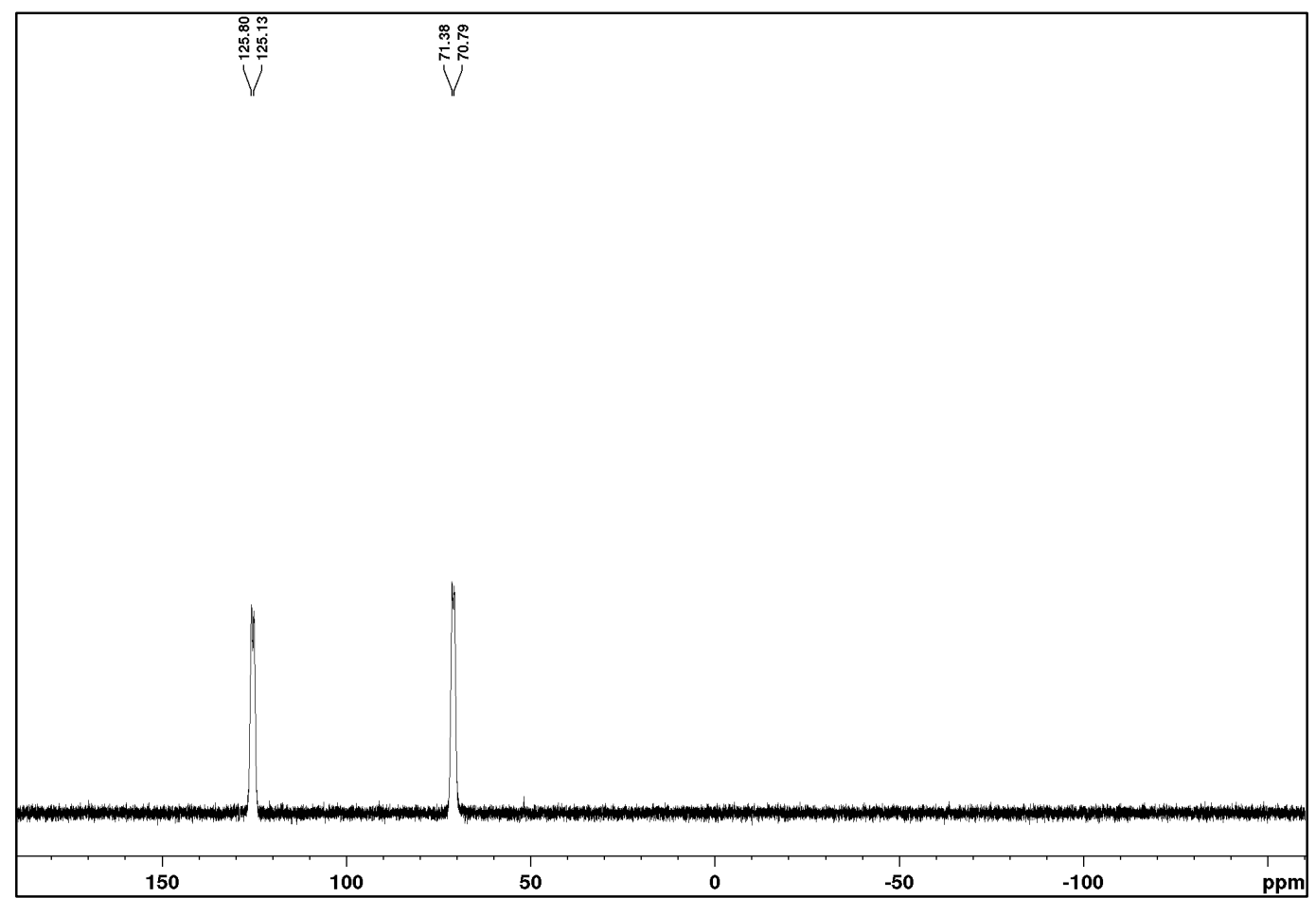

$\left(S_{\mathrm{p}}\right)-(-)-\mathrm{N}-[(1 R, 2 S), \quad$ 2-(Phenyl-o-biphenylphosphinito-borane)-1-methyl-2-phenylethyl], methylamino-diphenylphosphine-borane 21i.

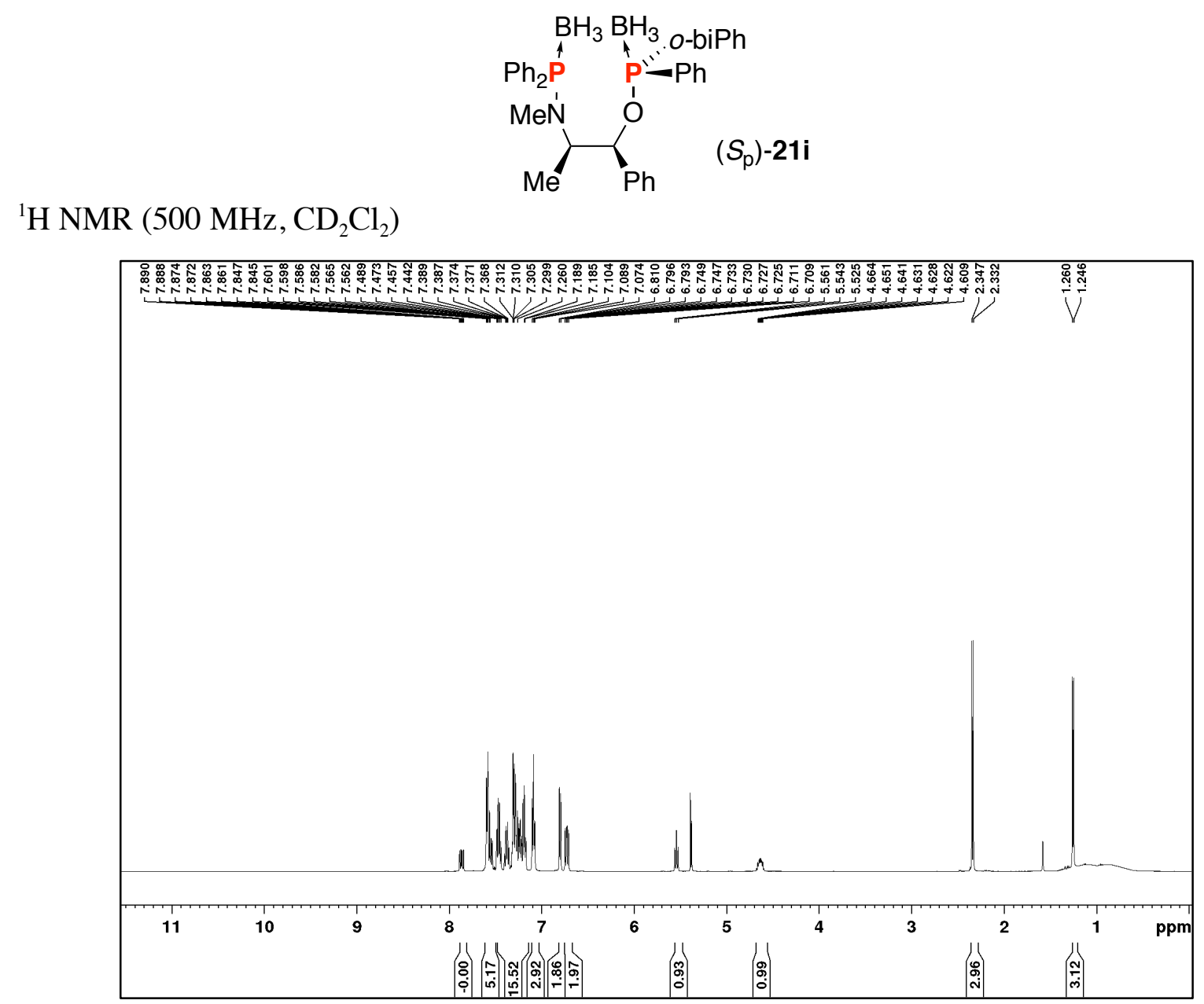


${ }^{13} \mathrm{C}\left\{{ }^{1} \mathrm{H}\right\} \mathrm{J}-\bmod$ NMR $\left(125.8 \mathrm{MHz}, \mathrm{CD}_{2} \mathrm{Cl}_{2}\right)$

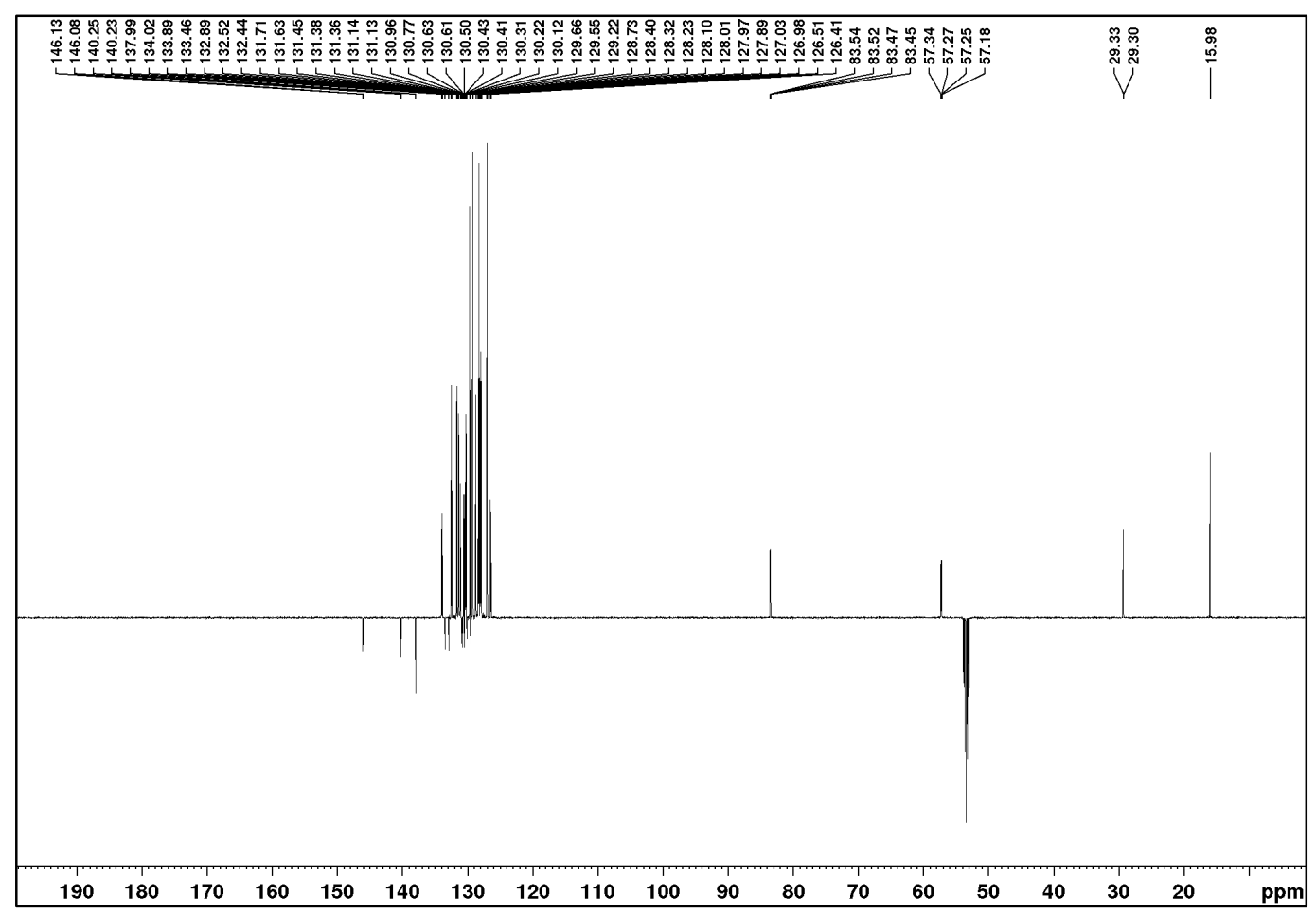

${ }^{31} \mathrm{P}\left\{{ }^{1} \mathrm{H}\right\}$ NMR $\left(202.5 \mathrm{MHz}, \mathrm{CD}_{2} \mathrm{Cl}_{2}\right)$

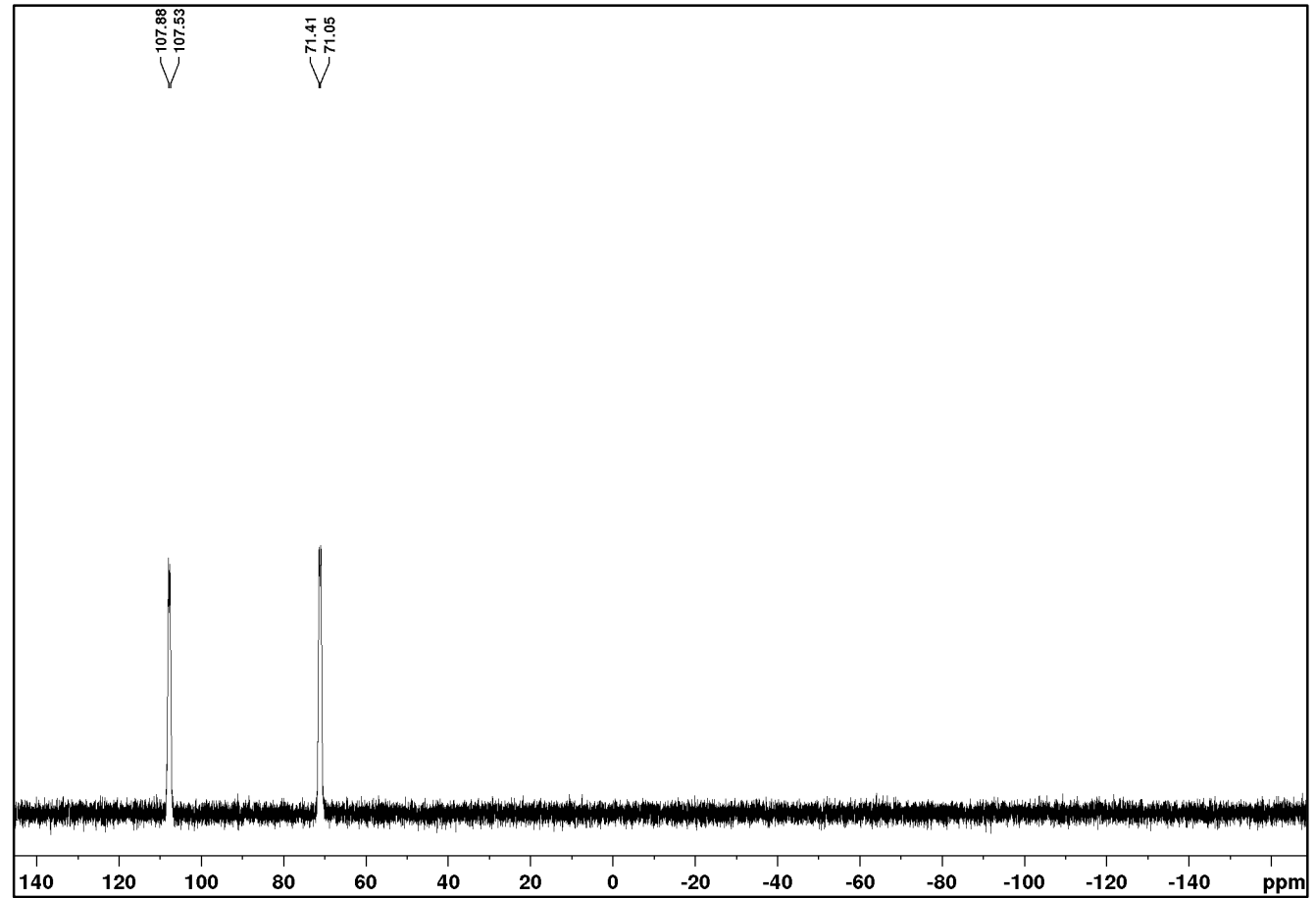


P-chirogenic aminophosphine-phosphinites 20.

$\left(R_{\mathrm{p}}\right)$-N-[(1R,2S)-2-(o-Anisylphenylphosphinito)-1-methyl-2-phenylethyl], N-methylamino diphenylphosphine 20a.

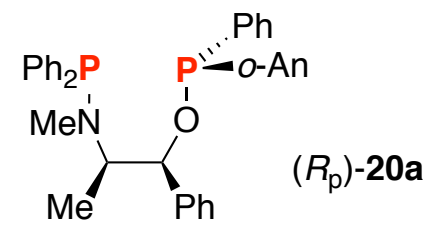

${ }^{1} \mathrm{H}$ NMR (500 MHz, $\mathrm{CDCl}_{3}$ )

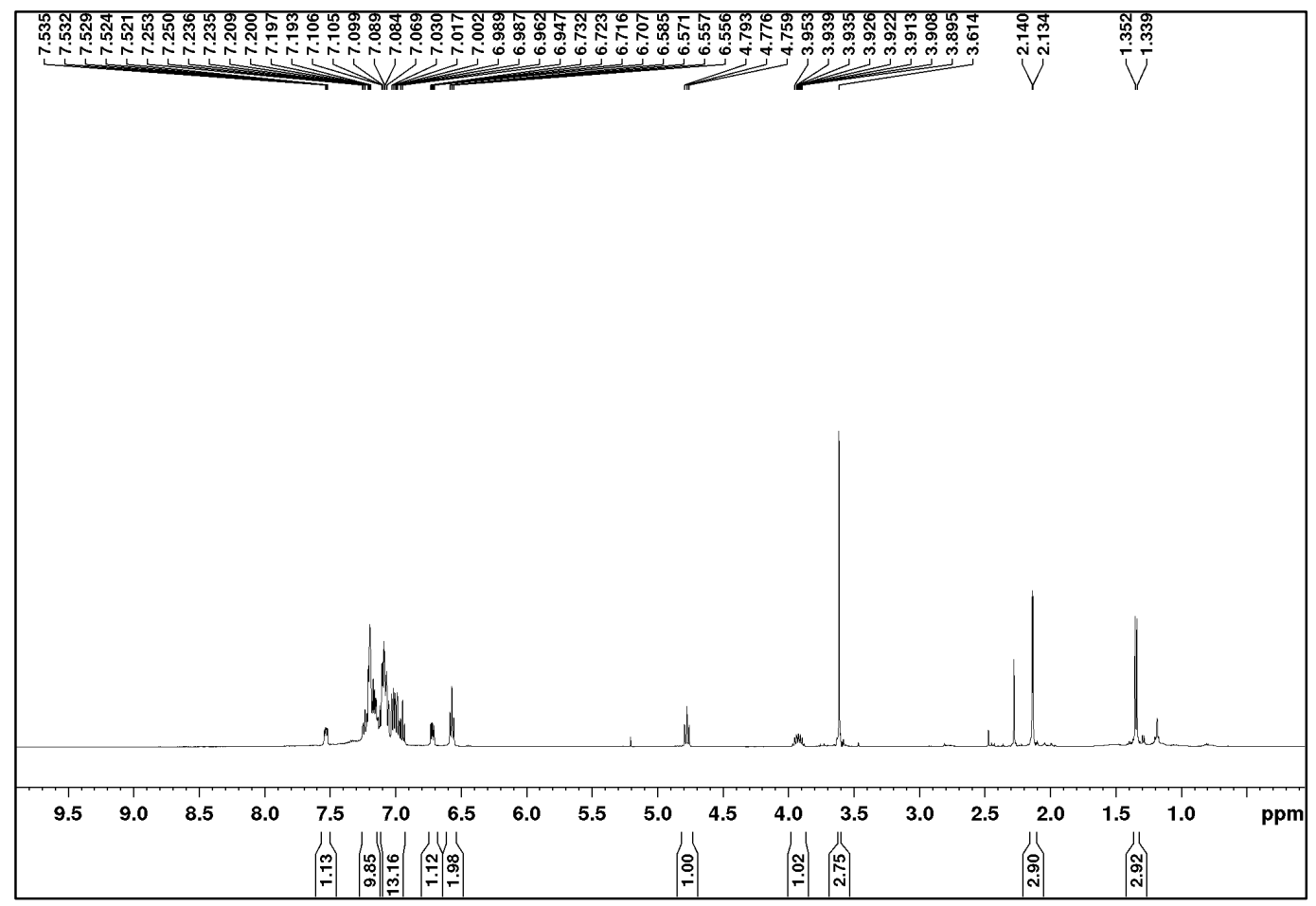

${ }^{31} \mathrm{P}\left\{{ }^{1} \mathrm{H}\right\} \mathrm{NMR}\left(202.5 \mathrm{MHz}, \mathrm{CDCl}_{3}\right)$

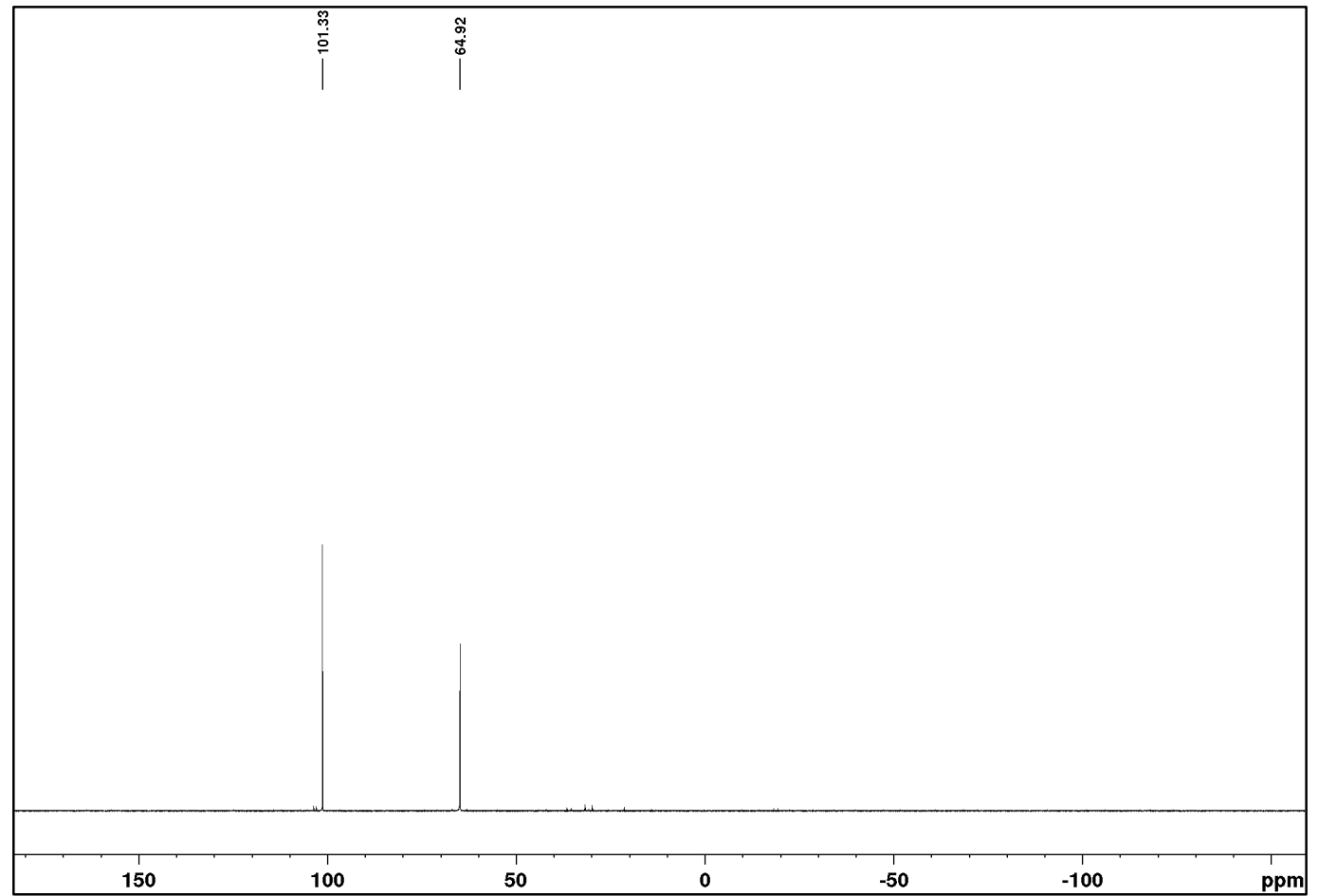


$\left(S_{\mathrm{p}}\right)-\mathrm{N}-[(1 S, 2 R)-2-(o-A n i s y l p h e n y l p h o s p h i n i t o)-1-m e t h y l-2-p h e n y l e t h y l]$,

diphenylphosphine 20a.

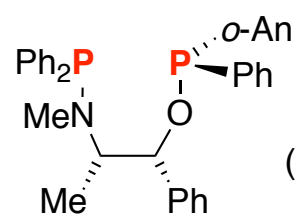

$\left(S_{\mathrm{p}}\right)-20 \mathrm{a}$

${ }^{1} \mathrm{H} \mathrm{NMR}\left(500 \mathrm{MHz}, \mathrm{CDCl}_{3}\right)$

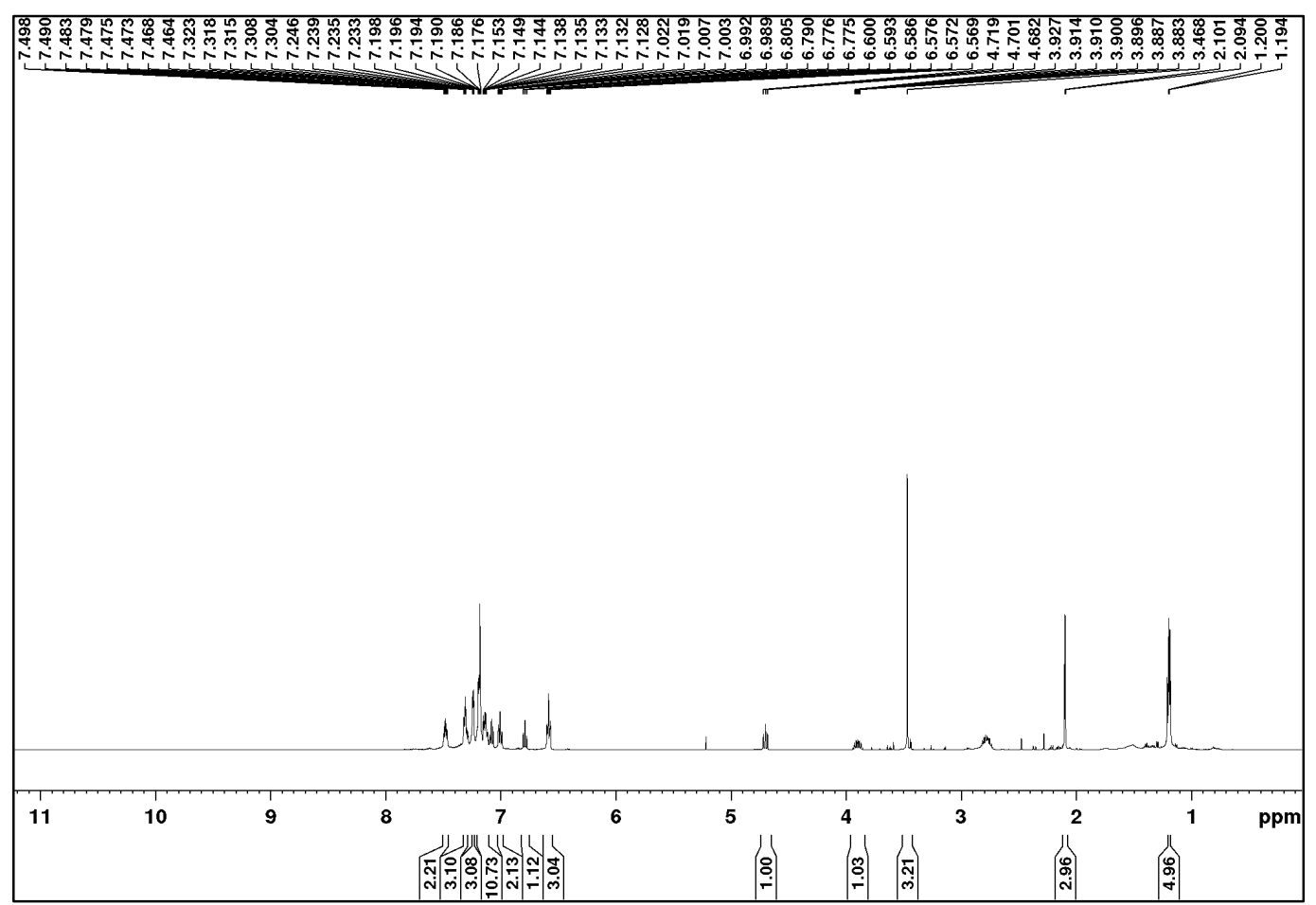

${ }^{31} \mathrm{P}\left\{{ }^{1} \mathrm{H}\right\}$ NMR $\left(202.5 \mathrm{MHz}, \mathrm{CDCl}_{3}\right)$

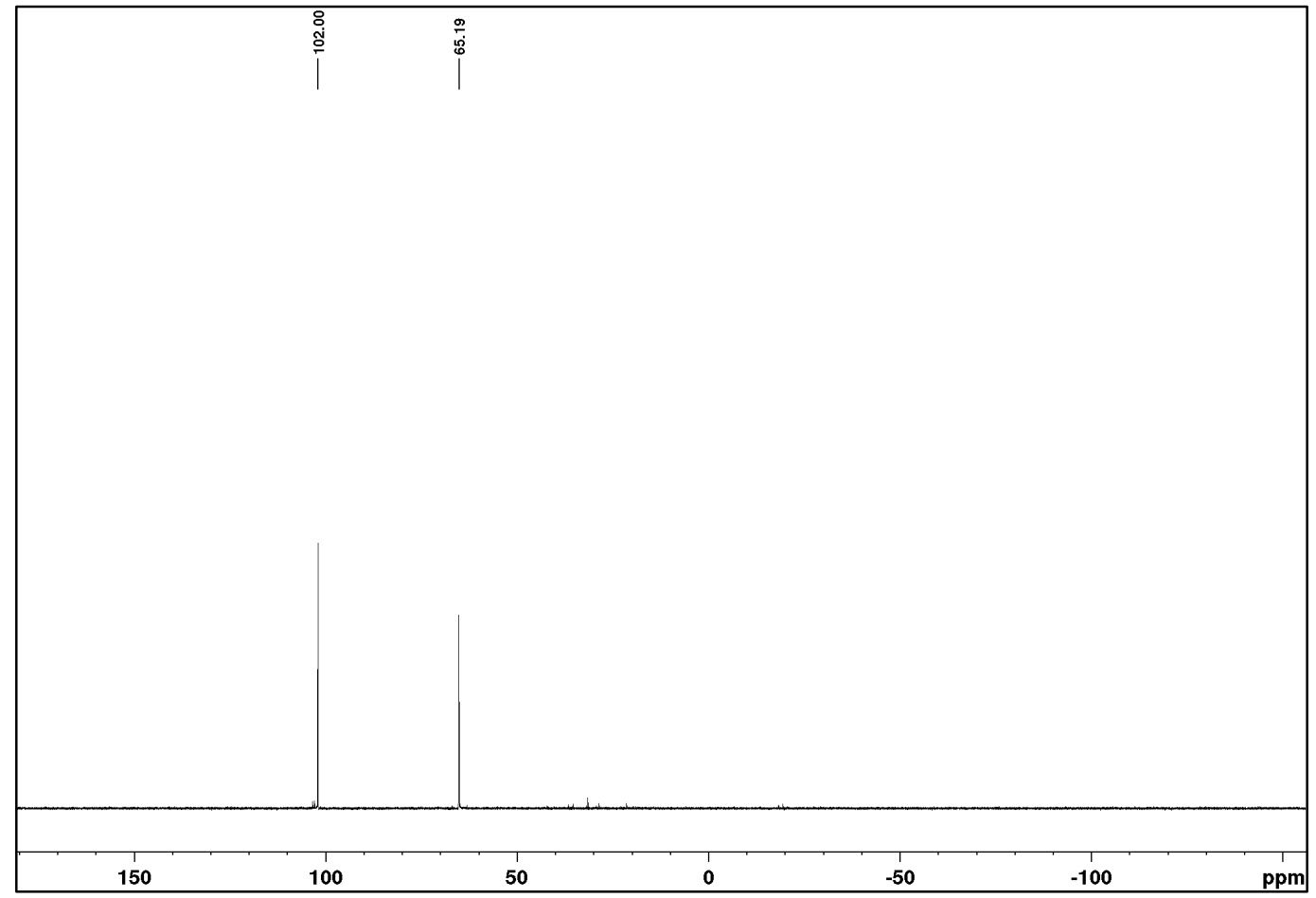


$\left(R_{\mathrm{p}}\right)-\mathrm{N}-[(1 R, 2 S)-2-(\alpha-N a p h t y l p h e n y l p h o s p h i n i t o)-1-m e t h y l-2-p h e n y l e t h y l], \quad$ N-methylamino diphenylphosphine $\mathbf{2 0 b}$.

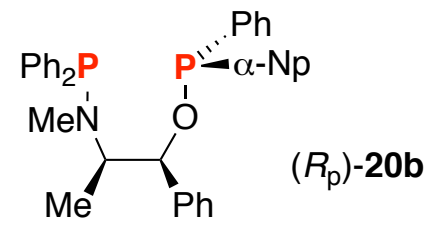

${ }^{1} \mathrm{H}$ NMR $\left(500 \mathrm{MHz}, \mathrm{CDCl}_{3}\right.$ )

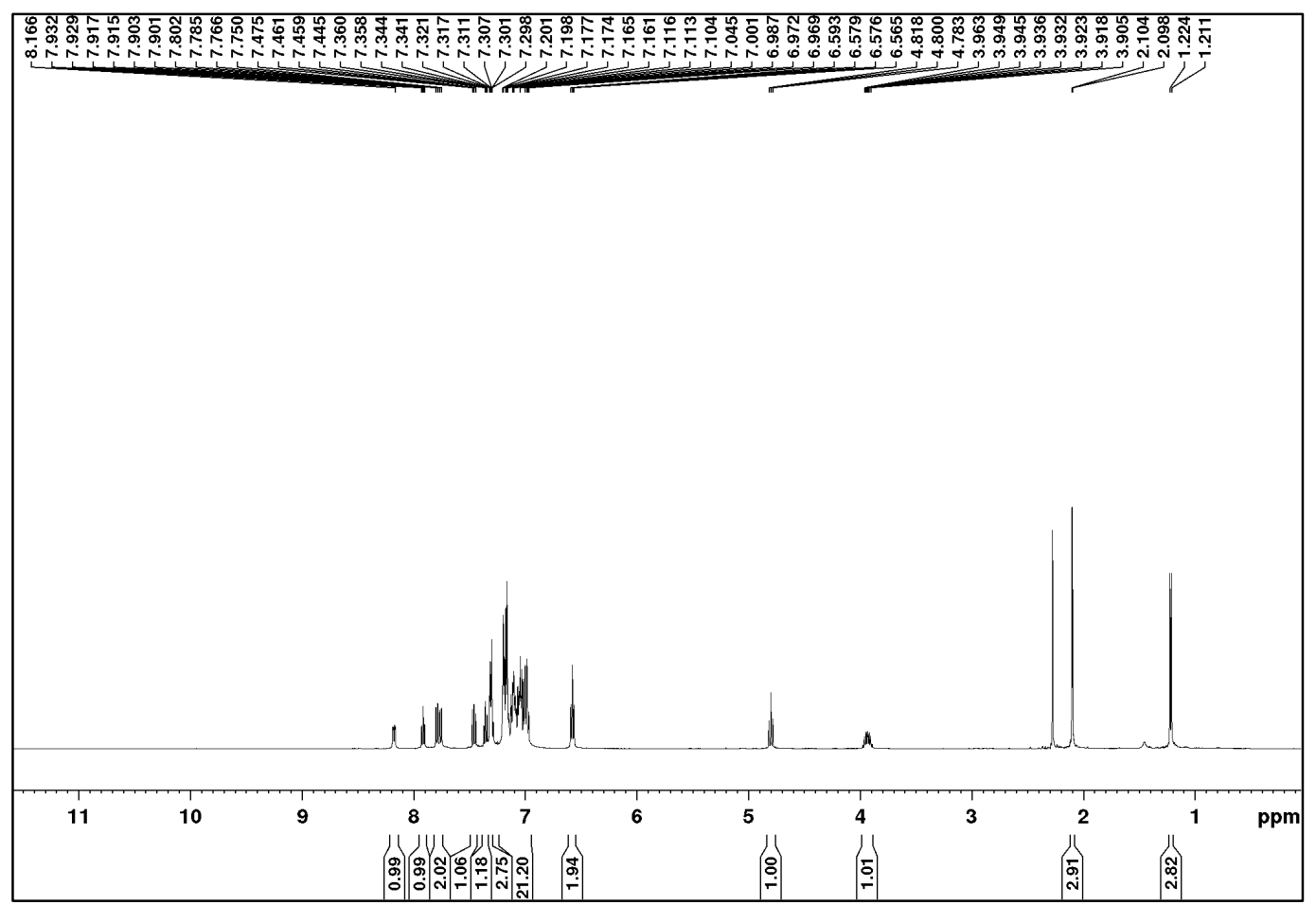

${ }^{31} \mathrm{P}\left\{{ }^{1} \mathrm{H}\right\}$ NMR $\left(202.5 \mathrm{MHz}, \mathrm{CDCl}_{3}\right)$

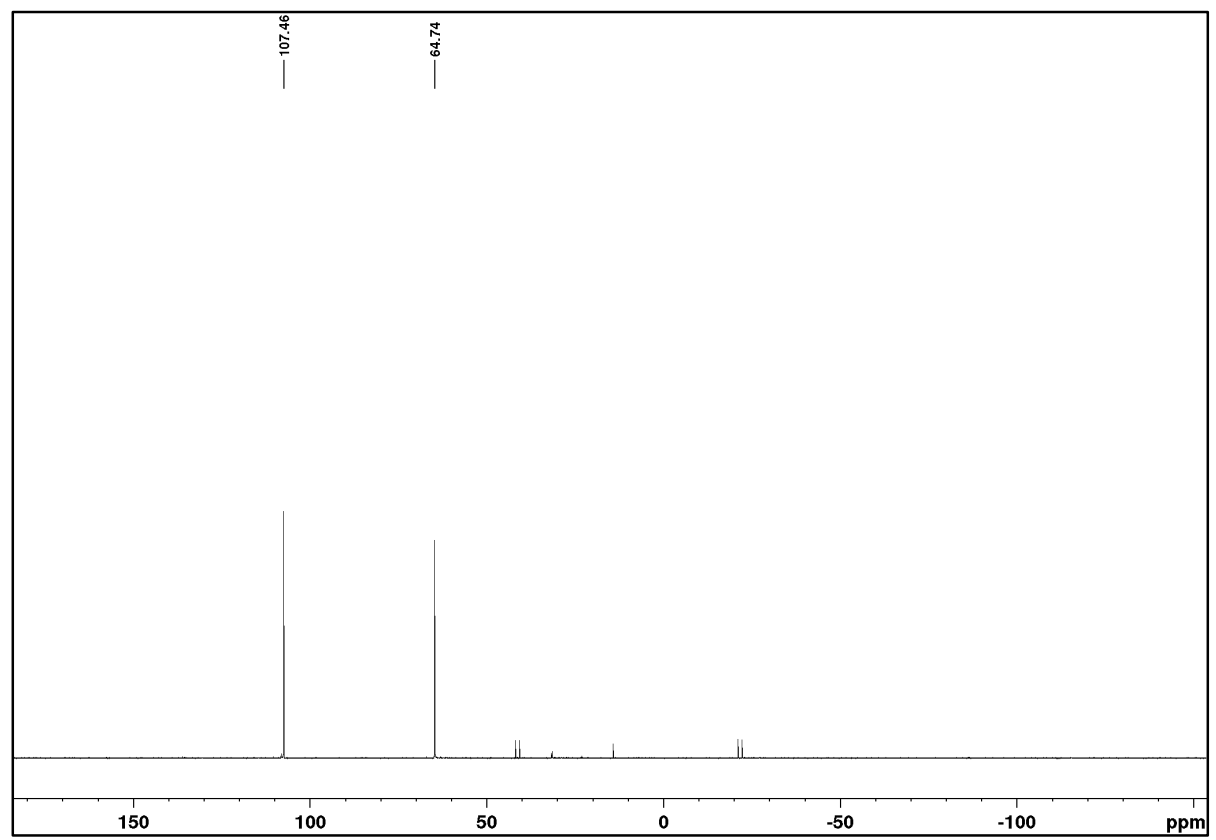


$\left(R_{\mathrm{p}}\right)-\mathrm{N}-[(1 R, 2 S)-2-(\beta-N a p h t y l p h e n y l p h o s p h i n i t o)-1-m e t h y l-2-p h e n y l e t h y l], \quad \mathrm{N}$-methylamino diphenylphosphine 20c.

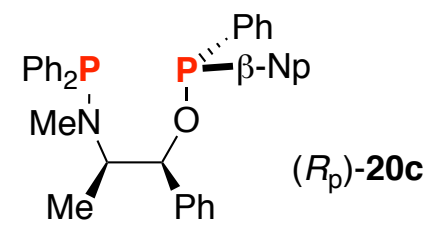

${ }^{1} \mathrm{H} \mathrm{NMR}\left(500 \mathrm{MHz}, \mathrm{CDCl}_{3}\right)$

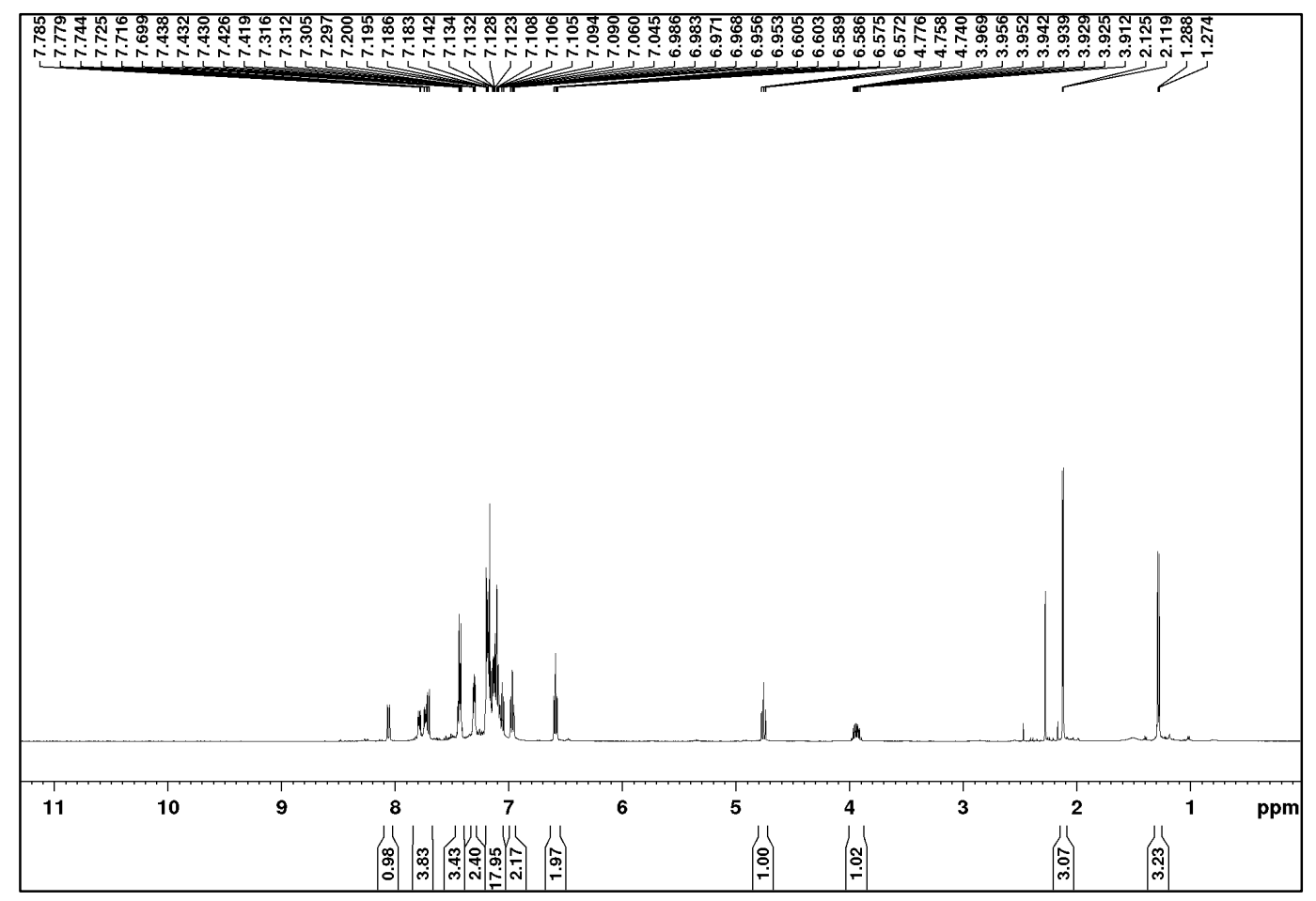

${ }^{31} \mathrm{P}\left\{{ }^{1} \mathrm{H}\right\}$ NMR $\left(202.5 \mathrm{MHz}, \mathrm{CDCl}_{3}\right)$

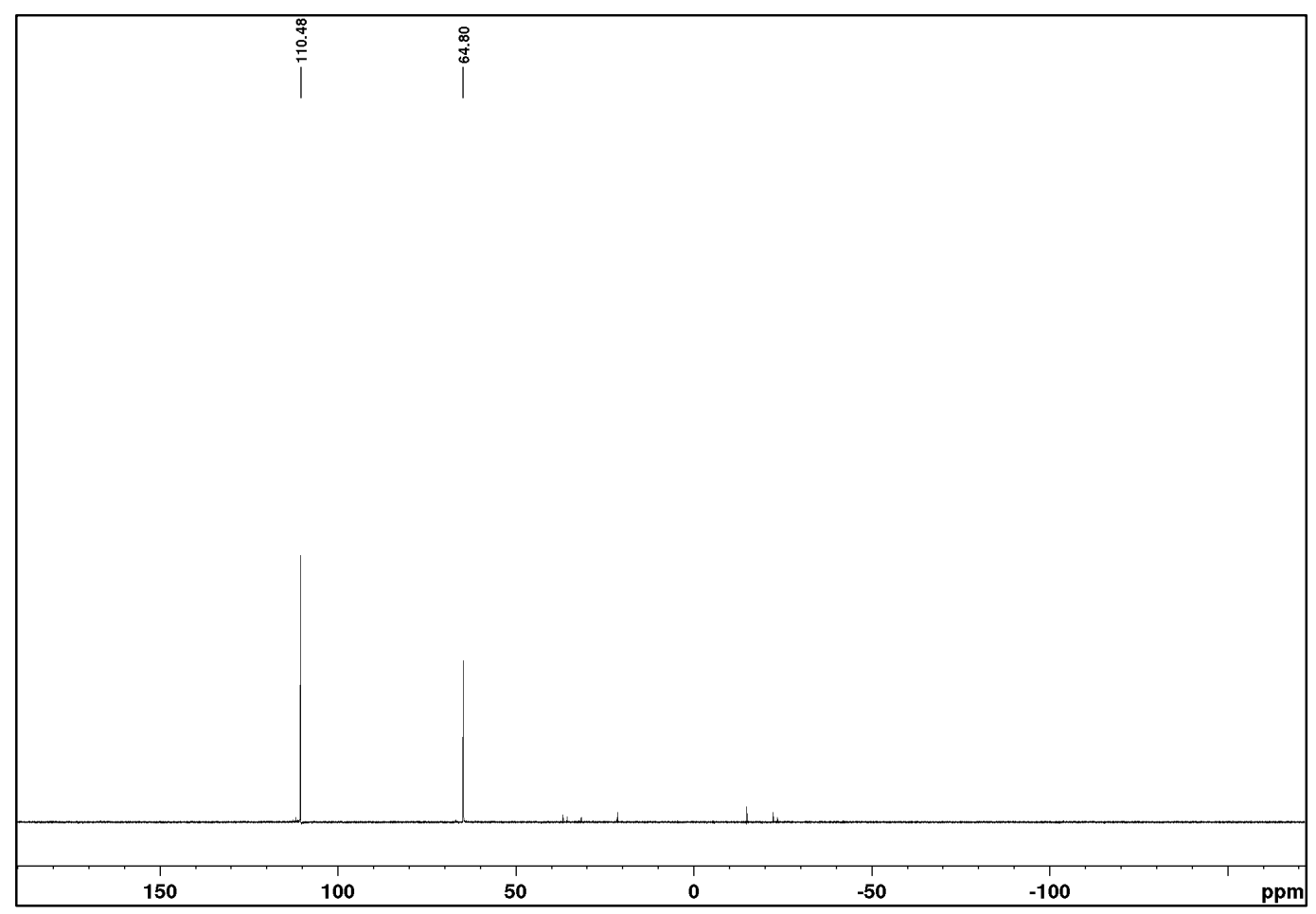


$\left(R_{\mathrm{p}}\right)-\mathrm{N}-[(1 R, 2 S)$-2-(Phenyl-o-tolylphosphinito)-1-methyl-2-phenylethyl], diphenylphosphine 20d.

$$
\mathrm{Ph}_{2} \mathrm{P}=\mathrm{Ph}_{\mathrm{Ph}}^{\mathrm{Ph}}\left(R_{\mathrm{p}}\right)-\mathbf{2 0 d}
$$

${ }^{1} \mathrm{H}$ NMR $\left(500 \mathrm{MHz}, \mathrm{CDCl}_{3}\right)$

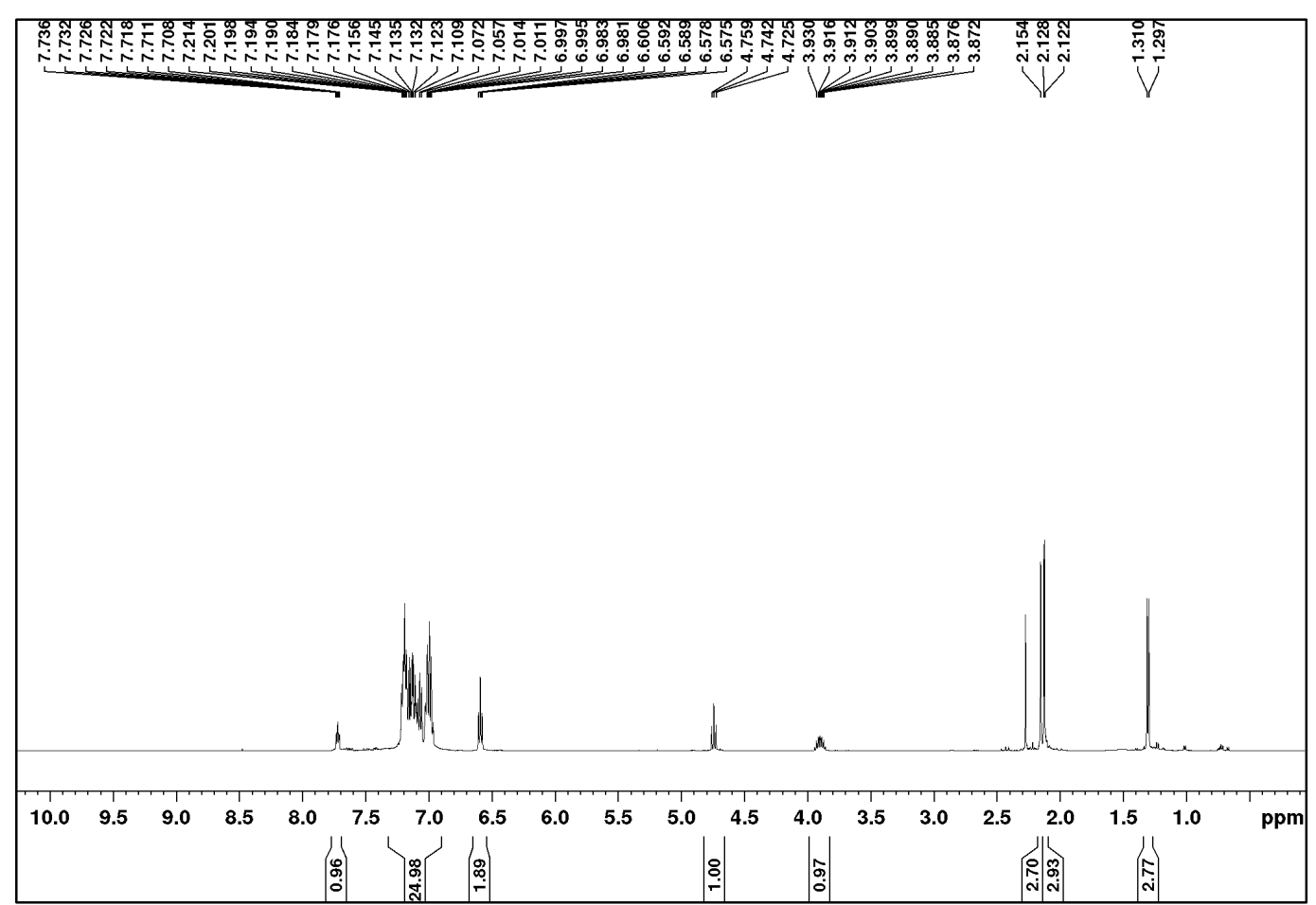

${ }^{31} \mathrm{P}\left\{{ }^{1} \mathrm{H}\right\} \mathrm{NMR}\left(202.5 \mathrm{MHz}, \mathrm{CDCl}_{3}\right)$

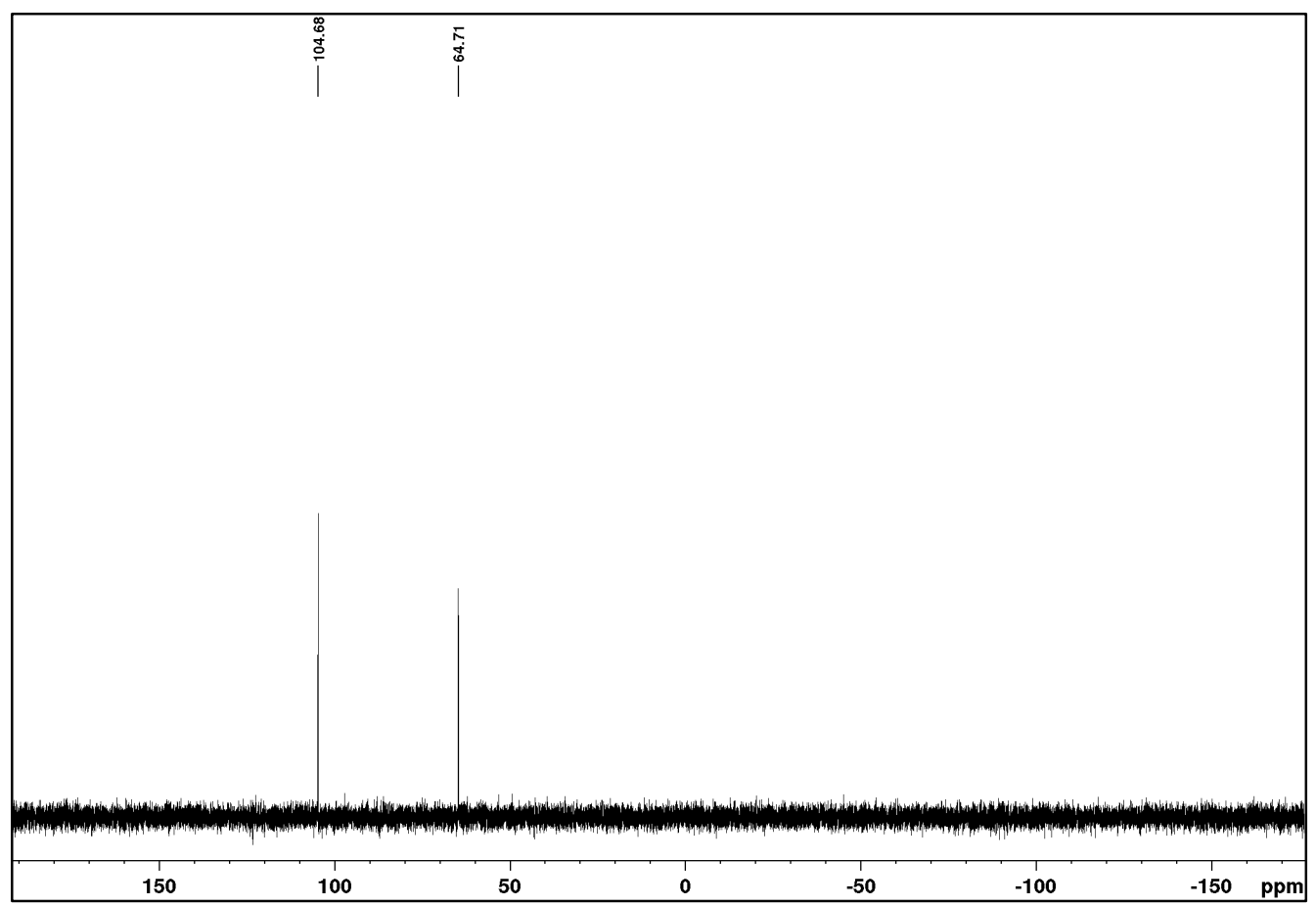


$\left(R_{\mathrm{p}}\right)-\mathrm{N}-[(1 R, 2 S), \quad$ 2-(Phenyl-o-biphenylphosphinito)-1-methyl-2-phenylethyl], N-methylamino diphenylphosphine $20 f$.

$$
\left.{ }_{\mathrm{Ph}}^{\mathrm{Ph}} \mathrm{Ph}_{2}^{\mathrm{P}} R_{\mathrm{p}}\right)-\mathbf{2 0 f}
$$

${ }^{1} \mathrm{H}$ NMR $\left(300 \mathrm{MHz}, \mathrm{CD}_{2} \mathrm{Cl}_{2}\right.$ )

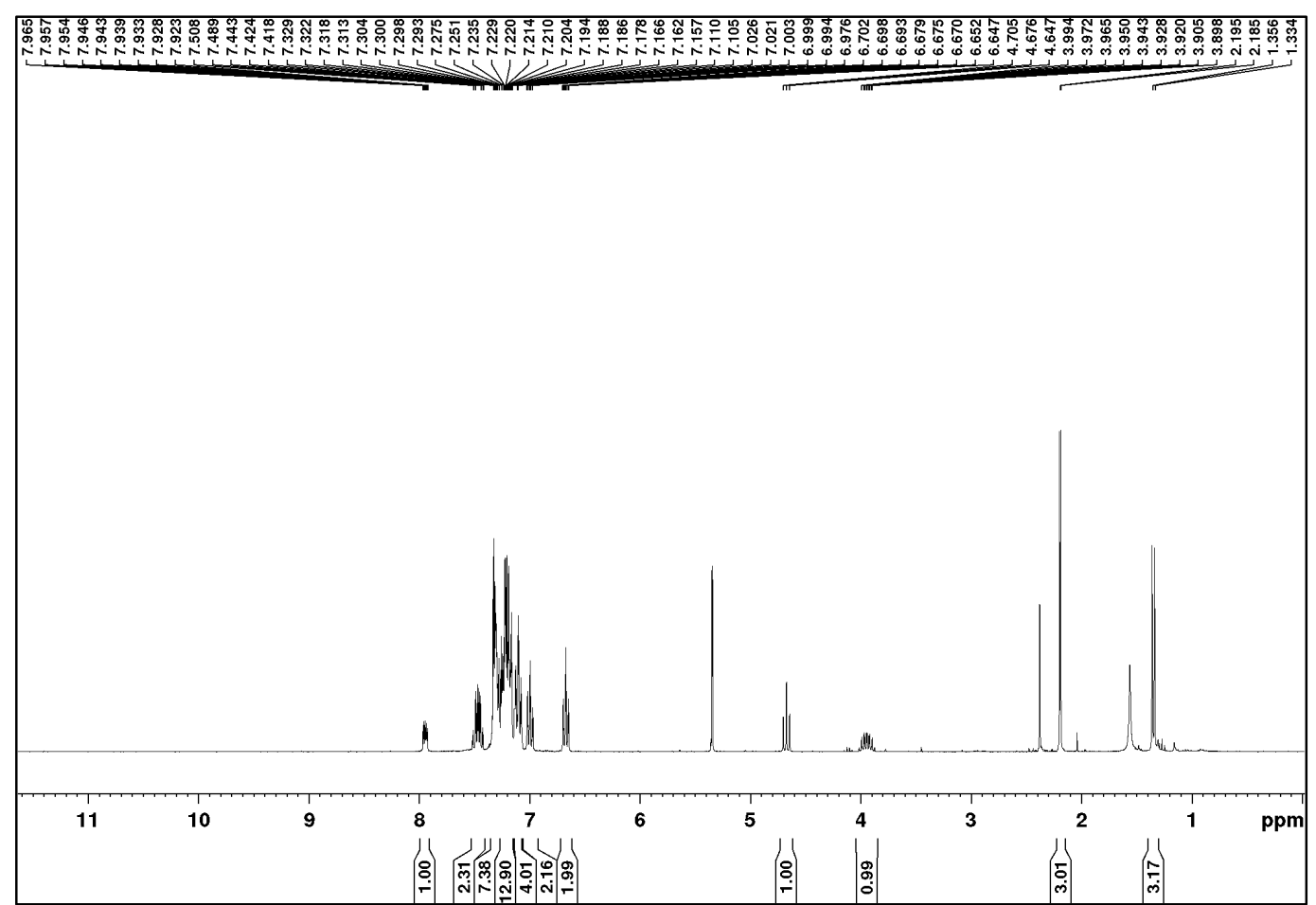

${ }^{31} \mathrm{P}\left\{{ }^{1} \mathrm{H}\right\} \mathrm{NMR}\left(121.5 \mathrm{MHz}, \mathrm{CD}_{2} \mathrm{Cl}_{2}\right)$

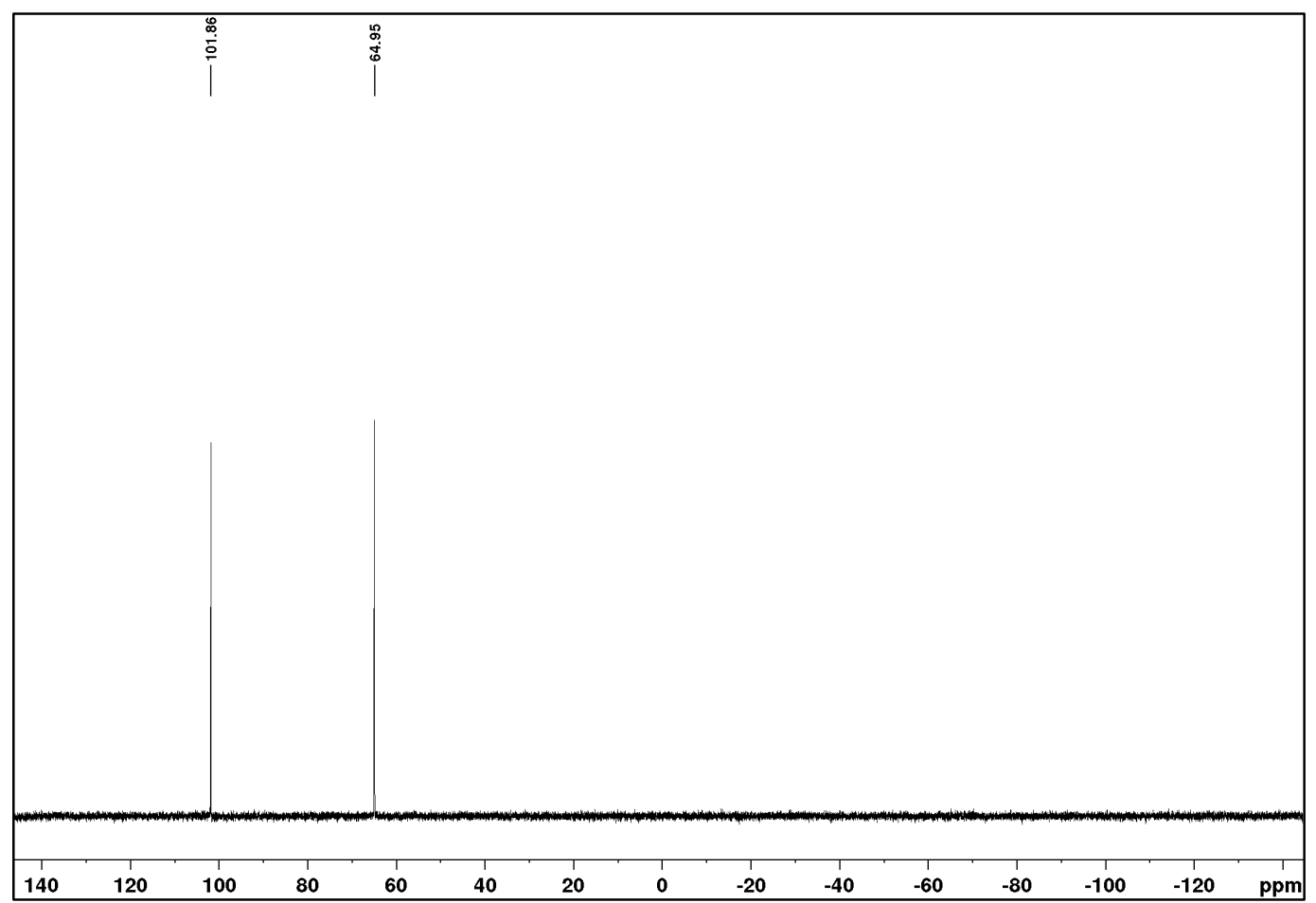


$\left(R_{\mathrm{p}}\right)-(+)-\mathrm{N}-[(1 R, 2 S), 2$-Ferrocenylphenylphosphinito)-1-methyl-2-phenylethyl], N-methylamino diphenylphosphine $\mathbf{2 0 g}$.

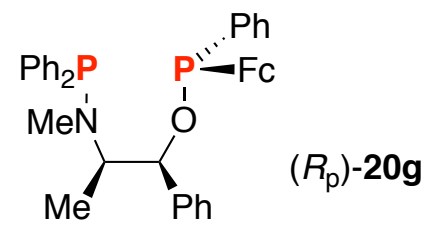

${ }^{1} \mathrm{H}$ NMR $\left(300 \mathrm{MHz}, \mathrm{CDCl}_{3}\right)$

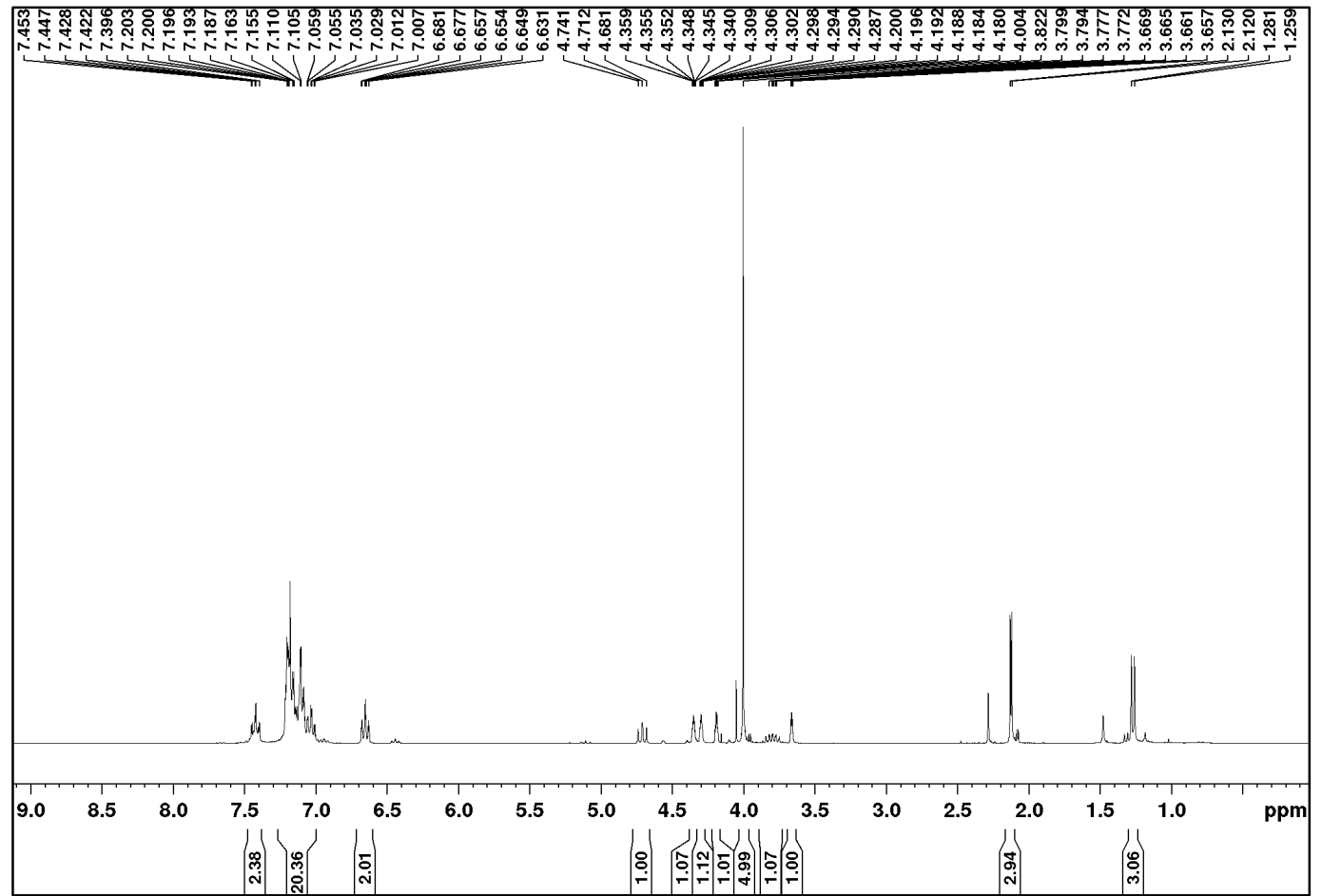

${ }^{31} \mathrm{P}\left\{{ }^{1} \mathrm{H}\right\}$ NMR $\left(121.5 \mathrm{MHz}, \mathrm{CDCl}_{3}\right)$

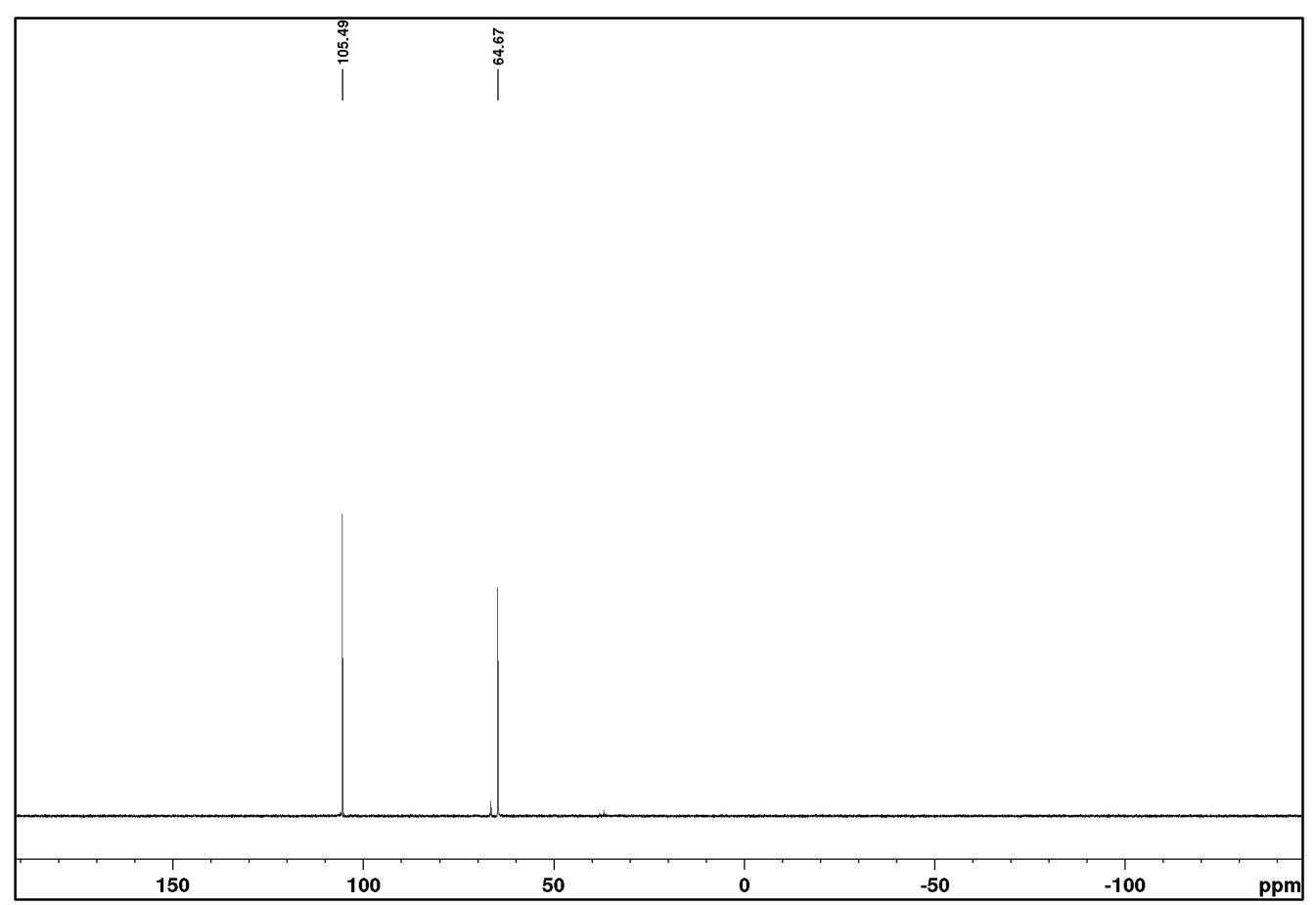


$\left(S_{\mathrm{p}}\right)$-N-[(1R,2S), 2-(Phenyl-o-biphenylphosphinito)-1-methyl-2-phenylethyl], N-methylamino diphenylphosphine $\mathbf{2 0 i}$.

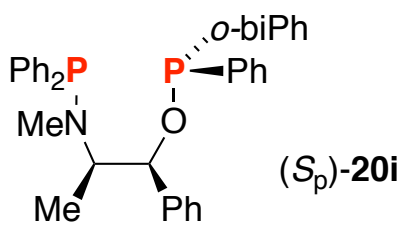

${ }^{1} \mathrm{H} \mathrm{NMR}\left(300 \mathrm{MHz}, \mathrm{CDCl}_{3}\right)$

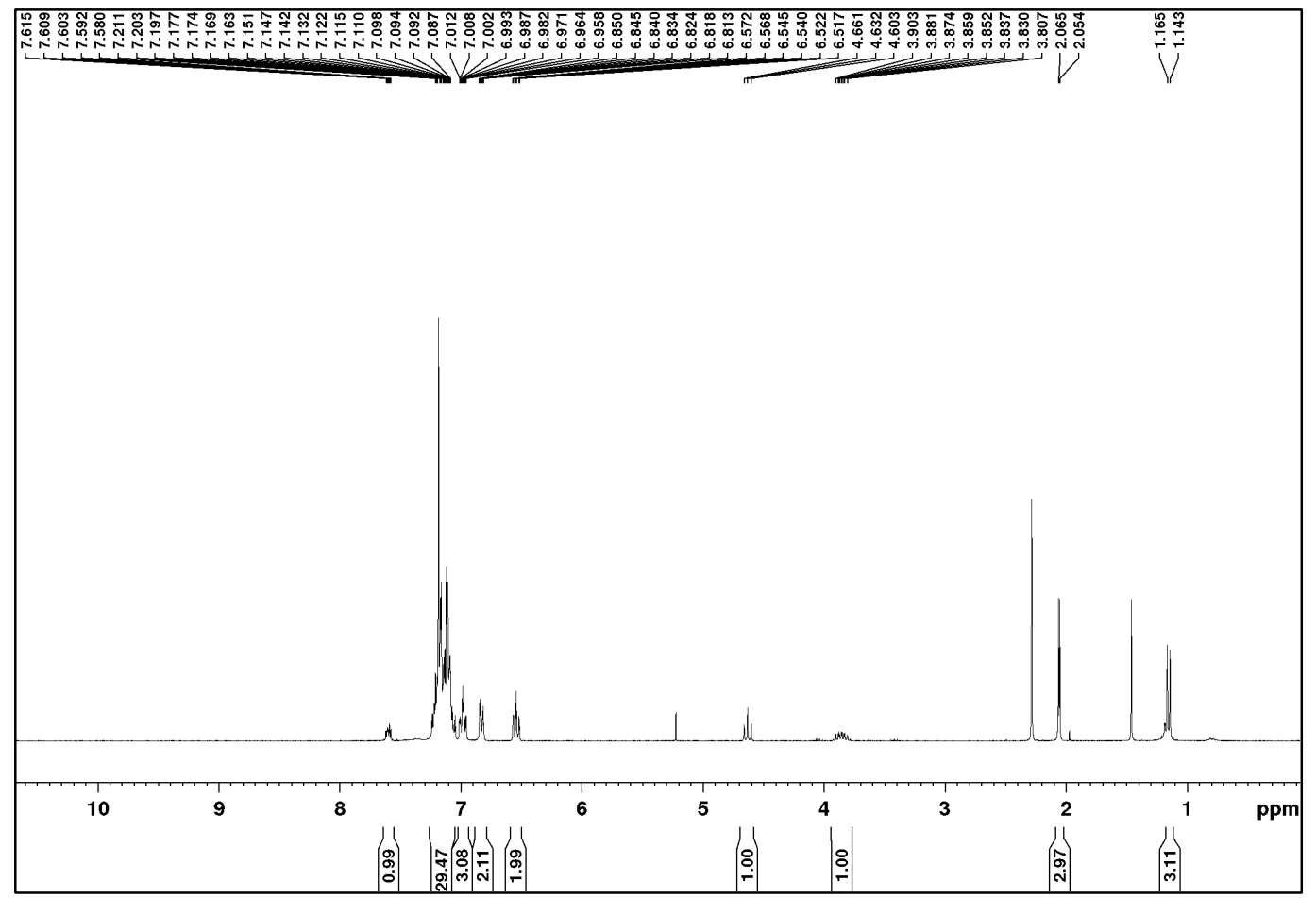

${ }^{31} \mathrm{P}\left\{{ }^{1} \mathrm{H}\right\}$ NMR (121.5 MHz, $\left.\mathrm{CDCl}_{3}\right)$

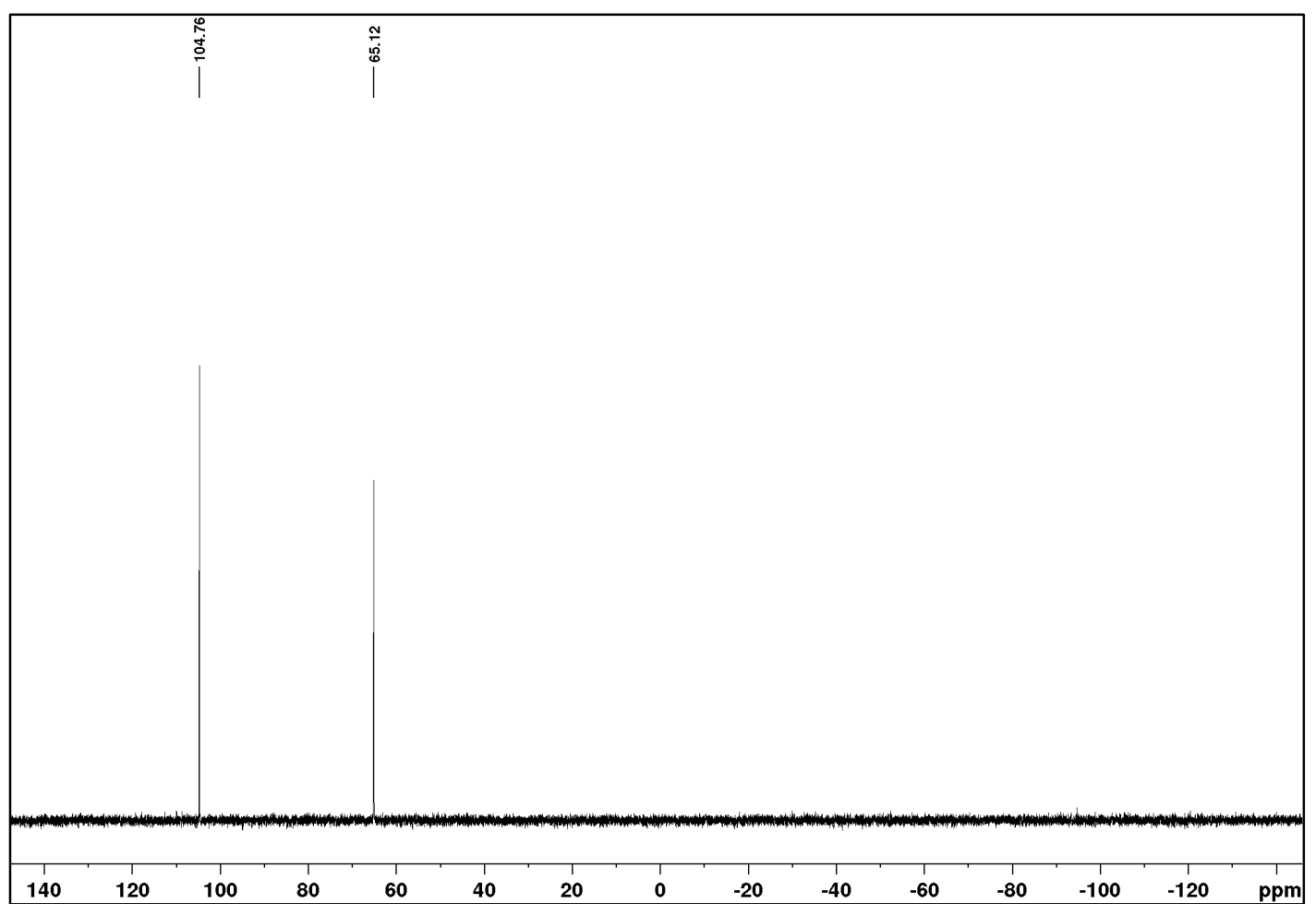


Dichloropalladium-AMPP* complexes.

$\mathrm{N}$-[(1R,2S)-(2-(Diphenylphosphinito)-1-methyl-2-phenylethyl], N-methylamino(o-biphenyl) phenylphosphine dichloropalladium complex 30.

${ }^{1} \mathrm{H} \mathrm{NMR}\left(400 \mathrm{MHz}, \mathrm{CD}_{2} \mathrm{Cl}_{2}\right)$<smiles>CC(N)C(O[PH](c1ccccc1)(c1ccccc1)c1ccccc1)P(Cl)(Cl)(Oc1ccccc1)c1ccccc1</smiles>

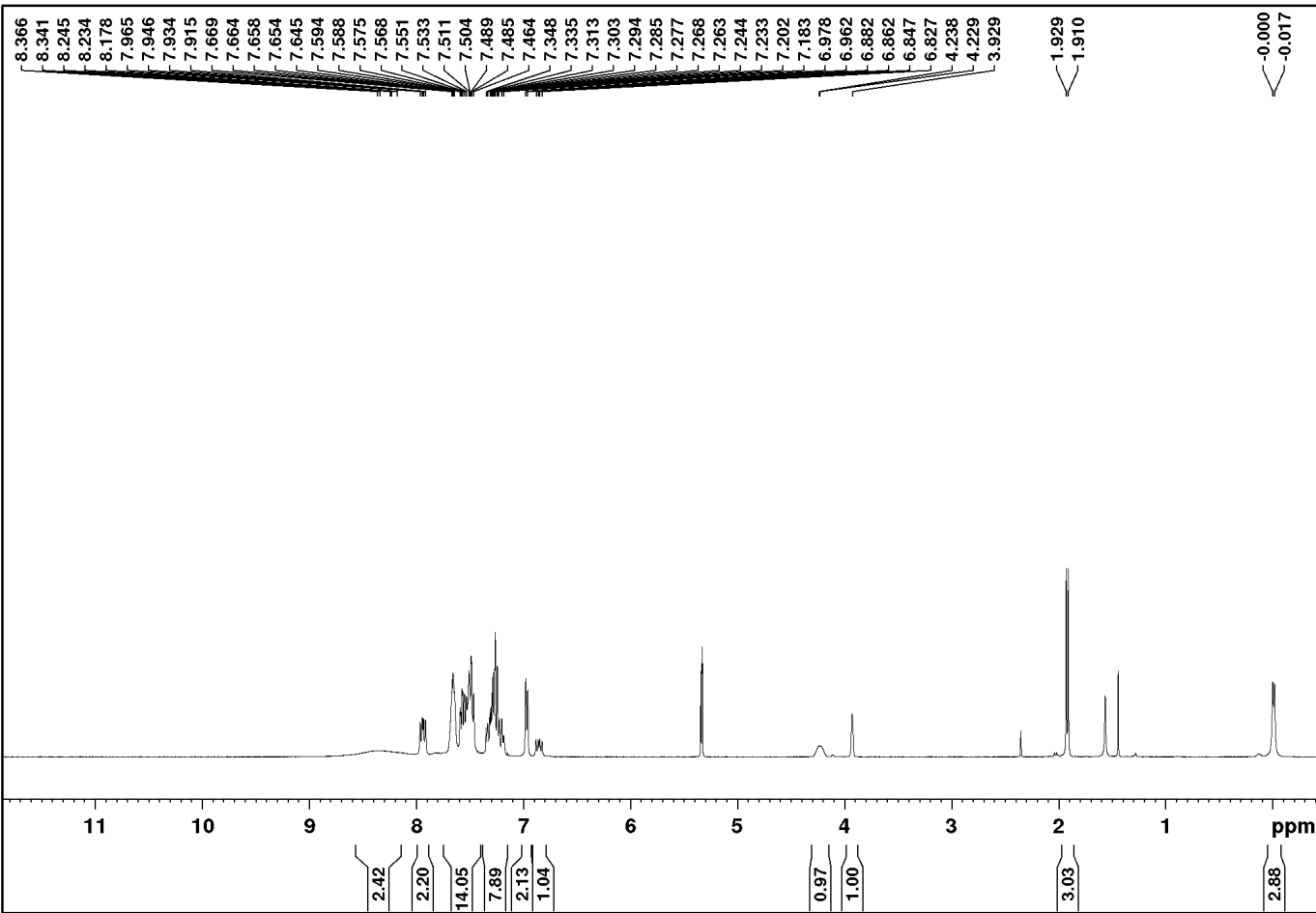

${ }^{13} \mathrm{C}\left\{{ }^{1} \mathrm{H}\right\}$ J-mod NMR (100.6 MHz, $\left.\mathrm{CD}_{2} \mathrm{Cl}_{2}\right)$

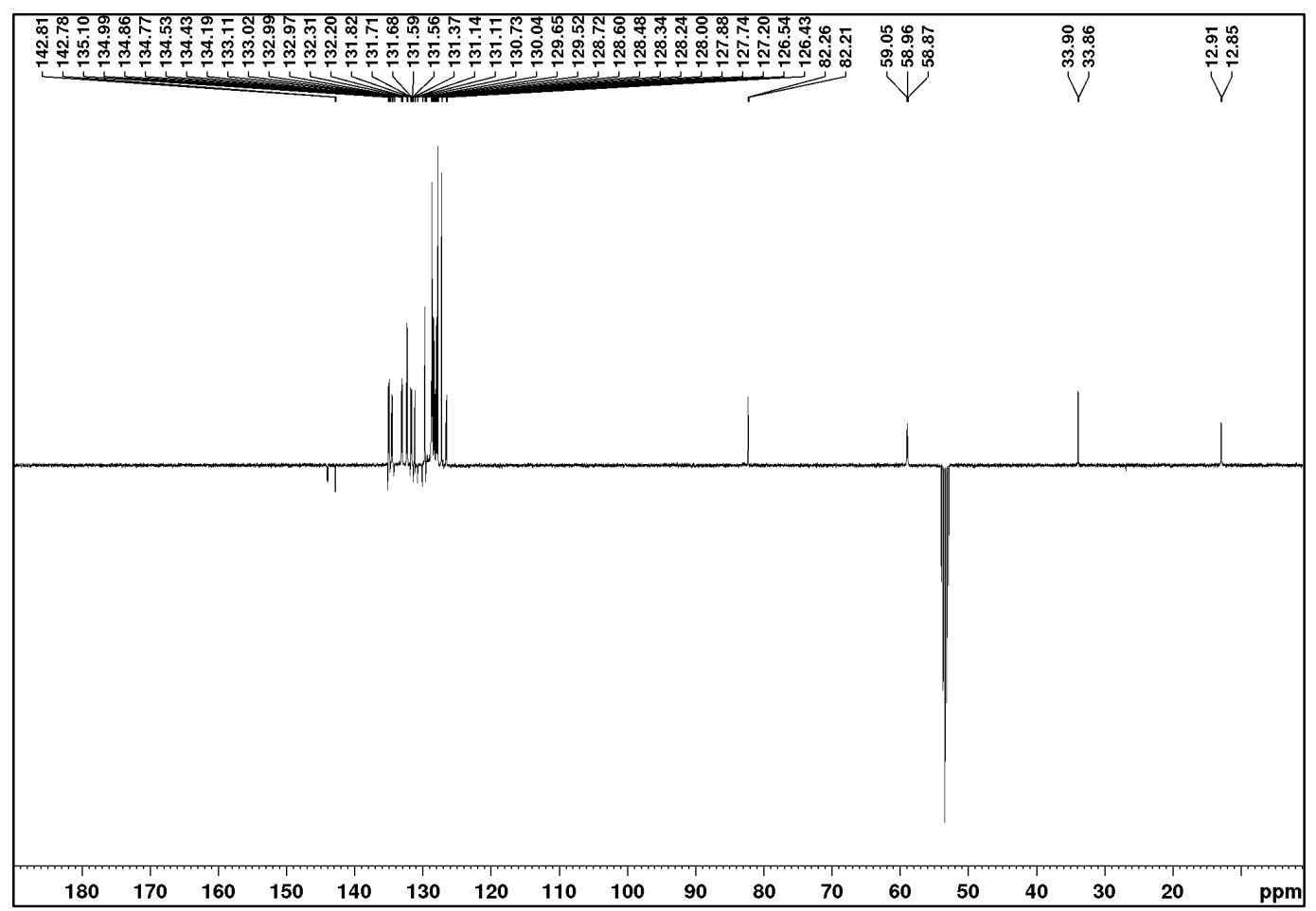




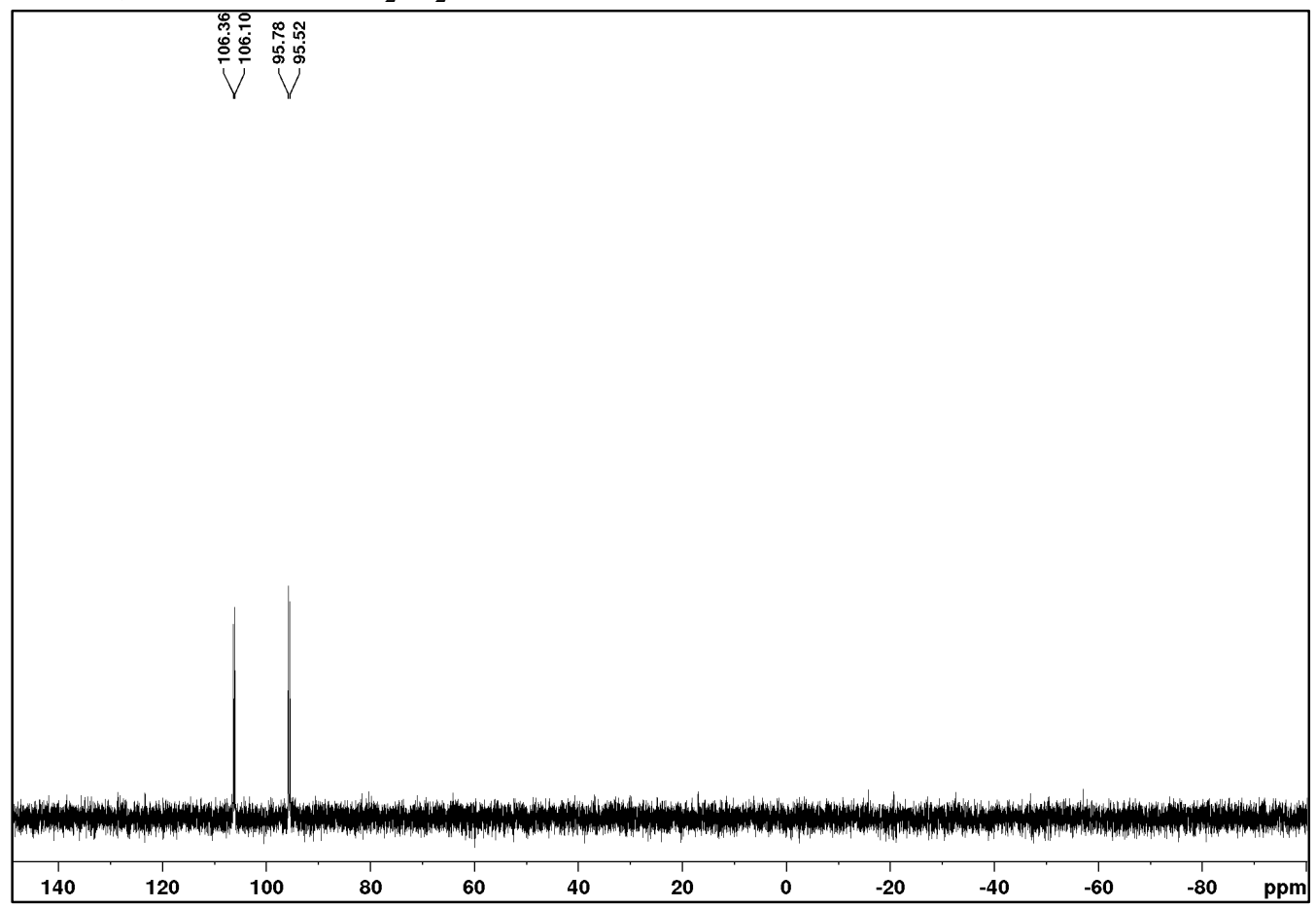

$\mathrm{N}$-[(1R,2S)-(2-(Diphenylphosphinito)-1-methyl-2-phenylethyl], N-methylaminoferrocenylphenyl phosphine dichloropalladium complex 31.

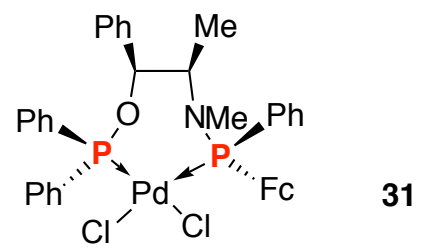

${ }^{1} \mathrm{H} \mathrm{NMR}\left(300 \mathrm{MHz} \mathrm{CDCl}_{3}\right)$

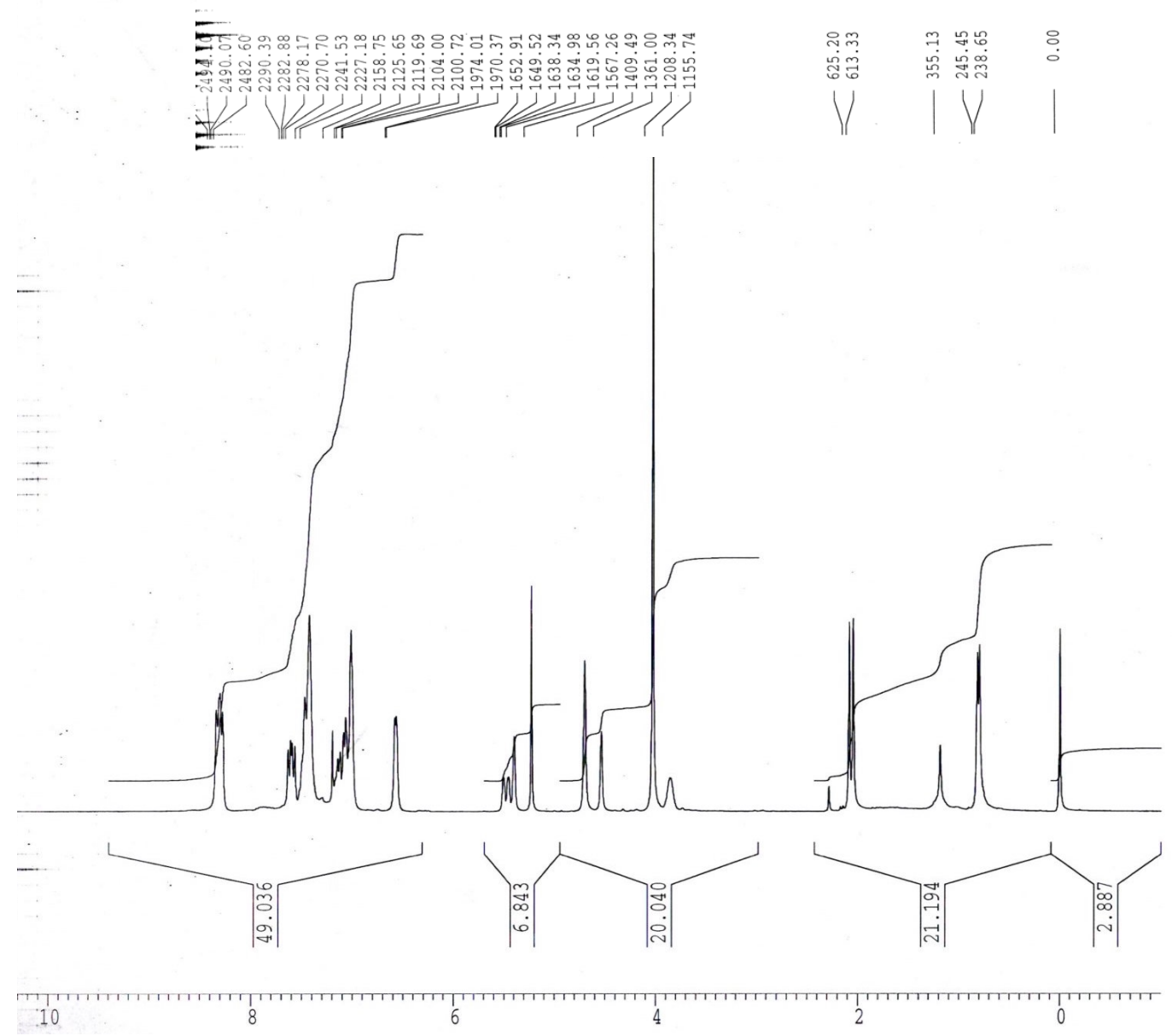


${ }^{13} \mathrm{C}\left\{{ }^{1} \mathrm{H}\right\}$ NMR (75.5 $\left.\mathrm{CDCl}_{3}\right)$

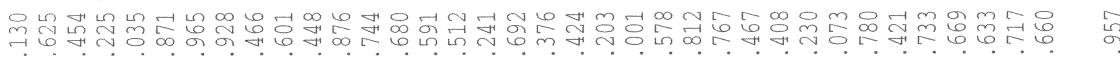

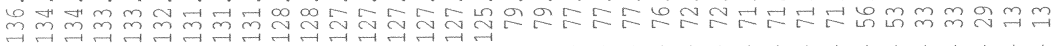

nit/

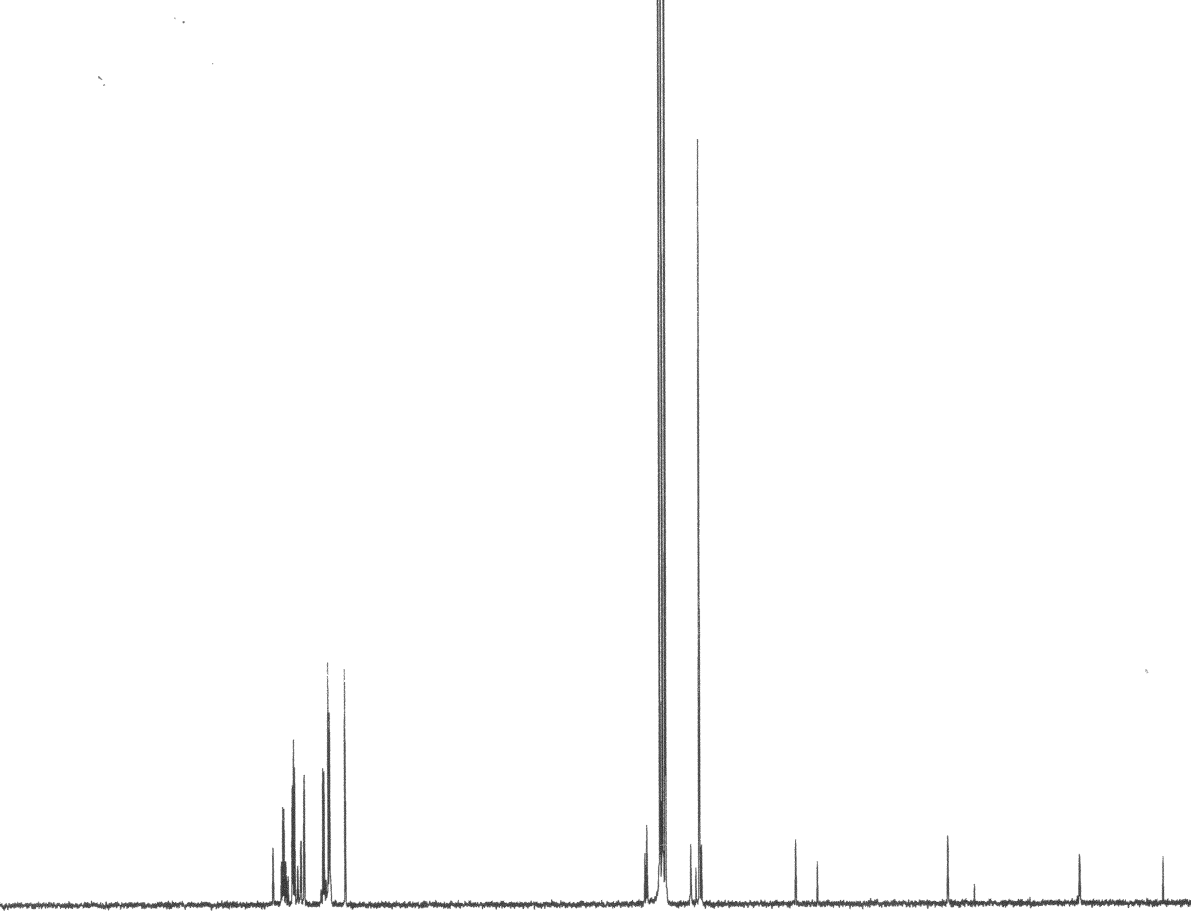

175

150

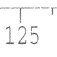

100

${ }^{31} \mathrm{P}\left\{{ }^{1} \mathrm{H}\right\} \mathrm{NMR}\left(121.5 \mathrm{CDCl}_{3}\right)$


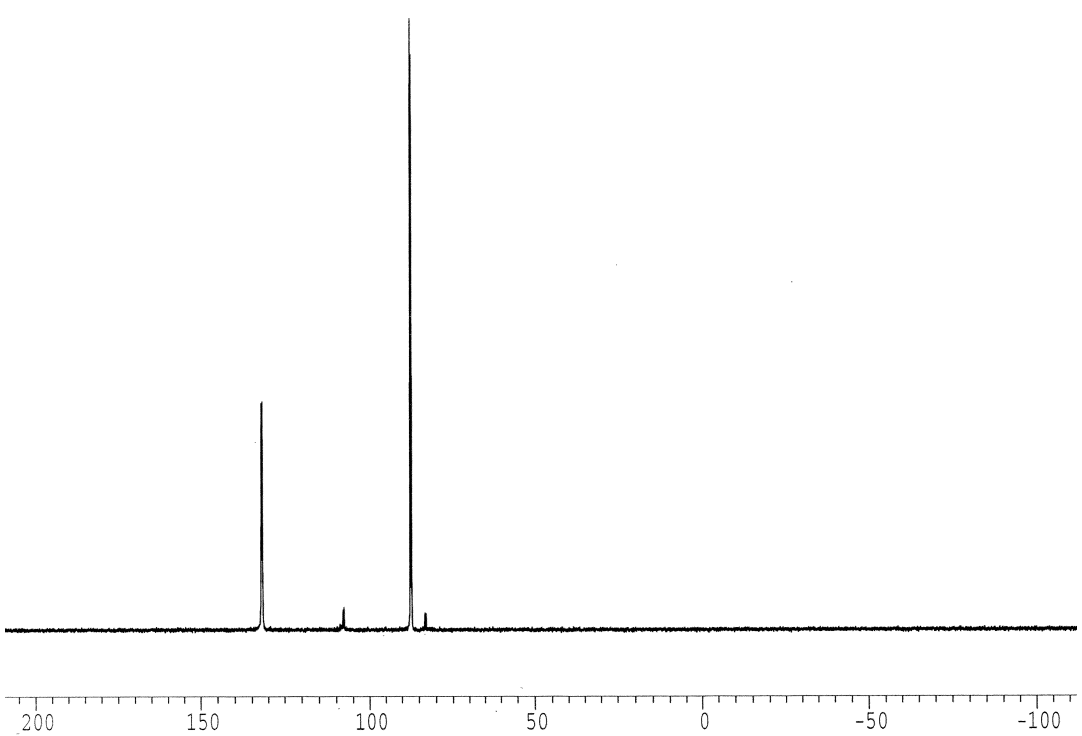
$\mathrm{N}-[(1 R, 2 S)-2-(\alpha-N a p h t y l p h e n y l p h o s p h i n i t o)-1-m e t h y l-2-p h e n y l e t h y l], \quad \mathrm{N}$-methylaminodiphenyl phosphine dichloropalladium complex 32 .

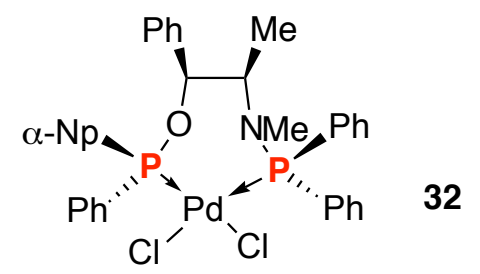

${ }^{1} \mathrm{H}$ NMR $\left(500 \mathrm{MHz}, \mathrm{CDCl}_{3}\right)$

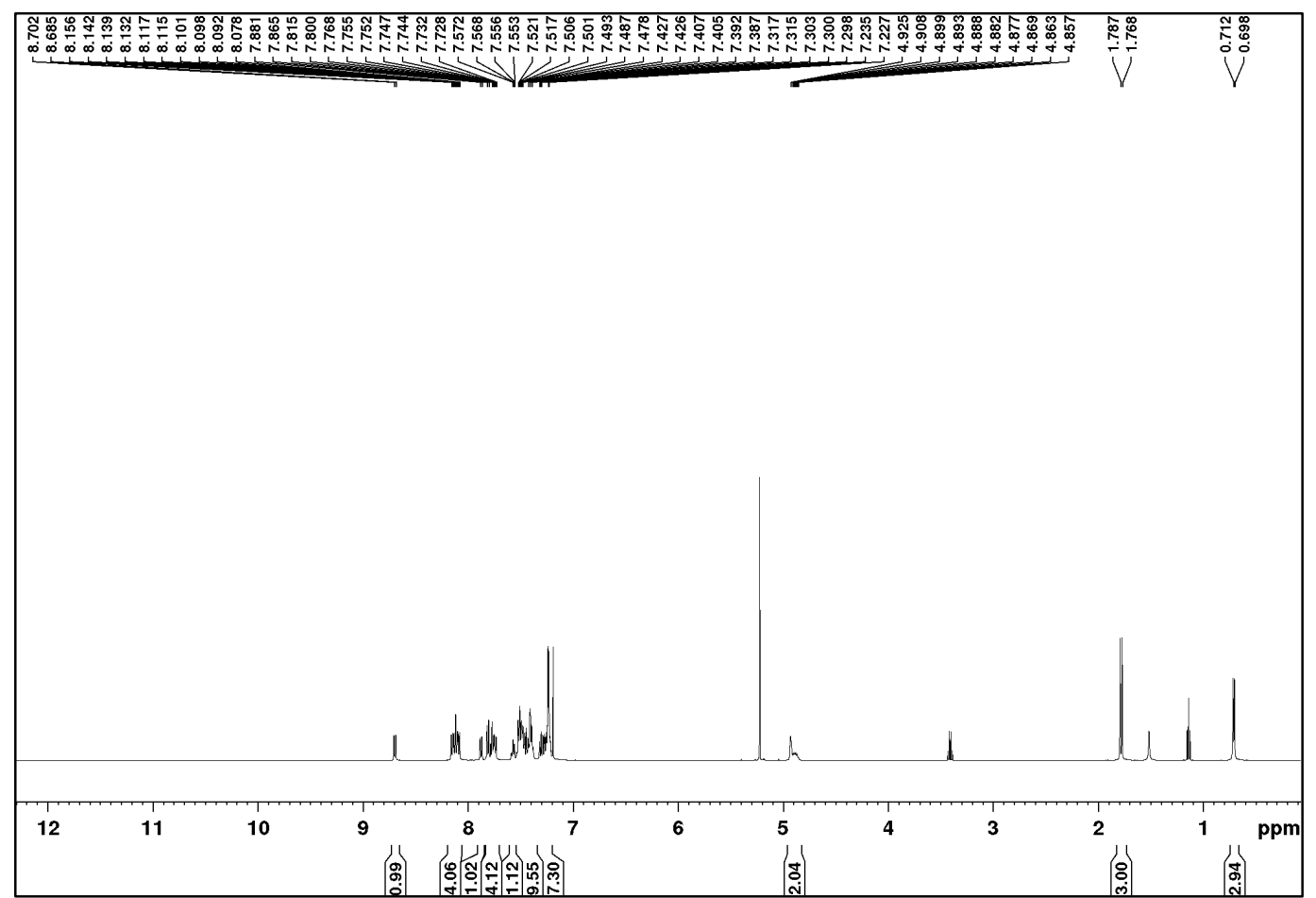

${ }^{13} \mathrm{C}\left\{{ }^{1} \mathrm{H}\right\} \mathrm{J}-\bmod \mathrm{NMR}\left(125.8 \mathrm{MHz}, \mathrm{CDCl}_{3}\right)$

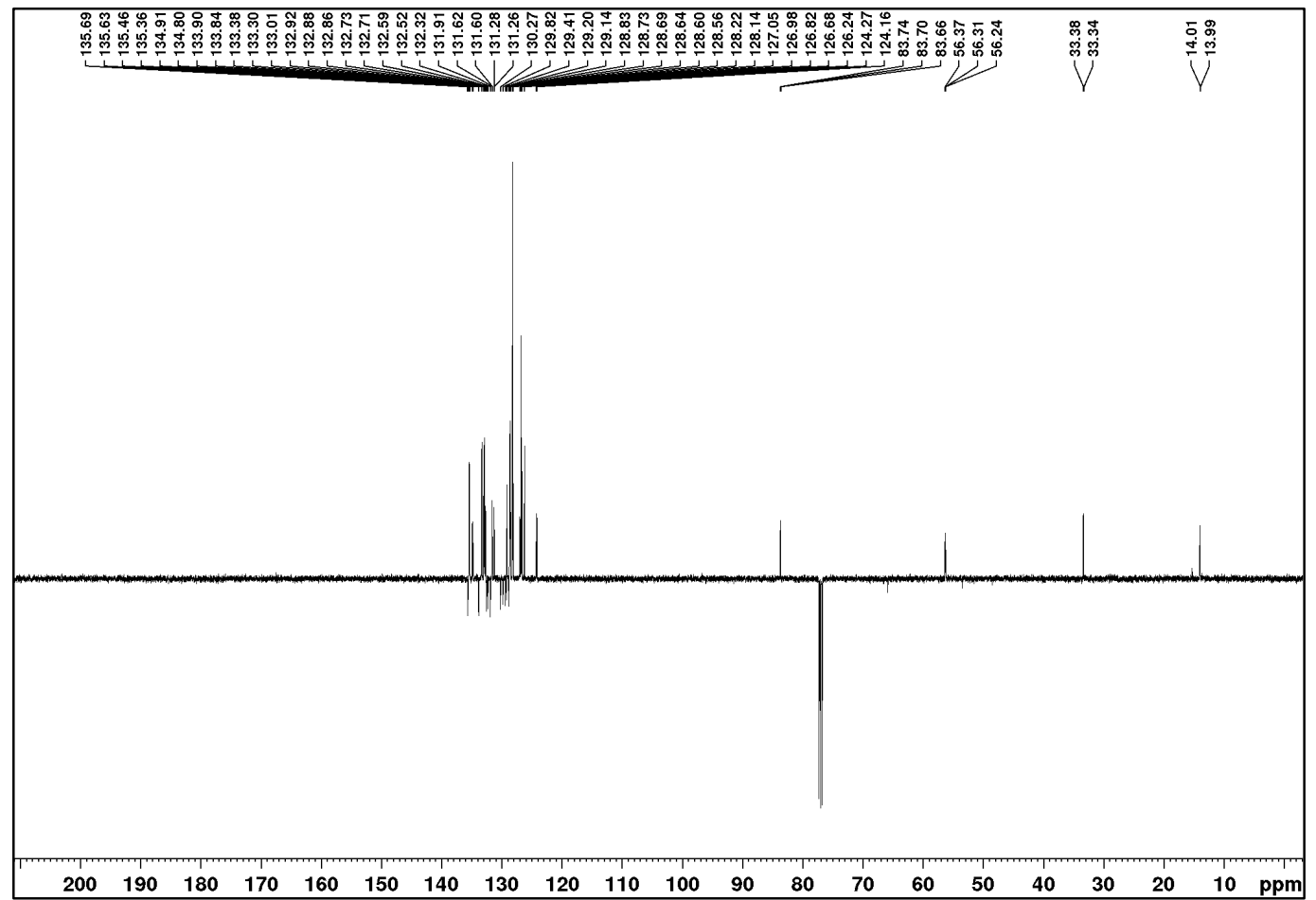


${ }^{31} \mathrm{P}$ NMR (202.5 MHz, $\left.\mathrm{CDCl}_{3}\right)$

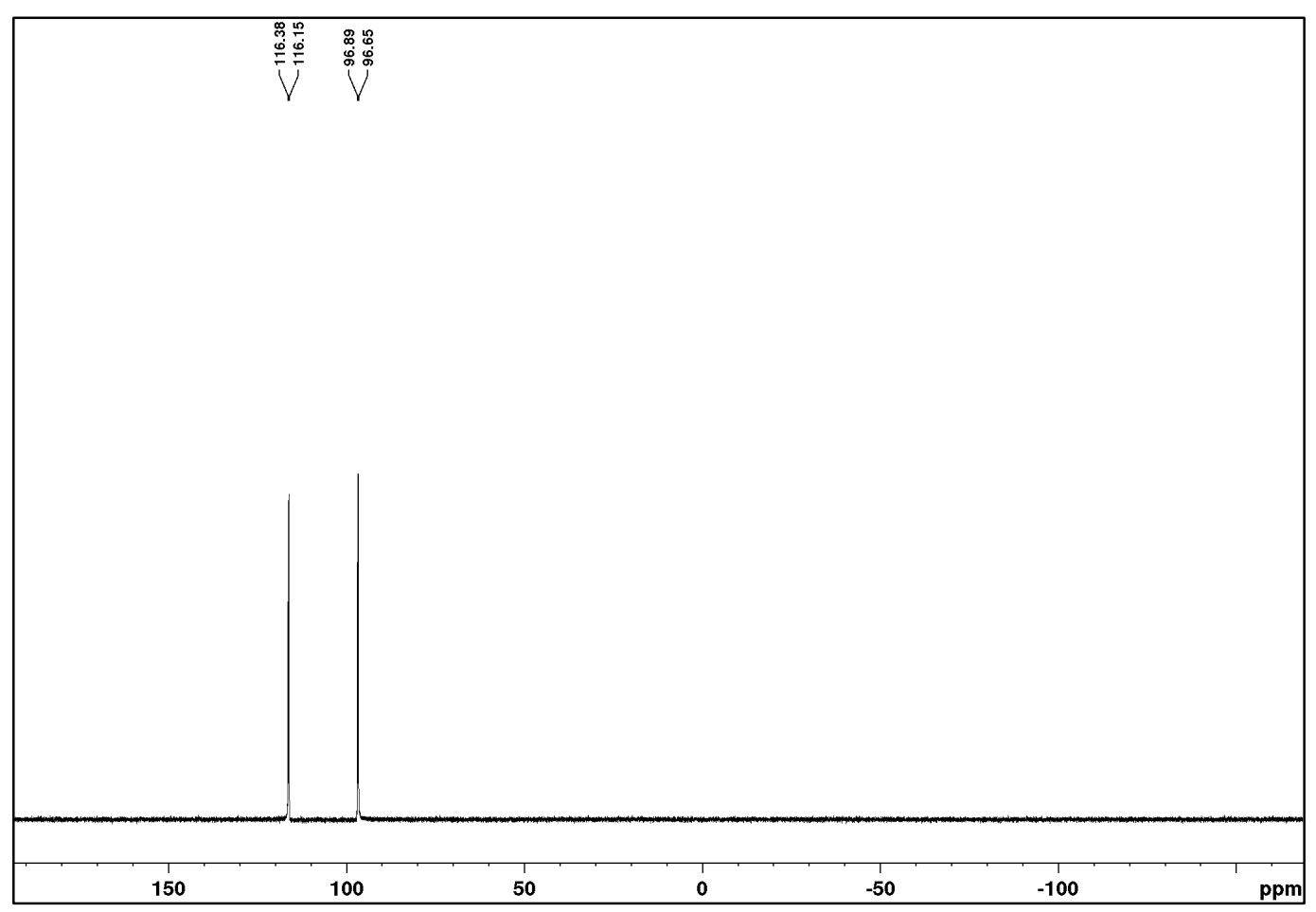




\section{Part B:}

\section{Crystallographic data}


Name: $\left(R_{\mathrm{p}}\right)-(-)-\mathrm{N}-[(1 R, 2 S)-2-\mathrm{Hydroxy}-1$-methyl-2-phenylethyl], N-methylamino(phenyl- $p$-tolyl) phosphine-borane 11e.

Submitted by: Antonin Jaillet

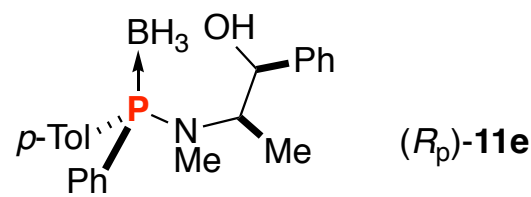

Solved by: Yoann Rousselin

Sample ID: $\quad$ 17AJ481

\section{Crystal Data and Experimental}

Figure S1 (thermal ellipsoïds are drawn at $50 \%$ probability plot)

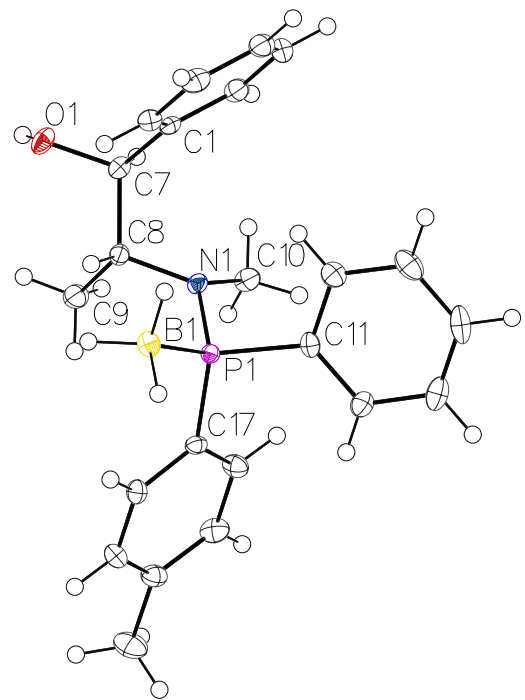

was 0.0743 (all data) and $R_{1}$ was 0.0348 (I > 2(I)).

Experimental. Single clear light colorless prism-shaped crystals of compound 11e were recrystallized from hexane by slow evaporation. A suitable crystal $0.36 \times 0.30 \times 0.28 \mathrm{~mm}^{3}$ was selected and mounted on a MITIGEN holder oil on a Bruker D8 Venture (Mo) diffractometer. The crystal was kept at a steady $T=$ 100.0(1) K during data collection. The structure was solved with the ShelXT (Sheldrick, 2015) structure solution program using the Intrinsic Phasing solution method and by using 0lex2 (Dolomanov et al., 2009) as the graphical interface. The model was refined with version 2018/3 of ShelXL (Sheldrick, 2015) using Least Squares minimization.

Crystal Data. $\mathrm{C}_{23} \mathrm{H}_{29} \mathrm{BNOP}, M_{r}=377.25$, monoclinic, $P 2_{1}$ (No. 4), $\mathrm{a}=7.9355(5) \AA, \quad \mathrm{b}=14.8222(10) \AA, \quad \mathrm{c}=$ 9.0656(6) $\AA, \quad \beta=101.1080(19)^{\circ}, \quad \alpha=\gamma=90^{\circ}, \quad V=$ 1046.33(12) $\AA^{3}, T=100.0(1) \mathrm{K}, Z=2, Z^{\prime}=1, \mu\left(\mathrm{MoK}_{\alpha}\right)=$ $0.144,56927$ reflections measured, 4794 unique $\left(R_{\text {int }}=\right.$ $0.0566)$ which were used in all calculations. The final $w R_{2}$ 


\begin{tabular}{|c|c|}
\hline Compound & 11e \\
\hline CCDC & 1982667 \\
\hline Formula & $\mathrm{C}_{23} \mathrm{H}_{29} \mathrm{BNOP}$ \\
\hline$D_{\text {calc. }} / \mathrm{g} \mathrm{cm}^{-3}$ & 1.197 \\
\hline$\mu / \mathrm{mm}^{-1}$ & 0.144 \\
\hline Formula Weight & 377.25 \\
\hline Color & clear light colorless \\
\hline Shape & prism \\
\hline Size $/ \mathrm{mm}^{3}$ & $0.36 \times 0.30 \times 0.28$ \\
\hline$T / \mathrm{K}$ & $100.0(1)$ \\
\hline Crystal System & monoclinic \\
\hline Flack Parameter & $0.00(2)$ \\
\hline Hooft Parameter & $0.01(2)$ \\
\hline Space Group & $P 2_{1}$ \\
\hline$a / \AA$ & $7.9355(5)$ \\
\hline$b / \AA ̊$ & $14.8222(10)$ \\
\hline$c / \AA$ & $9.0656(6)$ \\
\hline$\alpha /^{\circ}$ & 90 \\
\hline$\beta /^{\circ}$ & $101.1080(19)$ \\
\hline$\gamma /{ }^{\circ}$ & 90 \\
\hline $\mathrm{V} / \AA^{3}$ & $1046.33(12)$ \\
\hline$Z$ & 2 \\
\hline$Z^{\prime}$ & 1 \\
\hline Wavelength/Å & 0.71073 \\
\hline Radiation type & $\operatorname{MoK}_{\alpha}$ \\
\hline$\Theta_{\min } /^{\circ}$ & 2.955 \\
\hline$\Theta_{\max } /^{\circ}$ & 27.539 \\
\hline Measured Refl. & 56927 \\
\hline Independent Refl. & 4794 \\
\hline Reflections with I > & 4360 \\
\hline $2(\mathrm{I})$ & \\
\hline$R_{\text {int }}$ & 0.0566 \\
\hline Parameters & 250 \\
\hline Restraints & 1 \\
\hline Largest Peak & 0.204 \\
\hline Deepest Hole & -0.205 \\
\hline GooF & 1.059 \\
\hline$w R_{2}$ (all data) & 0.0743 \\
\hline$w R_{2}$ & 0.0714 \\
\hline$R_{1}$ (all data) & 0.0430 \\
\hline$R_{1}$ & 0.0348 \\
\hline
\end{tabular}


Reflections:

Refinement:

A clear light colorless prism-shaped crystal with dimensions $0.36 \times 0.30 \times 0.28 \mathrm{~mm}^{3}$ was mounted on a MITIGEN holder oil. Data were collected using a Bruker D8 Venture (Mo) diffractometer equipped with an Oxford Cryosystems low-temperature device operating at $T=100.0(1) \mathrm{K}$. Data were measured using $\phi$ and $\omega$ scans using $\mathrm{MoK}_{\alpha}$ radiation. The total number of runs and images was based on the strategy calculation from the program APEX3 (Bruker, 2015) The maximum resolution that was achieved was $\Theta$ $=27.539^{\circ}(0.77 \AA)$. The diffraction pattern was indexed. The total number of runs and images was based on the strategy calculation from the program APEX3 (Bruker, 2015) and the unit cell was refined using SAINT (Bruker, V8.37A, after 2013) on 9906 reflections, 17\% of the observed reflections. Data reduction, scaling and absorption corrections were performed using SAINT (Bruker, V8.37A, after 2013). The final completeness is $99.80 \%$ out to $27.539^{\circ}$ in $\Theta$. A multi-scan absorption correction was performed using SADABS-2016/2 (Bruker, 2016) was used for absorption correction. $w R_{2}$ (int) was 0.0641 before and 0.0590 after correction. The Ratio of minimum to maximum transmission is 0.9761 . The absorption coefficient $\mu$ of this material is $0.144 \mathrm{~mm}^{-1}$ at this wavelength $(\lambda=0.711 \AA)$ and the minimum and maximum transmissions are 0.854 and 0.875 . The structure was solved and the space group $P 2_{1}$ (\# 4) determined by the ShelXT (Sheldrick, 2015) structure solution program using Intrinsic Phasing and refined by Least Squares using version 2018/3 of ShelXL (Sheldrick, 2015). All nonhydrogen atoms were refined anisotropically. Hydrogen atom positions were calculated geometrically and refined using the riding model. Hydrogen atom on Boron atom were refined using an AFIX 138, which would allow the refinement of a common B-H distances, the same shifts being applied to all the B$\mathrm{H}$ bonds to the same atom, but still retain tetrahedral H-B-H angles within the group. There is a single molecule in the asymmetric unit, which is represented by the reported sum formula. In other words: $\mathrm{Z}$ is 2 and $Z^{\prime}$ is 1 . The Flack parameter was refined to $0.00(2)$. Determination of absolute structure using Bayesian statistics on Bijvoet differences using the Olex2 results in 0.01(2). Note: The Flack parameter is used to determine chirality of the crystal studied, the value should be near 0 , a value of 1 means that the stereochemistry is wrong and the model should be inverted. A value of 0.5 means that the crystal consists of a racemic mixture of the two enantiomers.

Table S1: Bond Lengths in Å for compound 11e.

\begin{tabular}{lll}
\hline Atom & Atom & Length/Å \\
\hline P1 & N1 & $1.6689(17)$ \\
P1 & C11 & $1.811(2)$ \\
P1 & C17 & $1.810(2)$ \\
P1 & B1 & $1.915(3)$ \\
O1 & C7 & $1.432(3)$ \\
N1 & C8 & $1.475(3)$ \\
N1 & C10 & $1.467(3)$ \\
C1 & C2 & $1.392(3)$ \\
C1 & C7 & $1.508(3)$ \\
C1 & C6 & $1.384(3)$ \\
C2 & C3 & $1.387(3)$ \\
C11 & C12 & $1.400(3)$ \\
C11 & C16 & $1.387(3)$ \\
C22 & C17 & $1.396(3)$ \\
C22 & C21 & $1.387(3)$
\end{tabular}

\begin{tabular}{lll}
\hline Atom & Atom & Length/Å \\
\hline C12 & C13 & $1.391(3)$ \\
C8 & C7 & $1.542(3)$ \\
C8 & C9 & $1.526(3)$ \\
C17 & C18 & $1.397(3)$ \\
C20 & C21 & $1.389(3)$ \\
C20 & C19 & $1.389(3)$ \\
C20 & C23 & $1.508(3)$ \\
C3 & C4 & $1.383(4)$ \\
C4 & C5 & $1.389(4)$ \\
C18 & C19 & $1.381(3)$ \\
C6 & C5 & $1.383(4)$ \\
C13 & C14 & $1.386(4)$ \\
C15 & C16 & $1.391(3)$ \\
C15 & C14 & $1.375(4)$
\end{tabular}


Table S2: Bond Angles in ${ }^{\circ}$ for compound $11 \mathrm{e}$.

\begin{tabular}{|c|c|c|c|c|c|c|c|}
\hline Atom & Atom & Atom & Angle $/^{\circ}$ & Atom & Atom & Atom & Angle $/^{\circ}$ \\
\hline $\mathrm{N} 1$ & P1 & C11 & $103.04(9)$ & $\mathrm{C} 22$ & C17 & P1 & $118.73(17)$ \\
\hline $\mathrm{N} 1$ & $\mathrm{P} 1$ & C17 & $109.46(10)$ & $\mathrm{C} 22$ & $\mathrm{C} 17$ & C18 & $118.3(2)$ \\
\hline N1 & $\mathrm{P} 1$ & B1 & 113.91(11) & C18 & C17 & P1 & $122.40(17)$ \\
\hline C11 & $\mathrm{P} 1$ & B1 & $109.99(11)$ & $\mathrm{C} 21$ & $\mathrm{C} 20$ & C19 & $118.4(2)$ \\
\hline C17 & $\mathrm{P} 1$ & C11 & $108.42(11)$ & $\mathrm{C} 21$ & $\mathrm{C} 20$ & $\mathrm{C} 23$ & $120.6(2)$ \\
\hline C17 & $\mathrm{P} 1$ & B1 & $111.57(11)$ & C19 & $\mathrm{C} 20$ & $\mathrm{C} 23$ & $121.0(2)$ \\
\hline C8 & N1 & $\mathrm{P} 1$ & $117.71(14)$ & $\mathrm{C} 22$ & $\mathrm{C} 21$ & $\mathrm{C} 20$ & $120.8(2)$ \\
\hline $\mathrm{C} 10$ & $\mathrm{~N} 1$ & $\mathrm{P} 1$ & $119.13(15)$ & $\mathrm{C} 4$ & $\mathrm{C} 3$ & $\mathrm{C} 2$ & $120.2(2)$ \\
\hline C10 & N1 & C8 & $115.79(17)$ & 01 & $\mathrm{C} 7$ & $\mathrm{C} 1$ & $108.27(18)$ \\
\hline $\mathrm{C} 2$ & $\mathrm{C} 1$ & $\mathrm{C} 7$ & $120.2(2)$ & 01 & $\mathrm{C} 7$ & C8 & $107.59(18)$ \\
\hline C6 & $\mathrm{C} 1$ & $\mathrm{C} 2$ & $118.8(2)$ & $\mathrm{C} 1$ & $\mathrm{C} 7$ & C8 & $112.86(18)$ \\
\hline C6 & $\mathrm{C} 1$ & $\mathrm{C} 7$ & $121.0(2)$ & $\mathrm{C} 3$ & $\mathrm{C} 4$ & $\mathrm{C} 5$ & $119.4(2)$ \\
\hline C3 & $\mathrm{C} 2$ & $\mathrm{C} 1$ & $120.5(2)$ & C19 & C18 & $\mathrm{C} 17$ & $120.5(2)$ \\
\hline C12 & $\mathrm{C} 11$ & $\mathrm{P} 1$ & $116.76(18)$ & $\mathrm{C} 5$ & C6 & $\mathrm{C} 1$ & $120.9(2)$ \\
\hline C16 & $\mathrm{C} 11$ & $\mathrm{P} 1$ & $123.57(18)$ & $\mathrm{C} 14$ & $\mathrm{C} 13$ & $\mathrm{C} 12$ & $120.4(2)$ \\
\hline $\mathrm{C} 16$ & $\mathrm{C} 11$ & $\mathrm{C} 12$ & $119.6(2)$ & $\mathrm{C} 14$ & C15 & $\mathrm{C} 16$ & $120.4(2)$ \\
\hline $\mathrm{C} 21$ & $\mathrm{C} 22$ & $\mathrm{C} 17$ & $120.7(2)$ & $\mathrm{C} 11$ & C16 & $\mathrm{C} 15$ & $120.1(2)$ \\
\hline C13 & $\mathrm{C} 12$ & C11 & $119.6(2)$ & $\mathrm{C} 15$ & C14 & $\mathrm{C} 13$ & $120.0(2)$ \\
\hline $\mathrm{N} 1$ & $\mathrm{C} 8$ & $\mathrm{C} 7$ & $110.97(17)$ & $\mathrm{C} 18$ & C19 & $\mathrm{C} 20$ & $121.3(2)$ \\
\hline N1 & $\mathrm{C} 8$ & C9 & $113.05(19)$ & C6 & $\mathrm{C} 5$ & $\mathrm{C} 4$ & $120.1(2)$ \\
\hline C9 & $\mathrm{C} 8$ & $\mathrm{C} 7$ & $111.45(18)$ & & & & \\
\hline
\end{tabular}

Table S3: Torsion Angles in ${ }^{\circ}$ for compound $11 e$.

\begin{tabular}{|c|c|c|c|c|}
\hline Atom & Atom & Atom & Atom & Angle $/^{\circ}$ \\
\hline$\overline{P 1}$ & N1 & $\mathrm{C} 8$ & C7 & $143.03(16)$ \\
\hline P1 & $\mathrm{N} 1$ & C8 & $\mathrm{C} 9$ & $-90.9(2)$ \\
\hline P1 & C11 & C12 & C13 & $177.57(18)$ \\
\hline P1 & C11 & C16 & C15 & $-177.36(18)$ \\
\hline P1 & $\mathrm{C} 17$ & C18 & C19 & -169.79(19) \\
\hline $\mathrm{N} 1$ & P1 & C11 & C12 & $58.12(19)$ \\
\hline $\mathrm{N} 1$ & P1 & C11 & C16 & $-125.2(2)$ \\
\hline $\mathrm{N} 1$ & $\mathrm{P} 1$ & C17 & $\mathrm{C} 22$ & $-117.31(18)$ \\
\hline $\mathrm{N} 1$ & $\mathrm{P} 1$ & C17 & C18 & $53.9(2)$ \\
\hline $\mathrm{N} 1$ & $\mathrm{C} 8$ & $\mathrm{C} 7$ & 01 & $-165.18(17)$ \\
\hline $\mathrm{N} 1$ & $\mathrm{C} 8$ & $\mathrm{C} 7$ & $\mathrm{C} 1$ & $-45.8(2)$ \\
\hline $\mathrm{C} 1$ & $\mathrm{C} 2$ & $\mathrm{C} 3$ & $\mathrm{C} 4$ & $0.3(3)$ \\
\hline $\mathrm{C} 1$ & C6 & $\mathrm{C} 5$ & $\mathrm{C} 4$ & $0.4(4)$ \\
\hline $\mathrm{C} 2$ & $\mathrm{C} 1$ & $\mathrm{C} 7$ & 01 & $49.4(3)$ \\
\hline $\mathrm{C} 2$ & $\mathrm{C} 1$ & $\mathrm{C} 7$ & $\mathrm{C} 8$ & $-69.6(3)$ \\
\hline $\mathrm{C} 2$ & $\mathrm{C} 1$ & C6 & $\mathrm{C} 5$ & $-0.3(3)$ \\
\hline $\mathrm{C} 2$ & $\mathrm{C} 3$ & $\mathrm{C} 4$ & $\mathrm{C} 5$ & $-0.1(4)$ \\
\hline C11 & $\mathrm{P} 1$ & $\mathrm{~N} 1$ & C8 & $-157.24(16)$ \\
\hline C11 & P1 & N1 & C10 & 53.98(18) \\
\hline C11 & P1 & C17 & $\mathrm{C} 22$ & $130.98(18)$ \\
\hline C11 & P1 & C17 & C18 & $-57.8(2)$ \\
\hline C11 & C12 & C13 & C14 & $-0.3(4)$ \\
\hline C22 & C17 & C18 & C19 & $1.4(3)$ \\
\hline C12 & C11 & C16 & C15 & $-0.7(3)$ \\
\hline C12 & C13 & C14 & C15 & $0.0(4)$ \\
\hline C17 & P1 & N1 & $\mathrm{C} 8$ & $87.56(18)$ \\
\hline C17 & $\mathrm{P} 1$ & $\mathrm{~N} 1$ & $\mathrm{C} 10$ & $-61.23(18)$ \\
\hline
\end{tabular}




\begin{tabular}{|c|c|c|c|c|}
\hline Atom & Atom & Atom & Atom & Angle ${ }^{\circ}$ \\
\hline$\overline{\mathrm{C} 17}$ & P1 & C11 & C12 & $174.07(17)$ \\
\hline C17 & P1 & C11 & C16 & $-9.2(2)$ \\
\hline C17 & C22 & C21 & C20 & $0.9(4)$ \\
\hline C17 & C18 & C19 & $\mathrm{C} 20$ & $0.5(4)$ \\
\hline C21 & C22 & C17 & P1 & 169.41(18) \\
\hline C21 & C22 & C17 & C18 & $-2.1(3)$ \\
\hline $\mathrm{C} 21$ & $\mathrm{C} 20$ & C19 & C18 & $-1.7(4)$ \\
\hline C3 & C4 & C5 & C6 & $-0.2(4)$ \\
\hline C7 & C1 & $\mathrm{C} 2$ & C3 & $-179.1(2)$ \\
\hline C7 & C1 & C6 & C5 & $178.7(2)$ \\
\hline C6 & C1 & $\mathrm{C} 2$ & C3 & $-0.1(3)$ \\
\hline C6 & $\mathrm{C} 1$ & $\mathrm{C} 7$ & 01 & $-129.6(2)$ \\
\hline C6 & C1 & C7 & C8 & $111.4(2)$ \\
\hline C10 & N1 & C8 & C7 & $-67.2(2)$ \\
\hline C10 & N1 & C8 & C9 & $58.9(2)$ \\
\hline C16 & C11 & C12 & C13 & $0.7(3)$ \\
\hline C16 & C15 & C14 & C13 & $0.0(4)$ \\
\hline C14 & C15 & C16 & C11 & $0.4(4)$ \\
\hline C19 & $\mathrm{C} 20$ & $\mathrm{C} 21$ & $\mathrm{C} 22$ & $1.0(4)$ \\
\hline C9 & C8 & $\mathrm{C} 7$ & 01 & $67.9(2)$ \\
\hline C9 & C8 & C7 & C1 & $-172.75(19)$ \\
\hline C23 & $\mathrm{C} 20$ & $\mathrm{C} 21$ & $\mathrm{C} 22$ & $-179.3(2)$ \\
\hline C23 & $\mathrm{C} 20$ & C19 & C18 & 178.6(3) \\
\hline B1 & P1 & N1 & C8 & $-38.13(19)$ \\
\hline B1 & P1 & N1 & C10 & $173.09(17)$ \\
\hline B1 & P1 & C11 & C12 & $-63.7(2)$ \\
\hline B1 & P1 & $\mathrm{C} 11$ & C16 & $113.0(2)$ \\
\hline B1 & P1 & $\mathrm{C} 17$ & $\mathrm{C} 22$ & $9.7(2)$ \\
\hline B1 & P1 & C17 & C18 & $-179.11(19)$ \\
\hline
\end{tabular}


Name: $(R p)-(-)-\mathrm{N}-[(1 S, 2 R)-2$-Hydroxy-1-methyl-2-phenylethyl], phosphine-borane $\mathbf{1 1 h}$.

Submitted by: Antonin Jaillet

Solved by: Yoann Rousselin<smiles>[B]P(CC(C)(C)C)(c1ccccc1)(N(C)C(C)C(O)c1ccccc1)C(C)(C)C</smiles>

Sample ID: $\quad$ 20180329AJ669

\section{Crystal Data and Experimental}

Figure S2 (thermal ellipsoïds are drawn at $50 \%$ probability plot)

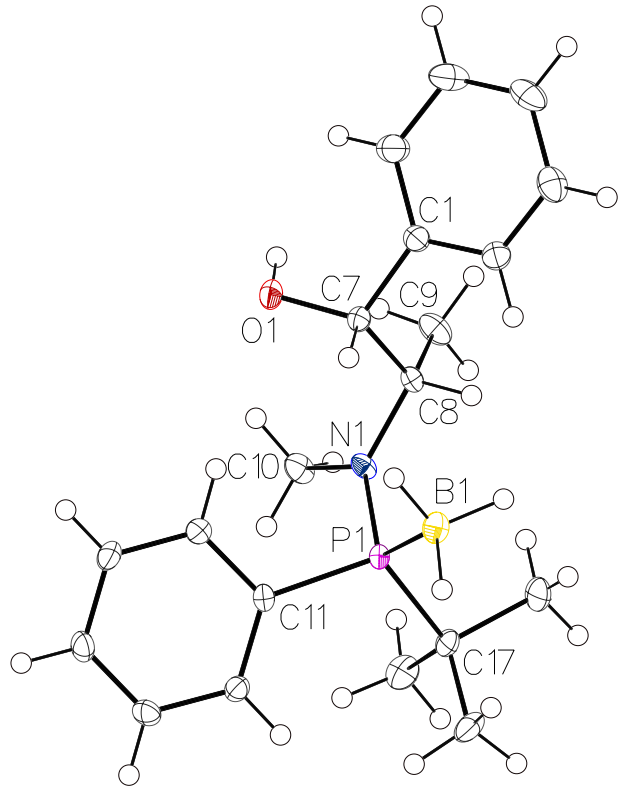

Experimental. Single clear light colorless prism-shaped crystals of compound $11 \mathrm{~h}$ were recrystallized from DCM by slow evaporation. A suitable crystal $0.34 \times 0.31 \times 0.21$ $\mathrm{mm}^{3}$ was selected and mounted on a MITIGEN holder oil on a Bruker D8 VENTURE diffractometer. The crystal was kept at a steady $T=100.0(1) \mathrm{K}$ during data collection. The structure was solved with the ShelXT (Sheldrick, 2015) structure solution program using the Intrinsic Phasing solution method and by using Olex2 (Dolomanov et al., 2009 ) as the graphical interface. The model was refined with version 2018/3 of ShelXL (Sheldrick, 2015) using Least Squares minimization.

Crystal Data. $\mathrm{C}_{20} \mathrm{H}_{31} \mathrm{BNOP}, M_{r}=343.24$, orthorhombic, $P 2{ }_{1} 2_{1} 2_{1}$ (No. 19), $\mathrm{a}=8.3440(5) \AA, \mathrm{b}=14.1396(8) \AA, \mathrm{c}=$ 16.7429(9) $\AA, \alpha=\beta=\gamma=90^{\circ}, V=1975.34(19) \AA^{3}, T=$ 100.0(1) K, $Z=4, \quad Z^{\prime}=1, \mu\left(\mathrm{MoK}_{\alpha}\right)=0.145,46813$ reflections measured, 4532 unique $\left(R_{\text {int }}=0.0386\right)$ which were used in all calculations. The final $w R_{2}$ was 0.0682 (all data) and $R_{1}$ was 0.0268 (I > 2(I)). 


\begin{tabular}{|c|c|}
\hline Compound & $11 \mathrm{~h}$ \\
\hline CCDC & 1982668 \\
\hline Formula & $\mathrm{C}_{20} \mathrm{H}_{31} \mathrm{BNOP}$ \\
\hline$D_{\text {calc. }} / \mathrm{g} \mathrm{cm}^{-3}$ & 1.154 \\
\hline$\mu / \mathrm{mm}^{-1}$ & 0.145 \\
\hline Formula Weight & 343.24 \\
\hline Color & clear light colorless \\
\hline Shape & prism \\
\hline Size $/ \mathrm{mm}^{3}$ & $0.34 \times 0.31 \times 0.21$ \\
\hline$T / \mathrm{K}$ & $100.0(1)$ \\
\hline Crystal System & orthorhombic \\
\hline Flack Parameter & $0.02(2)$ \\
\hline Hooft Parameter & $0.030(19)$ \\
\hline Space Group & $P 2{ }_{1} 2_{1} 2_{1}$ \\
\hline$a / \AA$ & $8.3440(5)$ \\
\hline$b / \AA$ & $14.1396(8)$ \\
\hline$c / \AA$ & $16.7429(9)$ \\
\hline$\alpha /^{\circ}$ & 90 \\
\hline$\beta /^{\circ}$ & 90 \\
\hline$\gamma /^{\circ}$ & 90 \\
\hline $\mathrm{V} / \AA^{3}$ & 1975.34(19) \\
\hline$Z$ & 4 \\
\hline$Z^{\prime}$ & 1 \\
\hline Wavelength/Å & 0.71073 \\
\hline Radiation type & $\operatorname{MoK}_{\alpha}$ \\
\hline$\Theta_{\min } /^{\circ}$ & 3.128 \\
\hline$\Theta_{\max } /^{\circ}$ & 27.565 \\
\hline Measured Refl. & 46813 \\
\hline Independent Refl. & 4532 \\
\hline Reflections with I > & 4349 \\
\hline $2(\mathrm{I})$ & \\
\hline$R_{\text {int }}$ & 0.0386 \\
\hline Parameters & 225 \\
\hline Restraints & 0 \\
\hline Largest Peak & 0.253 \\
\hline Deepest Hole & -0.227 \\
\hline GooF & 1.055 \\
\hline$w R_{2}$ (all data) & 0.0682 \\
\hline$w R_{2}$ & 0.0667 \\
\hline$R_{1}$ (all data) & 0.0292 \\
\hline$R_{1}$ & 0.0268 \\
\hline
\end{tabular}


Reflections:

A clear light colorless prism-shaped crystal with dimensions $0.34 \times 0.31 \times 0.21 \mathrm{~mm}^{3}$ was mounted on a MITIGEN holder oil. Data were collected using a Bruker D8 VENTURE diffractometer equipped with an Oxford Cryosystems low-temperature device operating at $T=100.0(1) \mathrm{K}$. Data were measured using $\phi$ and $\omega$ scans using $\operatorname{MoK}_{\alpha}$ radiation. The total number of runs and images was based on the strategy calculation from the program APEX3 (Bruker, 2015) The maximum resolution that was achieved was $\Theta$ $=27.565^{\circ}(0.77 \AA)$. The diffraction pattern was indexed. The total number of runs and images was based on the strategy calculation from the program APEX3 (Bruker, 2015) and the unit cell was refined using SAINT (Bruker, V8.38A, after 2013) on 9390 reflections, 20\% of the observed reflections. Data reduction, scaling and absorption corrections were performed using SAINT (Bruker, V8.38A, after 2013). The final completeness is $99.40 \%$ out to $27.565^{\circ}$ in $\Theta$. A multi-scan absorption correction was performed using SADABS-2016/2 (Bruker, 2016) was used for absorption correction. $w R_{2}$ (int) was 0.0568 before and 0.0532 after correction. The Ratio of minimum to maximum transmission is 0.9495 . The absorption coefficient $\mu$ of this material is $0.145 \mathrm{~mm}^{-1}$ at this wavelength $(\lambda=0.711 \AA)$ and the minimum and maximum transmissions are 0.910 and 0.958 . The structure was solved and the space group $P 2{ }_{1} 2_{1} 2_{1}$ (\# 19) determined by the ShelXT (Sheldrick, 2015) structure solution program using Intrinsic Phasing and refined by Least Squares using version 2018/3 of ShelXL (Sheldrick, 2015). All non-hydrogen atoms were refined anisotropically. Hydrogen atom positions were calculated geometrically and refined using the riding model. Hydrogen atom on Boron atom were refined using an AFIX 138, which would allow the refinement of a common B-H distances, the same shifts being applied to all the B-H bonds to the same atom, but still retain tetrahedral $\mathrm{H}-\mathrm{B}-\mathrm{H}$ angles within the group. There is a single molecule in the asymmetric unit, which is represented by the reported sum formula. In other words: $\mathrm{Z}$ is 4 and Z' is 1 . The Flack parameter was refined to 0.02(2). Determination of absolute structure using Bayesian statistics on Bijvoet differences using the Olex2 results in 0.030(19). Note: The Flack parameter is used to determine chirality of the crystal studied, the value should be near 0 , a value of 1 means that the stereochemistry is wrong and the model should be inverted. A value of 0.5 means that the crystal consists of a racemic mixture of the two enantiomers.

Table S4: Bond Lengths in Å for compound 11h.

\begin{tabular}{lll}
\hline Atom & Atom & Length/Å \\
\hline P1 & N1 & $1.6686(13)$ \\
P1 & C17 & $1.8629(16)$ \\
P1 & C11 & $1.8203(15)$ \\
P1 & B1 & $1.9290(18)$ \\
O1 & C7 & $1.4248(19)$ \\
N1 & C8 & $1.484(2)$ \\
N1 & C10 & $1.465(2)$ \\
C16 & C15 & $1.395(2)$ \\
C16 & C11 & $1.394(2)$ \\
C15 & C14 & $1.383(3)$ \\
C17 & C19 & $1.537(2)$ \\
C17 & C18 & $1.531(2)$ \\
C17 & C20 & $1.542(2)$
\end{tabular}

\begin{tabular}{lll}
\hline Atom & Atom & Length/Å \\
\hline C6 & C5 & $1.392(2)$ \\
C6 & C1 & $1.391(2)$ \\
C8 & C9 & $1.527(2)$ \\
C8 & C7 & $1.548(2)$ \\
C4 & C5 & $1.385(3)$ \\
C4 & C3 & $1.384(3)$ \\
C2 & C3 & $1.396(3)$ \\
C2 & C1 & $1.390(2)$ \\
C11 & C12 & $1.401(2)$ \\
C12 & C13 & $1.388(2)$ \\
C1 & C7 & $1.523(2)$ \\
C14 & C13 & $1.390(3)$
\end{tabular}


Table S5: Bond Angles in ${ }^{\circ}$ for compound $11 \mathrm{~h}$.

\begin{tabular}{llll}
\hline Atom & Atom & Atom & Angle $/^{\circ}$ \\
\hline N1 & P1 & C17 & $108.48(8)$ \\
N1 & P1 & C11 & $103.27(7)$ \\
N1 & P1 & B1 & $113.54(8)$ \\
C17 & P1 & B1 & $111.02(8)$ \\
C11 & P1 & C17 & $110.78(8)$ \\
C11 & P1 & B1 & $109.49(8)$ \\
C8 & N1 & P1 & $120.90(10)$ \\
C10 & N1 & P1 & $121.42(11)$ \\
C10 & N1 & C8 & $117.63(13)$ \\
C11 & C16 & C15 & $120.54(15)$ \\
C14 & C15 & C16 & $120.41(16)$ \\
C19 & C17 & P1 & $112.19(12)$ \\
C19 & C17 & C20 & $107.84(15)$ \\
C18 & C17 & P1 & $112.97(12)$ \\
C18 & C17 & C19 & $109.92(14)$ \\
C18 & C17 & C20 & $109.18(15)$ \\
C20 & C17 & P1 & $104.44(11)$ \\
C1 & C6 & C5 & $120.71(16)$ \\
N1 & C8 & C9 & $111.74(13)$
\end{tabular}

\begin{tabular}{lllc}
\hline Atom & Atom & Atom & Angle $^{\circ}$ \\
\hline N1 & C8 & C7 & $111.41(12)$ \\
C9 & C8 & C7 & $112.64(14)$ \\
C3 & C4 & C5 & $119.54(17)$ \\
C1 & C2 & C3 & $120.65(17)$ \\
C4 & C5 & C6 & $120.24(17)$ \\
C4 & C3 & C2 & $120.19(18)$ \\
C16 & C11 & P1 & $125.27(13)$ \\
C16 & C11 & C12 & $118.57(14)$ \\
C12 & C11 & P1 & $116.13(12)$ \\
C13 & C12 & C11 & $120.57(16)$ \\
C6 & C1 & C7 & $118.59(15)$ \\
C2 & C1 & C6 & $118.65(16)$ \\
C2 & C1 & C7 & $122.75(15)$ \\
O1 & C7 & C8 & $112.40(13)$ \\
01 & C7 & C1 & $114.07(14)$ \\
C1 & C7 & C8 & $111.05(13)$ \\
C15 & C14 & C13 & $119.47(16)$ \\
C12 & C13 & C14 & $120.42(16)$
\end{tabular}

Table S6: Torsion Angles in ${ }^{\circ}$ for compound $11 \mathrm{~h}$.

\begin{tabular}{llllc}
\hline Atom & Atom & Atom & Atom & Angle ${ }^{\circ}$ \\
\hline P1 & N1 & C8 & C9 & $146.37(12)$ \\
P1 & N1 & C8 & C7 & $-86.65(15)$ \\
P1 & C11 & C12 & C13 & $-178.73(14)$ \\
N1 & P1 & C17 & C19 & $177.17(11)$ \\
N1 & P1 & C17 & C18 & $-57.89(14)$ \\
N1 & P1 & C17 & C20 & $60.64(14)$ \\
N1 & P1 & C11 & C16 & $137.03(15)$ \\
N1 & P1 & C11 & C12 & $-44.99(14)$ \\
N1 & C8 & C7 & O1 & $-61.12(17)$ \\
N1 & C8 & C7 & C1 & $169.74(13)$ \\
C16 & C15 & C14 & C13 & $-0.3(3)$ \\
C16 & C11 & C12 & C13 & $-0.6(3)$ \\
C15 & C16 & C11 & P1 & $179.02(13)$ \\
C15 & C16 & C11 & C12 & $1.1(2)$ \\
C15 & C14 & C13 & C12 & $0.8(3)$ \\
C17 & P1 & N1 & C8 & $-108.12(13)$ \\
C17 & P1 & N1 & C10 & $74.40(14)$ \\
C17 & P1 & C11 & C16 & $21.06(17)$ \\
C17 & P1 & C11 & C12 & $-160.96(13)$ \\
C6 & C1 & C7 & 01 & $168.64(15)$ \\
C6 & C1 & C7 & C8 & $-63.1(2)$ \\
C9 & C8 & C7 & 01 & $65.37(17)$ \\
C9 & C8 & C7 & C1 & $-63.77(18)$ \\
C10 & N1 & C8 & C9 & $-36.1(2)$ \\
C10 & N1 & C8 & C7 & $90.93(17)$ \\
C2 & C1 & C7 & 01 & $-11.9(2)$ \\
C2 & C1 & C7 & C8 & $116.38(18)$ \\
C5 & C6 & C1 & C2 & $-1.0(3)$ \\
C5 & C6 & C1 & C7 & $178.55(15)$ \\
\end{tabular}




\begin{tabular}{llllc}
\hline Atom & Atom & Atom & Atom & Angle $^{\circ}$ \\
\hline C5 & C4 & C3 & C2 & $-0.2(3)$ \\
C3 & C4 & C5 & C6 & $0.0(3)$ \\
C3 & C2 & C1 & C6 & $0.7(3)$ \\
C3 & C2 & C1 & C7 & $-178.78(17)$ \\
C11 & P1 & N1 & C8 & $134.29(13)$ \\
C11 & P1 & N1 & C10 & $-43.19(15)$ \\
C11 & P1 & C17 & C19 & $-70.14(13)$ \\
C11 & P1 & C17 & C18 & $54.79(14)$ \\
C11 & P1 & C17 & C20 & $173.32(12)$ \\
C11 & C16 & C15 & C14 & $-0.6(3)$ \\
C11 & C12 & C13 & C14 & $-0.3(3)$ \\
C1 & C6 & C5 & C4 & $0.6(3)$ \\
C1 & C2 & C3 & C4 & $-0.1(3)$ \\
B1 & P1 & N1 & C8 & $15.82(15)$ \\
B1 & P1 & N1 & C10 & $-161.67(13)$ \\
B1 & P1 & C17 & C19 & $51.74(14)$ \\
B1 & P1 & C17 & C18 & $176.68(13)$ \\
B1 & P1 & C17 & C20 & $-64.79(15)$ \\
B1 & P1 & C11 & C16 & $-101.71(16)$ \\
B1 & P1 & C11 & C12 & $76.27(14)$
\end{tabular}


Name: (M, $\left.S_{\mathrm{p}}\right)-(+)-\mathrm{N}-[(1 S, 2 R)-2-($ Hydroxy-1-methyl-2-phenylethyl)], N-methylamino-2-(1,1'-binaphtyl phenyl)phosphine-borane 11v.

Submitted by: Jérôme Bayardon

Solved by: Yoann Rousselin

Sample ID: $\quad$ 11jb659

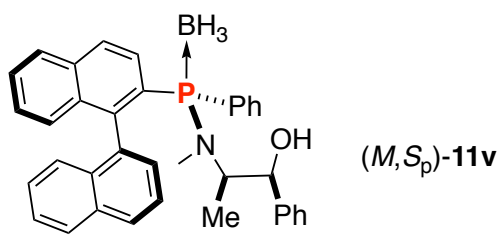

\section{Crystal Data and Experimental}

Figure S3 (thermal ellipsoïds are drawn at $50 \%$ probability plot)

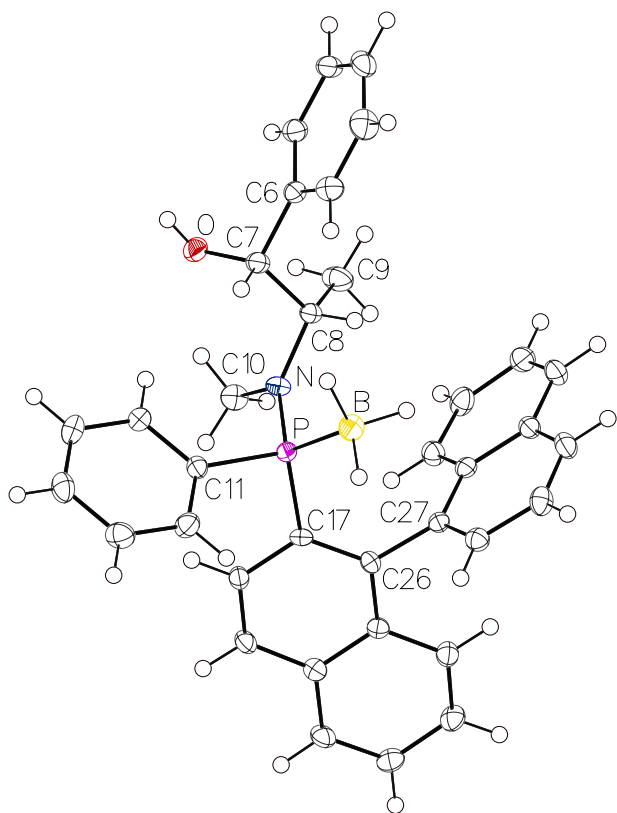

Experimental. Single clear light colorless prism-shaped crystals of compound $11 \mathbf{v}$ were recrystallized from ethyl acetate by slow evaporation. A suitable crystal $0.20 \times 0.17 \times 0.07 \mathrm{~mm}^{3}$ was selected and mounted on a glass fiber with grease on a Nonius Kappa APEX II diffractometer. The crystal was kept at a steady $T=$ 115.0(1) K during data collection. The structure was solved with the SUPERFLIP (Palatinus et al., 2007) structure solution program using the charge flipping methods solution method and by using olex2 (Dolomanov et al., 2009) as the graphical interface. The model was refined with version 2018/3 of ShelXL (Sheldrick, 2015) using Least Squares minimization.

Crystal Data. $\mathrm{C}_{36} \mathrm{H}_{35} \mathrm{BNOP}, M_{r}=539.43$, triclinic, $P 1$ (No. 1), $\mathrm{a}=7.6655(2) \AA, \mathrm{b}=10.2111(3) \AA, \mathrm{c}=10.5115(4) \AA$, $\alpha=99.6520(10)^{\circ}, \beta=111.305(2)^{\circ}, \gamma=103.266(2)^{\circ}, \quad V=$ 716.92(4) ̊ํ, $T=115.0(1) \mathrm{K}, Z=1, Z^{\prime}=1, \mu\left(\mathrm{MoK}_{\alpha}\right)=$ $0.126,6093$ reflections measured, 6093 unique $\left(R_{\text {int }}=0\right)$ which were used in all calculations. The final $w R_{2}$ was 0.0792 (all data) and $R_{1}$ was 0.0356 (I > 2(I)). 


\begin{tabular}{|c|c|}
\hline Compound & $11 v$ \\
\hline CCDC & 1982669 \\
\hline Formula & $\mathrm{C}_{36} \mathrm{H}_{35} \mathrm{BNOP}$ \\
\hline$D_{\text {calc. }} / \mathrm{g} \mathrm{cm}^{-3}$ & 1.249 \\
\hline$\mu / \mathrm{mm}^{-1}$ & 0.126 \\
\hline Formula Weight & 539.43 \\
\hline Color & $\begin{array}{l}\text { clear light } \\
\text { colorless }\end{array}$ \\
\hline Shape & prism \\
\hline Size $/ \mathrm{mm}^{3}$ & $0.20 \times 0.17 \times 0.07$ \\
\hline $\mathrm{T} / \mathrm{K}$ & $115.0(1)$ \\
\hline Crystal System & triclinic \\
\hline Flack Parameter & $0.00(9)$ \\
\hline Hooft Parameter & $0.03(2)$ \\
\hline Space Group & $P 1$ \\
\hline$a / \AA$ & $7.6655(2)$ \\
\hline$b / \AA$ & $10.2111(3)$ \\
\hline$c / \AA$ & $10.5115(4)$ \\
\hline$\alpha /^{\circ}$ & $99.6520(10)$ \\
\hline$\beta /^{\circ}$ & $111.305(2)$ \\
\hline$\gamma /{ }^{\circ}$ & $103.266(2)$ \\
\hline $\mathrm{V} / \AA^{3}$ & $716.92(4)$ \\
\hline$Z$ & 1 \\
\hline$Z^{\prime}$ & 1 \\
\hline Wavelength/Å & 0.71073 \\
\hline Radiation type & $\operatorname{MoK}_{\alpha}$ \\
\hline$\Theta_{\min } /^{\circ}$ & 3.366 \\
\hline$\Theta_{\max } /^{\circ}$ & 27.502 \\
\hline Measured Refl. & 6093 \\
\hline Independent Refl. & 6093 \\
\hline Reflections with I > 2(I) & 5946 \\
\hline$R_{\text {int }}$ & 0 \\
\hline Parameters & 367 \\
\hline Restraints & 3 \\
\hline Largest Peak & 0.264 \\
\hline Deepest Hole & -0.198 \\
\hline GooF & 1.073 \\
\hline$w R_{2}$ (all data) & 0.0792 \\
\hline$w R_{2}$ & 0.0773 \\
\hline$R_{1}$ (all data) & 0.0374 \\
\hline$R_{1}$ & 0.0356 \\
\hline
\end{tabular}


Reflections:

Refinement:
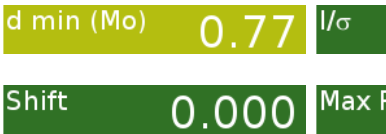

32.0

Rint Merged!

complete $\quad 95 \%$

Max Peak $\quad 0.3$

A clear light colorless prism-shaped crystal with dimensions $0.20 \times 0.17 \times 0.07 \mathrm{~mm}^{3}$ was mounted on a glass fiber with grease. Data were collected using a Nonius Kappa APEX II diffractometer equipped with an Oxford Cryosystems low-temperature device operating at $T=115.0(1) \mathrm{K}$. Data were measured using $\phi$ and $\omega$ scans using $\mathrm{MoK}_{\alpha}$ radiation. The total number of runs and images was based on the strategy calculation from the program Collect (Bruker, 1997-2004). The maximum resolution that was achieved was $\Theta=27.502^{\circ}(0.77 \AA)$. The diffraction pattern was indexed. The total number of runs and images was based on the strategy calculation from the program Collect (Bruker, 1997-2004) and the unit cell was refined using DENZO (Otwinowski \& Minor, 1997) on 2949 reflections, 48\% of the observed reflections. Data reduction, scaling and absorption corrections were performed using DENZO (Otwinowski \& Minor, 1997). The final completeness is $99.20 \%$ out to $27.502^{\circ}$ in $\Theta$. No absorption correction was performed. The absorption coefficient $\mu$ of this material is $0.126 \mathrm{~mm}^{-1}$ at this wavelength $(\lambda=0.711 \AA)$. The structure was solved and the space group P1 (\# 1) determined by the SUPERFLIP (Palatinus et al., 2007) structure solution program using charge flipping methods and refined by Least Squares using version 2018/3 of ShelXL (Sheldrick, 2015). All non-hydrogen atoms were refined anisotropically. Hydrogen atom positions were calculated geometrically and refined using the riding model. Most hydrogen atom positions were calculated geometrically and refined using the riding model, but some hydrogen atoms were refined freely. Hydrogen atom on Boron atom were refined using an AFIX 138, which would allow the refinement of a common B-H distances, the same shifts being applied to all the B$\mathrm{H}$ bonds to the same atom, but still retain tetrahedral $\mathrm{H}-\mathrm{B}-\mathrm{H}$ angles within the group.

Table S7: Bond Lengths in Å for compound 11v.

\begin{tabular}{|c|c|c|c|c|c|}
\hline Atom & Atom & Length/Å & Atom & Atom & Length/Å \\
\hline C1 & $\mathrm{C} 2$ & $1.396(4)$ & C19 & $\mathrm{C} 20$ & $1.414(3)$ \\
\hline $\mathrm{C} 1$ & C6 & $1.390(4)$ & $\mathrm{C} 20$ & $\mathrm{C} 21$ & $1.418(4)$ \\
\hline $\mathrm{C} 2$ & $\mathrm{C} 3$ & $1.381(4)$ & $\mathrm{C} 20$ & $\mathrm{C} 25$ & $1.424(3)$ \\
\hline C3 & $\mathrm{C} 4$ & $1.380(4)$ & $\mathrm{C} 21$ & $\mathrm{C} 22$ & $1.366(4)$ \\
\hline $\mathrm{C} 4$ & $\mathrm{C} 5$ & $1.391(4)$ & $\mathrm{C} 22$ & $\mathrm{C} 23$ & $1.405(4)$ \\
\hline C5 & $\mathrm{C} 6$ & $1.394(4)$ & $\mathrm{C} 23$ & $\mathrm{C} 24$ & $1.370(4)$ \\
\hline C6 & $\mathrm{C} 7$ & $1.523(3)$ & $\mathrm{C} 24$ & $\mathrm{C} 25$ & $1.423(3)$ \\
\hline C7 & $\mathrm{C} 8$ & $1.543(3)$ & $\mathrm{C} 25$ & $\mathrm{C} 26$ & $1.436(3)$ \\
\hline C7 & 0 & $1.434(3)$ & $\mathrm{C} 26$ & $\mathrm{C} 27$ & $1.498(3)$ \\
\hline C8 & $\mathrm{C} 9$ & $1.525(4)$ & $\mathrm{C} 27$ & $\mathrm{C} 28$ & $1.373(4)$ \\
\hline C8 & $\mathrm{N}$ & $1.482(3)$ & $\mathrm{C} 27$ & C36 & $1.424(3)$ \\
\hline C10 & $\mathrm{N}$ & $1.467(4)$ & $\mathrm{C} 28$ & $\mathrm{C} 29$ & $1.412(4)$ \\
\hline C11 & $\mathrm{C} 12$ & $1.390(3)$ & $\mathrm{C} 29$ & C30 & $1.364(4)$ \\
\hline C11 & C16 & $1.396(4)$ & C30 & C31 & $1.421(4)$ \\
\hline C11 & $\mathrm{P}$ & $1.828(2)$ & C31 & C32 & $1.419(4)$ \\
\hline C12 & C13 & $1.394(4)$ & C31 & C36 & $1.424(3)$ \\
\hline C13 & C14 & $1.377(4)$ & C32 & C33 & $1.365(4)$ \\
\hline C14 & C15 & $1.385(4)$ & C33 & C34 & $1.407(4)$ \\
\hline C15 & C16 & $1.394(4)$ & C34 & C35 & $1.368(4)$ \\
\hline C17 & C18 & $1.424(3)$ & C35 & C36 & $1.423(4)$ \\
\hline C17 & $\mathrm{C} 26$ & $1.390(3)$ & B & $\mathrm{P}$ & $1.925(3)$ \\
\hline C17 & $\mathrm{P}$ & $1.840(2)$ & $\mathrm{N}$ & $P$ & $1.662(2)$ \\
\hline C18 & C19 & $1.364(4)$ & & & \\
\hline
\end{tabular}


Table S8: Bond Angles in ${ }^{\circ}$ for compound 11v.

\begin{tabular}{|c|c|c|c|c|c|c|c|}
\hline Atom & Atom & Atom & Angle $/^{\circ}$ & Atom & Atom & Atom & Angle $/{ }^{\circ}$ \\
\hline$\overline{\mathrm{C} 6}$ & $\mathrm{C} 1$ & $\mathrm{C} 2$ & $120.6(3)$ & $\overline{\mathrm{C} 23}$ & C24 & $\mathrm{C} 25$ & $120.6(2)$ \\
\hline C3 & C2 & C1 & $119.6(3)$ & C20 & C25 & C26 & $119.8(2)$ \\
\hline C4 & C3 & $\mathrm{C} 2$ & $120.3(3)$ & C24 & $\mathrm{C} 25$ & $\mathrm{C} 20$ & $118.0(2)$ \\
\hline C3 & $\mathrm{C} 4$ & C5 & $120.4(3)$ & $\mathrm{C} 24$ & $\mathrm{C} 25$ & C26 & $122.2(2)$ \\
\hline C4 & C5 & C6 & $120.0(3)$ & C17 & C26 & $\mathrm{C} 25$ & $120.0(2)$ \\
\hline $\mathrm{C} 1$ & C6 & C5 & $119.1(2)$ & C17 & C26 & $\mathrm{C} 27$ & $122.7(2)$ \\
\hline C1 & C6 & $\mathrm{C} 7$ & $119.5(2)$ & $\mathrm{C} 25$ & $\mathrm{C} 26$ & $\mathrm{C} 27$ & $117.3(2)$ \\
\hline C5 & C6 & C7 & $121.4(2)$ & C28 & $\mathrm{C} 27$ & $\mathrm{C} 26$ & $121.3(2)$ \\
\hline C6 & $\mathrm{C} 7$ & C8 & $111.6(2)$ & $\mathrm{C} 28$ & $\mathrm{C} 27$ & C36 & $119.8(2)$ \\
\hline 0 & C7 & C6 & $112.0(2)$ & C36 & C27 & C26 & $118.9(2)$ \\
\hline 0 & $\mathrm{C} 7$ & C8 & $108.3(2)$ & $\mathrm{C} 27$ & $\mathrm{C} 28$ & C29 & $121.1(2)$ \\
\hline C9 & C8 & $\mathrm{C} 7$ & $112.4(2)$ & C30 & $\mathrm{C} 29$ & $\mathrm{C} 28$ & $120.0(3)$ \\
\hline $\mathrm{N}$ & $\mathrm{C} 8$ & $\mathrm{C} 7$ & $111.5(2)$ & $\mathrm{C} 29$ & C30 & C31 & $120.9(2)$ \\
\hline $\mathrm{N}$ & C8 & C9 & $111.3(2)$ & C30 & C31 & C36 & $118.8(2)$ \\
\hline C12 & C11 & C16 & $118.7(2)$ & C32 & C31 & C30 & $122.1(2)$ \\
\hline C12 & C11 & $\mathrm{P}$ & 121.14(19) & C32 & C31 & C36 & $119.0(2)$ \\
\hline C16 & C11 & $P$ & 119.97(19) & C33 & C32 & C31 & $120.8(3)$ \\
\hline C11 & C12 & C13 & $120.4(2)$ & C32 & C33 & C34 & 120.1(3) \\
\hline C14 & $\mathrm{C} 13$ & C12 & $120.5(3)$ & C35 & C34 & C33 & $121.0(3)$ \\
\hline C13 & C14 & C15 & $119.7(2)$ & C34 & C35 & C36 & $120.3(2)$ \\
\hline C14 & C15 & C16 & $120.2(3)$ & $\mathrm{C} 27$ & C36 & C31 & $119.2(2)$ \\
\hline C15 & C16 & C11 & $120.4(2)$ & C35 & C36 & $\mathrm{C} 27$ & $122.1(2)$ \\
\hline C18 & C17 & $\mathrm{P}$ & 118.27(18) & C35 & C36 & C31 & $118.6(2)$ \\
\hline C26 & C17 & C18 & $119.0(2)$ & C8 & $\mathrm{N}$ & $\mathrm{P}$ & $120.38(18)$ \\
\hline $\mathrm{C} 26$ & C17 & $\mathrm{P}$ & $122.70(18)$ & C10 & $\mathrm{N}$ & $\mathrm{C} 8$ & $118.6(2)$ \\
\hline C19 & C18 & C17 & $121.5(2)$ & C10 & $\mathrm{N}$ & $\mathrm{P}$ & $120.90(17)$ \\
\hline C18 & C19 & $\mathrm{C} 20$ & $121.2(2)$ & C11 & $P$ & $\mathrm{C} 17$ & $102.08(11)$ \\
\hline C19 & $\mathrm{C} 20$ & $\mathrm{C} 21$ & $121.9(2)$ & C11 & $P$ & B & $106.04(13)$ \\
\hline C19 & $\mathrm{C} 20$ & $\mathrm{C} 25$ & $118.4(2)$ & C17 & $\mathrm{P}$ & B & $119.18(12)$ \\
\hline $\mathrm{C} 21$ & $\mathrm{C} 20$ & $\mathrm{C} 25$ & $119.7(2)$ & $\mathrm{N}$ & $\mathrm{P}$ & C11 & $108.27(11)$ \\
\hline $\mathrm{C} 22$ & $\mathrm{C} 21$ & $\mathrm{C} 20$ & $120.6(2)$ & $\mathrm{N}$ & $P$ & C17 & $106.28(11)$ \\
\hline $\mathrm{C} 21$ & C22 & $\mathrm{C} 23$ & $120.0(2)$ & $\mathrm{N}$ & $P$ & B & $113.94(13)$ \\
\hline $\mathrm{C} 24$ & C23 & C22 & 121.1(2) & & & & \\
\hline
\end{tabular}

Table S9: Torsion Angles in ${ }^{\circ}$ for compound 11v.

\begin{tabular}{llllr}
\hline Atom & Atom & Atom & Atom & Angle ${ }^{\circ}$ \\
\hline C1 & C2 & C3 & C4 & $0.0(4)$ \\
C1 & C6 & C7 & C8 & $-89.7(3)$ \\
C1 & C6 & C7 & 0 & $148.6(2)$ \\
C2 & C1 & C6 & C5 & $0.7(4)$ \\
C2 & C1 & C6 & C7 & $-179.8(2)$ \\
C2 & C3 & C4 & C5 & $0.1(4)$ \\
C3 & C4 & C5 & C6 & $0.3(4)$ \\
C4 & C5 & C6 & C1 & $-0.7(4)$ \\
C4 & C5 & C6 & C7 & $179.8(2)$ \\
C5 & C6 & C7 & C8 & $89.8(3)$ \\
C5 & C6 & C7 & 0 & $-31.9(3)$ \\
C6 & C1 & C2 & C3 & $-0.4(4)$ \\
C6 & C7 & C8 & C9 & $-62.8(3)$ \\
C6 & C7 & C8 & N & $171.4(2)$
\end{tabular}




\begin{tabular}{|c|c|c|c|c|}
\hline Atom & Atom & Atom & Atom & Angle $/^{\circ}$ \\
\hline$\overline{\mathrm{C} 7}$ & $\mathrm{C} 8$ & $\mathrm{~N}$ & C10 & $88.3(3)$ \\
\hline $\mathrm{C} 7$ & $\mathrm{C} 8$ & $\mathrm{~N}$ & $\mathrm{P}$ & $-87.5(2)$ \\
\hline $\mathrm{C} 8$ & $\mathrm{~N}$ & $\mathrm{P}$ & C11 & $109.7(2)$ \\
\hline $\mathrm{C} 8$ & $\mathrm{~N}$ & $\mathrm{P}$ & C17 & $-141.28(19)$ \\
\hline $\mathrm{C} 8$ & $\mathrm{~N}$ & $\mathrm{P}$ & $\mathrm{B}$ & $-8.0(2)$ \\
\hline C9 & $\mathrm{C} 8$ & $\mathrm{~N}$ & C10 & $-38.1(3)$ \\
\hline $\mathrm{C} 9$ & $\mathrm{C} 8$ & $\mathrm{~N}$ & $\mathrm{P}$ & $146.1(2)$ \\
\hline C10 & $\mathrm{N}$ & $\mathrm{P}$ & C11 & $-66.0(2)$ \\
\hline C10 & $\mathrm{N}$ & $\mathrm{P}$ & C17 & $43.0(2)$ \\
\hline C10 & $\mathrm{N}$ & $\mathrm{P}$ & $\mathrm{B}$ & $176.3(2)$ \\
\hline C11 & $\mathrm{C} 12$ & C13 & C14 & $-0.3(4)$ \\
\hline C12 & C11 & C16 & C15 & $1.2(4)$ \\
\hline $\mathrm{C} 12$ & $\mathrm{C} 11$ & $\mathrm{P}$ & $\mathrm{C} 17$ & $-123.2(2)$ \\
\hline $\mathrm{C} 12$ & C11 & $\mathrm{P}$ & $\mathrm{B}$ & $111.3(2)$ \\
\hline $\mathrm{C} 12$ & C11 & $\mathrm{P}$ & $\mathrm{N}$ & $-11.3(2)$ \\
\hline C12 & C13 & C14 & C15 & $0.8(4)$ \\
\hline C13 & C14 & C15 & C16 & $-0.4(5)$ \\
\hline C14 & $\mathrm{C} 15$ & C16 & C11 & $-0.6(4)$ \\
\hline C16 & C11 & $\mathrm{C} 12$ & C13 & $-0.7(4)$ \\
\hline C16 & $\mathrm{C} 11$ & $\mathrm{P}$ & C17 & $61.3(2)$ \\
\hline C16 & C11 & $\mathrm{P}$ & $\mathrm{B}$ & $-64.2(2)$ \\
\hline C16 & C11 & $\mathrm{P}$ & $\mathrm{N}$ & $173.2(2)$ \\
\hline C17 & C18 & C19 & $\mathrm{C} 20$ & $-1.7(4)$ \\
\hline C17 & $\mathrm{C} 26$ & $\mathrm{C} 27$ & $\mathrm{C} 28$ & $94.3(3)$ \\
\hline C17 & $\mathrm{C} 26$ & $\mathrm{C} 27$ & C36 & $-88.4(3)$ \\
\hline C18 & C17 & $\mathrm{C} 26$ & $\mathrm{C} 25$ & $0.3(3)$ \\
\hline C18 & C17 & $\mathrm{C} 26$ & $\mathrm{C} 27$ & $179.0(2)$ \\
\hline C18 & C17 & $\mathrm{P}$ & C11 & $25.0(2)$ \\
\hline C18 & $\mathrm{C} 17$ & $\mathrm{P}$ & $\mathrm{B}$ & $141.3(2)$ \\
\hline C18 & C17 & $\mathrm{P}$ & $\mathrm{N}$ & $-88.4(2)$ \\
\hline C18 & C19 & $\mathrm{C} 20$ & $\mathrm{C} 21$ & $179.5(3)$ \\
\hline C18 & C19 & $\mathrm{C} 20$ & $\mathrm{C} 25$ & $-1.3(4)$ \\
\hline C19 & $\mathrm{C} 20$ & $\mathrm{C} 21$ & $\mathrm{C} 22$ & $177.3(3)$ \\
\hline C19 & $\mathrm{C} 20$ & $\mathrm{C} 25$ & $\mathrm{C} 24$ & $-176.4(2)$ \\
\hline C19 & $\mathrm{C} 20$ & $\mathrm{C} 25$ & $\mathrm{C} 26$ & $3.8(3)$ \\
\hline $\mathrm{C} 20$ & $\mathrm{C} 21$ & $\mathrm{C} 22$ & $\mathrm{C} 23$ & $-0.4(4)$ \\
\hline $\mathrm{C} 20$ & $\mathrm{C} 25$ & $\mathrm{C} 26$ & C17 & $-3.3(3)$ \\
\hline $\mathrm{C} 20$ & $\mathrm{C} 25$ & $\mathrm{C} 26$ & $\mathrm{C} 27$ & $177.9(2)$ \\
\hline $\mathrm{C} 21$ & $\mathrm{C} 20$ & $\mathrm{C} 25$ & $\mathrm{C} 24$ & $2.7(4)$ \\
\hline $\mathrm{C} 21$ & $\mathrm{C} 20$ & $\mathrm{C} 25$ & $\mathrm{C} 26$ & $-177.1(2)$ \\
\hline $\mathrm{C} 21$ & $\mathrm{C} 22$ & $\mathrm{C} 23$ & $\mathrm{C} 24$ & $1.7(4)$ \\
\hline $\mathrm{C} 22$ & $\mathrm{C} 23$ & $\mathrm{C} 24$ & $\mathrm{C} 25$ & $-0.7(4)$ \\
\hline $\mathrm{C} 23$ & $\mathrm{C} 24$ & $\mathrm{C} 25$ & $\mathrm{C} 20$ & $-1.5(4)$ \\
\hline $\mathrm{C} 23$ & $\mathrm{C} 24$ & $\mathrm{C} 25$ & $\mathrm{C} 26$ & $178.3(2)$ \\
\hline $\mathrm{C} 24$ & $\mathrm{C} 25$ & $\mathrm{C} 26$ & C17 & $176.9(2)$ \\
\hline $\mathrm{C} 24$ & $\mathrm{C} 25$ & $\mathrm{C} 26$ & $\mathrm{C} 27$ & $-1.8(3)$ \\
\hline $\mathrm{C} 25$ & $\mathrm{C} 20$ & $\mathrm{C} 21$ & $\mathrm{C} 22$ & $-1.8(4)$ \\
\hline $\mathrm{C} 25$ & $\mathrm{C} 26$ & $\mathrm{C} 27$ & $\mathrm{C} 28$ & $-87.0(3)$ \\
\hline $\mathrm{C} 25$ & $\mathrm{C} 26$ & $\mathrm{C} 27$ & C36 & $90.4(3)$ \\
\hline $\mathrm{C} 26$ & C17 & C18 & C19 & $2.2(4)$ \\
\hline $\mathrm{C} 26$ & C17 & $\mathrm{P}$ & C11 & $-156.7(2)$ \\
\hline C26 & C17 & $\mathrm{P}$ & B & $-40.4(3)$ \\
\hline $\mathrm{C} 26$ & C17 & $\mathrm{P}$ & $\mathrm{N}$ & $89.9(2)$ \\
\hline $\mathrm{C} 26$ & $\mathrm{C} 27$ & $\mathrm{C} 28$ & $\mathrm{C} 29$ & $-179.2(2)$ \\
\hline
\end{tabular}




\begin{tabular}{|c|c|c|c|c|}
\hline Atom & Atom & Atom & Atom & Angle $/^{\circ}$ \\
\hline$\overline{\mathrm{C} 26}$ & C27 & C36 & C31 & $177.7(2)$ \\
\hline $\mathrm{C} 26$ & $\mathrm{C} 27$ & C36 & C35 & $-5.0(3)$ \\
\hline C27 & C28 & C29 & C30 & $0.3(4)$ \\
\hline C28 & C27 & C36 & C31 & $-5.0(3)$ \\
\hline $\mathrm{C} 28$ & $\mathrm{C} 27$ & C36 & C35 & $172.4(2)$ \\
\hline $\mathrm{C} 28$ & C29 & C30 & C31 & $-2.8(4)$ \\
\hline $\mathrm{C} 29$ & C30 & C31 & C32 & $-176.7(3)$ \\
\hline C29 & C30 & C31 & C36 & $1.2(4)$ \\
\hline C30 & C31 & C32 & C33 & $175.8(3)$ \\
\hline C30 & C31 & C36 & $\mathrm{C} 27$ & $2.6(3)$ \\
\hline C30 & C31 & C36 & C35 & $-174.8(2)$ \\
\hline C31 & C32 & C33 & C34 & $-1.1(4)$ \\
\hline C32 & C31 & C36 & $\mathrm{C} 27$ & $-179.4(2)$ \\
\hline C32 & C31 & C36 & C35 & $3.1(3)$ \\
\hline C32 & C33 & C34 & C35 & $3.2(4)$ \\
\hline C33 & C34 & C35 & C36 & $-2.1(4)$ \\
\hline C34 & C35 & C36 & $\mathrm{C} 27$ & $-178.5(2)$ \\
\hline C34 & C35 & C36 & C31 & $-1.1(4)$ \\
\hline C36 & $\mathrm{C} 27$ & $\mathrm{C} 28$ & C29 & $3.5(4)$ \\
\hline C36 & C31 & C32 & C33 & $-2.1(4)$ \\
\hline 0 & C7 & C8 & C9 & $61.0(3)$ \\
\hline 0 & $\mathrm{C} 7$ & $\mathrm{C} 8$ & $\mathrm{~N}$ & $-64.8(3)$ \\
\hline$P$ & C11 & C12 & $\mathrm{C} 13$ & $-176.3(2)$ \\
\hline $\mathrm{P}$ & C11 & C16 & C15 & $176.8(2)$ \\
\hline $\mathrm{P}$ & C17 & C18 & C19 & $-179.4(2)$ \\
\hline $\mathrm{P}$ & C17 & $\mathrm{C} 26$ & $\mathrm{C} 25$ & $-177.98(18)$ \\
\hline $\mathrm{P}$ & C17 & $\mathrm{C} 26$ & $\mathrm{C} 27$ & $0.7(3)$ \\
\hline
\end{tabular}


Name: $\left(R_{\mathrm{p}}\right)-(-)-\mathrm{N}-[(1 R, 2 S)-2$-(Diphenylphosphinito-borane)-1-methyl-2-phenylethyl], N-methyl amino (o-biphenylphenyl)phosphine-borane 12f.

Submitted by: Jérôme Bayardon

Solved by: Yoann Rousselin

Sample ID: $\quad$ 16JB1309

\section{Crystal Data and Experimental}

Figure S4 (thermal ellipsoïds are drawn at $50 \%$ probability plot)

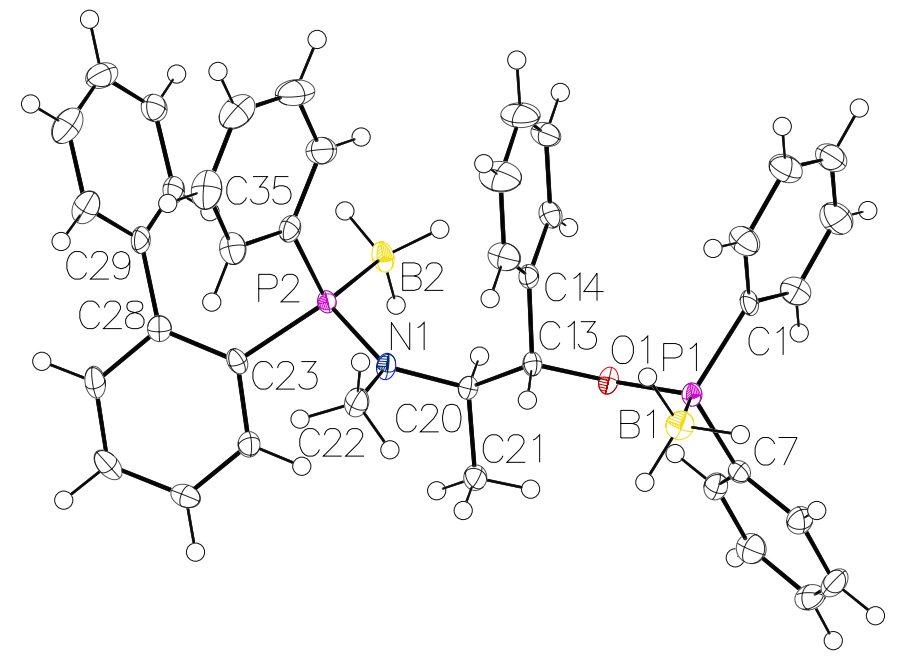

Experimental. Single clear light colorless prism-shaped crystals of compound 12f were recrystallized from a mixture of DCM and hexane by slow evaporation. A suitable crystal $0.82 \times 0.27 \times 0.25 \mathrm{~mm}^{3}$ was selected and mounted on a MITIGEN holder oil on a Bruker D8 Venture diffractometer. The crystal was kept at a steady $T=$ $100.0(1) \mathrm{K}$ during data collection. The structure was solved with the ShelXT (Sheldrick, 2015) structure solution program using the Intrinsic Phasing solution method and by using 0lex2 (Dolomanov et al., 2009) as the graphical interface. The model was refined with version 2018/3 of ShelXL (Sheldrick, 2015) using Least Squares minimization.

Crystal Data. $\mathrm{C}_{40} \mathrm{H}_{43} \mathrm{~B}_{2} \mathrm{NOP}_{2}, M_{r}=637.31$, monoclinic, $P 2{ }_{1}$ (No. 4), $\quad \mathrm{a}=9.3258(3) \AA, \quad \mathrm{b}=15.2698(6) \AA, \quad \mathrm{c}=$ 12.9060(5) $\AA, \quad \beta=90.285(2)^{\circ}, \quad \alpha=\gamma=90^{\circ}, \quad V=$ 1837.83(12) $\AA^{3}, T=100.0(1) \mathrm{K}, Z=2, Z^{\prime}=1, \mu\left(\mathrm{MoK}_{\alpha}\right)=$ $0.149,41082$ reflections measured, 8441 unique $\left(R_{\text {int }}=\right.$ $0.0498)$ which were used in all calculations. The final $w R_{2}$ was 0.0742 (all data) and $R_{1}$ was 0.0329 (I > 2(I)).

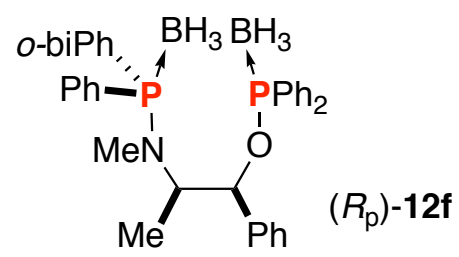




\begin{tabular}{|c|c|}
\hline Compound & $12 \mathrm{f}$ \\
\hline CCDC & 1982665 \\
\hline Formula & $\mathrm{C}_{40} \mathrm{H}_{43} \mathrm{~B}_{2} \mathrm{NOP}_{2}$ \\
\hline$D_{\text {calc. }} / \mathrm{g} \mathrm{cm}^{-3}$ & 1.152 \\
\hline$\mu / \mathrm{mm}^{-1}$ & 0.149 \\
\hline Formula Weight & 637.31 \\
\hline Color & clear light colorless \\
\hline Shape & prism \\
\hline Size $/ \mathrm{mm}^{3}$ & $0.82 \times 0.27 \times 0.25$ \\
\hline$T / \mathrm{K}$ & $100.0(1)$ \\
\hline Crystal System & monoclinic \\
\hline Flack Parameter & $-0.02(2)$ \\
\hline Hooft Parameter & $-0.01(2)$ \\
\hline Space Group & $P 2_{1}$ \\
\hline$a / \AA$ & $9.3258(3)$ \\
\hline$b / \AA$ & $15.2698(6)$ \\
\hline$c / \AA$ & $12.9060(5)$ \\
\hline$\alpha /^{\circ}$ & 90 \\
\hline$\beta /^{\circ}$ & $90.285(2)$ \\
\hline$\gamma /^{\circ}$ & 90 \\
\hline $\mathrm{V} / \AA^{3}$ & $1837.83(12)$ \\
\hline$Z$ & 2 \\
\hline$Z^{\prime}$ & 1 \\
\hline Wavelength/Å & 0.71073 \\
\hline Radiation type & $\operatorname{MoK}_{\alpha}$ \\
\hline$\Theta_{\min } /^{\circ}$ & 3.001 \\
\hline$\Theta_{\max } /^{\circ}$ & 27.529 \\
\hline Measured Refl. & 41082 \\
\hline Independent Refl. & 8441 \\
\hline Reflections with I > & 7697 \\
\hline $2(\mathrm{I})$ & \\
\hline$R_{\text {int }}$ & 0.0498 \\
\hline Parameters & 421 \\
\hline Restraints & 1 \\
\hline Largest Peak & 0.372 \\
\hline Deepest Hole & -0.201 \\
\hline GooF & 1.055 \\
\hline$w R_{2}$ (all data) & 0.0742 \\
\hline$w R_{2}$ & 0.0711 \\
\hline$R_{1}$ (all data) & 0.0399 \\
\hline$R_{1}$ & 0.0329 \\
\hline
\end{tabular}


Reflections:

Refinement:

A clear light colorless prism-shaped crystal with dimensions $0.82 \times 0.27 \times 0.25 \mathrm{~mm}^{3}$ was mounted on a MITIGEN holder oil. Data were collected using a Bruker D8 Venture diffractometer equipped with an Oxford Cryosystems low-temperature device operating at $T=100.0(1) \mathrm{K}$. Data were measured using $\phi$ and $\omega$ scans using $\mathrm{MoK}_{\alpha}$ radiation. The total number of runs and images was based on the strategy calculation from the program APEX3 (Bruker, 2015) The maximum resolution that was achieved was $\Theta$ $=27.529^{\circ}(0.77 \AA)$. The diffraction pattern was indexed. The total number of runs and images was based on the strategy calculation from the program APEX3 (Bruker, 2015) and the unit cell was refined using SAINT (Bruker, V8.37A, after 2013) on 9092 reflections, 22\% of the observed reflections. Data reduction, scaling and absorption corrections were performed using SAINT (Bruker, V8.37A, after 2013). The final completeness is $99.70 \%$ out to $27.529^{\circ}$ in $\Theta$. A multi-scan absorption correction was performed using SADABS-2014/5 (Bruker,2014) was used for absorption correction. $w R_{2}$ (int) was 0.0770 before and 0.0582 after correction. The Ratio of minimum to maximum transmission is 0.8957 . The $\lambda / 2$ correction factor is 0.00150 . The absorption coefficient $\mu$ of this material is $0.149 \mathrm{~mm}^{-1}$ at this wavelength $(\lambda=0.711 \AA)$ and the minimum and maximum transmissions are 0.668 and 0.746 . The structure was solved and the space group $P 2_{1}$ (\# 4) determined by the ShelXT (Sheldrick, 2015) structure solution program using the Intrinsic Phasing solution method and refined by Least Squares using version 2018/3 of ShelXL (Sheldrick, 2015). All non-hydrogen atoms were refined anisotropically. Hydrogen atom positions were calculated geometrically and refined using the riding model. Hydrogen atom on Boron atom were refined using an AFIX 138, which would allow the refinement of a common B$\mathrm{H}$ distances, the same shifts being applied to all the $\mathrm{B}-\mathrm{H}$ bonds to the same atom, but still retain tetrahedral H-B-H angles within the group. There is a single molecule in the asymmetric unit, which is represented by the reported sum formula. In other words: Z is 2 and Z' is 1 . The Flack parameter was refined to -0.02(2). Determination of absolute structure using Bayesian statistics on Bijvoet differences using the Olex2 results in -0.01(2). Note: The Flack parameter is used to determine chirality of the crystal studied, the value should be near 0 , a value of 1 means that the stereochemistry is wrong and the model should be inverted. A value of 0.5 means that the crystal consists of a racemic mixture of the two enantiomers.

Table S10: Bond Lengths in $\AA$ for compound 12f.

\begin{tabular}{lll}
\hline Atom & Atom & Length/A \\
\hline P2 & N1 & $1.6710(19)$ \\
P2 & C23 & $1.838(2)$ \\
P2 & C35 & $1.815(2)$ \\
P2 & B2 & $1.926(3)$ \\
P1 & O1 & $1.6097(16)$ \\
P1 & C1 & $1.808(2)$ \\
P1 & C7 & $1.804(2)$ \\
P1 & B1 & $1.912(3)$ \\
O1 & C13 & $1.457(3)$ \\
N1 & C20 & $1.464(3)$ \\
N1 & C22 & $1.469(3)$ \\
C1 & C6 & $1.390(3)$ \\
C1 & C2 & $1.395(3)$ \\
C6 & C5 & $1.387(3)$ \\
C7 & C12 & $1.395(3)$ \\
C7 & C8 & $1.397(3)$
\end{tabular}

\begin{tabular}{lll}
\hline Atom & Atom & Length/Å \\
\hline C24 & C23 & $1.393(3)$ \\
C24 & C25 & $1.388(3)$ \\
C34 & C29 & $1.389(3)$ \\
C34 & C33 & $1.389(3)$ \\
C20 & C13 & $1.537(3)$ \\
C20 & C21 & $1.527(3)$ \\
C29 & C28 & $1.494(3)$ \\
C29 & C30 & $1.393(3)$ \\
C23 & C28 & $1.408(3)$ \\
C35 & C40 & $1.388(3)$ \\
C35 & C36 & $1.395(3)$ \\
C12 & C11 & $1.387(3)$ \\
C33 & C32 & $1.385(4)$ \\
C13 & C14 & $1.510(3)$ \\
C15 & C14 & $1.389(3)$ \\
C15 & C16 & $1.386(3)$
\end{tabular}




\begin{tabular}{lll}
\hline Atom & Atom & Length/Å \\
\hline C25 & C26 & $1.383(4)$ \\
C5 & C4 & $1.383(4)$ \\
C14 & C19 & $1.385(3)$ \\
C28 & C27 & $1.404(3)$ \\
C2 & C3 & $1.391(3)$ \\
C8 & C9 & $1.390(4)$ \\
C17 & C16 & $1.375(4)$ \\
C17 & C18 & $1.392(4)$ \\
C26 & C27 & $1.385(3)$ \\
C40 & C39 & $1.383(4)$
\end{tabular}

\begin{tabular}{lll}
\hline Atom & Atom & Length/Å \\
\hline C9 & C10 & $1.382(4)$ \\
C19 & C18 & $1.382(4)$ \\
C36 & C37 & $1.391(4)$ \\
C3 & C4 & $1.374(4)$ \\
C38 & C37 & $1.384(4)$ \\
C38 & C39 & $1.377(4)$ \\
C10 & C11 & $1.388(4)$ \\
C31 & C32 & $1.384(4)$ \\
C31 & C30 & $1.380(4)$
\end{tabular}

Table S11: Bond Angles in ${ }^{\circ}$ for compound $12 f$.

\begin{tabular}{|c|c|c|c|c|c|c|c|}
\hline Atom & Atom & Atom & Angle $/^{\circ}$ & Atom & Atom & Atom & Angle $/^{\circ}$ \\
\hline N1 & P2 & $\mathrm{C} 23$ & $105.23(10)$ & $\overline{\mathrm{C} 11}$ & $\mathrm{C} 12$ & $\mathrm{C} 7$ & $120.4(2)$ \\
\hline N1 & $\mathrm{P} 2$ & $\mathrm{C} 35$ & $106.50(10)$ & $\mathrm{C} 32$ & $\mathrm{C} 33$ & C34 & $120.2(2)$ \\
\hline $\mathrm{N} 1$ & P2 & B2 & $111.10(11)$ & 01 & C13 & $\mathrm{C} 20$ & $102.95(16)$ \\
\hline C23 & $\mathrm{P} 2$ & B2 & $112.46(11)$ & 01 & C13 & C14 & $110.60(17)$ \\
\hline C35 & $\mathrm{P} 2$ & $\mathrm{C} 23$ & $105.08(10)$ & C14 & C13 & $\mathrm{C} 20$ & $114.61(18)$ \\
\hline C35 & $\mathrm{P} 2$ & B2 & $115.70(12)$ & C16 & C15 & C14 & $120.2(2)$ \\
\hline 01 & $\mathrm{P} 1$ & C1 & $104.37(9)$ & $\mathrm{C} 26$ & $\mathrm{C} 25$ & $\mathrm{C} 24$ & $119.3(2)$ \\
\hline 01 & $\mathrm{P} 1$ & $\mathrm{C} 7$ & $101.06(9)$ & $\mathrm{C} 4$ & $\mathrm{C} 5$ & C6 & $119.9(2)$ \\
\hline 01 & $\mathrm{P} 1$ & B1 & $115.23(11)$ & C15 & C14 & C13 & $120.4(2)$ \\
\hline $\mathrm{C} 1$ & $\mathrm{P} 1$ & B1 & $115.59(11)$ & C19 & C14 & C13 & $120.4(2)$ \\
\hline $\mathrm{C} 7$ & $\mathrm{P} 1$ & $\mathrm{C} 1$ & $105.84(10)$ & C19 & $\mathrm{C} 14$ & C15 & $119.2(2)$ \\
\hline $\mathrm{C} 7$ & $\mathrm{P} 1$ & B1 & $113.24(11)$ & $\mathrm{C} 23$ & $\mathrm{C} 28$ & $\mathrm{C} 29$ & $124.19(19)$ \\
\hline C13 & 01 & $\mathrm{P} 1$ & $121.90(13)$ & $\mathrm{C} 27$ & $\mathrm{C} 28$ & $\mathrm{C} 29$ & $117.3(2)$ \\
\hline $\mathrm{C} 20$ & N1 & P2 & $122.79(14)$ & $\mathrm{C} 27$ & $\mathrm{C} 28$ & $\mathrm{C} 23$ & $118.5(2)$ \\
\hline $\mathrm{C} 20$ & N1 & $\mathrm{C} 22$ & $117.70(18)$ & $\mathrm{C} 3$ & $\mathrm{C} 2$ & $\mathrm{C} 1$ & $119.9(2)$ \\
\hline C22 & N1 & P2 & $119.49(15)$ & C9 & C8 & $\mathrm{C} 7$ & $120.2(2)$ \\
\hline C6 & $\mathrm{C} 1$ & P1 & $122.50(18)$ & C16 & C17 & C18 & $119.9(2)$ \\
\hline C6 & $\mathrm{C} 1$ & $\mathrm{C} 2$ & $119.4(2)$ & $\mathrm{C} 25$ & $\mathrm{C} 26$ & $\mathrm{C} 27$ & $119.9(2)$ \\
\hline $\mathrm{C} 2$ & $\mathrm{C} 1$ & P1 & $118.07(18)$ & $\mathrm{C} 26$ & $\mathrm{C} 27$ & $\mathrm{C} 28$ & $121.4(2)$ \\
\hline $\mathrm{C} 5$ & $\mathrm{C} 6$ & $\mathrm{C} 1$ & $120.2(2)$ & C39 & $\mathrm{C} 40$ & C35 & $120.7(2)$ \\
\hline C12 & $\mathrm{C} 7$ & P1 & $121.99(17)$ & $\mathrm{C} 10$ & C9 & C8 & $120.1(2)$ \\
\hline C12 & $\mathrm{C} 7$ & $\mathrm{C} 8$ & 119.1(2) & C18 & C19 & C14 & $120.7(2)$ \\
\hline C8 & $\mathrm{C} 7$ & P1 & $118.84(18)$ & $\mathrm{C} 37$ & $\mathrm{C} 36$ & C35 & $120.1(2)$ \\
\hline C25 & $\mathrm{C} 24$ & $\mathrm{C} 23$ & $121.7(2)$ & $\mathrm{C} 4$ & $\mathrm{C} 3$ & $\mathrm{C} 2$ & $120.2(2)$ \\
\hline C33 & C34 & $\mathrm{C} 29$ & $120.3(2)$ & C39 & $\mathrm{C} 38$ & C37 & $120.1(2)$ \\
\hline N1 & $\mathrm{C} 20$ & C13 & $111.67(18)$ & C9 & C10 & C11 & $120.1(2)$ \\
\hline N1 & $\mathrm{C} 20$ & $\mathrm{C} 21$ & $111.99(18)$ & C17 & C16 & C15 & $120.3(2)$ \\
\hline C21 & $\mathrm{C} 20$ & C13 & $110.65(18)$ & $\mathrm{C} 12$ & $\mathrm{C} 11$ & C10 & $120.0(2)$ \\
\hline C34 & $\mathrm{C} 29$ & $\mathrm{C} 28$ & $120.4(2)$ & $\mathrm{C} 30$ & $\mathrm{C} 31$ & $\mathrm{C} 32$ & $120.3(3)$ \\
\hline C34 & $\mathrm{C} 29$ & C30 & $119.0(2)$ & $\mathrm{C} 38$ & $\mathrm{C} 37$ & C36 & $120.0(3)$ \\
\hline C30 & $\mathrm{C} 29$ & $\mathrm{C} 28$ & $120.5(2)$ & $\mathrm{C} 3$ & $\mathrm{C} 4$ & C5 & $120.4(2)$ \\
\hline C24 & $\mathrm{C} 23$ & $\mathrm{P} 2$ & $116.18(17)$ & C19 & C18 & C17 & $119.7(3)$ \\
\hline C24 & $\mathrm{C} 23$ & $\mathrm{C} 28$ & $119.0(2)$ & C31 & $\mathrm{C} 32$ & C33 & $119.6(2)$ \\
\hline C28 & $\mathrm{C} 23$ & P2 & $124.85(17)$ & C31 & $\mathrm{C} 30$ & $\mathrm{C} 29$ & $120.5(2)$ \\
\hline $\mathrm{C} 40$ & C35 & P2 & $118.28(18)$ & $\mathrm{C} 38$ & C39 & $\mathrm{C} 40$ & $120.1(2)$ \\
\hline $\mathrm{C} 40$ & C35 & C36 & $119.0(2)$ & & & & \\
\hline C36 & C35 & $\mathrm{P} 2$ & $122.70(18)$ & & & & \\
\hline
\end{tabular}

Table S12: Torsion Angles in ${ }^{\circ}$ for compound $12 f$. 


\begin{tabular}{|c|c|c|c|c|}
\hline Atom & Atom & Atom & Atom & Angle $/^{\circ}$ \\
\hline P2 & N1 & $\mathrm{C} 20$ & C13 & 119.03(18) \\
\hline P2 & N1 & $\mathrm{C} 20$ & $\mathrm{C} 21$ & $-116.24(19)$ \\
\hline P2 & $\mathrm{C} 23$ & $\mathrm{C} 28$ & C29 & $6.4(3)$ \\
\hline P2 & $\mathrm{C} 23$ & $\mathrm{C} 28$ & $\mathrm{C} 27$ & $-175.08(17)$ \\
\hline P2 & C35 & $\mathrm{C} 40$ & C39 & 179.8(2) \\
\hline P2 & C35 & C36 & C37 & $-179.83(19)$ \\
\hline P1 & 01 & C13 & C20 & $-158.93(13)$ \\
\hline P1 & 01 & C13 & C14 & $78.2(2)$ \\
\hline P1 & $\mathrm{C} 1$ & C6 & $\mathrm{C} 5$ & $177.9(2)$ \\
\hline P1 & $\mathrm{C} 1$ & $\mathrm{C} 2$ & $\mathrm{C} 3$ & $-178.0(2)$ \\
\hline P1 & $\mathrm{C} 7$ & C12 & C11 & $-177.25(18)$ \\
\hline P1 & $\mathrm{C} 7$ & $\mathrm{C} 8$ & C9 & $176.92(18)$ \\
\hline 01 & $\mathrm{P} 1$ & $\mathrm{C} 1$ & $\mathrm{C} 6$ & $-83.3(2)$ \\
\hline 01 & $\mathrm{P} 1$ & $\mathrm{C} 1$ & $\mathrm{C} 2$ & $94.57(19)$ \\
\hline 01 & $\mathrm{P} 1$ & $\mathrm{C} 7$ & C12 & $19.0(2)$ \\
\hline 01 & $\mathrm{P} 1$ & $\mathrm{C} 7$ & C8 & $-158.62(17)$ \\
\hline 01 & $\mathrm{C} 13$ & C14 & C15 & $46.2(3)$ \\
\hline 01 & $\mathrm{C} 13$ & C14 & C19 & $-133.4(2)$ \\
\hline N1 & P2 & $\mathrm{C} 23$ & $\mathrm{C} 24$ & $-27.3(2)$ \\
\hline N1 & $\mathrm{P} 2$ & $\mathrm{C} 23$ & C28 & $153.10(18)$ \\
\hline N1 & $\mathrm{P} 2$ & C35 & $\mathrm{C} 40$ & $107.40(19)$ \\
\hline $\mathrm{N} 1$ & $\mathrm{P} 2$ & C35 & C36 & $-71.3(2)$ \\
\hline $\mathrm{N} 1$ & $\mathrm{C} 20$ & C13 & 01 & $-173.31(16)$ \\
\hline $\mathrm{N} 1$ & $\mathrm{C} 20$ & C13 & C14 & $-53.2(2)$ \\
\hline C1 & P1 & 01 & C13 & $-106.78(16)$ \\
\hline C1 & $\mathrm{P} 1$ & $\mathrm{C} 7$ & C12 & $-89.5(2)$ \\
\hline C1 & $\mathrm{P} 1$ & $\mathrm{C} 7$ & $\mathrm{C} 8$ & $92.82(19)$ \\
\hline C1 & $\mathrm{C} 6$ & $\mathrm{C} 5$ & $\mathrm{C} 4$ & $0.0(4)$ \\
\hline $\mathrm{C} 1$ & $\mathrm{C} 2$ & $\mathrm{C} 3$ & $\mathrm{C} 4$ & $0.0(4)$ \\
\hline C6 & $\mathrm{C} 1$ & $\mathrm{C} 2$ & $\mathrm{C} 3$ & $-0.1(4)$ \\
\hline C6 & $\mathrm{C} 5$ & $\mathrm{C} 4$ & $\mathrm{C} 3$ & $-0.1(4)$ \\
\hline $\mathrm{C} 7$ & P1 & 01 & C13 & $143.52(15)$ \\
\hline $\mathrm{C} 7$ & $\mathrm{P} 1$ & $\mathrm{C} 1$ & C6 & $22.9(2)$ \\
\hline C7 & P1 & C1 & C2 & $-159.26(19)$ \\
\hline $\mathrm{C} 7$ & $\mathrm{C} 12$ & C11 & C10 & $0.7(3)$ \\
\hline C7 & C8 & C9 & C10 & $0.1(4)$ \\
\hline C24 & $\mathrm{C} 23$ & $\mathrm{C} 28$ & $\mathrm{C} 29$ & $-173.2(2)$ \\
\hline C24 & $\mathrm{C} 23$ & C28 & $\mathrm{C} 27$ & $5.4(3)$ \\
\hline C24 & $\mathrm{C} 25$ & $\mathrm{C} 26$ & $\mathrm{C} 27$ & $3.9(4)$ \\
\hline C34 & $\mathrm{C} 29$ & C28 & $\mathrm{C} 23$ & 65.1(3) \\
\hline C34 & $\mathrm{C} 29$ & $\mathrm{C} 28$ & $\mathrm{C} 27$ & $-113.5(2)$ \\
\hline C34 & $\mathrm{C} 29$ & C30 & C31 & $-2.8(4)$ \\
\hline C34 & C33 & C32 & C31 & $-1.9(4)$ \\
\hline C20 & C13 & C14 & C15 & $-69.6(3)$ \\
\hline C20 & C13 & C14 & C19 & $110.8(2)$ \\
\hline C29 & C34 & C33 & C32 & $0.3(3)$ \\
\hline C29 & C28 & C27 & C26 & $174.3(2)$ \\
\hline C23 & P2 & N1 & C20 & $118.90(18)$ \\
\hline C23 & $\mathrm{P} 2$ & N1 & $\mathrm{C} 22$ & $-62.82(19)$ \\
\hline C23 & $\mathrm{P} 2$ & C35 & $\mathrm{C} 40$ & $-141.28(19)$ \\
\hline C23 & $\mathrm{P} 2$ & C35 & C36 & $40.0(2)$ \\
\hline C23 & $\mathrm{C} 24$ & $\mathrm{C} 25$ & C26 & $-2.7(4)$ \\
\hline C23 & $\mathrm{C} 28$ & $\mathrm{C} 27$ & C26 & $-4.3(3)$ \\
\hline C35 & $\mathrm{P} 2$ & N1 & $\mathrm{C} 20$ & $-129.88(18)$ \\
\hline
\end{tabular}




\begin{tabular}{|c|c|c|c|c|}
\hline Atom & Atom & Atom & Atom & Angle $/^{\circ}$ \\
\hline C35 & P2 & N1 & $\mathrm{C} 22$ & $48.4(2)$ \\
\hline C35 & P2 & $\mathrm{C} 23$ & $\mathrm{C} 24$ & $-139.57(18)$ \\
\hline C35 & $\mathrm{P} 2$ & $\mathrm{C} 23$ & C28 & $40.9(2)$ \\
\hline C35 & $\mathrm{C} 40$ & C39 & C38 & $-0.3(4)$ \\
\hline C35 & C36 & C37 & C38 & $0.1(4)$ \\
\hline C12 & $\mathrm{C} 7$ & $\mathrm{C} 8$ & C9 & $-0.8(3)$ \\
\hline C33 & C34 & $\mathrm{C} 29$ & C28 & $177.8(2)$ \\
\hline C33 & C34 & $\mathrm{C} 29$ & C30 & $2.0(3)$ \\
\hline C13 & $\mathrm{C} 14$ & C19 & C18 & $-179.4(2)$ \\
\hline C15 & $\mathrm{C} 14$ & C19 & C18 & $0.9(4)$ \\
\hline C25 & $\mathrm{C} 24$ & $\mathrm{C} 23$ & P2 & $178.46(18)$ \\
\hline C25 & $\mathrm{C} 24$ & $\mathrm{C} 23$ & C28 & $-2.0(3)$ \\
\hline $\mathrm{C} 25$ & $\mathrm{C} 26$ & $\mathrm{C} 27$ & $\mathrm{C} 28$ & $-0.3(4)$ \\
\hline C14 & $\mathrm{C} 15$ & C16 & C17 & $0.6(4)$ \\
\hline C14 & C19 & C18 & C17 & $0.5(4)$ \\
\hline C28 & $\mathrm{C} 29$ & C30 & C31 & $-178.6(2)$ \\
\hline C2 & $\mathrm{C} 1$ & $\mathrm{C} 6$ & $\mathrm{C} 5$ & $0.0(4)$ \\
\hline C2 & $\mathrm{C} 3$ & $\mathrm{C} 4$ & $\mathrm{C} 5$ & $0.1(4)$ \\
\hline C8 & $\mathrm{C} 7$ & C12 & C11 & $0.4(3)$ \\
\hline C8 & C9 & C10 & C11 & $1.1(4)$ \\
\hline $\mathrm{C} 40$ & C35 & C36 & C37 & $1.5(4)$ \\
\hline C22 & N1 & $\mathrm{C} 20$ & C13 & $-59.3(2)$ \\
\hline C22 & N1 & $\mathrm{C} 20$ & $\mathrm{C} 21$ & $65.5(3)$ \\
\hline C9 & $\mathrm{C} 10$ & C11 & C12 & $-1.5(4)$ \\
\hline C36 & C35 & $\mathrm{C} 40$ & C39 & $-1.4(4)$ \\
\hline C16 & C15 & C14 & C13 & $178.9(2)$ \\
\hline C16 & $\mathrm{C} 15$ & C14 & C19 & $-1.5(3)$ \\
\hline C16 & $\mathrm{C} 17$ & C18 & C19 & $-1.3(5)$ \\
\hline C21 & $\mathrm{C} 20$ & C13 & 01 & $61.2(2)$ \\
\hline C21 & $\mathrm{C} 20$ & C13 & C14 & $-178.63(18)$ \\
\hline C37 & C38 & C39 & $\mathrm{C} 40$ & $1.9(4)$ \\
\hline C18 & $\mathrm{C} 17$ & C16 & C15 & $0.8(4)$ \\
\hline C32 & C31 & C30 & C29 & $1.2(4)$ \\
\hline B2 & P2 & N1 & $\mathrm{C} 20$ & $-3.1(2)$ \\
\hline B2 & P2 & N1 & $\mathrm{C} 22$ & $175.20(18)$ \\
\hline B2 & $\mathrm{P} 2$ & $\mathrm{C} 23$ & $\mathrm{C} 24$ & 93.74(19) \\
\hline B2 & $\mathrm{P} 2$ & $\mathrm{C} 23$ & C28 & $-85.8(2)$ \\
\hline B2 & $\mathrm{P} 2$ & C35 & $\mathrm{C} 40$ & $-16.6(2)$ \\
\hline B2 & $\mathrm{P} 2$ & C35 & C36 & $164.70(19)$ \\
\hline C30 & $\mathrm{C} 29$ & C28 & C23 & $-119.2(2)$ \\
\hline C30 & $\mathrm{C} 29$ & $\mathrm{C} 28$ & $\mathrm{C} 27$ & $62.3(3)$ \\
\hline C30 & C31 & C32 & C33 & 1.1(4) \\
\hline C39 & C38 & $\mathrm{C} 37$ & C36 & $-1.9(4)$ \\
\hline B1 & $\mathrm{P} 1$ & 01 & C13 & 21.07(19) \\
\hline B1 & P1 & $\mathrm{C} 1$ & C6 & 149.1(2) \\
\hline B1 & $\mathrm{P} 1$ & $\mathrm{C} 1$ & $\mathrm{C} 2$ & $-33.1(2)$ \\
\hline B1 & $\mathrm{P} 1$ & $\mathrm{C} 7$ & C12 & $142.87(19)$ \\
\hline B1 & P1 & $\mathrm{C} 7$ & C8 & $-34.8(2)$ \\
\hline
\end{tabular}


Name: $\left(R_{\mathrm{p}}\right)-(-)-\mathrm{N}-[(1 R, 2 S)-2-($ Diphenylphosphinito-borane)-1-methyl-2-phenylethyl], N-methyl amino(ferrocenylphenyl)phosphine-borane 12g.

Submitted by: Christophe Darcel

Solved by: Yoann Rousselin

Sample ID: $\quad$ darc8

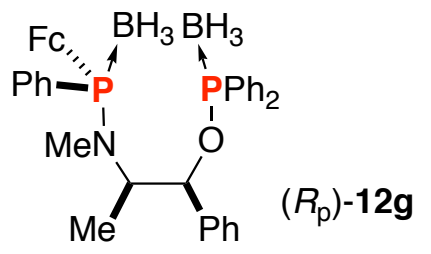

\section{Crystal Data and Experimental}

Figure S5 (thermal ellipsoïds are drawn at $50 \%$ probability plot)

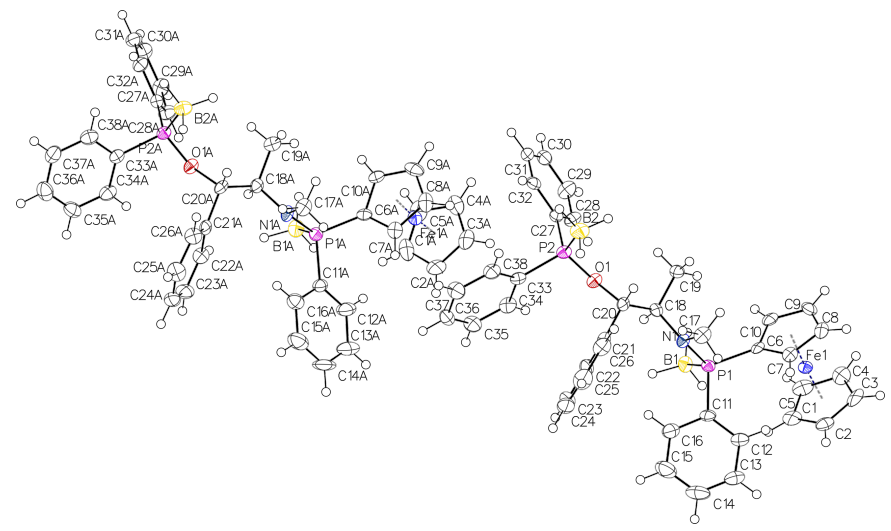

Experimental. Single clear light orange prism-shaped

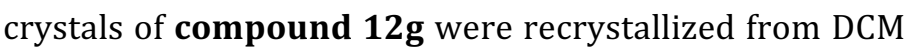
by slow evaporation. A suitable crystal $0.41 \times 0.32 \times 0.22$ $\mathrm{mm}^{3}$ was selected and mounted on a glass fiber with grease on a Nonius Kappa CCD diffractometer. The crystal was kept at a steady $T=110.0(1) \mathrm{K}$ during data collection. The structure was solved with the ShelXS-97 (Sheldrick, 1997) structure solution program using the direct methods solution method and by using olex2 (Dolomanov et al., 2009) as the graphical interface. The model was refined with version 2018/3 of ShelXL (Sheldrick, 2015) using Least Squares minimization.

Crystal Data. $\mathrm{C}_{38} \mathrm{H}_{43} \mathrm{~B}_{2} \mathrm{FeNOP}_{2}, M_{r}=669.14$, monoclinic, $P 2_{1}$ (No. 4), $\mathrm{a}=17.2276(3) \AA, \mathrm{b}=7.2764(2) \AA, \mathrm{c}=$ 28.2806(6) $\AA, \quad \beta=91.6200(10)^{\circ}, \quad \alpha=\gamma=90^{\circ}, \quad V=$ $3543.70(14) \AA^{3}, T=110.0(1) \mathrm{K}, Z=2, Z^{\prime}=2, \mu\left(\mathrm{MoK}_{\alpha}\right)=$ $0.546,16570$ reflections measured, 11303 unique $\left(R_{\text {int }}=\right.$ $0.0624)$ which were used in all calculations. The final $w R_{2}$ was 0.1200 (all data) and $R_{1}$ was 0.0531 (I > 2(I)). 


\begin{tabular}{|c|c|c|c|}
\hline Compound & $12 \mathrm{~g}$ & Radiation type & $\mathrm{MoK}_{\alpha}$ \\
\hline CCDC & 1982666 & $\Theta_{\min } /^{\circ}$ & 1.367 \\
\hline Formula & $\mathrm{C}_{38} \mathrm{H}_{43} \mathrm{~B}_{2} \mathrm{FeNOP}{ }_{2}$ & $\Theta_{\max } /^{\circ}$ & 24.999 \\
\hline$D_{\text {calc. }} / \mathrm{g} \mathrm{cm}^{-3}$ & 1.254 & Measured Refl. & 16570 \\
\hline$\mu / \mathrm{mm}^{-1}$ & 0.546 & Independent Refl. & 11303 \\
\hline Formula Weight & 669.14 & Reflections with I > & 9533 \\
\hline Color & clear light orange & $2(\mathrm{I})$ & \\
\hline Shape & prism & $R_{\text {int }}$ & 0.0624 \\
\hline $\mathrm{Size} / \mathrm{mm}^{3}$ & $0.41 \times 0.32 \times 0.22$ & Parameters & 824 \\
\hline$T / \mathrm{K}$ & $110.0(1)$ & Restraints & 1 \\
\hline Crystal System & monoclinic & Largest Peak & 0.377 \\
\hline Flack Parameter & $0.01(2)$ & Deepest Hole & -0.386 \\
\hline Hooft Parameter & $0.010(10)$ & GooF & 1.077 \\
\hline Space Group & $P 2_{1}$ & $w R_{2}$ (all data) & 0.1200 \\
\hline$a / \AA ̊$ & $17.2276(3)$ & $w R_{2}$ & 0.1100 \\
\hline$b / \AA ̊$ & $7.2764(2)$ & $R_{1}$ (all data) & 0.0723 \\
\hline$c / \AA$ & $28.2806(6)$ & $R_{1}$ & 0.0531 \\
\hline$\alpha /^{\circ}$ & $90.0000(7)$ & & \\
\hline$\beta /^{\circ}$ & $91.6203(7)$ & & \\
\hline$\gamma /^{\circ}$ & $90.0000(9)$ & & \\
\hline $\mathrm{V} / \AA^{3}$ & $3543.70(14)$ & & \\
\hline$Z$ & 2 & & \\
\hline$Z^{\prime}$ & 2 & & \\
\hline Wavelength/Å & 0.71073 & & \\
\hline
\end{tabular}

\section{Structure Quality Indicators}

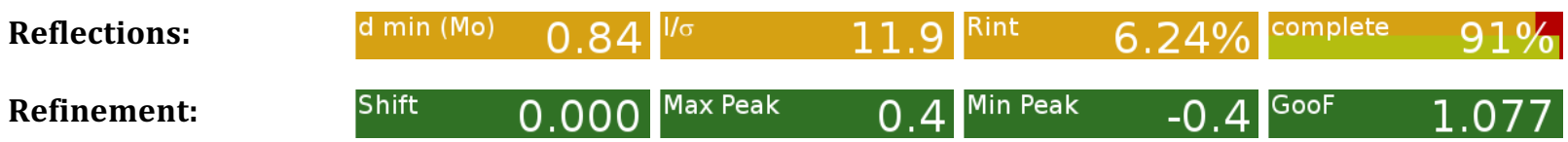

A clear light orange prism-shaped crystal with dimensions $0.41 \times 0.32 \times 0.22 \mathrm{~mm}^{3}$ was mounted on a glass fiber with grease. Data were collected using a Nonius Kappa CCD diffractometer equipped with an Oxford Cryosystems low-temperature device operating at $T=110.0(1) \mathrm{K}$. Data were measured using $\phi$ and $\omega$ scans using $\mathrm{MoK}_{\alpha}$ radiation. The maximum resolution that was achieved was $\Theta=24.999^{\circ}(0.84 \AA)$. The final completeness is $98.20 \%$ out to $24.999^{\circ}$ in $\Theta$. No absorption correction was performed. The absorption coefficient $\mu$ of this material is $0.546 \mathrm{~mm}^{-1}$ at this wavelength $(\lambda=0.711 \AA)$. The structure was solved and the space group $P 2_{1}$ (\# 4) determined by the ShelXS-97 (Sheldrick, 1997) structure solution program using direct methods and refined by Least Squares using version 2018/3 of ShelXL (Sheldrick, 2015). All non-hydrogen atoms were refined anisotropically. Hydrogen atom positions were calculated geometrically and refined using the riding model. Hydrogen atom on Boron atom were refined using an AFIX 138, which would allow the refinement of a common B-H distances, the same shifts being applied to all the B-H bonds to the same atom, but still retain tetrahedral $\mathrm{H}-\mathrm{B}-\mathrm{H}$ angles within the group. 
Table S13: Bond Lengths in Å for compound 12g.

\begin{tabular}{|c|c|c|c|c|c|}
\hline Atom & Atom & Length/Å & Atom & Atom & Length/Å \\
\hline$\overline{\mathrm{Fe} 1}$ & $\mathrm{C} 1$ & $2.066(7)$ & $\mathrm{C} 33$ & C34 & $1.400(9)$ \\
\hline $\mathrm{Fe} 1$ & $\mathrm{C} 2$ & $2.055(7)$ & C33 & C38 & $1.383(9)$ \\
\hline $\mathrm{Fe} 1$ & $\mathrm{C} 3$ & $2.057(6)$ & C34 & C35 & $1.371(9)$ \\
\hline $\mathrm{Fe} 1$ & $\mathrm{C} 4$ & $2.036(7)$ & C35 & C36 & $1.358(9)$ \\
\hline $\mathrm{Fe} 1$ & $\mathrm{C} 5$ & $2.043(7)$ & C36 & C37 & $1.398(10)$ \\
\hline $\mathrm{Fe} 1$ & C6 & $2.040(6)$ & C37 & C38 & $1.383(9)$ \\
\hline Fe1 & $\mathrm{C} 7$ & $2.050(6)$ & Fe1A & $\mathrm{C} 2 \mathrm{~A}$ & $2.053(7)$ \\
\hline $\mathrm{Fe} 1$ & $\mathrm{C} 8$ & $2.064(7)$ & Fe1A & $\mathrm{C} 3 \mathrm{~A}$ & $2.049(7)$ \\
\hline $\mathrm{Fe} 1$ & $\mathrm{C} 9$ & $2.046(7)$ & Fe1A & $\mathrm{C} 4 \mathrm{~A}$ & $2.051(7)$ \\
\hline $\mathrm{Fe} 1$ & C10 & $2.040(6)$ & Fe1A & C5A & $2.046(7)$ \\
\hline P1 & N1 & $1.684(5)$ & Fe1A & $\mathrm{C} 1 \mathrm{~A}$ & $2.040(7)$ \\
\hline P1 & B1 & $1.919(7)$ & Fe1A & C6A & $2.044(6)$ \\
\hline P1 & $\mathrm{C} 6$ & $1.799(6)$ & Fe1A & C7A & $2.045(6)$ \\
\hline P1 & C11 & $1.819(6)$ & Fe1A & $\mathrm{C} 8 \mathrm{~A}$ & $2.047(7)$ \\
\hline P2 & 01 & $1.606(4)$ & Fe1A & C9A & $2.040(7)$ \\
\hline P2 & B2 & $1.888(7)$ & Fe1A & $\mathrm{C} 10 \mathrm{~A}$ & $2.031(6)$ \\
\hline P2 & $\mathrm{C} 27$ & $1.801(6)$ & P1A & N1A & $1.677(5)$ \\
\hline P2 & C33 & $1.811(7)$ & P1A & B1A & $1.916(8)$ \\
\hline 01 & $\mathrm{C} 20$ & $1.475(7)$ & P1A & C6A & $1.800(6)$ \\
\hline N1 & C17 & $1.477(8)$ & P1A & $\mathrm{C} 11 \mathrm{~A}$ & $1.831(6)$ \\
\hline N1 & C18 & $1.469(8)$ & P2A & $01 \mathrm{~A}$ & $1.603(4)$ \\
\hline C1 & $\mathrm{C} 2$ & $1.433(10)$ & P2A & B2A & $1.900(8)$ \\
\hline C1 & C5 & $1.420(10)$ & P2A & C33A & $1.813(6)$ \\
\hline $\mathrm{C} 2$ & $\mathrm{C} 3$ & $1.425(10)$ & P2A & $\mathrm{C} 27 \mathrm{~A}$ & $1.801(6)$ \\
\hline C3 & $\mathrm{C} 4$ & $1.403(10)$ & $\mathrm{N} 1 \mathrm{~A}$ & C17A & $1.484(8)$ \\
\hline $\mathrm{C} 4$ & $\mathrm{C} 5$ & $1.421(10)$ & $\mathrm{N} 1 \mathrm{~A}$ & C18A & $1.475(8)$ \\
\hline C6 & $\mathrm{C} 7$ & $1.440(8)$ & $01 \mathrm{~A}$ & $\mathrm{C} 20 \mathrm{~A}$ & $1.462(7)$ \\
\hline C6 & $\mathrm{C} 10$ & $1.427(8)$ & $\mathrm{C} 2 \mathrm{~A}$ & $\mathrm{C} 3 \mathrm{~A}$ & $1.411(10)$ \\
\hline $\mathrm{C} 7$ & C8 & $1.414(9)$ & $\mathrm{C} 2 \mathrm{~A}$ & $\mathrm{C} 1 \mathrm{~A}$ & $1.431(11)$ \\
\hline C8 & $\mathrm{C} 9$ & $1.437(10)$ & $\mathrm{C} 3 \mathrm{~A}$ & $\mathrm{C} 4 \mathrm{~A}$ & $1.413(10)$ \\
\hline C9 & C10 & $1.414(9)$ & $\mathrm{C} 4 \mathrm{~A}$ & C5A & $1.418(9)$ \\
\hline C11 & $\mathrm{C} 12$ & $1.386(9)$ & C5A & $\mathrm{C} 1 \mathrm{~A}$ & $1.421(11)$ \\
\hline C11 & C16 & $1.387(9)$ & C6A & C7A & $1.422(9)$ \\
\hline C12 & C13 & $1.395(9)$ & C6A & $\mathrm{C} 10 \mathrm{~A}$ & $1.432(8)$ \\
\hline C13 & C14 & $1.362(11)$ & C7A & C8A & $1.419(9)$ \\
\hline C14 & C15 & $1.368(11)$ & C8A & C9A & $1.425(10)$ \\
\hline C15 & C16 & $1.389(10)$ & C9A & $\mathrm{C} 10 \mathrm{~A}$ & $1.415(9)$ \\
\hline C18 & C19 & $1.533(8)$ & C11A & $\mathrm{C} 12 \mathrm{~A}$ & $1.378(9)$ \\
\hline C18 & $\mathrm{C} 20$ & $1.528(8)$ & C11A & C16A & $1.376(9)$ \\
\hline $\mathrm{C} 20$ & $\mathrm{C} 21$ & $1.494(9)$ & C12A & C13A & $1.376(10)$ \\
\hline C21 & $\mathrm{C} 22$ & $1.408(9)$ & C13A & C14A & $1.384(11)$ \\
\hline $\mathrm{C} 21$ & $\mathrm{C} 26$ & $1.390(9)$ & C14A & C15A & $1.365(11)$ \\
\hline $\mathrm{C} 22$ & $\mathrm{C} 23$ & $1.376(9)$ & C15A & C16A & $1.394(10)$ \\
\hline C23 & $\mathrm{C} 24$ & $1.377(10)$ & C18A & C19A & $1.532(8)$ \\
\hline C24 & $\mathrm{C} 25$ & $1.377(12)$ & C18A & $\mathrm{C} 20 \mathrm{~A}$ & $1.525(8)$ \\
\hline $\mathrm{C} 25$ & $\mathrm{C} 26$ & $1.384(10)$ & $\mathrm{C} 20 \mathrm{~A}$ & $\mathrm{C} 21 \mathrm{~A}$ & $1.520(9)$ \\
\hline $\mathrm{C} 27$ & $\mathrm{C} 28$ & $1.391(9)$ & $\mathrm{C} 21 \mathrm{~A}$ & $\mathrm{C} 22 \mathrm{~A}$ & $1.400(9)$ \\
\hline C27 & C32 & $1.394(8)$ & $\mathrm{C} 21 \mathrm{~A}$ & $\mathrm{C} 26 \mathrm{~A}$ & $1.376(9)$ \\
\hline C28 & $\mathrm{C} 29$ & $1.385(9)$ & $\mathrm{C} 22 \mathrm{~A}$ & $\mathrm{C} 23 \mathrm{~A}$ & $1.380(9)$ \\
\hline C29 & C30 & $1.381(9)$ & $\mathrm{C} 23 \mathrm{~A}$ & $\mathrm{C} 24 \mathrm{~A}$ & $1.380(10)$ \\
\hline C30 & C31 & $1.371(10)$ & $\mathrm{C} 24 \mathrm{~A}$ & $\mathrm{C} 25 \mathrm{~A}$ & $1.378(11)$ \\
\hline C31 & C32 & $1.391(8)$ & $\mathrm{C} 25 \mathrm{~A}$ & $\mathrm{C} 26 \mathrm{~A}$ & $1.382(9)$ \\
\hline
\end{tabular}




\begin{tabular}{lll}
\hline Atom & Atom & Length/A \\
\hline C33A & C34A & $1.397(9)$ \\
C33A & C38A & $1.391(9)$ \\
C34A & C35A & $1.370(9)$ \\
C35A & C36A & $1.365(10)$ \\
C36A & C37A & $1.389(11)$ \\
C37A & C38A & $1.390(9)$
\end{tabular}

\begin{tabular}{lll}
\hline Atom & Atom & Length/Å \\
\hline C27A & C28A & $1.395(9)$ \\
C27A & C32A & $1.395(8)$ \\
C28A & C29A & $1.385(9)$ \\
C29A & C30A & $1.389(9)$ \\
C30A & C31A & $1.382(9)$ \\
C31A & C32A & $1.388(8)$
\end{tabular}

Table S14: Bond Angles in ${ }^{\circ}$ for compound 12g.

\begin{tabular}{|c|c|c|c|c|c|c|c|}
\hline Atom & Atom & Atom & Angle $/^{\circ}$ & Atom & Atom & Atom & Angle $/^{\circ}$ \\
\hline $\mathrm{C} 2$ & Fe1 & C1 & $40.7(3)$ & C10 & Fe1 & C9 & $40.5(3)$ \\
\hline C2 & $\mathrm{Fe} 1$ & C3 & $40.6(3)$ & N1 & P1 & B1 & $113.9(3)$ \\
\hline $\mathrm{C} 2$ & $\mathrm{Fe} 1$ & C8 & $122.7(3)$ & N1 & P1 & C6 & $108.4(3)$ \\
\hline C3 & Fe1 & C1 & 68.5(3) & N1 & P1 & C11 & $103.0(3)$ \\
\hline C3 & $\mathrm{Fe} 1$ & C8 & $105.8(3)$ & C6 & P1 & B1 & 111.1(3) \\
\hline C4 & $\mathrm{Fe} 1$ & $\mathrm{C} 1$ & $68.1(3)$ & C6 & P1 & C11 & 109.6(3) \\
\hline $\mathrm{C} 4$ & $\mathrm{Fe} 1$ & $\mathrm{C} 2$ & $67.7(3)$ & C11 & P1 & B1 & $110.5(3)$ \\
\hline $\mathrm{C} 4$ & $\mathrm{Fe} 1$ & C3 & $40.1(3)$ & 01 & P2 & B2 & $117.0(3)$ \\
\hline $\mathrm{C} 4$ & $\mathrm{Fe} 1$ & C5 & $40.8(3)$ & 01 & P2 & $\mathrm{C} 27$ & 101.1(3) \\
\hline $\mathrm{C} 4$ & $\mathrm{Fe} 1$ & C6 & $157.9(3)$ & 01 & P2 & C33 & $106.3(3)$ \\
\hline C4 & $\mathrm{Fe} 1$ & C7 & $157.5(3)$ & $\mathrm{C} 27$ & P2 & B2 & $113.4(3)$ \\
\hline $\mathrm{C} 4$ & $\mathrm{Fe} 1$ & C8 & $120.6(3)$ & $\mathrm{C} 27$ & P2 & C33 & $104.7(3)$ \\
\hline $\mathrm{C} 4$ & $\mathrm{Fe} 1$ & $\mathrm{C} 9$ & $104.4(3)$ & C33 & P2 & B2 & $113.0(3)$ \\
\hline $\mathrm{C} 4$ & $\mathrm{Fe} 1$ & C10 & $120.4(3)$ & $\mathrm{C} 20$ & 01 & P2 & $122.0(4)$ \\
\hline C5 & $\mathrm{Fe} 1$ & C1 & $40.4(3)$ & C17 & N1 & P1 & $116.7(4)$ \\
\hline C5 & $\mathrm{Fe} 1$ & C2 & 68.1(3) & C18 & N1 & P1 & $118.5(4)$ \\
\hline C5 & $\mathrm{Fe} 1$ & C3 & $68.3(3)$ & C18 & N1 & C17 & $117.0(5)$ \\
\hline C5 & $\mathrm{Fe} 1$ & C7 & 161.1(3) & $\mathrm{C} 2$ & $\mathrm{C} 1$ & $\mathrm{Fe} 1$ & $69.3(4)$ \\
\hline C5 & $\mathrm{Fe} 1$ & C8 & $157.1(3)$ & $\mathrm{C} 5$ & $\mathrm{C} 1$ & $\mathrm{Fe} 1$ & $68.9(4)$ \\
\hline C5 & $\mathrm{Fe} 1$ & C9 & 121.1(3) & C5 & $\mathrm{C} 1$ & $\mathrm{C} 2$ & 107.1(7) \\
\hline C6 & $\mathrm{Fe} 1$ & $\mathrm{C} 1$ & $110.4(3)$ & $\mathrm{C} 1$ & $\mathrm{C} 2$ & $\mathrm{Fe} 1$ & $70.0(4)$ \\
\hline C6 & $\mathrm{Fe} 1$ & $\mathrm{C} 2$ & $126.7(3)$ & C3 & $\mathrm{C} 2$ & $\mathrm{Fe} 1$ & $69.8(4)$ \\
\hline C6 & $\mathrm{Fe} 1$ & C3 & $161.6(3)$ & C3 & $\mathrm{C} 2$ & $\mathrm{C} 1$ & $108.5(6)$ \\
\hline C6 & $\mathrm{Fe} 1$ & C5 & $123.6(3)$ & $\mathrm{C} 2$ & $\mathrm{C} 3$ & $\mathrm{Fe} 1$ & $69.7(4)$ \\
\hline C6 & $\mathrm{Fe} 1$ & C7 & $41.2(2)$ & $\mathrm{C} 4$ & $\mathrm{C} 3$ & $\mathrm{Fe} 1$ & 69.1(4) \\
\hline C6 & $\mathrm{Fe} 1$ & C8 & $68.7(3)$ & $\mathrm{C} 4$ & C3 & $\mathrm{C} 2$ & 107.3(7) \\
\hline C6 & $\mathrm{Fe} 1$ & C9 & $68.7(3)$ & C3 & C4 & $\mathrm{Fe} 1$ & $70.8(4)$ \\
\hline C7 & $\mathrm{Fe} 1$ & $\mathrm{C} 1$ & $125.9(3)$ & C3 & $\mathrm{C} 4$ & $\mathrm{C} 5$ & $109.2(7)$ \\
\hline C7 & $\mathrm{Fe} 1$ & $\mathrm{C} 2$ & $110.1(3)$ & C5 & $\mathrm{C} 4$ & $\mathrm{Fe} 1$ & $69.9(4)$ \\
\hline C7 & $\mathrm{Fe} 1$ & C3 & 123.4(3) & $\mathrm{C} 1$ & C5 & $\mathrm{Fe} 1$ & $70.6(4)$ \\
\hline C7 & $\mathrm{Fe} 1$ & C8 & $40.2(3)$ & $\mathrm{C} 1$ & C5 & $\mathrm{C} 4$ & $107.9(7)$ \\
\hline C8 & $\mathrm{Fe} 1$ & $\mathrm{C} 1$ & $160.0(3)$ & $\mathrm{C} 4$ & $\mathrm{C} 5$ & $\mathrm{Fe} 1$ & $69.3(4)$ \\
\hline C9 & $\mathrm{Fe} 1$ & $\mathrm{C} 1$ & $158.8(3)$ & $\mathrm{P} 1$ & C6 & $\mathrm{Fe} 1$ & $130.3(3)$ \\
\hline C9 & $\mathrm{Fe} 1$ & $\mathrm{C} 2$ & $156.6(3)$ & $\mathrm{C} 7$ & C6 & $\mathrm{Fe} 1$ & $69.7(3)$ \\
\hline C9 & $\mathrm{Fe} 1$ & C3 & $119.3(3)$ & $\mathrm{C} 7$ & C6 & P1 & $130.8(5)$ \\
\hline C9 & $\mathrm{Fe} 1$ & C7 & $68.4(3)$ & C10 & C6 & $\mathrm{Fe} 1$ & $69.5(3)$ \\
\hline C9 & $\mathrm{Fe} 1$ & C8 & $40.9(3)$ & C10 & C6 & $\mathrm{P} 1$ & $121.7(5)$ \\
\hline C10 & $\mathrm{Fe} 1$ & $\mathrm{C} 1$ & $124.8(3)$ & C10 & C6 & $\mathrm{C} 7$ & $107.2(5)$ \\
\hline C10 & $\mathrm{Fe} 1$ & $\mathrm{C} 2$ & $162.5(3)$ & C6 & $\mathrm{C} 7$ & $\mathrm{Fe} 1$ & $69.0(3)$ \\
\hline C10 & $\mathrm{Fe} 1$ & C3 & $155.0(3)$ & $\mathrm{C} 8$ & $\mathrm{C} 7$ & $\mathrm{Fe} 1$ & $70.4(4)$ \\
\hline C10 & $\mathrm{Fe} 1$ & C5 & $107.0(3)$ & C8 & $\mathrm{C} 7$ & $\mathrm{C} 6$ & $108.4(6)$ \\
\hline C10 & $\mathrm{Fe} 1$ & C6 & $41.0(2)$ & $\mathrm{C} 7$ & $\mathrm{C} 8$ & $\mathrm{Fe} 1$ & $69.4(4)$ \\
\hline C10 & $\mathrm{Fe} 1$ & C7 & $68.7(3)$ & $\mathrm{C} 7$ & $\mathrm{C} 8$ & C9 & $107.7(6)$ \\
\hline C10 & $\mathrm{Fe} 1$ & C8 & $68.5(3)$ & C9 & C8 & $\mathrm{Fe} 1$ & $68.9(4)$ \\
\hline
\end{tabular}




\begin{tabular}{|c|c|c|c|c|c|c|c|}
\hline Atom & Atom & Atom & Angle $/^{\circ}$ & Atom & Atom & Atom & Angle $/^{\circ}$ \\
\hline$\overline{\mathrm{C} 8}$ & C9 & Fe1 & $70.2(4)$ & $\overline{\mathrm{C} 1 \mathrm{~A}}$ & Fe1A & $\mathrm{C} 5 \mathrm{~A}$ & $40.7(3)$ \\
\hline C10 & $\mathrm{C} 9$ & $\mathrm{Fe} 1$ & $69.5(4)$ & $\mathrm{C} 1 \mathrm{~A}$ & $\mathrm{Fe} 1 \mathrm{~A}$ & C6A & $110.9(3)$ \\
\hline C10 & C9 & C8 & $108.2(6)$ & $\mathrm{C} 1 \mathrm{~A}$ & $\mathrm{Fe} 1 \mathrm{~A}$ & C7A & $127.9(3)$ \\
\hline C6 & $\mathrm{C} 10$ & $\mathrm{Fe} 1$ & $69.5(3)$ & C1A & $\mathrm{Fe} 1 \mathrm{~A}$ & $\mathrm{C} 8 \mathrm{~A}$ & $162.9(3)$ \\
\hline C9 & $\mathrm{C} 10$ & $\mathrm{Fe} 1$ & $70.0(4)$ & $\mathrm{C} 1 \mathrm{~A}$ & $\mathrm{Fe} 1 \mathrm{~A}$ & C9A & $156.1(3)$ \\
\hline C9 & $\mathrm{C} 10$ & $\mathrm{C} 6$ & $108.4(6)$ & C6A & $\mathrm{Fe} 1 \mathrm{~A}$ & $\mathrm{C} 2 \mathrm{~A}$ & $125.7(3)$ \\
\hline C12 & C11 & P1 & $126.6(5)$ & C6A & $\mathrm{Fe} 1 \mathrm{~A}$ & $\mathrm{C} 3 \mathrm{~A}$ & $159.9(3)$ \\
\hline C12 & C11 & C16 & $118.4(6)$ & C6A & $\mathrm{Fe} 1 \mathrm{~A}$ & $\mathrm{C} 4 \mathrm{~A}$ & $159.5(3)$ \\
\hline C16 & C11 & $\mathrm{P} 1$ & $114.8(5)$ & C6A & $\mathrm{Fe} 1 \mathrm{~A}$ & C5A & $125.1(3)$ \\
\hline C11 & $\mathrm{C} 12$ & C13 & $119.7(6)$ & C6A & $\mathrm{Fe} 1 \mathrm{~A}$ & C7A & $40.7(2)$ \\
\hline C14 & C13 & C12 & $121.2(7)$ & C6A & $\mathrm{Fe} 1 \mathrm{~A}$ & $\mathrm{C} 8 \mathrm{~A}$ & $68.6(3)$ \\
\hline C13 & C14 & C15 & $119.6(7)$ & C7A & $\mathrm{Fe} 1 \mathrm{~A}$ & $\mathrm{C} 2 \mathrm{~A}$ & $110.8(3)$ \\
\hline C14 & C15 & C16 & $120.1(8)$ & $\mathrm{C} 7 \mathrm{~A}$ & Fe1A & $\mathrm{C} 3 \mathrm{~A}$ & $122.9(3)$ \\
\hline C11 & C16 & C15 & $120.9(7)$ & $\mathrm{C} 7 \mathrm{~A}$ & $\mathrm{Fe} 1 \mathrm{~A}$ & $\mathrm{C} 4 \mathrm{~A}$ & $156.0(3)$ \\
\hline N1 & C18 & C19 & $112.9(5)$ & C7A & $\mathrm{Fe} 1 \mathrm{~A}$ & C5A & $163.3(3)$ \\
\hline N1 & C18 & $\mathrm{C} 20$ & $110.8(5)$ & C7A & $\mathrm{Fe} 1 \mathrm{~A}$ & C8A & $40.6(3)$ \\
\hline C20 & C18 & C19 & $111.1(5)$ & $\mathrm{C} 8 \mathrm{~A}$ & $\mathrm{Fe} 1 \mathrm{~A}$ & $\mathrm{C} 2 \mathrm{~A}$ & $124.5(3)$ \\
\hline 01 & $\mathrm{C} 20$ & C18 & $103.2(5)$ & $\mathrm{C} 8 \mathrm{~A}$ & $\mathrm{Fe} 1 \mathrm{~A}$ & $\mathrm{C} 3 \mathrm{~A}$ & $106.2(3)$ \\
\hline 01 & $\mathrm{C} 20$ & $\mathrm{C} 21$ & $109.6(5)$ & $\mathrm{C} 8 \mathrm{~A}$ & $\mathrm{Fe} 1 \mathrm{~A}$ & $\mathrm{C} 4 \mathrm{~A}$ & $118.8(3)$ \\
\hline C21 & $\mathrm{C} 20$ & C18 & $117.1(5)$ & $\mathrm{C} 9 \mathrm{~A}$ & $\mathrm{Fe} 1 \mathrm{~A}$ & $\mathrm{C} 2 \mathrm{~A}$ & $158.5(3)$ \\
\hline $\mathrm{C} 22$ & $\mathrm{C} 21$ & $\mathrm{C} 20$ & $121.1(6)$ & C9A & $\mathrm{Fe} 1 \mathrm{~A}$ & $\mathrm{C} 3 \mathrm{~A}$ & $120.7(3)$ \\
\hline C26 & $\mathrm{C} 21$ & $\mathrm{C} 20$ & $121.2(6)$ & C9A & $\mathrm{Fe} 1 \mathrm{~A}$ & $\mathrm{C} 4 \mathrm{~A}$ & $103.6(3)$ \\
\hline C26 & $\mathrm{C} 21$ & $\mathrm{C} 22$ & $117.7(6)$ & C9A & $\mathrm{Fe} 1 \mathrm{~A}$ & $\mathrm{C} 5 \mathrm{~A}$ & $118.5(3)$ \\
\hline C23 & $\mathrm{C} 22$ & $\mathrm{C} 21$ & $120.7(6)$ & C9A & $\mathrm{Fe} 1 \mathrm{~A}$ & C6A & $68.8(3)$ \\
\hline C22 & $\mathrm{C} 23$ & $\mathrm{C} 24$ & $120.5(7)$ & $\mathrm{C} 9 \mathrm{~A}$ & $\mathrm{Fe} 1 \mathrm{~A}$ & C7A & $68.5(3)$ \\
\hline C25 & $\mathrm{C} 24$ & $\mathrm{C} 23$ & $119.8(7)$ & C9A & $\mathrm{Fe} 1 \mathrm{~A}$ & C8A & $40.8(3)$ \\
\hline C24 & $\mathrm{C} 25$ & $\mathrm{C} 26$ & $120.2(7)$ & C10A & $\mathrm{Fe} 1 \mathrm{~A}$ & $\mathrm{C} 2 \mathrm{~A}$ & $160.6(3)$ \\
\hline $\mathrm{C} 25$ & $\mathrm{C} 26$ & $\mathrm{C} 21$ & $121.1(7)$ & C10A & $\mathrm{Fe} 1 \mathrm{~A}$ & $\mathrm{C} 3 \mathrm{~A}$ & $156.9(3)$ \\
\hline C28 & $\mathrm{C} 27$ & P2 & $121.8(5)$ & $\mathrm{C} 10 \mathrm{~A}$ & $\mathrm{Fe} 1 \mathrm{~A}$ & $\mathrm{C} 4 \mathrm{~A}$ & $120.9(3)$ \\
\hline C28 & $\mathrm{C} 27$ & C32 & $119.6(6)$ & C10A & $\mathrm{Fe} 1 \mathrm{~A}$ & $\mathrm{C} 5 \mathrm{~A}$ & $106.1(3)$ \\
\hline C32 & $\mathrm{C} 27$ & $\mathrm{P} 2$ & $118.7(5)$ & C10A & $\mathrm{Fe} 1 \mathrm{~A}$ & $\mathrm{C} 1 \mathrm{~A}$ & $123.1(3)$ \\
\hline C29 & $\mathrm{C} 28$ & $\mathrm{C} 27$ & $119.9(6)$ & $\mathrm{C} 10 \mathrm{~A}$ & $\mathrm{Fe} 1 \mathrm{~A}$ & C6A & $41.1(2)$ \\
\hline C30 & $\mathrm{C} 29$ & $\mathrm{C} 28$ & $120.0(7)$ & C10A & $\mathrm{Fe} 1 \mathrm{~A}$ & C7A & $68.7(3)$ \\
\hline C31 & C30 & $\mathrm{C} 29$ & $120.8(7)$ & C10A & $\mathrm{Fe} 1 \mathrm{~A}$ & C8A & $68.6(3)$ \\
\hline C30 & C31 & C32 & $119.7(6)$ & C10A & $\mathrm{Fe} 1 \mathrm{~A}$ & C9A & $40.7(3)$ \\
\hline C31 & C32 & $\mathrm{C} 27$ & $120.0(6)$ & $\mathrm{N} 1 \mathrm{~A}$ & $\mathrm{P} 1 \mathrm{~A}$ & B1A & $113.0(3)$ \\
\hline C34 & C33 & $\mathrm{P} 2$ & $120.4(5)$ & $\mathrm{N} 1 \mathrm{~A}$ & P1A & C6A & $106.4(3)$ \\
\hline C38 & C33 & P2 & $120.7(5)$ & $\mathrm{N} 1 \mathrm{~A}$ & P1A & C11A & $105.6(3)$ \\
\hline C38 & C33 & C34 & $118.9(6)$ & C6A & P1A & B1A & $114.9(3)$ \\
\hline C35 & C34 & C33 & $119.3(6)$ & C6A & P1A & C11A & $104.5(3)$ \\
\hline C36 & C35 & C34 & $121.8(6)$ & C11A & P1A & B1A & $111.7(3)$ \\
\hline C35 & C36 & $\mathrm{C} 37$ & $120.0(6)$ & $01 \mathrm{~A}$ & $\mathrm{P} 2 \mathrm{~A}$ & B2A & $116.7(3)$ \\
\hline C38 & C37 & C36 & $118.6(6)$ & $01 \mathrm{~A}$ & $\mathrm{P} 2 \mathrm{~A}$ & C33A & $104.8(3)$ \\
\hline C37 & C38 & C33 & $121.4(6)$ & $01 \mathrm{~A}$ & $\mathrm{P} 2 \mathrm{~A}$ & $\mathrm{C} 27 \mathrm{~A}$ & 101.1(3) \\
\hline $\mathrm{C} 3 \mathrm{~A}$ & $\mathrm{Fe} 1 \mathrm{~A}$ & $\mathrm{C} 2 \mathrm{~A}$ & $40.2(3)$ & C33A & $\mathrm{P} 2 \mathrm{~A}$ & B2A & $112.3(3)$ \\
\hline $\mathrm{C} 3 \mathrm{~A}$ & Fe1A & $\mathrm{C} 4 \mathrm{~A}$ & $40.3(3)$ & C27A & $\mathrm{P} 2 \mathrm{~A}$ & $\mathrm{~B} 2 \mathrm{~A}$ & $115.2(3)$ \\
\hline $\mathrm{C} 4 \mathrm{~A}$ & Fe1A & $\mathrm{C} 2 \mathrm{~A}$ & $67.9(3)$ & C27A & P2A & C33A & $105.3(3)$ \\
\hline $\mathrm{C} 5 \mathrm{~A}$ & Fe1A & $\mathrm{C} 2 \mathrm{~A}$ & $68.6(3)$ & C17A & $\mathrm{N} 1 \mathrm{~A}$ & P1A & $118.0(4)$ \\
\hline C5A & Fe1A & $\mathrm{C} 3 \mathrm{~A}$ & $68.2(3)$ & C18A & $\mathrm{N} 1 \mathrm{~A}$ & P1A & $118.5(4)$ \\
\hline C5A & Fe1A & $\mathrm{C} 4 \mathrm{~A}$ & $40.5(3)$ & C18A & $\mathrm{N} 1 \mathrm{~A}$ & C17A & $116.4(5)$ \\
\hline C5A & $\mathrm{Fe} 1 \mathrm{~A}$ & C8A & $154.0(3)$ & $\mathrm{C} 20 \mathrm{~A}$ & $01 \mathrm{~A}$ & $\mathrm{P} 2 \mathrm{~A}$ & $123.2(4)$ \\
\hline C1A & Fe1A & $\mathrm{C} 2 \mathrm{~A}$ & $40.9(3)$ & $\mathrm{C} 3 \mathrm{~A}$ & $\mathrm{C} 2 \mathrm{~A}$ & Fe1A & $69.7(4)$ \\
\hline C1A & Fe1A & C3A & $68.1(3)$ & C3A & $\mathrm{C} 2 \mathrm{~A}$ & $\mathrm{C} 1 \mathrm{~A}$ & $107.4(7)$ \\
\hline C1A & Fe1A & $\mathrm{C} 4 \mathrm{~A}$ & $68.0(3)$ & $\mathrm{C} 1 \mathrm{~A}$ & $\mathrm{C} 2 \mathrm{~A}$ & $\mathrm{Fe} 1 \mathrm{~A}$ & $69.0(4)$ \\
\hline
\end{tabular}




\begin{tabular}{llll}
\hline Atom & Atom & Atom & \multicolumn{1}{c}{ Angle/ } \\
\hline C2A & C3A & Fe1A & $70.0(4)$ \\
C2A & C3A & C4A & $108.5(7)$ \\
C4A & C3A & Fe1A & $69.9(4)$ \\
C3A & C4A & Fe1A & $69.8(4)$ \\
C3A & C4A & C5A & $108.4(6)$ \\
C5A & C4A & Fe1A & $69.6(4)$ \\
C4A & C5A & Fe1A & $69.9(4)$ \\
C4A & C5A & C1A & $107.4(7)$ \\
C1A & C5A & Fe1A & $69.4(4)$ \\
C2A & C1A & Fe1A & $70.0(4)$ \\
C5A & C1A & Fe1A & $69.9(4)$ \\
C5A & C1A & C2A & $108.2(7)$ \\
P1A & C6A & Fe1A & $132.5(3)$ \\
C7A & C6A & Fe1A & $69.7(3)$ \\
C7A & C6A & P1A & $128.1(5)$ \\
C7A & C6A & C10A & $107.3(6)$ \\
C10A & C6A & Fe1A & $69.0(3)$ \\
C10A & C6A & P1A & $124.1(5)$ \\
C6A & C7A & Fe1A & $69.6(3)$ \\
C8A & C7A & Fe1A & $69.8(4)$ \\
C8A & C7A & C6A & $108.4(6)$ \\
C7A & C8A & Fe1A & $69.6(4)$ \\
C7A & C8A & C9A & $107.9(6)$ \\
C9A & C8A & Fe1A & $69.3(4)$ \\
C8A & C9A & Fe1A & $69.9(4)$ \\
C10A & C9A & Fe1A & $69.3(4)$ \\
C10A & C9A & C8A & $108.0(6)$ \\
C6A & C10A & Fe1A & $69.9(4)$ \\
C9A & C10A & Fe1A & $70.0(4)$ \\
C9A & C10A & C6A & $108.3(6)$ \\
C12A & C11A & P1A & $119.2(5)$ \\
C16A & C11A & P1A & $121.1(5)$ \\
C11A & C12A & $119.5(6)$ \\
C12A & C11A & $120.4(7)$ \\
C13A & C14A & $121.0(8)$ \\
& & & \\
C1A $1 A$ \\
C1A
\end{tabular}

\begin{tabular}{|c|c|c|c|}
\hline Atom & Atom & Atom & Angle $/^{\circ}$ \\
\hline C15A & C14A & C13A & $118.1(7)$ \\
\hline C14A & C15A & C16A & $121.8(7)$ \\
\hline $\mathrm{C} 11 \mathrm{~A}$ & C16A & C15A & $119.2(7)$ \\
\hline N1A & C18A & C19A & $112.9(5)$ \\
\hline $\mathrm{N} 1 \mathrm{~A}$ & C18A & $\mathrm{C} 20 \mathrm{~A}$ & $111.2(5)$ \\
\hline $\mathrm{C} 20 \mathrm{~A}$ & C18A & C19A & $110.8(5)$ \\
\hline $01 \mathrm{~A}$ & C20A & C18A & $103.9(5)$ \\
\hline $01 \mathrm{~A}$ & C20A & C21A & $108.3(5)$ \\
\hline $\mathrm{C} 21 \mathrm{~A}$ & $\mathrm{C} 20 \mathrm{~A}$ & C18A & $116.4(5)$ \\
\hline $\mathrm{C} 22 \mathrm{~A}$ & C21A & $\mathrm{C} 20 \mathrm{~A}$ & $120.5(6)$ \\
\hline $\mathrm{C} 26 \mathrm{~A}$ & C21A & C20A & $120.9(6)$ \\
\hline $\mathrm{C} 26 \mathrm{~A}$ & C21A & C22A & $118.5(6)$ \\
\hline $\mathrm{C} 23 \mathrm{~A}$ & C22A & C21A & $120.0(6)$ \\
\hline $\mathrm{C} 22 \mathrm{~A}$ & C23A & C24A & $120.6(6)$ \\
\hline $\mathrm{C} 25 \mathrm{~A}$ & $\mathrm{C} 24 \mathrm{~A}$ & $\mathrm{C} 23 \mathrm{~A}$ & $119.8(7)$ \\
\hline $\mathrm{C} 24 \mathrm{~A}$ & C25A & $\mathrm{C} 26 \mathrm{~A}$ & $119.5(7)$ \\
\hline $\mathrm{C} 21 \mathrm{~A}$ & C26A & $\mathrm{C} 25 \mathrm{~A}$ & $121.6(6)$ \\
\hline C34A & C33A & $\mathrm{P} 2 \mathrm{~A}$ & $122.4(5)$ \\
\hline C38A & C33A & $\mathrm{P} 2 \mathrm{~A}$ & $118.5(5)$ \\
\hline C38A & C33A & C34A & $119.1(6)$ \\
\hline C35A & C34A & C33A & $119.6(6)$ \\
\hline $\mathrm{C} 36 \mathrm{~A}$ & C35A & C34A & $121.7(7)$ \\
\hline C35A & C36A & C37A & $119.8(7)$ \\
\hline $\mathrm{C} 36 \mathrm{~A}$ & C37A & C38A & $119.4(7)$ \\
\hline C37A & C38A & C33A & $120.5(6)$ \\
\hline $\mathrm{C} 28 \mathrm{~A}$ & C27A & $\mathrm{P} 2 \mathrm{~A}$ & $121.7(5)$ \\
\hline $\mathrm{C} 32 \mathrm{~A}$ & C27A & P2A & $119.0(5)$ \\
\hline $\mathrm{C} 32 \mathrm{~A}$ & C27A & $\mathrm{C} 28 \mathrm{~A}$ & $119.3(6)$ \\
\hline C29A & $\mathrm{C} 28 \mathrm{~A}$ & C27A & $119.7(6)$ \\
\hline $\mathrm{C} 28 \mathrm{~A}$ & C29A & $\mathrm{C} 30 \mathrm{~A}$ & $120.7(6)$ \\
\hline C31A & $\mathrm{C} 30 \mathrm{~A}$ & C29A & $119.8(6)$ \\
\hline $\mathrm{C} 30 \mathrm{~A}$ & C31A & C32A & $119.8(6)$ \\
\hline C31A & C32A & C27A & $120.6(6)$ \\
\hline
\end{tabular}

Table S15: Torsion Angles in ${ }^{\circ}$ for compound 12g.

\begin{tabular}{llllr}
\hline Atom & Atom & Atom & Atom & Angle $^{\circ}$ \\
\hline Fe1 & C1 & C2 & C3 & $-59.4(4)$ \\
Fe1 & C1 & C5 & C4 & $59.6(4)$ \\
Fe1 & C2 & C3 & C4 & $-59.1(4)$ \\
Fe1 & C3 & C4 & C5 & $-59.5(5)$ \\
Fe1 & C4 & C5 & C1 & $-60.4(5)$ \\
Fe1 & C6 & C7 & C8 & $59.6(4)$ \\
Fe1 & C6 & C10 & C9 & $-59.4(4)$ \\
Fe1 & C7 & C8 & C9 & $58.4(5)$ \\
Fe1 & C8 & C9 & C10 & $59.3(5)$ \\
Fe1 & C9 & C10 & C6 & $59.1(4)$ \\
P1 & N1 & C18 & C19 & $-81.4(6)$ \\
P1 & N1 & C18 & C20 & $153.2(4)$ \\
P1 & C6 & C7 & Fe1 & $126.3(5)$ \\
P1 & C6 & C7 & C8 & $-174.1(5)$
\end{tabular}




\begin{tabular}{|c|c|c|c|c|}
\hline Atom & Atom & Atom & Atom & Angle $/{ }^{\circ}$ \\
\hline$\overline{\mathrm{P} 1}$ & $\mathrm{C} 6$ & C10 & Fe1 & $-125.5(4)$ \\
\hline P1 & $\mathrm{C} 6$ & $\mathrm{C} 10$ & C9 & $175.2(4)$ \\
\hline P1 & C11 & C12 & C13 & $-175.2(5)$ \\
\hline P1 & C11 & C16 & C15 & $174.5(6)$ \\
\hline P2 & 01 & $\mathrm{C} 20$ & C18 & $-153.9(4)$ \\
\hline P2 & 01 & $\mathrm{C} 20$ & $\mathrm{C} 21$ & $80.7(6)$ \\
\hline P2 & $\mathrm{C} 27$ & $\mathrm{C} 28$ & C29 & $-178.1(5)$ \\
\hline P2 & $\mathrm{C} 27$ & C32 & C31 & $179.5(5)$ \\
\hline P2 & C33 & C34 & C35 & $-178.4(5)$ \\
\hline P2 & C33 & C38 & C37 & $178.2(5)$ \\
\hline 01 & P2 & $\mathrm{C} 27$ & C28 & $4.9(6)$ \\
\hline 01 & $\mathrm{P} 2$ & $\mathrm{C} 27$ & C32 & $-174.4(5)$ \\
\hline 01 & $\mathrm{P} 2$ & C33 & C34 & $-31.2(6)$ \\
\hline 01 & P2 & C33 & C38 & $150.6(5)$ \\
\hline 01 & $\mathrm{C} 20$ & $\mathrm{C} 21$ & $\mathrm{C} 22$ & $60.4(8)$ \\
\hline 01 & $\mathrm{C} 20$ & $\mathrm{C} 21$ & $\mathrm{C} 26$ & $-118.8(6)$ \\
\hline $\mathrm{N} 1$ & P1 & C6 & $\mathrm{Fe} 1$ & $174.2(4)$ \\
\hline N1 & P1 & $\mathrm{C} 6$ & $\mathrm{C} 7$ & $76.7(6)$ \\
\hline N1 & P1 & C6 & C10 & $-96.6(5)$ \\
\hline N1 & P1 & C11 & C12 & $-128.4(6)$ \\
\hline N1 & $\mathrm{P} 1$ & C11 & C16 & $57.6(6)$ \\
\hline $\mathrm{N} 1$ & C18 & $\mathrm{C} 20$ & 01 & $-165.8(4)$ \\
\hline N1 & C18 & $\mathrm{C} 20$ & $\mathrm{C} 21$ & $-45.4(7)$ \\
\hline B1 & P1 & $\mathrm{N} 1$ & C17 & $-169.7(5)$ \\
\hline B1 & $\mathrm{P} 1$ & N1 & C18 & $-21.4(5)$ \\
\hline B1 & $\mathrm{P} 1$ & $\mathrm{C} 6$ & $\mathrm{Fe} 1$ & $-59.9(5)$ \\
\hline B1 & $\mathrm{P} 1$ & $\mathrm{C} 6$ & $\mathrm{C} 7$ & $-157.4(6)$ \\
\hline B1 & $\mathrm{P} 1$ & C6 & $\mathrm{C} 10$ & $29.2(6)$ \\
\hline B1 & $\mathrm{P} 1$ & C11 & $\mathrm{C} 12$ & $109.6(6)$ \\
\hline B1 & P1 & C11 & C16 & $-64.4(6)$ \\
\hline B2 & P2 & 01 & $\mathrm{C} 20$ & $27.9(6)$ \\
\hline B2 & P2 & $\mathrm{C} 27$ & $\mathrm{C} 28$ & $131.0(6)$ \\
\hline B2 & P2 & $\mathrm{C} 27$ & C32 & $-48.3(6)$ \\
\hline B2 & P2 & C33 & C34 & $-160.9(5)$ \\
\hline B2 & P2 & C33 & C38 & $20.9(6)$ \\
\hline C1 & $\mathrm{C} 2$ & C3 & Fe1 & $59.5(4)$ \\
\hline C1 & $\mathrm{C} 2$ & C3 & $\mathrm{C} 4$ & $0.4(7)$ \\
\hline $\mathrm{C} 2$ & $\mathrm{C} 1$ & $\mathrm{C} 5$ & $\mathrm{Fe} 1$ & $-59.0(4)$ \\
\hline $\mathrm{C} 2$ & $\mathrm{C} 1$ & $\mathrm{C} 5$ & $\mathrm{C} 4$ & $0.6(7)$ \\
\hline $\mathrm{C} 2$ & $\mathrm{C} 3$ & $\mathrm{C} 4$ & $\mathrm{Fe} 1$ & $59.4(4)$ \\
\hline $\mathrm{C} 2$ & $\mathrm{C} 3$ & $\mathrm{C} 4$ & $\mathrm{C} 5$ & $-0.1(7)$ \\
\hline C3 & $\mathrm{C} 4$ & $\mathrm{C} 5$ & $\mathrm{Fe} 1$ & $60.1(5)$ \\
\hline $\mathrm{C} 3$ & $\mathrm{C} 4$ & $\mathrm{C} 5$ & $\mathrm{C} 1$ & $-0.3(7)$ \\
\hline C5 & $\mathrm{C} 1$ & $\mathrm{C} 2$ & $\mathrm{Fe} 1$ & $58.8(4)$ \\
\hline C5 & $\mathrm{C} 1$ & $\mathrm{C} 2$ & $\mathrm{C} 3$ & $-0.6(7)$ \\
\hline C6 & $\mathrm{P} 1$ & N1 & C17 & $-45.5(5)$ \\
\hline C6 & $\mathrm{P} 1$ & N1 & C18 & $102.8(4)$ \\
\hline C6 & P1 & C11 & C12 & $-13.1(7)$ \\
\hline C6 & $\mathrm{P} 1$ & C11 & C16 & $172.9(5)$ \\
\hline C6 & $\mathrm{C} 7$ & C8 & $\mathrm{Fe} 1$ & $-58.8(4)$ \\
\hline C6 & $\mathrm{C} 7$ & $\mathrm{C} 8$ & C9 & $-0.4(7)$ \\
\hline $\mathrm{C} 7$ & $\mathrm{C} 6$ & C10 & $\mathrm{Fe} 1$ & $59.8(4)$ \\
\hline $\mathrm{C} 7$ & $\mathrm{C} 6$ & C10 & C9 & $0.4(7)$ \\
\hline $\mathrm{C} 7$ & $\mathrm{C} 8$ & C9 & $\mathrm{Fe} 1$ & $-58.7(5)$ \\
\hline
\end{tabular}




\begin{tabular}{|c|c|c|c|c|}
\hline Atom & Atom & Atom & Atom & Angle $/^{\circ}$ \\
\hline$\overline{\mathrm{C} 7}$ & $\mathrm{C} 8$ & C9 & C10 & $0.7(7)$ \\
\hline C8 & C9 & $\mathrm{C} 10$ & $\mathrm{Fe} 1$ & $-59.8(5)$ \\
\hline C8 & C9 & C10 & C6 & $-0.7(7)$ \\
\hline C10 & $\mathrm{C} 6$ & $\mathrm{C} 7$ & $\mathrm{Fe} 1$ & $-59.6(4)$ \\
\hline C10 & $\mathrm{C} 6$ & $\mathrm{C} 7$ & C8 & $0.0(7)$ \\
\hline C11 & $\mathrm{P} 1$ & N1 & C17 & $70.6(5)$ \\
\hline C11 & $\mathrm{P} 1$ & N1 & C18 & $-141.0(4)$ \\
\hline C11 & $\mathrm{P} 1$ & $\mathrm{C} 6$ & $\mathrm{Fe} 1$ & $62.5(5)$ \\
\hline C11 & $\mathrm{P} 1$ & $\mathrm{C} 6$ & $\mathrm{C} 7$ & $-35.0(6)$ \\
\hline C11 & $\mathrm{P} 1$ & C6 & C10 & $151.6(5)$ \\
\hline C11 & C12 & C13 & C14 & $1.6(11)$ \\
\hline C12 & C11 & C16 & C15 & $0.0(11)$ \\
\hline C12 & C13 & C14 & C15 & $-0.5(12)$ \\
\hline C13 & C14 & C15 & C16 & $-0.9(13)$ \\
\hline C14 & C15 & C16 & C11 & 1.1(13) \\
\hline C16 & C11 & $\mathrm{C} 12$ & C13 & $-1.4(10)$ \\
\hline C17 & N1 & C18 & C19 & $66.9(7)$ \\
\hline C17 & N1 & C18 & $\mathrm{C} 20$ & $-58.5(7)$ \\
\hline C18 & $\mathrm{C} 20$ & $\mathrm{C} 21$ & $\mathrm{C} 22$ & $-56.6(8)$ \\
\hline C18 & $\mathrm{C} 20$ & $\mathrm{C} 21$ & $\mathrm{C} 26$ & $124.2(7)$ \\
\hline C19 & C18 & $\mathrm{C} 20$ & 01 & $67.8(6)$ \\
\hline C19 & C18 & $\mathrm{C} 20$ & $\mathrm{C} 21$ & $-171.8(5)$ \\
\hline C20 & $\mathrm{C} 21$ & $\mathrm{C} 22$ & $\mathrm{C} 23$ & $-177.3(6)$ \\
\hline C20 & $\mathrm{C} 21$ & $\mathrm{C} 26$ & $\mathrm{C} 25$ & $177.7(6)$ \\
\hline C21 & $\mathrm{C} 22$ & $\mathrm{C} 23$ & $\mathrm{C} 24$ & $-1.8(10)$ \\
\hline C22 & $\mathrm{C} 21$ & $\mathrm{C} 26$ & $\mathrm{C} 25$ & $-1.6(10)$ \\
\hline C22 & $\mathrm{C} 23$ & $\mathrm{C} 24$ & $\mathrm{C} 25$ & $1.0(11)$ \\
\hline C23 & $\mathrm{C} 24$ & $\mathrm{C} 25$ & $\mathrm{C} 26$ & $-0.6(12)$ \\
\hline C24 & $\mathrm{C} 25$ & $\mathrm{C} 26$ & $\mathrm{C} 21$ & $0.9(11)$ \\
\hline C26 & $\mathrm{C} 21$ & $\mathrm{C} 22$ & $\mathrm{C} 23$ & $2.0(10)$ \\
\hline C27 & P2 & 01 & $\mathrm{C} 20$ & $151.5(4)$ \\
\hline C27 & P2 & C33 & C34 & $75.2(6)$ \\
\hline C27 & P2 & C33 & C38 & $-102.9(6)$ \\
\hline C27 & $\mathrm{C} 28$ & $\mathrm{C} 29$ & C30 & $-1.1(11)$ \\
\hline C28 & $\mathrm{C} 27$ & C32 & C31 & $0.2(9)$ \\
\hline C28 & $\mathrm{C} 29$ & C30 & C31 & $-0.4(11)$ \\
\hline C29 & C30 & C31 & C32 & $1.7(10)$ \\
\hline C30 & C31 & C32 & $\mathrm{C} 27$ & $-1.6(10)$ \\
\hline C32 & $\mathrm{C} 27$ & $\mathrm{C} 28$ & $\mathrm{C} 29$ & $1.2(10)$ \\
\hline C33 & P2 & 01 & $\mathrm{C} 20$ & $-99.5(5)$ \\
\hline C33 & P2 & $\mathrm{C} 27$ & $\mathrm{C} 28$ & $-105.4(6)$ \\
\hline C33 & P2 & $\mathrm{C} 27$ & C32 & 75.3(5) \\
\hline C33 & C34 & C35 & C36 & $0.1(10)$ \\
\hline C34 & C33 & C38 & C37 & $0.1(10)$ \\
\hline C34 & C35 & C36 & C37 & $0.4(11)$ \\
\hline C35 & C36 & C37 & C38 & $-0.6(11)$ \\
\hline C36 & C37 & C38 & C33 & $0.4(11)$ \\
\hline C38 & C33 & C34 & C35 & $-0.3(10)$ \\
\hline $\mathrm{Fe} 1 \mathrm{~A}$ & $\mathrm{C} 2 \mathrm{~A}$ & $\mathrm{C} 3 \mathrm{~A}$ & $\mathrm{C} 4 \mathrm{~A}$ & $-59.5(5)$ \\
\hline $\mathrm{Fe} 1 \mathrm{~A}$ & $\mathrm{C} 2 \mathrm{~A}$ & $\mathrm{C} 1 \mathrm{~A}$ & $\mathrm{C} 5 \mathrm{~A}$ & $59.7(5)$ \\
\hline $\mathrm{Fe} 1 \mathrm{~A}$ & C3A & $\mathrm{C} 4 \mathrm{~A}$ & $\mathrm{C} 5 \mathrm{~A}$ & $-59.0(5)$ \\
\hline $\mathrm{Fe} 1 \mathrm{~A}$ & $\mathrm{C} 4 \mathrm{~A}$ & C5A & C1A & $-59.5(5)$ \\
\hline $\mathrm{Fe} 1 \mathrm{~A}$ & $\mathrm{C} 5 \mathrm{~A}$ & $\mathrm{C} 1 \mathrm{~A}$ & $\mathrm{C} 2 \mathrm{~A}$ & $-59.8(5)$ \\
\hline $\mathrm{Fe} 1 \mathrm{~A}$ & C6A & C7A & C8A & $59.2(4)$ \\
\hline
\end{tabular}




\begin{tabular}{|c|c|c|c|c|}
\hline Atom & Atom & Atom & Atom & Angle $/{ }^{\circ}$ \\
\hline Fe1A & C6A & C10A & C9A & $-59.7(4)$ \\
\hline Fe1A & C7A & C8A & C9A & $58.9(4)$ \\
\hline Fe1A & $\mathrm{C} 8 \mathrm{~A}$ & C9A & C10A & $59.1(4)$ \\
\hline $\mathrm{Fe} 1 \mathrm{~A}$ & C9A & $\mathrm{C} 10 \mathrm{~A}$ & C6A & $59.6(4)$ \\
\hline P1A & $\mathrm{N} 1 \mathrm{~A}$ & C18A & C19A & $-86.3(6)$ \\
\hline P1A & $\mathrm{N} 1 \mathrm{~A}$ & C18A & $\mathrm{C} 20 \mathrm{~A}$ & $148.4(4)$ \\
\hline P1A & $\mathrm{C} 6 \mathrm{~A}$ & C7A & Fe1A & $128.7(5)$ \\
\hline $\mathrm{P} 1 \mathrm{~A}$ & C6A & C7A & C8A & $-172.1(5)$ \\
\hline $\mathrm{P} 1 \mathrm{~A}$ & C6A & $\mathrm{C} 10 \mathrm{~A}$ & Fe1A & $-127.9(5)$ \\
\hline $\mathrm{P} 1 \mathrm{~A}$ & C6A & $\mathrm{C} 10 \mathrm{~A}$ & $\mathrm{C} 9 \mathrm{~A}$ & $172.4(4)$ \\
\hline P1A & C11A & $\mathrm{C} 12 \mathrm{~A}$ & C13A & $-175.7(7)$ \\
\hline P1A & C11A & C16A & C15A & $176.2(5)$ \\
\hline $\mathrm{P} 2 \mathrm{~A}$ & $01 \mathrm{~A}$ & $\mathrm{C} 20 \mathrm{~A}$ & C18A & $-132.3(4)$ \\
\hline $\mathrm{P} 2 \mathrm{~A}$ & $01 \mathrm{~A}$ & $\mathrm{C} 20 \mathrm{~A}$ & $\mathrm{C} 21 \mathrm{~A}$ & $103.4(5)$ \\
\hline $\mathrm{P} 2 \mathrm{~A}$ & $\mathrm{C} 33 \mathrm{~A}$ & C34A & C35A & $177.6(5)$ \\
\hline $\mathrm{P} 2 \mathrm{~A}$ & C33A & C38A & C37A & $-178.6(5)$ \\
\hline $\mathrm{P} 2 \mathrm{~A}$ & $\mathrm{C} 27 \mathrm{~A}$ & $\mathrm{C} 28 \mathrm{~A}$ & C29A & $179.2(5)$ \\
\hline $\mathrm{P} 2 \mathrm{~A}$ & $\mathrm{C} 27 \mathrm{~A}$ & $\mathrm{C} 32 \mathrm{~A}$ & C31A & $-178.7(5)$ \\
\hline N1A & P1A & C6A & Fe1A & $-170.6(4)$ \\
\hline N1A & P1A & C6A & C7A & $92.4(6)$ \\
\hline $\mathrm{N} 1 \mathrm{~A}$ & P1A & C6A & $\mathrm{C} 10 \mathrm{~A}$ & $-78.7(6)$ \\
\hline N1A & P1A & $\mathrm{C} 11 \mathrm{~A}$ & $\mathrm{C} 12 \mathrm{~A}$ & $-172.0(6)$ \\
\hline $\mathrm{N} 1 \mathrm{~A}$ & $\mathrm{P} 1 \mathrm{~A}$ & $\mathrm{C} 11 \mathrm{~A}$ & C16A & $13.3(6)$ \\
\hline $\mathrm{N} 1 \mathrm{~A}$ & C18A & $\mathrm{C} 20 \mathrm{~A}$ & $01 \mathrm{~A}$ & $-165.7(5)$ \\
\hline N1A & C18A & $\mathrm{C} 20 \mathrm{~A}$ & $\mathrm{C} 21 \mathrm{~A}$ & $-46.7(7)$ \\
\hline $01 \mathrm{~A}$ & P2A & $\mathrm{C} 33 \mathrm{~A}$ & $\mathrm{C} 34 \mathrm{~A}$ & $-11.1(6)$ \\
\hline $01 \mathrm{~A}$ & P2A & C33A & C38A & $167.7(5)$ \\
\hline $01 \mathrm{~A}$ & $\mathrm{P} 2 \mathrm{~A}$ & $\mathrm{C} 27 \mathrm{~A}$ & $\mathrm{C} 28 \mathrm{~A}$ & $12.0(6)$ \\
\hline $01 \mathrm{~A}$ & $\mathrm{P} 2 \mathrm{~A}$ & C27A & $\mathrm{C} 32 \mathrm{~A}$ & $-168.5(5)$ \\
\hline $01 \mathrm{~A}$ & $\mathrm{C} 20 \mathrm{~A}$ & $\mathrm{C} 21 \mathrm{~A}$ & $\mathrm{C} 22 \mathrm{~A}$ & 70.8(7) \\
\hline $01 \mathrm{~A}$ & $\mathrm{C} 20 \mathrm{~A}$ & $\mathrm{C} 21 \mathrm{~A}$ & $\mathrm{C} 26 \mathrm{~A}$ & $-104.7(6)$ \\
\hline B1A & P1A & N1A & C17A & $-165.0(5)$ \\
\hline B1A & P1A & $\mathrm{N} 1 \mathrm{~A}$ & C18A & $-15.5(6)$ \\
\hline B1A & P1A & C6A & Fe1A & $-44.8(6)$ \\
\hline B1A & P1A & C6A & C7A & $-141.7(6)$ \\
\hline B1A & $\mathrm{P} 1 \mathrm{~A}$ & C6A & $\mathrm{C} 10 \mathrm{~A}$ & 47.1(6) \\
\hline B1A & $\mathrm{P} 1 \mathrm{~A}$ & $\mathrm{C} 11 \mathrm{~A}$ & $\mathrm{C} 12 \mathrm{~A}$ & $64.9(7)$ \\
\hline B1A & P1A & $\mathrm{C} 11 \mathrm{~A}$ & C16A & $-109.9(6)$ \\
\hline B2A & $\mathrm{P} 2 \mathrm{~A}$ & $01 \mathrm{~A}$ & $\mathrm{C} 20 \mathrm{~A}$ & 21.1(6) \\
\hline B2A & P2A & C33A & $\mathrm{C} 34 \mathrm{~A}$ & $-138.8(5)$ \\
\hline B2A & $\mathrm{P} 2 \mathrm{~A}$ & $\mathrm{C} 33 \mathrm{~A}$ & C38A & $40.0(6)$ \\
\hline B2A & $\mathrm{P} 2 \mathrm{~A}$ & $\mathrm{C} 27 \mathrm{~A}$ & $\mathrm{C} 28 \mathrm{~A}$ & $138.8(6)$ \\
\hline B2A & P2A & $\mathrm{C} 27 \mathrm{~A}$ & $\mathrm{C} 32 \mathrm{~A}$ & $-41.6(6)$ \\
\hline $\mathrm{C} 2 \mathrm{~A}$ & C3A & $\mathrm{C} 4 \mathrm{~A}$ & Fe1A & $59.6(4)$ \\
\hline $\mathrm{C} 2 \mathrm{~A}$ & C3A & $\mathrm{C} 4 \mathrm{~A}$ & C5A & $0.6(7)$ \\
\hline $\mathrm{C} 3 \mathrm{~A}$ & $\mathrm{C} 2 \mathrm{~A}$ & $\mathrm{C} 1 \mathrm{~A}$ & Fe1A & $-59.4(5)$ \\
\hline $\mathrm{C} 3 \mathrm{~A}$ & $\mathrm{C} 2 \mathrm{~A}$ & C1A & C5A & $0.4(8)$ \\
\hline $\mathrm{C} 3 \mathrm{~A}$ & $\mathrm{C} 4 \mathrm{~A}$ & C5A & Fe1A & $59.1(5)$ \\
\hline C3A & C4A & C5A & C1A & $-0.4(7)$ \\
\hline $\mathrm{C} 4 \mathrm{~A}$ & C5A & $\mathrm{C} 1 \mathrm{~A}$ & Fe1A & $59.8(5)$ \\
\hline $\mathrm{C} 4 \mathrm{~A}$ & C5A & $\mathrm{C} 1 \mathrm{~A}$ & $\mathrm{C} 2 \mathrm{~A}$ & $0.0(8)$ \\
\hline C1A & $\mathrm{C} 2 \mathrm{~A}$ & C3A & Fe1A & $59.0(5)$ \\
\hline C1A & $\mathrm{C} 2 \mathrm{~A}$ & C3A & $\mathrm{C} 4 \mathrm{~A}$ & $-0.6(7)$ \\
\hline C6A & P1A & $\mathrm{N} 1 \mathrm{~A}$ & C17A & $-38.0(5)$ \\
\hline
\end{tabular}




\begin{tabular}{|c|c|c|c|c|}
\hline Atom & Atom & Atom & Atom & Angle $/^{\circ}$ \\
\hline$\overline{\mathrm{C} 6 \mathrm{~A}}$ & P1A & N1A & C18A & $111.5(5)$ \\
\hline C6A & $\mathrm{P} 1 \mathrm{~A}$ & C11A & $\mathrm{C} 12 \mathrm{~A}$ & $-59.9(6)$ \\
\hline C6A & $\mathrm{P} 1 \mathrm{~A}$ & $\mathrm{C} 11 \mathrm{~A}$ & C16A & $125.3(6)$ \\
\hline C6A & C7A & $\mathrm{C} 8 \mathrm{~A}$ & Fe1A & $-59.1(4)$ \\
\hline C6A & C7A & C8A & $\mathrm{C} 9 \mathrm{~A}$ & $-0.1(7)$ \\
\hline C7A & C6A & C10A & Fe1A & $59.4(4)$ \\
\hline C7A & C6A & C10A & C9A & $-0.3(7)$ \\
\hline C7A & $\mathrm{C} 8 \mathrm{~A}$ & C9A & Fe1A & $-59.1(4)$ \\
\hline C7A & C8A & C9A & C10A & $-0.1(7)$ \\
\hline C8A & C9A & C10A & Fe1A & $-59.4(5)$ \\
\hline C8A & C9A & C10A & $\mathrm{C} 6 \mathrm{~A}$ & $0.2(7)$ \\
\hline C10A & C6A & C7A & Fe1A & $-58.9(4)$ \\
\hline C10A & C6A & C7A & $\mathrm{C} 8 \mathrm{~A}$ & $0.3(7)$ \\
\hline C11A & $\mathrm{P} 1 \mathrm{~A}$ & $\mathrm{~N} 1 \mathrm{~A}$ & C17A & $72.7(5)$ \\
\hline C11A & $\mathrm{P} 1 \mathrm{~A}$ & $\mathrm{~N} 1 \mathrm{~A}$ & C18A & $-137.8(5)$ \\
\hline C11A & $\mathrm{P} 1 \mathrm{~A}$ & C6A & Fe1A & $78.0(5)$ \\
\hline C11A & $\mathrm{P} 1 \mathrm{~A}$ & C6A & $\mathrm{C} 7 \mathrm{~A}$ & $-19.0(6)$ \\
\hline C11A & P1A & C6A & C10A & $169.8(5)$ \\
\hline C11A & C12A & C13A & C14A & $-0.8(14)$ \\
\hline C12A & C11A & C16A & C15A & $1.4(10)$ \\
\hline C12A & C13A & $\mathrm{C} 14 \mathrm{~A}$ & C15A & $1.9(13)$ \\
\hline C13A & C14A & C15A & C16A & $-1.3(12)$ \\
\hline C14A & C15A & C16A & C11A & $-0.4(11)$ \\
\hline C16A & C11A & $\mathrm{C} 12 \mathrm{~A}$ & C13A & $-0.9(12)$ \\
\hline C17A & $\mathrm{N} 1 \mathrm{~A}$ & C18A & C19A & $63.6(7)$ \\
\hline C17A & N1A & C18A & $\mathrm{C} 20 \mathrm{~A}$ & $-61.6(7)$ \\
\hline C18A & C20A & $\mathrm{C} 21 \mathrm{~A}$ & $\mathrm{C} 22 \mathrm{~A}$ & $-45.7(8)$ \\
\hline C18A & C20A & $\mathrm{C} 21 \mathrm{~A}$ & C26A & $138.7(6)$ \\
\hline C19A & C18A & $\mathrm{C} 20 \mathrm{~A}$ & $01 \mathrm{~A}$ & $68.0(6)$ \\
\hline C19A & C18A & $\mathrm{C} 20 \mathrm{~A}$ & $\mathrm{C} 21 \mathrm{~A}$ & $-173.1(5)$ \\
\hline $\mathrm{C} 20 \mathrm{~A}$ & $\mathrm{C} 21 \mathrm{~A}$ & $\mathrm{C} 22 \mathrm{~A}$ & $\mathrm{C} 23 \mathrm{~A}$ & $-176.6(6)$ \\
\hline C20A & $\mathrm{C} 21 \mathrm{~A}$ & $\mathrm{C} 26 \mathrm{~A}$ & $\mathrm{C} 25 \mathrm{~A}$ & $175.4(6)$ \\
\hline C21A & $\mathrm{C} 22 \mathrm{~A}$ & $\mathrm{C} 23 \mathrm{~A}$ & $\mathrm{C} 24 \mathrm{~A}$ & $1.5(10)$ \\
\hline C22A & $\mathrm{C} 21 \mathrm{~A}$ & $\mathrm{C} 26 \mathrm{~A}$ & $\mathrm{C} 25 \mathrm{~A}$ & $-0.3(10)$ \\
\hline C22A & C23A & $\mathrm{C} 24 \mathrm{~A}$ & $\mathrm{C} 25 \mathrm{~A}$ & $-0.9(11)$ \\
\hline C23A & $\mathrm{C} 24 \mathrm{~A}$ & $\mathrm{C} 25 \mathrm{~A}$ & $\mathrm{C} 26 \mathrm{~A}$ & $-0.2(11)$ \\
\hline $\mathrm{C} 24 \mathrm{~A}$ & $\mathrm{C} 25 \mathrm{~A}$ & $\mathrm{C} 26 \mathrm{~A}$ & $\mathrm{C} 21 \mathrm{~A}$ & $0.8(11)$ \\
\hline C26A & $\mathrm{C} 21 \mathrm{~A}$ & $\mathrm{C} 22 \mathrm{~A}$ & $\mathrm{C} 23 \mathrm{~A}$ & $-0.9(10)$ \\
\hline C33A & $\mathrm{P} 2 \mathrm{~A}$ & $01 \mathrm{~A}$ & $\mathrm{C} 20 \mathrm{~A}$ & $-103.8(5)$ \\
\hline C33A & P2A & $\mathrm{C} 27 \mathrm{~A}$ & C28A & $-96.9(6)$ \\
\hline C33A & P2A & $\mathrm{C} 27 \mathrm{~A}$ & C32A & $82.6(5)$ \\
\hline C33A & C34A & C35A & C36A & $0.9(10)$ \\
\hline C34A & C33A & C38A & C37A & $0.2(10)$ \\
\hline C34A & C35A & C36A & C37A & $0.4(11)$ \\
\hline C35A & C36A & C37A & C38A & $-1.4(11)$ \\
\hline C36A & C37A & C38A & C33A & $1.0(10)$ \\
\hline C38A & C33A & C34A & C35A & $-1.2(9)$ \\
\hline C27A & $\mathrm{P} 2 \mathrm{~A}$ & $01 \mathrm{~A}$ & $\mathrm{C} 20 \mathrm{~A}$ & $146.9(4)$ \\
\hline $\mathrm{C} 27 \mathrm{~A}$ & P2A & C33A & C34A & $95.1(6)$ \\
\hline $\mathrm{C} 27 \mathrm{~A}$ & $\mathrm{P} 2 \mathrm{~A}$ & C33A & C38A & $-86.1(5)$ \\
\hline C27A & C28A & C29A & $\mathrm{C} 30 \mathrm{~A}$ & $-0.2(10)$ \\
\hline C28A & C27A & $\mathrm{C} 32 \mathrm{~A}$ & C31A & $0.8(9)$ \\
\hline C28A & C29A & C30A & C31A & $0.2(10)$ \\
\hline C29A & C30A & C31A & $\mathrm{C} 32 \mathrm{~A}$ & $0.3(10)$ \\
\hline
\end{tabular}




\begin{tabular}{lllll}
\hline Atom & Atom & Atom & Atom & Angle $^{\circ}$ \\
\hline C30A & C31A & C32A & C27A & $-0.8(10)$ \\
C32A & C27A & C28A & C29A & $-0.3(10)$
\end{tabular}


Name: $\left(S_{\mathrm{p}}\right)-(-)-\mathrm{N}-[(1 \mathrm{R}, 2 \mathrm{~S})-2-($ Diphenylphosphinito-borane)-1-methyl-2-phenylethyl], N-methylamino (o-biphenylphenyl)phosphine-borane 12i.

Solved by: Yoann Rousselin

Sample ID: $\quad 16$ jb1308

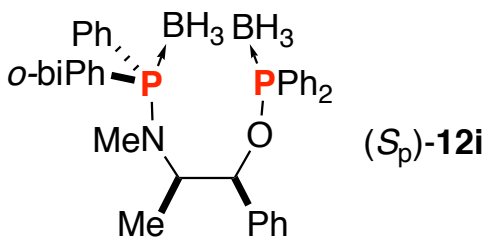

\section{Crystal Data and Experimental}

Figure S6 (thermal ellipsoïds are drawn at $50 \%$ probability plot)

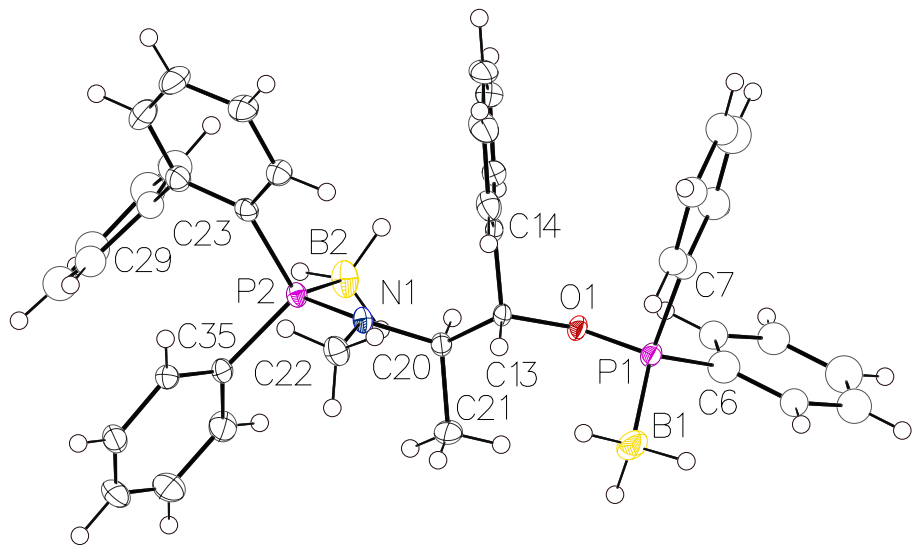

Experimental. Single clear light colorless prism-shaped crystals of compound 12i were recrystallized from a mixture DCM and hexane by slow evaporation. A suitable crystal $0.51 \times 0.46 \times 0.34 \mathrm{~mm}^{3}$ was selected and mounted on a MITIGEN holder oil on a Nonius Kappa APEX II diffractometer. The crystal was kept at a steady $T=$ 115.0(1) K during data collection. The structure was solved with the ShelXT (Sheldrick, 2015) structure solution program using the Intrinsic Phasing solution method and by using 0lex2 (Dolomanov et al., 2009) as the graphical interface. The model was refined with version 2018/3 of ShelXL (Sheldrick, 2015) using Least Squares minimization.

Crystal Data. $\mathrm{C}_{40} \mathrm{H}_{43} \mathrm{~B}_{2} \mathrm{NOP}_{2}, M_{r}=637.31$, orthorhombic, $P 2{ }_{1} 2_{1}{ }_{1}$ (No. 19), $\mathrm{a}=9.1390(4) \AA, \mathrm{b}=13.2373(6) \AA, \mathrm{c}=$ 30.6092(13) $\AA, \alpha=\beta=\gamma=90^{\circ}, V=3703.0(3) \AA^{3}, T=$ 115.0(1) K, $Z=4, \quad Z^{\prime}=1, \mu\left(\mathrm{MoK}_{\alpha}\right)=0.148,72028$ reflections measured, 8495 unique $\left(R_{\text {int }}=0.0438\right)$ which were used in all calculations. The final $w R_{2}$ was 0.1043 (all data) and $R_{1}$ was 0.0426 (I > 2(I)).

\begin{tabular}{|c|c|}
\hline Compound & $12 i$ \\
\hline CCDC & 1982663 \\
\hline Formula & $\mathrm{C}_{40} \mathrm{H}_{43} \mathrm{~B}_{2} \mathrm{NOP}_{2}$ \\
\hline$D_{\text {calc. }} / \mathrm{g} \mathrm{cm}^{-3}$ & 1.143 \\
\hline$\mu / \mathrm{mm}^{-1}$ & 0.148 \\
\hline Formula Weight & 637.31 \\
\hline Color & clear light colorless \\
\hline Shape & prism \\
\hline Size $/ \mathrm{mm}^{3}$ & $0.51 \times 0.46 \times 0.34$ \\
\hline$T / \mathrm{K}$ & $115.0(1)$ \\
\hline Crystal System & orthorhombic \\
\hline Flack Parameter & $0.001(18)$ \\
\hline Hooft Parameter & $0.010(17)$ \\
\hline Space Group & $P 2_{1} 2_{1} 2_{1}$ \\
\hline$a / \AA ̊$ & $9.1390(4)$ \\
\hline$b / \AA$ & $13.2373(6)$ \\
\hline$c / \AA ̊$ & $30.6092(13)$ \\
\hline$\alpha /^{\circ}$ & 90 \\
\hline$\beta /^{\circ}$ & 90 \\
\hline$\gamma /{ }^{\circ}$ & 90 \\
\hline $\mathrm{V} / \AA^{3}$ & $3703.0(3)$ \\
\hline$Z$ & 4 \\
\hline$Z^{\prime}$ & 1 \\
\hline Wavelength/Å & 0.71073 \\
\hline Radiation type & $\mathrm{MoK}_{\alpha}$ \\
\hline$\Theta_{\min } /^{\circ}$ & 2.789 \\
\hline$\Theta_{\max } /^{\circ}$ & 27.499 \\
\hline Measured Refl. & 72028 \\
\hline Independent Refl. & 8495 \\
\hline Reflections with I > & 8098 \\
\hline $2(\mathrm{I})$ & \\
\hline$R_{\text {int }}$ & 0.0438 \\
\hline Parameters & 343 \\
\hline Restraints & 0 \\
\hline Largest Peak & 0.594 \\
\hline Deepest Hole & -0.585 \\
\hline GooF & 1.059 \\
\hline$w R_{2}$ (all data) & 0.1043 \\
\hline$w R_{2}$ & 0.1023 \\
\hline$R_{1}$ (all data) & 0.0455 \\
\hline$R_{1}$ & 0.0426 \\
\hline
\end{tabular}


Reflections:

Refinement:

A clear light colorless prism-shaped crystal with dimensions $0.51 \times 0.46 \times 0.34 \mathrm{~mm}^{3}$ was mounted on a MITIGEN holder oil. Data were collected using a Nonius Kappa APEX II diffractometer equipped with an Oxford Cryosystems low-temperature device operating at $T=115.0(1) \mathrm{K}$. Data were measured using $\phi$ and $\omega$ scans using $\mathrm{MoK}_{\alpha}$ radiation. The total number of runs and images was based on the strategy calculation from the program APEX3 (Bruker, 2015) The maximum resolution that was achieved was $\Theta$ $=27.499^{\circ}(0.77 \AA)$. The diffraction pattern was indexed. The total number of runs and images was based on the strategy calculation from the program APEX3 (Bruker, 2015) and the unit cell was refined using SAINT (Bruker, V8.37A, after 2013) on 9944 reflections, 14\% of the observed reflections. Data reduction, scaling and absorption corrections were performed using SAINT (Bruker, V8.37A, after 2013). The final completeness is $99.70 \%$ out to $27.499^{\circ}$ in $\Theta$. A multi-scan absorption correction was performed using SADABS-2014/5 (Bruker, 2014) was used for absorption correction. $w R_{2}$ (int) was 0.0552 before and 0.0526 after correction. The Ratio of minimum to maximum transmission is 0.9256 . The $\lambda / 2$ correction factor is 0.00150 . The absorption coefficient $\mu$ of this material is $0.148 \mathrm{~mm}^{-1}$ at this wavelength $(\lambda=0.711 \AA)$ and the minimum and maximum transmissions are 0.690 and 0.746 . The structure was solved and the space group $P 2{ }_{1} 2_{1} 2_{1}$ (\# 19) determined by the ShelXT (Sheldrick, 2015) structure solution program using the Intrinsic Phasing solution method and refined by Least Squares using version 2018/3 of ShelXL (Sheldrick, 2015). All non-hydrogen atoms were refined anisotropically, excepted for disordered part. Some phenyl group were found disordered over two positions and their occupancies were fixed to 50\%. Hydrogen atom positions were calculated geometrically and refined using the riding model. Hydrogen atom on Boron atom were refined using an AFIX 138, which would allow the refinement of a common B-H distances, the same shifts being applied to all the B-H bonds to the same atom, but still retain tetrahedral H-B-H angles within the group. There is a single molecule in the asymmetric unit, which is represented by the reported sum formula. In other words: $\mathrm{Z}$ is 4 and $\mathrm{Z}$ ' is 1. The Flack parameter was refined to 0.001(18). Determination of absolute structure using Bayesian statistics on Bijvoet differences using the Olex2 results in 0.010(17). Note: The Flack parameter is used to determine chirality of the crystal studied, the value should be near 0 , a value of 1 means that the stereochemistry is wrong and the model should be inverted. A value of 0.5 means that the crystal consists of a racemic mixture of the two enantiomers.

Table S16: Bond Lengths in Å for compound 12i.

\begin{tabular}{lll}
\hline Atom & Atom & Length/Å \\
\hline P1 & O1 & $1.6146(18)$ \\
P1 & C6 & $1.806(4)$ \\
P1 & C7 & $1.839(3)$ \\
P1 & B1 & $1.894(4)$ \\
P1 & C1A & $1.789(3)$ \\
P1 & C7A & $1.768(3)$ \\
P2 & N1 & $1.671(2)$ \\
P2 & C23 & $1.829(3)$ \\
P2 & C35 & $1.819(3)$ \\
P2 & B2 & $1.919(3)$ \\
01 & C13 & $1.464(3)$ \\
C6 & C1 & 1.3900 \\
C6 & C2 & 1.3900 \\
C1 & C5 & 1.3900 \\
C5 & C4 & 1.3900
\end{tabular}

\begin{tabular}{lll}
\hline Atom & Atom & Length/Å \\
\hline C4 & C3 & 1.3900 \\
C3 & C2 & 1.3900 \\
N1 & C20 & $1.475(3)$ \\
N1 & C22 & $1.471(3)$ \\
C34 & C29 & $1.373(11)$ \\
C34 & C33 & $1.390(10)$ \\
C7 & C8 & 1.3900 \\
C7 & C12 & 1.3900 \\
C8 & C9 & 1.3900 \\
C9 & C10 & 1.3900 \\
C10 & C11 & 1.3900 \\
C11 & C12 & 1.3900 \\
C13 & C20 & $1.538(3)$ \\
C13 & C14 & $1.505(3)$ \\
C20 & C21 & $1.529(4)$
\end{tabular}




\begin{tabular}{lll}
\hline Atom & Atom & Length/A \\
\hline C23 & C28 & $1.410(4)$ \\
C23 & C24 & $1.400(4)$ \\
C28 & C29 & $1.520(7)$ \\
C28 & C27 & $1.396(4)$ \\
C28 & C29A & $1.490(4)$ \\
C29 & C30 & $1.412(10)$ \\
C33 & C32 & $1.390(10)$ \\
C32 & C31 & $1.389(10)$ \\
C30 & C31 & $1.395(9)$ \\
C24 & C25 & $1.391(4)$ \\
C25 & C26 & $1.376(4)$ \\
C26 & C27 & $1.388(4)$ \\
C14 & C15 & $1.397(4)$ \\
C14 & C19 & $1.391(4)$ \\
C15 & C16 & $1.384(4)$ \\
C16 & C17 & $1.380(5)$ \\
C17 & C18 & $1.379(5)$ \\
C18 & C19 & $1.391(4)$ \\
C35 & C40 & $1.398(4)$ \\
C35 & C36 & $1.399(4)$ \\
C40 & C39 & $1.394(4)$ \\
& & \\
\hline
\end{tabular}

\begin{tabular}{lll}
\hline Atom & Atom & Length/Å \\
\hline C39 & C38 & $1.375(5)$ \\
C38 & C37 & $1.378(5)$ \\
C37 & C36 & $1.387(4)$ \\
C2A & C3A & 1.3900 \\
C2A & C1A & 1.3900 \\
C3A & C4A & 1.3900 \\
C4A & C5A & 1.3900 \\
C5A & C6A & 1.3900 \\
C6A & C1A & 1.3900 \\
C7A & C8A & 1.3900 \\
C7A & C12A & 1.3900 \\
C8A & C9A & 1.3900 \\
C9A & C10A & 1.3900 \\
C10A & C11A & 1.3900 \\
C11A & C12A & 1.3900 \\
C29A & C30A & 1.3900 \\
C29A & C34A & 1.3900 \\
C30A & C31A & 1.3900 \\
C31A & C32A & 1.3900 \\
C32A & C33A & 1.3900 \\
C33A & C34A & 1.3900 \\
& & \\
\hline
\end{tabular}

Table S17: Bond Angles in ${ }^{\circ}$ for compound $12 \mathbf{i}$.

\begin{tabular}{llll}
\hline Atom & Atom & Atom & \multicolumn{1}{|c}{ Angle ${ }^{\circ}$} \\
\hline 01 & P1 & C6 & $100.11(17)$ \\
01 & P1 & C7 & $105.9(2)$ \\
01 & P1 & B1 & $113.61(14)$ \\
01 & P1 & C1A & $100.46(16)$ \\
O1 & P1 & C7A & $106.7(2)$ \\
C6 & P1 & C7 & $103.3(3)$ \\
C6 & P1 & B1 & $114.6(2)$ \\
C7 & P1 & B1 & $117.3(2)$ \\
C1A & P1 & B1 & $115.48(19)$ \\
C7A & P1 & B1 & $111.9(2)$ \\
C7A & P1 & C1A & $107.8(3)$ \\
N1 & P2 & C23 & $105.20(11)$ \\
N1 & P2 & C35 & $107.00(11)$ \\
N1 & P2 & B2 & $111.21(12)$ \\
C23 & P2 & B2 & $115.27(14)$ \\
C35 & P2 & C23 & $104.01(12)$ \\
C35 & P2 & B2 & $113.41(14)$ \\
C13 & O1 & P1 & $118.88(15)$ \\
C1 & C6 & P1 & $118.5(3)$ \\
C1 & C6 & C2 & 120.0 \\
C2 & C6 & P1 & $121.2(3)$ \\
C6 & C1 & C5 & 120.0 \\
C1 & C5 & C4 & 120.0 \\
C3 & C4 & C5 & 120.0 \\
C4 & C3 & C2 & 120.0 \\
C3 & C2 & C6 & 120.0 \\
C20 & N1 & P2 & $119.23(16)$ \\
C22 & N1 & P2 & $119.93(17)$ \\
C22 & N1 & C20 & $115.6(2)$ \\
& & &
\end{tabular}

\begin{tabular}{llll}
\hline Atom & Atom & Atom & \multicolumn{1}{c}{ Angle ${ }^{\circ}$} \\
\hline C29 & C34 & C33 & $120.5(8)$ \\
C8 & C7 & P1 & $120.1(3)$ \\
C8 & C7 & C12 & 120.0 \\
C12 & C7 & P1 & $119.7(3)$ \\
C7 & C8 & C9 & 120.0 \\
C8 & C9 & C10 & 120.0 \\
C11 & C10 & C9 & 120.0 \\
C12 & C11 & C10 & 120.0 \\
C11 & C12 & C7 & 120.0 \\
01 & C13 & C20 & $104.35(19)$ \\
01 & C13 & C14 & $109.40(19)$ \\
C14 & C13 & C20 & $114.1(2)$ \\
N1 & C20 & C13 & $109.44(19)$ \\
N1 & C20 & C21 & $113.2(2)$ \\
C21 & C20 & C13 & $111.9(2)$ \\
C28 & C23 & P2 & $122.4(2)$ \\
C24 & C23 & P2 & $118.34(19)$ \\
C24 & C23 & C28 & $119.2(2)$ \\
C23 & C28 & C29 & $121.2(4)$ \\
C23 & C28 & C29A & $126.8(3)$ \\
C27 & C28 & C23 & $118.2(3)$ \\
C27 & C28 & C29 & $119.8(4)$ \\
C27 & C28 & C29A & $115.0(3)$ \\
C34 & C29 & C28 & $120.4(6)$ \\
C34 & C29 & C30 & $118.8(6)$ \\
C30 & C29 & C28 & $120.3(6)$ \\
C32 & C33 & C34 & $119.4(7)$ \\
C31 & C32 & C33 & $122.1(7)$ \\
C31 & C30 & C29 & $122.0(7)$ \\
& & &
\end{tabular}




\begin{tabular}{llll}
\hline Atom & Atom & Atom & Angle $/^{\circ}$ \\
\hline C32 & C31 & C30 & $116.8(7)$ \\
C25 & C24 & C23 & $121.1(3)$ \\
C26 & C25 & C24 & $119.7(3)$ \\
C25 & C26 & C27 & $119.8(3)$ \\
C26 & C27 & C28 & $121.9(3)$ \\
C15 & C14 & C13 & $120.6(2)$ \\
C19 & C14 & C13 & $120.6(2)$ \\
C19 & C14 & C15 & $118.8(2)$ \\
C16 & C15 & C14 & $120.7(3)$ \\
C17 & C16 & C15 & $119.9(3)$ \\
C18 & C17 & C16 & $120.1(3)$ \\
C17 & C18 & C19 & $120.3(3)$ \\
C14 & C19 & C18 & $120.1(3)$ \\
C40 & C35 & P2 & $119.2(2)$ \\
C40 & C35 & C36 & $119.1(2)$ \\
C36 & C35 & P2 & $121.66(19)$ \\
C39 & C40 & C35 & $119.6(3)$ \\
C38 & C39 & C40 & $120.6(3)$ \\
C39 & C38 & C37 & $120.2(3)$ \\
C38 & C37 & C36 & $120.2(3)$ \\
C37 & C36 & C35 & $120.3(3)$ \\
C3A & C2A & C1A & 120.0 \\
C4A & C3A & C2A & 120.0
\end{tabular}

\begin{tabular}{llll}
\hline Atom & Atom & Atom & \multicolumn{1}{c}{ Angle $^{\circ}$} \\
\hline C3A & C4A & C5A & 120.0 \\
C6A & C5A & C4A & 120.0 \\
C5A & C6A & C1A & 120.0 \\
C2A & C1A & P1 & $120.9(3)$ \\
C6A & C1A & P1 & $119.1(3)$ \\
C6A & C1A & C2A & 120.0 \\
C8A & C7A & P1 & $121.1(3)$ \\
C8A & C7A & C12A & 120.0 \\
C12A & C7A & P1 & $118.8(3)$ \\
C9A & C8A & C7A & 120.0 \\
C10A & C9A & C8A & 120.0 \\
C9A & C10A & C11A & 120.0 \\
C12A & C11A & C10A & 120.0 \\
C11A & C12A & C7A & 120.0 \\
C30A & C29A & C28 & $122.6(3)$ \\
C30A & C29A & C34A & 120.0 \\
C34A & C29A & C28 & $117.4(3)$ \\
C31A & C30A & C29A & 120.0 \\
C30A & C31A & C32A & 120.0 \\
C33A & C32A & C31A & 120.0 \\
C32A & C33A & C34A & 120.0 \\
C33A & C34A & C29A & 120.0
\end{tabular}

Table S18: Torsion Angles in ${ }^{\circ}$ for compound 12i.

\begin{tabular}{|c|c|c|c|c|}
\hline Atom & Atom & Atom & Atom & Angle $/^{\circ}$ \\
\hline$\overline{P 1}$ & 01 & C13 & $\mathrm{C} 20$ & $-143.51(17)$ \\
\hline $\mathrm{P} 1$ & 01 & $\mathrm{C} 13$ & C14 & $94.0(2)$ \\
\hline P1 & $\mathrm{C} 6$ & $\mathrm{C} 1$ & $\mathrm{C} 5$ & $-173.7(5)$ \\
\hline P1 & $\mathrm{C} 6$ & $\mathrm{C} 2$ & $\mathrm{C} 3$ & $173.5(5)$ \\
\hline P1 & $\mathrm{C} 7$ & C8 & C9 & $174.9(4)$ \\
\hline P1 & $\mathrm{C} 7$ & $\mathrm{C} 12$ & C11 & $-174.9(4)$ \\
\hline P1 & C7A & C8A & C9A & $176.5(5)$ \\
\hline P1 & C7A & $\mathrm{C} 12 \mathrm{~A}$ & $\mathrm{C} 11 \mathrm{~A}$ & $-176.5(4)$ \\
\hline P2 & N1 & $\mathrm{C} 20$ & C13 & $143.85(18)$ \\
\hline P2 & N1 & $\mathrm{C} 20$ & $\mathrm{C} 21$ & $-90.5(3)$ \\
\hline $\mathrm{P} 2$ & $\mathrm{C} 23$ & $\mathrm{C} 28$ & $\mathrm{C} 29$ & $-11.1(5)$ \\
\hline P2 & $\mathrm{C} 23$ & $\mathrm{C} 28$ & $\mathrm{C} 27$ & $178.6(2)$ \\
\hline $\mathrm{P} 2$ & $\mathrm{C} 23$ & $\mathrm{C} 28$ & C29A & $0.7(4)$ \\
\hline P2 & $\mathrm{C} 23$ & $\mathrm{C} 24$ & $\mathrm{C} 25$ & $-179.0(2)$ \\
\hline P2 & C35 & $\mathrm{C} 40$ & C39 & $176.2(2)$ \\
\hline P2 & C35 & $\mathrm{C} 36$ & $\mathrm{C} 37$ & $-175.1(2)$ \\
\hline 01 & $\mathrm{P} 1$ & $\mathrm{C} 6$ & $\mathrm{C} 1$ & $-169.6(3)$ \\
\hline 01 & $\mathrm{P} 1$ & $\mathrm{C} 6$ & $\mathrm{C} 2$ & 16.8(3) \\
\hline 01 & $\mathrm{P} 1$ & $\mathrm{C} 7$ & $\mathrm{C} 8$ & $-64.7(3)$ \\
\hline 01 & $\mathrm{P} 1$ & $\mathrm{C} 7$ & $\mathrm{C} 12$ & $110.2(3)$ \\
\hline 01 & $\mathrm{P} 1$ & $\mathrm{C} 1 \mathrm{~A}$ & $\mathrm{C} 2 \mathrm{~A}$ & $-7.1(3)$ \\
\hline 01 & $\mathrm{P} 1$ & $\mathrm{C} 1 \mathrm{~A}$ & C6A & $172.2(3)$ \\
\hline 01 & $\mathrm{P} 1$ & C7A & C8A & $-68.1(3)$ \\
\hline 01 & P1 & C7A & $\mathrm{C} 12 \mathrm{~A}$ & 108.4(3) \\
\hline 01 & C13 & $\mathrm{C} 20$ & N1 & $-168.84(19)$ \\
\hline 01 & C13 & $\mathrm{C} 20$ & $\mathrm{C} 21$ & $64.8(3)$ \\
\hline 01 & $\mathrm{C} 13$ & $\mathrm{C} 14$ & C15 & $61.8(3)$ \\
\hline
\end{tabular}




\begin{tabular}{|c|c|c|c|c|}
\hline Atom & Atom & Atom & Atom & Angle $/^{\circ}$ \\
\hline 01 & $\mathrm{C} 13$ & C14 & C19 & $-116.6(2)$ \\
\hline C6 & $\mathrm{P} 1$ & 01 & $\mathrm{C} 13$ & $177.1(2)$ \\
\hline C6 & $\mathrm{P} 1$ & $\mathrm{C} 7$ & C8 & $40.1(4)$ \\
\hline C6 & $\mathrm{P} 1$ & $\mathrm{C} 7$ & C12 & $-145.0(3)$ \\
\hline C6 & $\mathrm{C} 1$ & $\mathrm{C} 5$ & $\mathrm{C} 4$ & 0.0 \\
\hline C1 & $\mathrm{C} 6$ & $\mathrm{C} 2$ & $\mathrm{C} 3$ & 0.0 \\
\hline C1 & $\mathrm{C} 5$ & $\mathrm{C} 4$ & $\mathrm{C} 3$ & 0.0 \\
\hline C5 & $\mathrm{C} 4$ & $\mathrm{C} 3$ & $\mathrm{C} 2$ & 0.0 \\
\hline $\mathrm{C} 4$ & $\mathrm{C} 3$ & $\mathrm{C} 2$ & $\mathrm{C} 6$ & 0.0 \\
\hline $\mathrm{C} 2$ & $\mathrm{C} 6$ & $\mathrm{C} 1$ & $\mathrm{C} 5$ & 0.0 \\
\hline N1 & P2 & $\mathrm{C} 23$ & $\mathrm{C} 28$ & $-169.4(2)$ \\
\hline N1 & $\mathrm{P} 2$ & $\mathrm{C} 23$ & $\mathrm{C} 24$ & $12.8(2)$ \\
\hline N1 & $\mathrm{P} 2$ & C35 & $\mathrm{C} 40$ & $-102.5(2)$ \\
\hline N1 & $\mathrm{P} 2$ & C35 & C36 & $73.9(2)$ \\
\hline C34 & $\mathrm{C} 29$ & C30 & C31 & $4.5(10)$ \\
\hline C34 & C33 & C32 & C31 & $3.5(11)$ \\
\hline C7 & $\mathrm{P} 1$ & 01 & C13 & $-75.8(2)$ \\
\hline C7 & $\mathrm{P} 1$ & $\mathrm{C} 6$ & $\mathrm{C} 1$ & $81.2(4)$ \\
\hline C7 & $\mathrm{P} 1$ & $\mathrm{C} 6$ & $\mathrm{C} 2$ & $-92.4(4)$ \\
\hline C7 & C8 & C9 & C10 & 0.0 \\
\hline C8 & $\mathrm{C} 7$ & C12 & C11 & 0.0 \\
\hline C8 & C9 & C10 & C11 & 0.0 \\
\hline C9 & C10 & C11 & $\mathrm{C} 12$ & 0.0 \\
\hline C10 & C11 & $\mathrm{C} 12$ & C7 & 0.0 \\
\hline C12 & $\mathrm{C} 7$ & C8 & C9 & 0.0 \\
\hline C13 & C14 & C15 & C16 & $-178.0(2)$ \\
\hline C13 & C14 & C19 & C18 & $178.4(2)$ \\
\hline $\mathrm{C} 20$ & C13 & C14 & C15 & $-54.7(3)$ \\
\hline C20 & $\mathrm{C} 13$ & C14 & C19 & $127.0(3)$ \\
\hline C23 & $\mathrm{P} 2$ & N1 & $\mathrm{C} 20$ & $-139.09(19)$ \\
\hline C23 & $\mathrm{P} 2$ & N1 & $\mathrm{C} 22$ & $67.5(2)$ \\
\hline C23 & $\mathrm{P} 2$ & C35 & $\mathrm{C} 40$ & $146.5(2)$ \\
\hline C23 & $\mathrm{P} 2$ & C35 & C36 & $-37.1(2)$ \\
\hline C23 & $\mathrm{C} 28$ & $\mathrm{C} 29$ & C34 & $-89.7(6)$ \\
\hline C23 & $\mathrm{C} 28$ & $\mathrm{C} 29$ & C30 & $99.0(7)$ \\
\hline C23 & $\mathrm{C} 28$ & $\mathrm{C} 27$ & C26 & $1.3(5)$ \\
\hline C23 & $\mathrm{C} 28$ & C29A & $\mathrm{C} 30 \mathrm{~A}$ & $99.5(4)$ \\
\hline C23 & $\mathrm{C} 28$ & C29A & $\mathrm{C} 34 \mathrm{~A}$ & $-80.5(4)$ \\
\hline C23 & $\mathrm{C} 24$ & $\mathrm{C} 25$ & $\mathrm{C} 26$ & $-0.1(4)$ \\
\hline C28 & $\mathrm{C} 23$ & $\mathrm{C} 24$ & $\mathrm{C} 25$ & $3.1(4)$ \\
\hline C28 & C29 & C30 & C31 & $175.9(6)$ \\
\hline C28 & $\mathrm{C} 29 \mathrm{~A}$ & $\mathrm{C} 30 \mathrm{~A}$ & $\mathrm{C} 31 \mathrm{~A}$ & $-180.0(5)$ \\
\hline C28 & $\mathrm{C} 29 \mathrm{~A}$ & $\mathrm{C} 34 \mathrm{~A}$ & $\mathrm{C} 33 \mathrm{~A}$ & $180.0(5)$ \\
\hline C29 & C34 & C33 & C32 & 2.8(10) \\
\hline C29 & $\mathrm{C} 28$ & $\mathrm{C} 27$ & $\mathrm{C} 26$ & $-169.1(5)$ \\
\hline C29 & C30 & C31 & C32 & $1.4(10)$ \\
\hline C33 & C34 & $\mathrm{C} 29$ & C28 & $-178.0(6)$ \\
\hline C33 & C34 & $\mathrm{C} 29$ & C30 & $-6.6(10)$ \\
\hline C33 & C32 & C31 & C30 & $-5.4(10)$ \\
\hline C24 & $\mathrm{C} 23$ & $\mathrm{C} 28$ & $\mathrm{C} 29$ & $166.6(4)$ \\
\hline C24 & $\mathrm{C} 23$ & $\mathrm{C} 28$ & $\mathrm{C} 27$ & $-3.6(4)$ \\
\hline C24 & $\mathrm{C} 23$ & $\mathrm{C} 28$ & C29A & $178.5(3)$ \\
\hline C24 & $\mathrm{C} 25$ & $\mathrm{C} 26$ & C27 & $-2.2(5)$ \\
\hline $\mathrm{C} 25$ & $\mathrm{C} 26$ & $\mathrm{C} 27$ & $\mathrm{C} 28$ & $1.7(5)$ \\
\hline
\end{tabular}




\begin{tabular}{|c|c|c|c|c|}
\hline Atom & Atom & Atom & Atom & Angle $/^{\circ}$ \\
\hline $\mathrm{C} 27$ & $\mathrm{C} 28$ & $\mathrm{C} 29$ & C34 & $80.4(7)$ \\
\hline C27 & $\mathrm{C} 28$ & $\mathrm{C} 29$ & C30 & $-90.9(6)$ \\
\hline C27 & $\mathrm{C} 28$ & C29A & $\mathrm{C} 30 \mathrm{~A}$ & $-78.5(4)$ \\
\hline C27 & $\mathrm{C} 28$ & C29A & C34A & $101.5(4)$ \\
\hline C14 & C13 & $\mathrm{C} 20$ & N1 & $-49.5(3)$ \\
\hline C14 & C13 & $\mathrm{C} 20$ & $\mathrm{C} 21$ & $-175.8(2)$ \\
\hline C14 & C15 & C16 & C17 & $-0.9(4)$ \\
\hline C15 & C14 & C19 & C18 & $0.0(4)$ \\
\hline C15 & C16 & C17 & C18 & $0.9(5)$ \\
\hline C16 & $\mathrm{C} 17$ & C18 & C19 & $-0.5(5)$ \\
\hline C17 & C18 & C19 & C14 & $0.1(4)$ \\
\hline C19 & C14 & C15 & C16 & $0.4(4)$ \\
\hline C22 & N1 & $\mathrm{C} 20$ & C13 & $-61.6(3)$ \\
\hline C22 & N1 & $\mathrm{C} 20$ & $\mathrm{C} 21$ & $64.0(3)$ \\
\hline C35 & $\mathrm{P} 2$ & N1 & $\mathrm{C} 20$ & $110.70(19)$ \\
\hline C35 & $\mathrm{P} 2$ & N1 & $\mathrm{C} 22$ & $-42.7(2)$ \\
\hline C35 & $\mathrm{P} 2$ & $\mathrm{C} 23$ & $\mathrm{C} 28$ & $-57.1(2)$ \\
\hline C35 & P2 & $\mathrm{C} 23$ & $\mathrm{C} 24$ & $125.1(2)$ \\
\hline C35 & $\mathrm{C} 40$ & C39 & C38 & $-0.7(5)$ \\
\hline C40 & C35 & C36 & C37 & $1.3(4)$ \\
\hline C40 & C39 & C38 & C37 & $0.8(5)$ \\
\hline C39 & C38 & $\mathrm{C} 37$ & C36 & $0.2(5)$ \\
\hline C38 & C37 & C36 & C35 & $-1.2(4)$ \\
\hline C36 & C35 & $\mathrm{C} 40$ & C39 & $-0.3(4)$ \\
\hline B1 & $\mathrm{P} 1$ & 01 & C13 & $54.5(2)$ \\
\hline B1 & $\mathrm{P} 1$ & $\mathrm{C} 6$ & $\mathrm{C} 1$ & $-47.6(4)$ \\
\hline B1 & $\mathrm{P} 1$ & C6 & $\mathrm{C} 2$ & $138.7(3)$ \\
\hline B1 & $\mathrm{P} 1$ & $\mathrm{C} 7$ & $\mathrm{C} 8$ & $167.3(3)$ \\
\hline B1 & $\mathrm{P} 1$ & $\mathrm{C} 7$ & C12 & $-17.8(4)$ \\
\hline B1 & $\mathrm{P} 1$ & $\mathrm{C} 1 \mathrm{~A}$ & $\mathrm{C} 2 \mathrm{~A}$ & $115.6(3)$ \\
\hline B1 & $\mathrm{P} 1$ & $\mathrm{C} 1 \mathrm{~A}$ & C6A & $-65.2(3)$ \\
\hline B1 & P1 & C7A & C8A & $167.1(3)$ \\
\hline B1 & $\mathrm{P} 1$ & C7A & $\mathrm{C} 12 \mathrm{~A}$ & $-16.4(4)$ \\
\hline B2 & $\mathrm{P} 2$ & N1 & $\mathrm{C} 20$ & $-13.6(2)$ \\
\hline B2 & $\mathrm{P} 2$ & N1 & $\mathrm{C} 22$ & $-167.1(2)$ \\
\hline B2 & $\mathrm{P} 2$ & $\mathrm{C} 23$ & C28 & $67.7(3)$ \\
\hline B2 & $\mathrm{P} 2$ & $\mathrm{C} 23$ & $\mathrm{C} 24$ & $-110.1(2)$ \\
\hline B2 & P2 & C35 & $\mathrm{C} 40$ & $20.5(3)$ \\
\hline B2 & $\mathrm{P} 2$ & C35 & C36 & $-163.1(2)$ \\
\hline $\mathrm{C} 2 \mathrm{~A}$ & C3A & $\mathrm{C} 4 \mathrm{~A}$ & C5A & 0.0 \\
\hline C3A & $\mathrm{C} 2 \mathrm{~A}$ & C1A & P1 & $179.3(4)$ \\
\hline C3A & $\mathrm{C} 2 \mathrm{~A}$ & $\mathrm{C} 1 \mathrm{~A}$ & C6A & 0.0 \\
\hline C3A & $\mathrm{C} 4 \mathrm{~A}$ & C5A & C6A & 0.0 \\
\hline C4A & $\mathrm{C} 5 \mathrm{~A}$ & C6A & $\mathrm{C} 1 \mathrm{~A}$ & 0.0 \\
\hline C5A & C6A & $\mathrm{C} 1 \mathrm{~A}$ & P1 & $-179.3(4)$ \\
\hline C5A & C6A & $\mathrm{C} 1 \mathrm{~A}$ & $\mathrm{C} 2 \mathrm{~A}$ & 0.0 \\
\hline C1A & P1 & 01 & C13 & $178.4(2)$ \\
\hline C1A & $\mathrm{P} 1$ & C7A & C8A & $39.1(4)$ \\
\hline C1A & P1 & C7A & $\mathrm{C} 12 \mathrm{~A}$ & $-144.4(3)$ \\
\hline C1A & $\mathrm{C} 2 \mathrm{~A}$ & $\mathrm{C} 3 \mathrm{~A}$ & $\mathrm{C} 4 \mathrm{~A}$ & 0.0 \\
\hline C7A & P1 & 01 & C13 & $-69.3(3)$ \\
\hline C7A & P1 & $\mathrm{C} 1 \mathrm{~A}$ & $\mathrm{C} 2 \mathrm{~A}$ & $-118.6(3)$ \\
\hline C7A & P1 & $\mathrm{C} 1 \mathrm{~A}$ & C6A & $60.7(4)$ \\
\hline C7A & C8A & $\mathrm{C} 9 \mathrm{~A}$ & C10A & 0.0 \\
\hline
\end{tabular}




\begin{tabular}{llllc}
\hline Atom & Atom & Atom & Atom & Angle $^{\circ}$ \\
\hline C8A & C7A & C12A & C11A & 0.0 \\
C8A & C9A & C10A & C11A & 0.0 \\
C9A & C10A & C11A & C12A & 0.0 \\
C10A & C11A & C12A & C7A & 0.0 \\
C12A & C7A & C8A & C9A & 0.0 \\
C29A & C28 & C27 & C26 & $179.5(3)$ \\
C29A & C30A & C31A & C32A & 0.0 \\
C30A & C29A & C34A & C33A & 0.0 \\
C30A & C31A & C32A & C33A & 0.0 \\
C31A & C32A & C33A & C34A & 0.0 \\
C32A & C33A & C34A & C29A & 0.0 \\
C34A & C29A & C30A & C31A & 0.0
\end{tabular}

Table S19: Atomic Occupancies for all atoms that are not fully occupied in compound 12i.

\begin{tabular}{|c|c|c|c|c|c|c|c|}
\hline & & & \\
\hline Atom & Occupancy & Atom & Occupancy & Atom & Occupancy & Atom & Occupancy \\
\hline$\overline{\mathrm{C} 6}$ & 0.5 & H9 & 0.5 & $\overline{\mathrm{H} 2 \mathrm{AA}}$ & $\overline{0.5}$ & $\overline{\mathrm{C} 11 \mathrm{~A}}$ & 0.5 \\
\hline C1 & 0.5 & C10 & 0.5 & $\mathrm{C} 3 \mathrm{~A}$ & 0.5 & $\mathrm{H} 11 \mathrm{~A}$ & 0.5 \\
\hline H1 & 0.5 & $\mathrm{H} 10$ & 0.5 & H3A & 0.5 & $\mathrm{C} 12 \mathrm{~A}$ & 0.5 \\
\hline $\mathrm{C} 5$ & 0.5 & C11 & 0.5 & $\mathrm{C} 4 \mathrm{~A}$ & 0.5 & $\mathrm{H} 12 \mathrm{~A}$ & 0.5 \\
\hline H5 & 0.5 & H11 & 0.5 & $\mathrm{H} 4 \mathrm{~A}$ & 0.5 & C29A & 0.5 \\
\hline $\mathrm{C} 4$ & 0.5 & C12 & 0.5 & $\mathrm{C} 5 \mathrm{~A}$ & 0.5 & $\mathrm{C} 30 \mathrm{~A}$ & 0.5 \\
\hline $\mathrm{H} 4$ & 0.5 & $\mathrm{H} 12$ & 0.5 & $\mathrm{H} 5 \mathrm{~A}$ & 0.5 & H30A & 0.5 \\
\hline C3 & 0.5 & $\mathrm{C} 29$ & 0.5 & $\mathrm{C} 6 \mathrm{~A}$ & 0.5 & C31A & 0.5 \\
\hline H3 & 0.5 & C33 & 0.5 & H6A & 0.5 & H31A & 0.5 \\
\hline $\mathrm{C} 2$ & 0.5 & H33 & 0.5 & $\mathrm{C} 1 \mathrm{~A}$ & 0.5 & $\mathrm{C} 32 \mathrm{~A}$ & 0.5 \\
\hline $\mathrm{H} 2$ & 0.5 & $\mathrm{C} 32$ & 0.5 & $\mathrm{C} 7 \mathrm{~A}$ & 0.5 & $\mathrm{H} 32 \mathrm{~A}$ & 0.5 \\
\hline C34 & 0.5 & H32 & 0.5 & $\mathrm{C} 8 \mathrm{~A}$ & 0.5 & $\mathrm{C} 33 \mathrm{~A}$ & 0.5 \\
\hline H34 & 0.5 & $\mathrm{C} 30$ & 0.5 & $\mathrm{H} 8 \mathrm{~A}$ & 0.5 & H33A & 0.5 \\
\hline C7 & 0.5 & H30 & 0.5 & $\mathrm{C} 9 \mathrm{~A}$ & 0.5 & $\mathrm{C} 34 \mathrm{~A}$ & 0.5 \\
\hline C8 & 0.5 & C31 & 0.5 & H9A & 0.5 & H34A & 0.5 \\
\hline H8 & 0.5 & H31 & 0.5 & C10A & 0.5 & & \\
\hline C9 & 0.5 & $\mathrm{C} 2 \mathrm{~A}$ & 0.5 & $\mathrm{H} 10 \mathrm{~A}$ & 0.5 & & \\
\hline
\end{tabular}


Name: $\left(S_{p}\right)-(+)-\mathrm{N}-\left\{(1 R, 2 S)-2-\left[\left(R_{\mathrm{p}}\right)-\mathrm{o}-\right.\right.$ Anisylphenylphosphinito-borane]-1-methyl-2-phenylethyl $\}$, methylamino(o-anisylphenyl)phosphine-borane $\mathbf{1 5 e}$.

Submitted by: Sylvain Jugé

Solved by: Yoann Rousselin

Sample ID: $\quad 20180525 M D D 265$

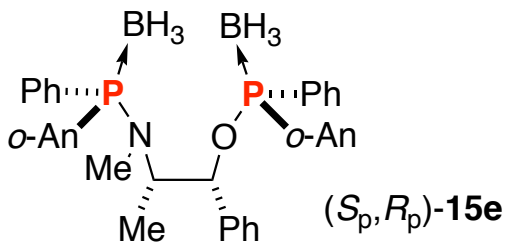

\section{Crystal Data and Experimental}

Figure S7 (thermal ellipsoïds are drawn at $50 \%$ probability plot)

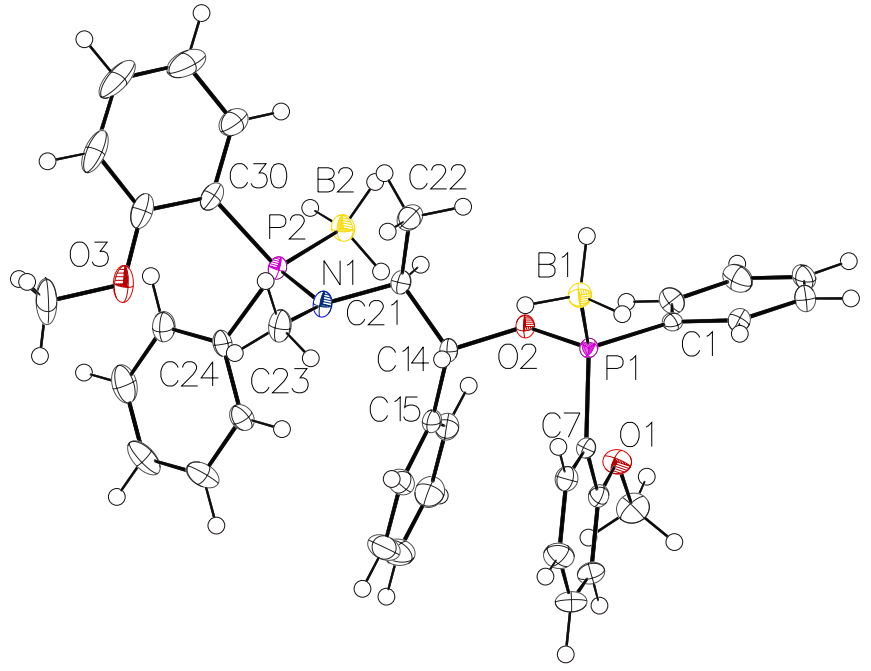

Experimental. Single clear light colorless block-shaped crystals of compound 15e were recrystallized from DCM by slow evaporation. A suitable crystal $(0.65 \times 0.55 \times 0.41) \mathrm{mm}^{3}$ was selected and mounted on a MITIGEN holder oil on a Bruker D8 VENTURE diffractometer. The crystal was kept at $T=100(1) \mathrm{K}$ during data collection. Using Olex2 (Dolomanov et al., 2009), the structure was solved with the ShelXT (Sheldrick, 2015) structure solution program, using the Intrinsic Phasing solution method. The model was refined with version 2018/3 of ShelXL (Sheldrick, 2015) using Least Squares minimization.

Crystal Data. $\mathrm{C}_{36} \mathrm{H}_{43} \mathrm{~B}_{2} \mathrm{NO}_{3} \mathrm{P}_{2}, M_{r}=621.27$, orthorhombic, $\mathrm{P} 2{ }_{1} 2_{1} 2_{1}$ (No. 19), $\mathrm{a}=9.0680(7) \AA, \mathrm{b}=13.4673(10) \AA, \mathrm{c}=$ 29.121(2) ̊, $\alpha=\beta=\gamma=90^{\circ}, \quad V=3556.3(5) \AA^{3}, \quad T=$ $100(1) \mathrm{K}, \quad Z=4, \quad Z^{\prime}=1, \mu\left(\mathrm{MoK}_{\alpha}\right)=0.156,134942$ reflections measured, 8184 unique $\left(R_{\text {int }}=0.0324\right)$ which were used in all calculations. The final $w R_{2}$ was 0.0707 (all data) and $R_{1}$ was 0.0268 (I > 2(I)). 


\begin{tabular}{|c|c|c|c|}
\hline Compound & $15 e$ & Wavelength/Å & 0.710760 \\
\hline CCDC & 1982664 & Radiation type & $\mathrm{MoK}_{\alpha}$ \\
\hline Formula & $\mathrm{C}_{36} \mathrm{H}_{43} \mathrm{~B}_{2} \mathrm{NO}_{3} \mathrm{P}_{2}$ & $\Theta_{\min } /^{\circ}$ & 2.797 \\
\hline$D_{\text {calc. }} / \mathrm{g} \mathrm{cm}^{-3}$ & 1.160 & $\Theta_{\max } /^{\circ}$ & 27.527 \\
\hline$\mu / \mathrm{mm}^{-1}$ & 0.156 & Measured Refl. & 134942 \\
\hline Formula Weight & 621.27 & Independent Refl. & 8184 \\
\hline Color & clear light colorless & Reflections Used & 7975 \\
\hline Shape & block & $R_{\text {int }}$ & 0.0324 \\
\hline $\mathrm{Size} / \mathrm{mm}^{3}$ & $0.65 \times 0.55 \times 0.41$ & Parameters & 405 \\
\hline$T / \mathrm{K}$ & $100(1)$ & Restraints & 0 \\
\hline Crystal System & orthorhombic & Largest Peak & 0.319 \\
\hline Flack Parameter & $0.004(9)$ & Deepest Hole & -0.197 \\
\hline Hooft Parameter & $0.017(9)$ & GooF & 1.075 \\
\hline Space Group & $\mathrm{P} 2{ }_{1} 2{ }_{1} 2_{1}$ & $w R_{2}$ (all data) & 0.0707 \\
\hline$a / \AA ̊$ & $9.0680(7)$ & $w R_{2}$ & 0.0700 \\
\hline$b / \AA$ & $13.4673(10)$ & $R_{1}$ (all data) & 0.0278 \\
\hline$c / \AA$ & $29.121(2)$ & $R_{1}$ & 0.0268 \\
\hline$\alpha /^{\circ}$ & 90 & & \\
\hline$\beta /^{\circ}$ & 90 & & \\
\hline$\gamma /^{\circ}$ & 90 & & \\
\hline $\mathrm{V} / \AA^{3}$ & $3556.3(5)$ & & \\
\hline$Z$ & 4 & & \\
\hline$Z^{\prime}$ & 1 & & \\
\hline
\end{tabular}

\section{Structure Quality Indicators}

Reflections:

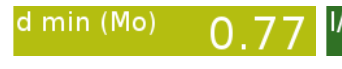

$87.0^{\text {R }}$

Rint

$3.24 \%$

complete $\quad 100 \%$

Refinement:

A clear light colorless block-shaped crystal with dimensions $0.65 \times 0.55 \times 0.41 \mathrm{~mm}^{3}$ was mounted on a MITIGEN holder oil. X-ray diffraction data were collected using a Bruker D8 VENTURE diffractometer equipped with an Oxford Cryosystems low-temperature device, operating at $T=100(1) \mathrm{K}$. Data were measured using $\phi$ and $\omega$ scans of $0.30^{\circ}$ per frame for $60 \mathrm{~s}$ using $\mathrm{MoK}_{\alpha}$ radiation (X-ray tube, $50 \mathrm{kV}, 30$ $\mathrm{mA}$ ). The total number of runs and images was based on the strategy calculation from the program APEX3 (Bruker, 2015). The maximum resolution achieved was $\Theta=27.527^{\circ}$. Cell parameters were retrieved using the SAINT (Bruker, V8.38A, after 2013) software and refined using SAINT (Bruker, V8.38A, after 2013) on 9396 reflections, 7 \% of the observed reflections. Data reduction was performed using the SAINT (Bruker, V8.38A, after 2013) software which corrects for Lorentz polarization. The final completeness is $99.70 \%$ out to $27.527^{\circ}$ in $\Theta$. A multi-scan absorption correction was performed using SADABS-2016/2 (Bruker, 2016) was used for absorption correction. $w R_{2}$ (int) was 0.0604 before and 0.0517 after correction. The Ratio of minimum to maximum transmission is 0.9415 . The absorption coefficient $\mu$ of this material is $0.156 \mathrm{~mm}^{-1}$ at this wavelength $(\lambda=0.71073 \AA)$ and the minimum and maximum transmissions are 0.8738 and 0.9281 . The structure was solved in the space group $\mathrm{P} 2{ }_{1} 2_{1} 2_{1}$ (\# 19) by Intrinsic Phasing using the ShelXT (Sheldrick, 2015) structure solution program and refined by Least Squares using version 2018/3 of ShelXL (Sheldrick, 2015). All non-hydrogen atoms were refined anisotropically. Hydrogen atom on Boron atom were refined using an AFIX 138, which would allow the refinement of a common $\mathrm{B}-\mathrm{H}$ distances, the same shifts being applied to all the $\mathrm{B}-\mathrm{H}$ bonds to the same atom, but still retain tetrahedral H-B-H angles within the group. SADABS-2016/2 (Bruker,2016) was used for absorption correction. $w R_{2}$ (int) was 0.0604 before and 0.0517 after correction. The Ratio of minimum to maximum transmission is 0.9415 . There is a single molecule in the asymmetric unit, which 
is represented by the reported sum formula. In other words: $\mathrm{Z}$ is 4 and $\mathrm{Z}$ ' is 1 . The Flack parameter was refined to 0.004(9). Determination of absolute structure using Bayesian statistics on Bijvoet differences using the Olex2 results in 0.017(9). Note: The Flack parameter is used to determine chirality of the crystal studied, the value should be near 0 , a value of 1 means that the stereochemistry is wrong and the model should be inverted. A value of 0.5 means that the crystal consists of a racemic mixture of the two enantiomers.

Table S20: Bond Lengths in Å for compound 15e.

\begin{tabular}{|c|c|c|}
\hline Atom & Atom & Length/Å \\
\hline P1 & 02 & $1.6121(11)$ \\
\hline P1 & C1 & $1.8009(15)$ \\
\hline P1 & $\mathrm{C} 7$ & $1.7969(16)$ \\
\hline P1 & B1 & $1.9031(17)$ \\
\hline P2 & N1 & $1.6683(14)$ \\
\hline P2 & $\mathrm{C} 24$ & $1.8098(17)$ \\
\hline P2 & C30 & $1.8221(16)$ \\
\hline P2 & B2 & $1.9243(19)$ \\
\hline 01 & C12 & $1.356(2)$ \\
\hline 01 & C13 & $1.4319(19)$ \\
\hline 02 & C14 & $1.4580(18)$ \\
\hline 03 & C35 & $1.360(3)$ \\
\hline 03 & C36 & $1.417(2)$ \\
\hline N1 & $\mathrm{C} 21$ & $1.470(2)$ \\
\hline N1 & C23 & $1.470(2)$ \\
\hline $\mathrm{C} 1$ & $\mathrm{C} 2$ & $1.393(2)$ \\
\hline C1 & C6 & $1.391(2)$ \\
\hline $\mathrm{C} 2$ & C3 & $1.394(2)$ \\
\hline C3 & $\mathrm{C} 4$ & $1.383(3)$ \\
\hline $\mathrm{C} 4$ & C5 & $1.383(3)$ \\
\hline C5 & C6 & $1.389(2)$ \\
\hline C7 & C8 & $1.397(2)$ \\
\hline C7 & C12 & $1.407(2)$ \\
\hline $\mathrm{C} 8$ & C9 & $1.392(3)$ \\
\hline
\end{tabular}

\begin{tabular}{lll}
\hline Atom & Atom & Length/ \\
\hline C9 & C10 & $1.385(3)$ \\
C10 & C11 & $1.384(3)$ \\
C11 & C12 & $1.395(2)$ \\
C14 & C15 & $1.509(2)$ \\
C14 & C21 & $1.545(2)$ \\
C15 & C16 & $1.388(2)$ \\
C15 & C20 & $1.399(2)$ \\
C16 & C17 & $1.395(3)$ \\
C17 & C18 & $1.384(3)$ \\
C18 & C19 & $1.385(3)$ \\
C19 & C20 & $1.387(3)$ \\
C21 & C22 & $1.530(2)$ \\
C24 & C25 & $1.395(2)$ \\
C24 & C29 & $1.389(2)$ \\
C25 & C26 & $1.386(3)$ \\
C26 & C27 & $1.385(3)$ \\
C27 & C28 & $1.384(3)$ \\
C28 & C29 & $1.396(3)$ \\
C30 & C31 & $1.399(3)$ \\
C30 & C35 & $1.402(2)$ \\
C31 & C32 & $1.392(3)$ \\
C32 & C33 & $1.379(4)$ \\
C33 & C34 & $1.372(4)$ \\
C34 & C35 & $1.398(3)$
\end{tabular}

Table S21: Bond Angles in ${ }^{\circ}$ for compound $15 e$.

\begin{tabular}{llll}
\hline Atom & Atom & Atom & Angle $/^{\circ}$ \\
\hline O2 & P1 & C1 & $101.23(7)$ \\
O2 & P1 & C7 & $106.60(7)$ \\
O2 & P1 & B1 & $113.42(7)$ \\
C1 & P1 & B1 & $111.46(8)$ \\
C7 & P1 & C1 & $109.26(7)$ \\
C7 & P1 & B1 & $114.00(8)$ \\
N1 & P2 & C24 & $106.25(7)$ \\
N1 & P2 & C30 & $110.01(7)$ \\
N1 & P2 & B2 & $113.42(8)$ \\
C24 & P2 & C30 & $106.90(8)$ \\
C24 & P2 & B2 & $109.70(8)$ \\
C30 & P2 & B2 & $110.28(8)$ \\
C12 & O1 & C13 & $117.93(14)$ \\
C14 & O2 & P1 & $118.25(9)$ \\
C35 & O3 & C36 & $117.57(17)$ \\
C21 & N1 & P2 & $117.92(10)$ \\
C21 & N1 & C23 & $116.98(13)$
\end{tabular}

\begin{tabular}{lllr}
\hline Atom & Atom & Atom & Angle $^{\circ}$ \\
\hline C23 & N1 & P2 & $119.25(11)$ \\
C2 & C1 & P1 & $117.66(12)$ \\
C6 & C1 & P1 & $122.51(13)$ \\
C6 & C1 & C2 & $119.78(15)$ \\
C1 & C2 & C3 & $119.89(16)$ \\
C4 & C3 & C2 & $119.96(17)$ \\
C3 & C4 & C5 & $120.24(16)$ \\
C4 & C5 & C6 & $120.23(17)$ \\
C5 & C6 & C1 & $119.89(17)$ \\
C8 & C7 & P1 & $119.09(12)$ \\
C8 & C7 & C12 & $119.13(14)$ \\
C12 & C7 & P1 & $121.67(12)$ \\
C9 & C8 & C7 & $120.70(16)$ \\
C10 & C9 & C8 & $119.06(16)$ \\
C11 & C10 & C9 & $121.64(16)$ \\
C10 & C11 & C12 & $119.29(16)$ \\
01 & C12 & C7 & $115.65(14)$ \\
& & & S102
\end{tabular}




\begin{tabular}{llll}
\hline Atom & Atom & Atom & Angle $^{\circ}$ \\
\hline O1 & C12 & C11 & $124.17(15)$ \\
C11 & C12 & C7 & $120.18(15)$ \\
O2 & C14 & C15 & $110.04(12)$ \\
O2 & C14 & C21 & $103.85(12)$ \\
C15 & C14 & C21 & $114.28(13)$ \\
C16 & C15 & C14 & $119.70(15)$ \\
C16 & C15 & C20 & $119.26(16)$ \\
C20 & C15 & C14 & $121.03(15)$ \\
C15 & C16 & C17 & $120.57(18)$ \\
C18 & C17 & C16 & $119.76(19)$ \\
C17 & C18 & C19 & $119.96(18)$ \\
C18 & C19 & C20 & $120.53(18)$ \\
C19 & C20 & C15 & $119.90(18)$ \\
N1 & C21 & C14 & $110.89(13)$ \\
N1 & C21 & C22 & $112.89(13)$ \\
C22 & C21 & C14 & $110.96(13)$ \\
C25 & C24 & P2 & $119.50(14)$
\end{tabular}

\begin{tabular}{llll}
\hline Atom & Atom & Atom & \multicolumn{1}{c}{ Angle $^{\circ}$} \\
\hline C29 & C24 & P2 & $120.67(12)$ \\
C29 & C24 & C25 & $119.60(16)$ \\
C26 & C25 & C24 & $120.13(19)$ \\
C27 & C26 & C25 & $120.07(18)$ \\
C28 & C27 & C26 & $120.27(19)$ \\
C27 & C28 & C29 & $119.9(2)$ \\
C24 & C29 & C28 & $120.06(17)$ \\
C31 & C30 & P2 & $116.49(14)$ \\
C31 & C30 & C35 & $118.68(16)$ \\
C35 & C30 & P2 & $124.82(14)$ \\
C32 & C31 & C30 & $120.9(2)$ \\
C33 & C32 & C31 & $119.3(2)$ \\
C34 & C33 & C32 & $121.05(19)$ \\
C33 & C34 & C35 & $120.2(2)$ \\
03 & C35 & C30 & $117.11(16)$ \\
03 & C35 & C34 & $123.09(18)$ \\
C34 & C35 & C30 & $119.80(19)$
\end{tabular}

Table S22: Torsion Angles in ${ }^{\circ}$ for compound $15 e$.

\begin{tabular}{|c|c|c|c|c|}
\hline Atom & Atom & Atom & Atom & Angle $/^{\circ}$ \\
\hline $\mathrm{P} 1$ & 02 & C14 & C15 & $-97.87(13)$ \\
\hline P1 & 02 & C14 & $\mathrm{C} 21$ & $139.40(10)$ \\
\hline P1 & $\mathrm{C} 1$ & $\mathrm{C} 2$ & $\mathrm{C} 3$ & $-178.42(13)$ \\
\hline P1 & $\mathrm{C} 1$ & $\mathrm{C} 6$ & $\mathrm{C} 5$ & $177.40(13)$ \\
\hline P1 & $\mathrm{C} 7$ & C8 & C9 & $175.60(13)$ \\
\hline P1 & $\mathrm{C} 7$ & C12 & 01 & $3.9(2)$ \\
\hline P1 & $\mathrm{C} 7$ & C12 & C11 & $-175.56(13)$ \\
\hline P2 & N1 & $\mathrm{C} 21$ & $\mathrm{C} 14$ & $-138.84(11)$ \\
\hline P2 & N1 & $\mathrm{C} 21$ & $\mathrm{C} 22$ & $95.93(15)$ \\
\hline P2 & $\mathrm{C} 24$ & $\mathrm{C} 25$ & $\mathrm{C} 26$ & $175.67(15)$ \\
\hline P2 & $\mathrm{C} 24$ & $\mathrm{C} 29$ & $\mathrm{C} 28$ & $-174.79(14)$ \\
\hline P2 & C30 & C31 & C32 & $177.85(16)$ \\
\hline P2 & C30 & C35 & 03 & $2.5(2)$ \\
\hline P2 & C30 & C35 & $\mathrm{C} 34$ & $-177.54(13)$ \\
\hline 02 & $\mathrm{P} 1$ & $\mathrm{C} 1$ & $\mathrm{C} 2$ & $168.80(12)$ \\
\hline 02 & $\mathrm{P} 1$ & $\mathrm{C} 1$ & $\mathrm{C} 6$ & $-8.69(15)$ \\
\hline 02 & $\mathrm{P} 1$ & $\mathrm{C} 7$ & C8 & $-116.15(13)$ \\
\hline 02 & $\mathrm{P} 1$ & $\mathrm{C} 7$ & $\mathrm{C} 12$ & $59.82(14)$ \\
\hline 02 & C14 & C15 & C16 & $129.36(15)$ \\
\hline 02 & $\mathrm{C} 14$ & C15 & $\mathrm{C} 20$ & $-50.72(19)$ \\
\hline 02 & C14 & $\mathrm{C} 21$ & N1 & $162.28(12)$ \\
\hline 02 & C14 & $\mathrm{C} 21$ & $\mathrm{C} 22$ & $-71.41(16)$ \\
\hline N1 & $\mathrm{P} 2$ & $\mathrm{C} 24$ & $\mathrm{C} 25$ & $162.81(13)$ \\
\hline $\mathrm{N} 1$ & $\mathrm{P} 2$ & $\mathrm{C} 24$ & $\mathrm{C} 29$ & $-22.72(15)$ \\
\hline N1 & $\mathrm{P} 2$ & C30 & C31 & $103.07(14)$ \\
\hline N1 & $\mathrm{P} 2$ & C30 & C35 & $-78.10(15)$ \\
\hline C1 & P1 & 02 & $\mathrm{C} 14$ & $170.44(11)$ \\
\hline C1 & $\mathrm{P} 1$ & $\mathrm{C} 7$ & C8 & $135.23(13)$ \\
\hline C1 & $\mathrm{P} 1$ & $\mathrm{C} 7$ & $\mathrm{C} 12$ & $-48.80(15)$ \\
\hline C1 & $\mathrm{C} 2$ & $\mathrm{C} 3$ & $\mathrm{C} 4$ & $1.5(3)$ \\
\hline C2 & $\mathrm{C} 1$ & $\mathrm{C} 6$ & $\mathrm{C} 5$ & $0.0(3)$ \\
\hline C2 & $\mathrm{C} 3$ & $\mathrm{C} 4$ & $\mathrm{C} 5$ & $-1.3(3)$ \\
\hline C3 & $\mathrm{C} 4$ & $\mathrm{C} 5$ & $\mathrm{C} 6$ & $0.4(3)$ \\
\hline
\end{tabular}




\begin{tabular}{|c|c|c|c|c|}
\hline Atom & Atom & Atom & Atom & Angle $/^{\circ}$ \\
\hline $\mathrm{C} 4$ & $\mathrm{C} 5$ & C6 & $\mathrm{C} 1$ & $0.3(3)$ \\
\hline C6 & $\mathrm{C} 1$ & $\mathrm{C} 2$ & $\mathrm{C} 3$ & $-0.9(2)$ \\
\hline $\mathrm{C} 7$ & $\mathrm{P} 1$ & 02 & C14 & $56.23(12)$ \\
\hline $\mathrm{C} 7$ & $\mathrm{P} 1$ & $\mathrm{C} 1$ & $\mathrm{C} 2$ & $-78.99(13)$ \\
\hline C7 & $\mathrm{P} 1$ & $\mathrm{C} 1$ & $\mathrm{C} 6$ & $103.52(14)$ \\
\hline C7 & C8 & C9 & C10 & $0.1(3)$ \\
\hline C8 & $\mathrm{C} 7$ & $\mathrm{C} 12$ & 01 & $179.85(14)$ \\
\hline C8 & $\mathrm{C} 7$ & $\mathrm{C} 12$ & C11 & $0.4(2)$ \\
\hline C8 & C9 & $\mathrm{C} 10$ & C11 & $0.3(3)$ \\
\hline C9 & $\mathrm{C} 10$ & C11 & C12 & $-0.3(3)$ \\
\hline C10 & C11 & C12 & 01 & $-179.40(16)$ \\
\hline C10 & C11 & $\mathrm{C} 12$ & $\mathrm{C} 7$ & $0.0(3)$ \\
\hline C12 & $\mathrm{C} 7$ & C8 & C9 & $-0.5(2)$ \\
\hline C13 & 01 & C12 & $\mathrm{C} 7$ & $178.04(15)$ \\
\hline C13 & 01 & C12 & C11 & $-2.5(2)$ \\
\hline C14 & C15 & C16 & C17 & 178.78(17) \\
\hline C14 & C15 & $\mathrm{C} 20$ & C19 & $-178.91(16)$ \\
\hline C15 & $\mathrm{C} 14$ & $\mathrm{C} 21$ & N1 & 42.38(18) \\
\hline C15 & C14 & $\mathrm{C} 21$ & $\mathrm{C} 22$ & $168.69(14)$ \\
\hline C15 & $\mathrm{C} 16$ & $\mathrm{C} 17$ & C18 & $0.5(3)$ \\
\hline C16 & $\mathrm{C} 15$ & $\mathrm{C} 20$ & C19 & $1.0(3)$ \\
\hline C16 & $\mathrm{C} 17$ & C18 & C19 & $0.2(3)$ \\
\hline C17 & C18 & C19 & $\mathrm{C} 20$ & $-0.4(3)$ \\
\hline C18 & C19 & $\mathrm{C} 20$ & C15 & $-0.3(3)$ \\
\hline C20 & C15 & C16 & C17 & $-1.1(3)$ \\
\hline C21 & C14 & C15 & C16 & $-114.28(17)$ \\
\hline C21 & C14 & C15 & $\mathrm{C} 20$ & 65.64(19) \\
\hline C23 & N1 & $\mathrm{C} 21$ & C14 & $68.26(18)$ \\
\hline C23 & N1 & $\mathrm{C} 21$ & $\mathrm{C} 22$ & $-56.97(19)$ \\
\hline C24 & $\mathrm{P} 2$ & N1 & $\mathrm{C} 21$ & $138.87(12)$ \\
\hline C24 & $\mathrm{P} 2$ & N1 & $\mathrm{C} 23$ & $-68.86(14)$ \\
\hline C24 & $\mathrm{P} 2$ & C30 & C31 & $-141.98(14)$ \\
\hline C24 & $\mathrm{P} 2$ & C30 & C35 & $36.85(16)$ \\
\hline C24 & $\mathrm{C} 25$ & $\mathrm{C} 26$ & $\mathrm{C} 27$ & $-1.0(3)$ \\
\hline $\mathrm{C} 25$ & $\mathrm{C} 24$ & $\mathrm{C} 29$ & $\mathrm{C} 28$ & $-0.3(2)$ \\
\hline C25 & $\mathrm{C} 26$ & $\mathrm{C} 27$ & $\mathrm{C} 28$ & $0.0(3)$ \\
\hline C26 & $\mathrm{C} 27$ & $\mathrm{C} 28$ & C29 & $0.8(3)$ \\
\hline C27 & $\mathrm{C} 28$ & $\mathrm{C} 29$ & $\mathrm{C} 24$ & $-0.6(3)$ \\
\hline C29 & $\mathrm{C} 24$ & $\mathrm{C} 25$ & C26 & 1.1(3) \\
\hline C30 & P2 & N1 & $\mathrm{C} 21$ & $-105.77(13)$ \\
\hline C30 & $\mathrm{P} 2$ & N1 & $\mathrm{C} 23$ & 46.51(15) \\
\hline C30 & $\mathrm{P} 2$ & $\mathrm{C} 24$ & $\mathrm{C} 25$ & $45.36(15)$ \\
\hline C30 & $\mathrm{P} 2$ & $\mathrm{C} 24$ & $\mathrm{C} 29$ & $-140.18(13)$ \\
\hline C30 & C31 & C32 & C33 & $-0.3(3)$ \\
\hline C31 & C30 & C35 & 03 & $-178.74(16)$ \\
\hline C31 & C30 & C35 & C34 & $1.3(2)$ \\
\hline C31 & C32 & C33 & C34 & $1.4(3)$ \\
\hline C32 & $\mathrm{C} 33$ & $\mathrm{C} 34$ & $\mathrm{C} 35$ & $-1.2(3)$ \\
\hline C33 & C34 & C35 & 03 & $179.85(18)$ \\
\hline C33 & C34 & C35 & C30 & $-0.2(3)$ \\
\hline C35 & C30 & C31 & C32 & $-1.1(3)$ \\
\hline C36 & 03 & C35 & C30 & $-157.85(19)$ \\
\hline C36 & 03 & C35 & C34 & 22.1(3) \\
\hline B1 & P1 & 02 & C14 & $-70.05(12)$ \\
\hline
\end{tabular}




\begin{tabular}{llllr}
\hline Atom & Atom & Atom & Atom & \multicolumn{1}{c}{ Angle $^{\circ}$} \\
\hline B1 & P1 & C1 & C2 & $47.91(15)$ \\
B1 & P1 & C1 & C6 & $-129.58(14)$ \\
B1 & P1 & C7 & C8 & $9.79(15)$ \\
B1 & P1 & C7 & C12 & $-174.25(13)$ \\
B2 & P2 & N1 & C21 & $18.28(15)$ \\
B2 & P2 & N1 & C23 & $170.55(13)$ \\
B2 & P2 & C24 & C25 & $-74.22(15)$ \\
B2 & P2 & C24 & C29 & $100.24(14)$ \\
B2 & P2 & C30 & C31 & $-22.77(16)$ \\
B2 & P2 & C30 & C35 & $156.06(14)$
\end{tabular}


Name: $\left(S_{\mathrm{p}}\right)-(+)-\mathrm{N}-[(1 S, 2 R)-2-(o-A n i s y l p h e n y l p h o s p h i n i t o-b o r a n e)-1-m e t h y l-2-p h e n y l e t h y l], \mathrm{N}-m e t h y l$ aminodiphenylphosphine-borane $\mathbf{2 1 a}$.

Submitted by: Antonin Jaillet

Solved by: Yoann Rousselin

Sample ID: $\quad$ 15AJ242

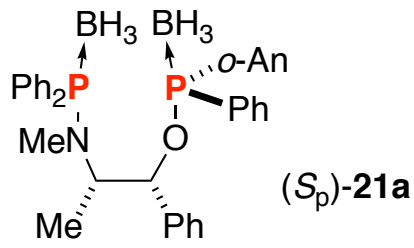

\section{Crystal Data and Experimental}

Figure S8 (thermal ellipsoïds are drawn at $50 \%$ probability plot)

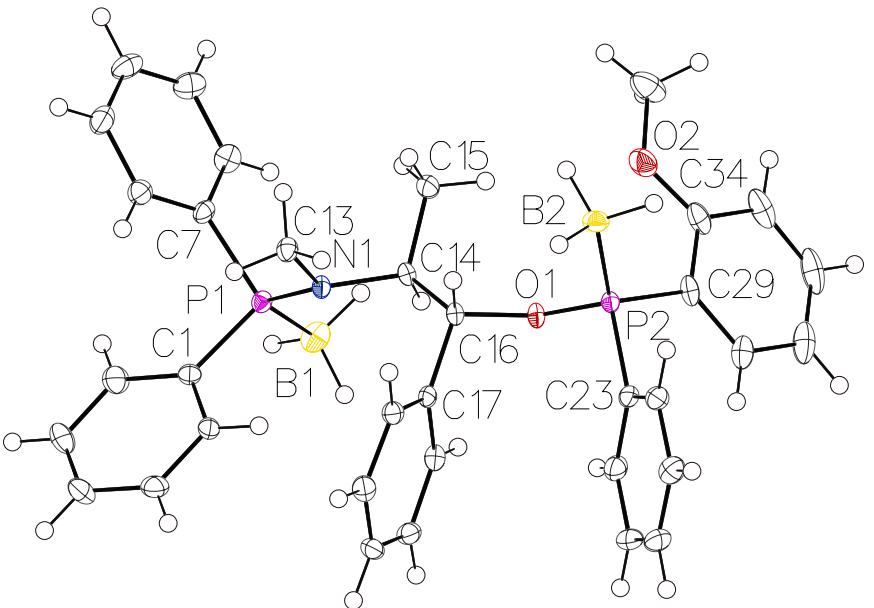

Experimental. Single clear light colorless prism-shaped crystals of compound 21a were recrystallized from DCM by slow evaporation. A suitable crystal $0.56 \times 0.32 \times 0.24$ $\mathrm{mm}^{3}$ was selected and mounted on a MITIGEN holder oil on a Bruker D8 Venture (Mo) diffractometer. The crystal was kept at a steady $T=100.0(1) \mathrm{K}$ during data collection. The structure was solved with the ShelXT (Sheldrick, 2015) structure solution program using the Intrinsic Phasing solution method and by using Olex2 (Dolomanov et al., 2009) as the graphical interface. The model was refined with version 2018/3 of ShelXL (Sheldrick, 2015) using Least Squares minimization.

Crystal Data. $\mathrm{C}_{35} \mathrm{H}_{41} \mathrm{~B}_{2} \mathrm{NO}_{2} \mathrm{P}_{2}, M_{r}=591.25$, monoclinic, $P 2_{1}$ (No. 4), $\mathrm{a}=12.7888(6) \AA, \mathrm{b}=$ 9.3942(5) $\AA, \mathrm{c}=$ 14.3603(8) $\AA, \quad \beta=109.9970(10)^{\circ}, \quad \alpha=\gamma=90^{\circ}, \quad V=$ 1621.24(15) $\AA^{3}, T=100.0(1) \mathrm{K}, Z=2, Z^{\prime}=1, \mu\left(\mathrm{MoK}_{\alpha}\right)=$ $0.166,101922$ reflections measured, 7476 unique $\left(R_{\text {int }}=\right.$ 0.0683 ) which were used in all calculations. The final $w R_{2}$ was 0.0663 (all data) and $R_{1}$ was 0.0289 (I > 2(I)). 


\begin{tabular}{|c|c|}
\hline Compound & 21a \\
\hline CCDC & 1982661 \\
\hline Formula & $\mathrm{C}_{35} \mathrm{H}_{41} \mathrm{~B}_{2} \mathrm{NO}_{2} \mathrm{P}_{2}$ \\
\hline$D_{\text {calc. }} / \mathrm{g} \mathrm{cm}^{-3}$ & 1.211 \\
\hline$\mu / \mathrm{mm}^{-1}$ & 0.166 \\
\hline Formula Weight & 591.25 \\
\hline Color & clear light colorless \\
\hline Shape & prism \\
\hline Size $/ \mathrm{mm}^{3}$ & $0.56 \times 0.32 \times 0.24$ \\
\hline$T / \mathrm{K}$ & $100.0(1)$ \\
\hline Crystal System & monoclinic \\
\hline Flack Parameter & $-0.017(19)$ \\
\hline Hooft Parameter & $0.001(17)$ \\
\hline Space Group & $P 2_{1}$ \\
\hline$a / \AA$ & $12.7888(6)$ \\
\hline$b / \AA$ & $9.3942(5)$ \\
\hline$c / \AA$ & $14.3603(8)$ \\
\hline$\alpha /^{\circ}$ & 90 \\
\hline$\beta /^{\circ}$ & $109.9970(10)$ \\
\hline$\gamma /^{\circ}$ & 90 \\
\hline $\mathrm{V} / \AA^{3}$ & $1621.24(15)$ \\
\hline$Z$ & 2 \\
\hline$Z^{\prime}$ & 1 \\
\hline Wavelength/Å & 0.71073 \\
\hline Radiation type & $\operatorname{MoK}_{\alpha}$ \\
\hline$\Theta_{\min } /^{\circ}$ & 2.913 \\
\hline$\Theta_{\max } /^{\circ}$ & 27.528 \\
\hline Measured Refl. & 101922 \\
\hline Independent Refl. & 7476 \\
\hline Reflections with I > & 6928 \\
\hline $2(\mathrm{I})$ & \\
\hline$R_{\text {int }}$ & 0.0683 \\
\hline Parameters & 386 \\
\hline Restraints & 1 \\
\hline Largest Peak & 0.323 \\
\hline Deepest Hole & -0.191 \\
\hline GooF & 1.047 \\
\hline$w R_{2}$ (all data) & 0.0663 \\
\hline$w R_{2}$ & 0.0635 \\
\hline$R_{1}$ (all data) & 0.0349 \\
\hline$R_{1}$ & 0.0289 \\
\hline
\end{tabular}


Reflections:

Refinement:

A clear light colorless prism-shaped crystal with dimensions $0.56 \times 0.32 \times 0.24 \mathrm{~mm}^{3}$ was mounted on a MITIGEN holder oil. Data were collected using a Bruker D8 Venture (Mo) diffractometer equipped with an Oxford Cryosystems low-temperature device operating at $T=100.0(1) \mathrm{K}$. Data were measured using $\phi$ and $\omega$ scans using $\mathrm{MoK}_{\alpha}$ radiation. The total number of runs and images was based on the strategy calculation from the program APEX3 (Bruker, 2015) The maximum resolution that was achieved was $\Theta$ $=27.528^{\circ}(0.77 \AA)$. The diffraction pattern was indexed. The total number of runs and images was based on the strategy calculation from the program APEX3 (Bruker, 2015) and the unit cell was refined using SAINT (Bruker, V8.34A, after 2013) on 9758 reflections, 10\% of the observed reflections. Data reduction, scaling and absorption corrections were performed using SAINT (Bruker, V8.34A, after 2013). The final completeness is $99.80 \%$ out to $27.528^{\circ}$ in $\Theta$. A multi-scan absorption correction was performed using SADABS-2014/5 (Bruker, 2014) was used for absorption correction. $w R_{2}$ (int) was 0.0768 before and 0.0630 after correction. The Ratio of minimum to maximum transmission is 0.9455 . The $\lambda / 2$ correction factor is 0.00150 . The absorption coefficient $\mu$ of this material is $0.166 \mathrm{~mm}^{-1}$ at this wavelength $(\lambda=0.711 \AA)$ and the minimum and maximum transmissions are 0.705 and 0.746 . The structure was solved and the space group $P 2_{1}$ (\# 4) determined by the ShelXT (Sheldrick, 2015) structure solution program using the Intrinsic Phasing solution method and refined by Least Squares using version 2018/3 of ShelXL (Sheldrick, 2015). All non-hydrogen atoms were refined anisotropically. Hydrogen atom positions were calculated geometrically and refined using the riding model. Hydrogen atom on Boron atom were refined using an AFIX 138, which would allow the refinement of a common B$\mathrm{H}$ distances, the same shifts being applied to all the $\mathrm{B}-\mathrm{H}$ bonds to the same atom, but still retain tetrahedral H-B-H angles within the group. There is a single molecule in the asymmetric unit, which is represented by the reported sum formula. In other words: Z is 2 and Z' is 1 . The Flack parameter was refined to -0.017(19). Determination of absolute structure using Bayesian statistics on Bijvoet differences using the Olex2 results in 0.001(17). Note: The Flack parameter is used to determine chirality of the crystal studied, the value should be near 0 , a value of 1 means that the stereochemistry is wrong and the model should be inverted. A value of 0.5 means that the crystal consists of a racemic mixture of the two enantiomers.

Table S23: Bond Lengths in Å for compound 21a.

\begin{tabular}{lll}
\hline Atom & Atom & Length/Å \\
\hline P1 & N1 & $1.6733(16)$ \\
P1 & C7 & $1.820(2)$ \\
P1 & C1 & $1.817(2)$ \\
P1 & B1 & $1.916(2)$ \\
P2 & 01 & $1.6181(14)$ \\
P2 & C23 & $1.807(2)$ \\
P2 & C29 & $1.806(2)$ \\
P2 & B2 & $1.915(2)$ \\
O1 & C16 & $1.462(2)$ \\
O2 & C34 & $1.360(3)$ \\
02 & C35 & $1.434(3)$ \\
N1 & C14 & $1.477(2)$ \\
N1 & C13 & $1.469(2)$ \\
C11 & C10 & $1.381(3)$ \\
C11 & C12 & $1.386(3)$ \\
C10 & C9 & $1.385(3)$
\end{tabular}

\begin{tabular}{lll}
\hline Atom & Atom & Length/Å \\
\hline C3 & C4 & $1.386(3)$ \\
C3 & C2 & $1.393(3)$ \\
C12 & C7 & $1.398(3)$ \\
C7 & C8 & $1.391(3)$ \\
C14 & C16 & $1.544(3)$ \\
C14 & C15 & $1.526(3)$ \\
C16 & C17 & $1.509(3)$ \\
C23 & C28 & $1.401(3)$ \\
C23 & C24 & $1.397(3)$ \\
C28 & C27 & $1.383(3)$ \\
C27 & C26 & $1.386(3)$ \\
C26 & C25 & $1.381(3)$ \\
C4 & C5 & $1.385(4)$ \\
C5 & C6 & $1.390(3)$ \\
C6 & C1 & $1.403(3)$ \\
C1 & C2 & $1.391(3)$
\end{tabular}




\begin{tabular}{lll}
\hline Atom & Atom & Length/Å \\
\hline C9 & C8 & $1.396(3)$ \\
C17 & C22 & $1.389(3)$ \\
C17 & C18 & $1.399(3)$ \\
C22 & C21 & $1.395(3)$ \\
C21 & C20 & $1.387(3)$ \\
C20 & C19 & $1.391(3)$ \\
C19 & C18 & $1.386(3)$
\end{tabular}

\begin{tabular}{lll}
\hline Atom & Atom & Length/Å \\
\hline C25 & C24 & $1.392(3)$ \\
C29 & C30 & $1.395(3)$ \\
C29 & C34 & $1.404(3)$ \\
C30 & C31 & $1.394(3)$ \\
C31 & C32 & $1.374(4)$ \\
C32 & C33 & $1.383(4)$ \\
C33 & C34 & $1.395(3)$
\end{tabular}

Table S24: Bond Angles in ${ }^{\circ}$ for compound 21 a.

\begin{tabular}{|c|c|c|c|c|c|c|c|}
\hline Atom & Atom & Atom & Angle $/^{\circ}$ & Atom & Atom & Atom & Angle $/^{\circ}$ \\
\hline N1 & P1 & $\mathrm{C} 7$ & $107.77(9)$ & $\mathrm{C} 27$ & $\mathrm{C} 28$ & $\mathrm{C} 23$ & $120.0(2)$ \\
\hline $\mathrm{N} 1$ & $\mathrm{P} 1$ & $\mathrm{C} 1$ & $105.06(9)$ & $\mathrm{C} 28$ & $\mathrm{C} 27$ & $\mathrm{C} 26$ & $120.3(2)$ \\
\hline N1 & $\mathrm{P} 1$ & B1 & $114.17(10)$ & $\mathrm{C} 25$ & $\mathrm{C} 26$ & $\mathrm{C} 27$ & $120.3(2)$ \\
\hline $\mathrm{C} 7$ & P1 & B1 & $113.63(11)$ & $\mathrm{C} 5$ & $\mathrm{C} 4$ & C3 & $120.0(2)$ \\
\hline C1 & $\mathrm{P} 1$ & $\mathrm{C} 7$ & $104.22(10)$ & $\mathrm{C} 4$ & $\mathrm{C} 5$ & C6 & $120.4(2)$ \\
\hline C1 & $\mathrm{P} 1$ & B1 & $111.19(11)$ & $\mathrm{C} 5$ & $\mathrm{C} 6$ & $\mathrm{C} 1$ & $119.9(2)$ \\
\hline 01 & $\mathrm{P} 2$ & $\mathrm{C} 23$ & 106.24(9) & $\mathrm{C} 6$ & $\mathrm{C} 1$ & $\mathrm{P} 1$ & $120.06(17)$ \\
\hline 01 & P2 & C29 & $100.49(8)$ & $\mathrm{C} 2$ & $\mathrm{C} 1$ & $\mathrm{P} 1$ & $120.62(15)$ \\
\hline 01 & P2 & B2 & 115.34(9) & $\mathrm{C} 2$ & $\mathrm{C} 1$ & C6 & 119.19(19) \\
\hline C23 & P2 & B2 & $110.34(10)$ & C10 & C9 & $\mathrm{C} 8$ & $120.0(2)$ \\
\hline C29 & $\mathrm{P} 2$ & $\mathrm{C} 23$ & $104.75(10)$ & C7 & $\mathrm{C} 8$ & C9 & $120.3(2)$ \\
\hline C29 & P2 & B2 & $118.40(10)$ & $\mathrm{C} 22$ & $\mathrm{C} 17$ & C16 & $120.21(18)$ \\
\hline C16 & 01 & P2 & $120.36(12)$ & $\mathrm{C} 22$ & C17 & C18 & 119.13(19) \\
\hline C34 & 02 & C35 & 119.35(19) & $\mathrm{C} 18$ & $\mathrm{C} 17$ & C16 & $120.65(18)$ \\
\hline C14 & N1 & P1 & $119.41(12)$ & $\mathrm{C} 17$ & $\mathrm{C} 22$ & $\mathrm{C} 21$ & $120.4(2)$ \\
\hline C13 & N1 & P1 & $117.92(13)$ & $\mathrm{C} 20$ & $\mathrm{C} 21$ & $\mathrm{C} 22$ & $120.0(2)$ \\
\hline C13 & N1 & C14 & $116.20(15)$ & $\mathrm{C} 21$ & $\mathrm{C} 20$ & C19 & $119.9(2)$ \\
\hline C10 & C11 & C12 & $120.3(2)$ & C18 & C19 & $\mathrm{C} 20$ & $120.1(2)$ \\
\hline C11 & $\mathrm{C} 10$ & C9 & $120.0(2)$ & C19 & C18 & $\mathrm{C} 17$ & $120.43(19)$ \\
\hline $\mathrm{C} 4$ & C3 & $\mathrm{C} 2$ & $120.0(2)$ & $\mathrm{C} 26$ & $\mathrm{C} 25$ & $\mathrm{C} 24$ & $119.9(2)$ \\
\hline C11 & $\mathrm{C} 12$ & $\mathrm{C} 7$ & $120.4(2)$ & $\mathrm{C} 25$ & $\mathrm{C} 24$ & $\mathrm{C} 23$ & $120.2(2)$ \\
\hline C12 & $\mathrm{C} 7$ & $\mathrm{P} 1$ & $122.07(17)$ & C30 & $\mathrm{C} 29$ & $\mathrm{P} 2$ & $121.27(18)$ \\
\hline C8 & $\mathrm{C} 7$ & P1 & $118.78(16)$ & C30 & C29 & C34 & 119.12(19) \\
\hline C8 & $\mathrm{C} 7$ & $\mathrm{C} 12$ & $119.0(2)$ & C34 & $\mathrm{C} 29$ & $\mathrm{P} 2$ & 119.61(17) \\
\hline N1 & $\mathrm{C} 14$ & C16 & $110.06(16)$ & C31 & C30 & $\mathrm{C} 29$ & $120.8(2)$ \\
\hline N1 & C14 & C15 & $112.63(17)$ & C32 & C31 & C30 & $119.0(3)$ \\
\hline C15 & $\mathrm{C} 14$ & C16 & $111.66(16)$ & C31 & $\mathrm{C} 32$ & C33 & $121.8(2)$ \\
\hline 01 & C16 & C14 & $104.29(15)$ & C32 & C33 & C34 & $119.4(2)$ \\
\hline 01 & C16 & C17 & $110.30(16)$ & 02 & C34 & $\mathrm{C} 29$ & 115.62(19) \\
\hline C17 & C16 & C14 & $113.39(16)$ & 02 & C34 & C33 & $124.4(2)$ \\
\hline C28 & $\mathrm{C} 23$ & P2 & $121.60(16)$ & C33 & C34 & $\mathrm{C} 29$ & $119.9(2)$ \\
\hline C24 & $\mathrm{C} 23$ & P2 & $119.18(16)$ & $\mathrm{C} 1$ & $\mathrm{C} 2$ & C3 & $120.4(2)$ \\
\hline C24 & $\mathrm{C} 23$ & C28 & $119.22(19)$ & & & & \\
\hline
\end{tabular}

Table S25: Torsion Angles in ${ }^{\circ}$ for compound 21a.

\begin{tabular}{llllc}
\hline Atom & Atom & Atom & Atom & Angle $^{\circ}$ \\
\hline P1 & N1 & C14 & C16 & $-143.45(15)$ \\
P1 & N1 & C14 & C15 & $91.2(2)$ \\
P1 & C7 & C8 & C9 & $-173.62(17)$ \\
P1 & C1 & C2 & C3 & $-176.74(16)$ \\
P2 & 01 & C16 & C14 & $143.63(14)$ \\
P2 & 01 & C16 & C17 & $-94.29(17)$
\end{tabular}




\begin{tabular}{|c|c|c|c|c|}
\hline Atom & Atom & Atom & Atom & Angle $/^{\circ}$ \\
\hline $\mathrm{P} 2$ & $\mathrm{C} 23$ & $\mathrm{C} 28$ & $\mathrm{C} 27$ & $-179.35(16)$ \\
\hline P2 & $\mathrm{C} 23$ & $\mathrm{C} 24$ & $\mathrm{C} 25$ & $-179.90(16)$ \\
\hline P2 & $\mathrm{C} 29$ & $\mathrm{C} 30$ & $\mathrm{C} 31$ & $-179.67(17)$ \\
\hline P2 & $\mathrm{C} 29$ & C34 & 02 & $-3.3(3)$ \\
\hline P2 & $\mathrm{C} 29$ & C34 & C33 & $178.41(17)$ \\
\hline 01 & $\mathrm{P} 2$ & $\mathrm{C} 23$ & $\mathrm{C} 28$ & $32.90(19)$ \\
\hline 01 & $\mathrm{P} 2$ & $\mathrm{C} 23$ & $\mathrm{C} 24$ & $-147.38(16)$ \\
\hline 01 & $\mathrm{P} 2$ & $\mathrm{C} 29$ & C30 & $-102.12(17)$ \\
\hline 01 & $\mathrm{P} 2$ & $\mathrm{C} 29$ & C34 & $77.84(17)$ \\
\hline 01 & C16 & $\mathrm{C} 17$ & $\mathrm{C} 22$ & $130.46(18)$ \\
\hline 01 & C16 & $\mathrm{C} 17$ & C18 & $-50.9(2)$ \\
\hline N1 & $\mathrm{P} 1$ & $\mathrm{C} 7$ & C12 & $-75.89(19)$ \\
\hline N1 & $\mathrm{P} 1$ & $\mathrm{C} 7$ & C8 & $99.67(17)$ \\
\hline N1 & $\mathrm{P} 1$ & $\mathrm{C} 1$ & $\mathrm{C} 6$ & $156.90(18)$ \\
\hline N1 & $\mathrm{P} 1$ & $\mathrm{C} 1$ & $\mathrm{C} 2$ & $-27.29(19)$ \\
\hline N1 & $\mathrm{C} 14$ & C16 & 01 & $165.33(15)$ \\
\hline $\mathrm{N} 1$ & $\mathrm{C} 14$ & C16 & C17 & $45.3(2)$ \\
\hline C11 & $\mathrm{C} 10$ & $\mathrm{C} 9$ & $\mathrm{C} 8$ & $-0.4(3)$ \\
\hline C11 & $\mathrm{C} 12$ & $\mathrm{C} 7$ & $\mathrm{P} 1$ & $174.03(17)$ \\
\hline C11 & $\mathrm{C} 12$ & $\mathrm{C} 7$ & $\mathrm{C} 8$ & $-1.5(3)$ \\
\hline C10 & $\mathrm{C} 11$ & $\mathrm{C} 12$ & $\mathrm{C} 7$ & $0.0(3)$ \\
\hline C10 & C9 & $\mathrm{C} 8$ & $\mathrm{C} 7$ & $-1.1(3)$ \\
\hline C3 & $\mathrm{C} 4$ & $\mathrm{C} 5$ & $\mathrm{C} 6$ & $-0.8(4)$ \\
\hline C12 & C11 & $\mathrm{C} 10$ & C9 & $1.0(3)$ \\
\hline C12 & $\mathrm{C} 7$ & $\mathrm{C} 8$ & $\mathrm{C} 9$ & $2.1(3)$ \\
\hline C7 & $\mathrm{P} 1$ & N1 & C14 & $-106.19(17)$ \\
\hline C7 & $\mathrm{P} 1$ & N1 & C13 & $44.55(18)$ \\
\hline C7 & $\mathrm{P} 1$ & $\mathrm{C} 1$ & $\mathrm{C} 6$ & $43.7(2)$ \\
\hline $\mathrm{C} 7$ & $\mathrm{P} 1$ & $\mathrm{C} 1$ & $\mathrm{C} 2$ & $-140.51(17)$ \\
\hline C14 & C16 & $\mathrm{C} 17$ & $\mathrm{C} 22$ & $-113.0(2)$ \\
\hline C14 & C16 & $\mathrm{C} 17$ & C18 & $65.7(2)$ \\
\hline C16 & $\mathrm{C} 17$ & $\mathrm{C} 22$ & $\mathrm{C} 21$ & $177.03(18)$ \\
\hline C16 & $\mathrm{C} 17$ & C18 & C19 & $-179.48(18)$ \\
\hline C23 & $\mathrm{P} 2$ & 01 & C16 & 81.92(16) \\
\hline C23 & $\mathrm{P} 2$ & $\mathrm{C} 29$ & C30 & 7.92(19) \\
\hline C23 & $\mathrm{P} 2$ & $\mathrm{C} 29$ & C34 & $-172.11(16)$ \\
\hline C23 & $\mathrm{C} 28$ & $\mathrm{C} 27$ & $\mathrm{C} 26$ & $-0.6(3)$ \\
\hline C28 & $\mathrm{C} 23$ & $\mathrm{C} 24$ & $\mathrm{C} 25$ & $-0.2(3)$ \\
\hline C28 & $\mathrm{C} 27$ & $\mathrm{C} 26$ & $\mathrm{C} 25$ & $-0.5(4)$ \\
\hline C27 & $\mathrm{C} 26$ & $\mathrm{C} 25$ & $\mathrm{C} 24$ & $1.2(3)$ \\
\hline C26 & $\mathrm{C} 25$ & $\mathrm{C} 24$ & $\mathrm{C} 23$ & $-0.9(3)$ \\
\hline $\mathrm{C} 4$ & $\mathrm{C} 3$ & $\mathrm{C} 2$ & $\mathrm{C} 1$ & $0.2(3)$ \\
\hline $\mathrm{C} 4$ & $\mathrm{C} 5$ & $\mathrm{C} 6$ & $\mathrm{C} 1$ & $0.1(4)$ \\
\hline C5 & $\mathrm{C} 6$ & $\mathrm{C} 1$ & $\mathrm{P} 1$ & $176.58(19)$ \\
\hline C5 & $\mathrm{C} 6$ & $\mathrm{C} 1$ & $\mathrm{C} 2$ & $0.7(3)$ \\
\hline C6 & $\mathrm{C} 1$ & $\mathrm{C} 2$ & $\mathrm{C} 3$ & $-0.9(3)$ \\
\hline $\mathrm{C} 1$ & $\mathrm{P} 1$ & N1 & C14 & $143.13(16)$ \\
\hline $\mathrm{C} 1$ & $\mathrm{P} 1$ & N1 & C13 & $-66.14(17)$ \\
\hline $\mathrm{C} 1$ & $\mathrm{P} 1$ & $\mathrm{C} 7$ & C12 & $35.4(2)$ \\
\hline C1 & $\mathrm{P} 1$ & $\mathrm{C} 7$ & C8 & $-149.07(17)$ \\
\hline C17 & $\mathrm{C} 22$ & $\mathrm{C} 21$ & $\mathrm{C} 20$ & $2.5(3)$ \\
\hline C22 & $\mathrm{C} 17$ & C18 & C19 & $-0.8(3)$ \\
\hline C22 & $\mathrm{C} 21$ & $\mathrm{C} 20$ & C19 & $-0.8(3)$ \\
\hline C21 & $\mathrm{C} 20$ & C19 & C18 & $-1.6(3)$ \\
\hline
\end{tabular}




\begin{tabular}{|c|c|c|c|c|}
\hline Atom & Atom & Atom & Atom & Angle $/^{\circ}$ \\
\hline$\overline{\mathrm{C} 20}$ & C19 & C18 & C17 & $2.5(3)$ \\
\hline C18 & $\mathrm{C} 17$ & $\mathrm{C} 22$ & $\mathrm{C} 21$ & $-1.7(3)$ \\
\hline C24 & $\mathrm{C} 23$ & $\mathrm{C} 28$ & $\mathrm{C} 27$ & $0.9(3)$ \\
\hline C29 & P2 & 01 & $\mathrm{C} 16$ & $-169.20(15)$ \\
\hline C29 & $\mathrm{P} 2$ & $\mathrm{C} 23$ & $\mathrm{C} 28$ & $-72.93(19)$ \\
\hline C29 & $\mathrm{P} 2$ & $\mathrm{C} 23$ & $\mathrm{C} 24$ & $106.78(17)$ \\
\hline C29 & C30 & C31 & C32 & $0.6(3)$ \\
\hline C30 & $\mathrm{C} 29$ & C34 & 02 & $176.69(19)$ \\
\hline C30 & $\mathrm{C} 29$ & $\mathrm{C} 34$ & C33 & $-1.6(3)$ \\
\hline C30 & C31 & $\mathrm{C} 32$ & C33 & $-0.4(4)$ \\
\hline C31 & C32 & C33 & C34 & $-0.9(4)$ \\
\hline C32 & C33 & C34 & 02 & $-176.3(2)$ \\
\hline C32 & C33 & C34 & C29 & $1.9(3)$ \\
\hline C34 & $\mathrm{C} 29$ & C30 & C31 & $0.4(3)$ \\
\hline C35 & 02 & C34 & $\mathrm{C} 29$ & $180.00(19)$ \\
\hline C35 & 02 & C34 & C33 & $-1.8(3)$ \\
\hline C15 & C14 & C16 & 01 & $-68.8(2)$ \\
\hline C15 & C14 & C16 & $\mathrm{C} 17$ & $171.17(16)$ \\
\hline C13 & N1 & C14 & $\mathrm{C} 16$ & $65.3(2)$ \\
\hline C13 & N1 & C14 & $\mathrm{C} 15$ & $-60.0(2)$ \\
\hline C2 & $\mathrm{C} 3$ & $\mathrm{C} 4$ & $\mathrm{C} 5$ & $0.6(4)$ \\
\hline B2 & $\mathrm{P} 2$ & 01 & C16 & $-40.67(18)$ \\
\hline B2 & $\mathrm{P} 2$ & $\mathrm{C} 23$ & $\mathrm{C} 28$ & $158.59(18)$ \\
\hline B2 & $\mathrm{P} 2$ & $\mathrm{C} 23$ & $\mathrm{C} 24$ & $-21.69(19)$ \\
\hline B2 & $\mathrm{P} 2$ & C29 & C30 & $131.36(17)$ \\
\hline B2 & $\mathrm{P} 2$ & C29 & C34 & $-48.7(2)$ \\
\hline B1 & $\mathrm{P} 1$ & N1 & C14 & $21.1(2)$ \\
\hline B1 & $\mathrm{P} 1$ & N1 & $\mathrm{C} 13$ & $171.79(16)$ \\
\hline B1 & $\mathrm{P} 1$ & $\mathrm{C} 7$ & $\mathrm{C} 12$ & $156.56(18)$ \\
\hline B1 & $\mathrm{P} 1$ & $\mathrm{C} 7$ & C8 & $-27.9(2)$ \\
\hline B1 & $\mathrm{P} 1$ & $\mathrm{C} 1$ & $\mathrm{C} 6$ & $-79.1(2)$ \\
\hline B1 & $\mathrm{P} 1$ & $\mathrm{C} 1$ & $\mathrm{C} 2$ & $96.70(19)$ \\
\hline
\end{tabular}


Name: $\left(S_{\mathrm{p}}\right)-(+)-\mathrm{N}-[(1 S, 2 R)-2-(\alpha-N a p h t y l p h e n y l p h o s p h i n i t o-b o r a n e)-1-m e t h y l-2-p h e n y l e t h y l]$, methylaminodiphenylphosphine-borane $\mathbf{2 1 b}$.

Submitted by: Antonin Jaillet

Solved by: Yoann Rousselin

Sample ID: $\quad 15 \mathrm{AJ} 245$

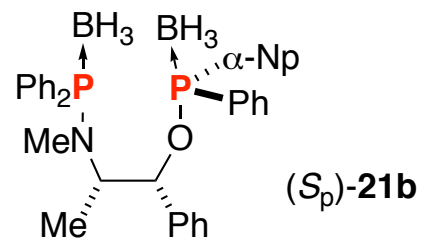

\section{Crystal Data and Experimental}

Figure S9 (thermal ellipsoïds are drawn at $50 \%$ probability plot)

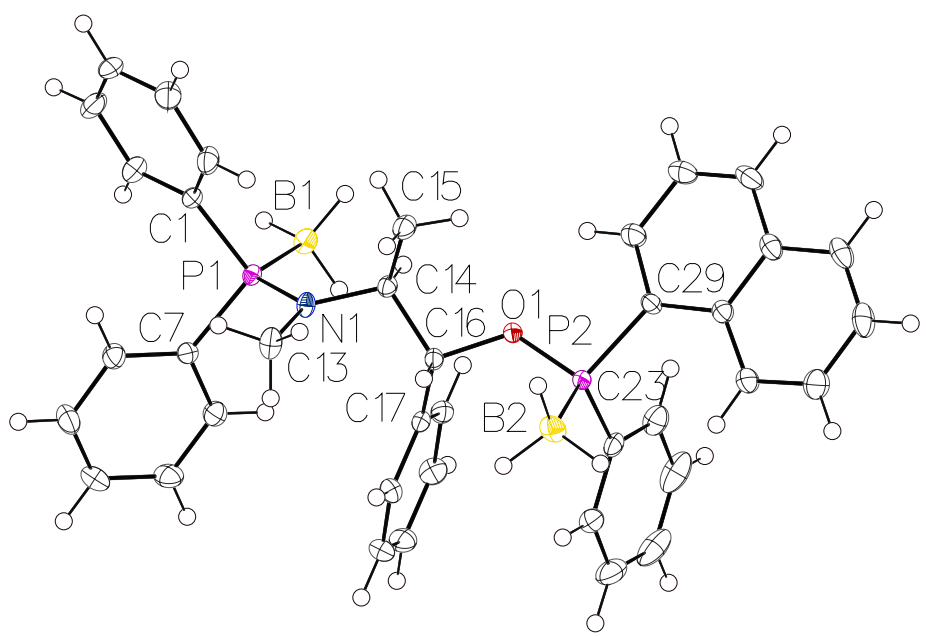

Experimental. Single clear light colorless prism-shaped crystals of compound $\mathbf{2 1 b}$ were recrystallized from DCM by slow evaporation. A suitable crystal $0.23 \times 0.22 \times 0.12$ $\mathrm{mm}^{3}$ was selected and mounted on a MITIGEN holder oil on a Bruker D8 VENTURE diffractometer. The crystal was kept at a steady $T=100.0(1) \mathrm{K}$ during data collection. The structure was solved with the ShelXT (Sheldrick, 2015) structure solution program using the direct solution method and by using 0lex2 (Dolomanov et al., 2009) as the graphical interface. The model was refined with version 2018/3 of ShelXL (Sheldrick, 2015) using Least Squares minimization.

Crystal Data. $\mathrm{C}_{38} \mathrm{H}_{41} \mathrm{~B}_{2} \mathrm{NOP}_{2}, M_{r}=611.28$, monoclinic, $P 2_{1}$ (No. 4), $\quad \mathrm{a}=7.7579(5) \AA, \quad \mathrm{b}=13.9598(8) \AA, \quad \mathrm{c}=$ 16.1051(9) $\AA, \quad \beta=98.966(2)^{\circ}, \quad \alpha=\quad \gamma=90^{\circ}, \quad V=$ 1722.85(18) $\AA^{3}, T=100.0(1) \mathrm{K}, Z=2, Z^{\prime}=1, \mu\left(\mathrm{MoK}_{\alpha}\right)=$ $0.156,87823$ reflections measured, 7906 unique $\left(R_{\text {int }}=\right.$ 0.0490 ) which were used in all calculations. The final $w R_{2}$ was 0.0667 (all data) and $R_{1}$ was 0.0267 (I > 2(I)). 


\begin{tabular}{|c|c|}
\hline Compound & $21 b$ \\
\hline CCDC & 1982662 \\
\hline Formula & $\mathrm{C}_{38} \mathrm{H}_{41} \mathrm{~B}_{2} \mathrm{NOP}_{2}$ \\
\hline$D_{\text {calc. }} / \mathrm{g} \mathrm{cm}^{-3}$ & 1.178 \\
\hline$\mu / \mathrm{mm}^{-1}$ & 0.156 \\
\hline Formula Weight & 611.28 \\
\hline Color & clear light colorless \\
\hline Shape & prism \\
\hline Size $/ \mathrm{mm}^{3}$ & $0.23 \times 0.22 \times 0.12$ \\
\hline$T / \mathrm{K}$ & $100.0(1)$ \\
\hline Crystal System & monoclinic \\
\hline Flack Parameter & $-0.036(17)$ \\
\hline Hooft Parameter & $-0.022(15)$ \\
\hline Space Group & $P 2_{1}$ \\
\hline$a / \AA$ & $7.7579(5)$ \\
\hline$b / \AA$ & $13.9598(8)$ \\
\hline$c / \AA$ & $16.1051(9)$ \\
\hline$\alpha /^{\circ}$ & 90 \\
\hline$\beta /^{\circ}$ & $98.966(2)$ \\
\hline$\gamma /{ }^{\circ}$ & 90 \\
\hline $\mathrm{V} / \AA^{3}$ & $1722.85(18)$ \\
\hline$Z$ & 2 \\
\hline$Z^{\prime}$ & 1 \\
\hline Wavelength/Å & 0.71073 \\
\hline Radiation type & $\mathrm{MoK}_{\alpha}$ \\
\hline$\Theta_{\min } /^{\circ}$ & 2.918 \\
\hline$\Theta_{\max } /^{\circ}$ & 27.524 \\
\hline Measured Refl. & 87823 \\
\hline Independent Refl. & 7906 \\
\hline Reflections with I > & 7426 \\
\hline $2(\mathrm{I})$ & \\
\hline$R_{\text {int }}$ & 0.0490 \\
\hline Parameters & 403 \\
\hline Restraints & 1 \\
\hline Largest Peak & 0.267 \\
\hline Deepest Hole & -0.173 \\
\hline GooF & 1.049 \\
\hline$w R_{2}$ (all data) & 0.0667 \\
\hline$w R_{2}$ & 0.0641 \\
\hline$R_{1}$ (all data) & 0.0310 \\
\hline$R_{1}$ & 0.0267 \\
\hline
\end{tabular}


Reflections:

A clear light colorless prism-shaped crystal with dimensions $0.23 \times 0.22 \times 0.12 \mathrm{~mm}^{3}$ was mounted on a MITIGEN holder oil. Data were collected using a Bruker D8 VENTURE diffractometer equipped with an Oxford Cryosystems low-temperature device operating at $T=100.0(1) \mathrm{K}$. Data were measured using $\phi$ and $\omega$ scans using $\operatorname{MoK}_{\alpha}$ radiation. The total number of runs and images was based on the strategy calculation from the program APEX3 (Bruker, 2015) The maximum resolution that was achieved was $\Theta$ $=27.524^{\circ}(0.77 \AA)$. The diffraction pattern was indexed. The total number of runs and images was based on the strategy calculation from the program APEX3 (Bruker, 2015) and the unit cell was refined using SAINT (Bruker, V8.34A, after 2013) on 9703 reflections, 11\% of the observed reflections. Data reduction, scaling and absorption corrections were performed using SAINT (Bruker, V8.34A, after 2013). The final completeness is $99.80 \%$ out to $27.524^{\circ}$ in $\Theta$. A multi-scan absorption correction was performed using SADABS-2014/5 (Bruker,2014) was used for absorption correction. $w R_{2}$ (int) was 0.0600 before and 0.0562 after correction. The Ratio of minimum to maximum transmission is 0.9371 . The $\lambda / 2$ correction factor is 0.00150 . The absorption coefficient $\mu$ of this material is $0.156 \mathrm{~mm}^{-1}$ at this wavelength $(\lambda=0.711 \AA)$ and the minimum and maximum transmissions are 0.699 and 0.746 . The structure was solved and the space group $P 2_{1}$ (\# 4) determined by the ShelXT (Sheldrick, 2015) structure solution program using direct and refined by Least Squares using version 2018/3 of ShelXL (Sheldrick, 2015). All non-hydrogen atoms were refined anisotropically. Hydrogen atom positions were calculated geometrically and refined using the riding model. Hydrogen atom on Boron atom were refined using an AFIX 138, which would allow the refinement of a common B-H distances, the same shifts being applied to all the B-H bonds to the same atom, but still retain tetrahedral $\mathrm{H}-\mathrm{B}-\mathrm{H}$ angles within the group. There is a single molecule in the asymmetric unit, which is represented by the reported sum formula. In other words: Z is 2 and Z' is 1 . The Flack parameter was refined to -0.036(17). Determination of absolute structure using Bayesian statistics on Bijvoet differences using the Olex2 results in -0.022(15). Note: The Flack parameter is used to determine chirality of the crystal studied, the value should be near 0 , a value of 1 means that the stereochemistry is wrong and the model should be inverted. A value of 0.5 means that the crystal consists of a racemic mixture of the two enantiomers.

Table S26: Bond Lengths in Å for compound 21b.

\begin{tabular}{lll}
\hline Atom & Atom & Length/Å \\
\hline P1 & N1 & $1.6603(16)$ \\
P1 & C1 & $1.8190(19)$ \\
P1 & C7 & $1.8166(19)$ \\
P1 & B1 & $1.908(2)$ \\
P2 & O1 & $1.6134(14)$ \\
P2 & C29 & $1.817(2)$ \\
P2 & C23 & $1.806(2)$ \\
P2 & B2 & $1.912(2)$ \\
O1 & C16 & $1.460(2)$ \\
N1 & C14 & $1.472(2)$ \\
N1 & C13 & $1.465(2)$ \\
C4 & C3 & $1.387(3)$ \\
C4 & C5 & $1.383(3)$ \\
C3 & C2 & $1.390(3)$ \\
C2 & C1 & $1.398(3)$ \\
C1 & C6 & $1.395(3)$ \\
C14 & C16 & $1.537(3)$
\end{tabular}

\begin{tabular}{lll}
\hline Atom & Atom & Length/Å \\
\hline C14 & C15 & $1.535(3)$ \\
C16 & C17 & $1.508(3)$ \\
C29 & C34 & $1.440(3)$ \\
C29 & C30 & $1.381(3)$ \\
C34 & C35 & $1.420(3)$ \\
C34 & C33 & $1.425(3)$ \\
C35 & C36 & $1.377(3)$ \\
C36 & C37 & $1.405(3)$ \\
C37 & C38 & $1.365(3)$ \\
C10 & C11 & $1.390(3)$ \\
C10 & C9 & $1.378(3)$ \\
C11 & C12 & $1.390(3)$ \\
C12 & C7 & $1.400(3)$ \\
C7 & C8 & $1.392(3)$ \\
C23 & C28 & $1.393(3)$ \\
C23 & C24 & $1.394(3)$ \\
C28 & C27 & $1.390(3)$
\end{tabular}




\begin{tabular}{lll}
\hline Atom & Atom & Length/Å \\
\hline C27 & C26 & $1.383(4)$ \\
C26 & C25 & $1.380(4)$ \\
C25 & C24 & $1.387(3)$ \\
C30 & C31 & $1.410(3)$ \\
C31 & C32 & $1.362(3)$ \\
C32 & C33 & $1.420(3)$ \\
C33 & C38 & $1.422(3)$ \\
C17 & C18 & $1.397(3)$
\end{tabular}

\begin{tabular}{lll}
\hline Atom & Atom & Length/Å \\
\hline C17 & C22 & $1.392(3)$ \\
C18 & C19 & $1.387(3)$ \\
C19 & C20 & $1.393(3)$ \\
C20 & C21 & $1.384(3)$ \\
C21 & C22 & $1.392(3)$ \\
C5 & C6 & $1.391(3)$ \\
C8 & C9 & $1.398(3)$
\end{tabular}

Table S27: Bond Angles in ${ }^{\circ}$ for compound 21b.

\begin{tabular}{|c|c|c|c|c|c|c|c|}
\hline Atom & Atom & Atom & Angle $/^{\circ}$ & Atom & Atom & Atom & Angle $/^{\circ}$ \\
\hline N1 & P1 & $\mathrm{C} 1$ & $108.65(9)$ & $\overline{\text { C38 }}$ & C37 & C36 & $119.8(2)$ \\
\hline $\mathrm{N} 1$ & P1 & $\mathrm{C} 7$ & $105.19(8)$ & C9 & C10 & C11 & $120.1(2)$ \\
\hline $\mathrm{N} 1$ & $\mathrm{P} 1$ & B1 & $115.56(9)$ & C12 & C11 & C10 & $120.20(19)$ \\
\hline $\mathrm{C} 1$ & $\mathrm{P} 1$ & B1 & $108.62(10)$ & C11 & $\mathrm{C} 12$ & $\mathrm{C} 7$ & $120.03(19)$ \\
\hline $\mathrm{C} 7$ & $\mathrm{P} 1$ & $\mathrm{C} 1$ & $105.39(9)$ & C12 & $\mathrm{C} 7$ & $\mathrm{P} 1$ & $117.75(15)$ \\
\hline $\mathrm{C} 7$ & $\mathrm{P} 1$ & B1 & $112.89(10)$ & C8 & $\mathrm{C} 7$ & $\mathrm{P} 1$ & $122.83(14)$ \\
\hline 01 & P2 & $\mathrm{C} 29$ & $98.43(8)$ & $\mathrm{C} 8$ & $\mathrm{C} 7$ & C12 & $119.37(18)$ \\
\hline 01 & P2 & $\mathrm{C} 23$ & 105.71(8) & C28 & $\mathrm{C} 23$ & P2 & $121.82(16)$ \\
\hline 01 & P2 & B2 & $112.28(9)$ & C28 & $\mathrm{C} 23$ & $\mathrm{C} 24$ & 119.71(19) \\
\hline C29 & $\mathrm{P} 2$ & B2 & $118.54(10)$ & $\mathrm{C} 24$ & $\mathrm{C} 23$ & P2 & $118.34(16)$ \\
\hline C23 & P2 & $\mathrm{C} 29$ & $106.58(9)$ & $\mathrm{C} 27$ & $\mathrm{C} 28$ & $\mathrm{C} 23$ & $119.3(2)$ \\
\hline $\mathrm{C} 23$ & $\mathrm{P} 2$ & B2 & $113.62(10)$ & $\mathrm{C} 26$ & $\mathrm{C} 27$ & $\mathrm{C} 28$ & $120.7(2)$ \\
\hline C16 & 01 & $\mathrm{P} 2$ & $121.89(11)$ & $\mathrm{C} 25$ & $\mathrm{C} 26$ & $\mathrm{C} 27$ & $120.2(2)$ \\
\hline C14 & N1 & $\mathrm{P} 1$ & $121.74(13)$ & $\mathrm{C} 26$ & $\mathrm{C} 25$ & $\mathrm{C} 24$ & $119.7(2)$ \\
\hline C13 & N1 & $\mathrm{P} 1$ & $119.51(13)$ & $\mathrm{C} 25$ & $\mathrm{C} 24$ & $\mathrm{C} 23$ & $120.4(2)$ \\
\hline C13 & N1 & $\mathrm{C} 14$ & $118.39(16)$ & $\mathrm{C} 29$ & $\mathrm{C} 30$ & C31 & $121.4(2)$ \\
\hline $\mathrm{C} 5$ & $\mathrm{C} 4$ & $\mathrm{C} 3$ & 119.61(19) & C32 & C31 & C30 & $120.2(2)$ \\
\hline $\mathrm{C} 4$ & $\mathrm{C} 3$ & $\mathrm{C} 2$ & $120.5(2)$ & $\mathrm{C} 31$ & $\mathrm{C} 32$ & C33 & $120.68(19)$ \\
\hline C3 & $\mathrm{C} 2$ & $\mathrm{C} 1$ & $120.2(2)$ & $\mathrm{C} 32$ & C33 & C34 & 119.81(19) \\
\hline $\mathrm{C} 2$ & $\mathrm{C} 1$ & $\mathrm{P} 1$ & $121.11(15)$ & C32 & C33 & C38 & 120.71(19) \\
\hline C6 & $\mathrm{C} 1$ & P1 & $119.71(15)$ & C38 & C33 & C34 & $119.46(19)$ \\
\hline C6 & $\mathrm{C} 1$ & $\mathrm{C} 2$ & $118.80(18)$ & C37 & C38 & C33 & $120.9(2)$ \\
\hline $\mathrm{N} 1$ & C14 & C16 & $110.17(15)$ & C18 & $\mathrm{C} 17$ & C16 & $120.75(17)$ \\
\hline $\mathrm{N} 1$ & C14 & C15 & $112.13(16)$ & $\mathrm{C} 22$ & C17 & C16 & 119.85(17) \\
\hline C15 & C14 & C16 & $110.54(16)$ & $\mathrm{C} 22$ & C17 & C18 & $119.34(18)$ \\
\hline 01 & C16 & C14 & $103.53(14)$ & C19 & C18 & $\mathrm{C} 17$ & $120.10(18)$ \\
\hline 01 & C16 & $\mathrm{C} 17$ & $109.64(15)$ & C18 & C19 & $\mathrm{C} 20$ & 119.97(19) \\
\hline C17 & C16 & C14 & $114.99(15)$ & $\mathrm{C} 21$ & $\mathrm{C} 20$ & C19 & $120.5(2)$ \\
\hline C34 & $\mathrm{C} 29$ & P2 & $122.41(14)$ & $\mathrm{C} 20$ & $\mathrm{C} 21$ & $\mathrm{C} 22$ & 119.42(19) \\
\hline C30 & $\mathrm{C} 29$ & P2 & $118.11(16)$ & $\mathrm{C} 17$ & $\mathrm{C} 22$ & $\mathrm{C} 21$ & 120.71(19) \\
\hline C30 & $\mathrm{C} 29$ & C34 & $119.45(18)$ & $\mathrm{C} 4$ & $\mathrm{C} 5$ & $\mathrm{C} 6$ & $120.3(2)$ \\
\hline C35 & C34 & $\mathrm{C} 29$ & $123.47(18)$ & $\mathrm{C} 5$ & $\mathrm{C} 6$ & $\mathrm{C} 1$ & $120.5(2)$ \\
\hline C35 & C34 & C33 & $118.16(18)$ & $\mathrm{C} 7$ & C8 & $\mathrm{C} 9$ & $120.14(19)$ \\
\hline C33 & C34 & $\mathrm{C} 29$ & $118.37(17)$ & C10 & $\mathrm{C} 9$ & C8 & $120.2(2)$ \\
\hline C36 & C35 & C34 & $120.6(2)$ & & & & \\
\hline C35 & C36 & C37 & $121.1(2)$ & & & & \\
\hline
\end{tabular}


Table S28: Torsion Angles in ${ }^{\circ}$ for compound $21 \mathbf{b}$.

\begin{tabular}{|c|c|c|c|c|}
\hline Atom & Atom & Atom & Atom & Angle ${ }^{\circ}$ \\
\hline $\mathrm{P} 1$ & N1 & C14 & C16 & $-129.13(15)$ \\
\hline $\mathrm{P} 1$ & N1 & C14 & $\mathrm{C} 15$ & 107.31(17) \\
\hline $\mathrm{P} 1$ & $\mathrm{C} 1$ & C6 & $\mathrm{C} 5$ & $-172.95(17)$ \\
\hline $\mathrm{P} 1$ & $\mathrm{C} 7$ & $\mathrm{C} 8$ & $\mathrm{C} 9$ & $-177.08(17)$ \\
\hline P2 & 01 & C16 & $\mathrm{C} 14$ & $158.69(12)$ \\
\hline P2 & 01 & C16 & $\mathrm{C} 17$ & $-78.14(18)$ \\
\hline $\mathrm{P} 2$ & $\mathrm{C} 29$ & $\mathrm{C} 34$ & $\mathrm{C} 35$ & $5.1(3)$ \\
\hline $\mathrm{P} 2$ & $\mathrm{C} 29$ & $\mathrm{C} 34$ & $\mathrm{C} 33$ & $-175.37(14)$ \\
\hline P2 & $\mathrm{C} 29$ & $\mathrm{C} 30$ & $\mathrm{C} 31$ & $176.39(18)$ \\
\hline P2 & $\mathrm{C} 23$ & $\mathrm{C} 28$ & $\mathrm{C} 27$ & $-176.99(17)$ \\
\hline P2 & $\mathrm{C} 23$ & $\mathrm{C} 24$ & $\mathrm{C} 25$ & $176.43(17)$ \\
\hline 01 & $\mathrm{P} 2$ & $\mathrm{C} 29$ & $\mathrm{C} 34$ & $171.04(15)$ \\
\hline 01 & $\mathrm{P} 2$ & $\mathrm{C} 29$ & $\mathrm{C} 30$ & $-7.13(18)$ \\
\hline 01 & $\mathrm{P} 2$ & $\mathrm{C} 23$ & $\mathrm{C} 28$ & $73.88(18)$ \\
\hline 01 & $\mathrm{P} 2$ & $\mathrm{C} 23$ & $\mathrm{C} 24$ & $-101.94(17)$ \\
\hline 01 & C16 & C17 & C18 & $-50.4(2)$ \\
\hline 01 & C16 & C17 & $\mathrm{C} 22$ & $126.64(18)$ \\
\hline N1 & $\mathrm{P} 1$ & $\mathrm{C} 1$ & $\mathrm{C} 2$ & $15.7(2)$ \\
\hline N1 & $\mathrm{P} 1$ & C1 & $\mathrm{C} 6$ & $-171.46(16)$ \\
\hline N1 & $\mathrm{P} 1$ & $\mathrm{C} 7$ & $\mathrm{C} 12$ & $-80.23(16)$ \\
\hline N1 & $\mathrm{P} 1$ & $\mathrm{C} 7$ & C8 & $97.29(17)$ \\
\hline N1 & $\mathrm{C} 14$ & C16 & 01 & $174.54(14)$ \\
\hline N1 & C14 & C16 & $\mathrm{C} 17$ & $55.0(2)$ \\
\hline $\mathrm{C} 4$ & $\mathrm{C} 3$ & $\mathrm{C} 2$ & $\mathrm{C} 1$ & $1.4(4)$ \\
\hline $\mathrm{C} 4$ & $\mathrm{C} 5$ & C6 & $\mathrm{C} 1$ & $1.2(3)$ \\
\hline $\mathrm{C} 3$ & $\mathrm{C} 4$ & $\mathrm{C} 5$ & $\mathrm{C} 6$ & $-1.2(3)$ \\
\hline $\mathrm{C} 3$ & $\mathrm{C} 2$ & $\mathrm{C} 1$ & $\mathrm{P} 1$ & $171.59(17)$ \\
\hline $\mathrm{C} 3$ & $\mathrm{C} 2$ & $\mathrm{C} 1$ & $\mathrm{C} 6$ & $-1.3(3)$ \\
\hline $\mathrm{C} 2$ & $\mathrm{C} 1$ & C6 & $\mathrm{C} 5$ & $0.1(3)$ \\
\hline $\mathrm{C} 1$ & $\mathrm{P} 1$ & N1 & C14 & $-105.46(15)$ \\
\hline $\mathrm{C} 1$ & $\mathrm{P} 1$ & N1 & $\mathrm{C} 13$ & 67.61(17) \\
\hline $\mathrm{C} 1$ & $\mathrm{P} 1$ & $\mathrm{C} 7$ & $\mathrm{C} 12$ & $165.03(15)$ \\
\hline C1 & $\mathrm{P} 1$ & $\mathrm{C} 7$ & C8 & $-17.45(18)$ \\
\hline C14 & C16 & C17 & C18 & $65.7(2)$ \\
\hline C14 & C16 & C17 & $\mathrm{C} 22$ & $-117.2(2)$ \\
\hline C16 & C17 & C18 & C19 & $176.97(18)$ \\
\hline C16 & C17 & $\mathrm{C} 22$ & $\mathrm{C} 21$ & $-176.95(18)$ \\
\hline C29 & $\mathrm{P} 2$ & 01 & $\mathrm{C} 16$ & $-160.50(14)$ \\
\hline $\mathrm{C} 29$ & $\mathrm{P} 2$ & $\mathrm{C} 23$ & $\mathrm{C} 28$ & $-30.16(19)$ \\
\hline C29 & $\mathrm{P} 2$ & $\mathrm{C} 23$ & $\mathrm{C} 24$ & $154.01(16)$ \\
\hline $\mathrm{C} 29$ & C34 & $\mathrm{C} 35$ & $\mathrm{C} 36$ & $180.0(2)$ \\
\hline $\mathrm{C} 29$ & C34 & C33 & $\mathrm{C} 32$ & $-1.8(3)$ \\
\hline $\mathrm{C} 29$ & C34 & C33 & $\mathrm{C} 38$ & $179.41(18)$ \\
\hline $\mathrm{C} 29$ & $\mathrm{C} 30$ & C31 & $\mathrm{C} 32$ & $-0.2(4)$ \\
\hline C34 & $\mathrm{C} 29$ & C30 & C31 & $-1.8(3)$ \\
\hline C34 & $\mathrm{C} 35$ & C36 & $\mathrm{C} 37$ & $0.5(3)$ \\
\hline C34 & $\mathrm{C} 33$ & $\mathrm{C} 38$ & $\mathrm{C} 37$ & $0.7(3)$ \\
\hline C35 & $\mathrm{C} 34$ & C33 & $\mathrm{C} 32$ & $177.77(19)$ \\
\hline C35 & C34 & C33 & $\mathrm{C} 38$ & $-1.0(3)$ \\
\hline C35 & $\mathrm{C} 36$ & $\mathrm{C} 37$ & $\mathrm{C} 38$ & $-0.8(4)$ \\
\hline C36 & $\mathrm{C} 37$ & $\mathrm{C} 38$ & $\mathrm{C} 33$ & $0.2(3)$ \\
\hline C10 & C11 & C12 & $\mathrm{C} 7$ & $-0.7(3)$ \\
\hline
\end{tabular}




\begin{tabular}{|c|c|c|c|c|}
\hline Atom & Atom & Atom & Atom & Angle $/^{\circ}$ \\
\hline C11 & $\mathrm{C} 10$ & $\mathrm{C} 9$ & $\mathrm{C} 8$ & $0.8(3)$ \\
\hline C11 & $\mathrm{C} 12$ & $\mathrm{C} 7$ & $\mathrm{P} 1$ & $178.04(16)$ \\
\hline C11 & $\mathrm{C} 12$ & $\mathrm{C} 7$ & $\mathrm{C} 8$ & $0.4(3)$ \\
\hline C12 & $\mathrm{C} 7$ & $\mathrm{C} 8$ & C9 & $0.4(3)$ \\
\hline C7 & $\mathrm{P} 1$ & N1 & C14 & $142.09(15)$ \\
\hline $\mathrm{C} 7$ & $\mathrm{P} 1$ & N1 & C13 & $-44.83(17)$ \\
\hline C7 & $\mathrm{P} 1$ & $\mathrm{C} 1$ & $\mathrm{C} 2$ & $127.99(17)$ \\
\hline $\mathrm{C} 7$ & $\mathrm{P} 1$ & $\mathrm{C} 1$ & C6 & $-59.14(18)$ \\
\hline $\mathrm{C} 7$ & $\mathrm{C} 8$ & $\mathrm{C} 9$ & C10 & $-1.0(3)$ \\
\hline C13 & N1 & C14 & C16 & $57.7(2)$ \\
\hline C13 & N1 & C14 & C15 & $-65.8(2)$ \\
\hline C23 & $\mathrm{P} 2$ & 01 & C16 & $89.53(15)$ \\
\hline C23 & $\mathrm{P} 2$ & $\mathrm{C} 29$ & C34 & $-79.71(17)$ \\
\hline C23 & P2 & $\mathrm{C} 29$ & C30 & $102.12(17)$ \\
\hline C23 & $\mathrm{C} 28$ & $\mathrm{C} 27$ & $\mathrm{C} 26$ & $0.6(3)$ \\
\hline C28 & $\mathrm{C} 23$ & $\mathrm{C} 24$ & $\mathrm{C} 25$ & $0.5(3)$ \\
\hline C28 & $\mathrm{C} 27$ & $\mathrm{C} 26$ & $\mathrm{C} 25$ & $0.7(4)$ \\
\hline C27 & $\mathrm{C} 26$ & $\mathrm{C} 25$ & $\mathrm{C} 24$ & $-1.4(4)$ \\
\hline C26 & $\mathrm{C} 25$ & $\mathrm{C} 24$ & C23 & $0.8(3)$ \\
\hline C24 & $\mathrm{C} 23$ & $\mathrm{C} 28$ & $\mathrm{C} 27$ & $-1.2(3)$ \\
\hline C30 & $\mathrm{C} 29$ & C34 & C35 & $-176.77(19)$ \\
\hline C30 & $\mathrm{C} 29$ & C34 & C33 & $2.8(3)$ \\
\hline C30 & C31 & C32 & C33 & $1.2(4)$ \\
\hline C31 & C32 & C33 & C34 & $-0.2(3)$ \\
\hline C31 & C32 & C33 & C38 & $178.6(2)$ \\
\hline C32 & C33 & C38 & C37 & $-178.1(2)$ \\
\hline C33 & C34 & C35 & C36 & $0.5(3)$ \\
\hline C17 & C18 & C19 & $\mathrm{C} 20$ & $0.0(3)$ \\
\hline C18 & $\mathrm{C} 17$ & $\mathrm{C} 22$ & C21 & $0.1(3)$ \\
\hline C18 & C19 & $\mathrm{C} 20$ & $\mathrm{C} 21$ & $0.0(3)$ \\
\hline C19 & $\mathrm{C} 20$ & $\mathrm{C} 21$ & $\mathrm{C} 22$ & $0.1(3)$ \\
\hline C20 & $\mathrm{C} 21$ & $\mathrm{C} 22$ & C17 & $-0.1(3)$ \\
\hline C22 & C17 & C18 & C19 & $-0.1(3)$ \\
\hline C15 & C14 & C16 & 01 & $-60.98(19)$ \\
\hline C15 & C14 & C16 & $\mathrm{C} 17$ & $179.45(16)$ \\
\hline C5 & $\mathrm{C} 4$ & $\mathrm{C} 3$ & $\mathrm{C} 2$ & $-0.1(4)$ \\
\hline C9 & $\mathrm{C} 10$ & C11 & $\mathrm{C} 12$ & $0.0(3)$ \\
\hline B1 & $\mathrm{P} 1$ & N1 & C14 & $16.88(18)$ \\
\hline B1 & $\mathrm{P} 1$ & N1 & C13 & $-170.04(15)$ \\
\hline B1 & $\mathrm{P} 1$ & $\mathrm{C} 1$ & $\mathrm{C} 2$ & $-110.78(18)$ \\
\hline B1 & $\mathrm{P} 1$ & $\mathrm{C} 1$ & C6 & 62.08(19) \\
\hline B1 & $\mathrm{P} 1$ & $\mathrm{C} 7$ & C12 & $46.63(18)$ \\
\hline B1 & $\mathrm{P} 1$ & $\mathrm{C} 7$ & $\mathrm{C} 8$ & $-135.85(17)$ \\
\hline B2 & $\mathrm{P} 2$ & 01 & C16 & $-34.87(17)$ \\
\hline B2 & $\mathrm{P} 2$ & $\mathrm{C} 29$ & C34 & $49.9(2)$ \\
\hline B2 & $\mathrm{P} 2$ & $\mathrm{C} 29$ & C30 & $-128.25(17)$ \\
\hline B2 & $\mathrm{P} 2$ & $\mathrm{C} 23$ & C28 & $-162.55(17)$ \\
\hline B2 & P2 & $\mathrm{C} 23$ & $\mathrm{C} 24$ & $21.6(2)$ \\
\hline
\end{tabular}


Name: $\left(R_{\mathrm{p}}\right)-(-)-\mathrm{N}-[(1 R, 2 S)-2-(\beta-N a p h t y l p h e n y l p h o s p h i n i t o-b o r a n e)-1-m e t h y l-2-p h e n y l e t h y l], \mathrm{N}-m e t h y l$ aminodiphenylphosphine-borane $\mathbf{2 1 c}$.

Submitted by: Antonin Jaillet

Solved by: Yoann Rousselin

Sample ID: $\quad$ 16AJ361

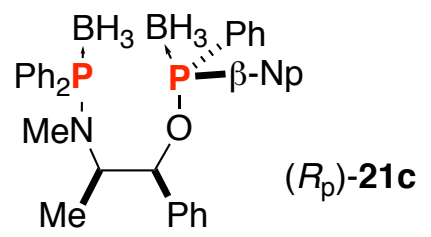

\section{Crystal Data and Experimental}

Figure S10 (thermal ellipsoïds are drawn at $50 \%$ probability plot)

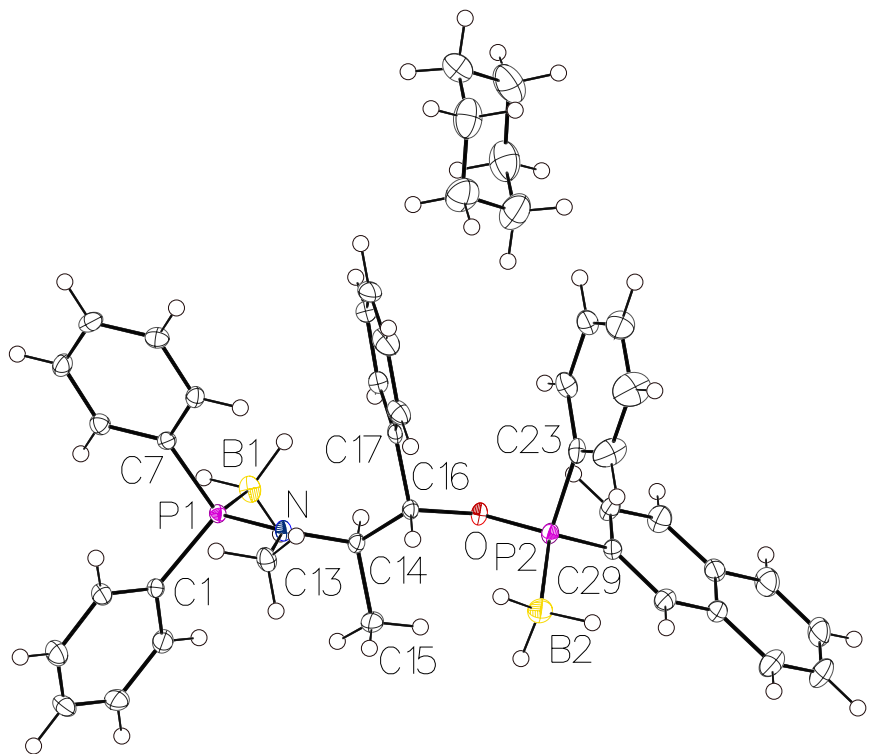

Experimental. Single clear light colorless needle-shaped crystals of compound 21c were recrystallized from cyclohexane by slow evaporation. A suitable crystal $0.30 \times 0.25 \times 0.16 \mathrm{~mm}^{3}$ was selected and mounted on a MITIGEN holder oil on a Bruker D8 VENTURE diffractometer. The crystal was kept at a steady $T=$ 100.0(2) K during data collection. The structure was solved with the ShelXT (Sheldrick, 2015) structure solution program using the direct solution method and by using Olex2 (Dolomanov et al., 2009) as the graphical interface. The model was refined with version 2018/3 of ShelXL (Sheldrick, 2015) using Least Squares minimization.

Crystal Data. $\mathrm{C}_{44} \mathrm{H}_{53} \mathrm{~B}_{2} \mathrm{NOP}_{2}, M_{r}=695.43$, orthorhombic, $P 2{ }_{1} 2_{1} 2_{1}$ (No. 19), $\mathrm{a}=9.0483(4) \AA, \mathrm{b}=13.0779(6) \AA, \mathrm{c}=$ 34.0273(17) $\AA, \alpha=\beta=\gamma=90^{\circ}, V=4026.5(3) \AA^{3}, T=$ $100.0(2) \mathrm{K}, \quad Z=4, Z^{\prime}=1, \mu\left(\mathrm{MoK}_{\alpha}\right)=0.142,94746$ reflections measured, 9219 unique $\left(R_{\text {int }}=0.0373\right)$ which were used in all calculations. The final $w R_{2}$ was 0.0720 (all data) and $R_{1}$ was 0.0300 (I > 2(I)). 


\begin{tabular}{|c|c|c|c|}
\hline Compound & $21 c$ & Radiation type & $\mathrm{MoK}_{\alpha}$ \\
\hline CCDC & 1982659 & $\Theta_{\min } /^{\circ}$ & 2.856 \\
\hline Formula & $\mathrm{C}_{44} \mathrm{H}_{53} \mathrm{~B}_{2} \mathrm{NOP}_{2}$ & $\Theta_{\max } /^{\circ}$ & 27.516 \\
\hline$D_{\text {calc. }} / \mathrm{g} \mathrm{cm}^{-3}$ & 1.147 & Measured Refl. & 94746 \\
\hline$\mu / \mathrm{mm}^{-1}$ & 0.142 & Independent Refl. & 9219 \\
\hline Formula Weight & 695.43 & Reflections with I > & 8650 \\
\hline Color & clear light colorless & $2(\mathrm{I})$ & \\
\hline Shape & needle & $R_{\text {int }}$ & 0.0373 \\
\hline Size $/ \mathrm{mm}^{3}$ & $0.30 \times 0.25 \times 0.16$ & Parameters & 457 \\
\hline$T / \mathrm{K}$ & $100.0(2)$ & Restraints & 0 \\
\hline Crystal System & orthorhombic & Largest Peak & 0.306 \\
\hline Flack Parameter & $0.005(14)$ & Deepest Hole & -0.182 \\
\hline Hooft Parameter & $0.020(13)$ & GooF & 1.048 \\
\hline Space Group & $P 2_{1} 2_{1} 2_{1}$ & $w R_{2}$ (all data) & 0.0720 \\
\hline$a / \AA ̊$ & $9.0483(4)$ & $w R_{2}$ & 0.0698 \\
\hline$b / \AA$ & $13.0779(6)$ & $R_{1}$ (all data) & 0.0345 \\
\hline$c / \AA$ & $34.0273(17)$ & $R_{1}$ & 0.0300 \\
\hline$\alpha /^{\circ}$ & 90 & & \\
\hline$\beta /^{\circ}$ & 90 & & \\
\hline$\gamma /^{\circ}$ & 90 & & \\
\hline $\mathrm{V} / \AA^{3}$ & $4026.5(3)$ & & \\
\hline$Z$ & 4 & & \\
\hline$Z^{\prime}$ & 1 & & \\
\hline Wavelength/Å & 0.71073 & & \\
\hline
\end{tabular}

\section{Structure Quality Indicators}

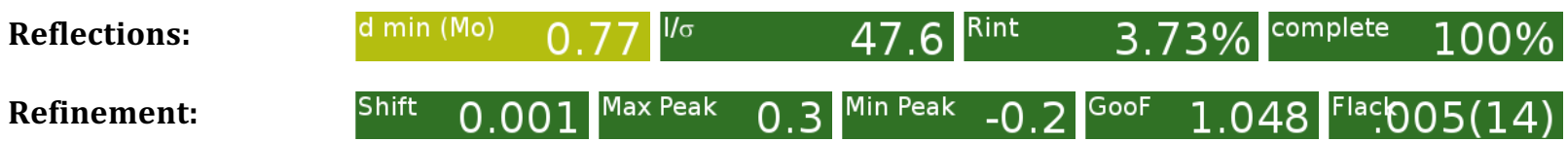

A clear light colorless needle-shaped crystal with dimensions $0.30 \times 0.25 \times 0.16 \mathrm{~mm}^{3}$ was mounted on a MITIGEN holder oil. Data were collected using a Bruker D8 VENTURE diffractometer equipped with an Oxford Cryosystems low-temperature device operating at $T=100.0(2) \mathrm{K}$. Data were measured using $\phi$ and $\omega$ scans using $\operatorname{MoK}_{\alpha}$ radiation. The total number of runs and images was based on the strategy calculation from the program APEX3 (Bruker, 2015) The maximum resolution that was achieved was $\Theta$ $=27.516^{\circ}(0.77 \AA)$. The diffraction pattern was indexed. The total number of runs and images was based on the strategy calculation from the program APEX3 (Bruker, 2015) and the unit cell was refined using SAINT (Bruker, V8.37A, after 2013) on 9506 reflections, 10\% of the observed reflections. Data reduction, scaling and absorption corrections were performed using SAINT (Bruker, V8.37A, after 2013). The final completeness is $99.80 \%$ out to $27.516^{\circ}$ in $\Theta$. A multi-scan absorption correction was performed using SADABS-2014/5 (Bruker, 2014) was used for absorption correction. $w R_{2}$ (int) was 0.0543 before and 0.0508 after correction. The Ratio of minimum to maximum transmission is 0.9382 . The $\lambda / 2$ correction factor is 0.00150 . The absorption coefficient $\mu$ of this material is $0.142 \mathrm{~mm}^{-1}$ at this wavelength $(\lambda=0.711 \AA)$ and the minimum and maximum transmissions are 0.700 and 0.746 . The structure was solved and the space group $P 2{ }_{1} 2_{1} 2_{1}$ (\# 19) determined by the ShelXT (Sheldrick, 2015) structure solution program using direct and refined by Least Squares using version 2018/3 of ShelXL (Sheldrick, 2015). All non-hydrogen atoms were refined anisotropically. Hydrogen atom positions were calculated geometrically and refined using the riding model. Hydrogen atom on Boron atom were refined using an AFIX 138, which would allow the refinement of a common B-H distances, the same 
shifts being applied to all the B-H bonds to the same atom, but still retain tetrahedral $\mathrm{H}-\mathrm{B}-\mathrm{H}$ angles within the group.

Table S29: Bond Lengths in Å for compound 21c.

\begin{tabular}{|c|c|c|}
\hline Atom & Atom & Length/Å \\
\hline P1 & $\mathrm{N}$ & $1.6671(15)$ \\
\hline P1 & C1 & $1.8185(18)$ \\
\hline P1 & $\mathrm{C} 7$ & $1.8129(18)$ \\
\hline P1 & B1 & $1.910(2)$ \\
\hline P2 & 0 & $1.6178(12)$ \\
\hline P2 & $\mathrm{C} 23$ & $1.805(2)$ \\
\hline P2 & $\mathrm{C} 29$ & $1.8014(19)$ \\
\hline P2 & B2 & $1.896(2)$ \\
\hline 0 & C16 & $1.469(2)$ \\
\hline $\mathrm{N}$ & $\mathrm{C} 13$ & $1.467(2)$ \\
\hline N & C14 & $1.478(2)$ \\
\hline C1 & $\mathrm{C} 2$ & $1.395(3)$ \\
\hline $\mathrm{C} 1$ & C6 & $1.399(3)$ \\
\hline C2 & C3 & $1.395(3)$ \\
\hline C3 & $\mathrm{C} 4$ & $1.378(3)$ \\
\hline $\mathrm{C} 4$ & C5 & $1.381(3)$ \\
\hline C5 & C6 & $1.388(3)$ \\
\hline C7 & C8 & $1.403(2)$ \\
\hline C7 & $\mathrm{C} 12$ & $1.391(3)$ \\
\hline C8 & C9 & $1.387(3)$ \\
\hline C9 & C10 & $1.388(3)$ \\
\hline $\mathrm{C} 10$ & C11 & $1.390(3)$ \\
\hline C11 & $\mathrm{C} 12$ & $1.390(3)$ \\
\hline C14 & C15 & $1.529(3)$ \\
\hline C14 & C16 & $1.537(2)$ \\
\hline C16 & C17 & $1.508(2)$ \\
\hline C17 & C18 & $1.389(3)$ \\
\hline C17 & C22 & $1.393(3)$ \\
\hline
\end{tabular}

\begin{tabular}{lll}
\hline Atom & Atom & Length/A \\
\hline C18 & C19 & $1.392(3)$ \\
C19 & C20 & $1.384(3)$ \\
C20 & C21 & $1.386(3)$ \\
C21 & C22 & $1.391(3)$ \\
C23 & C24 & $1.393(3)$ \\
C23 & C28 & $1.391(3)$ \\
C24 & C25 & $1.381(3)$ \\
C25 & C26 & $1.382(3)$ \\
C26 & C27 & $1.371(4)$ \\
C27 & C28 & $1.394(3)$ \\
C29 & C30 & $1.417(3)$ \\
C29 & C38 & $1.375(3)$ \\
C30 & C31 & $1.367(3)$ \\
C31 & C32 & $1.418(3)$ \\
C32 & C33 & $1.420(3)$ \\
C32 & C37 & $1.417(3)$ \\
C33 & C34 & $1.364(3)$ \\
C34 & C35 & $1.406(4)$ \\
C35 & C36 & $1.368(3)$ \\
C36 & C37 & $1.418(3)$ \\
C37 & C38 & $1.418(3)$ \\
C39 & C40 & $1.522(4)$ \\
C39 & C44 & $1.519(4)$ \\
C40 & C41 & $1.511(4)$ \\
C41 & C42 & $1.510(4)$ \\
C42 & C43 & $1.526(4)$ \\
C43 & C44 & $1.510(4)$ \\
& &
\end{tabular}

Table S30: Bond Angles in ${ }^{\circ}$ for compound 21c.

\begin{tabular}{llll}
\hline Atom & Atom & Atom & Angle $^{\circ}$ \\
\hline $\mathrm{N}$ & $\mathrm{P} 1$ & $\mathrm{C} 1$ & $108.96(8)$ \\
$\mathrm{N}$ & $\mathrm{P} 1$ & $\mathrm{C} 7$ & $106.24(8)$ \\
$\mathrm{N}$ & $\mathrm{P} 1$ & $\mathrm{~B} 1$ & $113.96(8)$ \\
$\mathrm{C} 1$ & $\mathrm{P} 1$ & $\mathrm{~B} 1$ & $112.78(9)$ \\
$\mathrm{C} 7$ & $\mathrm{P} 1$ & $\mathrm{C} 1$ & $104.05(8)$ \\
$\mathrm{C} 7$ & $\mathrm{P} 1$ & $\mathrm{~B} 1$ & $110.20(9)$ \\
$\mathrm{O}$ & $\mathrm{P} 2$ & $\mathrm{C} 23$ & $105.28(8)$ \\
$\mathrm{O}$ & $\mathrm{P} 2$ & $\mathrm{C} 29$ & $100.44(7)$ \\
$\mathrm{O}$ & $\mathrm{P} 2$ & $\mathrm{~B} 2$ & $115.22(9)$ \\
$\mathrm{C} 23$ & $\mathrm{P} 2$ & $\mathrm{~B} 2$ & $114.15(10)$ \\
$\mathrm{C} 29$ & $\mathrm{P} 2$ & $\mathrm{C} 23$ & $106.17(9)$ \\
$\mathrm{C} 29$ & $\mathrm{P} 2$ & $\mathrm{~B} 2$ & $114.20(10)$ \\
$\mathrm{C} 16$ & $\mathrm{O}$ & $\mathrm{P} 2$ & $118.87(10)$ \\
$\mathrm{C} 13$ & $\mathrm{~N}$ & $\mathrm{P} 1$ & $119.77(12)$ \\
$\mathrm{C} 13$ & $\mathrm{~N}$ & $\mathrm{C} 14$ & $116.93(14)$ \\
$\mathrm{C} 14$ & $\mathrm{~N}$ & $\mathrm{P} 1$ & $119.37(11)$
\end{tabular}

\begin{tabular}{llll}
\hline Atom & Atom & Atom & \multicolumn{1}{c}{ Angle $^{\circ}$} \\
\hline C2 & C1 & P1 & $119.11(14)$ \\
C2 & C1 & C6 & $119.17(17)$ \\
C6 & C1 & P1 & $121.72(14)$ \\
C1 & C2 & C3 & $119.88(19)$ \\
C4 & C3 & C2 & $120.4(2)$ \\
C3 & C4 & C5 & $120.04(18)$ \\
C4 & C5 & C6 & $120.3(2)$ \\
C5 & C6 & C1 & $120.18(19)$ \\
C8 & C7 & P1 & $118.49(14)$ \\
C12 & C7 & P1 & $121.53(13)$ \\
C12 & C7 & C8 & $119.71(16)$ \\
C9 & C8 & C7 & $120.05(17)$ \\
C8 & C9 & C10 & $119.88(17)$ \\
C9 & C10 & C11 & $120.34(17)$ \\
C12 & C11 & C10 & $120.02(18)$ \\
C11 & C12 & C7 & $119.99(17)$
\end{tabular}




\begin{tabular}{llll}
\hline Atom & Atom & Atom & Angle ${ }^{\circ}$ \\
\hline $\mathrm{N}$ & $\mathrm{C} 14$ & $\mathrm{C} 15$ & $112.87(15)$ \\
$\mathrm{N}$ & $\mathrm{C} 14$ & $\mathrm{C} 16$ & $109.14(14)$ \\
$\mathrm{C} 15$ & $\mathrm{C} 14$ & $\mathrm{C} 16$ & $111.74(15)$ \\
$\mathrm{O}$ & $\mathrm{C} 16$ & $\mathrm{C} 14$ & $104.87(13)$ \\
$\mathrm{O}$ & $\mathrm{C} 16$ & $\mathrm{C} 17$ & $109.86(14)$ \\
$\mathrm{C} 17$ & $\mathrm{C} 16$ & $\mathrm{C} 14$ & $114.76(14)$ \\
$\mathrm{C} 18$ & $\mathrm{C} 17$ & $\mathrm{C} 16$ & $119.77(17)$ \\
$\mathrm{C} 18$ & $\mathrm{C} 17$ & $\mathrm{C} 22$ & $119.01(18)$ \\
$\mathrm{C} 22$ & $\mathrm{C} 17$ & $\mathrm{C} 16$ & $121.21(16)$ \\
$\mathrm{C} 17$ & $\mathrm{C} 18$ & $\mathrm{C} 19$ & $120.5(2)$ \\
$\mathrm{C} 20$ & $\mathrm{C} 19$ & $\mathrm{C} 18$ & $120.2(2)$ \\
$\mathrm{C} 19$ & $\mathrm{C} 20$ & $\mathrm{C} 21$ & $119.8(2)$ \\
$\mathrm{C} 20$ & $\mathrm{C} 21$ & $\mathrm{C} 22$ & $120.0(2)$ \\
$\mathrm{C} 21$ & $\mathrm{C} 22$ & $\mathrm{C} 17$ & $120.50(19)$ \\
$\mathrm{C} 24$ & $\mathrm{C} 23$ & $\mathrm{P} 2$ & $121.89(14)$ \\
$\mathrm{C} 28$ & $\mathrm{C} 23$ & $\mathrm{P} 2$ & $119.47(16)$ \\
$\mathrm{C} 28$ & $\mathrm{C} 23$ & $\mathrm{C} 24$ & $118.64(19)$ \\
$\mathrm{C} 25$ & $\mathrm{C} 24$ & $\mathrm{C} 23$ & $120.99(18)$ \\
$\mathrm{C} 24$ & $\mathrm{C} 25$ & $\mathrm{C} 26$ & $119.67(19)$ \\
$\mathrm{C} 27$ & $\mathrm{C} 26$ & $\mathrm{C} 25$ & $120.4(2)$ \\
$\mathrm{C} 26$ & $\mathrm{C} 27$ & $\mathrm{C} 28$ & $120.2(2)$ \\
$\mathrm{C} 23$ & $\mathrm{C} 28$ & $\mathrm{C} 27$ & $120.1(2)$
\end{tabular}

\begin{tabular}{llll}
\hline Atom & Atom & Atom & \multicolumn{1}{c}{ Angle $^{\circ}$} \\
\hline C30 & C29 & P2 & $120.80(14)$ \\
C38 & C29 & P2 & $119.35(14)$ \\
C38 & C29 & C30 & $119.82(17)$ \\
C31 & C30 & C29 & $120.34(17)$ \\
C30 & C31 & C32 & $121.13(19)$ \\
C31 & C32 & C33 & $122.3(2)$ \\
C37 & C32 & C31 & $118.63(18)$ \\
C37 & C32 & C33 & $119.05(19)$ \\
C34 & C33 & C32 & $120.5(2)$ \\
C33 & C34 & C35 & $120.6(2)$ \\
C36 & C35 & C34 & $120.4(2)$ \\
C35 & C36 & C37 & $120.6(2)$ \\
C32 & C37 & C36 & $118.90(19)$ \\
C32 & C37 & C38 & $119.35(17)$ \\
C38 & C37 & C36 & $121.75(19)$ \\
C29 & C38 & C37 & $120.72(18)$ \\
C44 & C39 & C40 & $111.4(2)$ \\
C41 & C40 & C39 & $111.9(2)$ \\
C42 & C41 & C40 & $111.0(2)$ \\
C41 & C42 & C43 & $111.5(2)$ \\
C44 & C43 & C42 & $111.0(2)$ \\
C43 & C44 & C39 & $111.7(2)$
\end{tabular}

Table S31: Torsion Angles in ${ }^{\circ}$ for compound 21c.

\begin{tabular}{|c|c|c|c|c|}
\hline Atom & Atom & Atom & Atom & Angle $/^{\circ}$ \\
\hline $\mathrm{P} 1$ & $\mathrm{~N}$ & C14 & C15 & $-92.30(17)$ \\
\hline P1 & $\mathrm{N}$ & C14 & C16 & $142.81(13)$ \\
\hline P1 & $\mathrm{C} 1$ & $\mathrm{C} 2$ & C3 & $178.26(15)$ \\
\hline P1 & C1 & C6 & C5 & $-177.98(14)$ \\
\hline P1 & $\mathrm{C} 7$ & C8 & C9 & $-174.53(14)$ \\
\hline $\mathrm{P} 1$ & C7 & C12 & C11 & $174.27(14)$ \\
\hline P2 & 0 & C16 & C14 & $-140.86(12)$ \\
\hline P2 & 0 & C16 & C17 & $95.32(15)$ \\
\hline P2 & $\mathrm{C} 23$ & C24 & $\mathrm{C} 25$ & $179.97(15)$ \\
\hline $\mathrm{P} 2$ & $\mathrm{C} 23$ & C28 & $\mathrm{C} 27$ & 179.1(2) \\
\hline P2 & C29 & C30 & C31 & $177.27(16)$ \\
\hline P2 & C29 & C38 & C37 & $-176.96(14)$ \\
\hline 0 & P2 & $\mathrm{C} 23$ & $\mathrm{C} 24$ & $-32.85(18)$ \\
\hline 0 & P2 & $\mathrm{C} 23$ & $\mathrm{C} 28$ & $147.43(17)$ \\
\hline 0 & P2 & C29 & C30 & $35.58(17)$ \\
\hline 0 & P2 & C29 & C38 & $-146.47(15)$ \\
\hline 0 & C16 & C17 & C18 & $-108.15(18)$ \\
\hline 0 & C16 & C17 & $\mathrm{C} 22$ & $70.4(2)$ \\
\hline $\mathrm{N}$ & P1 & $\mathrm{C} 1$ & $\mathrm{C} 2$ & $-95.69(16)$ \\
\hline $\mathrm{N}$ & P1 & C1 & C6 & $83.09(16)$ \\
\hline $\mathrm{N}$ & P1 & C7 & C8 & $-167.53(14)$ \\
\hline $\mathrm{N}$ & P1 & C7 & C12 & $18.42(17)$ \\
\hline $\mathrm{N}$ & C14 & C16 & 0 & $-170.71(13)$ \\
\hline $\mathrm{N}$ & C14 & C16 & C17 & $-50.1(2)$ \\
\hline C1 & P1 & $\mathrm{N}$ & C13 & $-44.75(16)$ \\
\hline $\mathrm{C} 1$ & $\mathrm{P} 1$ & $\mathrm{~N}$ & C14 & $112.29(14)$ \\
\hline $\mathrm{C} 1$ & P1 & $\mathrm{C} 7$ & C8 & $-52.59(16)$ \\
\hline
\end{tabular}




\begin{tabular}{|c|c|c|c|c|}
\hline Atom & Atom & Atom & Atom & Angle $/^{\circ}$ \\
\hline $\mathrm{C} 1$ & $\mathrm{P} 1$ & C7 & C12 & $133.35(15)$ \\
\hline C1 & $\mathrm{C} 2$ & $\mathrm{C} 3$ & $\mathrm{C} 4$ & $-0.1(3)$ \\
\hline $\mathrm{C} 2$ & $\mathrm{C} 1$ & C6 & $\mathrm{C} 5$ & $0.8(3)$ \\
\hline $\mathrm{C} 2$ & $\mathrm{C} 3$ & $\mathrm{C} 4$ & $\mathrm{C} 5$ & $0.6(3)$ \\
\hline C3 & $\mathrm{C} 4$ & $\mathrm{C} 5$ & $\mathrm{C} 6$ & $-0.3(3)$ \\
\hline $\mathrm{C} 4$ & $\mathrm{C} 5$ & C6 & $\mathrm{C} 1$ & $-0.4(3)$ \\
\hline C6 & $\mathrm{C} 1$ & $\mathrm{C} 2$ & $\mathrm{C} 3$ & $-0.6(3)$ \\
\hline $\mathrm{C} 7$ & $\mathrm{P} 1$ & $\mathrm{~N}$ & $\mathrm{C} 13$ & $66.80(15)$ \\
\hline $\mathrm{C} 7$ & $\mathrm{P} 1$ & $\mathrm{~N}$ & C14 & $-136.16(13)$ \\
\hline $\mathrm{C} 7$ & $\mathrm{P} 1$ & $\mathrm{C} 1$ & $\mathrm{C} 2$ & 151.31(15) \\
\hline $\mathrm{C} 7$ & $\mathrm{P} 1$ & $\mathrm{C} 1$ & $\mathrm{C} 6$ & $-29.91(17)$ \\
\hline C7 & C8 & C9 & C10 & $0.0(3)$ \\
\hline C8 & $\mathrm{C} 7$ & C12 & C11 & $0.3(3)$ \\
\hline C8 & C9 & C10 & $\mathrm{C} 11$ & $0.5(3)$ \\
\hline C9 & $\mathrm{C} 10$ & C11 & $\mathrm{C} 12$ & $-0.5(3)$ \\
\hline C10 & C11 & $\mathrm{C} 12$ & $\mathrm{C} 7$ & $0.2(3)$ \\
\hline C12 & $\mathrm{C} 7$ & $\mathrm{C} 8$ & C9 & $-0.4(3)$ \\
\hline C13 & $\mathrm{N}$ & C14 & C15 & $65.4(2)$ \\
\hline C13 & $\mathrm{N}$ & C14 & C16 & $-59.5(2)$ \\
\hline C14 & C16 & C17 & C18 & $134.01(17)$ \\
\hline C14 & C16 & C17 & $\mathrm{C} 22$ & $-47.4(2)$ \\
\hline C15 & $\mathrm{C} 14$ & C16 & 0 & $63.75(18)$ \\
\hline C15 & C14 & C16 & $\mathrm{C} 17$ & $-175.62(15)$ \\
\hline C16 & $\mathrm{C} 17$ & C18 & C19 & $178.89(17)$ \\
\hline C16 & $\mathrm{C} 17$ & $\mathrm{C} 22$ & $\mathrm{C} 21$ & $-178.01(16)$ \\
\hline C17 & C18 & C19 & $\mathrm{C} 20$ & $-0.5(3)$ \\
\hline C18 & C17 & $\mathrm{C} 22$ & $\mathrm{C} 21$ & $0.6(3)$ \\
\hline C18 & C19 & $\mathrm{C} 20$ & $\mathrm{C} 21$ & $-0.1(3)$ \\
\hline C19 & $\mathrm{C} 20$ & $\mathrm{C} 21$ & $\mathrm{C} 22$ & $1.0(3)$ \\
\hline C20 & $\mathrm{C} 21$ & $\mathrm{C} 22$ & $\mathrm{C} 17$ & $-1.2(3)$ \\
\hline C22 & $\mathrm{C} 17$ & C18 & C19 & $0.3(3)$ \\
\hline C23 & P2 & 0 & $\mathrm{C} 16$ & $-80.96(13)$ \\
\hline C23 & $\mathrm{P} 2$ & $\mathrm{C} 29$ & C30 & $-73.83(17)$ \\
\hline C23 & $\mathrm{P} 2$ & $\mathrm{C} 29$ & $\mathrm{C} 38$ & $104.12(16)$ \\
\hline C23 & $\mathrm{C} 24$ & $\mathrm{C} 25$ & $\mathrm{C} 26$ & $0.7(3)$ \\
\hline C24 & $\mathrm{C} 23$ & $\mathrm{C} 28$ & $\mathrm{C} 27$ & $-0.6(3)$ \\
\hline C24 & $\mathrm{C} 25$ & $\mathrm{C} 26$ & $\mathrm{C} 27$ & $-0.1(4)$ \\
\hline C25 & $\mathrm{C} 26$ & $\mathrm{C} 27$ & $\mathrm{C} 28$ & $-0.8(4)$ \\
\hline C26 & $\mathrm{C} 27$ & $\mathrm{C} 28$ & $\mathrm{C} 23$ & $1.2(4)$ \\
\hline C28 & $\mathrm{C} 23$ & $\mathrm{C} 24$ & $\mathrm{C} 25$ & $-0.3(3)$ \\
\hline C29 & $\mathrm{P} 2$ & 0 & C16 & $168.92(13)$ \\
\hline C29 & P2 & $\mathrm{C} 23$ & $\mathrm{C} 24$ & $73.10(17)$ \\
\hline C29 & $\mathrm{P} 2$ & $\mathrm{C} 23$ & $\mathrm{C} 28$ & $-106.62(18)$ \\
\hline C29 & C30 & C31 & C32 & $-0.2(3)$ \\
\hline C30 & $\mathrm{C} 29$ & C38 & C37 & $1.0(3)$ \\
\hline C30 & C31 & C32 & C33 & $-179.9(2)$ \\
\hline C30 & C31 & C32 & C37 & $0.7(3)$ \\
\hline C31 & C32 & C33 & C34 & $-179.6(2)$ \\
\hline C31 & C32 & C37 & C36 & $179.30(19)$ \\
\hline C31 & C32 & $\mathrm{C} 37$ & C38 & $-0.4(3)$ \\
\hline C32 & C33 & C34 & C35 & $0.4(3)$ \\
\hline C32 & C37 & C38 & $\mathrm{C} 29$ & $-0.5(3)$ \\
\hline C33 & C32 & C37 & C36 & $-0.1(3)$ \\
\hline C33 & C32 & C37 & C38 & $-179.82(18)$ \\
\hline
\end{tabular}




\begin{tabular}{llllc}
\hline Atom & Atom & Atom & Atom & Angle $/^{\circ}$ \\
\hline C33 & C34 & C35 & C36 & $-0.3(4)$ \\
C34 & C35 & C36 & C37 & $-0.1(4)$ \\
C35 & C36 & C37 & C32 & $0.3(3)$ \\
C35 & C36 & C37 & C38 & $180.0(2)$ \\
C36 & C37 & C38 & C29 & $179.8(2)$ \\
C37 & C32 & C33 & C34 & $-0.2(3)$ \\
C38 & C29 & C30 & C31 & $-0.7(3)$ \\
B1 & P1 & N & C13 & $-171.66(14)$ \\
B1 & P1 & N & C14 & $-14.63(17)$ \\
B1 & P1 & C1 & C2 & $31.90(18)$ \\
B1 & P1 & C1 & C6 & $-149.32(15)$ \\
B1 & P1 & C7 & C8 & $68.56(16)$ \\
B1 & P1 & C7 & C12 & $-105.50(16)$ \\
B2 & P2 & O & C16 & $45.73(16)$ \\
B2 & P2 & C23 & C24 & $-160.19(16)$ \\
B2 & P2 & C23 & C28 & $20.1(2)$ \\
B2 & P2 & C29 & C30 & $159.49(16)$ \\
B2 & P2 & C29 & C38 & $-22.56(19)$ \\
C39 & C40 & C41 & C42 & $-54.8(3)$ \\
C40 & C39 & C44 & C43 & $-54.0(3)$ \\
C40 & C41 & C42 & C43 & $55.7(3)$ \\
C41 & C42 & C43 & C44 & $-55.9(3)$ \\
C42 & C43 & C44 & C39 & $54.9(3)$ \\
C44 & C39 & C40 & C41 & $53.9(3)$
\end{tabular}


Name: $\left(R_{\mathrm{p}}\right)-(-)-\mathrm{N}-[(1 R, 2 S), \quad$ 2-(Phenyl-o-tolylphosphinito-borane)-1-methyl-2-phenylethyl], N-methyl aminodiphenylphosphine-borane $\mathbf{2 1 d}$.

Submitted by: Antonin Jaillet

Solved by: Yoann Rousselin

Sample ID: $\quad$ 15AJ264

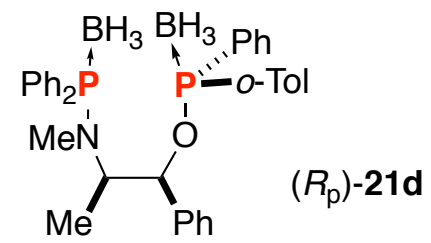

\section{Crystal Data and Experimental}

Figure S11 (thermal ellipsoïds are drawn at $50 \%$ probability plot)

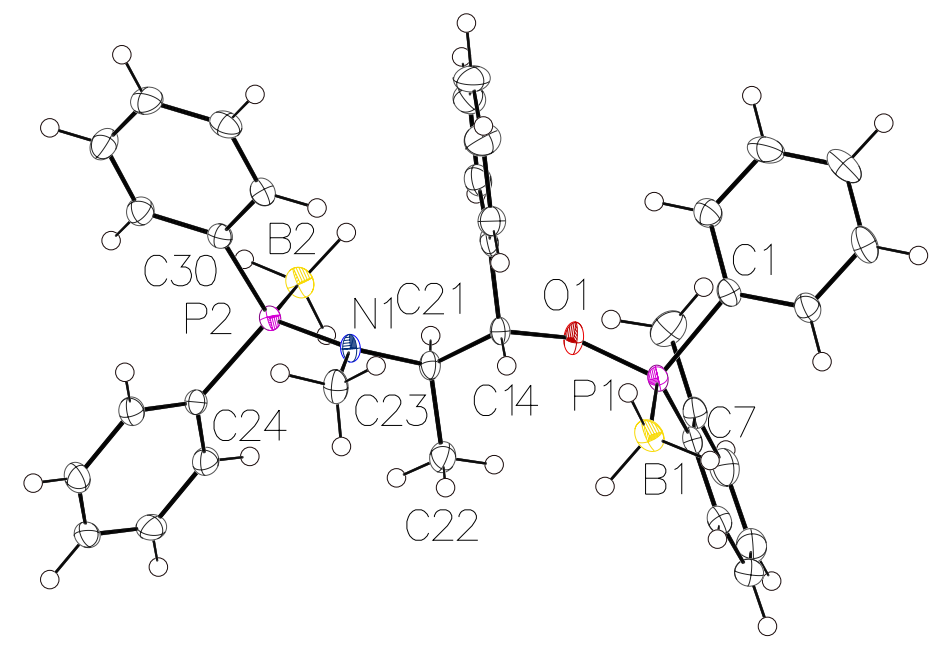

Experimental. Single clear light colorless prism-shaped crystals of compound 21d $(\boldsymbol{R})$ were recrystallized from hexane by slow evaporation. A suitable crystal $0.60 \times 0.54 \times 0.48 \mathrm{~mm}^{3}$ was selected and mounted on a MITIGEN holder oil on a Nonius Kappa APEX II diffractometer. The crystal was kept at a steady $T=$ 115.0(1) K during data collection. The structure was solved with the ShelXT (Sheldrick, 2015) structure solution program using the direct solution method and by using Olex2 (Dolomanov et al., 2009) as the graphical interface. The model was refined with version 2018/3 of ShelXL (Sheldrick, 2015) using Least Squares minimization.

Crystal Data. $\mathrm{C}_{35} \mathrm{H}_{41} \mathrm{~B}_{2} \mathrm{NOP}_{2}, M_{r}=575.25$, orthorhombic, $P 2{ }_{1} 2_{1} 2_{1}$ (No. 19), $\mathrm{a}=13.4215(7) \AA, \mathrm{b}=13.8198(7) \AA \AA, \mathrm{c}=$ 17.5737(9) $\AA, \alpha=\beta=\gamma=90^{\circ}, \quad V=3259.6(3) \AA^{3}, \quad T=$ 115.0(1) $\mathrm{K}, \quad Z=4, Z^{\prime}=1, \mu\left(\mathrm{MoK}_{\alpha}\right)=0.161,124578$ reflections measured, 7478 unique $\left(R_{\text {int }}=0.0349\right)$ which were used in all calculations. The final $w R_{2}$ was 0.0740 (all data) and $R_{1}$ was 0.0274 (I > 2(I)). 


\begin{tabular}{|c|c|}
\hline Compound & $21 \mathrm{~d}(R)$ \\
\hline CCDC & 1982660 \\
\hline Formula & $\mathrm{C}_{35} \mathrm{H}_{41} \mathrm{~B}_{2} \mathrm{NOP}_{2}$ \\
\hline$D_{\text {calc. }} / \mathrm{g} \mathrm{cm}^{-3}$ & 1.172 \\
\hline$\mu / \mathrm{mm}^{-1}$ & 0.161 \\
\hline Formula Weight & 575.25 \\
\hline Color & clear light colorless \\
\hline Shape & prism \\
\hline Size $/ \mathrm{mm}^{3}$ & $0.60 \times 0.54 \times 0.48$ \\
\hline$T / \mathrm{K}$ & $115.0(1)$ \\
\hline Crystal System & orthorhombic \\
\hline Flack Parameter & $-0.022(10)$ \\
\hline Hooft Parameter & $-0.013(10)$ \\
\hline Space Group & $P 2{ }_{1} 2{ }_{1}{ }_{1}$ \\
\hline$a / \AA$ & $13.4215(7)$ \\
\hline$b / \AA$ & $13.8198(7)$ \\
\hline$c / \AA$ & $17.5737(9)$ \\
\hline$\alpha /^{\circ}$ & 90 \\
\hline$\beta /^{\circ}$ & 90 \\
\hline$\gamma /^{\circ}$ & 90 \\
\hline $\mathrm{V} / \AA^{3}$ & $3259.6(3)$ \\
\hline$Z$ & 4 \\
\hline$Z^{\prime}$ & 1 \\
\hline Wavelength/Å & 0.71073 \\
\hline Radiation type & $\mathrm{MoK}_{\alpha}$ \\
\hline$\Theta_{\min } /^{\circ}$ & 1.875 \\
\hline$\Theta_{\max } /^{\circ}$ & 27.526 \\
\hline Measured Refl. & 124578 \\
\hline Independent Refl. & 7478 \\
\hline Reflections with I > & 7038 \\
\hline $2(\mathrm{I})$ & \\
\hline$R_{\text {int }}$ & 0.0349 \\
\hline Parameters & 377 \\
\hline Restraints & 0 \\
\hline Largest Peak & 0.262 \\
\hline Deepest Hole & -0.215 \\
\hline GooF & 1.068 \\
\hline$w R_{2}$ (all data) & 0.0740 \\
\hline$w R_{2}$ & 0.0705 \\
\hline$R_{1}$ (all data) & 0.0316 \\
\hline$R_{1}$ & 0.0274 \\
\hline
\end{tabular}


Reflections:

Refinement:

A clear light colorless prism-shaped crystal with dimensions $0.60 \times 0.54 \times 0.48 \mathrm{~mm}^{3}$ was mounted on a MITIGEN holder oil. Data were collected using a Nonius Kappa APEX II diffractometer equipped with an Oxford Cryosystems low-temperature device operating at $T=115.0(1) \mathrm{K}$. Data were measured using $\phi$ and $\omega$ scans using $\mathrm{MoK}_{\alpha}$ radiation. The total number of runs and images was based on the strategy calculation from the program APEX3 (Bruker, 2015) The maximum resolution that was achieved was $\Theta$ $=27.526^{\circ}(0.77 \AA)$. The diffraction pattern was indexed. The total number of runs and images was based on the strategy calculation from the program APEX3 (Bruker, 2015) and the unit cell was refined using SAINT (Bruker, V8.34A, after 2013) on 9003 reflections, 7\% of the observed reflections. Data reduction, scaling and absorption corrections were performed using SAINT (Bruker, V8.34A, after 2013). The final completeness is $99.80 \%$ out to $27.526^{\circ}$ in $\Theta$. A multi-scan absorption correction was performed using SADABS-2014/5 (Bruker,2014) was used for absorption correction. $w R_{2}$ (int) was 0.0542 before and 0.0461 after correction. The Ratio of minimum to maximum transmission is 0.9500 . The $\lambda / 2$ correction factor is 0.00150 . The absorption coefficient $\mu$ of this material is $0.161 \mathrm{~mm}^{-1}$ at this wavelength $(\lambda=$ $0.711 \AA$ ) and the minimum and maximum transmissions are 0.708 and 0.746 . The structure was solved and the space group $P 2{ }_{1} 2_{1} 2_{1}$ (\# 19) determined by the ShelXT (Sheldrick, 2015) structure solution program using direct method and refined by Least Squares using version 2018/3 of ShelXL (Sheldrick, 2015). All non-hydrogen atoms were refined anisotropically. Hydrogen atom positions were calculated geometrically and refined using the riding model. Hydrogen atom on Boron atom were refined using an AFIX 138, which would allow the refinement of a common B-H distances, the same shifts being applied to all the $\mathrm{B}-\mathrm{H}$ bonds to the same atom, but still retain tetrahedral $\mathrm{H}-\mathrm{B}-\mathrm{H}$ angles within the group. There is a single molecule in the asymmetric unit, which is represented by the reported sum formula. In other words: $\mathrm{Z}$ is 4 and $\mathrm{Z}$ ' is 1 . The Flack parameter was refined to $-0.022(10)$. Determination of absolute structure using Bayesian statistics on Bijvoet differences using the Olex2 results in -0.013(10). Note: The Flack parameter is used to determine chirality of the crystal studied, the value should be near 0 , a value of 1 means that the stereochemistry is wrong and the model should be inverted. A value of 0.5 means that the crystal consists of a racemic mixture of the two enantiomers.

Table 32: Bond Lengths in $\AA$ for compound 21d (R).

\begin{tabular}{lll}
\hline Atom & Atom & Length/Å \\
\hline P1 & O1 & $1.6081(13)$ \\
P1 & C1 & $1.8102(18)$ \\
P1 & C7 & $1.807(2)$ \\
P1 & B1 & $1.908(2)$ \\
P2 & N1 & $1.6666(15)$ \\
P2 & C30 & $1.8127(18)$ \\
P2 & C24 & $1.8196(18)$ \\
P2 & B2 & $1.911(2)$ \\
O1 & C14 & $1.463(2)$ \\
N1 & C23 & $1.466(2)$ \\
N1 & C21 & $1.472(2)$ \\
C4 & C5 & $1.382(3)$ \\
C4 & C3 & $1.382(3)$ \\
C5 & C6 & $1.385(3)$ \\
C11 & C12 & $1.400(3)$ \\
C11 & C10 & $1.383(3)$ \\
C9 & C10 & $1.378(4)$
\end{tabular}

\begin{tabular}{lll}
\hline Atom & Atom & Length/Å \\
\hline C9 & C8 & $1.386(3)$ \\
C6 & C1 & $1.393(3)$ \\
C1 & C2 & $1.393(3)$ \\
C14 & C21 & $1.539(2)$ \\
C14 & C15 & $1.509(3)$ \\
C21 & C22 & $1.528(3)$ \\
C30 & C31 & $1.402(2)$ \\
C30 & C35 & $1.393(2)$ \\
C31 & C32 & $1.387(3)$ \\
C32 & C33 & $1.387(3)$ \\
C33 & C34 & $1.384(3)$ \\
C27 & C26 & $1.382(3)$ \\
C27 & C28 & $1.379(3)$ \\
C26 & C25 & $1.396(3)$ \\
C25 & C24 & $1.393(3)$ \\
C24 & C29 & $1.399(3)$ \\
C3 & C2 & $1.393(3)$
\end{tabular}




\begin{tabular}{|c|c|c|c|c|c|}
\hline Atom & Atom & Length/Å & Atom & Atom & Length/Å \\
\hline $\mathrm{C} 7$ & $\mathrm{C} 12$ & $1.410(3)$ & $\mathrm{C} 17$ & C18 & $1.376(4)$ \\
\hline C7 & C8 & $1.401(3)$ & C18 & C19 & $1.382(4)$ \\
\hline C12 & C13 & $1.497(3)$ & C19 & $\mathrm{C} 20$ & $1.388(3)$ \\
\hline C15 & C16 & $1.388(3)$ & C35 & C34 & $1.393(3)$ \\
\hline C15 & $\mathrm{C} 20$ & $1.393(3)$ & $\mathrm{C} 29$ & $\mathrm{C} 28$ & $1.391(3)$ \\
\hline C16 & $\mathrm{C} 17$ & $1.388(3)$ & & & \\
\hline
\end{tabular}

Table S33: Bond Angles in ${ }^{\circ}$ for compound 21d (R).

\begin{tabular}{|c|c|c|c|c|c|c|c|}
\hline Atom & Atom & Atom & Angle $/^{\circ}$ & Atom & Atom & Atom & Angle ${ }^{\circ}$ \\
\hline 01 & P1 & $\mathrm{C} 1$ & $104.67(8)$ & $\mathrm{C} 32$ & $\mathrm{C} 31$ & $\mathrm{C} 30$ & $120.17(18)$ \\
\hline 01 & P1 & $\mathrm{C} 7$ & $103.30(8)$ & C31 & $\mathrm{C} 32$ & C33 & $120.10(19)$ \\
\hline 01 & $\mathrm{P} 1$ & B1 & $115.30(9)$ & C34 & $\mathrm{C} 33$ & C32 & $120.02(18)$ \\
\hline C1 & $\mathrm{P} 1$ & B1 & 111.71(9) & $\mathrm{C} 28$ & $\mathrm{C} 27$ & $\mathrm{C} 26$ & $120.15(18)$ \\
\hline C7 & $\mathrm{P} 1$ & $\mathrm{C} 1$ & $106.14(8)$ & $\mathrm{C} 27$ & $\mathrm{C} 26$ & $\mathrm{C} 25$ & $120.26(19)$ \\
\hline $\mathrm{C} 7$ & $\mathrm{P} 1$ & B1 & $114.70(10)$ & $\mathrm{C} 24$ & $\mathrm{C} 25$ & $\mathrm{C} 26$ & $120.13(18)$ \\
\hline N1 & $\mathrm{P} 2$ & $\mathrm{C} 30$ & $106.69(8)$ & $\mathrm{C} 25$ & $\mathrm{C} 24$ & P2 & $123.53(14)$ \\
\hline N1 & $\mathrm{P} 2$ & $\mathrm{C} 24$ & 108.51(8) & $\mathrm{C} 25$ & $\mathrm{C} 24$ & $\mathrm{C} 29$ & $118.86(17)$ \\
\hline $\mathrm{N} 1$ & $\mathrm{P} 2$ & B2 & $113.29(9)$ & $\mathrm{C} 29$ & $\mathrm{C} 24$ & P2 & $117.59(14)$ \\
\hline C30 & $\mathrm{P} 2$ & $\mathrm{C} 24$ & $105.79(8)$ & $\mathrm{C} 4$ & $\mathrm{C} 3$ & $\mathrm{C} 2$ & $120.3(2)$ \\
\hline C30 & P2 & B2 & $110.87(9)$ & $\mathrm{C} 1$ & $\mathrm{C} 2$ & $\mathrm{C} 3$ & $119.73(19)$ \\
\hline $\mathrm{C} 24$ & $\mathrm{P} 2$ & B2 & 111.31(9) & $\mathrm{C} 12$ & $\mathrm{C} 7$ & $\mathrm{P} 1$ & $124.05(14)$ \\
\hline C14 & 01 & $\mathrm{P} 1$ & $122.31(11)$ & C8 & $\mathrm{C} 7$ & $\mathrm{P} 1$ & $116.71(15)$ \\
\hline $\mathrm{C} 23$ & N1 & $\mathrm{P} 2$ & $119.10(12)$ & $\mathrm{C} 8$ & $\mathrm{C} 7$ & C12 & $119.23(18)$ \\
\hline C23 & N1 & $\mathrm{C} 21$ & $117.04(14)$ & C11 & $\mathrm{C} 12$ & C7 & 117.94(19) \\
\hline $\mathrm{C} 21$ & N1 & P2 & $119.93(12)$ & C11 & $\mathrm{C} 12$ & C13 & $118.38(19)$ \\
\hline C5 & $\mathrm{C} 4$ & $\mathrm{C} 3$ & $120.21(19)$ & $\mathrm{C} 7$ & $\mathrm{C} 12$ & C13 & $123.68(18)$ \\
\hline C4 & $\mathrm{C} 5$ & $\mathrm{C} 6$ & 119.88(19) & C9 & C10 & C11 & $119.9(2)$ \\
\hline C10 & C11 & $\mathrm{C} 12$ & $122.0(2)$ & $\mathrm{C} 9$ & C8 & C7 & $121.4(2)$ \\
\hline C10 & C9 & $\mathrm{C} 8$ & $119.4(2)$ & C16 & C15 & C14 & $119.30(17)$ \\
\hline C5 & C6 & C1 & $120.50(18)$ & C16 & C15 & $\mathrm{C} 20$ & $119.20(18)$ \\
\hline C6 & C1 & P1 & $118.03(14)$ & $\mathrm{C} 20$ & C15 & C14 & $121.49(18)$ \\
\hline $\mathrm{C} 2$ & $\mathrm{C} 1$ & $\mathrm{P} 1$ & $122.57(14)$ & C15 & C16 & $\mathrm{C} 17$ & $120.3(2)$ \\
\hline $\mathrm{C} 2$ & $\mathrm{C} 1$ & $\mathrm{C} 6$ & $119.40(17)$ & C18 & $\mathrm{C} 17$ & C16 & $120.3(2)$ \\
\hline 01 & C14 & $\mathrm{C} 21$ & $104.67(14)$ & C17 & C18 & C19 & $119.9(2)$ \\
\hline 01 & C14 & C15 & $108.92(14)$ & C18 & C19 & $\mathrm{C} 20$ & $120.2(2)$ \\
\hline C15 & C14 & $\mathrm{C} 21$ & $114.45(15)$ & C19 & $\mathrm{C} 20$ & C15 & $120.1(2)$ \\
\hline N1 & $\mathrm{C} 21$ & C14 & $109.24(14)$ & $\mathrm{C} 30$ & $\mathrm{C} 35$ & C34 & $119.88(17)$ \\
\hline N1 & $\mathrm{C} 21$ & $\mathrm{C} 22$ & $112.93(15)$ & C33 & $\mathrm{C} 34$ & C35 & $120.40(18)$ \\
\hline C22 & $\mathrm{C} 21$ & C14 & $111.22(15)$ & $\mathrm{C} 28$ & $\mathrm{C} 29$ & $\mathrm{C} 24$ & $120.55(19)$ \\
\hline C31 & C30 & $\mathrm{P} 2$ & $119.65(13)$ & $\mathrm{C} 27$ & $\mathrm{C} 28$ & $\mathrm{C} 29$ & $120.04(19)$ \\
\hline C35 & $\mathrm{C} 30$ & P2 & $120.63(13)$ & & & & \\
\hline C35 & C30 & C31 & $119.43(16)$ & & & & \\
\hline
\end{tabular}

Table S34: Torsion Angles in ${ }^{\circ}$ for compound $21 \mathrm{~d}(\boldsymbol{R})$.

\begin{tabular}{llllc}
\hline Atom & Atom & Atom & Atom & Angle $^{\circ}$ \\
\hline P1 & O1 & C14 & C21 & $-131.10(13)$ \\
P1 & O1 & C14 & C15 & $106.09(15)$ \\
P1 & C1 & C2 & C3 & $-179.08(16)$ \\
P1 & C7 & C12 & C11 & $179.03(14)$ \\
P1 & C7 & C12 & C13 & $-1.0(3)$ \\
P1 & C7 & C8 & C9 & $-178.41(15)$ \\
P2 & N1 & C21 & C14 & $139.11(13)$
\end{tabular}




\begin{tabular}{|c|c|c|c|c|}
\hline Atom & Atom & Atom & Atom & Angle ${ }^{\circ}$ \\
\hline $\mathrm{P} 2$ & N1 & $\mathrm{C} 21$ & $\mathrm{C} 22$ & $-96.56(17)$ \\
\hline P2 & C30 & C31 & $\mathrm{C} 32$ & $-174.74(16)$ \\
\hline P2 & C30 & C35 & C34 & $174.40(14)$ \\
\hline P2 & $\mathrm{C} 24$ & $\mathrm{C} 29$ & $\mathrm{C} 28$ & $179.82(15)$ \\
\hline 01 & P1 & $\mathrm{C} 1$ & $\mathrm{C} 6$ & $177.20(15)$ \\
\hline 01 & $\mathrm{P} 1$ & $\mathrm{C} 1$ & $\mathrm{C} 2$ & $-2.33(18)$ \\
\hline 01 & $\mathrm{P} 1$ & $\mathrm{C} 7$ & $\mathrm{C} 12$ & $46.97(17)$ \\
\hline 01 & $\mathrm{P} 1$ & $\mathrm{C} 7$ & C8 & $-134.41(14)$ \\
\hline 01 & C14 & $\mathrm{C} 21$ & N1 & $-170.25(13)$ \\
\hline 01 & C14 & $\mathrm{C} 21$ & $\mathrm{C} 22$ & $64.43(19)$ \\
\hline 01 & C14 & C15 & C16 & $-118.28(17)$ \\
\hline 01 & C14 & C15 & $\mathrm{C} 20$ & $60.8(2)$ \\
\hline N1 & P2 & C30 & C31 & $-165.25(14)$ \\
\hline N1 & $\mathrm{P} 2$ & C30 & C35 & $20.93(17)$ \\
\hline N1 & P2 & $\mathrm{C} 24$ & $\mathrm{C} 25$ & $91.52(16)$ \\
\hline N1 & $\mathrm{P} 2$ & $\mathrm{C} 24$ & $\mathrm{C} 29$ & $-87.19(15)$ \\
\hline $\mathrm{C} 4$ & $\mathrm{C} 5$ & C6 & $\mathrm{C} 1$ & $0.1(3)$ \\
\hline $\mathrm{C} 4$ & $\mathrm{C} 3$ & $\mathrm{C} 2$ & $\mathrm{C} 1$ & $-0.3(3)$ \\
\hline C23 & N1 & $\mathrm{C} 21$ & C14 & $-63.4(2)$ \\
\hline C23 & N1 & $\mathrm{C} 21$ & $\mathrm{C} 22$ & $60.9(2)$ \\
\hline C5 & $\mathrm{C} 4$ & $\mathrm{C} 3$ & $\mathrm{C} 2$ & $-0.9(3)$ \\
\hline C5 & C6 & $\mathrm{C} 1$ & $\mathrm{P} 1$ & $179.17(16)$ \\
\hline C5 & $\mathrm{C} 6$ & $\mathrm{C} 1$ & $\mathrm{C} 2$ & $-1.3(3)$ \\
\hline C6 & $\mathrm{C} 1$ & $\mathrm{C} 2$ & $\mathrm{C} 3$ & $1.4(3)$ \\
\hline C1 & $\mathrm{P} 1$ & 01 & $\mathrm{C} 14$ & $-110.78(13)$ \\
\hline C1 & $\mathrm{P} 1$ & $\mathrm{C} 7$ & $\mathrm{C} 12$ & $-62.87(17)$ \\
\hline $\mathrm{C} 1$ & $\mathrm{P} 1$ & $\mathrm{C} 7$ & $\mathrm{C} 8$ & $115.76(15)$ \\
\hline C14 & $\mathrm{C} 15$ & C16 & $\mathrm{C} 17$ & $179.63(19)$ \\
\hline C14 & $\mathrm{C} 15$ & $\mathrm{C} 20$ & C19 & $-179.12(18)$ \\
\hline $\mathrm{C} 21$ & C14 & C15 & C16 & $124.99(18)$ \\
\hline C21 & C14 & C15 & $\mathrm{C} 20$ & $-55.9(2)$ \\
\hline C30 & P2 & N1 & $\mathrm{C} 23$ & $65.20(15)$ \\
\hline C30 & $\mathrm{P} 2$ & N1 & $\mathrm{C} 21$ & $-137.77(13)$ \\
\hline C30 & $\mathrm{P} 2$ & $\mathrm{C} 24$ & $\mathrm{C} 25$ & $-22.64(17)$ \\
\hline C30 & $\mathrm{P} 2$ & $\mathrm{C} 24$ & $\mathrm{C} 29$ & $158.64(14)$ \\
\hline C30 & C31 & C32 & C33 & $0.6(3)$ \\
\hline C30 & C35 & C34 & C33 & $0.0(3)$ \\
\hline C31 & C30 & C35 & C34 & $0.6(3)$ \\
\hline C31 & C32 & C33 & C34 & $0.0(3)$ \\
\hline C32 & C33 & C34 & C35 & $-0.3(3)$ \\
\hline $\mathrm{C} 27$ & $\mathrm{C} 26$ & $\mathrm{C} 25$ & $\mathrm{C} 24$ & $0.6(3)$ \\
\hline C26 & $\mathrm{C} 27$ & $\mathrm{C} 28$ & $\mathrm{C} 29$ & $-0.7(3)$ \\
\hline C26 & $\mathrm{C} 25$ & $\mathrm{C} 24$ & P2 & $-179.99(15)$ \\
\hline C26 & $\mathrm{C} 25$ & $\mathrm{C} 24$ & $\mathrm{C} 29$ & $-1.3(3)$ \\
\hline $\mathrm{C} 25$ & $\mathrm{C} 24$ & $\mathrm{C} 29$ & $\mathrm{C} 28$ & $1.0(3)$ \\
\hline C24 & $\mathrm{P} 2$ & N1 & $\mathrm{C} 23$ & $-48.37(16)$ \\
\hline C24 & $\mathrm{P} 2$ & N1 & $\mathrm{C} 21$ & $108.65(14)$ \\
\hline C24 & $\mathrm{P} 2$ & C30 & C31 & $-49.83(17)$ \\
\hline C24 & $\mathrm{P} 2$ & C30 & C35 & $136.34(15)$ \\
\hline $\mathrm{C} 24$ & $\mathrm{C} 29$ & $\mathrm{C} 28$ & $\mathrm{C} 27$ & $-0.1(3)$ \\
\hline C3 & $\mathrm{C} 4$ & $\mathrm{C} 5$ & $\mathrm{C} 6$ & 1.1(3) \\
\hline C7 & $\mathrm{P} 1$ & 01 & C14 & $138.29(13)$ \\
\hline C7 & $\mathrm{P} 1$ & $\mathrm{C} 1$ & C6 & $-73.94(17)$ \\
\hline $\mathrm{C} 7$ & $\mathrm{P} 1$ & $\mathrm{C} 1$ & $\mathrm{C} 2$ & $106.54(17)$ \\
\hline
\end{tabular}




\begin{tabular}{|c|c|c|c|c|}
\hline Atom & Atom & Atom & Atom & Angle $/^{\circ}$ \\
\hline $\mathrm{C} 12$ & C11 & C10 & C9 & $0.8(3)$ \\
\hline C12 & C7 & $\mathrm{C} 8$ & C9 & $0.3(3)$ \\
\hline C10 & C11 & C12 & C7 & $-1.0(3)$ \\
\hline C10 & C11 & C12 & C13 & $179.0(2)$ \\
\hline C10 & C9 & $\mathrm{C} 8$ & C7 & $-0.5(3)$ \\
\hline C8 & C9 & C10 & C11 & $-0.1(3)$ \\
\hline C8 & C7 & C12 & C11 & $0.4(3)$ \\
\hline C8 & C7 & C12 & C13 & -179.54(19) \\
\hline C15 & C14 & $\mathrm{C} 21$ & N1 & $-51.1(2)$ \\
\hline C15 & C14 & $\mathrm{C} 21$ & $\mathrm{C} 22$ & $-176.42(16)$ \\
\hline C15 & C16 & C17 & C18 & $-1.0(3)$ \\
\hline C16 & C15 & $\mathrm{C} 20$ & C19 & $0.0(3)$ \\
\hline C16 & C17 & C18 & C19 & $1.0(4)$ \\
\hline C17 & C18 & C19 & $\mathrm{C} 20$ & $-0.5(4)$ \\
\hline C18 & C19 & C20 & C15 & $0.0(3)$ \\
\hline C20 & C15 & C16 & C17 & $0.5(3)$ \\
\hline C35 & C30 & C31 & C32 & $-0.8(3)$ \\
\hline $\mathrm{C} 28$ & $\mathrm{C} 27$ & C26 & $\mathrm{C} 25$ & $0.4(3)$ \\
\hline B2 & P2 & $\mathrm{N} 1$ & $\mathrm{C} 23$ & $-172.52(14)$ \\
\hline B2 & P2 & $\mathrm{N} 1$ & $\mathrm{C} 21$ & $-15.50(16)$ \\
\hline B2 & P2 & C30 & C31 & $70.97(17)$ \\
\hline B2 & P2 & C30 & C35 & $-102.85(16)$ \\
\hline B2 & P2 & $\mathrm{C} 24$ & $\mathrm{C} 25$ & $-143.16(16)$ \\
\hline B2 & $\mathrm{P} 2$ & $\mathrm{C} 24$ & C29 & $38.12(17)$ \\
\hline B1 & P1 & 01 & C14 & $12.36(17)$ \\
\hline B1 & P1 & C1 & C6 & $51.76(18)$ \\
\hline B1 & P1 & C1 & $\mathrm{C} 2$ & $-127.76(17)$ \\
\hline B1 & $\mathrm{P} 1$ & $\mathrm{C} 7$ & $\mathrm{C} 12$ & $173.28(15)$ \\
\hline B1 & P1 & C7 & C8 & $-8.09(18)$ \\
\hline
\end{tabular}


Name: $\left(S_{\mathrm{p}}\right)-(-)-\mathrm{N}-[(1 S, 2 R), \quad$ 2-(Phenyl-o-tolylphosphinito-borane)-1-methyl-2-phenylethyl], N-methyl aminodiphenylphosphine-borane 21d.

Submitted by: Antonin Jaillet

Solved by: Yoann Rousselin

Sample ID: $\quad$ 16aj359

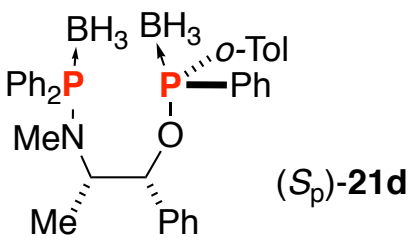

\section{Crystal Data and Experimental}

Figure S12 (thermal ellipsoïds are drawn at $50 \%$ probability plot)

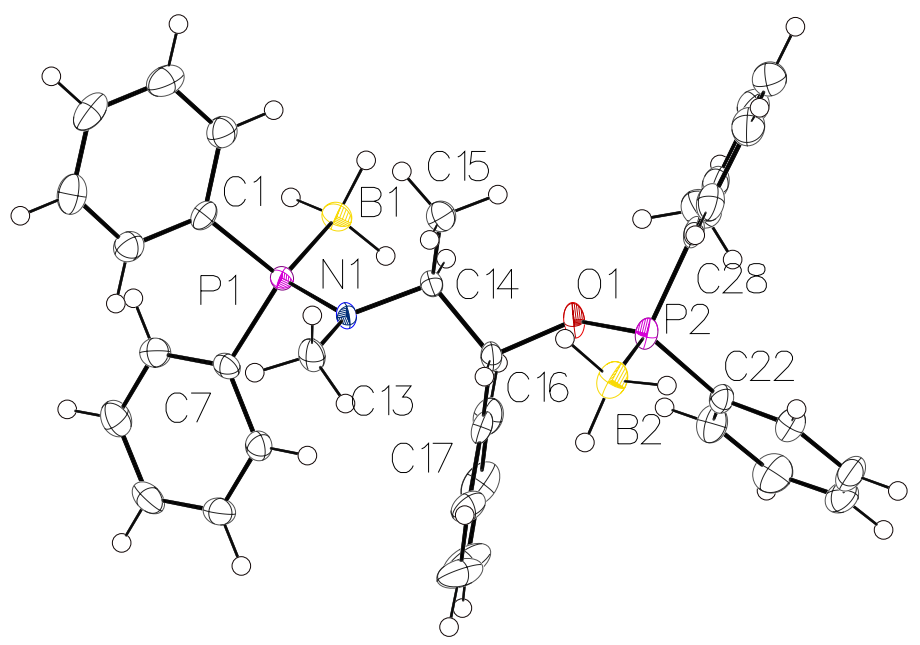

Experimental. Single clear light colorless prism-shaped crystals of compound 21d $(S)$ were recrystallized from hexane by slow evaporation. A suitable crystal $0.84 \times 0.57 \times 0.16 \mathrm{~mm}^{3}$ was selected and mounted on a MITIGEN holder oil on an Nonius Kappa APEX II diffractometer. The crystal was kept at a steady $T=$ 115.0(1) K during data collection. The structure was solved with the ShelXT (Sheldrick, 2015) structure solution program using the Intrinsic Phasing solution method and by using 0lex2 (Dolomanov et al., 2009) as the graphical interface. The model was refined with version 2018/3 of ShelXL (Sheldrick, 2015) using Least Squares minimization.

Crystal Data. $\mathrm{C}_{35} \mathrm{H}_{41} \mathrm{~B}_{2} \mathrm{NOP}_{2}, M_{r}=575.25$, orthorhombic, $P 2{ }_{1} 2_{1} 2_{1}$ (No. 19), $\mathrm{a}=13.4313(5) \AA, \mathrm{b}=13.8152(6) \AA, \mathrm{c}=$ 17.5341(7) $\AA, \alpha=\beta=\gamma=90^{\circ}, \quad V=3253.6(2) \AA^{3}, \quad T=$ 115.0(1) K, $Z=4, Z^{\prime}=1, \mu\left(\mathrm{MoK}_{\alpha}\right)=0.161,61086$ reflections measured, 7485 unique $\left(R_{\text {int }}=0.0489\right)$ which were used in all calculations. The final $w R_{2}$ was 0.0899 (all data) and $R_{1}$ was 0.0349 (I > 2(I)). 


\begin{tabular}{|c|c|}
\hline Compound & compound $21 \mathrm{~d}(S)$ \\
\hline CCDC & 1982656 \\
\hline Formula & $\mathrm{C}_{35} \mathrm{H}_{41} \mathrm{~B}_{2} \mathrm{NOP}_{2}$ \\
\hline$D_{\text {calc. }} / \mathrm{g} \mathrm{cm}^{-3}$ & 1.174 \\
\hline$\mu / \mathrm{mm}^{-1}$ & 0.161 \\
\hline Formula Weight & 575.25 \\
\hline Color & clear light colorless \\
\hline Shape & prism \\
\hline Size $/ \mathrm{mm}^{3}$ & $0.84 \times 0.57 \times 0.16$ \\
\hline$T / \mathrm{K}$ & $115.0(1)$ \\
\hline Crystal System & orthorhombic \\
\hline Flack Parameter & $-0.03(2)$ \\
\hline Hooft Parameter & $-0.02(2)$ \\
\hline Space Group & $P 2{ }_{1} 2_{1} 2_{1}$ \\
\hline$a / \AA$ & $13.4313(5)$ \\
\hline$b / \AA$ & $13.8152(6)$ \\
\hline$c / \AA$ & $17.5341(7)$ \\
\hline$\alpha /^{\circ}$ & 90 \\
\hline$\beta /^{\circ}$ & 90 \\
\hline$\gamma /{ }^{\circ}$ & 90 \\
\hline $\mathrm{V} / \AA^{3}$ & $3253.6(2)$ \\
\hline$Z$ & 4 \\
\hline$Z^{\prime}$ & 1 \\
\hline Wavelength/Å & 0.71073 \\
\hline Radiation type & $\mathrm{MoK}_{\alpha}$ \\
\hline$\Theta_{\min } /^{\circ}$ & 1.877 \\
\hline$\Theta_{\max } /^{\circ}$ & 27.558 \\
\hline Measured Refl. & 61086 \\
\hline Independent Refl. & 7485 \\
\hline Reflections with I > & 6677 \\
\hline $2(I)$ & \\
\hline$R_{\text {int }}$ & 0.0489 \\
\hline Parameters & 377 \\
\hline Restraints & 0 \\
\hline Largest Peak & 0.304 \\
\hline Deepest Hole & -0.200 \\
\hline GooF & 1.074 \\
\hline$w R_{2}$ (all data) & 0.0899 \\
\hline$w R_{2}$ & 0.0832 \\
\hline$R_{1}$ (all data) & 0.0449 \\
\hline$R_{1}$ & 0.0349 \\
\hline
\end{tabular}


Reflections:

A clear light colorless prism-shaped crystal with dimensions $0.84 \times 0.57 \times 0.16 \mathrm{~mm}^{3}$ was mounted on a MITIGEN holder oil. Data were collected using an Nonius Kappa APEX II diffractometer equipped with an Oxford Cryosystems low-temperature device operating at $T=115.0(1) \mathrm{K}$. Data were measured using $\phi$ and $\omega$ scans using $\mathrm{MoK}_{\alpha}$ radiation. The total number of runs and images was based on the strategy calculation from the program APEX3 (Bruker, 2015) The maximum resolution that was achieved was $\Theta$ $=27.558^{\circ}(0.77 \AA)$. The diffraction pattern was indexed. The total number of runs and images was based on the strategy calculation from the program APEX3 (Bruker, 2015) and the unit cell was refined using SAINT (Bruker, V8.37A, after 2013) on 9994 reflections, 16\% of the observed reflections. Data reduction, scaling and absorption corrections were performed using SAINT (Bruker, V8.37A, after 2013). The final completeness is $99.90 \%$ out to $27.558^{\circ}$ in $\Theta$. A multi-scan absorption correction was performed using SADABS-2014/5 (Bruker,2014) was used for absorption correction. $w R_{2}$ (int) was 0.0613 before and 0.0533 after correction. The Ratio of minimum to maximum transmission is 0.8997 . The $\lambda / 2$ correction factor is 0.00150 . The absorption coefficient $\mu$ of this material is $0.161 \mathrm{~mm}^{-1}$ at this wavelength $(\lambda=0.711 \AA)$ and the minimum and maximum transmissions are 0.671 and 0.746 . The structure was solved and the space group $P 2{ }_{1} 2_{1} 2_{1}$ (\# 19) determined by the ShelXT (Sheldrick, 2015) structure solution program using the Intrinsic Phasing solution method and refined by Least Squares using version 2018/3 of ShelXL (Sheldrick, 2015). All non-hydrogen atoms were refined anisotropically. Hydrogen atom positions were calculated geometrically and refined using the riding model. Hydrogen atom on Boron atom were refined using an AFIX 138, which would allow the refinement of a common B$\mathrm{H}$ distances, the same shifts being applied to all the $\mathrm{B}-\mathrm{H}$ bonds to the same atom, but still retain tetrahedral H-B-H angles within the group. There is a single molecule in the asymmetric unit, which is represented by the reported sum formula. In other words: $\mathrm{Z}$ is 4 and $\mathrm{Z}^{\prime}$ is 1 . The Flack parameter was refined to -0.03(2). Determination of absolute structure using Bayesian statistics on Bijvoet differences using the Olex2 results in -0.02(2). Note: The Flack parameter is used to determine chirality of the crystal studied, the value should be near 0 , a value of 1 means that the stereochemistry is wrong and the model should be inverted. A value of 0.5 means that the crystal consists of a racemic mixture of the two enantiomers.

Table S35: Bond Lengths in Å for compound 21d (S).

\begin{tabular}{lll}
\hline Atom & Atom & Length/Å \\
\hline P1 & N1 & $1.6648(19)$ \\
P1 & C1 & $1.822(2)$ \\
P1 & C7 & $1.810(2)$ \\
P1 & B1 & $1.908(3)$ \\
P2 & O1 & $1.6049(17)$ \\
P2 & C22 & $1.810(2)$ \\
P2 & C28 & $1.810(3)$ \\
P2 & B2 & $1.905(3)$ \\
01 & C16 & $1.464(3)$ \\
N1 & C13 & $1.466(3)$ \\
N1 & C14 & $1.470(3)$ \\
C1 & C2 & $1.394(4)$ \\
C1 & C6 & $1.387(4)$ \\
C2 & C3 & $1.389(4)$ \\
C3 & C4 & $1.372(4)$ \\
C4 & C5 & $1.378(4)$
\end{tabular}

\begin{tabular}{lll}
\hline Atom & Atom & Length/A \\
\hline C5 & C6 & $1.399(4)$ \\
C7 & C8 & $1.391(3)$ \\
C7 & C12 & $1.399(3)$ \\
C7AA & C17 & $1.390(4)$ \\
C7AA & C21 & $1.385(4)$ \\
C8 & C9 & $1.389(4)$ \\
C9 & C10 & $1.382(4)$ \\
C10 & C11 & $1.384(4)$ \\
C11 & C12 & $1.385(4)$ \\
C14 & C15 & $1.526(4)$ \\
C14 & C16 & $1.535(3)$ \\
C16 & C17 & $1.505(3)$ \\
C17 & C18 & $1.391(4)$ \\
C18 & C19 & $1.386(4)$ \\
C19 & C20 & $1.375(5)$ \\
C20 & C21 & $1.374(5)$
\end{tabular}




\begin{tabular}{|c|c|c|c|c|c|}
\hline Atom & Atom & Length/Å & Atom & Atom & Length/Å \\
\hline $\mathrm{C} 22$ & $\mathrm{C} 23$ & $1.389(4)$ & $\overline{\mathrm{C} 28}$ & C33 & $1.398(4)$ \\
\hline $\mathrm{C} 22$ & $\mathrm{C} 27$ & $1.389(3)$ & $\mathrm{C} 29$ & C30 & $1.404(4)$ \\
\hline $\mathrm{C} 23$ & $\mathrm{C} 24$ & $1.396(4)$ & $\mathrm{C} 29$ & C34 & $1.488(4)$ \\
\hline C24 & $\mathrm{C} 25$ & $1.382(4)$ & C30 & C31 & $1.376(5)$ \\
\hline $\mathrm{C} 25$ & $\mathrm{C} 26$ & $1.370(4)$ & C31 & C32 & $1.374(5)$ \\
\hline C26 & $\mathrm{C} 27$ & $1.387(4)$ & C32 & C33 & $1.382(4)$ \\
\hline C28 & $\mathrm{C} 29$ & $1.405(4)$ & & & \\
\hline
\end{tabular}

Table S36: Bond Angles in ${ }^{\circ}$ for compound 21d (S).

\begin{tabular}{|c|c|c|c|c|c|c|c|}
\hline Atom & Atom & Atom & Angle $/^{\circ}$ & Atom & Atom & Atom & Angle $/^{\circ}$ \\
\hline$\overline{\mathrm{N} 1}$ & P1 & $\mathrm{C} 1$ & $108.29(10)$ & $\overline{\mathrm{C} 11}$ & C12 & C7 & $120.2(2)$ \\
\hline N1 & $\mathrm{P} 1$ & $\mathrm{C} 7$ & $106.80(10)$ & N1 & $\mathrm{C} 14$ & C15 & $112.8(2)$ \\
\hline N1 & $\mathrm{P} 1$ & B1 & $113.26(12)$ & N1 & $\mathrm{C} 14$ & C16 & $109.23(18)$ \\
\hline $\mathrm{C} 1$ & $\mathrm{P} 1$ & B1 & $111.15(12)$ & C15 & C14 & C16 & $111.4(2)$ \\
\hline C7 & $\mathrm{P} 1$ & C1 & $105.82(11)$ & 01 & C16 & C14 & $104.62(18)$ \\
\hline C7 & $\mathrm{P} 1$ & B1 & $111.14(12)$ & 01 & C16 & C17 & 108.92(19) \\
\hline 01 & P2 & $\mathrm{C} 22$ & $104.55(11)$ & $\mathrm{C} 17$ & C16 & C14 & $114.5(2)$ \\
\hline 01 & $\mathrm{P} 2$ & $\mathrm{C} 28$ & $103.35(11)$ & C7AA & C17 & C16 & $119.1(2)$ \\
\hline 01 & $\mathrm{P} 2$ & B2 & $115.34(12)$ & C7AA & C17 & C18 & $119.2(2)$ \\
\hline $\mathrm{C} 22$ & $\mathrm{P} 2$ & B2 & $111.80(12)$ & C18 & C17 & C16 & $121.7(2)$ \\
\hline $\mathrm{C} 28$ & $\mathrm{P} 2$ & $\mathrm{C} 22$ & $105.99(11)$ & C19 & C18 & C17 & $120.0(3)$ \\
\hline $\mathrm{C} 28$ & $\mathrm{P} 2$ & B2 & $114.76(13)$ & $\mathrm{C} 20$ & C19 & C18 & $120.3(3)$ \\
\hline C16 & 01 & P2 & $122.48(15)$ & $\mathrm{C} 21$ & $\mathrm{C} 20$ & C19 & $120.2(3)$ \\
\hline C13 & N1 & $\mathrm{P} 1$ & $119.06(16)$ & $\mathrm{C} 20$ & $\mathrm{C} 21$ & C7AA & $120.2(3)$ \\
\hline C13 & N1 & C14 & $117.00(19)$ & $\mathrm{C} 23$ & $\mathrm{C} 22$ & $\mathrm{P} 2$ & $122.51(19)$ \\
\hline C14 & N1 & $\mathrm{P} 1$ & $120.04(15)$ & $\mathrm{C} 27$ & $\mathrm{C} 22$ & $\mathrm{P} 2$ & $118.00(19)$ \\
\hline $\mathrm{C} 2$ & $\mathrm{C} 1$ & $\mathrm{P} 1$ & $117.89(19)$ & $\mathrm{C} 27$ & $\mathrm{C} 22$ & $\mathrm{C} 23$ & $119.5(2)$ \\
\hline C6 & $\mathrm{C} 1$ & $\mathrm{P} 1$ & $123.5(2)$ & $\mathrm{C} 22$ & $\mathrm{C} 23$ & $\mathrm{C} 24$ & $119.6(3)$ \\
\hline $\mathrm{C} 6$ & $\mathrm{C} 1$ & $\mathrm{C} 2$ & $118.6(2)$ & $\mathrm{C} 25$ & $\mathrm{C} 24$ & $\mathrm{C} 23$ & $120.1(3)$ \\
\hline C3 & $\mathrm{C} 2$ & $\mathrm{C} 1$ & $120.9(3)$ & $\mathrm{C} 26$ & $\mathrm{C} 25$ & $\mathrm{C} 24$ & $120.3(3)$ \\
\hline $\mathrm{C} 4$ & $\mathrm{C} 3$ & $\mathrm{C} 2$ & 120.1(3) & $\mathrm{C} 25$ & $\mathrm{C} 26$ & $\mathrm{C} 27$ & 120.1(3) \\
\hline C3 & $\mathrm{C} 4$ & $\mathrm{C} 5$ & $119.9(3)$ & $\mathrm{C} 26$ & $\mathrm{C} 27$ & $\mathrm{C} 22$ & $120.4(3)$ \\
\hline $\mathrm{C} 4$ & $\mathrm{C} 5$ & C6 & $120.4(3)$ & $\mathrm{C} 29$ & $\mathrm{C} 28$ & P2 & $123.8(2)$ \\
\hline C1 & C6 & $\mathrm{C} 5$ & $120.1(3)$ & C33 & $\mathrm{C} 28$ & P2 & $116.8(2)$ \\
\hline C8 & $\mathrm{C} 7$ & P1 & $120.83(18)$ & C33 & $\mathrm{C} 28$ & $\mathrm{C} 29$ & $119.4(2)$ \\
\hline C8 & $\mathrm{C} 7$ & $\mathrm{C} 12$ & 119.3(2) & $\mathrm{C} 28$ & $\mathrm{C} 29$ & C34 & $124.1(3)$ \\
\hline C12 & $\mathrm{C} 7$ & $\mathrm{P} 1$ & 119.61(18) & $\mathrm{C} 30$ & $\mathrm{C} 29$ & $\mathrm{C} 28$ & $117.8(3)$ \\
\hline $\mathrm{C} 21$ & C7AA & $\mathrm{C} 17$ & $120.1(3)$ & C30 & $\mathrm{C} 29$ & C34 & $118.2(3)$ \\
\hline C9 & C8 & $\mathrm{C} 7$ & $120.1(2)$ & C31 & $\mathrm{C} 30$ & $\mathrm{C} 29$ & $121.7(3)$ \\
\hline C10 & C9 & $\mathrm{C} 8$ & $120.2(2)$ & $\mathrm{C} 32$ & $\mathrm{C} 31$ & C30 & $120.4(3)$ \\
\hline C9 & C10 & C11 & $120.2(2)$ & C31 & C32 & C33 & $119.3(3)$ \\
\hline C10 & C11 & C12 & $120.0(3)$ & $\mathrm{C} 32$ & C33 & $\mathrm{C} 28$ & $121.4(3)$ \\
\hline
\end{tabular}

Table S37: Torsion Angles in ${ }^{\circ}$ for compound 21d $(S)$.

\begin{tabular}{lllll}
\hline Atom & Atom & Atom & Atom & Angle $^{\circ}$ \\
\hline P1 & N1 & C14 & C15 & $96.6(2)$ \\
P1 & N1 & C14 & C16 & $-138.87(18)$ \\
P1 & C1 & C2 & C3 & $-179.8(2)$ \\
P1 & C1 & C6 & C5 & $180.0(2)$ \\
P1 & C7 & C8 & C9 & $-174.37(19)$ \\
P1 & C7 & C12 & C11 & $174.9(2)$ \\
P2 & 01 & C16 & C14 & $130.74(18)$
\end{tabular}




\begin{tabular}{|c|c|c|c|c|}
\hline Atom & Atom & Atom & Atom & Angle $/^{\circ}$ \\
\hline $\mathrm{P} 2$ & 01 & C16 & C17 & $-106.4(2)$ \\
\hline P2 & $\mathrm{C} 22$ & $\mathrm{C} 23$ & $\mathrm{C} 24$ & $179.1(2)$ \\
\hline P2 & $\mathrm{C} 22$ & $\mathrm{C} 27$ & $\mathrm{C} 26$ & $-178.8(2)$ \\
\hline P2 & $\mathrm{C} 28$ & $\mathrm{C} 29$ & C30 & $-179.0(2)$ \\
\hline P2 & $\mathrm{C} 28$ & $\mathrm{C} 29$ & C34 & $1.0(4)$ \\
\hline P2 & $\mathrm{C} 28$ & C33 & C32 & $178.4(2)$ \\
\hline 01 & $\mathrm{P} 2$ & $\mathrm{C} 22$ & $\mathrm{C} 23$ & $2.8(2)$ \\
\hline 01 & $\mathrm{P} 2$ & $\mathrm{C} 22$ & $\mathrm{C} 27$ & $-176.85(19)$ \\
\hline 01 & $\mathrm{P} 2$ & $\mathrm{C} 28$ & $\mathrm{C} 29$ & $-46.7(2)$ \\
\hline 01 & $\mathrm{P} 2$ & $\mathrm{C} 28$ & C33 & $134.4(2)$ \\
\hline 01 & C16 & $\mathrm{C} 17$ & C7AA & $118.2(2)$ \\
\hline 01 & C16 & $\mathrm{C} 17$ & C18 & $-60.6(3)$ \\
\hline N1 & $\mathrm{P} 1$ & C1 & $\mathrm{C} 2$ & $87.2(2)$ \\
\hline N1 & $\mathrm{P} 1$ & $\mathrm{C} 1$ & $\mathrm{C} 6$ & $-91.1(2)$ \\
\hline N1 & $\mathrm{P} 1$ & $\mathrm{C} 7$ & $\mathrm{C} 8$ & $-20.8(2)$ \\
\hline N1 & $\mathrm{P} 1$ & $\mathrm{C} 7$ & C12 & $165.09(19)$ \\
\hline N1 & $\mathrm{C} 14$ & C16 & 01 & $170.25(18)$ \\
\hline N1 & $\mathrm{C} 14$ & C16 & $\mathrm{C} 17$ & 51.1(3) \\
\hline C1 & $\mathrm{P} 1$ & N1 & C13 & $48.4(2)$ \\
\hline $\mathrm{C} 1$ & $\mathrm{P} 1$ & N1 & C14 & $-108.69(18)$ \\
\hline C1 & $\mathrm{P} 1$ & $\mathrm{C} 7$ & $\mathrm{C} 8$ & $-136.05(19)$ \\
\hline $\mathrm{C} 1$ & $\mathrm{P} 1$ & $\mathrm{C} 7$ & C12 & $49.9(2)$ \\
\hline $\mathrm{C} 1$ & $\mathrm{C} 2$ & $\mathrm{C} 3$ & $\mathrm{C} 4$ & $0.0(4)$ \\
\hline $\mathrm{C} 2$ & $\mathrm{C} 1$ & $\mathrm{C} 6$ & $\mathrm{C} 5$ & $1.7(4)$ \\
\hline $\mathrm{C} 2$ & $\mathrm{C} 3$ & $\mathrm{C} 4$ & $\mathrm{C} 5$ & $1.1(4)$ \\
\hline C3 & $\mathrm{C} 4$ & $\mathrm{C} 5$ & $\mathrm{C} 6$ & $-0.8(4)$ \\
\hline $\mathrm{C} 4$ & $\mathrm{C} 5$ & $\mathrm{C} 6$ & $\mathrm{C} 1$ & $-0.6(4)$ \\
\hline C6 & $\mathrm{C} 1$ & $\mathrm{C} 2$ & $\mathrm{C} 3$ & $-1.5(4)$ \\
\hline $\mathrm{C} 7$ & $\mathrm{P} 1$ & N1 & C13 & $-65.2(2)$ \\
\hline C7 & $\mathrm{P} 1$ & N1 & C14 & $137.75(18)$ \\
\hline $\mathrm{C} 7$ & $\mathrm{P} 1$ & $\mathrm{C} 1$ & $\mathrm{C} 2$ & $-158.63(19)$ \\
\hline $\mathrm{C} 7$ & $\mathrm{P} 1$ & $\mathrm{C} 1$ & C6 & 23.1(2) \\
\hline C7 & $\mathrm{C} 8$ & $\mathrm{C} 9$ & C10 & $-0.1(4)$ \\
\hline C7AA & $\mathrm{C} 17$ & C18 & C19 & $0.4(4)$ \\
\hline C8 & $\mathrm{C} 7$ & $\mathrm{C} 12$ & C11 & $0.7(4)$ \\
\hline C8 & C9 & $\mathrm{C} 10$ & C11 & $0.1(4)$ \\
\hline C9 & $\mathrm{C} 10$ & C11 & C12 & $0.3(4)$ \\
\hline C10 & C11 & $\mathrm{C} 12$ & $\mathrm{C} 7$ & $-0.7(4)$ \\
\hline C12 & $\mathrm{C} 7$ & $\mathrm{C} 8$ & C9 & $-0.3(4)$ \\
\hline C13 & N1 & C14 & C15 & $-61.0(3)$ \\
\hline C13 & N1 & $\mathrm{C} 14$ & C16 & $63.6(3)$ \\
\hline C14 & C16 & $\mathrm{C} 17$ & C7AA & $-125.1(2)$ \\
\hline C14 & C16 & $\mathrm{C} 17$ & C18 & $56.2(3)$ \\
\hline C15 & $\mathrm{C} 14$ & C16 & 01 & $-64.4(2)$ \\
\hline C15 & $\mathrm{C} 14$ & C16 & C17 & $176.4(2)$ \\
\hline C16 & $\mathrm{C} 17$ & C18 & C19 & $179.2(2)$ \\
\hline C17 & C7AA & $\mathrm{C} 21$ & $\mathrm{C} 20$ & $1.6(5)$ \\
\hline C17 & C18 & C19 & $\mathrm{C} 20$ & $-0.5(4)$ \\
\hline C18 & C19 & $\mathrm{C} 20$ & $\mathrm{C} 21$ & $1.0(5)$ \\
\hline C19 & $\mathrm{C} 20$ & $\mathrm{C} 21$ & C7AA & $-1.6(5)$ \\
\hline C21 & C7AA & $\mathrm{C} 17$ & C16 & $-179.8(3)$ \\
\hline C21 & C7AA & C17 & C18 & $-1.0(4)$ \\
\hline C22 & P2 & 01 & C16 & 111.11(18) \\
\hline C22 & P2 & $\mathrm{C} 28$ & $\mathrm{C} 29$ & $62.9(2)$ \\
\hline
\end{tabular}




\begin{tabular}{|c|c|c|c|c|}
\hline Atom & Atom & Atom & Atom & Angle $/^{\circ}$ \\
\hline$\overline{\mathrm{C} 22}$ & P2 & C28 & C33 & $-116.0(2)$ \\
\hline $\mathrm{C} 22$ & $\mathrm{C} 23$ & $\mathrm{C} 24$ & $\mathrm{C} 25$ & $-0.1(4)$ \\
\hline C23 & C22 & $\mathrm{C} 27$ & $\mathrm{C} 26$ & $1.5(4)$ \\
\hline $\mathrm{C} 23$ & C24 & C25 & $\mathrm{C} 26$ & $1.2(5)$ \\
\hline $\mathrm{C} 24$ & $\mathrm{C} 25$ & $\mathrm{C} 26$ & $\mathrm{C} 27$ & $-1.0(4)$ \\
\hline C25 & C26 & $\mathrm{C} 27$ & $\mathrm{C} 22$ & $-0.4(4)$ \\
\hline $\mathrm{C} 27$ & C22 & $\mathrm{C} 23$ & $\mathrm{C} 24$ & $-1.2(4)$ \\
\hline C28 & P2 & 01 & C16 & $-138.15(18)$ \\
\hline C28 & P2 & C22 & $\mathrm{C} 23$ & $-106.0(2)$ \\
\hline $\mathrm{C} 28$ & P2 & $\mathrm{C} 22$ & $\mathrm{C} 27$ & $74.3(2)$ \\
\hline C28 & C29 & C30 & C31 & $0.7(4)$ \\
\hline C29 & C28 & C33 & C32 & $-0.5(4)$ \\
\hline C29 & C30 & C31 & C32 & $-0.6(5)$ \\
\hline C30 & C31 & C32 & C33 & $-0.1(5)$ \\
\hline C31 & C32 & C33 & C28 & $0.7(4)$ \\
\hline C33 & C28 & C29 & C30 & $-0.1(4)$ \\
\hline C33 & C28 & $\mathrm{C} 29$ & C34 & $179.8(3)$ \\
\hline C34 & $\mathrm{C} 29$ & C30 & C31 & $-179.3(3)$ \\
\hline B1 & P1 & N1 & C13 & $172.17(18)$ \\
\hline B1 & P1 & N1 & C14 & 15.1(2) \\
\hline B1 & P1 & C1 & $\mathrm{C} 2$ & $-37.9(2)$ \\
\hline B1 & P1 & $\mathrm{C} 1$ & C6 & $143.9(2)$ \\
\hline B1 & P1 & $\mathrm{C} 7$ & $\mathrm{C} 8$ & $103.2(2)$ \\
\hline B1 & P1 & $\mathrm{C} 7$ & C12 & $-70.9(2)$ \\
\hline B2 & P2 & 01 & C16 & $-12.1(2)$ \\
\hline B2 & P2 & $\mathrm{C} 22$ & $\mathrm{C} 23$ & $128.2(2)$ \\
\hline B2 & P2 & $\mathrm{C} 22$ & $\mathrm{C} 27$ & $-51.4(2)$ \\
\hline B2 & P2 & $\mathrm{C} 28$ & $\mathrm{C} 29$ & $-173.2(2)$ \\
\hline B2 & P2 & C28 & C33 & $7.9(2)$ \\
\hline
\end{tabular}


Name: $\left(R_{\mathrm{p}}\right)-(-)-\mathrm{N}-[(1 R, 2 S), \quad 2$-(Phenyl- $p$-tolylphosphinito-borane)-1-methyl-2-phenylethyl], N-methyl aminodiphenylphosphine-borane $\mathbf{2 1 e}$.

Submitted by: Antonin Jaillet

Solved by: Yoann Rousselin

Sample ID: $\quad$ 17AJ602

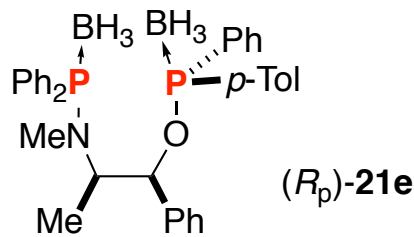

\section{Crystal Data and Experimental}

Figure S13 (thermal ellipsoïds are drawn at $50 \%$ probability plot)

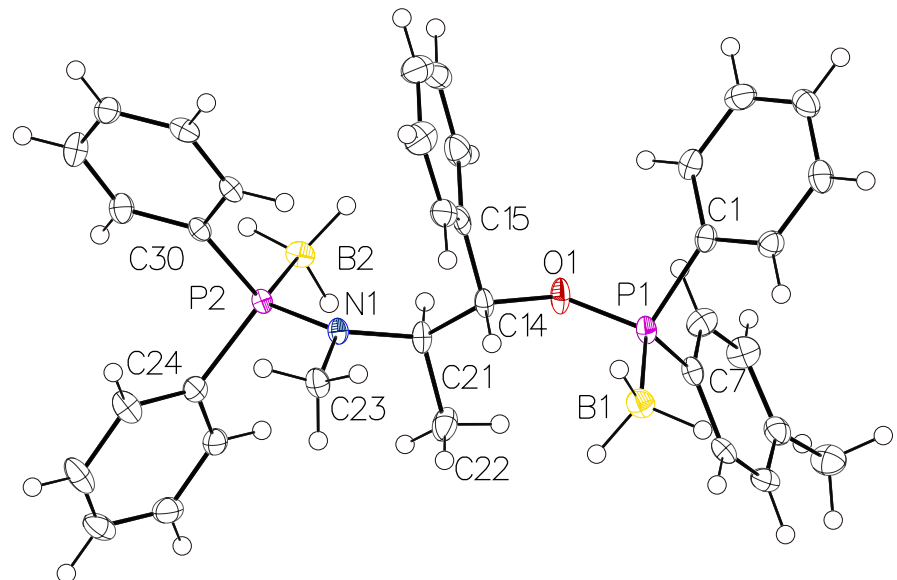

Experimental. Single clear light colorless prism-shaped crystals of compound 21e were recrystallized from diethyl ether by slow evaporation. A suitable crystal $0.18 \times 0.17 \times 0.12 \mathrm{~mm}^{3}$ was selected and mounted on a MITIGEN holder oil on a Bruker D8 VENTURE diffractometer. The crystal was kept at a steady $T=$ 100.0(1) K during data collection. The structure was solved with the ShelXT (Sheldrick, 2015) structure solution program using the Intrinsic Phasing solution method and by using 0lex2 (Dolomanov et al., 2009) as the graphical interface. The model was refined with version 2018/3 of ShelXL (Sheldrick, 2015) using Least Squares minimization.

Crystal Data. $\mathrm{C}_{35} \mathrm{H}_{41} \mathrm{~B}_{2} \mathrm{NOP}_{2}, M_{r}=575.25$, orthorhombic, $P 2{ }_{1} 2_{1} 2_{1}$ (No. 19), $\mathrm{a}=13.3946(4) \AA, \mathrm{b}=13.6712(3) \AA, \mathrm{c}=$ 17.7075(5) $\AA, \alpha=\beta=\gamma=90^{\circ}, V=3242.60(15) \AA^{3}, T=$ $100.0(1) \mathrm{K}, \quad Z=4, \quad Z^{\prime}=1, \mu\left(\mathrm{CuK}_{\alpha}\right)=1.418,21485$ reflections measured, 5727 unique $\left(R_{\text {int }}=0.0495\right)$ which were used in all calculations. The final $w R_{2}$ was 0.0710 (all data) and $R_{1}$ was 0.0321 (I > 2(I)). 


\begin{tabular}{|c|c|}
\hline Compound & compound $21 \mathrm{e}$ \\
\hline CCDC & 1982657 \\
\hline Formula & $\mathrm{C}_{35} \mathrm{H}_{41} \mathrm{~B}_{2} \mathrm{NOP}_{2}$ \\
\hline$D_{\text {calc. }} / \mathrm{g} \mathrm{cm}^{-3}$ & 1.178 \\
\hline$\mu / \mathrm{mm}^{-1}$ & 1.418 \\
\hline Formula Weight & 575.25 \\
\hline Color & clear light colorless \\
\hline Shape & prism \\
\hline Size $/ \mathrm{mm}^{3}$ & $0.18 \times 0.17 \times 0.12$ \\
\hline$T / \mathrm{K}$ & $100.0(1)$ \\
\hline Crystal System & orthorhombic \\
\hline Flack Parameter & $0.016(10)$ \\
\hline Hooft Parameter & $0.016(11)$ \\
\hline Space Group & $P 2{ }_{1} 2_{1} 2_{1}$ \\
\hline$a / \AA$ & $13.3946(4)$ \\
\hline$b / \AA ̊$ & $13.6712(3)$ \\
\hline$c / \AA$ & $17.7075(5)$ \\
\hline$\alpha /^{\circ}$ & 90 \\
\hline$\beta /^{\circ}$ & 90 \\
\hline$\gamma /{ }^{\circ}$ & 90 \\
\hline $\mathrm{V} / \AA^{3}$ & $3242.60(15)$ \\
\hline$Z$ & 4 \\
\hline$Z^{\prime}$ & 1 \\
\hline Wavelength/Å & 1.54178 \\
\hline Radiation type & $\mathrm{CuK}_{\alpha}$ \\
\hline$\Theta_{\min } /^{\circ}$ & 4.085 \\
\hline$\Theta_{\max } /^{\circ}$ & 66.760 \\
\hline Measured Refl. & 21485 \\
\hline Independent Refl. & 5727 \\
\hline Reflections with I > & 5261 \\
\hline $2(\mathrm{I})$ & \\
\hline$R_{\text {int }}$ & 0.0495 \\
\hline Parameters & 377 \\
\hline Restraints & 0 \\
\hline Largest Peak & 0.216 \\
\hline Deepest Hole & -0.263 \\
\hline GooF & 1.056 \\
\hline$w R_{2}$ (all data) & 0.0710 \\
\hline$w R_{2}$ & 0.0687 \\
\hline$R_{1}$ (all data) & 0.0374 \\
\hline$R_{1}$ & 0.0321 \\
\hline
\end{tabular}


Reflections:

A clear light colorless prism-shaped crystal with dimensions $0.18 \times 0.17 \times 0.12 \mathrm{~mm}^{3}$ was mounted on a MITIGEN holder oil. Data were collected using a Bruker D8 VENTURE diffractometer equipped with an Oxford Cryosystems low-temperature device operating at $T=100.0(1) \mathrm{K}$. Data were measured using $\phi$ and $\omega$ scans using $\mathrm{CuK}_{\alpha}$ radiation. The total number of runs and images was based on the strategy calculation from the program APEX3 (Bruker, 2015) The maximum resolution that was achieved was $\Theta$ $=66.760^{\circ}(0.84 \AA)$. The diffraction pattern was indexed. The total number of runs and images was based on the strategy calculation from the program APEX3 (Bruker, 2015) and the unit cell was refined using SAINT (Bruker, V8.38A, after 2013) on 9917 reflections, 46\% of the observed reflections. Data reduction, scaling and absorption corrections were performed using SAINT (Bruker, V8.38A, after 2013). The final completeness is $99.90 \%$ out to $66.760^{\circ}$ in $\Theta$. A multi-scan absorption correction was performed using SADABS-2016/2 (Bruker, 2016) was used for absorption correction. $w R_{2}$ (int) was 0.0787 before and 0.0695 after correction. The Ratio of minimum to maximum transmission is 0.8836 . The absorption coefficient $\mu$ of this material is $1.418 \mathrm{~mm}^{-1}$ at this wavelength $(\lambda=1.542 \AA)$ and the minimum and maximum transmissions are 0.722 and 0.817 . The structure was solved and the space group $P 2{ }_{1} 2_{1} 2_{1}$ ( \# 19) determined by the ShelXT (Sheldrick, 2015) structure solution program using Intrinsic Phasing and refined by Least Squares using version 2018/3 of ShelXL (Sheldrick, 2015). All non-hydrogen atoms were refined anisotropically. Hydrogen atom positions were calculated geometrically and refined using the riding model. Hydrogen atom on Boron atom were refined using an AFIX 138, which would allow the refinement of a common B-H distances, the same shifts being applied to all the $\mathrm{B}-\mathrm{H}$ bonds to the same atom, but still retain tetrahedral $\mathrm{H}-\mathrm{B}-\mathrm{H}$ angles within the group. There is a single molecule in the asymmetric unit, which is represented by the reported sum formula. In other words: Z is 4 and Z' is 1 . The Flack parameter was refined to $0.016(10)$. Determination of absolute structure using Bayesian statistics on Bijvoet differences using the Olex2 results in 0.016(11). Note: The Flack parameter is used to determine chirality of the crystal studied, the value should be near 0 , a value of 1 means that the stereochemistry is wrong and the model should be inverted. A value of 0.5 means that the crystal consists of a racemic mixture of the two enantiomers.

Table S38: Bond Lengths in Å for compound 21e.

\begin{tabular}{lll}
\hline Atom & Atom & Length/Å \\
\hline P2 & N1 & $1.667(2)$ \\
P2 & C30 & $1.812(3)$ \\
P2 & C24 & $1.825(3)$ \\
P2 & B2 & $1.909(3)$ \\
P1 & O1 & $1.6088(18)$ \\
P1 & C1 & $1.813(3)$ \\
P1 & C7 & $1.806(3)$ \\
P1 & B1 & $1.908(3)$ \\
01 & C14 & $1.462(3)$ \\
N1 & C21 & $1.468(3)$ \\
N1 & C23 & $1.466(3)$ \\
C30 & C31 & $1.395(4)$ \\
C30 & C35 & $1.396(4)$ \\
C21 & C14 & $1.545(4)$ \\
C21 & C22 & $1.524(4)$ \\
C31 & C32 & $1.387(4)$ \\
C2 & C1 & $1.387(4)$
\end{tabular}

\begin{tabular}{lll}
\hline Atom & Atom & Length/Å \\
\hline C2 & C3 & $1.386(4)$ \\
C1 & C6 & $1.394(4)$ \\
C9 & C8 & $1.385(4)$ \\
C9 & C10 & $1.388(4)$ \\
C8 & C7 & $1.390(4)$ \\
C29 & C24 & $1.389(4)$ \\
C29 & C28 & $1.389(4)$ \\
C35 & C34 & $1.383(4)$ \\
C32 & C33 & $1.376(4)$ \\
C33 & C34 & $1.386(4)$ \\
C15 & C14 & $1.506(4)$ \\
C15 & C20 & $1.392(4)$ \\
C15 & C16 & $1.391(4)$ \\
C10 & C11 & $1.395(4)$ \\
C10 & C13 & $1.505(4)$ \\
C6 & C5 & $1.384(4)$ \\
C24 & C25 & $1.399(4)$
\end{tabular}




\begin{tabular}{lll}
\hline Atom & Atom & Length/Å \\
\hline C26 & C25 & $1.382(4)$ \\
C26 & C27 & $1.385(5)$ \\
C7 & C12 & $1.393(4)$ \\
C28 & C27 & $1.376(5)$ \\
C12 & C11 & $1.385(4)$ \\
C17 & C16 & $1.386(4)$
\end{tabular}

\begin{tabular}{lll}
\hline Atom & Atom & Length/Å \\
\hline C17 & C18 & $1.380(5)$ \\
C5 & C4 & $1.395(4)$ \\
C20 & C19 & $1.381(4)$ \\
C4 & C3 & $1.378(4)$ \\
C18 & C19 & $1.390(5)$
\end{tabular}

Table S39: Bond Angles in ${ }^{\circ}$ for compound $21 \mathrm{e}$.

\begin{tabular}{|c|c|c|c|}
\hline Atom & Atom & Atom & Angle $/^{\circ}$ \\
\hline N1 & P2 & C30 & $107.11(12)$ \\
\hline N1 & $\mathrm{P} 2$ & $\mathrm{C} 24$ & $108.21(12)$ \\
\hline $\mathrm{N} 1$ & P2 & B2 & $113.13(13)$ \\
\hline C30 & P2 & $\mathrm{C} 24$ & $104.87(12)$ \\
\hline C30 & P2 & B2 & $111.47(13)$ \\
\hline C24 & P2 & B2 & $111.61(13)$ \\
\hline 01 & P1 & $\mathrm{C} 1$ & $104.64(12)$ \\
\hline 01 & $\mathrm{P} 1$ & $\mathrm{C} 7$ & $101.75(12)$ \\
\hline 01 & $\mathrm{P} 1$ & B1 & $116.28(12)$ \\
\hline $\mathrm{C} 1$ & $\mathrm{P} 1$ & B1 & $113.08(13)$ \\
\hline C7 & $\mathrm{P} 1$ & C1 & $104.07(12)$ \\
\hline $\mathrm{C} 7$ & $\mathrm{P} 1$ & B1 & $115.50(14)$ \\
\hline C14 & 01 & $\mathrm{P} 1$ & 122.01(17) \\
\hline C21 & N1 & $\mathrm{P} 2$ & $119.55(18)$ \\
\hline C23 & N1 & P2 & $118.30(17)$ \\
\hline C23 & N1 & $\mathrm{C} 21$ & $117.1(2)$ \\
\hline C31 & C30 & P2 & $120.7(2)$ \\
\hline C31 & C30 & C35 & $119.1(3)$ \\
\hline C35 & C30 & P2 & $120.0(2)$ \\
\hline $\mathrm{N} 1$ & $\mathrm{C} 21$ & $\mathrm{C} 14$ & $110.3(2)$ \\
\hline $\mathrm{N} 1$ & $\mathrm{C} 21$ & $\mathrm{C} 22$ & $113.0(2)$ \\
\hline C22 & $\mathrm{C} 21$ & $\mathrm{C} 14$ & $111.0(2)$ \\
\hline C32 & C31 & C30 & $119.9(3)$ \\
\hline C3 & $\mathrm{C} 2$ & $\mathrm{C} 1$ & $120.1(3)$ \\
\hline $\mathrm{C} 2$ & $\mathrm{C} 1$ & $\mathrm{P} 1$ & $118.6(2)$ \\
\hline $\mathrm{C} 2$ & $\mathrm{C} 1$ & C6 & $119.6(2)$ \\
\hline C6 & $\mathrm{C} 1$ & $\mathrm{P} 1$ & $121.6(2)$ \\
\hline $\mathrm{C} 8$ & C9 & C10 & $121.3(3)$ \\
\hline C9 & $\mathrm{C} 8$ & $\mathrm{C} 7$ & $120.2(3)$ \\
\hline $\mathrm{C} 24$ & $\mathrm{C} 29$ & $\mathrm{C} 28$ & $120.1(3)$ \\
\hline C34 & C35 & C30 & $120.4(3)$ \\
\hline C33 & C32 & C31 & $120.6(3)$ \\
\hline C32 & C33 & C34 & $120.0(3)$ \\
\hline
\end{tabular}

\begin{tabular}{|c|c|c|c|}
\hline Atom & Atom & Atom & Angle $/^{\circ}$ \\
\hline $\mathrm{C} 20$ & C15 & C14 & 121.5(3) \\
\hline C16 & C15 & C14 & $119.9(2)$ \\
\hline C16 & C15 & $\mathrm{C} 20$ & $118.6(3)$ \\
\hline C9 & C10 & C11 & 118.3(3) \\
\hline C9 & C10 & C13 & $121.3(3)$ \\
\hline C11 & C10 & C13 & $120.4(3)$ \\
\hline C5 & C6 & $\mathrm{C} 1$ & 120.1(3) \\
\hline C29 & $\mathrm{C} 24$ & P2 & $123.4(2)$ \\
\hline $\mathrm{C} 29$ & C24 & $\mathrm{C} 25$ & $118.9(3)$ \\
\hline C25 & C24 & P2 & $117.7(2)$ \\
\hline $\mathrm{C} 25$ & C26 & $\mathrm{C} 27$ & $120.0(3)$ \\
\hline C8 & $\mathrm{C} 7$ & P1 & $120.3(2)$ \\
\hline C8 & C7 & C12 & $118.9(3)$ \\
\hline C12 & C7 & P1 & $120.7(2)$ \\
\hline $\mathrm{C} 27$ & C28 & C29 & $120.5(3)$ \\
\hline C11 & C12 & C7 & $120.5(3)$ \\
\hline 01 & C14 & $\mathrm{C} 21$ & $103.7(2)$ \\
\hline 01 & C14 & C15 & $109.6(2)$ \\
\hline C15 & C14 & $\mathrm{C} 21$ & $114.5(2)$ \\
\hline C18 & C17 & C16 & $119.9(3)$ \\
\hline $\mathrm{C} 26$ & C25 & $\mathrm{C} 24$ & $120.6(3)$ \\
\hline C6 & C5 & $\mathrm{C} 4$ & $119.9(3)$ \\
\hline C19 & C20 & C15 & $120.5(3)$ \\
\hline C3 & C4 & $\mathrm{C} 5$ & $119.9(3)$ \\
\hline C12 & C11 & C10 & $120.7(3)$ \\
\hline C35 & C34 & C33 & $120.1(3)$ \\
\hline $\mathrm{C} 17$ & C16 & C15 & $120.9(3)$ \\
\hline C17 & C18 & C19 & $119.7(3)$ \\
\hline $\mathrm{C} 4$ & C3 & $\mathrm{C} 2$ & $120.4(3)$ \\
\hline $\mathrm{C} 28$ & $\mathrm{C} 27$ & $\mathrm{C} 26$ & $119.9(3)$ \\
\hline C20 & C19 & C18 & $120.3(3)$ \\
\hline
\end{tabular}

Table S40: Torsion Angles in ${ }^{\circ}$ for compound 21 e.

\begin{tabular}{llllr}
\hline Atom & Atom & Atom & Atom & Angle $^{\circ}$ \\
\hline P2 & N1 & C21 & C14 & $138.3(2)$ \\
P2 & N1 & C21 & C22 & $-96.7(3)$ \\
P2 & C30 & C31 & C32 & $175.4(2)$ \\
P2 & C30 & C35 & C34 & $-175.7(2)$ \\
P2 & C24 & C25 & C26 & $179.0(2)$ \\
P1 & 01 & C14 & C21 & $-132.2(2)$ \\
P1 & 01 & C14 & C15 & $105.1(2)$
\end{tabular}




\begin{tabular}{|c|c|c|c|c|}
\hline Atom & Atom & Atom & Atom & Angle $/{ }^{\circ}$ \\
\hline$\overline{\mathrm{P} 1}$ & $\mathrm{C} 1$ & $\mathrm{C} 6$ & $\mathrm{C} 5$ & $-175.9(2)$ \\
\hline P1 & $\mathrm{C} 7$ & C12 & C11 & $174.8(2)$ \\
\hline 01 & $\mathrm{P} 1$ & C1 & $\mathrm{C} 2$ & $158.7(2)$ \\
\hline 01 & $\mathrm{P} 1$ & $\mathrm{C} 1$ & $\mathrm{C} 6$ & $-24.6(2)$ \\
\hline 01 & P1 & $\mathrm{C} 7$ & C8 & $-129.2(2)$ \\
\hline 01 & P1 & C7 & C12 & $54.6(2)$ \\
\hline N1 & P2 & C30 & C31 & $20.1(2)$ \\
\hline N1 & $\mathrm{P} 2$ & C30 & C35 & $-165.0(2)$ \\
\hline N1 & P2 & $\mathrm{C} 24$ & $\mathrm{C} 29$ & $91.5(2)$ \\
\hline N1 & P2 & $\mathrm{C} 24$ & $\mathrm{C} 25$ & $-87.2(2)$ \\
\hline N1 & $\mathrm{C} 21$ & C14 & 01 & $-167.7(2)$ \\
\hline N1 & $\mathrm{C} 21$ & C14 & C15 & $-48.3(3)$ \\
\hline C30 & P2 & N1 & $\mathrm{C} 21$ & $-136.9(2)$ \\
\hline C30 & P2 & N1 & $\mathrm{C} 23$ & $69.0(2)$ \\
\hline C30 & $\mathrm{P} 2$ & $\mathrm{C} 24$ & C29 & $-22.6(3)$ \\
\hline C30 & P2 & $\mathrm{C} 24$ & $\mathrm{C} 25$ & $158.7(2)$ \\
\hline C30 & C31 & C32 & C33 & $0.1(4)$ \\
\hline C30 & C35 & C34 & C33 & $0.3(4)$ \\
\hline C31 & C30 & C35 & C34 & $-0.7(4)$ \\
\hline C31 & C32 & C33 & C34 & $-0.4(4)$ \\
\hline $\mathrm{C} 2$ & $\mathrm{C} 1$ & $\mathrm{C} 6$ & $\mathrm{C} 5$ & $0.7(4)$ \\
\hline C1 & $\mathrm{P} 1$ & 01 & C14 & $-110.1(2)$ \\
\hline C1 & $\mathrm{P} 1$ & $\mathrm{C} 7$ & $\mathrm{C} 8$ & $122.3(2)$ \\
\hline $\mathrm{C} 1$ & $\mathrm{P} 1$ & $\mathrm{C} 7$ & $\mathrm{C} 12$ & $-53.9(2)$ \\
\hline C1 & $\mathrm{C} 2$ & $\mathrm{C} 3$ & $\mathrm{C} 4$ & $0.7(4)$ \\
\hline $\mathrm{C} 1$ & $\mathrm{C} 6$ & $\mathrm{C} 5$ & $\mathrm{C} 4$ & $-0.4(4)$ \\
\hline C9 & $\mathrm{C} 8$ & $\mathrm{C} 7$ & $\mathrm{P} 1$ & $-174.9(2)$ \\
\hline C9 & $\mathrm{C} 8$ & $\mathrm{C} 7$ & $\mathrm{C} 12$ & $1.4(4)$ \\
\hline C9 & C10 & C11 & $\mathrm{C} 12$ & $3.0(4)$ \\
\hline C23 & N1 & $\mathrm{C} 21$ & C14 & $-67.3(3)$ \\
\hline C23 & N1 & $\mathrm{C} 21$ & $\mathrm{C} 22$ & $57.7(3)$ \\
\hline C8 & C9 & C10 & C11 & $-3.1(4)$ \\
\hline C8 & C9 & C10 & C13 & $174.5(3)$ \\
\hline C8 & C7 & C12 & C11 & $-1.4(4)$ \\
\hline C29 & $\mathrm{C} 24$ & $\mathrm{C} 25$ & $\mathrm{C} 26$ & $0.2(4)$ \\
\hline C29 & C28 & $\mathrm{C} 27$ & C26 & $0.1(5)$ \\
\hline C35 & C30 & C31 & C32 & $0.5(4)$ \\
\hline C32 & C33 & C34 & C35 & $0.2(5)$ \\
\hline C15 & $\mathrm{C} 20$ & C19 & C18 & $-1.4(4)$ \\
\hline C10 & C9 & $\mathrm{C} 8$ & $\mathrm{C} 7$ & $0.9(4)$ \\
\hline C6 & $\mathrm{C} 5$ & $\mathrm{C} 4$ & $\mathrm{C} 3$ & $0.2(4)$ \\
\hline C24 & P2 & N1 & $\mathrm{C} 21$ & $110.5(2)$ \\
\hline C24 & P2 & $\mathrm{N} 1$ & $\mathrm{C} 23$ & $-43.6(2)$ \\
\hline C24 & $\mathrm{P} 2$ & C30 & C31 & $134.9(2)$ \\
\hline C24 & P2 & C30 & C35 & $-50.2(2)$ \\
\hline C24 & $\mathrm{C} 29$ & C28 & $\mathrm{C} 27$ & $0.8(5)$ \\
\hline C7 & P1 & 01 & C14 & $141.8(2)$ \\
\hline C7 & P1 & $\mathrm{C} 1$ & C2 & $-94.9(2)$ \\
\hline $\mathrm{C} 7$ & P1 & $\mathrm{C} 1$ & C6 & $81.8(2)$ \\
\hline C7 & C12 & C11 & C10 & $-0.8(4)$ \\
\hline C28 & $\mathrm{C} 29$ & $\mathrm{C} 24$ & P2 & $-179.6(2)$ \\
\hline C28 & C29 & $\mathrm{C} 24$ & $\mathrm{C} 25$ & $-0.9(4)$ \\
\hline C14 & C15 & $\mathrm{C} 20$ & C19 & $179.7(2)$ \\
\hline C14 & $\mathrm{C} 15$ & C16 & C17 & $-178.1(3)$ \\
\hline
\end{tabular}




\begin{tabular}{|c|c|c|c|c|}
\hline Atom & Atom & Atom & Atom & Angle $/^{\circ}$ \\
\hline$\overline{\mathrm{C} 17}$ & C18 & C19 & C20 & $1.2(5)$ \\
\hline $\mathrm{C} 25$ & C26 & $\mathrm{C} 27$ & $\mathrm{C} 28$ & $-0.8(4)$ \\
\hline C5 & $\mathrm{C} 4$ & C3 & $\mathrm{C} 2$ & $-0.4(4)$ \\
\hline $\mathrm{C} 20$ & C15 & C14 & 01 & $51.2(3)$ \\
\hline $\mathrm{C} 20$ & C15 & C14 & $\mathrm{C} 21$ & $-64.8(3)$ \\
\hline $\mathrm{C} 20$ & C15 & C16 & C17 & $1.6(4)$ \\
\hline C16 & C15 & C14 & 01 & $-129.1(2)$ \\
\hline C16 & C15 & C14 & $\mathrm{C} 21$ & $114.9(3)$ \\
\hline C16 & C15 & $\mathrm{C} 20$ & C19 & $0.0(4)$ \\
\hline C16 & C17 & C18 & C19 & $0.4(5)$ \\
\hline C18 & C17 & C16 & C15 & $-1.8(4)$ \\
\hline C3 & $\mathrm{C} 2$ & $\mathrm{C} 1$ & P1 & $175.8(2)$ \\
\hline C3 & $\mathrm{C} 2$ & $\mathrm{C} 1$ & $\mathrm{C} 6$ & $-0.9(4)$ \\
\hline $\mathrm{C} 13$ & C10 & C11 & C12 & $-174.6(3)$ \\
\hline $\mathrm{C} 27$ & C26 & $\mathrm{C} 25$ & C24 & $0.6(4)$ \\
\hline $\mathrm{C} 22$ & $\mathrm{C} 21$ & C14 & 01 & $66.2(3)$ \\
\hline $\mathrm{C} 22$ & $\mathrm{C} 21$ & C14 & C15 & $-174.3(2)$ \\
\hline B2 & P2 & N1 & $\mathrm{C} 21$ & $-13.7(2)$ \\
\hline \multirow[t]{2}{*}{ B2 } & P2 & N1 & $\mathrm{C} 23$ & - \\
\hline & & & & 167.77(19) \\
\hline B2 & P2 & C30 & C31 & $-104.2(2)$ \\
\hline B2 & P2 & C30 & C35 & $70.7(3)$ \\
\hline B2 & P2 & C24 & C29 & $-143.4(2)$ \\
\hline B2 & P2 & C24 & $\mathrm{C} 25$ & $37.9(2)$ \\
\hline B1 & P1 & 01 & C14 & $15.4(3)$ \\
\hline B1 & P1 & C1 & $\mathrm{C} 2$ & $31.2(3)$ \\
\hline B1 & P1 & $\mathrm{C} 1$ & $\mathrm{C} 6$ & $-152.1(2)$ \\
\hline B1 & P1 & $\mathrm{C} 7$ & $\mathrm{C} 8$ & $-2.3(3)$ \\
\hline B1 & P1 & C7 & C12 & $-178.5(2)$ \\
\hline
\end{tabular}


Name: $\left(R_{\mathrm{p}}\right)-(-)-\mathrm{N}-[(1 R, 2 S), 2$-(Phenyl-o-biphenylphosphinito-borane)-1-methyl-2-phenylethyl], Nmethylamino-diphenylphosphine-borane 21f.

Submitted by: Jérôme Bayardon

Solved by: Yoann Rousselin

Sample ID: $\quad$ 15JB1277

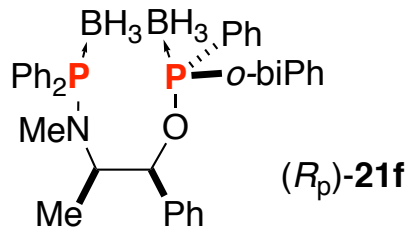

\section{Crystal Data and Experimental}

Figure S14 (thermal ellipsoïds are drawn at $50 \%$ probability plot)

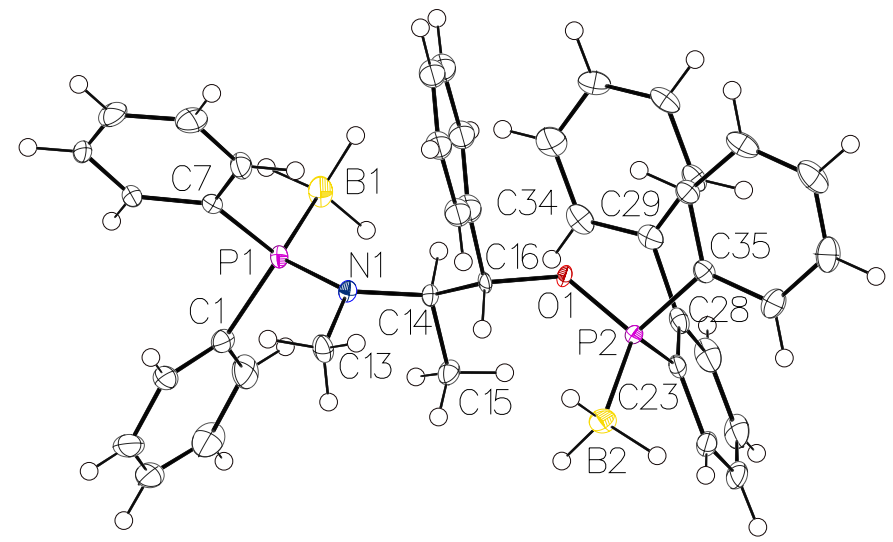

Experimental. Single clear light colorless prism-shaped crystals of compound $21 \mathrm{f}$ were recrystallized from DCM by slow evaporation. A suitable crystal $0.69 \times 0.61 \times 0.30$ $\mathrm{mm}^{3}$ was selected and mounted on a MITIGEN holder oil on an Nonius Kappa APEX II diffractometer. The crystal was kept at a steady $T=115 \mathrm{~K}$ during data collection. The structure was solved with the ShelXT (Sheldrick, 2015) structure solution program using the Intrinsic Phasing solution method and by using 0lex2 (Dolomanov et al., 2009 ) as the graphical interface. The model was refined with version 2018/3 of ShelXL (Sheldrick, 2015) using Least Squares minimization.

Crystal Data. $\mathrm{C}_{40} \mathrm{H}_{43} \mathrm{~B}_{2} \mathrm{NOP}_{2}, M_{r}=637.31$, monoclinic, $P 2_{1}$ (No. 4), $a=10.6504(7) \AA, \quad b=15.5326(8) \AA, \quad c=$ $21.6939(14) \AA, \quad \beta=96.532(4)^{\circ}, \quad \alpha=\quad \gamma=90^{\circ}, \quad V=$ $3565.5(4) \AA^{3}, T=115 \mathrm{~K}, Z=4, Z^{\prime}=2, \mu\left(\mathrm{MoK}_{\alpha}\right)=0.154$, 129114 reflections measured, 16478 unique $\left(R_{\text {int }}=\right.$ 0.0482 ) which were used in all calculations. The final $w R_{2}$ was 0.1687 (all data) and $R_{1}$ was 0.0562 (I > 2(I)). 


\begin{tabular}{|c|c|}
\hline Compound & 21f \\
\hline CCDC & 1982658 \\
\hline Formula & $\mathrm{C}_{40} \mathrm{H}_{43} \mathrm{~B}_{2} \mathrm{NOP}_{2}$ \\
\hline$D_{\text {calc. }} / \mathrm{g} \mathrm{cm}^{-3}$ & 1.187 \\
\hline$\mu / \mathrm{mm}^{-1}$ & 0.154 \\
\hline Formula Weight & 637.31 \\
\hline Color & clear light colorless \\
\hline Shape & prism \\
\hline Size $/ \mathrm{mm}^{3}$ & $0.69 \times 0.61 \times 0.30$ \\
\hline$T / \mathrm{K}$ & 115 \\
\hline Crystal System & monoclinic \\
\hline Flack Parameter & $0.007(14)$ \\
\hline Hooft Parameter & $0.021(17)$ \\
\hline Space Group & $P 2_{1}$ \\
\hline$a / \AA$ & $10.6504(7)$ \\
\hline$b / \AA$ & $15.5326(8)$ \\
\hline$c / \AA$ & $21.6939(14)$ \\
\hline$\alpha /^{\circ}$ & 90 \\
\hline$\beta /^{\circ}$ & $96.532(4)$ \\
\hline$\gamma /^{\circ}$ & 90 \\
\hline $\mathrm{V} / \AA^{3}$ & $3565.5(4)$ \\
\hline$Z$ & 4 \\
\hline$Z^{\prime}$ & 2 \\
\hline Wavelength/Å & 0.71073 \\
\hline Radiation type & $\operatorname{MoK}_{\alpha}$ \\
\hline$\Theta_{\min } /^{\circ}$ & 0.945 \\
\hline$\Theta_{\max } /^{\circ}$ & 27.612 \\
\hline Measured Refl. & 129114 \\
\hline Independent Refl. & 16478 \\
\hline Reflections with I > & 15889 \\
\hline $2(\mathrm{I})$ & \\
\hline$R_{\text {int }}$ & 0.0482 \\
\hline Parameters & 782 \\
\hline Restraints & 1 \\
\hline Largest Peak & 0.913 \\
\hline Deepest Hole & -0.392 \\
\hline GooF & 1.030 \\
\hline$w R_{2}$ (all data) & 0.1687 \\
\hline$w R_{2}$ & 0.1615 \\
\hline$R_{1}$ (all data) & 0.0598 \\
\hline$R_{1}$ & 0.0562 \\
\hline
\end{tabular}


Reflections:

Refinement:

A clear light colorless prism-shaped crystal with dimensions $0.69 \times 0.61 \times 0.30 \mathrm{~mm}^{3}$ was mounted on a MITIGEN holder oil. Data were collected using a Nonius Kappa APEX II diffractometer equipped with an Oxford Cryosystems low-temperature device operating at $T=115 \mathrm{~K}$. Data were measured using $\phi$ and $\omega$ scans using $\mathrm{MoK}_{\alpha}$ radiation. The total number of runs and images was based on the strategy calculation from the program APEX3 (Bruker, 2015) The maximum resolution that was achieved was $\Theta=27.612^{\circ}$ $(0.77 \AA)$. The diffraction pattern was indexed. The total number of runs and images was based on the strategy calculation from the program APEX3 (Bruker, 2015) and the unit cell was refined using SAINT (Bruker, V8.34A, after 2013) on 9297 reflections, 7\% of the observed reflections. Data reduction, scaling and absorption corrections were performed using SAINT (Bruker, V8.34A, after 2013). The final completeness is $99.90 \%$ out to $27.612^{\circ}$ in $\Theta$. A multi-scan absorption correction was performed using SADABS-2014/5 (Bruker,2014) was used for absorption correction. $w R_{2}$ (int) was 0.1175 before and 0.0652 after correction. The Ratio of minimum to maximum transmission is 0.8723 . The $\lambda / 2$ correction factor is 0.00150 . The absorption coefficient $\mu$ of this material is $0.154 \mathrm{~mm}^{-1}$ at this wavelength $(\lambda=$ $0.711 \AA$ ) and the minimum and maximum transmissions are 0.650 and 0.746 . The structure was solved and the space group $P 2_{1}$ (\# 4) determined by the ShelXT (Sheldrick, 2015) structure solution program using the Intrinsic Phasing solution method and refined by Least Squares using version 2018/3 of ShelXL (Sheldrick, 2015). All non-hydrogen atoms were refined anisotropically. Hydrogen atom positions were calculated geometrically and refined using the riding model. Hydrogen atom on Boron atom were refined using an AFIX 138, which would allow the refinement of a common B-H distances, the same shifts being applied to all the B-H bonds to the same atom, but still retain tetrahedral $\mathrm{H}-\mathrm{B}-\mathrm{H}$ angles within the group. The value of $\mathrm{Z}^{\prime}$ is 2 . This means that there are two independent molecules in the asymmetric unit. The Flack parameter was refined to 0.007(14). Determination of absolute structure using Bayesian statistics on Bijvoet differences using the Olex2 results in 0.021(17). Note: The Flack parameter is used to determine chirality of the crystal studied, the value should be near 0 , a value of 1 means that the stereochemistry is wrong and the model should be inverted. A value of 0.5 means that the crystal consists of a racemic mixture of the two enantiomers. The structure displays a merohedral twinning and the twin law was found by using TwinRotMat implemented in Platon (Spek, 2009). The

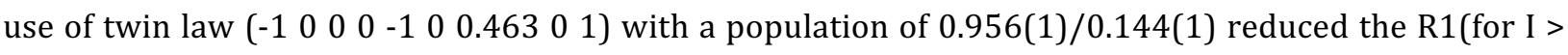
2sigma(I)) and Flack parameter from 10.04\%, 0.131(14) to 5.62\%, 0.007 (14).

Table S41: Bond Lengths in Å for compound 21f.

\begin{tabular}{lll}
\hline Atom & Atom & Length/Å \\
\hline P1 & C7 & $1.808(5)$ \\
P1 & N1 & $1.677(4)$ \\
P1 & C1 & $1.832(6)$ \\
P1 & B1 & $1.912(7)$ \\
P2 & O1 & $1.602(3)$ \\
P2 & C23 & $1.816(6)$ \\
P2 & C35 & $1.806(5)$ \\
P2 & B2 & $1.894(6)$ \\
P1A & N1A & $1.668(4)$ \\
P1A & C1A & $1.810(5)$ \\
P1A & C7A & $1.806(5)$ \\
P1A & B1A & $1.915(6)$ \\
P2A & 01A & $1.598(3)$ \\
P2A & C23A & $1.805(5)$
\end{tabular}

\begin{tabular}{lll}
\hline Atom & Atom & Length/Å \\
\hline P2A & B2A & $1.896(6)$ \\
P2A & C35A & $1.804(5)$ \\
01 & C16 & $1.462(6)$ \\
C40 & C35 & $1.390(7)$ \\
C40 & C39 & $1.392(8)$ \\
01A & C16A & $1.458(5)$ \\
C31 & C30 & $1.410(8)$ \\
C31 & C32 & $1.365(9)$ \\
C13 & N1 & $1.463(7)$ \\
N1A & C14A & $1.467(6)$ \\
N1A & C13A & $1.464(6)$ \\
C10 & C9 & $1.377(9)$ \\
C10 & C11 & $1.377(10)$ \\
C9 & C8 & $1.394(8)$
\end{tabular}




\begin{tabular}{|c|c|c|}
\hline Atom & Atom & Length/Å \\
\hline $\mathrm{C} 8$ & $\mathrm{C} 7$ & $1.389(8)$ \\
\hline $\mathrm{C} 7$ & $\mathrm{C} 12$ & $1.404(7)$ \\
\hline $\mathrm{N} 1$ & C14 & $1.477(6)$ \\
\hline C14 & C16 & $1.541(7)$ \\
\hline C14 & C15 & $1.525(7)$ \\
\hline C16 & C17 & $1.503(7)$ \\
\hline C23 & $\mathrm{C} 28$ & $1.405(8)$ \\
\hline C23 & $\mathrm{C} 24$ & $1.405(8)$ \\
\hline $\mathrm{C} 28$ & $\mathrm{C} 29$ & $1.498(8)$ \\
\hline C28 & $\mathrm{C} 27$ & $1.408(7)$ \\
\hline C29 & C30 & $1.382(8)$ \\
\hline C29 & C34 & $1.383(8)$ \\
\hline C32 & C33 & $1.391(9)$ \\
\hline C17 & $\mathrm{C} 22$ & $1.409(9)$ \\
\hline C17 & C18 & $1.372(9)$ \\
\hline C22 & $\mathrm{C} 21$ & $1.397(8)$ \\
\hline C21 & $\mathrm{C} 20$ & $1.381(9)$ \\
\hline C20 & C19 & $1.373(9)$ \\
\hline C18 & C19 & $1.401(8)$ \\
\hline C27 & $\mathrm{C} 26$ & $1.385(10)$ \\
\hline C26 & $\mathrm{C} 25$ & $1.367(12)$ \\
\hline C25 & $\mathrm{C} 24$ & $1.385(9)$ \\
\hline C33 & C34 & $1.401(9)$ \\
\hline C35 & C36 & $1.382(7)$ \\
\hline C39 & C38 & $1.386(9)$ \\
\hline C36 & C37 & $1.405(9)$ \\
\hline C1 & C6 & $1.374(9)$ \\
\hline C1 & $\mathrm{C} 2$ & $1.390(9)$ \\
\hline C6 & $\mathrm{C} 5$ & $1.398(8)$ \\
\hline C5 & $\mathrm{C} 4$ & $1.371(12)$ \\
\hline $\mathrm{C} 2$ & C3 & $1.394(9)$ \\
\hline C12 & C11 & $1.394(8)$ \\
\hline C4A & C3A & $1.380(9)$ \\
\hline C4A & C5A & $1.395(9)$ \\
\hline $\mathrm{C} 3 \mathrm{~A}$ & $\mathrm{C} 2 \mathrm{~A}$ & $1.392(8)$ \\
\hline $\mathrm{C} 2 \mathrm{~A}$ & $\mathrm{C} 1 \mathrm{~A}$ & $1.390(8)$ \\
\hline C1A & C6A & $1.403(8)$ \\
\hline
\end{tabular}

\begin{tabular}{|c|c|c|}
\hline Atom & Atom & Length/Å \\
\hline$\overline{C 14 A}$ & C16A & $1.536(7)$ \\
\hline C14A & C15A & $1.526(7)$ \\
\hline C16A & C17A & $1.514(7)$ \\
\hline C23A & $\mathrm{C} 28 \mathrm{~A}$ & $1.412(7)$ \\
\hline C23A & $\mathrm{C} 24 \mathrm{~A}$ & $1.410(7)$ \\
\hline C28A & C29A & $1.499(6)$ \\
\hline C28A & $\mathrm{C} 27 \mathrm{~A}$ & $1.393(7)$ \\
\hline C29A & C34A & $1.392(7)$ \\
\hline C29A & $\mathrm{C} 30 \mathrm{~A}$ & $1.392(7)$ \\
\hline C34A & C33A & $1.387(8)$ \\
\hline C33A & C32A & $1.394(9)$ \\
\hline C32A & C31A & $1.370(9)$ \\
\hline C10A & C9A & $1.391(7)$ \\
\hline C10A & C11A & $1.383(8)$ \\
\hline C12A & C7A & $1.404(7)$ \\
\hline C12A & $\mathrm{C} 11 \mathrm{~A}$ & $1.387(8)$ \\
\hline C7A & C8A & $1.392(7)$ \\
\hline $\mathrm{C} 8 \mathrm{~A}$ & C9A & $1.389(7)$ \\
\hline C5A & C6A & $1.402(8)$ \\
\hline C17A & $\mathrm{C} 22 \mathrm{~A}$ & $1.394(8)$ \\
\hline C17A & C18A & $1.383(8)$ \\
\hline $\mathrm{C} 22 \mathrm{~A}$ & $\mathrm{C} 21 \mathrm{~A}$ & $1.390(7)$ \\
\hline $\mathrm{C} 21 \mathrm{~A}$ & $\mathrm{C} 20 \mathrm{~A}$ & $1.393(7)$ \\
\hline $\mathrm{C} 20 \mathrm{~A}$ & C19A & $1.379(8)$ \\
\hline C19A & C18A & $1.399(7)$ \\
\hline C35A & $\mathrm{C} 36 \mathrm{~A}$ & $1.397(8)$ \\
\hline C35A & $\mathrm{C} 40 \mathrm{~A}$ & $1.396(8)$ \\
\hline C36A & C37A & $1.400(8)$ \\
\hline C37A & C38A & $1.390(9)$ \\
\hline C38A & C39A & $1.366(9)$ \\
\hline C39A & $\mathrm{C} 40 \mathrm{~A}$ & $1.400(8)$ \\
\hline $\mathrm{C} 24 \mathrm{~A}$ & $\mathrm{C} 25 \mathrm{~A}$ & $1.385(7)$ \\
\hline $\mathrm{C} 25 \mathrm{~A}$ & $\mathrm{C} 26 \mathrm{~A}$ & $1.389(8)$ \\
\hline C26A & $\mathrm{C} 27 \mathrm{~A}$ & $1.391(8)$ \\
\hline C31A & $\mathrm{C} 30 \mathrm{~A}$ & $1.386(7)$ \\
\hline C38 & C37 & $1.361(10)$ \\
\hline C4 & $\mathrm{C} 3$ & $1.376(13)$ \\
\hline
\end{tabular}

Table S42: Bond Angles in ${ }^{\circ}$ for compound $21 f$.

\begin{tabular}{lllr}
\hline Atom & Atom & Atom & Angle $/^{\circ}$ \\
\hline C7 & P1 & C1 & $105.6(2)$ \\
C7 & P1 & B1 & $112.4(3)$ \\
N1 & P1 & C7 & $103.2(2)$ \\
N1 & P1 & C1 & $108.1(2)$ \\
N1 & P1 & B1 & $114.1(3)$ \\
C1 & P1 & B1 & $112.7(3)$ \\
01 & P2 & C23 & $104.3(2)$ \\
O1 & P2 & C35 & $104.2(2)$ \\
01 & P2 & B2 & $115.4(2)$ \\
C23 & P2 & B2 & $112.0(3)$ \\
C35 & P2 & C23 & $107.9(2)$ \\
C35 & P2 & B2 & $112.4(3)$ \\
N1A & P1A & C1A & $104.6(2)$
\end{tabular}

\begin{tabular}{lllr}
\hline Atom & Atom & Atom & Angle \\
\hline N1A & P1A & C7A & $105.5(2)$ \\
N1A & P1A & B1A & $114.9(3)$ \\
C1A & P1A & B1A & $113.4(3)$ \\
C7A & P1A & C1A & $105.8(2)$ \\
C7A & P1A & B1A & $111.8(3)$ \\
01A & P2A & C23A & $104.4(2)$ \\
01A & P2A & B2A & $115.5(2)$ \\
01A & P2A & C35A & $104.5(2)$ \\
C23A & P2A & B2A & $113.0(2)$ \\
C35A & P2A & C23A & $108.7(2)$ \\
C35A & P2A & B2A & $110.2(2)$ \\
C16 & 01 & P2 & $121.9(3)$ \\
C35 & C40 & C39 & $119.7(5)$
\end{tabular}




\begin{tabular}{|c|c|c|c|}
\hline Atom & Atom & Atom & Angle $/{ }^{\circ}$ \\
\hline$\overline{\mathrm{C} 16 \mathrm{~A}}$ & 01A & P2A & $122.3(3)$ \\
\hline C32 & C31 & C30 & $120.2(5)$ \\
\hline C14A & $\mathrm{N} 1 \mathrm{~A}$ & $\mathrm{P} 1 \mathrm{~A}$ & $121.4(3)$ \\
\hline $\mathrm{C} 13 \mathrm{~A}$ & $\mathrm{~N} 1 \mathrm{~A}$ & P1A & $118.3(3)$ \\
\hline C13A & $\mathrm{N} 1 \mathrm{~A}$ & $\mathrm{C} 14 \mathrm{~A}$ & $117.5(4)$ \\
\hline C9 & $\mathrm{C} 10$ & C11 & $121.0(6)$ \\
\hline C10 & C9 & C8 & $119.6(6)$ \\
\hline $\mathrm{C} 7$ & $\mathrm{C} 8$ & C9 & $120.5(5)$ \\
\hline C8 & $\mathrm{C} 7$ & $\mathrm{P} 1$ & $119.7(4)$ \\
\hline C8 & $\mathrm{C} 7$ & $\mathrm{C} 12$ & $119.2(5)$ \\
\hline C12 & $\mathrm{C} 7$ & $\mathrm{P} 1$ & $121.0(4)$ \\
\hline C13 & N1 & $\mathrm{P} 1$ & $118.0(3)$ \\
\hline C13 & N1 & C14 & $117.3(4)$ \\
\hline C14 & N1 & $\mathrm{P} 1$ & $118.0(3)$ \\
\hline $\mathrm{N} 1$ & C14 & $\mathrm{C} 16$ & $108.2(4)$ \\
\hline N1 & C14 & C15 & $113.0(4)$ \\
\hline C15 & C14 & C16 & $111.7(4)$ \\
\hline 01 & C16 & $\mathrm{C} 14$ & $105.4(4)$ \\
\hline 01 & C16 & C17 & $108.3(4)$ \\
\hline C17 & C16 & C14 & $114.5(4)$ \\
\hline C28 & $\mathrm{C} 23$ & P2 & $124.3(4)$ \\
\hline $\mathrm{C} 24$ & $\mathrm{C} 23$ & $\mathrm{P} 2$ & $116.6(5)$ \\
\hline $\mathrm{C} 24$ & $\mathrm{C} 23$ & $\mathrm{C} 28$ & $119.0(5)$ \\
\hline C23 & $\mathrm{C} 28$ & $\mathrm{C} 29$ & $122.9(5)$ \\
\hline $\mathrm{C} 23$ & $\mathrm{C} 28$ & $\mathrm{C} 27$ & $118.8(5)$ \\
\hline C27 & C28 & $\mathrm{C} 29$ & $118.3(5)$ \\
\hline C30 & $\mathrm{C} 29$ & $\mathrm{C} 28$ & $119.8(5)$ \\
\hline C30 & C29 & C34 & $120.1(5)$ \\
\hline C34 & $\mathrm{C} 29$ & $\mathrm{C} 28$ & $120.1(5)$ \\
\hline C29 & C30 & C31 & $119.8(5)$ \\
\hline C31 & C32 & C33 & $120.0(6)$ \\
\hline $\mathrm{C} 22$ & $\mathrm{C} 17$ & $\mathrm{C} 16$ & $121.1(5)$ \\
\hline C18 & $\mathrm{C} 17$ & $\mathrm{C} 16$ & $120.1(6)$ \\
\hline C18 & $\mathrm{C} 17$ & $\mathrm{C} 22$ & $118.8(6)$ \\
\hline C21 & $\mathrm{C} 22$ & C17 & $119.6(6)$ \\
\hline C20 & $\mathrm{C} 21$ & $\mathrm{C} 22$ & $120.4(6)$ \\
\hline C19 & $\mathrm{C} 20$ & $\mathrm{C} 21$ & $120.2(6)$ \\
\hline C17 & C18 & C19 & $121.4(6)$ \\
\hline C26 & $\mathrm{C} 27$ & $\mathrm{C} 28$ & $120.6(6)$ \\
\hline $\mathrm{C} 25$ & $\mathrm{C} 26$ & $\mathrm{C} 27$ & $120.7(6)$ \\
\hline $\mathrm{C} 26$ & $\mathrm{C} 25$ & $\mathrm{C} 24$ & $119.9(6)$ \\
\hline C32 & C33 & $\mathrm{C} 34$ & $120.1(6)$ \\
\hline C29 & C34 & C33 & $119.7(5)$ \\
\hline $\mathrm{C} 40$ & C35 & $\mathrm{P} 2$ & $120.8(4)$ \\
\hline C36 & C35 & $\mathrm{P} 2$ & 119.1(4) \\
\hline C36 & C35 & $\mathrm{C} 40$ & $120.0(5)$ \\
\hline C38 & C39 & $\mathrm{C} 40$ & $120.1(6)$ \\
\hline C35 & C36 & C37 & $119.5(6)$ \\
\hline C6 & C1 & $\mathrm{P} 1$ & $122.4(4)$ \\
\hline C6 & $\mathrm{C} 1$ & $\mathrm{C} 2$ & $119.8(5)$ \\
\hline $\mathrm{C} 2$ & $\mathrm{C} 1$ & $\mathrm{P} 1$ & $117.5(5)$ \\
\hline C1 & $\mathrm{C} 6$ & $\mathrm{C} 5$ & $120.0(6)$ \\
\hline $\mathrm{C} 4$ & $\mathrm{C} 5$ & $\mathrm{C} 6$ & $120.0(7)$ \\
\hline C1 & $\mathrm{C} 2$ & $\mathrm{C} 3$ & $120.0(7)$ \\
\hline
\end{tabular}

\begin{tabular}{|c|c|c|c|}
\hline Atom & Atom & Atom & Angle $/{ }^{\circ}$ \\
\hline C11 & C12 & C7 & $119.9(5)$ \\
\hline C10 & C11 & $\mathrm{C} 12$ & $119.9(5)$ \\
\hline $\mathrm{C} 3 \mathrm{~A}$ & $\mathrm{C} 4 \mathrm{~A}$ & C5A & $119.9(5)$ \\
\hline $\mathrm{C} 4 \mathrm{~A}$ & C3A & $\mathrm{C} 2 \mathrm{~A}$ & $120.5(6)$ \\
\hline $\mathrm{C} 1 \mathrm{~A}$ & $\mathrm{C} 2 \mathrm{~A}$ & $\mathrm{C} 3 \mathrm{~A}$ & $120.6(5)$ \\
\hline $\mathrm{C} 2 \mathrm{~A}$ & C1A & $\mathrm{P} 1 \mathrm{~A}$ & $123.8(4)$ \\
\hline $\mathrm{C} 2 \mathrm{~A}$ & C1A & C6A & $118.9(5)$ \\
\hline $\mathrm{C} 6 \mathrm{~A}$ & C1A & $\mathrm{P} 1 \mathrm{~A}$ & $117.0(4)$ \\
\hline N1A & C14A & C16A & $108.6(4)$ \\
\hline $\mathrm{N} 1 \mathrm{~A}$ & C14A & C15A & $111.9(4)$ \\
\hline C15A & C14A & $\mathrm{C} 16 \mathrm{~A}$ & $111.9(4)$ \\
\hline $01 \mathrm{~A}$ & C16A & $\mathrm{C} 14 \mathrm{~A}$ & $107.3(4)$ \\
\hline $01 \mathrm{~A}$ & C16A & C17A & $107.8(4)$ \\
\hline C17A & C16A & C14A & $114.4(4)$ \\
\hline C28A & $\mathrm{C} 23 \mathrm{~A}$ & $\mathrm{P} 2 \mathrm{~A}$ & $124.4(4)$ \\
\hline C24A & $\mathrm{C} 23 \mathrm{~A}$ & P2A & $116.1(4)$ \\
\hline C24A & $\mathrm{C} 23 \mathrm{~A}$ & $\mathrm{C} 28 \mathrm{~A}$ & $119.2(4)$ \\
\hline C23A & $\mathrm{C} 28 \mathrm{~A}$ & $\mathrm{C} 29 \mathrm{~A}$ & $122.7(4)$ \\
\hline C27A & C28A & $\mathrm{C} 23 \mathrm{~A}$ & $118.5(4)$ \\
\hline C27A & $\mathrm{C} 28 \mathrm{~A}$ & $\mathrm{C} 29 \mathrm{~A}$ & $118.8(4)$ \\
\hline C34A & C29A & $\mathrm{C} 28 \mathrm{~A}$ & $119.9(4)$ \\
\hline C34A & C29A & $\mathrm{C} 30 \mathrm{~A}$ & $119.4(5)$ \\
\hline C30A & C29A & $\mathrm{C} 28 \mathrm{~A}$ & $120.7(4)$ \\
\hline C33A & C34A & C29A & $120.4(5)$ \\
\hline C34A & C33A & $\mathrm{C} 32 \mathrm{~A}$ & $119.6(5)$ \\
\hline C31A & $\mathrm{C} 32 \mathrm{~A}$ & C33A & $119.9(5)$ \\
\hline C11A & C10A & C9A & $120.2(5)$ \\
\hline C11A & $\mathrm{C} 12 \mathrm{~A}$ & C7A & $119.8(5)$ \\
\hline C12A & C7A & P1A & $120.3(4)$ \\
\hline C8A & C7A & P1A & $120.2(4)$ \\
\hline C8A & C7A & $\mathrm{C} 12 \mathrm{~A}$ & $119.4(5)$ \\
\hline C9A & C8A & C7A & $120.4(5)$ \\
\hline C8A & C9A & $\mathrm{C} 10 \mathrm{~A}$ & $119.8(5)$ \\
\hline $\mathrm{C} 4 \mathrm{~A}$ & C5A & C6A & $119.7(5)$ \\
\hline $\mathrm{C} 5 \mathrm{~A}$ & $\mathrm{C} 6 \mathrm{~A}$ & C1A & $120.3(6)$ \\
\hline C22A & C17A & C16A & $120.7(5)$ \\
\hline C18A & C17A & C16A & 119.1(5) \\
\hline C18A & C17A & $\mathrm{C} 22 \mathrm{~A}$ & $120.2(5)$ \\
\hline C21A & $\mathrm{C} 22 \mathrm{~A}$ & C17A & $120.0(5)$ \\
\hline $\mathrm{C} 22 \mathrm{~A}$ & $\mathrm{C} 21 \mathrm{~A}$ & $\mathrm{C} 20 \mathrm{~A}$ & $119.6(5)$ \\
\hline C19A & $\mathrm{C} 20 \mathrm{~A}$ & $\mathrm{C} 21 \mathrm{~A}$ & $120.5(5)$ \\
\hline $\mathrm{C} 20 \mathrm{~A}$ & C19A & C18A & $120.0(5)$ \\
\hline $\mathrm{C} 17 \mathrm{~A}$ & C18A & C19A & $119.8(5)$ \\
\hline C36A & C35A & P2A & $120.8(4)$ \\
\hline C36A & C35A & $\mathrm{C} 40 \mathrm{~A}$ & $119.9(5)$ \\
\hline C40A & C35A & P2A & $119.1(4)$ \\
\hline C35A & $\mathrm{C} 36 \mathrm{~A}$ & C37A & $119.6(5)$ \\
\hline C38A & C37A & $\mathrm{C} 36 \mathrm{~A}$ & $119.9(6)$ \\
\hline C39A & C38A & C37A & $120.5(5)$ \\
\hline C38A & C39A & $\mathrm{C} 40 \mathrm{~A}$ & $120.5(6)$ \\
\hline C35A & $\mathrm{C} 40 \mathrm{~A}$ & C39A & $119.6(5)$ \\
\hline C25A & $\mathrm{C} 24 \mathrm{~A}$ & $\mathrm{C} 23 \mathrm{~A}$ & $121.0(5)$ \\
\hline $\mathrm{C} 24 \mathrm{~A}$ & $\mathrm{C} 25 \mathrm{~A}$ & $\mathrm{C} 26 \mathrm{~A}$ & $119.7(5)$ \\
\hline C25A & $\mathrm{C} 26 \mathrm{~A}$ & $\mathrm{C} 27 \mathrm{~A}$ & $119.7(5)$ \\
\hline
\end{tabular}




\begin{tabular}{llll}
\hline Atom & Atom & Atom & Angle $^{\circ}$ \\
\hline C26A & C27A & C28A & $121.8(5)$ \\
C32A & C31A & C30A & $121.0(5)$ \\
C31A & C30A & C29A & $119.7(5)$ \\
C10A & C11A & C12A & $120.4(5)$ \\
C25 & C24 & C23 & $121.0(6)$
\end{tabular}

\begin{tabular}{lllc}
\hline Atom & Atom & Atom & Angle $^{\circ}$ \\
\hline C37 & C38 & C39 & $120.2(6)$ \\
C38 & C37 & C36 & $120.5(6)$ \\
C20 & C19 & C18 & $119.6(6)$ \\
C5 & C4 & C3 & $120.5(6)$ \\
C4 & C3 & C2 & $119.7(7)$
\end{tabular}

Table S43: Torsion Angles in ${ }^{\circ}$ for compound $21 f$.

\begin{tabular}{|c|c|c|c|c|}
\hline Atom & Atom & Atom & Atom & Angle $/^{\circ}$ \\
\hline P1 & $\mathrm{C} 7$ & C12 & C11 & $-176.9(4)$ \\
\hline P1 & N1 & C14 & C16 & $148.7(3)$ \\
\hline P1 & N1 & C14 & C15 & $-87.1(5)$ \\
\hline P1 & $\mathrm{C} 1$ & C6 & $\mathrm{C} 5$ & $-174.8(4)$ \\
\hline P1 & $\mathrm{C} 1$ & $\mathrm{C} 2$ & C3 & $174.9(5)$ \\
\hline P2 & 01 & C16 & C14 & $-121.7(4)$ \\
\hline P2 & 01 & C16 & C17 & $115.3(4)$ \\
\hline P2 & $\mathrm{C} 23$ & C28 & C29 & $5.3(7)$ \\
\hline $\mathrm{P} 2$ & $\mathrm{C} 23$ & C28 & $\mathrm{C} 27$ & $-173.8(4)$ \\
\hline P2 & $\mathrm{C} 23$ & $\mathrm{C} 24$ & $\mathrm{C} 25$ & $176.2(4)$ \\
\hline P2 & C35 & C36 & C37 & $-179.3(6)$ \\
\hline P1A & N1A & C14A & $\mathrm{C} 16 \mathrm{~A}$ & $143.3(3)$ \\
\hline P1A & $\mathrm{N} 1 \mathrm{~A}$ & C14A & C15A & $-92.6(5)$ \\
\hline $\mathrm{P} 1 \mathrm{~A}$ & C1A & C6A & $\mathrm{C} 5 \mathrm{~A}$ & $171.2(4)$ \\
\hline $\mathrm{P} 1 \mathrm{~A}$ & C7A & C8A & C9A & $177.6(4)$ \\
\hline P2A & $01 \mathrm{~A}$ & C16A & C14A & $-116.7(4)$ \\
\hline $\mathrm{P} 2 \mathrm{~A}$ & $01 \mathrm{~A}$ & C16A & C17A & $119.7(4)$ \\
\hline $\mathrm{P} 2 \mathrm{~A}$ & $\mathrm{C} 23 \mathrm{~A}$ & $\mathrm{C} 28 \mathrm{~A}$ & C29A & $6.8(7)$ \\
\hline P2A & $\mathrm{C} 23 \mathrm{~A}$ & $\mathrm{C} 28 \mathrm{~A}$ & $\mathrm{C} 27 \mathrm{~A}$ & $-172.3(4)$ \\
\hline P2A & $\mathrm{C} 23 \mathrm{~A}$ & $\mathrm{C} 24 \mathrm{~A}$ & $\mathrm{C} 25 \mathrm{~A}$ & $173.9(4)$ \\
\hline $\mathrm{P} 2 \mathrm{~A}$ & C35A & C36A & C37A & $172.9(5)$ \\
\hline P2A & C35A & $\mathrm{C} 40 \mathrm{~A}$ & C39A & $-173.8(5)$ \\
\hline 01 & P2 & $\mathrm{C} 23$ & C28 & 29.7(5) \\
\hline 01 & $\mathrm{P} 2$ & $\mathrm{C} 23$ & $\mathrm{C} 24$ & $-146.1(4)$ \\
\hline 01 & P2 & C35 & $\mathrm{C} 40$ & $4.3(5)$ \\
\hline 01 & P2 & C35 & C36 & $-178.0(5)$ \\
\hline 01 & C16 & C17 & $\mathrm{C} 22$ & $66.3(7)$ \\
\hline 01 & C16 & C17 & C18 & $-114.9(6)$ \\
\hline $\mathrm{C} 40$ & C35 & C36 & C37 & $-1.6(10)$ \\
\hline $\mathrm{C} 40$ & C39 & C38 & C37 & $-0.5(10)$ \\
\hline $01 \mathrm{~A}$ & $\mathrm{P} 2 \mathrm{~A}$ & $\mathrm{C} 23 \mathrm{~A}$ & C28A & $26.7(5)$ \\
\hline $01 \mathrm{~A}$ & $\mathrm{P} 2 \mathrm{~A}$ & $\mathrm{C} 23 \mathrm{~A}$ & $\mathrm{C} 24 \mathrm{~A}$ & $-147.3(4)$ \\
\hline $01 \mathrm{~A}$ & $\mathrm{P} 2 \mathrm{~A}$ & C35A & C36A & $16.0(5)$ \\
\hline $01 \mathrm{~A}$ & $\mathrm{P} 2 \mathrm{~A}$ & C35A & $\mathrm{C} 40 \mathrm{~A}$ & $-170.0(4)$ \\
\hline $01 \mathrm{~A}$ & C16A & C17A & $\mathrm{C} 22 \mathrm{~A}$ & $67.2(6)$ \\
\hline $01 \mathrm{~A}$ & C16A & C17A & C18A & $-112.9(5)$ \\
\hline C31 & C32 & C33 & C34 & $1.2(9)$ \\
\hline C13 & N1 & C14 & C16 & $-60.5(6)$ \\
\hline C13 & $\mathrm{N} 1$ & C14 & C15 & $63.8(6)$ \\
\hline N1A & P1A & C1A & $\mathrm{C} 2 \mathrm{~A}$ & $100.5(5)$ \\
\hline $\mathrm{N} 1 \mathrm{~A}$ & $\mathrm{P} 1 \mathrm{~A}$ & C1A & C6A & $-73.2(4)$ \\
\hline N1A & P1A & C7A & $\mathrm{C} 12 \mathrm{~A}$ & $-171.5(4)$ \\
\hline $\mathrm{N} 1 \mathrm{~A}$ & $\mathrm{P} 1 \mathrm{~A}$ & C7A & C8A & $12.0(5)$ \\
\hline $\mathrm{N} 1 \mathrm{~A}$ & C14A & C16A & $01 \mathrm{~A}$ & $-175.0(4)$ \\
\hline $\mathrm{N} 1 \mathrm{~A}$ & C14A & C16A & C17A & $-55.5(5)$ \\
\hline
\end{tabular}




\begin{tabular}{|c|c|c|c|c|}
\hline Atom & Atom & Atom & Atom & Angle $/^{\circ}$ \\
\hline C10 & C9 & $\mathrm{C} 8$ & $\mathrm{C} 7$ & $1.1(10)$ \\
\hline C9 & $\mathrm{C} 10$ & C11 & C12 & $-0.2(9)$ \\
\hline C9 & $\mathrm{C} 8$ & $\mathrm{C} 7$ & P1 & $175.8(5)$ \\
\hline C9 & $\mathrm{C} 8$ & $\mathrm{C} 7$ & C12 & $0.0(9)$ \\
\hline C8 & $\mathrm{C} 7$ & C12 & C11 & $-1.2(8)$ \\
\hline C7 & P1 & N1 & C13 & $64.6(4)$ \\
\hline C7 & P1 & N1 & C14 & $-144.8(4)$ \\
\hline $\mathrm{C} 7$ & P1 & $\mathrm{C} 1$ & C6 & $-32.6(5)$ \\
\hline $\mathrm{C} 7$ & P1 & $\mathrm{C} 1$ & $\mathrm{C} 2$ & $153.4(5)$ \\
\hline $\mathrm{C} 7$ & $\mathrm{C} 12$ & C11 & C10 & $1.3(9)$ \\
\hline N1 & P1 & C7 & C8 & $33.1(5)$ \\
\hline N1 & P1 & $\mathrm{C} 7$ & C12 & $-151.2(4)$ \\
\hline N1 & P1 & $\mathrm{C} 1$ & C6 & 77.3(5) \\
\hline N1 & P1 & $\mathrm{C} 1$ & $\mathrm{C} 2$ & $-96.6(5)$ \\
\hline N1 & C14 & C16 & 01 & $-172.2(4)$ \\
\hline N1 & C14 & C16 & C17 & $-53.4(6)$ \\
\hline C14 & C16 & C17 & $\mathrm{C} 22$ & $-50.9(7)$ \\
\hline C14 & C16 & C17 & C18 & $127.8(6)$ \\
\hline C16 & C17 & $\mathrm{C} 22$ & C21 & $177.2(5)$ \\
\hline C16 & $\mathrm{C} 17$ & C18 & C19 & $-177.2(5)$ \\
\hline C23 & $\mathrm{P} 2$ & 01 & C16 & $132.3(4)$ \\
\hline C23 & P2 & C35 & C40 & $114.8(5)$ \\
\hline C23 & P2 & C35 & C36 & $-67.6(5)$ \\
\hline C23 & $\mathrm{C} 28$ & $\mathrm{C} 29$ & C30 & $72.2(7)$ \\
\hline $\mathrm{C} 23$ & $\mathrm{C} 28$ & $\mathrm{C} 29$ & C34 & $-107.1(6)$ \\
\hline C23 & $\mathrm{C} 28$ & $\mathrm{C} 27$ & C26 & $-2.4(8)$ \\
\hline C28 & $\mathrm{C} 23$ & $\mathrm{C} 24$ & $\mathrm{C} 25$ & $0.2(8)$ \\
\hline C28 & $\mathrm{C} 29$ & C30 & C31 & $-176.5(5)$ \\
\hline C28 & $\mathrm{C} 29$ & C34 & C33 & $175.9(5)$ \\
\hline C28 & $\mathrm{C} 27$ & $\mathrm{C} 26$ & $\mathrm{C} 25$ & $0.8(9)$ \\
\hline C29 & $\mathrm{C} 28$ & $\mathrm{C} 27$ & C26 & $178.4(5)$ \\
\hline C30 & C31 & C32 & C33 & $-1.8(9)$ \\
\hline C30 & $\mathrm{C} 29$ & C34 & C33 & $-3.4(8)$ \\
\hline C32 & C31 & C30 & C29 & $-0.2(8)$ \\
\hline C32 & C33 & C34 & C29 & $1.4(9)$ \\
\hline C17 & $\mathrm{C} 22$ & $\mathrm{C} 21$ & $\mathrm{C} 20$ & $1.4(9)$ \\
\hline C17 & C18 & C19 & $\mathrm{C} 20$ & $-1.4(9)$ \\
\hline C22 & C17 & C18 & C19 & $1.5(9)$ \\
\hline C22 & $\mathrm{C} 21$ & $\mathrm{C} 20$ & C19 & $-1.2(9)$ \\
\hline C21 & $\mathrm{C} 20$ & C19 & C18 & $1.2(9)$ \\
\hline C18 & C17 & $\mathrm{C} 22$ & C21 & $-1.5(9)$ \\
\hline C27 & C28 & $\mathrm{C} 29$ & C30 & $-108.8(6)$ \\
\hline $\mathrm{C} 27$ & C28 & C29 & C34 & $72.0(7)$ \\
\hline C27 & C26 & $\mathrm{C} 25$ & $\mathrm{C} 24$ & $1.3(9)$ \\
\hline C26 & $\mathrm{C} 25$ & $\mathrm{C} 24$ & C23 & $-1.8(9)$ \\
\hline C34 & C29 & C30 & C31 & $2.8(8)$ \\
\hline C35 & P2 & 01 & C16 & $-114.6(4)$ \\
\hline C35 & P2 & $\mathrm{C} 23$ & C28 & $-80.6(5)$ \\
\hline C35 & P2 & C23 & $\mathrm{C} 24$ & $103.5(4)$ \\
\hline C35 & C40 & C39 & C38 & $-0.9(9)$ \\
\hline C35 & C36 & C37 & C38 & $0.2(11)$ \\
\hline C39 & C40 & C35 & P2 & $179.6(4)$ \\
\hline C39 & C40 & C35 & C36 & $2.0(8)$ \\
\hline C39 & C38 & C37 & C36 & $0.9(12)$ \\
\hline
\end{tabular}




\begin{tabular}{|c|c|c|c|c|}
\hline Atom & Atom & Atom & Atom & Angle ${ }^{\circ}$ \\
\hline$\overline{C 15}$ & C14 & C16 & 01 & $62.7(5)$ \\
\hline C15 & C14 & C16 & $\mathrm{C} 17$ & $-178.4(5)$ \\
\hline C1 & P1 & $\mathrm{C} 7$ & C8 & $146.4(5)$ \\
\hline $\mathrm{C} 1$ & $\mathrm{P} 1$ & $\mathrm{C} 7$ & $\mathrm{C} 12$ & $-37.8(5)$ \\
\hline $\mathrm{C} 1$ & P1 & N1 & C13 & $-47.0(5)$ \\
\hline $\mathrm{C} 1$ & $\mathrm{P} 1$ & N1 & C14 & $103.7(4)$ \\
\hline $\mathrm{C} 1$ & $\mathrm{C} 6$ & $\mathrm{C} 5$ & $\mathrm{C} 4$ & $-0.3(10)$ \\
\hline $\mathrm{C} 1$ & $\mathrm{C} 2$ & $\mathrm{C} 3$ & $\mathrm{C} 4$ & $0.7(11)$ \\
\hline $\mathrm{C} 6$ & $\mathrm{C} 1$ & $\mathrm{C} 2$ & $\mathrm{C} 3$ & $0.8(9)$ \\
\hline $\mathrm{C} 6$ & $\mathrm{C} 5$ & $\mathrm{C} 4$ & $\mathrm{C} 3$ & $1.8(10)$ \\
\hline $\mathrm{C} 5$ & $\mathrm{C} 4$ & $\mathrm{C} 3$ & $\mathrm{C} 2$ & $-2.0(11)$ \\
\hline $\mathrm{C} 2$ & $\mathrm{C} 1$ & C6 & $\mathrm{C} 5$ & $-1.0(9)$ \\
\hline C11 & C10 & $\mathrm{C} 9$ & C8 & $-1.0(10)$ \\
\hline C4A & C3A & $\mathrm{C} 2 \mathrm{~A}$ & $\mathrm{C} 1 \mathrm{~A}$ & $-0.9(9)$ \\
\hline C4A & C5A & C6A & C1A & $1.5(9)$ \\
\hline C3A & $\mathrm{C} 4 \mathrm{~A}$ & $\mathrm{C} 5 \mathrm{~A}$ & C6A & $0.1(9)$ \\
\hline C3A & $\mathrm{C} 2 \mathrm{~A}$ & C1A & P1A & $-171.1(4)$ \\
\hline C3A & $\mathrm{C} 2 \mathrm{~A}$ & C1A & C6A & $2.5(8)$ \\
\hline $\mathrm{C} 2 \mathrm{~A}$ & C1A & C6A & C5A & $-2.8(8)$ \\
\hline C1A & P1A & $\mathrm{N} 1 \mathrm{~A}$ & C14A & $120.1(4)$ \\
\hline C1A & P1A & $\mathrm{N} 1 \mathrm{~A}$ & C13A & $-40.7(4)$ \\
\hline C1A & P1A & $\mathrm{C} 7 \mathrm{~A}$ & C12A & $-61.0(5)$ \\
\hline C1A & P1A & C7A & C8A & $122.5(4)$ \\
\hline C14A & C16A & C17A & $\mathrm{C} 22 \mathrm{~A}$ & $-52.0(6)$ \\
\hline C14A & C16A & C17A & C18A & $127.9(5)$ \\
\hline C16A & C17A & $\mathrm{C} 22 \mathrm{~A}$ & $\mathrm{C} 21 \mathrm{~A}$ & $177.8(5)$ \\
\hline C16A & C17A & C18A & C19A & $-177.2(5)$ \\
\hline $\mathrm{C} 23 \mathrm{~A}$ & P2A & $01 \mathrm{~A}$ & C16A & $127.4(4)$ \\
\hline $\mathrm{C} 23 \mathrm{~A}$ & $\mathrm{P} 2 \mathrm{~A}$ & C35A & C36A & $127.0(4)$ \\
\hline $\mathrm{C} 23 \mathrm{~A}$ & $\mathrm{P} 2 \mathrm{~A}$ & C35A & $\mathrm{C} 40 \mathrm{~A}$ & $-59.0(5)$ \\
\hline $\mathrm{C} 23 \mathrm{~A}$ & C28A & C29A & C34A & $-108.8(6)$ \\
\hline $\mathrm{C} 23 \mathrm{~A}$ & C28A & C29A & $\mathrm{C} 30 \mathrm{~A}$ & $70.0(7)$ \\
\hline $\mathrm{C} 23 \mathrm{~A}$ & $\mathrm{C} 28 \mathrm{~A}$ & C27A & $\mathrm{C} 26 \mathrm{~A}$ & $-0.8(8)$ \\
\hline $\mathrm{C} 23 \mathrm{~A}$ & $\mathrm{C} 24 \mathrm{~A}$ & $\mathrm{C} 25 \mathrm{~A}$ & $\mathrm{C} 26 \mathrm{~A}$ & $-1.3(8)$ \\
\hline $\mathrm{C} 28 \mathrm{~A}$ & $\mathrm{C} 23 \mathrm{~A}$ & $\mathrm{C} 24 \mathrm{~A}$ & $\mathrm{C} 25 \mathrm{~A}$ & $-0.4(7)$ \\
\hline C28A & C29A & C34A & C33A & $178.5(5)$ \\
\hline C28A & C29A & C30A & C31A & $-178.5(5)$ \\
\hline C29A & C28A & $\mathrm{C} 27 \mathrm{~A}$ & $\mathrm{C} 26 \mathrm{~A}$ & $-180.0(5)$ \\
\hline C29A & C34A & C33A & C32A & $0.6(8)$ \\
\hline C34A & C29A & $\mathrm{C} 30 \mathrm{~A}$ & C31A & $0.3(8)$ \\
\hline C34A & C33A & C32A & C31A & $-0.8(9)$ \\
\hline C33A & C32A & C31A & $\mathrm{C} 30 \mathrm{~A}$ & $0.8(9)$ \\
\hline C32A & C31A & C30A & $\mathrm{C} 29 \mathrm{~A}$ & $-0.5(9)$ \\
\hline $\mathrm{C} 12 \mathrm{~A}$ & C7A & C8A & C9A & 1.1(7) \\
\hline C7A & P1A & $\mathrm{N} 1 \mathrm{~A}$ & C14A & $-128.6(4)$ \\
\hline C7A & $\mathrm{P} 1 \mathrm{~A}$ & $\mathrm{~N} 1 \mathrm{~A}$ & C13A & $70.7(4)$ \\
\hline C7A & P1A & C1A & $\mathrm{C} 2 \mathrm{~A}$ & $-10.6(5)$ \\
\hline C7A & P1A & C1A & C6A & $175.7(4)$ \\
\hline C7A & $\mathrm{C} 12 \mathrm{~A}$ & C11A & $\mathrm{C} 10 \mathrm{~A}$ & $-0.1(8)$ \\
\hline C7A & C8A & C9A & $\mathrm{C} 10 \mathrm{~A}$ & $0.1(8)$ \\
\hline C9A & $\mathrm{C} 10 \mathrm{~A}$ & $\mathrm{C} 11 \mathrm{~A}$ & $\mathrm{C} 12 \mathrm{~A}$ & $1.4(9)$ \\
\hline C5A & $\mathrm{C} 4 \mathrm{~A}$ & C3A & $\mathrm{C} 2 \mathrm{~A}$ & $-0.4(9)$ \\
\hline C13A & $\mathrm{N} 1 \mathrm{~A}$ & C14A & C16A & $-55.8(5)$ \\
\hline C13A & $\mathrm{N} 1 \mathrm{~A}$ & C14A & C15A & $68.3(5)$ \\
\hline
\end{tabular}




\begin{tabular}{|c|c|c|c|c|}
\hline Atom & Atom & Atom & Atom & Angle $/^{\circ}$ \\
\hline$\overline{C 17 A}$ & $\mathrm{C} 22 \mathrm{~A}$ & $\mathrm{C} 21 \mathrm{~A}$ & $\mathrm{C} 20 \mathrm{~A}$ & $0.8(8)$ \\
\hline C22A & C17A & C18A & C19A & $2.7(8)$ \\
\hline C22A & $\mathrm{C} 21 \mathrm{~A}$ & $\mathrm{C} 20 \mathrm{~A}$ & C19A & $-0.1(8)$ \\
\hline C21A & $\mathrm{C} 20 \mathrm{~A}$ & C19A & C18A & $0.7(8)$ \\
\hline C20A & C19A & C18A & C17A & $-2.0(8)$ \\
\hline C18A & C17A & $\mathrm{C} 22 \mathrm{~A}$ & $\mathrm{C} 21 \mathrm{~A}$ & $-2.0(8)$ \\
\hline B2A & $\mathrm{P} 2 \mathrm{~A}$ & $01 \mathrm{~A}$ & C16A & $2.7(4)$ \\
\hline B2A & P2A & $\mathrm{C} 23 \mathrm{~A}$ & C28A & $152.9(4)$ \\
\hline B2A & P2A & $\mathrm{C} 23 \mathrm{~A}$ & $\mathrm{C} 24 \mathrm{~A}$ & $-21.1(5)$ \\
\hline B2A & $\mathrm{P} 2 \mathrm{~A}$ & C35A & C36A & $-108.6(5)$ \\
\hline B2A & $\mathrm{P} 2 \mathrm{~A}$ & C35A & $\mathrm{C} 40 \mathrm{~A}$ & $65.4(5)$ \\
\hline C35A & P2A & $01 \mathrm{~A}$ & C16A & $-118.5(4)$ \\
\hline C35A & P2A & $\mathrm{C} 23 \mathrm{~A}$ & C28A & $-84.4(4)$ \\
\hline C35A & P2A & $\mathrm{C} 23 \mathrm{~A}$ & $\mathrm{C} 24 \mathrm{~A}$ & $101.6(4)$ \\
\hline C35A & $\mathrm{C} 36 \mathrm{~A}$ & C37A & C38A & $0.5(9)$ \\
\hline $\mathrm{C} 36 \mathrm{~A}$ & C35A & $\mathrm{C} 40 \mathrm{~A}$ & C39A & $0.2(9)$ \\
\hline $\mathrm{C} 36 \mathrm{~A}$ & C37A & C38A & C39A & $0.7(10)$ \\
\hline C37A & C38A & C39A & $\mathrm{C} 40 \mathrm{~A}$ & $-1.5(10)$ \\
\hline C38A & C39A & $\mathrm{C} 40 \mathrm{~A}$ & C35A & $1.0(9)$ \\
\hline $\mathrm{C} 40 \mathrm{~A}$ & C35A & C36A & C37A & $-1.0(8)$ \\
\hline C24A & $\mathrm{C} 23 \mathrm{~A}$ & C28A & C29A & $-179.4(4)$ \\
\hline $\mathrm{C} 24 \mathrm{~A}$ & $\mathrm{C} 23 \mathrm{~A}$ & $\mathrm{C} 28 \mathrm{~A}$ & C27A & $1.5(7)$ \\
\hline $\mathrm{C} 24 \mathrm{~A}$ & $\mathrm{C} 25 \mathrm{~A}$ & $\mathrm{C} 26 \mathrm{~A}$ & $\mathrm{C} 27 \mathrm{~A}$ & $1.9(8)$ \\
\hline $\mathrm{C} 25 \mathrm{~A}$ & $\mathrm{C} 26 \mathrm{~A}$ & $\mathrm{C} 27 \mathrm{~A}$ & $\mathrm{C} 28 \mathrm{~A}$ & $-0.9(8)$ \\
\hline $\mathrm{C} 27 \mathrm{~A}$ & $\mathrm{C} 28 \mathrm{~A}$ & C29A & $\mathrm{C} 34 \mathrm{~A}$ & $70.3(6)$ \\
\hline C27A & $\mathrm{C} 28 \mathrm{~A}$ & C29A & $\mathrm{C} 30 \mathrm{~A}$ & $-110.9(6)$ \\
\hline $\mathrm{C} 30 \mathrm{~A}$ & C29A & C34A & $\mathrm{C} 33 \mathrm{~A}$ & $-0.3(8)$ \\
\hline C15A & $\mathrm{C} 14 \mathrm{~A}$ & C16A & $01 \mathrm{~A}$ & $61.0(5)$ \\
\hline C15A & C14A & C16A & C17A & $-179.5(4)$ \\
\hline B2 & $\mathrm{P} 2$ & 01 & C16 & $9.1(4)$ \\
\hline B2 & P2 & $\mathrm{C} 23$ & $\mathrm{C} 28$ & $155.2(4)$ \\
\hline B2 & P2 & $\mathrm{C} 23$ & $\mathrm{C} 24$ & $-20.6(5)$ \\
\hline B2 & P2 & C35 & C40 & $-121.3(5)$ \\
\hline B2 & $\mathrm{P} 2$ & C35 & C36 & $56.3(6)$ \\
\hline B1 & P1 & $\mathrm{C} 7$ & C8 & $-90.3(5)$ \\
\hline B1 & P1 & C7 & C12 & 85.4(5) \\
\hline B1 & $\mathrm{P} 1$ & N1 & C13 & $-173.2(4)$ \\
\hline B1 & $\mathrm{P} 1$ & N1 & C14 & $-22.5(5)$ \\
\hline B1 & $\mathrm{P} 1$ & $\mathrm{C} 1$ & $\mathrm{C} 6$ & $-155.6(5)$ \\
\hline B1 & $\mathrm{P} 1$ & $\mathrm{C} 1$ & $\mathrm{C} 2$ & $30.4(6)$ \\
\hline C11A & C10A & C9A & C8A & $-1.4(8)$ \\
\hline C11A & $\mathrm{C} 12 \mathrm{~A}$ & C7A & P1A & $-177.6(4)$ \\
\hline C11A & $\mathrm{C} 12 \mathrm{~A}$ & C7A & $\mathrm{C} 8 \mathrm{~A}$ & $-1.1(8)$ \\
\hline B1A & $\mathrm{P} 1 \mathrm{~A}$ & $\mathrm{~N} 1 \mathrm{~A}$ & C14A & $-5.0(5)$ \\
\hline B1A & P1A & $\mathrm{N} 1 \mathrm{~A}$ & $\mathrm{C} 13 \mathrm{~A}$ & $-165.7(4)$ \\
\hline B1A & P1A & C1A & $\mathrm{C} 2 \mathrm{~A}$ & $-133.5(5)$ \\
\hline B1A & P1A & C1A & C6A & $52.7(5)$ \\
\hline B1A & P1A & C7A & C12A & $62.9(5)$ \\
\hline B1A & P1A & C7A & C8A & $-113.6(4)$ \\
\hline C24 & C23 & C28 & C29 & $-179.0(5)$ \\
\hline C24 & $\mathrm{C} 23$ & $\mathrm{C} 28$ & $\mathrm{C} 27$ & $1.9(7)$ \\
\hline
\end{tabular}


Name: $\left(R_{\mathrm{p}}\right)-(+)-\mathrm{N}-[(1 R, 2 S), 2$-Ferrocenylphenylphosphinito-borane)-1-methyl-2-phenylethyl], Nmethylamino diphenylphosphine-borane $\mathbf{2 1 g}$.

Submitted by: Antonin Jaillet

Solved by: Yoann Rousselin

Sample ID: $\quad$ 15AK243

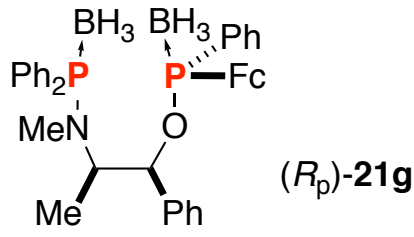

\section{Crystal Data and Experimental}

Figure S15 (thermal ellipsoïds are drawn at $50 \%$ probability plot)

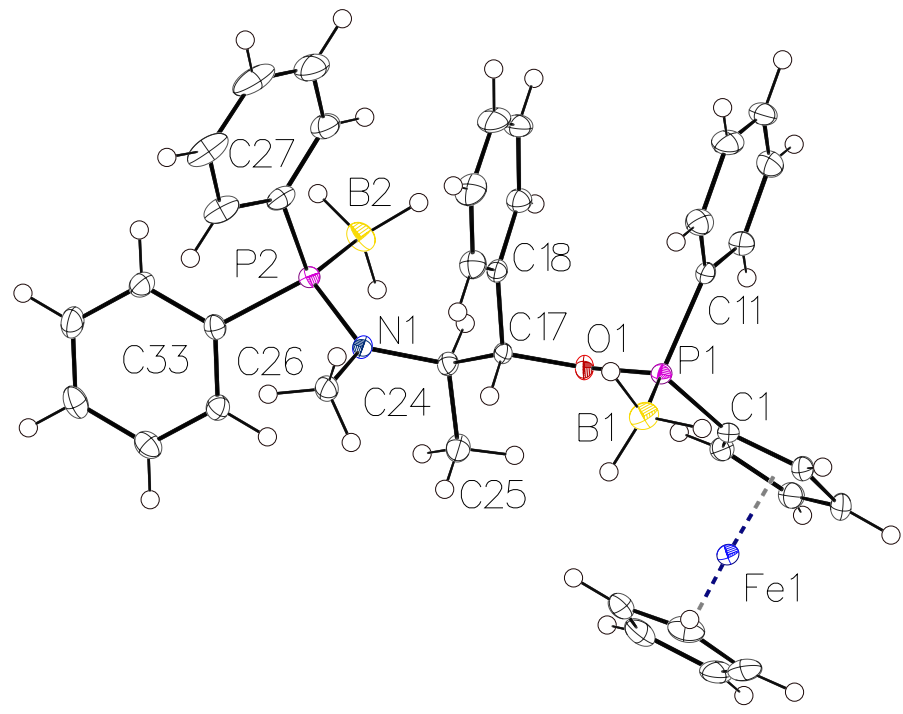

Experimental. Single clear light orange prism-shaped crystals of compound $\mathbf{2 1 g}$ were recrystallized from DCM by slow evaporation. A suitable crystal $0.33 \times 0.29 \times 0.28$ $\mathrm{mm}^{3}$ was selected and mounted on a MITIGEN holder oil on a Bruker D8 Venture (Mo) diffractometer. The crystal was kept at a steady $T=100.0(1) \mathrm{K}$ during data collection. The structure was solved with the ShelXT (Sheldrick, 2015) structure solution program using the Intrinsic Phasing solution method and by using Olex2 (Dolomanov et al., 2009) as the graphical interface. The model was refined with version 2018/3 of ShelXL (Sheldrick, 2015) using Least Squares minimization.

Crystal Data. $\quad \mathrm{C}_{38} \mathrm{H}_{43} \mathrm{~B}_{2} \mathrm{FeNOP}_{2}, \quad M_{r}=669.14$, orthorhombic, $P 2{ }_{1} 2{ }_{1} 2_{1}$ (No. 19), $a=7.9290(9) \AA, b=$ 9.6907(11) $, \quad \mathrm{c}=45.127(5) \AA, \quad \alpha=\beta=\gamma=90^{\circ}, \quad V=$ 3467.5(7) $\AA^{3}, T=100.0(1) \mathrm{K}, Z=4, Z^{\prime}=1, \mu\left(\mathrm{MoK}_{\alpha}\right)=$ $0.558,53809$ reflections measured, 7981 unique $\left(R_{\text {int }}=\right.$ $0.0222)$ which were used in all calculations. The final $w R_{2}$ was 0.0578 (all data) and $R_{1}$ was 0.0230 (I > 2(I)). 


\begin{tabular}{|c|c|}
\hline Compound & $21 g$ \\
\hline CCDC & 1982652 \\
\hline Formula & $\mathrm{C}_{38} \mathrm{H}_{43} \mathrm{~B}_{2} \mathrm{FeNOP}$ \\
\hline$D_{\text {calc. }} / \mathrm{g} \mathrm{cm}^{-3}$ & 1.282 \\
\hline$\mu / \mathrm{mm}^{-1}$ & 0.558 \\
\hline Formula Weight & 669.14 \\
\hline Color & clear light orange \\
\hline Shape & prism \\
\hline $\mathrm{Size} / \mathrm{mm}^{3}$ & $0.33 \times 0.29 \times 0.28$ \\
\hline$T / \mathrm{K}$ & $100.0(1)$ \\
\hline Crystal System & orthorhombic \\
\hline Flack Parameter & $0.008(10)$ \\
\hline Hooft Parameter & $0.015(3)$ \\
\hline Space Group & $P 2{ }_{1}{ }_{1} 2_{1}$ \\
\hline$a / \AA ̊$ & $7.9290(9)$ \\
\hline$b / \AA ̊$ & $9.6907(11)$ \\
\hline$c / \AA$ & $45.127(5)$ \\
\hline$\alpha /^{\circ}$ & 90 \\
\hline$\beta /^{\circ}$ & 90 \\
\hline$\gamma /^{\circ}$ & 90 \\
\hline $\mathrm{V} / \AA^{3}$ & $3467.5(7)$ \\
\hline$Z$ & 4 \\
\hline$Z^{\prime}$ & 1 \\
\hline Wavelength/Å & 0.71073 \\
\hline Radiation type & $\operatorname{MoK}_{\alpha}$ \\
\hline$\Theta_{\min } /^{\circ}$ & 2.904 \\
\hline$\Theta_{\max } /^{\circ}$ & 27.547 \\
\hline Measured Refl. & 53809 \\
\hline Independent Refl. & 7981 \\
\hline Reflections with I > & 7679 \\
\hline $2(\mathrm{I})$ & \\
\hline$R_{\text {int }}$ & 0.0222 \\
\hline Parameters & 413 \\
\hline Restraints & 0 \\
\hline Largest Peak & 0.284 \\
\hline Deepest Hole & -0.184 \\
\hline GooF & 1.068 \\
\hline$w R_{2}$ (all data) & 0.0578 \\
\hline$w R_{2}$ & 0.0570 \\
\hline$R_{1}$ (all data) & 0.0247 \\
\hline$R_{1}$ & 0.0230 \\
\hline
\end{tabular}


Reflections:
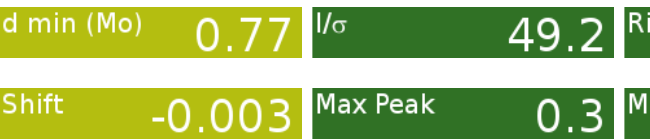

Refinement:

A clear light orange prism-shaped crystal with dimensions $0.33 \times 0.29 \times 0.28 \mathrm{~mm}^{3}$ was mounted on a MITIGEN holder oil. Data were collected using a Bruker D8 Venture (Mo) diffractometer equipped with an Oxford Cryosystems low-temperature device operating at $T=100.0(1) \mathrm{K}$. Data were measured using $\phi$ and $\omega$ scans using $\mathrm{MoK}_{\alpha}$ radiation. The total number of runs and images was based on the strategy calculation from the program APEX3 (Bruker, 2015) The maximum resolution that was achieved was $\Theta$ $=27.547^{\circ}(0.77 \AA)$. The diffraction pattern was indexed. The total number of runs and images was based on the strategy calculation from the program APEX3 (Bruker, 2015) and the unit cell was refined using SAINT (Bruker, V8.34A, after 2013) on 9585 reflections, 18\% of the observed reflections. Data reduction, scaling and absorption corrections were performed using SAINT (Bruker, V8.34A, after 2013). The final completeness is $99.80 \%$ out to $27.547^{\circ}$ in $\Theta$. A multi-scan absorption correction was performed using SADABS-2014/5 (Bruker,2014) was used for absorption correction. $w R_{2}$ (int) was 0.0578 before and 0.0526 after correction. The Ratio of minimum to maximum transmission is 0.9225 . The $\lambda / 2$ correction factor is 0.00150 . The absorption coefficient $\mu$ of this material is $0.558 \mathrm{~mm}^{-1}$ at this wavelength $(\lambda=0.711 \AA)$ and the minimum and maximum transmissions are 0.688 and 0.746 . The structure was solved and the space group $P 2{ }_{1} 2{ }_{1} 2_{1}$ (\# 19) determined by the ShelXT (Sheldrick, 2015) structure solution program using the Intrinsic Phasing solution method and refined by Least Squares using version 2018/3 of ShelXL (Sheldrick, 2015). All non-hydrogen atoms were refined anisotropically. Hydrogen atom positions were calculated geometrically and refined using the riding model. Hydrogen atom on Boron atom were refined using an AFIX 138, which would allow the refinement of a common B$\mathrm{H}$ distances, the same shifts being applied to all the $\mathrm{B}-\mathrm{H}$ bonds to the same atom, but still retain tetrahedral H-B-H angles within the group. There is a single molecule in the asymmetric unit, which is represented by the reported sum formula. In other words: Z is 4 and Z' is 1 . The Flack parameter was refined to 0.008(10). Determination of absolute structure using Bayesian statistics on Bijvoet differences using the Olex2 results in 0.015(3). Note: The Flack parameter is used to determine chirality of the crystal studied, the value should be near 0 , a value of 1 means that the stereochemistry is wrong and the model should be inverted. A value of 0.5 means that the crystal consists of a racemic mixture of the two enantiomers.

Table S44: Bond Lengths in Å for compound 21g.

\begin{tabular}{lll}
\hline Atom & Atom & Length/A \\
\hline Fe1 & C8 & $2.045(2)$ \\
Fe1 & C9 & $2.052(2)$ \\
Fe1 & C10 & $2.0561(19)$ \\
Fe1 & C6 & $2.054(2)$ \\
Fe1 & C7 & $2.046(2)$ \\
Fe1 & C5 & $2.043(2)$ \\
Fe1 & C4 & $2.0462(19)$ \\
Fe1 & C3 & $2.039(2)$ \\
Fe1 & C2 & $2.0448(19)$ \\
Fe1 & C1 & $2.0378(18)$ \\
P1 & O1 & $1.6169(13)$ \\
P1 & C1 & $1.7880(19)$ \\
P1 & C11 & $1.814(2)$ \\
P1 & B1 & $1.900(2)$ \\
P2 & N1 & $1.6628(16)$ \\
P2 & C33 & $1.8190(19)$
\end{tabular}

\begin{tabular}{lll}
\hline Atom & Atom & Length/Å \\
\hline P2 & C27 & $1.815(2)$ \\
P2 & B2 & $1.915(2)$ \\
O1 & C17 & $1.461(2)$ \\
C8 & C9 & $1.419(3)$ \\
C8 & C7 & $1.417(4)$ \\
C9 & C10 & $1.425(3)$ \\
C10 & C6 & $1.421(3)$ \\
C6 & C7 & $1.422(3)$ \\
C5 & C4 & $1.422(3)$ \\
C5 & C1 & $1.440(3)$ \\
C4 & C3 & $1.418(3)$ \\
C3 & C2 & $1.428(3)$ \\
C2 & C1 & $1.435(3)$ \\
C11 & C12 & $1.398(3)$ \\
C11 & C16 & $1.395(3)$ \\
C12 & C13 & $1.391(3)$
\end{tabular}




\begin{tabular}{lll}
\hline Atom & Atom & Length/Å \\
\hline $\mathrm{C} 13$ & $\mathrm{C} 14$ & $1.384(3)$ \\
$\mathrm{C} 14$ & $\mathrm{C} 15$ & $1.384(3)$ \\
$\mathrm{C} 15$ & $\mathrm{C} 16$ & $1.398(3)$ \\
$\mathrm{C} 17$ & $\mathrm{C} 18$ & $1.511(3)$ \\
$\mathrm{C} 17$ & $\mathrm{C} 24$ & $1.541(3)$ \\
$\mathrm{C} 18$ & $\mathrm{C} 19$ & $1.398(3)$ \\
$\mathrm{C} 18$ & $\mathrm{C} 23$ & $1.392(3)$ \\
$\mathrm{C} 19$ & $\mathrm{C} 20$ & $1.386(3)$ \\
$\mathrm{C} 20$ & $\mathrm{C} 21$ & $1.390(3)$ \\
$\mathrm{C} 21$ & $\mathrm{C} 22$ & $1.392(3)$ \\
$\mathrm{C} 22$ & $\mathrm{C} 23$ & $1.389(3)$ \\
$\mathrm{C} 24$ & $\mathrm{C} 25$ & $1.525(3)$ \\
$\mathrm{C} 24$ & $\mathrm{~N} 1$ & $1.475(2)$
\end{tabular}

\begin{tabular}{lll}
\hline Atom & Atom & Length/A \\
\hline $\mathrm{N} 1$ & C26 & $1.467(2)$ \\
C33 & C34 & $1.399(3)$ \\
C33 & C38 & $1.397(3)$ \\
C34 & C35 & $1.392(3)$ \\
C35 & C36 & $1.387(3)$ \\
C36 & C37 & $1.382(3)$ \\
C37 & C38 & $1.395(3)$ \\
C27 & C28 & $1.393(3)$ \\
C27 & C32 & $1.401(3)$ \\
C28 & C29 & $1.396(3)$ \\
C29 & C30 & $1.377(4)$ \\
C30 & C31 & $1.382(4)$ \\
C31 & C32 & $1.393(3)$
\end{tabular}

Table S45: Bond Angles in ${ }^{\circ}$ for compound 21g.

\begin{tabular}{|c|c|c|c|c|c|c|c|}
\hline Atom & Atom & Atom & Angle ${ }^{\circ}$ & Atom & Atom & Atom & Angle ${ }^{\circ}$ \\
\hline $\mathrm{C} 8$ & Fe1 & C9 & $40.51(10)$ & $\mathrm{C} 1$ & Fe1 & C9 & $118.35(9)$ \\
\hline C8 & $\mathrm{Fe} 1$ & C10 & 68.15(9) & $\mathrm{C} 1$ & $\mathrm{Fe} 1$ & C10 & 111.08(8) \\
\hline C8 & $\mathrm{Fe} 1$ & C6 & $68.21(10)$ & $\mathrm{C} 1$ & $\mathrm{Fe} 1$ & C6 & $132.30(8)$ \\
\hline C8 & $\mathrm{Fe} 1$ & C7 & $40.53(10)$ & $\mathrm{C} 1$ & $\mathrm{Fe} 1$ & C7 & 169.76(9) \\
\hline C8 & $\mathrm{Fe} 1$ & $\mathrm{C} 4$ & $106.60(9)$ & $\mathrm{C} 1$ & $\mathrm{Fe} 1$ & $\mathrm{C} 5$ & $41.33(7)$ \\
\hline C9 & $\mathrm{Fe} 1$ & C10 & $40.60(9)$ & $\mathrm{C} 1$ & $\mathrm{Fe} 1$ & $\mathrm{C} 4$ & $68.96(8)$ \\
\hline C9 & $\mathrm{Fe} 1$ & C6 & $68.23(10)$ & C1 & $\mathrm{Fe} 1$ & C3 & $69.06(8)$ \\
\hline C6 & $\mathrm{Fe} 1$ & C10 & $40.46(9)$ & C1 & $\mathrm{Fe} 1$ & $\mathrm{C} 2$ & $41.15(8)$ \\
\hline C7 & $\mathrm{Fe} 1$ & C9 & $68.20(10)$ & 01 & P1 & C1 & $99.78(8)$ \\
\hline $\mathrm{C} 7$ & $\mathrm{Fe} 1$ & C10 & $68.14(9)$ & 01 & P1 & C11 & $104.50(8)$ \\
\hline $\mathrm{C} 7$ & $\mathrm{Fe} 1$ & C6 & $40.59(9)$ & 01 & P1 & B1 & $117.87(9)$ \\
\hline C7 & $\mathrm{Fe} 1$ & $\mathrm{C} 4$ & $113.66(9)$ & C1 & P1 & C11 & $104.05(9)$ \\
\hline C5 & $\mathrm{Fe} 1$ & C8 & $115.44(9)$ & $\mathrm{C} 1$ & P1 & B1 & $114.80(10)$ \\
\hline C5 & $\mathrm{Fe} 1$ & $\mathrm{C} 9$ & $109.54(9)$ & C11 & P1 & B1 & $113.96(10)$ \\
\hline C5 & $\mathrm{Fe} 1$ & C10 & $133.05(9)$ & $\mathrm{N} 1$ & P2 & C33 & $105.16(8)$ \\
\hline C5 & $\mathrm{Fe} 1$ & C6 & $172.08(9)$ & N1 & P2 & $\mathrm{C} 27$ & $108.40(8)$ \\
\hline C5 & $\mathrm{Fe} 1$ & $\mathrm{C} 7$ & $146.55(9)$ & $\mathrm{N} 1$ & P2 & B2 & $114.45(9)$ \\
\hline C5 & Fe1 & $\mathrm{C} 4$ & $40.69(9)$ & C33 & P2 & B2 & 109.20(9) \\
\hline C5 & $\mathrm{Fe} 1$ & $\mathrm{C} 2$ & 69.17(8) & $\mathrm{C} 27$ & P2 & C33 & $105.47(9)$ \\
\hline $\mathrm{C} 4$ & $\mathrm{Fe} 1$ & C9 & $130.23(9)$ & $\mathrm{C} 27$ & P2 & B2 & $113.47(10)$ \\
\hline $\mathrm{C} 4$ & $\mathrm{Fe} 1$ & C10 & $170.28(9)$ & C17 & 01 & P1 & $120.95(11)$ \\
\hline $\mathrm{C} 4$ & $\mathrm{Fe} 1$ & C6 & $146.54(9)$ & C9 & $\mathrm{C} 8$ & $\mathrm{Fe} 1$ & $70.02(12)$ \\
\hline C3 & $\mathrm{Fe} 1$ & C8 & $128.16(9)$ & $\mathrm{C} 7$ & $\mathrm{C} 8$ & $\mathrm{Fe} 1$ & $69.77(12)$ \\
\hline $\mathrm{C} 3$ & $\mathrm{Fe} 1$ & C9 & $167.58(9)$ & $\mathrm{C} 7$ & C8 & C9 & $108.3(2)$ \\
\hline C3 & $\mathrm{Fe} 1$ & C10 & $149.03(9)$ & C8 & $\mathrm{C} 9$ & $\mathrm{Fe} 1$ & $69.46(13)$ \\
\hline C3 & $\mathrm{Fe} 1$ & C6 & $115.16(9)$ & C8 & $\mathrm{C} 9$ & C10 & $107.8(2)$ \\
\hline C3 & $\mathrm{Fe} 1$ & $\mathrm{C} 7$ & $106.07(9)$ & $\mathrm{C} 10$ & C9 & $\mathrm{Fe} 1$ & $69.84(12)$ \\
\hline C3 & $\mathrm{Fe} 1$ & $\mathrm{C} 5$ & $68.74(9)$ & C9 & $\mathrm{C} 10$ & $\mathrm{Fe} 1$ & $69.56(12)$ \\
\hline C3 & $\mathrm{Fe} 1$ & $\mathrm{C} 4$ & $40.62(9)$ & C6 & C10 & $\mathrm{Fe} 1$ & $69.69(11)$ \\
\hline C3 & $\mathrm{Fe} 1$ & $\mathrm{C} 2$ & $40.92(8)$ & $\mathrm{C} 6$ & C10 & C9 & $108.0(2)$ \\
\hline $\mathrm{C} 2$ & $\mathrm{Fe} 1$ & C8 & $167.45(10)$ & C10 & $\mathrm{C} 6$ & $\mathrm{Fe} 1$ & $69.84(12)$ \\
\hline $\mathrm{C} 2$ & $\mathrm{Fe} 1$ & C9 & $151.04(9)$ & $\mathrm{C} 10$ & C6 & $\mathrm{C} 7$ & $107.8(2)$ \\
\hline $\mathrm{C} 2$ & $\mathrm{Fe} 1$ & C10 & 118.14(8) & $\mathrm{C} 7$ & C6 & $\mathrm{Fe} 1$ & $69.40(12)$ \\
\hline $\mathrm{C} 2$ & $\mathrm{Fe} 1$ & C6 & $108.85(9)$ & C8 & $\mathrm{C} 7$ & $\mathrm{Fe} 1$ & $69.70(12)$ \\
\hline $\mathrm{C} 2$ & $\mathrm{Fe} 1$ & $\mathrm{C} 7$ & $129.45(9)$ & C8 & $\mathrm{C} 7$ & C6 & $108.1(2)$ \\
\hline $\mathrm{C} 2$ & $\mathrm{Fe} 1$ & $\mathrm{C} 4$ & $68.71(8)$ & C6 & $\mathrm{C} 7$ & $\mathrm{Fe} 1$ & $70.01(12)$ \\
\hline C1 & $\mathrm{Fe} 1$ & $\mathrm{C} 8$ & $149.48(9)$ & $\mathrm{C} 4$ & $\mathrm{C} 5$ & $\mathrm{Fe} 1$ & 69.78(11) \\
\hline
\end{tabular}




\begin{tabular}{|c|c|c|c|c|c|c|c|}
\hline Atom & Atom & Atom & Angle $/^{\circ}$ & Atom & Atom & Atom & Angle $/^{\circ}$ \\
\hline$\overline{\mathrm{C} 4}$ & C5 & $\mathrm{C} 1$ & $107.78(18)$ & $\overline{\mathrm{C} 23}$ & C18 & C19 & 119.03(19) \\
\hline $\mathrm{C} 1$ & $\mathrm{C} 5$ & $\mathrm{Fe} 1$ & 69.14(11) & $\mathrm{C} 20$ & C19 & C18 & $120.50(19)$ \\
\hline C5 & $\mathrm{C} 4$ & $\mathrm{Fe} 1$ & $69.53(11)$ & C19 & $\mathrm{C} 20$ & $\mathrm{C} 21$ & $120.1(2)$ \\
\hline C3 & $\mathrm{C} 4$ & $\mathrm{Fe} 1$ & $69.42(11)$ & $\mathrm{C} 20$ & $\mathrm{C} 21$ & $\mathrm{C} 22$ & $119.8(2)$ \\
\hline C3 & $\mathrm{C} 4$ & C5 & 108.47(17) & $\mathrm{C} 23$ & $\mathrm{C} 22$ & C21 & $120.1(2)$ \\
\hline $\mathrm{C} 4$ & C3 & $\mathrm{Fe} 1$ & 69.95(12) & $\mathrm{C} 22$ & $\mathrm{C} 23$ & C18 & 120.51(19) \\
\hline C4 & C3 & $\mathrm{C} 2$ & 108.44(18) & C25 & C24 & C17 & $110.88(15)$ \\
\hline $\mathrm{C} 2$ & $\mathrm{C} 3$ & $\mathrm{Fe} 1$ & $69.75(11)$ & N1 & $\mathrm{C} 24$ & C17 & $111.21(15)$ \\
\hline C3 & $\mathrm{C} 2$ & $\mathrm{Fe} 1$ & 69.33(11) & $\mathrm{N} 1$ & $\mathrm{C} 24$ & $\mathrm{C} 25$ & $111.55(15)$ \\
\hline C3 & $\mathrm{C} 2$ & C1 & 107.67(17) & $\mathrm{C} 24$ & N1 & P2 & $121.47(12)$ \\
\hline $\mathrm{C} 1$ & $\mathrm{C} 2$ & $\mathrm{Fe} 1$ & 69.16(10) & $\mathrm{C} 26$ & N1 & P2 & $120.00(12)$ \\
\hline P1 & C1 & $\mathrm{Fe} 1$ & $128.77(10)$ & C26 & N1 & C24 & $118.31(15)$ \\
\hline C5 & $\mathrm{C} 1$ & $\mathrm{Fe} 1$ & 69.53(11) & C34 & C33 & P2 & $118.68(14)$ \\
\hline C5 & $\mathrm{C} 1$ & P1 & $124.27(15)$ & C38 & C33 & P2 & $121.73(15)$ \\
\hline $\mathrm{C} 2$ & $\mathrm{C} 1$ & $\mathrm{Fe} 1$ & 69.69(11) & C38 & C33 & C34 & 119.21(17) \\
\hline $\mathrm{C} 2$ & $\mathrm{C} 1$ & P1 & $128.00(13)$ & C35 & $\mathrm{C} 34$ & C33 & $120.13(18)$ \\
\hline C2 & C1 & C5 & 107.64(17) & C36 & C35 & C34 & $120.17(19)$ \\
\hline C12 & C11 & P1 & 119.98(15) & C37 & C36 & C35 & $120.16(18)$ \\
\hline C16 & C11 & P1 & $120.73(16)$ & C36 & C37 & C38 & $120.13(18)$ \\
\hline C16 & C11 & $\mathrm{C} 12$ & 119.15(19) & C37 & C38 & C33 & $120.21(19)$ \\
\hline C13 & C12 & C11 & $120.50(19)$ & $\mathrm{C} 28$ & $\mathrm{C} 27$ & P2 & $118.05(16)$ \\
\hline C14 & $\mathrm{C} 13$ & C12 & $119.9(2)$ & $\mathrm{C} 28$ & $\mathrm{C} 27$ & C32 & $119.3(2)$ \\
\hline C15 & C14 & C13 & $120.2(2)$ & C32 & $\mathrm{C} 27$ & P2 & $122.38(16)$ \\
\hline C14 & C15 & C16 & $120.2(2)$ & $\mathrm{C} 27$ & $\mathrm{C} 28$ & C29 & $120.1(2)$ \\
\hline C11 & C16 & C15 & $120.0(2)$ & C30 & C29 & $\mathrm{C} 28$ & $120.1(2)$ \\
\hline 01 & C17 & C18 & $111.00(14)$ & $\mathrm{C} 29$ & C30 & C31 & $120.4(2)$ \\
\hline 01 & C17 & $\mathrm{C} 24$ & $103.74(14)$ & C30 & C31 & C32 & $120.2(2)$ \\
\hline C18 & $\mathrm{C} 17$ & $\mathrm{C} 24$ & $114.41(15)$ & C31 & C32 & $\mathrm{C} 27$ & $119.8(2)$ \\
\hline C19 & C18 & C17 & 120.96(18) & & & & \\
\hline $\mathrm{C} 23$ & C18 & C17 & 119.96(17) & & & & \\
\hline
\end{tabular}

Table S46: Torsion Angles in ${ }^{\circ}$ for compound 21g.

\begin{tabular}{llllr}
\hline Atom & Atom & Atom & Atom & \multicolumn{1}{c}{ Angle $^{\circ}$} \\
\hline $\mathrm{Fe} 1$ & $\mathrm{C} 8$ & $\mathrm{C} 9$ & $\mathrm{C} 10$ & $59.56(15)$ \\
$\mathrm{Fe} 1$ & $\mathrm{C} 8$ & $\mathrm{C} 7$ & $\mathrm{C} 6$ & $-59.71(15)$ \\
$\mathrm{Fe} 1$ & $\mathrm{C} 9$ & $\mathrm{C} 10$ & $\mathrm{C} 6$ & $59.29(14)$ \\
$\mathrm{Fe} 1$ & $\mathrm{C} 10$ & $\mathrm{C} 6$ & $\mathrm{C} 7$ & $59.22(14)$ \\
$\mathrm{Fe} 1$ & $\mathrm{C} 6$ & $\mathrm{C} 7$ & $\mathrm{C} 8$ & $59.51(15)$ \\
$\mathrm{Fe} 1$ & $\mathrm{C} 5$ & $\mathrm{C} 4$ & $\mathrm{C} 3$ & $58.65(14)$ \\
$\mathrm{Fe} 1$ & $\mathrm{C} 5$ & $\mathrm{C} 1$ & $\mathrm{P} 1$ & $123.67(14)$ \\
$\mathrm{Fe} 1$ & $\mathrm{C} 5$ & $\mathrm{C} 1$ & $\mathrm{C} 2$ & $-59.50(13)$ \\
$\mathrm{Fe} 1$ & $\mathrm{C} 4$ & $\mathrm{C} 3$ & $\mathrm{C} 2$ & $59.33(13)$ \\
$\mathrm{Fe} 1$ & $\mathrm{C} 3$ & $\mathrm{C} 2$ & $\mathrm{C} 1$ & $58.72(13)$ \\
$\mathrm{Fe} 1$ & $\mathrm{C} 2$ & $\mathrm{C} 1$ & $\mathrm{P} 1$ & $-123.93(15)$ \\
$\mathrm{Fe} 1$ & $\mathrm{C} 2$ & $\mathrm{C} 1$ & $\mathrm{C} 5$ & $59.40(13)$ \\
$\mathrm{P} 1$ & 01 & $\mathrm{C} 17$ & $\mathrm{C} 18$ & $64.54(18)$ \\
$\mathrm{P} 1$ & 01 & $\mathrm{C} 17$ & $\mathrm{C} 24$ & $-172.14(11)$ \\
$\mathrm{P} 1$ & $\mathrm{C} 11$ & $\mathrm{C} 12$ & $\mathrm{C} 13$ & $174.91(15)$ \\
$\mathrm{P} 1$ & $\mathrm{C} 11$ & $\mathrm{C} 16$ & $\mathrm{C} 15$ & $-174.55(15)$ \\
$\mathrm{P} 2$ & $\mathrm{C} 33$ & $\mathrm{C} 34$ & $\mathrm{C} 35$ & $-173.47(15)$ \\
$\mathrm{P} 2$ & $\mathrm{C} 33$ & $\mathrm{C} 38$ & $\mathrm{C} 37$ & $172.74(16)$ \\
$\mathrm{P} 2$ & $\mathrm{C} 27$ & $\mathrm{C} 28$ & $\mathrm{C} 29$ & $-172.88(18)$ \\
$\mathrm{P} 2$ & $\mathrm{C} 27$ & $\mathrm{C} 32$ & $\mathrm{C} 31$ & $173.50(16)$
\end{tabular}




\begin{tabular}{|c|c|c|c|c|}
\hline Atom & Atom & Atom & Atom & Angle ${ }^{\circ}$ \\
\hline 01 & P1 & $\mathrm{C} 1$ & Fe1 & $-79.01(13)$ \\
\hline 01 & $\mathrm{P} 1$ & $\mathrm{C} 1$ & $\mathrm{C} 5$ & $-169.27(16)$ \\
\hline 01 & $\mathrm{P} 1$ & $\mathrm{C} 1$ & $\mathrm{C} 2$ & $14.58(18)$ \\
\hline 01 & $\mathrm{P} 1$ & C11 & $\mathrm{C} 12$ & $-67.15(16)$ \\
\hline 01 & P1 & C11 & $\mathrm{C} 16$ & $108.58(17)$ \\
\hline 01 & C17 & C18 & C19 & $54.5(2)$ \\
\hline 01 & $\mathrm{C} 17$ & C18 & $\mathrm{C} 23$ & $-122.89(18)$ \\
\hline 01 & $\mathrm{C} 17$ & $\mathrm{C} 24$ & $\mathrm{C} 25$ & 58.28(18) \\
\hline 01 & $\mathrm{C} 17$ & $\mathrm{C} 24$ & N1 & $-176.99(14)$ \\
\hline C8 & C9 & $\mathrm{C} 10$ & Fe1 & $-59.32(15)$ \\
\hline C8 & C9 & C10 & $\mathrm{C} 6$ & $0.0(2)$ \\
\hline C9 & $\mathrm{C} 8$ & $\mathrm{C} 7$ & Fe1 & $59.67(15)$ \\
\hline C9 & $\mathrm{C} 8$ & $\mathrm{C} 7$ & $\mathrm{C} 6$ & $0.0(2)$ \\
\hline C9 & $\mathrm{C} 10$ & $\mathrm{C} 6$ & $\mathrm{Fe} 1$ & $-59.21(14)$ \\
\hline C9 & $\mathrm{C} 10$ & $\mathrm{C} 6$ & $\mathrm{C} 7$ & $0.0(2)$ \\
\hline $\mathrm{C} 10$ & C6 & $\mathrm{C} 7$ & Fe1 & $-59.49(14)$ \\
\hline C10 & $\mathrm{C} 6$ & $\mathrm{C} 7$ & C8 & $0.0(2)$ \\
\hline C7 & $\mathrm{C} 8$ & C9 & Fe1 & $-59.52(15)$ \\
\hline C7 & $\mathrm{C} 8$ & C9 & C10 & $0.0(2)$ \\
\hline C5 & $\mathrm{C} 4$ & $\mathrm{C} 3$ & Fe1 & $-58.72(14)$ \\
\hline C5 & $\mathrm{C} 4$ & $\mathrm{C} 3$ & $\mathrm{C} 2$ & $0.6(2)$ \\
\hline C4 & $\mathrm{C} 5$ & $\mathrm{C} 1$ & Fe1 & $59.30(14)$ \\
\hline $\mathrm{C} 4$ & $\mathrm{C} 5$ & $\mathrm{C} 1$ & $\mathrm{P} 1$ & $-177.03(14)$ \\
\hline $\mathrm{C} 4$ & $\mathrm{C} 5$ & $\mathrm{C} 1$ & $\mathrm{C} 2$ & $-0.2(2)$ \\
\hline $\mathrm{C} 4$ & $\mathrm{C} 3$ & $\mathrm{C} 2$ & $\mathrm{Fe} 1$ & $-59.46(14)$ \\
\hline C4 & $\mathrm{C} 3$ & $\mathrm{C} 2$ & $\mathrm{C} 1$ & $-0.7(2)$ \\
\hline C3 & $\mathrm{C} 2$ & $\mathrm{C} 1$ & Fe1 & $-58.82(13)$ \\
\hline C3 & $\mathrm{C} 2$ & $\mathrm{C} 1$ & $\mathrm{P} 1$ & $177.25(14)$ \\
\hline C3 & $\mathrm{C} 2$ & $\mathrm{C} 1$ & $\mathrm{C} 5$ & $0.6(2)$ \\
\hline $\mathrm{C} 1$ & $\mathrm{P} 1$ & 01 & C17 & $155.93(13)$ \\
\hline $\mathrm{C} 1$ & $\mathrm{P} 1$ & C11 & $\mathrm{C} 12$ & $37.06(17)$ \\
\hline $\mathrm{C} 1$ & P1 & C11 & C16 & $-147.21(17)$ \\
\hline C1 & $\mathrm{C} 5$ & $\mathrm{C} 4$ & Fe1 & $-58.90(13)$ \\
\hline C1 & $\mathrm{C} 5$ & $\mathrm{C} 4$ & $\mathrm{C} 3$ & $-0.2(2)$ \\
\hline C11 & $\mathrm{P} 1$ & 01 & C17 & $-96.68(14)$ \\
\hline C11 & $\mathrm{P} 1$ & $\mathrm{C} 1$ & Fe1 & $173.23(12)$ \\
\hline C11 & $\mathrm{P} 1$ & $\mathrm{C} 1$ & $\mathrm{C} 5$ & $82.98(17)$ \\
\hline C11 & P1 & C1 & $\mathrm{C} 2$ & $-93.18(18)$ \\
\hline C11 & C12 & C13 & C14 & $0.4(3)$ \\
\hline C12 & C11 & C16 & C15 & $1.2(3)$ \\
\hline C12 & C13 & C14 & C15 & $-0.2(3)$ \\
\hline C13 & C14 & C15 & C16 & $0.6(3)$ \\
\hline C14 & C15 & C16 & C11 & $-1.1(3)$ \\
\hline C16 & C11 & C12 & C13 & $-0.9(3)$ \\
\hline C17 & C18 & C19 & $\mathrm{C} 20$ & $-176.28(17)$ \\
\hline C17 & C18 & $\mathrm{C} 23$ & $\mathrm{C} 22$ & $177.23(18)$ \\
\hline C17 & $\mathrm{C} 24$ & N1 & $\mathrm{P} 2$ & $123.23(15)$ \\
\hline C17 & $\mathrm{C} 24$ & N1 & $\mathrm{C} 26$ & $-51.3(2)$ \\
\hline C18 & C17 & $\mathrm{C} 24$ & $\mathrm{C} 25$ & $179.35(16)$ \\
\hline C18 & $\mathrm{C} 17$ & $\mathrm{C} 24$ & N1 & $-55.9(2)$ \\
\hline C18 & C19 & $\mathrm{C} 20$ & $\mathrm{C} 21$ & $-0.8(3)$ \\
\hline C19 & C18 & $\mathrm{C} 23$ & $\mathrm{C} 22$ & $-0.2(3)$ \\
\hline C19 & $\mathrm{C} 20$ & $\mathrm{C} 21$ & $\mathrm{C} 22$ & $-0.3(3)$ \\
\hline $\mathrm{C} 20$ & $\mathrm{C} 21$ & $\mathrm{C} 22$ & $\mathrm{C} 23$ & $1.3(3)$ \\
\hline
\end{tabular}




\begin{tabular}{|c|c|c|c|c|}
\hline Atom & Atom & Atom & Atom & Angle $/^{\circ}$ \\
\hline$\overline{\mathrm{C} 21}$ & $\mathrm{C} 22$ & $\mathrm{C} 23$ & C18 & $-1.0(3)$ \\
\hline $\mathrm{C} 23$ & C18 & C19 & $\mathrm{C} 20$ & 1.1(3) \\
\hline $\mathrm{C} 24$ & C17 & C18 & C19 & $-62.5(2)$ \\
\hline $\mathrm{C} 24$ & C17 & C18 & $\mathrm{C} 23$ & $120.14(18)$ \\
\hline $\mathrm{C} 25$ & $\mathrm{C} 24$ & N1 & P2 & $-112.42(17)$ \\
\hline $\mathrm{C} 25$ & $\mathrm{C} 24$ & N1 & $\mathrm{C} 26$ & $73.0(2)$ \\
\hline N1 & $\mathrm{P} 2$ & C33 & $\mathrm{C} 34$ & $-40.23(17)$ \\
\hline $\mathrm{N} 1$ & $\mathrm{P} 2$ & C33 & $\mathrm{C} 38$ & $146.95(16)$ \\
\hline N1 & $\mathrm{P} 2$ & $\mathrm{C} 27$ & $\mathrm{C} 28$ & $101.71(17)$ \\
\hline $\mathrm{N} 1$ & $\mathrm{P} 2$ & $\mathrm{C} 27$ & $\mathrm{C} 32$ & $-72.66(18)$ \\
\hline C33 & $\mathrm{P} 2$ & N1 & $\mathrm{C} 24$ & $130.97(15)$ \\
\hline C33 & $\mathrm{P} 2$ & N1 & $\mathrm{C} 26$ & $-54.56(17)$ \\
\hline C33 & $\mathrm{P} 2$ & $\mathrm{C} 27$ & $\mathrm{C} 28$ & $-146.08(16)$ \\
\hline C33 & $\mathrm{P} 2$ & $\mathrm{C} 27$ & $\mathrm{C} 32$ & $39.54(18)$ \\
\hline C33 & $\mathrm{C} 34$ & C35 & $\mathrm{C} 36$ & $0.5(3)$ \\
\hline $\mathrm{C} 34$ & $\mathrm{C} 33$ & $\mathrm{C} 38$ & $\mathrm{C} 37$ & $0.0(3)$ \\
\hline C34 & C35 & $\mathrm{C} 36$ & $\mathrm{C} 37$ & $0.0(3)$ \\
\hline C35 & C36 & $\mathrm{C} 37$ & $\mathrm{C} 38$ & $-0.5(3)$ \\
\hline C36 & C37 & $\mathrm{C} 38$ & C33 & $0.5(3)$ \\
\hline C38 & C33 & $\mathrm{C} 34$ & $\mathrm{C} 35$ & $-0.5(3)$ \\
\hline $\mathrm{C} 27$ & $\mathrm{P} 2$ & N1 & $\mathrm{C} 24$ & $-116.62(15)$ \\
\hline $\mathrm{C} 27$ & $\mathrm{P} 2$ & N1 & $\mathrm{C} 26$ & $57.85(17)$ \\
\hline $\mathrm{C} 27$ & $\mathrm{P} 2$ & C33 & $\mathrm{C} 34$ & $-154.70(15)$ \\
\hline $\mathrm{C} 27$ & $\mathrm{P} 2$ & C33 & $\mathrm{C} 38$ & $32.48(18)$ \\
\hline $\mathrm{C} 27$ & $\mathrm{C} 28$ & $\mathrm{C} 29$ & $\mathrm{C} 30$ & $-0.8(4)$ \\
\hline $\mathrm{C} 28$ & $\mathrm{C} 27$ & $\mathrm{C} 32$ & C31 & $-0.8(3)$ \\
\hline $\mathrm{C} 28$ & $\mathrm{C} 29$ & $\mathrm{C} 30$ & C31 & $-1.0(4)$ \\
\hline $\mathrm{C} 29$ & $\mathrm{C} 30$ & C31 & $\mathrm{C} 32$ & $1.9(3)$ \\
\hline C30 & $\mathrm{C} 31$ & $\mathrm{C} 32$ & $\mathrm{C} 27$ & $-1.0(3)$ \\
\hline C32 & $\mathrm{C} 27$ & $\mathrm{C} 28$ & $\mathrm{C} 29$ & $1.7(3)$ \\
\hline B2 & $\mathrm{P} 2$ & N1 & $\mathrm{C} 24$ & $11.14(18)$ \\
\hline B2 & $\mathrm{P} 2$ & N1 & $\mathrm{C} 26$ & $-174.39(15)$ \\
\hline B2 & $\mathrm{P} 2$ & $\mathrm{C} 33$ & C34 & 83.03(17) \\
\hline B2 & $\mathrm{P} 2$ & $\mathrm{C} 33$ & C38 & $-89.78(18)$ \\
\hline B2 & $\mathrm{P} 2$ & $\mathrm{C} 27$ & $\mathrm{C} 28$ & $-26.60(19)$ \\
\hline B2 & $\mathrm{P} 2$ & $\mathrm{C} 27$ & C32 & $159.02(16)$ \\
\hline B1 & $\mathrm{P} 1$ & 01 & C17 & $31.00(17)$ \\
\hline B1 & $\mathrm{P} 1$ & $\mathrm{C} 1$ & $\mathrm{Fe} 1$ & $48.01(15)$ \\
\hline B1 & $\mathrm{P} 1$ & $\mathrm{C} 1$ & $\mathrm{C} 5$ & $-42.24(19)$ \\
\hline B1 & $\mathrm{P} 1$ & $\mathrm{C} 1$ & $\mathrm{C} 2$ & $141.60(17)$ \\
\hline B1 & $\mathrm{P} 1$ & C11 & $\mathrm{C} 12$ & $162.82(15)$ \\
\hline B1 & $\mathrm{P} 1$ & C11 & C16 & $-21.5(2)$ \\
\hline
\end{tabular}


Name: $\left(S_{\mathrm{p}}\right)-(-)-\mathrm{N}-[(1 R, 2 S)-2-t$-Butylphenylphosphinito-borane)-1-methyl-2-phenylethyl], N-methyl aminodiphenyl phosphine-borane $\mathbf{2 1 h}$.

Submitted by: Antonin Jaillet

Solved by: Yoann Rousselin

Sample ID: $\quad$ 15AJ265

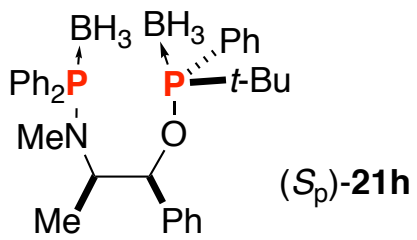

\section{Crystal Data and Experimental}

Figure S16 (thermal ellipsoïds are drawn at $50 \%$ probability plot)

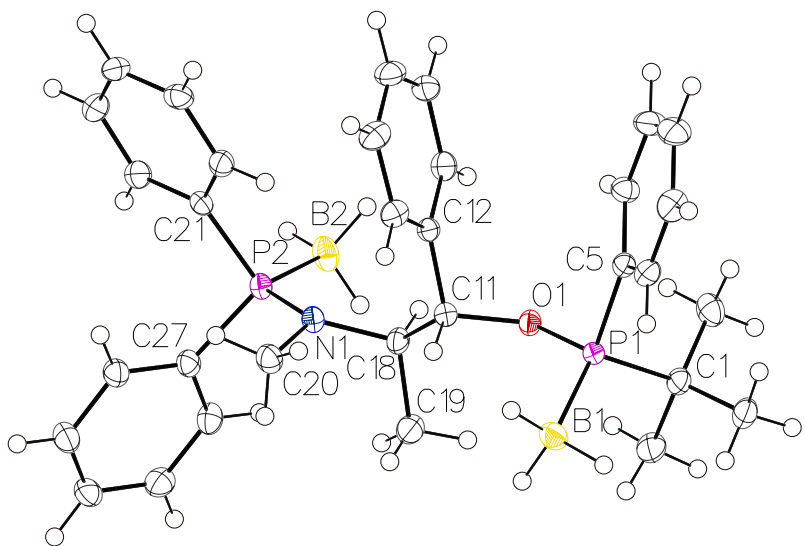

Experimental. Single clear light colorless needle-shaped crystals of compound $21 \mathrm{~h}$ were recrystallized from hexane by slow evaporation. A suitable crystal $0.53 \times 0.23 \times 0.09 \mathrm{~mm}^{3}$ was selected and mounted on a MITIGEN holder oil on a Nonius Kappa APEX II diffractometer. The crystal was kept at a steady $T=$ $115.0(1) \mathrm{K}$ during data collection. The structure was solved with the ShelXT (Sheldrick, 2015) structure solution program using the Intrinsic Phasing solution method and by using 0lex2 (Dolomanov et al., 2009) as the graphical interface. The model was refined with version 2018/3 of ShelXL (Sheldrick, 2015) using Least Squares minimization.

Crystal Data. $\mathrm{C}_{32} \mathrm{H}_{43} \mathrm{~B}_{2} \mathrm{NOP}_{2}, M_{r}=541.23$, orthorhombic, $P 22_{1} 2_{1}$ (No. 19), $a=9.4813(3) \AA, b=14.7256(5) \AA, c=$ 22.4494(6) $\AA, \alpha=\beta=\gamma=90^{\circ}, V=3134.34(17) \AA^{3}, T=$ 115.0(1) $\mathrm{K}, \quad Z=4, Z^{\prime}=1, \mu\left(\mathrm{MoK}_{\alpha}\right)=0.163,58399$ reflections measured, 7223 unique $\left(R_{\text {int }}=0.0721\right)$ which were used in all calculations. The final $w R_{2}$ was 0.0755 (all data) and $R_{1}$ was 0.0334 (I > 2(I)). 


\begin{tabular}{|c|c|}
\hline Compound & $21 \mathrm{~h}$ \\
\hline CCDC & 1982653 \\
\hline Formula & $\mathrm{C}_{32} \mathrm{H}_{43} \mathrm{~B}_{2} \mathrm{NOP}_{2}$ \\
\hline$D_{\text {calc. }} / \mathrm{g} \mathrm{cm}^{-3}$ & 1.147 \\
\hline$\mu / \mathrm{mm}^{-1}$ & 0.163 \\
\hline Formula Weight & 541.23 \\
\hline Color & clear light colorless \\
\hline Shape & needle \\
\hline Size $/ \mathrm{mm}^{3}$ & $0.53 \times 0.23 \times 0.09$ \\
\hline$T / \mathrm{K}$ & $115.0(1)$ \\
\hline Crystal System & orthorhombic \\
\hline Flack Parameter & $0.01(3)$ \\
\hline Hooft Parameter & $0.01(3)$ \\
\hline Space Group & $P 2{ }_{1} 2_{1} 2_{1}$ \\
\hline$a / \AA$ & $9.4813(3)$ \\
\hline$b / \AA$ & $14.7256(5)$ \\
\hline$c / \AA$ & $22.4494(6)$ \\
\hline$\alpha /^{\circ}$ & 90 \\
\hline$\beta /^{\circ}$ & 90 \\
\hline$\gamma /^{\circ}$ & 90 \\
\hline $\mathrm{V} / \AA^{3}$ & $3134.34(17)$ \\
\hline$Z$ & 4 \\
\hline$Z^{\prime}$ & 1 \\
\hline Wavelength/Å & 0.71073 \\
\hline Radiation type & $\mathrm{MoK}_{\alpha}$ \\
\hline$\Theta_{\min } /^{\circ}$ & 1.814 \\
\hline$\Theta_{\max } /^{\circ}$ & 27.554 \\
\hline Measured Refl. & 58399 \\
\hline Independent Refl. & 7223 \\
\hline Reflections with I > & 6310 \\
\hline $2(\mathrm{I})$ & \\
\hline$R_{\text {int }}$ & 0.0721 \\
\hline Parameters & 352 \\
\hline Restraints & 0 \\
\hline Largest Peak & 0.244 \\
\hline Deepest Hole & -0.221 \\
\hline GooF & 1.021 \\
\hline$w R_{2}$ (all data) & 0.0755 \\
\hline$w R_{2}$ & 0.0714 \\
\hline$R_{1}$ (all data) & 0.0438 \\
\hline$R_{1}$ & 0.0334 \\
\hline
\end{tabular}


Reflections:

A clear light colorless needle-shaped crystal with dimensions $0.53 \times 0.23 \times 0.09 \mathrm{~mm}^{3}$ was mounted on a MITIGEN holder oil. Data were collected using an Nonius Kappa APEX II diffractometer equipped with an Oxford Cryosystems low-temperature device operating at $T=115.0(1) \mathrm{K}$. Data were measured using $\phi$ and $\omega$ scans using $\mathrm{MoK}_{\alpha}$ radiation. The total number of runs and images was based on the strategy calculation from the program APEX3 (Bruker, 2015) The maximum resolution that was achieved was $\Theta$ $=27.554^{\circ}(0.77 \AA)$. The diffraction pattern was indexed. The total number of runs and images was based on the strategy calculation from the program APEX3 (Bruker, 2015) and the unit cell was refined using SAINT (Bruker, V8.34A, after 2013) on 9995 reflections, 17\% of the observed reflections. Data reduction, scaling and absorption corrections were performed using SAINT (Bruker, V8.34A, after 2013). The final completeness is $99.80 \%$ out to $27.554^{\circ}$ in $\Theta$. A multi-scan absorption correction was performed using SADABS-2014/5 (Bruker,2014) was used for absorption correction. $w R_{2}$ (int) was 0.0655 before and 0.0610 after correction. The Ratio of minimum to maximum transmission is 0.8836 . The $\lambda / 2$ correction factor is 0.00150 . The absorption coefficient $\mu$ of this material is $0.163 \mathrm{~mm}^{-1}$ at this wavelength $(\lambda=0.711 \AA)$ and the minimum and maximum transmissions are 0.659 and 0.746 . The structure was solved and the space group $P 2{ }_{1} 2_{1} 2_{1}$ (\# 19) determined by the ShelXT (Sheldrick, 2015) structure solution program using the Intrinsic Phasing solution method and refined by Least Squares using version 2018/3 of ShelXL (Sheldrick, 2015). All non-hydrogen atoms were refined anisotropically. Hydrogen atom positions were calculated geometrically and refined using the riding model. Hydrogen atom on Boron atom were refined using an AFIX 138, which would allow the refinement of a common B$\mathrm{H}$ distances, the same shifts being applied to all the $\mathrm{B}-\mathrm{H}$ bonds to the same atom, but still retain tetrahedral H-B-H angles within the group. There is a single molecule in the asymmetric unit, which is represented by the reported sum formula. In other words: $\mathrm{Z}$ is 4 and $\mathrm{Z}^{\prime}$ is 1 . The Flack parameter was refined to 0.01(3). Determination of absolute structure using Bayesian statistics on Bijvoet differences using the Olex2 results in 0.01(3). Note: The Flack parameter is used to determine chirality of the crystal studied, the value should be near 0 , a value of 1 means that the stereochemistry is wrong and the model should be inverted. A value of 0.5 means that the crystal consists of a racemic mixture of the two enantiomers.

Table S47: Bond Lengths in Å for compound 21h.

\begin{tabular}{lll}
\hline Atom & Atom & Length/Å \\
\hline P2 & C27 & $1.821(2)$ \\
P2 & N1 & $1.6702(18)$ \\
P2 & C21 & $1.814(2)$ \\
P2 & B2 & $1.912(3)$ \\
P1 & 01 & $1.6186(15)$ \\
P1 & C5 & $1.808(2)$ \\
P1 & C1 & $1.839(2)$ \\
P1 & B1 & $1.902(3)$ \\
01 & C11 & $1.461(3)$ \\
C30 & C29 & $1.378(4)$ \\
C30 & C31 & $1.394(4)$ \\
C29 & C28 & $1.392(3)$ \\
C28 & C27 & $1.389(3)$ \\
C27 & C32 & $1.395(3)$ \\
N1 & C18 & $1.473(3)$ \\
N1 & C20 & $1.467(3)$
\end{tabular}

\begin{tabular}{lll}
\hline Atom & Atom & Length/A \\
\hline C18 & C11 & $1.541(3)$ \\
C18 & C19 & $1.522(3)$ \\
C11 & C12 & $1.504(3)$ \\
C5 & C10 & $1.390(3)$ \\
C5 & C6 & $1.398(3)$ \\
C10 & C9 & $1.384(3)$ \\
C9 & C8 & $1.389(3)$ \\
C8 & C7 & $1.379(4)$ \\
C1 & C2 & $1.541(3)$ \\
C1 & C4 & $1.535(3)$ \\
C1 & C3 & $1.523(3)$ \\
C31 & C32 & $1.387(4)$ \\
C21 & C22 & $1.399(3)$ \\
C21 & C26 & $1.385(3)$ \\
C22 & C23 & $1.385(4)$ \\
C23 & C24 & $1.382(4)$
\end{tabular}




\begin{tabular}{lll}
\hline Atom & Atom & Length/Å \\
\hline C24 & C25 & $1.386(3)$ \\
C25 & C26 & $1.387(3)$ \\
C12 & C13 & $1.389(3)$ \\
C12 & C17 & $1.391(3)$ \\
C13 & C14 & $1.389(3)$
\end{tabular}

\begin{tabular}{lll}
\hline Atom & Atom & Length/Å \\
\hline C14 & C15 & $1.379(4)$ \\
C15 & C16 & $1.391(4)$ \\
C16 & C17 & $1.384(3)$ \\
C6 & C7 & $1.390(3)$
\end{tabular}

Table S48: Bond Angles in ${ }^{\circ}$ for compound 21h.

\begin{tabular}{llll}
\hline Atom & Atom & Atom & \multicolumn{1}{|c}{ Angle $/^{\circ}$} \\
\hline C27 & P2 & B2 & $112.92(12)$ \\
N1 & P2 & C27 & $107.49(10)$ \\
N1 & P2 & C21 & $105.73(10)$ \\
N1 & P2 & B2 & $114.13(11)$ \\
C21 & P2 & C27 & $105.00(10)$ \\
C21 & P2 & B2 & $110.93(12)$ \\
01 & P1 & C5 & $105.97(9)$ \\
01 & P1 & C1 & $101.06(9)$ \\
01 & P1 & B1 & $113.58(11)$ \\
C5 & P1 & C1 & $106.63(11)$ \\
C5 & P1 & B1 & $113.09(12)$ \\
C1 & P1 & B1 & $115.40(13)$ \\
C11 & 01 & P1 & $119.51(13)$ \\
C29 & C30 & C31 & $119.7(2)$ \\
C30 & C29 & C28 & $120.3(2)$ \\
C27 & C28 & C29 & $120.5(2)$ \\
C28 & C27 & P2 & $122.99(18)$ \\
C28 & C27 & C32 & $118.9(2)$ \\
C32 & C27 & P2 & $117.92(18)$ \\
C18 & N1 & P2 & $119.57(14)$ \\
C20 & N1 & P2 & $118.41(15)$ \\
C20 & N1 & C18 & $116.98(18)$ \\
N1 & C18 & C11 & $109.69(17)$ \\
N1 & C18 & C19 & $112.80(19)$ \\
C19 & C18 & C11 & $112.04(18)$ \\
01 & C11 & C18 & $105.55(16)$ \\
01 & C11 & C12 & $109.98(17)$ \\
C12 & C11 & C18 & $113.25(17)$ \\
C10 & C5 & P1 & $121.43(17)$ \\
C10 & C5 & C6 & $119.4(2)$ \\
& C5 & P1 & $119.19(18)$
\end{tabular}

\begin{tabular}{|c|c|c|c|}
\hline Atom & Atom & Atom & Angle $/^{\circ}$ \\
\hline C9 & C10 & C5 & $120.6(2)$ \\
\hline C10 & C9 & C8 & $119.8(2)$ \\
\hline C7 & $\mathrm{C} 8$ & C9 & $120.0(2)$ \\
\hline $\mathrm{C} 2$ & $\mathrm{C} 1$ & P1 & $106.69(15)$ \\
\hline C4 & C1 & P1 & $108.33(16)$ \\
\hline $\mathrm{C} 4$ & $\mathrm{C} 1$ & $\mathrm{C} 2$ & $110.3(2)$ \\
\hline C3 & C1 & P1 & $111.58(17)$ \\
\hline $\mathrm{C} 3$ & C1 & $\mathrm{C} 2$ & $109.8(2)$ \\
\hline C3 & C1 & $\mathrm{C} 4$ & $110.16(19)$ \\
\hline C32 & C31 & C30 & $120.0(2)$ \\
\hline C31 & C32 & $\mathrm{C} 27$ & $120.5(2)$ \\
\hline $\mathrm{C} 22$ & $\mathrm{C} 21$ & P2 & $119.07(18)$ \\
\hline $\mathrm{C} 26$ & $\mathrm{C} 21$ & P2 & $121.37(17)$ \\
\hline $\mathrm{C} 26$ & $\mathrm{C} 21$ & C22 & $119.3(2)$ \\
\hline $\mathrm{C} 23$ & $\mathrm{C} 22$ & $\mathrm{C} 21$ & $119.7(2)$ \\
\hline $\mathrm{C} 24$ & $\mathrm{C} 23$ & C22 & $120.5(2)$ \\
\hline $\mathrm{C} 23$ & C24 & C25 & $120.1(2)$ \\
\hline C26 & C25 & C24 & $119.6(2)$ \\
\hline C21 & C26 & C25 & $120.8(2)$ \\
\hline C13 & C12 & C11 & $120.4(2)$ \\
\hline C13 & C12 & C17 & $118.9(2)$ \\
\hline C17 & C12 & C11 & $120.7(2)$ \\
\hline C14 & C13 & C12 & $120.4(2)$ \\
\hline C15 & C14 & C13 & $120.1(2)$ \\
\hline C14 & C15 & C16 & $120.1(2)$ \\
\hline C17 & C16 & C15 & $119.5(2)$ \\
\hline $\mathrm{C} 16$ & C17 & $\mathrm{C} 12$ & $120.9(2)$ \\
\hline C7 & C6 & $\mathrm{C} 5$ & $119.6(2)$ \\
\hline C8 & C7 & C6 & $120.6(2)$ \\
\hline
\end{tabular}

Table S49: Torsion Angles in ${ }^{\circ}$ for compound $21 \mathrm{~h}$.

\begin{tabular}{lllll}
\hline Atom & Atom & Atom & Atom & Angle $^{\circ}$ \\
\hline P2 & C27 & C32 & C31 & $175.09(18)$ \\
P2 & N1 & C18 & C11 & $141.67(16)$ \\
P2 & N1 & C18 & C19 & $-92.7(2)$ \\
P2 & C21 & C22 & C23 & $-175.3(2)$ \\
P2 & C21 & C26 & C25 & $175.48(18)$ \\
P1 & 01 & C11 & C18 & $-143.02(15)$ \\
P1 & O1 & C11 & C12 & $94.48(18)$ \\
P1 & C5 & C10 & C9 & $-178.48(19)$ \\
P1 & C5 & C6 & C7 & $178.71(18)$ \\
01 & P1 & C5 & C10 & $-29.7(2)$
\end{tabular}




\begin{tabular}{|c|c|c|c|c|}
\hline Atom & Atom & Atom & Atom & Angle ${ }^{\circ}$ \\
\hline 01 & P1 & $\mathrm{C} 5$ & $\mathrm{C} 6$ & $150.95(17)$ \\
\hline 01 & $\mathrm{P} 1$ & $\mathrm{C} 1$ & $\mathrm{C} 2$ & $-179.22(16)$ \\
\hline 01 & $\mathrm{P} 1$ & $\mathrm{C} 1$ & $\mathrm{C} 4$ & $-60.52(18)$ \\
\hline 01 & $\mathrm{P} 1$ & $\mathrm{C} 1$ & $\mathrm{C} 3$ & $60.91(18)$ \\
\hline 01 & C11 & C12 & $\mathrm{C} 13$ & $-124.6(2)$ \\
\hline 01 & C11 & C12 & $\mathrm{C} 17$ & $57.4(3)$ \\
\hline C30 & $\mathrm{C} 29$ & $\mathrm{C} 28$ & $\mathrm{C} 27$ & $-0.9(4)$ \\
\hline C30 & C31 & C32 & $\mathrm{C} 27$ & $-1.3(4)$ \\
\hline C29 & C30 & C31 & $\mathrm{C} 32$ & $2.0(4)$ \\
\hline C29 & $\mathrm{C} 28$ & $\mathrm{C} 27$ & P2 & $-173.79(18)$ \\
\hline C29 & $\mathrm{C} 28$ & $\mathrm{C} 27$ & C32 & $1.5(3)$ \\
\hline C28 & $\mathrm{C} 27$ & C32 & C31 & $-0.5(3)$ \\
\hline C27 & P2 & N1 & C18 & $110.69(18)$ \\
\hline C27 & $\mathrm{P} 2$ & N1 & $\mathrm{C} 20$ & $-43.5(2)$ \\
\hline C27 & $\mathrm{P} 2$ & $\mathrm{C} 21$ & $\mathrm{C} 22$ & $-51.9(2)$ \\
\hline C27 & $\mathrm{P} 2$ & $\mathrm{C} 21$ & $\mathrm{C} 26$ & 133.71(19) \\
\hline N1 & $\mathrm{P} 2$ & $\mathrm{C} 27$ & $\mathrm{C} 28$ & $81.2(2)$ \\
\hline N1 & $\mathrm{P} 2$ & $\mathrm{C} 27$ & $\mathrm{C} 32$ & $-94.14(19)$ \\
\hline N1 & $\mathrm{P} 2$ & $\mathrm{C} 21$ & $\mathrm{C} 22$ & $-165.44(18)$ \\
\hline N1 & $\mathrm{P} 2$ & $\mathrm{C} 21$ & $\mathrm{C} 26$ & $20.2(2)$ \\
\hline N1 & C18 & C11 & 01 & $-168.67(17)$ \\
\hline N1 & C18 & C11 & $\mathrm{C} 12$ & $-48.3(2)$ \\
\hline C18 & C11 & C12 & C13 & $117.6(2)$ \\
\hline C18 & C11 & $\mathrm{C} 12$ & $\mathrm{C} 17$ & $-60.4(3)$ \\
\hline C11 & C12 & C13 & C14 & $-176.5(2)$ \\
\hline C11 & C12 & C17 & $\mathrm{C} 16$ & $177.9(2)$ \\
\hline C5 & $\mathrm{P} 1$ & 01 & C11 & $-85.22(17)$ \\
\hline $\mathrm{C} 5$ & $\mathrm{P} 1$ & $\mathrm{C} 1$ & $\mathrm{C} 2$ & $70.24(19)$ \\
\hline C5 & $\mathrm{P} 1$ & $\mathrm{C} 1$ & $\mathrm{C} 4$ & $-171.06(16)$ \\
\hline $\mathrm{C} 5$ & $\mathrm{P} 1$ & $\mathrm{C} 1$ & $\mathrm{C} 3$ & $-49.63(19)$ \\
\hline C5 & C10 & C9 & $\mathrm{C} 8$ & $-0.4(4)$ \\
\hline $\mathrm{C} 5$ & $\mathrm{C} 6$ & $\mathrm{C} 7$ & C8 & $0.0(4)$ \\
\hline C10 & $\mathrm{C} 5$ & $\mathrm{C} 6$ & $\mathrm{C} 7$ & $-0.6(3)$ \\
\hline C10 & C9 & $\mathrm{C} 8$ & $\mathrm{C} 7$ & $-0.2(4)$ \\
\hline C9 & $\mathrm{C} 8$ & $\mathrm{C} 7$ & C6 & $0.4(4)$ \\
\hline C1 & $\mathrm{P} 1$ & 01 & C11 & $163.73(16)$ \\
\hline C1 & $\mathrm{P} 1$ & $\mathrm{C} 5$ & C10 & $77.4(2)$ \\
\hline C1 & P1 & $\mathrm{C} 5$ & C6 & $-101.97(19)$ \\
\hline B1 & P1 & 01 & C11 & 39.52(19) \\
\hline B1 & $\mathrm{P} 1$ & $\mathrm{C} 5$ & C10 & $-154.75(19)$ \\
\hline B1 & $\mathrm{P} 1$ & $\mathrm{C} 5$ & C6 & $25.9(2)$ \\
\hline B1 & $\mathrm{P} 1$ & $\mathrm{C} 1$ & $\mathrm{C} 2$ & $-56.3(2)$ \\
\hline B1 & $\mathrm{P} 1$ & $\mathrm{C} 1$ & $\mathrm{C} 4$ & $62.4(2)$ \\
\hline B1 & $\mathrm{P} 1$ & $\mathrm{C} 1$ & $\mathrm{C} 3$ & $-176.13(17)$ \\
\hline C31 & C30 & $\mathrm{C} 29$ & $\mathrm{C} 28$ & $-0.9(4)$ \\
\hline C21 & $\mathrm{P} 2$ & $\mathrm{C} 27$ & $\mathrm{C} 28$ & $-31.0(2)$ \\
\hline C21 & $\mathrm{P} 2$ & $\mathrm{C} 27$ & C32 & $153.60(18)$ \\
\hline C21 & $\mathrm{P} 2$ & N1 & C18 & $-137.55(17)$ \\
\hline C21 & $\mathrm{P} 2$ & N1 & $\mathrm{C} 20$ & 68.29(19) \\
\hline C21 & $\mathrm{C} 22$ & $\mathrm{C} 23$ & $\mathrm{C} 24$ & $0.0(4)$ \\
\hline C22 & $\mathrm{C} 21$ & $\mathrm{C} 26$ & $\mathrm{C} 25$ & $1.2(3)$ \\
\hline C22 & $\mathrm{C} 23$ & $\mathrm{C} 24$ & $\mathrm{C} 25$ & $0.5(4)$ \\
\hline C23 & $\mathrm{C} 24$ & $\mathrm{C} 25$ & $\mathrm{C} 26$ & $-0.1(4)$ \\
\hline $\mathrm{C} 24$ & $\mathrm{C} 25$ & $\mathrm{C} 26$ & $\mathrm{C} 21$ & $-0.7(3)$ \\
\hline
\end{tabular}




\begin{tabular}{llllc}
\hline Atom & Atom & Atom & Atom & Angle $^{\circ}$ \\
\hline C26 & C21 & C22 & C23 & $-0.8(3)$ \\
C20 & N1 & C18 & C11 & $-63.8(2)$ \\
C20 & N1 & C18 & C19 & $61.8(3)$ \\
C19 & C18 & C11 & 01 & $65.3(2)$ \\
C19 & C18 & C11 & C12 & $-174.37(19)$ \\
C12 & C13 & C14 & C15 & $-1.4(4)$ \\
C13 & C12 & C17 & C16 & $-0.1(3)$ \\
C13 & C14 & C15 & C16 & $-0.1(4)$ \\
C14 & C15 & C16 & C17 & $1.5(4)$ \\
C15 & C16 & C17 & C12 & $-1.4(3)$ \\
C17 & C12 & C13 & C14 & $1.4(3)$ \\
C6 & C5 & C10 & C9 & $0.9(3)$ \\
B2 & P2 & C27 & C28 & $-152.0(2)$ \\
B2 & P2 & C27 & C32 & $32.6(2)$ \\
B2 & P2 & N1 & C18 & $-15.4(2)$ \\
B2 & P2 & N1 & C20 & $-169.52(18)$ \\
B2 & P2 & C21 & C22 & $70.3(2)$ \\
B2 & P2 & C21 & C26 & $-104.0(2)$
\end{tabular}


Name: N-[(1R,2S)-(2-(Diphenylphosphinito)-1-methyl-2-phenylethyl],

phenylphosphine dichloropalladium complex $\mathbf{3 0 .}$

Submitted by: Christophe Darcel<smiles>CC(N)C(O[PH](c1ccccc1)(c1ccccc1)c1ccccc1)[PH](Cl)(Oc1ccccc1)c1ccccc1</smiles>

Solved by: Yoann Rousselin

Sample ID: $\quad$ darc4

\section{Crystal Data and Experimental}

Figure S17 (thermal ellipsoïds are drawn at $50 \%$ probability plot)

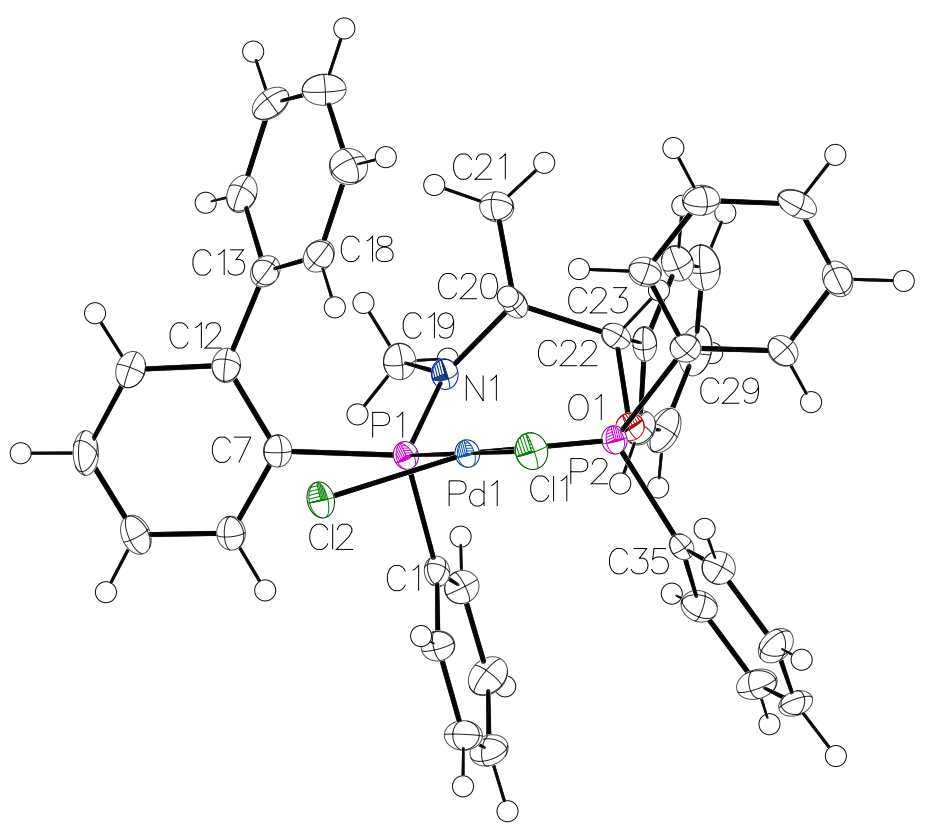

Experimental. Single clear light orange prism-shaped crystals of compound $\mathbf{3 0}$ were recrystallized from DCM by slow evaporation. A suitable crystal $(0.15 \times 0.15 \times 0.05) \mathrm{mm}^{3}$ was selected and mounted on a glass fiber with grease on a Nonius Kappa CCD diffractometer. The crystal was kept at $T=110(1) \mathrm{K}$ during data collection. The structure was solved with the ShelXS-97 (Sheldrick, 1997) structure solution program, using the direct methods solution method. The model was refined with version 2018/3 of ShelXL (Sheldrick, 2015) using Least Squares minimization.

Crystal Data. $\mathrm{C}_{40} \mathrm{H}_{37} \mathrm{Cl}_{2} \mathrm{NOP}_{2} \mathrm{Pd}, M_{r}=786.94$, monoclinic, P2 1 (No. 4), $\mathrm{a}=10.7036(2) \AA, \mathrm{b}=16.6047(3) \AA, \mathrm{c}=$ $11.0373(3) \AA, \quad \beta=116.4910(10)^{\circ}, \quad \alpha=\gamma=90^{\circ}, \quad V=$ 1755.69(7) $\AA^{3}, T=110(1) \mathrm{K}, Z=2, Z^{\prime}=1, \mu\left(\mathrm{MoK}_{\alpha}\right)=$ $0.806,11666$ reflections measured, 7988 unique $\left(R_{\text {int }}=\right.$ $0.0287)$ which were used in all calculations. The final $w R_{2}$ was 0.0675 (all data) and $R_{1}$ was 0.0349 (I > 2(I)). 


\begin{tabular}{|c|c|}
\hline Compound & 30 \\
\hline CCDC & 1982654 \\
\hline Formula & $\mathrm{C}_{40} \mathrm{H}_{37} \mathrm{Cl}_{2} \mathrm{NOP}_{2} \mathrm{Pd}$ \\
\hline$D_{\text {calc. }} / \mathrm{g} \mathrm{cm}^{-3}$ & 1.489 \\
\hline$\mu / \mathrm{mm}^{-1}$ & 0.806 \\
\hline Formula Weight & 786.94 \\
\hline Color & clear light orange \\
\hline Shape & prism \\
\hline Size $/ \mathrm{mm}^{3}$ & $0.15 \times 0.15 \times 0.05$ \\
\hline$T / \mathrm{K}$ & $110(1)$ \\
\hline Crystal System & monoclinic \\
\hline Flack Parameter & $-0.02(3)$ \\
\hline Hooft Parameter & $-0.031(14)$ \\
\hline Space Group & $\mathrm{P} 2_{1}$ \\
\hline$a / \AA$ & $10.7036(2)$ \\
\hline$b / \AA$ & $16.6047(3)$ \\
\hline$c / \AA$ & $11.0373(3)$ \\
\hline$\alpha /^{\circ}$ & $90.0000(8)$ \\
\hline$\beta /^{\circ}$ & $116.4910(8)$ \\
\hline$\gamma /^{\circ}$ & $90.0000(10)$ \\
\hline $\mathrm{V} / \AA^{3}$ & $1755.69(7)$ \\
\hline$Z$ & 2 \\
\hline$Z^{\prime}$ & 1 \\
\hline Wavelength/Å & 0.71073 \\
\hline Radiation type & $\mathrm{MoK}_{\alpha}$ \\
\hline$\Theta_{\min } /^{\circ}$ & 2.523 \\
\hline$\Theta_{\max } /^{\circ}$ & 27.485 \\
\hline Measured Refl. & 11666 \\
\hline Independent Refl. & 7988 \\
\hline Reflections Used & 7214 \\
\hline$R_{\text {int }}$ & 0.0287 \\
\hline Parameters & 427 \\
\hline Restraints & 1 \\
\hline Largest Peak & 0.551 \\
\hline Deepest Hole & -0.546 \\
\hline GooF & 1.028 \\
\hline$w R_{2}$ (all data) & 0.0675 \\
\hline$w R_{2}$ & 0.0641 \\
\hline$R_{1}$ (all data) & 0.0433 \\
\hline$R_{1}$ & 0.0349 \\
\hline
\end{tabular}


Reflections:

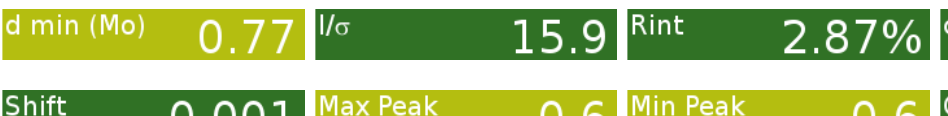

complete $\quad 100 \%$

Refinement:

A clear colorless prism-shaped crystal with dimensions $0.15 \times 0.15 \times 0.05 \mathrm{~mm}^{3}$ was mounted on a glass fiber with grease. X-ray diffraction data were collected using a Nonius Kappa CCD diffractometer equipped with an Oxford Cryosystems low-temperature device, operating at $T=110(1) \mathrm{K}$. Data were measured using $\phi$ and $\omega$ scans using $\mathrm{MoK}_{\alpha}$ radiation (X-ray tube, $50 \mathrm{kV}, 32 \mathrm{~mA}$ ). The total number of runs and images was based on the strategy calculation from the program Collect (Nonius BV, 19972000). The maximum resolution achieved was $\Theta=27.485^{\circ}$. Cell parameters were retrieved using the HKL Scalepack (Otwinowski \& Minor 1997) software and refined using HKL Denzo and Scalepack (Otwinowski \& Minor 1997) on 5075 reflections, $44 \%$ of the observed reflections. Data reduction was performed using the HKL Denzo and Scalepack (Otwinowski \& Minor 1997) software which corrects for Lorentz polarization. The final completeness is $99.80 \%$ out to $27.485^{\circ}$ in $\Theta$. No absorption correction was performed. The absorption coefficient $\mu$ of this material is $0.806 \mathrm{~mm}^{-1}$ at this wavelength $(\lambda=$ $0.71073 \AA$ ) . The structure was solved in the space group P2 1 (\# 4) by direct methods using the ShelXS97 (Sheldrick, 1997) structure solution program and refined by Least Squares using version 2018/3 of ShelXL (Sheldrick, 2015). All non-hydrogen atoms were refined anisotropically. Hydrogen atom positions were calculated geometrically and refined using the riding model. There is a single molecule in the asymmetric unit, which is represented by the reported sum formula. In other words: $\mathrm{Z}$ is 2 and $\mathrm{Z}^{\prime}$ is 1. The Flack parameter was refined to $-0.02(3)$. Determination of absolute structure using Bayesian statistics on Bijvoet differences using the Olex2 results in -0.031(14). Note: The Flack parameter is used to determine chirality of the crystal studied, the value should be near 0 , a value of 1 means that the stereochemistry is wrong and the model should be inverted. A value of 0.5 means that the crystal consists of a racemic mixture of the two enantiomers.

Table S50: Bond Lengths in Å for compound 30.

\begin{tabular}{|c|c|c|c|c|c|}
\hline Atom & Atom & Length/Å & Atom & Atom & Length/Å \\
\hline $\mathrm{Pd} 1$ & $\mathrm{Cl} 1$ & $2.3339(12)$ & C9 & C10 & $1.367(8)$ \\
\hline Pd1 & $\mathrm{Cl} 2$ & $2.3591(12)$ & C10 & C11 & $1.388(8)$ \\
\hline $\operatorname{Pd} 1$ & $\mathrm{P} 1$ & $2.2579(12)$ & C11 & C12 & $1.402(7)$ \\
\hline Pd1 & $\mathrm{P} 2$ & $2.2330(12)$ & $\mathrm{C} 12$ & C13 & $1.492(7)$ \\
\hline P1 & N1 & $1.655(4)$ & C13 & C14 & $1.393(7)$ \\
\hline P1 & $\mathrm{C} 1$ & $1.823(5)$ & C13 & C18 & $1.404(7)$ \\
\hline P1 & $\mathrm{C} 7$ & $1.839(5)$ & C14 & C15 & $1.394(8)$ \\
\hline P2 & 01 & $1.615(3)$ & C15 & C16 & $1.376(8)$ \\
\hline P2 & $\mathrm{C} 29$ & $1.809(4)$ & C16 & C17 & $1.395(8)$ \\
\hline P2 & $\mathrm{C} 35$ & $1.813(5)$ & $\mathrm{C} 17$ & C18 & $1.383(7)$ \\
\hline $\mathrm{N} 1$ & C19 & $1.478(6)$ & $\mathrm{C} 20$ & $\mathrm{C} 21$ & $1.517(7)$ \\
\hline $\mathrm{N} 1$ & $\mathrm{C} 20$ & $1.476(6)$ & $\mathrm{C} 20$ & $\mathrm{C} 22$ & $1.541(6)$ \\
\hline 01 & $\mathrm{C} 22$ & $1.450(5)$ & $\mathrm{C} 22$ & $\mathrm{C} 23$ & $1.510(6)$ \\
\hline C1 & $\mathrm{C} 2$ & $1.384(7)$ & $\mathrm{C} 23$ & $\mathrm{C} 24$ & $1.399(7)$ \\
\hline C1 & $\mathrm{C} 6$ & $1.395(7)$ & $\mathrm{C} 23$ & $\mathrm{C} 28$ & $1.389(7)$ \\
\hline $\mathrm{C} 2$ & $\mathrm{C} 3$ & $1.392(7)$ & $\mathrm{C} 24$ & $\mathrm{C} 25$ & $1.388(7)$ \\
\hline $\mathrm{C} 3$ & $\mathrm{C} 4$ & $1.378(7)$ & $\mathrm{C} 25$ & $\mathrm{C} 26$ & $1.380(8)$ \\
\hline $\mathrm{C} 4$ & $\mathrm{C} 5$ & $1.390(8)$ & $\mathrm{C} 26$ & $\mathrm{C} 27$ & $1.378(8)$ \\
\hline $\mathrm{C} 5$ & $\mathrm{C} 6$ & $1.388(7)$ & $\mathrm{C} 27$ & $\mathrm{C} 28$ & $1.391(7)$ \\
\hline $\mathrm{C} 7$ & $\mathrm{C} 8$ & $1.383(7)$ & $\mathrm{C} 29$ & C30 & $1.395(6)$ \\
\hline C7 & C12 & $1.425(7)$ & $\mathrm{C} 29$ & C34 & $1.400(6)$ \\
\hline C8 & C9 & $1.388(7)$ & C30 & $\mathrm{C} 31$ & $1.376(7)$ \\
\hline
\end{tabular}




\begin{tabular}{lll}
\hline Atom & Atom & Length/Å \\
\hline C31 & C32 & $1.380(7)$ \\
C32 & C33 & $1.375(7)$ \\
C33 & C34 & $1.378(6)$ \\
C35 & C36 & $1.393(6)$ \\
C35 & C40 & $1.388(7)$
\end{tabular}

\begin{tabular}{lll}
\hline Atom & Atom & Length/Å \\
\hline C36 & C37 & $1.386(7)$ \\
C37 & C38 & $1.394(8)$ \\
C38 & C39 & $1.379(8)$ \\
C39 & C40 & $1.391(7)$
\end{tabular}

Table S51: Bond Angles in ${ }^{\circ}$ for compound 30.

\begin{tabular}{|c|c|c|c|c|c|c|c|}
\hline Atom & Atom & Atom & Angle $/^{\circ}$ & Atom & Atom & Atom & Angle $/^{\circ}$ \\
\hline $\mathrm{Cl} 1$ & $\mathrm{Pd} 1$ & $\mathrm{Cl} 2$ & $90.57(4)$ & C14 & C13 & C12 & $119.3(5)$ \\
\hline P1 & $\operatorname{Pd} 1$ & $\mathrm{Cl} 1$ & $179.67(5)$ & C14 & C13 & C18 & $118.0(5)$ \\
\hline P1 & $\operatorname{Pd} 1$ & $\mathrm{Cl} 2$ & $89.75(4)$ & C18 & C13 & C12 & $122.5(5)$ \\
\hline $\mathrm{P} 2$ & Pd1 & $\mathrm{Cl} 1$ & $86.37(4)$ & C13 & C14 & C15 & $121.3(5)$ \\
\hline $\mathrm{P} 2$ & Pd1 & $\mathrm{Cl} 2$ & $168.29(5)$ & C16 & C15 & C14 & $120.0(5)$ \\
\hline $\mathrm{P} 2$ & $\operatorname{Pd} 1$ & $\mathrm{P} 1$ & $93.34(4)$ & C15 & C16 & $\mathrm{C} 17$ & $119.5(5)$ \\
\hline N1 & P1 & $\mathrm{Pd} 1$ & $116.31(15)$ & C18 & C17 & C16 & $120.6(5)$ \\
\hline N1 & $\mathrm{P} 1$ & $\mathrm{C} 1$ & $109.4(2)$ & $\mathrm{C} 17$ & C18 & C13 & $120.5(5)$ \\
\hline N1 & $\mathrm{P} 1$ & $\mathrm{C} 7$ & $104.1(2)$ & N1 & $\mathrm{C} 20$ & $\mathrm{C} 21$ & $113.4(4)$ \\
\hline $\mathrm{C} 1$ & $\mathrm{P} 1$ & $\operatorname{Pd} 1$ & $103.80(16)$ & N1 & $\mathrm{C} 20$ & $\mathrm{C} 22$ & $112.1(4)$ \\
\hline C1 & $\mathrm{P} 1$ & $\mathrm{C} 7$ & $103.7(2)$ & $\mathrm{C} 21$ & $\mathrm{C} 20$ & $\mathrm{C} 22$ & $111.7(4)$ \\
\hline C7 & $\mathrm{P} 1$ & Pd1 & $118.57(16)$ & 01 & $\mathrm{C} 22$ & $\mathrm{C} 20$ & $109.3(4)$ \\
\hline 01 & $\mathrm{P} 2$ & Pd1 & $117.81(12)$ & 01 & $\mathrm{C} 22$ & $\mathrm{C} 23$ & $108.5(4)$ \\
\hline 01 & $\mathrm{P} 2$ & $\mathrm{C} 29$ & $103.6(2)$ & $\mathrm{C} 23$ & $\mathrm{C} 22$ & $\mathrm{C} 20$ & $115.8(4)$ \\
\hline 01 & P2 & $\mathrm{C} 35$ & $98.58(18)$ & $\mathrm{C} 24$ & $\mathrm{C} 23$ & $\mathrm{C} 22$ & $118.6(5)$ \\
\hline C29 & P2 & Pd1 & $117.01(15)$ & $\mathrm{C} 28$ & $\mathrm{C} 23$ & $\mathrm{C} 22$ & $122.2(4)$ \\
\hline C29 & $\mathrm{P} 2$ & C35 & $105.1(2)$ & $\mathrm{C} 28$ & $\mathrm{C} 23$ & $\mathrm{C} 24$ & $119.3(5)$ \\
\hline C35 & P2 & Pd1 & $112.42(15)$ & $\mathrm{C} 25$ & $\mathrm{C} 24$ & $\mathrm{C} 23$ & $119.9(5)$ \\
\hline C19 & N1 & $\mathrm{P} 1$ & $120.1(3)$ & $\mathrm{C} 26$ & $\mathrm{C} 25$ & $\mathrm{C} 24$ & $120.3(5)$ \\
\hline $\mathrm{C} 20$ & N1 & $\mathrm{P} 1$ & $121.8(3)$ & $\mathrm{C} 27$ & $\mathrm{C} 26$ & $\mathrm{C} 25$ & $120.0(5)$ \\
\hline $\mathrm{C} 20$ & N1 & C19 & $117.5(4)$ & $\mathrm{C} 26$ & $\mathrm{C} 27$ & $\mathrm{C} 28$ & $120.4(6)$ \\
\hline C22 & 01 & P2 & $120.8(3)$ & $\mathrm{C} 23$ & $\mathrm{C} 28$ & $\mathrm{C} 27$ & $120.0(5)$ \\
\hline $\mathrm{C} 2$ & $\mathrm{C} 1$ & $\mathrm{P} 1$ & $118.4(4)$ & C30 & $\mathrm{C} 29$ & P2 & $121.5(3)$ \\
\hline $\mathrm{C} 2$ & $\mathrm{C} 1$ & $\mathrm{C} 6$ & $118.9(5)$ & C30 & $\mathrm{C} 29$ & C34 & $119.4(4)$ \\
\hline C6 & $\mathrm{C} 1$ & $\mathrm{P} 1$ & $122.7(4)$ & C34 & $\mathrm{C} 29$ & P2 & $119.0(3)$ \\
\hline $\mathrm{C} 1$ & $\mathrm{C} 2$ & $\mathrm{C} 3$ & $120.7(5)$ & C31 & C30 & $\mathrm{C} 29$ & $119.8(4)$ \\
\hline $\mathrm{C} 4$ & $\mathrm{C} 3$ & $\mathrm{C} 2$ & $120.1(5)$ & C30 & C31 & C32 & $120.5(5)$ \\
\hline C3 & $\mathrm{C} 4$ & $\mathrm{C} 5$ & $119.8(5)$ & C33 & C32 & C31 & $120.2(5)$ \\
\hline C6 & $\mathrm{C} 5$ & $\mathrm{C} 4$ & $120.1(5)$ & C32 & C33 & C34 & $120.4(4)$ \\
\hline C5 & $\mathrm{C} 6$ & $\mathrm{C} 1$ & $120.4(5)$ & C33 & C34 & C29 & $119.8(4)$ \\
\hline C8 & $\mathrm{C} 7$ & $\mathrm{P} 1$ & $118.1(4)$ & C36 & C35 & P2 & $119.6(4)$ \\
\hline C8 & $\mathrm{C} 7$ & $\mathrm{C} 12$ & $119.3(4)$ & $\mathrm{C} 40$ & C35 & P2 & $120.7(4)$ \\
\hline C12 & $\mathrm{C} 7$ & P1 & $122.4(4)$ & $\mathrm{C} 40$ & C35 & C36 & $119.6(4)$ \\
\hline C7 & $\mathrm{C} 8$ & $\mathrm{C} 9$ & $122.1(5)$ & C37 & C36 & C35 & $119.9(5)$ \\
\hline C10 & C9 & $\mathrm{C} 8$ & $119.5(5)$ & C36 & C37 & C38 & $120.1(5)$ \\
\hline $\mathrm{C} 9$ & C10 & C11 & $119.6(5)$ & C39 & C38 & C37 & $120.0(5)$ \\
\hline $\mathrm{C} 10$ & C11 & $\mathrm{C} 12$ & $122.6(5)$ & C38 & C39 & $\mathrm{C} 40$ & $119.9(5)$ \\
\hline C7 & $\mathrm{C} 12$ & $\mathrm{C} 13$ & $126.0(4)$ & C35 & $\mathrm{C} 40$ & C39 & $120.3(5)$ \\
\hline C11 & C12 & $\mathrm{C} 7$ & $116.9(5)$ & & & & \\
\hline C11 & $\mathrm{C} 12$ & C13 & $117.1(5)$ & & & & \\
\hline
\end{tabular}


Table S52: Torsion Angles in ${ }^{\circ}$ for compound 30.

\begin{tabular}{|c|c|c|c|c|}
\hline Atom & Atom & Atom & Atom & Angle $/{ }^{\circ}$ \\
\hline $\mathrm{Pd} 1$ & $\mathrm{P} 1$ & N1 & C19 & $-171.5(3)$ \\
\hline Pd1 & $\mathrm{P} 1$ & N1 & $\mathrm{C} 20$ & $-1.0(4)$ \\
\hline $\operatorname{Pd} 1$ & $\mathrm{P} 1$ & $\mathrm{C} 1$ & $\mathrm{C} 2$ & $35.3(4)$ \\
\hline $\operatorname{Pd} 1$ & P1 & $\mathrm{C} 1$ & C6 & $-143.2(4)$ \\
\hline $\operatorname{Pd} 1$ & P1 & $\mathrm{C} 7$ & $\mathrm{C} 8$ & $-103.5(4)$ \\
\hline $\operatorname{Pd} 1$ & $\mathrm{P} 1$ & $\mathrm{C} 7$ & C12 & $81.6(4)$ \\
\hline $\operatorname{Pd} 1$ & P2 & 01 & $\mathrm{C} 22$ & $78.8(3)$ \\
\hline Pd1 & $\mathrm{P} 2$ & $\mathrm{C} 29$ & C30 & $-19.8(5)$ \\
\hline $\operatorname{Pd} 1$ & P2 & $\mathrm{C} 29$ & C34 & $164.3(4)$ \\
\hline $\operatorname{Pd} 1$ & $\mathrm{P} 2$ & C35 & C36 & $-79.1(4)$ \\
\hline $\operatorname{Pd} 1$ & P2 & C35 & $\mathrm{C} 40$ & $103.0(4)$ \\
\hline P1 & N1 & $\mathrm{C} 20$ & $\mathrm{C} 21$ & $-142.0(4)$ \\
\hline P1 & N1 & $\mathrm{C} 20$ & $\mathrm{C} 22$ & $90.4(4)$ \\
\hline P1 & $\mathrm{C} 1$ & $\mathrm{C} 2$ & $\mathrm{C} 3$ & $-178.4(4)$ \\
\hline P1 & $\mathrm{C} 1$ & $\mathrm{C} 6$ & $\mathrm{C} 5$ & $177.7(4)$ \\
\hline P1 & $\mathrm{C} 7$ & $\mathrm{C} 8$ & $\mathrm{C} 9$ & $-173.1(4)$ \\
\hline P1 & $\mathrm{C} 7$ & $\mathrm{C} 12$ & C11 & $172.5(4)$ \\
\hline P1 & $\mathrm{C} 7$ & C12 & C13 & $-6.5(7)$ \\
\hline P2 & 01 & $\mathrm{C} 22$ & $\mathrm{C} 20$ & $-58.4(4)$ \\
\hline P2 & 01 & $\mathrm{C} 22$ & $\mathrm{C} 23$ & $174.6(3)$ \\
\hline P2 & $\mathrm{C} 29$ & C30 & C31 & $-175.9(4)$ \\
\hline P2 & $\mathrm{C} 29$ & C34 & C33 & $176.6(4)$ \\
\hline P2 & C35 & C36 & C37 & $-175.2(4)$ \\
\hline P2 & C35 & $\mathrm{C} 40$ & C39 & $175.0(4)$ \\
\hline N1 & $\mathrm{P} 1$ & $\mathrm{C} 1$ & $\mathrm{C} 2$ & $160.1(4)$ \\
\hline N1 & $\mathrm{P} 1$ & $\mathrm{C} 1$ & $\mathrm{C} 6$ & $-18.4(5)$ \\
\hline N1 & $\mathrm{P} 1$ & $\mathrm{C} 7$ & $\mathrm{C} 8$ & $125.4(4)$ \\
\hline N1 & $\mathrm{P} 1$ & $\mathrm{C} 7$ & C12 & $-49.5(5)$ \\
\hline N1 & $\mathrm{C} 20$ & $\mathrm{C} 22$ & 01 & $-53.9(5)$ \\
\hline N1 & $\mathrm{C} 20$ & $\mathrm{C} 22$ & $\mathrm{C} 23$ & $68.9(5)$ \\
\hline 01 & P2 & $\mathrm{C} 29$ & C30 & $111.7(5)$ \\
\hline 01 & P2 & $\mathrm{C} 29$ & C34 & $-64.2(5)$ \\
\hline 01 & P2 & C35 & C36 & $155.9(4)$ \\
\hline 01 & P2 & C35 & $\mathrm{C} 40$ & $-22.0(4)$ \\
\hline 01 & $\mathrm{C} 22$ & $\mathrm{C} 23$ & $\mathrm{C} 24$ & $-151.8(4)$ \\
\hline 01 & $\mathrm{C} 22$ & $\mathrm{C} 23$ & $\mathrm{C} 28$ & $28.1(6)$ \\
\hline C1 & P1 & N1 & C19 & $71.3(4)$ \\
\hline $\mathrm{C} 1$ & $\mathrm{P} 1$ & N1 & $\mathrm{C} 20$ & $-118.2(4)$ \\
\hline $\mathrm{C} 1$ & $\mathrm{P} 1$ & $\mathrm{C} 7$ & C8 & $10.8(5)$ \\
\hline $\mathrm{C} 1$ & P1 & $\mathrm{C} 7$ & $\mathrm{C} 12$ & $-164.0(4)$ \\
\hline $\mathrm{C} 1$ & $\mathrm{C} 2$ & $\mathrm{C} 3$ & $\mathrm{C} 4$ & $0.8(8)$ \\
\hline $\mathrm{C} 2$ & $\mathrm{C} 1$ & $\mathrm{C} 6$ & $\mathrm{C} 5$ & $-0.8(7)$ \\
\hline $\mathrm{C} 2$ & $\mathrm{C} 3$ & $\mathrm{C} 4$ & $\mathrm{C} 5$ & $-1.1(8)$ \\
\hline C3 & $\mathrm{C} 4$ & $\mathrm{C} 5$ & $\mathrm{C} 6$ & $0.5(8)$ \\
\hline $\mathrm{C} 4$ & $\mathrm{C} 5$ & $\mathrm{C} 6$ & $\mathrm{C} 1$ & $0.5(8)$ \\
\hline C6 & $\mathrm{C} 1$ & $\mathrm{C} 2$ & $\mathrm{C} 3$ & $0.1(7)$ \\
\hline $\mathrm{C} 7$ & P1 & N1 & C19 & $-39.1(4)$ \\
\hline C7 & P1 & N1 & $\mathrm{C} 20$ & $131.4(4)$ \\
\hline C7 & $\mathrm{P} 1$ & $\mathrm{C} 1$ & $\mathrm{C} 2$ & $-89.3(4)$ \\
\hline $\mathrm{C} 7$ & P1 & $\mathrm{C} 1$ & $\mathrm{C} 6$ & $92.2(4)$ \\
\hline $\mathrm{C} 7$ & $\mathrm{C} 8$ & C9 & C10 & $-0.7(8)$ \\
\hline $\mathrm{C7}$ & C12 & C13 & C14 & $133.9(5)$ \\
\hline
\end{tabular}




\begin{tabular}{|c|c|c|c|c|}
\hline Atom & Atom & Atom & Atom & Angle $/^{\circ}$ \\
\hline $\begin{array}{l}\mathrm{C} 7 \\
\mathrm{C}\end{array}$ & C12 & C13 & C18 & $-51.5(7)$ \\
\hline C8 & $\mathrm{C} 7$ & C12 & C11 & $-2.3(7)$ \\
\hline C8 & C7 & C12 & C13 & $178.7(5)$ \\
\hline C8 & C9 & C10 & C11 & $-0.1(8)$ \\
\hline C9 & C10 & C11 & C12 & $-0.5(9)$ \\
\hline C10 & $\mathrm{C} 11$ & $\mathrm{C} 12$ & $\mathrm{C} 7$ & $1.7(8)$ \\
\hline C10 & C11 & C12 & C13 & $-179.2(5)$ \\
\hline C11 & $\mathrm{C} 12$ & C13 & C14 & $-45.1(7)$ \\
\hline C11 & C12 & C13 & C18 & $129.5(5)$ \\
\hline C12 & $\mathrm{C} 7$ & C8 & C9 & $1.9(8)$ \\
\hline C12 & $\mathrm{C} 13$ & C14 & C15 & $174.9(5)$ \\
\hline C12 & C13 & C18 & C17 & $-175.8(5)$ \\
\hline C13 & C14 & C15 & C16 & $0.8(8)$ \\
\hline C14 & $\mathrm{C} 13$ & C18 & C17 & $-1.2(7)$ \\
\hline C14 & C15 & C16 & C17 & $-0.7(8)$ \\
\hline C15 & C16 & C17 & C18 & $-0.4(8)$ \\
\hline C16 & C17 & C18 & C13 & $1.3(7)$ \\
\hline C18 & $\mathrm{C} 13$ & C14 & C15 & $0.1(7)$ \\
\hline C19 & $\mathrm{N} 1$ & $\mathrm{C} 20$ & $\mathrm{C} 21$ & $28.8(6)$ \\
\hline C19 & $\mathrm{N} 1$ & $\mathrm{C} 20$ & $\mathrm{C} 22$ & $-98.8(5)$ \\
\hline $\mathrm{C} 20$ & $\mathrm{C} 22$ & $\mathrm{C} 23$ & $\mathrm{C} 24$ & $85.0(5)$ \\
\hline $\mathrm{C} 20$ & $\mathrm{C} 22$ & $\mathrm{C} 23$ & $\mathrm{C} 28$ & $-95.1(5)$ \\
\hline C21 & $\mathrm{C} 20$ & $\mathrm{C} 22$ & 01 & $177.6(4)$ \\
\hline C21 & C20 & C22 & C23 & $-59.6(5)$ \\
\hline C22 & C23 & C24 & C25 & $-178.8(4)$ \\
\hline $\mathrm{C} 22$ & $\mathrm{C} 23$ & $\mathrm{C} 28$ & $\mathrm{C} 27$ & $177.5(4)$ \\
\hline $\mathrm{C} 23$ & $\mathrm{C} 24$ & $\mathrm{C} 25$ & C26 & $0.3(8)$ \\
\hline $\mathrm{C} 24$ & $\mathrm{C} 23$ & $\mathrm{C} 28$ & $\mathrm{C} 27$ & $-2.6(7)$ \\
\hline $\mathrm{C} 24$ & $\mathrm{C} 25$ & C26 & $\mathrm{C} 27$ & $-0.7(8)$ \\
\hline $\mathrm{C} 25$ & C26 & $\mathrm{C} 27$ & $\mathrm{C} 28$ & $-0.6(8)$ \\
\hline C26 & $\mathrm{C} 27$ & $\mathrm{C} 28$ & $\mathrm{C} 23$ & $2.3(7)$ \\
\hline C28 & $\mathrm{C} 23$ & $\mathrm{C} 24$ & $\mathrm{C} 25$ & $1.3(7)$ \\
\hline C29 & P2 & 01 & C22 & $-52.2(3)$ \\
\hline $\mathrm{C} 29$ & P2 & C35 & C36 & $49.2(4)$ \\
\hline $\mathrm{C} 29$ & P2 & C35 & $\mathrm{C} 40$ & $-128.7(4)$ \\
\hline $\mathrm{C} 29$ & C30 & C31 & C32 & $-0.7(8)$ \\
\hline C30 & C29 & C34 & C33 & $0.7(9)$ \\
\hline C30 & C31 & C32 & C33 & $0.9(8)$ \\
\hline C31 & C32 & C33 & C34 & $-0.3(8)$ \\
\hline C32 & C33 & C34 & C29 & $-0.4(9)$ \\
\hline C34 & C29 & C30 & C31 & $-0.1(8)$ \\
\hline C35 & P2 & 01 & $\mathrm{C} 22$ & $-160.2(3)$ \\
\hline C35 & P2 & C29 & C30 & $-145.4(5)$ \\
\hline C35 & P2 & C29 & C34 & $38.8(5)$ \\
\hline C35 & C36 & C37 & C38 & $-0.7(8)$ \\
\hline C36 & C35 & C40 & C39 & $-2.9(7)$ \\
\hline C36 & C37 & C38 & C39 & $-1.2(8)$ \\
\hline C37 & C38 & C39 & C40 & $1.1(8)$ \\
\hline C38 & C39 & C40 & C35 & $1.0(7)$ \\
\hline C40 & C35 & C36 & C37 & $2.7(7)$ \\
\hline
\end{tabular}


Name: N-[(1R,2S)-(2-(Diphenylphosphinito)-1-methyl-2-phenylethyl],

phenylphosphine dichloropalladium complex $\mathbf{3 1}$.

Submitted by: Christophe Darcel

Solved by: Yoann Rousselin

Sample ID: $\quad$ darc10<smiles>CC(NC(OP(c1ccccc1)c1ccccc1)P(Cl)(Cl)(Cl)c1ccccc1)P(F)(=P)c1ccccc1</smiles>

\section{Crystal Data and Experimental}

Figure S18 (thermal ellipsoïds are drawn at $50 \%$ probability plot)

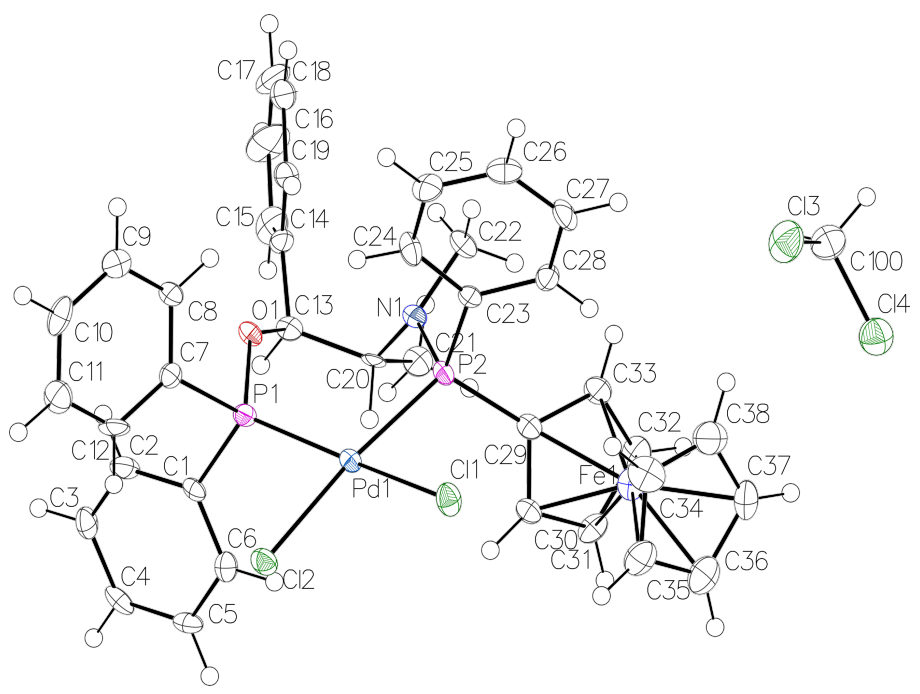

Experimental. Single clear light orange prism-shaped crystals of compound 31 were recrystallized from DCM by slow evaporation. A suitable crystal $0.28 \times 0.16 \times 0.12$ $\mathrm{mm}^{3}$ was selected and mounted on a glass fiber with grease on a Nonius Kappa CCD diffractometer. The crystal was kept at a steady $T=110.0(1) \mathrm{K}$ during data collection. The structure was solved with the ShelXS-97 (Sheldrick, 1997) structure solution program using the direct methods solution method. The model was refined with version 2018/3 of ShelXL (Sheldrick, 2015) using Least Squares minimization.

Crystal Data. $\mathrm{C}_{39} \mathrm{H}_{39} \mathrm{Cl}_{4} \mathrm{FeNOP}{ }_{2} \mathrm{Pd}, \quad M_{r}=903.70$, orthorhombic, $P 2{ }_{1} 2_{1} 2_{1}$ (No. 19), $a=9.9851(3) \AA, b=$ 17.9215(5) $\AA, \quad \mathrm{c}=20.6101(8) \AA, \quad \alpha=\beta=\gamma=90^{\circ}, \quad V=$ $3688.1(2) \AA^{3}, T=110.0(1) \mathrm{K}, Z=4, Z^{\prime}=1, \mu\left(\mathrm{MoK}_{\alpha}\right)=$ $1.291,15458$ reflections measured, 6475 unique $\left(R_{\text {int }}=\right.$ $0.0757)$ which were used in all calculations. The final $w R_{2}$ was 0.0710 (all data) and $R_{1}$ was 0.0472 (I > 2(I)). 


\begin{tabular}{|c|c|}
\hline Compound & 31 \\
\hline CCDC & 1982650 \\
\hline Formula & $\mathrm{C}_{39} \mathrm{H}_{39} \mathrm{Cl}_{4} \mathrm{FeNOP}_{2} \mathrm{Pd}$ \\
\hline$D_{\text {calc. }} / \mathrm{g} \mathrm{cm}^{-3}$ & 1.628 \\
\hline$\mu / \mathrm{mm}^{-1}$ & 1.291 \\
\hline Formula Weight & 903.70 \\
\hline Color & clear light orange \\
\hline Shape & prism \\
\hline Size $/ \mathrm{mm}^{3}$ & $0.28 \times 0.16 \times 0.12$ \\
\hline$T / \mathrm{K}$ & $110.0(1)$ \\
\hline Crystal System & orthorhombic \\
\hline Flack Parameter & $0.00(3)$ \\
\hline Hooft Parameter & $0.00(3)$ \\
\hline Space Group & $P 2{ }_{1} 2_{1} 2_{1}$ \\
\hline$a / \AA$ & $9.9851(3)$ \\
\hline$b / \AA ̊$ & $17.9215(5)$ \\
\hline$c / \AA$ & 20.6101(8) \\
\hline$\alpha /^{\circ}$ & $90.0000(10)$ \\
\hline$\beta /^{\circ}$ & $90.0000(10)$ \\
\hline$\gamma /{ }^{\circ}$ & $90.000(2)$ \\
\hline $\mathrm{V} / \AA^{3}$ & $3688.1(2)$ \\
\hline$Z$ & 4 \\
\hline$Z^{\prime}$ & 1 \\
\hline Wavelength/Å & 0.71073 \\
\hline Radiation type & $\operatorname{MoK}_{\alpha}$ \\
\hline$\Theta_{\min } /^{\circ}$ & 3.013 \\
\hline$\Theta_{\max } /^{\circ}$ & 24.998 \\
\hline Measured Refl. & 15458 \\
\hline Independent Refl. & 6475 \\
\hline Reflections with I > & 4712 \\
\hline $2(\mathrm{I})$ & \\
\hline$R_{\text {int }}$ & 0.0757 \\
\hline Parameters & 445 \\
\hline Restraints & 444 \\
\hline Largest Peak & 0.536 \\
\hline Deepest Hole & -0.521 \\
\hline GooF & 0.958 \\
\hline$w R_{2}$ (all data) & 0.0710 \\
\hline$w R_{2}$ & 0.0636 \\
\hline$R_{1}$ (all data) & 0.0832 \\
\hline$R_{1}$ & 0.0472 \\
\hline
\end{tabular}


Reflections:

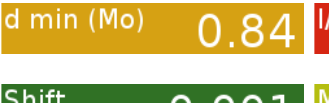

Refinement:

A clear light orange prism-shaped crystal with dimensions $0.28 \times 0.16 \times 0.12 \mathrm{~mm}^{3}$ was mounted on a glass fiber with grease. Data were collected using an Nonius Kappa CCD diffractometer equipped with an Oxford Cryosystems low-temperature device operating at $T=110.0(1) \mathrm{K}$. Data were measured using $\phi$ and $\omega$ scans using $\mathrm{MoK}_{\alpha}$ radiation. The maximum resolution that was achieved was $\Theta=24.998^{\circ}(0.84 \AA)$. The diffraction pattern was indexed and the unit cell was refined on 5409 reflections, 35\% of the observed reflections. Data reduction, scaling and absorption corrections were performed. The final completeness is $99.70 \%$ out to $24.998^{\circ}$ in $\Theta$. No absorption correction was performed. The absorption coefficient $\mu$ of this material is $1.291 \mathrm{~mm}^{-1}$ at this wavelength $(\lambda=0.711 \AA)$. The structure was solved and the space group $P 2{ }_{1} 2_{1} 2_{1}$ (\# 19) determined by the ShelXS-97 (Sheldrick, 1997) structure solution program using direct methods and refined by Least Squares using version 2018/3 of ShelXL (Sheldrick, 2015). All non-hydrogen atoms were refined anisotropically. Hydrogen atom positions were calculated geometrically and refined using the riding model. Hydrogen atom positions were calculated geometrically and refined using the riding model. There is a single molecule in the asymmetric unit, which is represented by the reported sum formula. In other words: Z is 4 and Z' is 1 . The Flack parameter was refined to 0.00(3). Determination of absolute structure using Bayesian statistics on Bijvoet differences using the Olex2 results in $0.00(3)$. Note: The Flack parameter is used to determine chirality of the crystal studied, the value should be near 0 , a value of 1 means that the stereochemistry is wrong and the model should be inverted. A value of 0.5 means that the crystal consists of a racemic mixture of the two enantiomers.

Table S53: Bond Lengths in Å for compound 31.

\begin{tabular}{|c|c|c|c|c|c|}
\hline Atom & Atom & Length/Å & Atom & Atom & Length/Å \\
\hline$\overline{P d 1}$ & $\mathrm{Cl} 1$ & $2.341(2)$ & $\overline{\mathrm{C} 14}$ & C15 & $1.394(10)$ \\
\hline $\operatorname{Pd} 1$ & $\mathrm{Cl} 2$ & $2.3700(19)$ & C14 & C19 & $1.393(10)$ \\
\hline $\operatorname{Pd} 1$ & $\mathrm{P} 1$ & $2.246(2)$ & C15 & C16 & $1.416(12)$ \\
\hline $\mathrm{Pd} 1$ & $\mathrm{P} 2$ & $2.270(2)$ & C16 & C17 & $1.382(12)$ \\
\hline 01 & $\mathrm{P} 1$ & $1.613(5)$ & C17 & C18 & $1.373(12)$ \\
\hline 01 & C13 & $1.456(8)$ & C18 & C19 & $1.390(10)$ \\
\hline N1 & $\mathrm{C} 20$ & $1.478(9)$ & $\mathrm{C} 20$ & C21 & $1.521(9)$ \\
\hline N1 & $\mathrm{C} 22$ & $1.482(9)$ & C23 & $\mathrm{C} 24$ & $1.394(10)$ \\
\hline N1 & P2 & $1.680(6)$ & $\mathrm{C} 23$ & C28 & $1.388(9)$ \\
\hline $\mathrm{C} 1$ & $\mathrm{C} 2$ & $1.390(10)$ & $\mathrm{C} 23$ & P2 & $1.797(8)$ \\
\hline $\mathrm{C} 1$ & $\mathrm{C} 6$ & $1.384(9)$ & $\mathrm{C} 24$ & $\mathrm{C} 25$ & $1.368(10)$ \\
\hline $\mathrm{C} 1$ & $\mathrm{P} 1$ & $1.820(8)$ & $\mathrm{C} 25$ & $\mathrm{C} 26$ & $1.360(11)$ \\
\hline $\mathrm{C} 2$ & $\mathrm{C} 3$ & $1.375(9)$ & $\mathrm{C} 26$ & $\mathrm{C} 27$ & $1.376(11)$ \\
\hline C3 & $\mathrm{C} 4$ & $1.389(10)$ & $\mathrm{C} 27$ & $\mathrm{C} 28$ & $1.368(9)$ \\
\hline $\mathrm{C} 4$ & $\mathrm{C} 5$ & $1.366(10)$ & P2 & $\mathrm{C} 29$ & $1.797(8)$ \\
\hline C5 & $\mathrm{C} 6$ & $1.393(9)$ & C29 & C30 & $1.423(10)$ \\
\hline P1 & C7 & $1.825(8)$ & $\mathrm{C} 29$ & C33 & $1.453(10)$ \\
\hline $\mathrm{C} 7$ & $\mathrm{C} 8$ & $1.373(10)$ & $\mathrm{C} 29$ & $\mathrm{Fe} 1$ & $2.046(8)$ \\
\hline $\mathrm{C} 7$ & $\mathrm{C} 12$ & $1.381(11)$ & C30 & $\mathrm{C} 31$ & $1.399(10)$ \\
\hline $\mathrm{C} 8$ & C9 & $1.371(9)$ & C30 & $\mathrm{Fe} 1$ & $2.039(8)$ \\
\hline C9 & C10 & $1.387(10)$ & C31 & C32 & $1.436(10)$ \\
\hline C10 & C11 & $1.367(10)$ & C31 & Fe1 & $2.057(8)$ \\
\hline C11 & C12 & $1.390(10)$ & C32 & C33 & $1.428(10)$ \\
\hline C13 & C14 & $1.497(10)$ & C32 & $\mathrm{Fe} 1$ & $2.044(8)$ \\
\hline C13 & $\mathrm{C} 20$ & $1.525(9)$ & C33 & $\mathrm{Fe} 1$ & $2.037(8)$ \\
\hline
\end{tabular}




\begin{tabular}{lll}
\hline Atom & Atom & Length/Å \\
\hline Fe1 & C34 & $2.045(8)$ \\
Fe1 & C35 & $2.058(8)$ \\
Fe1 & C36 & $2.065(8)$ \\
Fe1 & C37 & $2.040(8)$ \\
Fe1 & C38 & $2.040(8)$ \\
C34 & C35 & $1.409(10)$
\end{tabular}

\begin{tabular}{lll}
\hline Atom & Atom & Length/Å \\
\hline C34 & C38 & $1.398(11)$ \\
C35 & C36 & $1.434(10)$ \\
C36 & C37 & $1.434(11)$ \\
C37 & C38 & $1.421(11)$ \\
C100 & Cl3 & $1.753(9)$ \\
C100 & Cl4 & $1.768(8)$
\end{tabular}

Table S54: Bond Angles in ${ }^{\circ}$ for compound 31.

\begin{tabular}{|c|c|c|c|}
\hline Atom & Atom & Atom & Angle $/^{\circ}$ \\
\hline $\mathrm{Cl} 1$ & $\mathrm{Pd} 1$ & $\mathrm{Cl} 2$ & $90.68(7)$ \\
\hline P1 & $\mathrm{Pd} 1$ & $\mathrm{Cl} 1$ & $169.80(8)$ \\
\hline P1 & Pd1 & $\mathrm{Cl} 2$ & $85.55(8)$ \\
\hline P1 & $\mathrm{Pd} 1$ & $\mathrm{P} 2$ & $99.74(8)$ \\
\hline P2 & $\operatorname{Pd} 1$ & $\mathrm{Cl} 1$ & $84.52(7)$ \\
\hline P2 & Pd1 & $\mathrm{Cl} 2$ & $174.09(8)$ \\
\hline C13 & 01 & $\mathrm{P} 1$ & $125.5(4)$ \\
\hline $\mathrm{C} 20$ & N1 & $\mathrm{C} 22$ & $117.3(6)$ \\
\hline $\mathrm{C} 20$ & N1 & $\mathrm{P} 2$ & $116.5(5)$ \\
\hline C22 & N1 & $\mathrm{P} 2$ & $118.2(5)$ \\
\hline $\mathrm{C} 2$ & $\mathrm{C} 1$ & P1 & $120.0(6)$ \\
\hline C6 & $\mathrm{C} 1$ & $\mathrm{C} 2$ & $118.9(8)$ \\
\hline C6 & C1 & $\mathrm{P} 1$ & $121.0(7)$ \\
\hline C3 & $\mathrm{C} 2$ & C1 & $120.5(8)$ \\
\hline $\mathrm{C} 2$ & C3 & $\mathrm{C} 4$ & $119.7(8)$ \\
\hline C5 & $\mathrm{C} 4$ & $\mathrm{C} 3$ & $120.8(8)$ \\
\hline $\mathrm{C} 4$ & C5 & C6 & $119.3(8)$ \\
\hline $\mathrm{C} 1$ & C6 & $\mathrm{C} 5$ & $120.8(8)$ \\
\hline 01 & $\mathrm{P} 1$ & Pd1 & $120.7(2)$ \\
\hline 01 & P1 & $\mathrm{C} 1$ & $103.8(3)$ \\
\hline 01 & P1 & $\mathrm{C} 7$ & $96.4(3)$ \\
\hline C1 & P1 & Pd1 & $116.9(3)$ \\
\hline C1 & $\mathrm{P} 1$ & $\mathrm{C} 7$ & $106.3(4)$ \\
\hline C7 & $\mathrm{P} 1$ & Pd1 & $110.2(3)$ \\
\hline C8 & $\mathrm{C} 7$ & $\mathrm{P} 1$ & $120.5(6)$ \\
\hline C8 & $\mathrm{C} 7$ & C12 & $119.3(8)$ \\
\hline C12 & $\mathrm{C} 7$ & P1 & $120.1(6)$ \\
\hline C9 & $\mathrm{C} 8$ & $\mathrm{C} 7$ & $121.1(7)$ \\
\hline C8 & $\mathrm{C} 9$ & C10 & $119.7(8)$ \\
\hline C11 & C10 & C9 & $119.7(8)$ \\
\hline C10 & C11 & C12 & $120.4(8)$ \\
\hline $\mathrm{C} 7$ & C12 & C11 & $119.8(8)$ \\
\hline 01 & C13 & C14 & $106.9(6)$ \\
\hline 01 & C13 & $\mathrm{C} 20$ & $109.0(6)$ \\
\hline C14 & C13 & $\mathrm{C} 20$ & $115.8(6)$ \\
\hline C15 & C14 & C13 & $119.4(8)$ \\
\hline C19 & C14 & C13 & $121.5(8)$ \\
\hline C19 & C14 & C15 & 119.1(8) \\
\hline C14 & C15 & C16 & $119.7(9)$ \\
\hline C17 & C16 & C15 & $119.7(9)$ \\
\hline C18 & C17 & C16 & $120.6(9)$ \\
\hline C17 & C18 & C19 & $120.0(9)$ \\
\hline C18 & C19 & C14 & $120.8(9)$ \\
\hline N1 & $\mathrm{C} 20$ & C13 & $109.9(6)$ \\
\hline
\end{tabular}

\begin{tabular}{|c|c|c|c|}
\hline Atom & Atom & Atom & Angle $/{ }^{\circ}$ \\
\hline N1 & $\mathrm{C} 20$ & $\mathrm{C} 21$ & $114.5(6)$ \\
\hline $\mathrm{C} 21$ & $\mathrm{C} 20$ & C13 & $112.6(6)$ \\
\hline $\mathrm{C} 24$ & $\mathrm{C} 23$ & P2 & $118.9(6)$ \\
\hline C28 & $\mathrm{C} 23$ & $\mathrm{C} 24$ & $117.4(7)$ \\
\hline C28 & $\mathrm{C} 23$ & P2 & $123.6(6)$ \\
\hline $\mathrm{C} 25$ & $\mathrm{C} 24$ & $\mathrm{C} 23$ & $121.3(8)$ \\
\hline C26 & $\mathrm{C} 25$ & $\mathrm{C} 24$ & $119.6(8)$ \\
\hline $\mathrm{C} 25$ & $\mathrm{C} 26$ & $\mathrm{C} 27$ & $120.9(8)$ \\
\hline C28 & $\mathrm{C} 27$ & $\mathrm{C} 26$ & $119.3(8)$ \\
\hline $\mathrm{C} 27$ & $\mathrm{C} 28$ & $\mathrm{C} 23$ & $121.5(8)$ \\
\hline N1 & P2 & $\mathrm{Pd} 1$ & $114.8(2)$ \\
\hline N1 & P2 & $\mathrm{C} 23$ & $102.9(3)$ \\
\hline $\mathrm{N} 1$ & P2 & C29 & $104.0(3)$ \\
\hline C23 & $\mathrm{P} 2$ & $\mathrm{Pd} 1$ & $111.4(2)$ \\
\hline $\mathrm{C} 23$ & $\mathrm{P} 2$ & $\mathrm{C} 29$ & $110.9(4)$ \\
\hline $\mathrm{C} 29$ & $\mathrm{P} 2$ & $\mathrm{Pd} 1$ & $112.2(3)$ \\
\hline P2 & $\mathrm{C} 29$ & $\mathrm{Fe} 1$ & $144.1(4)$ \\
\hline C30 & $\mathrm{C} 29$ & P2 & $123.4(6)$ \\
\hline C30 & $\mathrm{C} 29$ & C33 & $105.8(7)$ \\
\hline C30 & $\mathrm{C} 29$ & $\mathrm{Fe} 1$ & $69.3(4)$ \\
\hline C33 & $\mathrm{C} 29$ & P2 & $126.7(6)$ \\
\hline C33 & $\mathrm{C} 29$ & $\mathrm{Fe} 1$ & $68.8(4)$ \\
\hline C29 & $\mathrm{C} 30$ & $\mathrm{Fe} 1$ & $69.9(4)$ \\
\hline C31 & C30 & $\mathrm{C} 29$ & $110.7(7)$ \\
\hline C31 & $\mathrm{C} 30$ & $\mathrm{Fe} 1$ & $70.7(5)$ \\
\hline C30 & $\mathrm{C} 31$ & C32 & $107.5(7)$ \\
\hline C30 & $\mathrm{C} 31$ & $\mathrm{Fe} 1$ & $69.3(5)$ \\
\hline C32 & C31 & $\mathrm{Fe} 1$ & $69.0(4)$ \\
\hline C31 & C32 & $\mathrm{Fe} 1$ & $70.0(4)$ \\
\hline C33 & C32 & C31 & $107.8(8)$ \\
\hline C33 & $\mathrm{C} 32$ & $\mathrm{Fe} 1$ & $69.3(5)$ \\
\hline C29 & C33 & $\mathrm{Fe} 1$ & $69.5(5)$ \\
\hline C32 & C33 & $\mathrm{C} 29$ & $108.2(7)$ \\
\hline C32 & C33 & Fe1 & $69.8(5)$ \\
\hline C29 & $\mathrm{Fe} 1$ & C31 & $68.9(3)$ \\
\hline C29 & $\mathrm{Fe} 1$ & C35 & $123.8(3)$ \\
\hline C29 & $\mathrm{Fe} 1$ & C36 & $158.5(3)$ \\
\hline C30 & $\mathrm{Fe} 1$ & $\mathrm{C} 29$ & $40.8(3)$ \\
\hline C30 & $\mathrm{Fe} 1$ & C31 & $40.0(3)$ \\
\hline C30 & Fe1 & C32 & $68.1(3)$ \\
\hline C30 & $\mathrm{Fe} 1$ & C34 & $126.5(3)$ \\
\hline C30 & $\mathrm{Fe} 1$ & C35 & $108.8(3)$ \\
\hline C30 & $\mathrm{Fe} 1$ & C36 & $121.7(3)$ \\
\hline C30 & $\mathrm{Fe} 1$ & C37 & $156.3(3)$ \\
\hline
\end{tabular}




\begin{tabular}{llll}
\hline Atom & Atom & Atom & \multicolumn{1}{c}{ Angle $^{\circ}$} \\
\hline C30 & Fe1 & C38 & $162.1(3)$ \\
C31 & Fe1 & C35 & $122.1(3)$ \\
C31 & Fe1 & C36 & $105.0(3)$ \\
C32 & Fe1 & C29 & $69.6(3)$ \\
C32 & Fe1 & C31 & $41.0(3)$ \\
C32 & Fe1 & C34 & $159.1(3)$ \\
C32 & Fe1 & C35 & $157.3(3)$ \\
C32 & Fe1 & C36 & $120.2(3)$ \\
C33 & Fe1 & C29 & $41.7(3)$ \\
C33 & Fe1 & C30 & $68.5(3)$ \\
C33 & Fe1 & C31 & $68.9(3)$ \\
C33 & Fe1 & C32 & $41.0(3)$ \\
C33 & Fe1 & C34 & $125.1(3)$ \\
C33 & Fe1 & C35 & $160.9(3)$ \\
C33 & Fe1 & C36 & $157.2(3)$ \\
C33 & Fe1 & C37 & $121.8(4)$ \\
C33 & Fe1 & C38 & $108.1(3)$ \\
C34 & Fe1 & C29 & $110.6(3)$ \\
C34 & Fe1 & C31 & $159.8(3)$ \\
C34 & Fe1 & C35 & $40.2(3)$ \\
C34 & Fe1 & C36 & $67.6(4)$ \\
C35 & Fe1 & C36 & $40.7(3)$ \\
C37 & Fe1 & C29 & $160.1(3)$ \\
C37 & Fe1 & C31 & $119.9(3)$ \\
C37 & Fe1 & C32 & $104.7(4)$ \\
C37 & Fe1 & C34 & $67.7(4)$ \\
& & & \\
& &
\end{tabular}

\begin{tabular}{llll}
\hline Atom & Atom & Atom & \multicolumn{1}{c}{ Angle $/^{\circ}$} \\
\hline C37 & Fe1 & C35 & $68.6(4)$ \\
C37 & Fe1 & C36 & $40.9(3)$ \\
C37 & Fe1 & C38 & $40.8(3)$ \\
C38 & Fe1 & C29 & $125.0(3)$ \\
C38 & Fe1 & C31 & $156.9(3)$ \\
C38 & Fe1 & C32 & $121.7(4)$ \\
C38 & Fe1 & C34 & $40.0(3)$ \\
C38 & Fe1 & C35 & $68.2(3)$ \\
C38 & Fe1 & C36 & $68.4(3)$ \\
C35 & C34 & Fe1 & $70.4(5)$ \\
C38 & C34 & Fe1 & $69.8(5)$ \\
C38 & C34 & C35 & $109.9(8)$ \\
C34 & C35 & Fe1 & $69.4(5)$ \\
C34 & C35 & C36 & $107.2(8)$ \\
C36 & C35 & Fe1 & $69.9(5)$ \\
C35 & C36 & Fe1 & $69.4(5)$ \\
C37 & C36 & Fe1 & $68.6(5)$ \\
C37 & C36 & C35 & $107.3(8)$ \\
C36 & C37 & Fe1 & $70.5(5)$ \\
C38 & C37 & Fe1 & $69.6(5)$ \\
C38 & C37 & C36 & $108.0(9)$ \\
C34 & C38 & Fe1 & $70.2(5)$ \\
C34 & C38 & C37 & $107.7(9)$ \\
C37 & C38 & Fe1 & $69.6(5)$ \\
C13 & C100 & Cl4 & $112.3(5)$
\end{tabular}

Table S55: Torsion Angles in ${ }^{\circ}$ for compound 31.

\begin{tabular}{llllc}
\hline Atom & Atom & Atom & Atom & Angle ${ }^{\circ}$ \\
\hline Pd1 & P1 & C7 & C8 & $89.9(6)$ \\
Pd1 & P1 & C7 & C12 & $-88.9(7)$ \\
Pd1 & P2 & C29 & C30 & $22.0(7)$ \\
Pd1 & P2 & C29 & C33 & $176.0(6)$ \\
Pd1 & P2 & C29 & Fe1 & $-78.9(7)$ \\
O1 & P1 & C7 & C8 & $-36.1(7)$ \\
01 & P1 & C7 & C12 & $145.1(7)$ \\
O1 & C13 & C14 & C15 & $-144.8(7)$ \\
O1 & C13 & C14 & C19 & $37.5(10)$ \\
01 & C13 & C20 & N1 & $-53.9(7)$ \\
O1 & C13 & C20 & C21 & $177.2(6)$ \\
N1 & P2 & C29 & C30 & $-102.7(7)$ \\
N1 & P2 & C29 & C33 & $51.3(8)$ \\
N1 & P2 & C29 & Fe1 & $156.4(7)$ \\
C1 & C2 & C3 & C4 & $-1.4(12)$ \\
C1 & P1 & C7 & C8 & $-142.5(7)$ \\
C1 & P1 & C7 & C12 & $38.7(8)$ \\
C2 & C1 & C6 & C5 & $-1.7(12)$ \\
C2 & C1 & P1 & Pd1 & $174.5(5)$ \\
C2 & C1 & P1 & O1 & $-50.0(7)$ \\
C2 & C1 & P1 & C7 & $51.1(7)$ \\
C2 & C3 & C4 & C5 & $1.0(12)$ \\
C3 & C4 & C5 & C6 & $-0.9(11)$ \\
C4 & C5 & C6 & C1 & $1.3(12)$
\end{tabular}




\begin{tabular}{|c|c|c|c|c|}
\hline Atom & Atom & Atom & Atom & Angle $/^{\circ}$ \\
\hline$\overline{\mathrm{C} 6}$ & $\mathrm{C} 1$ & $\mathrm{C} 2$ & $\mathrm{C} 3$ & $1.8(12)$ \\
\hline C6 & $\mathrm{C} 1$ & $\mathrm{P} 1$ & $\mathrm{Pd} 1$ & $-8.6(8)$ \\
\hline C6 & $\mathrm{C} 1$ & P1 & 01 & $126.9(7)$ \\
\hline C6 & $\mathrm{C} 1$ & P1 & $\mathrm{C} 7$ & $-132.0(7)$ \\
\hline P1 & 01 & $\mathrm{C} 13$ & C14 & $-178.8(5)$ \\
\hline P1 & 01 & C13 & $\mathrm{C} 20$ & $-52.9(8)$ \\
\hline P1 & $\mathrm{C} 1$ & $\mathrm{C} 2$ & C3 & $178.7(6)$ \\
\hline P1 & $\mathrm{C} 1$ & $\mathrm{C} 6$ & $\mathrm{C} 5$ & $-178.6(5)$ \\
\hline P1 & $\mathrm{C} 7$ & $\mathrm{C} 8$ & C9 & $-178.9(6)$ \\
\hline P1 & $\mathrm{C} 7$ & $\mathrm{C} 12$ & C11 & $179.7(6)$ \\
\hline C7 & $\mathrm{C} 8$ & C9 & C10 & $0.3(12)$ \\
\hline C8 & $\mathrm{C} 7$ & C12 & C11 & $0.9(12)$ \\
\hline C8 & C9 & C10 & C11 & $-1.4(12)$ \\
\hline C9 & C10 & C11 & C12 & $2.2(12)$ \\
\hline C10 & C11 & C12 & $\mathrm{C} 7$ & $-2.0(13)$ \\
\hline C12 & $\mathrm{C} 7$ & C8 & C9 & $-0.1(12)$ \\
\hline C13 & 01 & P1 & Pd1 & $74.2(6)$ \\
\hline C13 & 01 & P1 & $\mathrm{C} 1$ & $-59.2(6)$ \\
\hline C13 & 01 & $\mathrm{P} 1$ & $\mathrm{C} 7$ & $-167.7(6)$ \\
\hline C13 & C14 & C15 & C16 & $-177.0(8)$ \\
\hline C13 & C14 & C19 & C18 & $176.5(7)$ \\
\hline C14 & C13 & $\mathrm{C} 20$ & N1 & 66.7(9) \\
\hline C14 & C13 & $\mathrm{C} 20$ & $\mathrm{C} 21$ & $-62.2(9)$ \\
\hline C14 & $\mathrm{C} 15$ & C16 & C17 & $-0.9(14)$ \\
\hline C15 & C14 & C19 & C18 & $-1.3(12)$ \\
\hline C15 & C16 & C17 & C18 & $1.4(14)$ \\
\hline C16 & C17 & C18 & C19 & $-1.8(14)$ \\
\hline C17 & C18 & C19 & C14 & $1.8(12)$ \\
\hline C19 & C14 & C15 & C16 & $0.8(13)$ \\
\hline $\mathrm{C} 20$ & N1 & P2 & Pd1 & $-46.8(6)$ \\
\hline $\mathrm{C} 20$ & N1 & P2 & $\mathrm{C} 23$ & $-168.0(5)$ \\
\hline $\mathrm{C} 20$ & N1 & P2 & $\mathrm{C} 29$ & $76.2(6)$ \\
\hline $\mathrm{C} 20$ & C13 & C14 & C15 & $93.5(10)$ \\
\hline C20 & C13 & C14 & C19 & $-84.2(9)$ \\
\hline C22 & N1 & $\mathrm{C} 20$ & C13 & $-95.0(8)$ \\
\hline C22 & N1 & $\mathrm{C} 20$ & $\mathrm{C} 21$ & $32.9(9)$ \\
\hline C22 & N1 & P2 & Pd1 & $165.2(5)$ \\
\hline C22 & N1 & P2 & $\mathrm{C} 23$ & $44.0(6)$ \\
\hline C22 & N1 & P2 & $\mathrm{C} 29$ & $-71.8(6)$ \\
\hline C23 & $\mathrm{C} 24$ & $\mathrm{C} 25$ & $\mathrm{C} 26$ & 2.1(13) \\
\hline C23 & P2 & $\mathrm{C} 29$ & C30 & $147.3(6)$ \\
\hline C23 & $\mathrm{P} 2$ & $\mathrm{C} 29$ & C33 & $-58.7(8)$ \\
\hline C23 & P2 & $\mathrm{C} 29$ & $\mathrm{Fe} 1$ & $46.4(8)$ \\
\hline C24 & $\mathrm{C} 23$ & $\mathrm{C} 28$ & $\mathrm{C} 27$ & $0.6(11)$ \\
\hline C24 & $\mathrm{C} 23$ & P2 & Pd1 & $-53.8(7)$ \\
\hline C24 & $\mathrm{C} 23$ & P2 & N1 & $69.7(7)$ \\
\hline C24 & C23 & $\mathrm{P} 2$ & C29 & $-179.6(6)$ \\
\hline C24 & $\mathrm{C} 25$ & $\mathrm{C} 26$ & $\mathrm{C} 27$ & $-0.6(13)$ \\
\hline $\mathrm{C} 25$ & C26 & $\mathrm{C} 27$ & C28 & $-0.9(13)$ \\
\hline C26 & $\mathrm{C} 27$ & $\mathrm{C} 28$ & $\mathrm{C} 23$ & $0.8(12)$ \\
\hline C28 & C23 & $\mathrm{C} 24$ & $\mathrm{C} 25$ & $-2.1(12)$ \\
\hline C28 & C23 & P2 & Pd1 & $131.0(6)$ \\
\hline C28 & C23 & P2 & N1 & $-105.4(7)$ \\
\hline C28 & $\mathrm{C} 23$ & P2 & C29 & $5.3(8)$ \\
\hline
\end{tabular}




\begin{tabular}{|c|c|c|c|c|}
\hline Atom & Atom & Atom & Atom & Angle $/^{\circ}$ \\
\hline P2 & N1 & $\mathrm{C} 20$ & C13 & $116.7(6)$ \\
\hline P2 & N1 & $\mathrm{C} 20$ & $\mathrm{C} 21$ & $-115.4(6)$ \\
\hline P2 & $\mathrm{C} 23$ & $\mathrm{C} 24$ & $\mathrm{C} 25$ & $-177.5(6)$ \\
\hline P2 & $\mathrm{C} 23$ & C28 & C27 & $175.9(6)$ \\
\hline P2 & C29 & $\mathrm{C} 30$ & C31 & $158.9(6)$ \\
\hline P2 & C29 & C30 & $\mathrm{Fe} 1$ & $-142.0(6)$ \\
\hline P2 & C29 & C33 & C32 & $-158.2(6)$ \\
\hline P2 & $\mathrm{C} 29$ & C33 & $\mathrm{Fe} 1$ & $142.6(7)$ \\
\hline C29 & C30 & C31 & C32 & $-0.1(9)$ \\
\hline $\mathrm{C} 29$ & C30 & C31 & $\mathrm{Fe} 1$ & $58.6(6)$ \\
\hline C30 & C29 & C33 & C32 & $-0.6(9)$ \\
\hline C30 & C29 & C33 & $\mathrm{Fe} 1$ & $-59.8(5)$ \\
\hline C30 & C31 & C32 & C33 & $-0.3(9)$ \\
\hline C30 & C31 & C32 & $\mathrm{Fe} 1$ & $58.9(5)$ \\
\hline C31 & C32 & C33 & C29 & $0.5(9)$ \\
\hline C31 & C32 & C33 & $\mathrm{Fe} 1$ & $59.6(5)$ \\
\hline C33 & $\mathrm{C} 29$ & C30 & C31 & $0.4(9)$ \\
\hline C33 & $\mathrm{C} 29$ & C30 & $\mathrm{Fe} 1$ & $59.5(5)$ \\
\hline $\mathrm{Fe} 1$ & C29 & C30 & C31 & $-59.1(6)$ \\
\hline $\mathrm{Fe} 1$ & $\mathrm{C} 29$ & C33 & C32 & $59.2(6)$ \\
\hline $\mathrm{Fe} 1$ & C30 & C31 & C32 & $-58.7(5)$ \\
\hline $\mathrm{Fe} 1$ & C31 & C32 & C33 & $-59.1(5)$ \\
\hline $\mathrm{Fe} 1$ & C32 & C33 & C29 & $-59.1(6)$ \\
\hline $\mathrm{Fe} 1$ & C34 & C35 & C36 & $60.0(6)$ \\
\hline $\mathrm{Fe} 1$ & C34 & C38 & C37 & $-59.8(6)$ \\
\hline $\mathrm{Fe} 1$ & C35 & C36 & C37 & $58.3(6)$ \\
\hline $\mathrm{Fe} 1$ & C36 & C37 & C38 & $59.8(6)$ \\
\hline $\mathrm{Fe} 1$ & C37 & C38 & C34 & $60.1(6)$ \\
\hline C34 & C35 & C36 & $\mathrm{Fe} 1$ & $-59.7(6)$ \\
\hline C34 & C35 & C36 & C37 & $-1.4(9)$ \\
\hline C35 & C34 & C38 & $\mathrm{Fe} 1$ & 59.1(6) \\
\hline C35 & C34 & C38 & C37 & $-0.7(10)$ \\
\hline C35 & C36 & C37 & $\mathrm{Fe} 1$ & $-58.8(6)$ \\
\hline C35 & C36 & C37 & C38 & $1.0(10)$ \\
\hline C36 & C37 & C38 & Fe1 & $-60.4(6)$ \\
\hline C36 & C37 & C38 & C34 & $-0.2(10)$ \\
\hline C38 & C34 & C35 & $\mathrm{Fe} 1$ & $-58.8(6)$ \\
\hline C38 & C34 & C35 & C36 & 1.3(10) \\
\hline
\end{tabular}


Name: N-[(1R,2S)-2-( $\alpha$-Naphtylphenylphosphinito)-1-methyl-2-phenylethyl], N-methylaminodiphenyl phosphine dichloropalladium complex 32.

Submitted by: Antonin Jaillet

Solved by: Yoann Rousselin

Sample ID: $\quad$ 17aj640<smiles>CC(N)C(O[PH]([N+]#N)(c1ccccc1)c1ccccc1)P(Cl)(Cl)(c1ccccc1)c1ccccc1</smiles>

\section{Crystal Data and Experimental}

Figure S19 (thermal ellipsoïds are drawn at $50 \%$ probability plot)

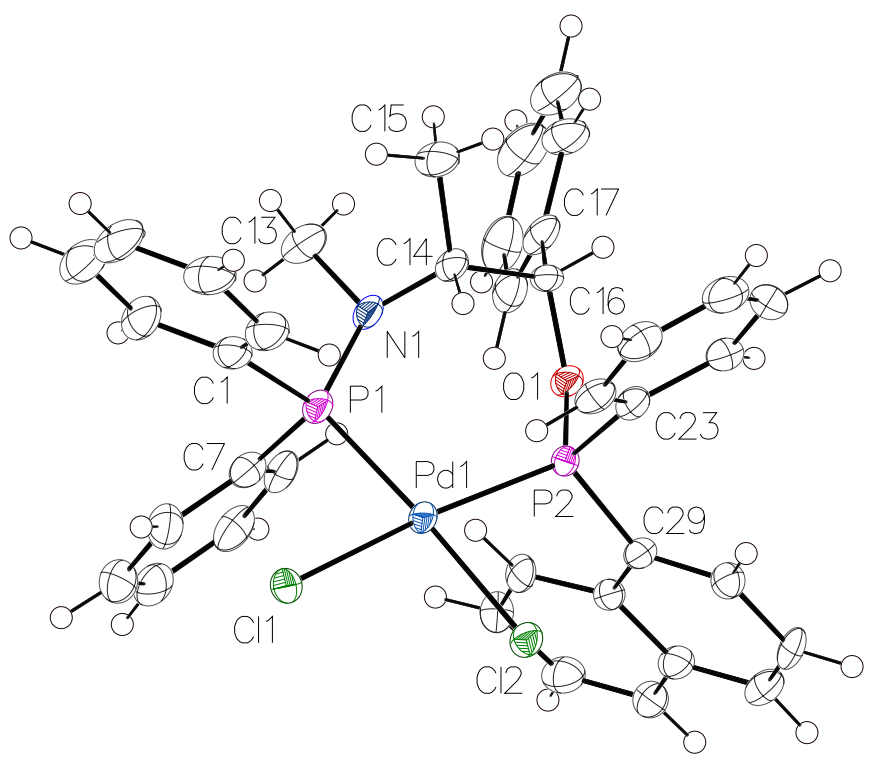

Experimental. Single clear light-yellow prism-shaped crystals of compound $\mathbf{3 2}$ were recrystallized from a mixture of DCM and hexane by slow evaporation. A suitable crystal $(0.32 \times 0.10 \times 0.08) \mathrm{mm}^{3}$ was selected and mounted on a MITIGEN holder oil on a Bruker D8 VENTURE diffractometer. The crystal was kept at $T=$ 100(1) K during data collection. Using Olex2 (Dolomanov et al., 2009), the structure was solved with the ShelXT (Sheldrick, 2015) structure solution program, using the Intrinsic Phasing solution method. The model was refined with version 2017/1 of ShelXL (Sheldrick, 2015) using Least Squares minimization.

Crystal Data. $\mathrm{C}_{38} \mathrm{H}_{35} \mathrm{Cl}_{2} \mathrm{NOP}_{2} \mathrm{Pd}, M_{r}=760.91$, monoclinic, $\mathrm{P} 21_{1}$ (No. 4), $\mathrm{a}=11.2567(4) \AA, \mathrm{b}=15.6082(6) \AA, \mathrm{c}=$ $12.6693(5) \AA, \quad \beta=108.843(2)^{\circ}, \quad \alpha=\gamma=90^{\circ}, \quad V=$ 2106.67(14) $\AA^{3}, T=100(1) \mathrm{K}, Z=2, Z^{\prime}=1, \mu\left(\mathrm{CuK}_{\alpha}\right)=$ $5.635,65144$ reflections measured, 7446 unique $\left(R_{\text {int }}=\right.$ $0.1041)$ which were used in all calculations. The final $w R_{2}$ was 0.0843 (all data) and $R_{1}$ was 0.0354 (I > 2(I)). 


\begin{tabular}{|c|c|c|c|}
\hline Compound & 32 & Wavelength/Å & 1.541840 \\
\hline CCDC & 1982651 & Radiation type & $\mathrm{CuK}_{\alpha}$ \\
\hline Formula & $\mathrm{C}_{38} \mathrm{H}_{35} \mathrm{Cl}_{2} \mathrm{NOP}_{2} \mathrm{Pd}$ & $\Theta_{\min } /^{\circ}$ & 3.686 \\
\hline$D_{\text {calc. }} / \mathrm{g} \mathrm{cm}^{-3}$ & 1.200 & $\Theta_{\max } /^{\circ}$ & 66.830 \\
\hline$\mu / \mathrm{mm}^{-1}$ & 5.635 & Measured Refl. & 65144 \\
\hline Formula Weight & 760.91 & Independent Refl. & 7446 \\
\hline Color & clear light yellow & Reflections Used & 6747 \\
\hline Shape & prism & $R_{\text {int }}$ & 0.1041 \\
\hline Size $/ \mathrm{mm}^{3}$ & $0.32 \times 0.10 \times 0.08$ & Parameters & 409 \\
\hline$T / \mathrm{K}$ & $100(1)$ & Restraints & 1 \\
\hline Crystal System & monoclinic & Largest Peak & 0.649 \\
\hline Flack Parameter & $0.133(10)$ & Deepest Hole & -0.483 \\
\hline Hooft Parameter & $0.165(5)$ & GooF & 1.038 \\
\hline Space Group & $\mathrm{P} 2{ }_{1}$ & $w R_{2}$ (all data) & 0.0843 \\
\hline$a / \AA$ & $11.2567(4)$ & $w R_{2}$ & 0.0811 \\
\hline$b / \AA$ & $15.6082(6)$ & $R_{1}$ (all data) & 0.0425 \\
\hline$c / \AA$ & $12.6693(5)$ & $R_{1}$ & 0.0354 \\
\hline$\alpha /^{\circ}$ & 90 & & \\
\hline$\beta /^{\circ}$ & $108.843(2)$ & & \\
\hline$\gamma /^{\circ}$ & 90 & & \\
\hline $\mathrm{V} / \AA^{3}$ & $2106.67(14)$ & & \\
\hline$Z$ & 2 & & \\
\hline$Z^{\prime}$ & 1 & & \\
\hline
\end{tabular}

\section{Structure Quality Indicators}

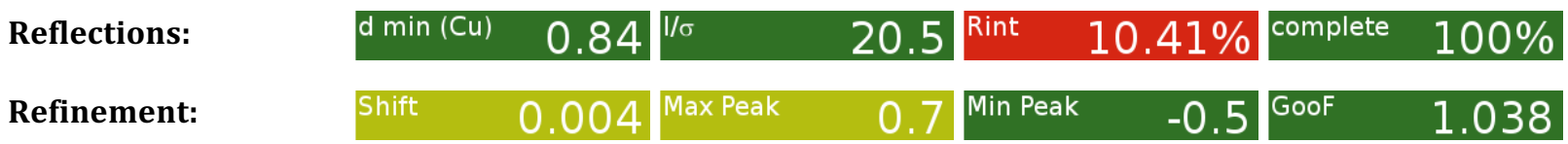

A clear light-yellow prism-shaped crystal with dimensions $0.32 \times 0.10 \times 0.08 \mathrm{~mm}^{3}$ was mounted on a MITIGEN holder oil. X-ray diffraction data were collected using a Bruker D8 VENTURE diffractometer equipped with a Oxford Cryosystems low-temperature device, operating at $T=100$ (1) K. Data were measured using $\phi$ and $\omega$ scans of $1.00^{\circ}$ per frame for $20.00 \mathrm{~s}$ using $\mathrm{CuK}_{\alpha}$ radiation (sealed X-ray tube, 50 $\mathrm{kV}, 1 \mathrm{~mA}$ ). The total number of runs and images was based on the strategy calculation from the program APEX3 (Bruker, 2015). The maximum resolution achieved was $\Theta=66.830^{\circ}$. Cell parameters were retrieved using the SAINT (Bruker, V8.38A, after 2013) software and refined using SAINT (Bruker, V8.38A, after 2013) on 9678 reflections, $15 \%$ of the observed reflections. Data reduction was performed using the SAINT (Bruker, V8.38A, after 2013) software which corrects for Lorentz polarization. The final completeness is $99.70 \%$ out to $66.830^{\circ}$ in $\Theta$. A multi-scan absorption correction was performed using SADABS-2016/2 (Bruker,2016) was used for absorption correction. $w R_{2}$ (int) was 0.1243 before and 0.0990 after correction. The Ratio of minimum to maximum transmission is 0.5759 . The absorption coefficient $\mu$ of this material is $5.635 \mathrm{~mm}^{-1}$ at this wavelength $(\lambda=1.54178 \AA$ ) and the minimum and maximum transmissions are 0.2995 and 0.5201 . The structure was solved in the space group P2 (\# 4) by Intrinsic Phasing using the ShelXT (Sheldrick, 2015) structure solution program and refined by Least Squares using version 2017/1 of ShelXL (Sheldrick, 2015). All non-hydrogen atoms were refined anisotropically. Hydrogen atom positions were calculated geometrically and refined using the riding model. Refined as a 2-component inversion twin. SADABS-2016/2 (Bruker,2016) was used for absorption correction. $w R_{2}$ (int) was 0.1243 before and 0.0990 after correction. The Ratio of minimum to maximum transmission is 0.5759 . There is a single molecule in the asymmetric unit, which is represented by the reported sum formula. In other words: Z is 2 and $\mathrm{Z}^{\prime}$ is 1 . The Flack parameter was refined to 0.133(10). Determination of absolute structure using Bayesian statistics on Bijvoet differences using the Olex2 results in $0.165(5)$. Note: The Flack parameter is used to determine chirality 
of the crystal studied, the value should be near 0 , a value of 1 means that the stereochemistry is wrong and the model should be inverted. A value of 0.5 means that the crystal consists of a racemic mixture of the two enantiomers. Trials to refine DCM solvate molecules led to a very complicated solvate model (partial occupancy of molecules in several positions) and, thus, solvate molecules were squeezed using PLATON. A number of squeezed electrons (213 e-) well correspond with electron count belonging to the found number (5) of disordered DCM molecules (5 x $42 \mathrm{e}-=210 \mathrm{e}-)$ in the previous model.

Table S56: Bond Lengths in Å for compound 32.

\begin{tabular}{lll}
\hline Atom & Atom & Length/Å \\
\hline Pd1 & Cl2 & $2.3570(16)$ \\
Pd1 & Cl1 & $2.3404(15)$ \\
Pd1 & P2 & $2.2430(15)$ \\
Pd1 & P1 & $2.2582(17)$ \\
P2 & 01 & $1.614(5)$ \\
P2 & C23 & $1.794(7)$ \\
P2 & C29 & $1.816(7)$ \\
P1 & N1 & $1.666(6)$ \\
P1 & C1 & $1.813(7)$ \\
P1 & C7 & $1.805(8)$ \\
O1 & C16 & $1.438(7)$ \\
N1 & C14 & $1.469(8)$ \\
N1 & C13 & $1.474(10)$ \\
C38 & C37 & $1.399(9)$ \\
C38 & C29 & $1.427(9)$ \\
C38 & C33 & $1.445(9)$ \\
C15 & C14 & $1.520(10)$ \\
C32 & C31 & $1.369(11)$ \\
C32 & C33 & $1.388(10)$ \\
C36 & C37 & $1.361(10)$ \\
C36 & C35 & $1.412(10)$ \\
C31 & C30 & $1.414(9)$ \\
C9 & C8 & $1.374(11)$ \\
C9 & C10 & $1.376(12)$ \\
C30 & C29 & $1.376(10)$ \\
C8 & C7 & $1.396(10)$ \\
& &
\end{tabular}

\begin{tabular}{lll}
\hline Atom & Atom & Length/Å \\
\hline C28 & C23 & $1.450(10)$ \\
C28 & C27 & $1.383(11)$ \\
C16 & C14 & $1.558(8)$ \\
C16 & C17 & $1.508(10)$ \\
C23 & C24 & $1.379(10)$ \\
C6 & C1 & $1.394(11)$ \\
C6 & C5 & $1.400(11)$ \\
C27 & C26 & $1.364(12)$ \\
C34 & C35 & $1.373(10)$ \\
C34 & C33 & $1.424(10)$ \\
C4 & C3 & $1.384(14)$ \\
C4 & C5 & $1.401(17)$ \\
C18 & C17 & $1.391(13)$ \\
C18 & C19 & $1.394(11)$ \\
C24 & C25 & $1.378(11)$ \\
C1 & C2 & $1.406(11)$ \\
C17 & C22 & $1.389(10)$ \\
C3 & C2 & $1.379(11)$ \\
C21 & C20 & $1.375(15)$ \\
C21 & C22 & $1.369(11)$ \\
C20 & C19 & $1.330(13)$ \\
C10 & C11 & $1.370(12)$ \\
C26 & C25 & $1.366(12)$ \\
C11 & C12 & $1.380(11)$ \\
C12 & C7 & $1.395(10)$ \\
& &
\end{tabular}

Table S57: Bond Angles in ${ }^{\circ}$ for compound 32.

\begin{tabular}{llll}
\hline Atom & Atom & Atom & Angle/d \\
\hline Cl1 & Pd1 & Cl2 & $91.24(5)$ \\
P2 & Pd1 & Cl2 & $82.25(6)$ \\
P2 & Pd1 & Cl1 & $172.79(6)$ \\
P2 & Pd1 & P1 & $100.16(6)$ \\
P1 & Pd1 & Cl2 & $176.90(7)$ \\
P1 & Pd1 & Cl1 & $86.46(6)$ \\
01 & P2 & Pd1 & $121.06(17)$ \\
O1 & P2 & C23 & $104.0(3)$ \\
01 & P2 & C29 & $97.0(3)$ \\
C23 & P2 & Pd1 & $108.8(2)$ \\
C23 & P2 & C29 & $110.4(3)$ \\
C29 & P2 & Pd1 & $114.6(2)$ \\
N1 & P1 & Pd1 & $116.2(2)$ \\
N1 & P1 & C1 & $105.4(3)$ \\
N1 & P1 & C7 & $104.3(3)$
\end{tabular}

\begin{tabular}{lllc}
\hline Atom & Atom & Atom & Angle/d \\
\hline C1 & P1 & Pd1 & $115.0(3)$ \\
C7 & P1 & Pd1 & $106.8(2)$ \\
C7 & P1 & C1 & $108.2(4)$ \\
C16 & 01 & P2 & $125.0(4)$ \\
C14 & N1 & P1 & $116.0(5)$ \\
C14 & N1 & C13 & $114.1(6)$ \\
C13 & N1 & P1 & $119.0(5)$ \\
C37 & C38 & C29 & $125.1(6)$ \\
C37 & C38 & C33 & $118.3(6)$ \\
C29 & C38 & C33 & $116.6(6)$ \\
C31 & C32 & C33 & $122.0(6)$ \\
C37 & C36 & C35 & $119.9(7)$ \\
C36 & C37 & C38 & $122.5(6)$ \\
C32 & C31 & C30 & $118.6(7)$ \\
C8 & C9 & C10 & $122.1(8)$
\end{tabular}




\begin{tabular}{|c|c|c|c|}
\hline Atom & Atom & Atom & Angle/d \\
\hline C29 & C30 & C31 & $121.6(7)$ \\
\hline C9 & C8 & $\mathrm{C} 7$ & $120.0(7)$ \\
\hline $\mathrm{C} 27$ & C28 & $\mathrm{C} 23$ & 117.4(7) \\
\hline 01 & C16 & C14 & $109.3(5)$ \\
\hline 01 & C16 & C17 & $107.0(5)$ \\
\hline C17 & C16 & C14 & $114.3(5)$ \\
\hline N1 & C14 & C15 & $116.5(6)$ \\
\hline N1 & C14 & C16 & $110.4(5)$ \\
\hline C15 & C14 & C16 & $112.1(6)$ \\
\hline $\mathrm{C} 28$ & $\mathrm{C} 23$ & P2 & 119.7(5) \\
\hline $\mathrm{C} 24$ & $\mathrm{C} 23$ & P2 & $121.6(5)$ \\
\hline $\mathrm{C} 24$ & $\mathrm{C} 23$ & C28 & $118.4(6)$ \\
\hline $\mathrm{C} 1$ & C6 & $\mathrm{C} 5$ & $120.5(9)$ \\
\hline C26 & C27 & C28 & $121.0(7)$ \\
\hline C35 & C34 & C33 & $121.5(6)$ \\
\hline C3 & C4 & C5 & $120.4(7)$ \\
\hline C17 & C18 & C19 & $117.9(7)$ \\
\hline C25 & $\mathrm{C} 24$ & $\mathrm{C} 23$ & $122.6(7)$ \\
\hline C6 & C1 & P1 & $121.0(7)$ \\
\hline C6 & C1 & $\mathrm{C} 2$ & $120.0(7)$ \\
\hline $\mathrm{C} 2$ & $\mathrm{C} 1$ & P1 & 118.1(6) \\
\hline C34 & C35 & C36 & $119.9(7)$ \\
\hline C18 & C17 & C16 & $121.0(6)$ \\
\hline $\mathrm{C} 22$ & C17 & C16 & $120.0(8)$ \\
\hline $\mathrm{C} 22$ & C17 & C18 & $118.9(7)$ \\
\hline $\mathrm{C} 2$ & C3 & $\mathrm{C} 4$ & 121.1(9) \\
\hline C6 & C5 & C4 & 118.8(8) \\
\hline $\mathrm{C} 22$ & $\mathrm{C} 21$ & $\mathrm{C} 20$ & 118.4(8) \\
\hline C19 & $\mathrm{C} 20$ & $\mathrm{C} 21$ & $121.2(8)$ \\
\hline $\mathrm{C} 20$ & C19 & C18 & $121.9(9)$ \\
\hline C38 & $\mathrm{C} 29$ & P2 & $120.0(5)$ \\
\hline C30 & C29 & P2 & $119.2(6)$ \\
\hline C30 & C29 & C38 & $120.7(6)$ \\
\hline C3 & C2 & C1 & 119.2(8) \\
\hline $\mathrm{C} 21$ & $\mathrm{C} 22$ & C17 & 121.6(9) \\
\hline C32 & C33 & C38 & $120.5(7)$ \\
\hline C32 & C33 & C34 & $121.6(6)$ \\
\hline C34 & C33 & C38 & $117.8(6)$ \\
\hline C11 & C10 & C9 & 118.2(8) \\
\hline C27 & C26 & C25 & $122.7(8)$ \\
\hline C10 & C11 & C12 & $121.0(8)$ \\
\hline C11 & C12 & C7 & $120.9(8)$ \\
\hline C8 & C7 & P1 & $119.9(6)$ \\
\hline C12 & C7 & P1 & $122.1(6)$ \\
\hline C12 & C7 & C8 & $117.7(7)$ \\
\hline C26 & C25 & C24 & $117.7(8)$ \\
\hline
\end{tabular}


Table S58: Torsion Angles in ${ }^{\circ}$ for compound 32.

\begin{tabular}{|c|c|c|c|c|}
\hline Atom & Atom & Atom & Atom & Angle $/ \infty$ \\
\hline$\overline{\mathrm{Pd} 1}$ & $\mathrm{P} 2$ & 01 & C16 & $76.4(5)$ \\
\hline Pd1 & P2 & $\mathrm{C} 23$ & C28 & $-175.8(5)$ \\
\hline Pd1 & P2 & $\mathrm{C} 23$ & $\mathrm{C} 24$ & $-1.5(6)$ \\
\hline Pd1 & P2 & C29 & C38 & $69.2(6)$ \\
\hline Pd1 & P2 & C29 & C30 & $-112.3(5)$ \\
\hline Pd1 & P1 & N1 & C14 & $-44.9(5)$ \\
\hline Pd1 & P1 & N1 & C13 & $172.8(5)$ \\
\hline Pd1 & P1 & $\mathrm{C} 1$ & C6 & $-152.6(5)$ \\
\hline Pd1 & P1 & $\mathrm{C} 1$ & $\mathrm{C} 2$ & $38.1(7)$ \\
\hline Pd1 & P1 & $\mathrm{C} 7$ & C8 & $-90.1(6)$ \\
\hline Pd1 & P1 & C7 & C12 & $83.0(6)$ \\
\hline P2 & 01 & C16 & C14 & $-47.1(7)$ \\
\hline P2 & 01 & C16 & C17 & $-171.4(5)$ \\
\hline P2 & $\mathrm{C} 23$ & $\mathrm{C} 24$ & C25 & $-171.4(6)$ \\
\hline $\mathrm{P} 1$ & $\mathrm{~N} 1$ & C14 & C15 & $-115.1(6)$ \\
\hline P1 & N1 & C14 & C16 & $115.6(5)$ \\
\hline P1 & $\mathrm{C} 1$ & $\mathrm{C} 2$ & C3 & $169.0(6)$ \\
\hline 01 & P2 & $\mathrm{C} 23$ & C28 & $-45.4(6)$ \\
\hline 01 & P2 & $\mathrm{C} 23$ & C24 & $128.8(6)$ \\
\hline 01 & P2 & C29 & C38 & $-59.6(6)$ \\
\hline 01 & P2 & C29 & C30 & $118.8(6)$ \\
\hline 01 & C16 & C14 & $\mathrm{N} 1$ & $-60.0(7)$ \\
\hline 01 & C16 & C14 & C15 & $168.4(6)$ \\
\hline 01 & C16 & C17 & C18 & 29.7(8) \\
\hline 01 & C16 & C17 & $\mathrm{C} 22$ & $-151.6(6)$ \\
\hline N1 & P1 & $\mathrm{C} 1$ & $\mathrm{C} 6$ & $77.9(7)$ \\
\hline N1 & P1 & $\mathrm{C} 1$ & $\mathrm{C} 2$ & $-91.3(7)$ \\
\hline N1 & P1 & $\mathrm{C} 7$ & C8 & $33.5(6)$ \\
\hline N1 & P1 & C7 & C12 & $-153.4(6)$ \\
\hline C32 & C31 & C30 & C29 & $-0.2(10)$ \\
\hline C37 & C38 & C29 & P2 & $-3.1(10)$ \\
\hline C37 & C38 & C29 & C30 & $178.4(6)$ \\
\hline C37 & C38 & C33 & C32 & $-178.9(6)$ \\
\hline C37 & C38 & C33 & C34 & $3.5(9)$ \\
\hline C37 & C36 & C35 & C34 & $2.4(11)$ \\
\hline C31 & C32 & C33 & C38 & $-0.7(11)$ \\
\hline C31 & C32 & C33 & C34 & $176.9(6)$ \\
\hline C31 & C30 & C29 & P2 & $-176.7(5)$ \\
\hline C31 & C30 & C29 & C38 & $1.7(10)$ \\
\hline C9 & C8 & C7 & P1 & $172.9(6)$ \\
\hline C9 & C8 & $\mathrm{C} 7$ & C12 & $-0.5(11)$ \\
\hline C9 & C10 & C11 & C12 & $-2.4(12)$ \\
\hline C8 & C9 & C10 & C11 & $1.3(12)$ \\
\hline C28 & $\mathrm{C} 23$ & $\mathrm{C} 24$ & C25 & $2.9(10)$ \\
\hline C28 & C27 & C26 & C25 & $3.1(13)$ \\
\hline C16 & C17 & $\mathrm{C} 22$ & $\mathrm{C} 21$ & $-176.1(7)$ \\
\hline C14 & C16 & C17 & C18 & $-91.4(7)$ \\
\hline C14 & C16 & C17 & C22 & $87.2(8)$ \\
\hline $\mathrm{C} 23$ & P2 & 01 & C16 & $-46.3(5)$ \\
\hline C23 & P2 & C29 & C38 & $-167.5(5)$ \\
\hline
\end{tabular}




\begin{tabular}{|c|c|c|c|c|}
\hline Atom & Atom & Atom & Atom & Angle $/ \infty$ \\
\hline$\overline{\mathrm{C} 23}$ & $\mathrm{P} 2$ & C29 & C30 & $11.0(7)$ \\
\hline $\mathrm{C} 23$ & $\mathrm{C} 28$ & $\mathrm{C} 27$ & C26 & $-1.6(11)$ \\
\hline $\mathrm{C} 23$ & C24 & $\mathrm{C} 25$ & C26 & $-1.6(11)$ \\
\hline C6 & C1 & C2 & C3 & $-0.3(11)$ \\
\hline $\mathrm{C} 27$ & $\mathrm{C} 28$ & $\mathrm{C} 23$ & $\mathrm{P} 2$ & $173.2(5)$ \\
\hline $\mathrm{C} 27$ & $\mathrm{C} 28$ & $\mathrm{C} 23$ & $\mathrm{C} 24$ & $-1.2(10)$ \\
\hline $\mathrm{C} 27$ & C26 & $\mathrm{C} 25$ & $\mathrm{C} 24$ & $-1.5(12)$ \\
\hline C4 & C3 & C2 & C1 & $0.6(12)$ \\
\hline C18 & C17 & C22 & $\mathrm{C} 21$ & $2.6(11)$ \\
\hline $\mathrm{C} 1$ & $\mathrm{P} 1$ & $\mathrm{~N} 1$ & C14 & $83.8(6)$ \\
\hline $\mathrm{C} 1$ & $\mathrm{P} 1$ & $\mathrm{~N} 1$ & $\mathrm{C} 13$ & $-58.4(6)$ \\
\hline $\mathrm{C} 1$ & P1 & $\mathrm{C} 7$ & $\mathrm{C} 8$ & $145.4(6)$ \\
\hline $\mathrm{C} 1$ & $\mathrm{P} 1$ & $\mathrm{C} 7$ & $\mathrm{C} 12$ & $-41.5(7)$ \\
\hline C1 & C6 & C5 & C4 & $-0.2(12)$ \\
\hline C35 & C36 & C37 & C38 & $-1.3(11)$ \\
\hline C35 & C34 & C33 & C38 & $-2.4(10)$ \\
\hline C35 & C34 & C33 & C32 & $179.9(7)$ \\
\hline C17 & C16 & C14 & N1 & $59.9(8)$ \\
\hline C17 & C16 & C14 & C15 & $-71.7(8)$ \\
\hline C17 & C18 & C19 & $\mathrm{C} 20$ & $-0.4(11)$ \\
\hline $\mathrm{C} 3$ & $\mathrm{C} 4$ & $\mathrm{C} 5$ & $\mathrm{C} 6$ & $0.5(13)$ \\
\hline C5 & C6 & C1 & P1 & $-168.9(6)$ \\
\hline C5 & C6 & $\mathrm{C} 1$ & $\mathrm{C} 2$ & $0.1(11)$ \\
\hline C5 & C4 & C3 & C2 & $-0.7(12)$ \\
\hline $\mathrm{C} 21$ & $\mathrm{C} 20$ & C19 & C18 & $1.4(13)$ \\
\hline $\mathrm{C} 20$ & $\mathrm{C} 21$ & $\mathrm{C} 22$ & C17 & $-1.7(12)$ \\
\hline C19 & C18 & C17 & C16 & $177.2(6)$ \\
\hline C19 & C18 & C17 & $\mathrm{C} 22$ & $-1.5(10)$ \\
\hline $\mathrm{C} 29$ & P2 & 01 & C16 & $-159.4(5)$ \\
\hline $\mathrm{C} 29$ & P2 & $\mathrm{C} 23$ & $\mathrm{C} 28$ & $57.7(6)$ \\
\hline $\mathrm{C} 29$ & P2 & $\mathrm{C} 23$ & $\mathrm{C} 24$ & $-128.0(6)$ \\
\hline C29 & C38 & C37 & C36 & 177.3(7) \\
\hline C29 & C38 & C33 & C32 & 2.1(10) \\
\hline $\mathrm{C} 29$ & C38 & C33 & C34 & $-175.6(6)$ \\
\hline C22 & C21 & C20 & C19 & $-0.3(12)$ \\
\hline C33 & C38 & C37 & C36 & $-1.7(10)$ \\
\hline C33 & C38 & C29 & P2 & $175.9(5)$ \\
\hline C33 & C38 & C29 & $\mathrm{C} 30$ & $-2.6(10)$ \\
\hline C33 & C32 & C31 & C30 & $-0.3(11)$ \\
\hline C33 & C34 & C35 & C36 & $-0.5(11)$ \\
\hline C10 & C9 & C8 & $\mathrm{C} 7$ & $0.1(11)$ \\
\hline C10 & C11 & $\mathrm{C} 12$ & $\mathrm{C} 7$ & $2.1(12)$ \\
\hline C11 & C12 & $\mathrm{C} 7$ & P1 & $-173.8(6)$ \\
\hline C11 & $\mathrm{C} 12$ & $\mathrm{C} 7$ & $\mathrm{C} 8$ & $-0.6(11)$ \\
\hline C13 & N1 & C14 & C15 & 28.9(8) \\
\hline C13 & $\mathrm{N} 1$ & C14 & C16 & $-100.4(7)$ \\
\hline C7 & $\mathrm{P} 1$ & $\mathrm{~N} 1$ & C14 & $-162.3(5)$ \\
\hline C7 & P1 & N1 & C13 & $55.5(6)$ \\
\hline C7 & P1 & $\mathrm{C} 1$ & $\mathrm{C} 6$ & $-33.3(7)$ \\
\hline C7 & P1 & C1 & C2 & $157.5(6)$ \\
\hline
\end{tabular}


Table S59: Solvent masking (PLATON/SQUEEZE) information for compound 32.

\begin{tabular}{lllrrrr}
\hline No & \multicolumn{1}{c}{$\mathbf{x}$} & $\mathbf{y}$ & \multicolumn{1}{c}{$\mathbf{z}$} & $\mathbf{V}$ & $\mathbf{e}$ & Content \\
\hline 1 & -0.000 & 0.147 & -0.000 & 564 & 213 & \\
2 & 0.361 & 0.714 & 0.166 & 8 & 1 & \\
3 & 0.638 & 0.215 & 0.834 & 8 & 1 &
\end{tabular}




\section{Crystallographic data references}

Dolomanov, L. J; Bourhis, L. J.; Gildea, R. J.; Howard, J. A. K.; Puschmann, H. Olex2: A complete structure solution, refinement and analysis program. J. Appl. Cryst. 2009, 42, 339-341.

Sheldrick, G. M. Crystal structure refinement with ShelXL. Acta Cryst. C 2015, 71, 3-8.

Sheldrick, G. M. ShelXT-Integrated space-group and crystal-structure determination. Acta Cryst. A 2015, 71, 3-8.

Software for the Integration of CCD Detector System Bruker Analytical X-ray Systems, Bruker AXS, Madison, WI (after 2013).

Sheldrick, G. M. A short history of ShelX. Acta Cryst. A 2008, 64, 339-341.

Otwinowski, Z.; Minor, W. Processing of X-ray Diffraction Data Collected in Oscillation Mode. Methods in Enzymology 1997, 276, 307-326.

Palatinus, L. The Charge-Flipping Algorithm in Crystallography. Acta Cryst. B 2013, 69, 1-16.

Sheldrick, G. M. SHELXS-97, Program for X-Ray Crystal Structure Solution and Refinement; University of Gottingen, 1997.

Spek, A. PLATON SQUEEZE: A Tool for the Calculation of the Disordered Solvent Contribution to the Calculated Structure Factors. Acta Cryst. C 2015, 71, 9-18.

Spek, A. PLATON, An Integrated Tool for the Analysis of the Results of a Single Crystal Structure Determination. Acta Cryst. A 1990, 46, c34. 


\section{Part C:}




\section{HPLC chromatograms on chiral column}

Figure S20

Allylic alkylation of ( \pm )-1,3-diphenylpropenyl acetate 24 with dimethylmalonate into compound $(R)-28$

Pd-AMPP* 13i: chromatogram HPLC: detn 254nm, Chiralpak IA, $1 \mathrm{~mL} / \mathrm{min}, \mathrm{Hex} / i-\mathrm{PrOH}$ (90:10): 91\% e.e. $(R)$

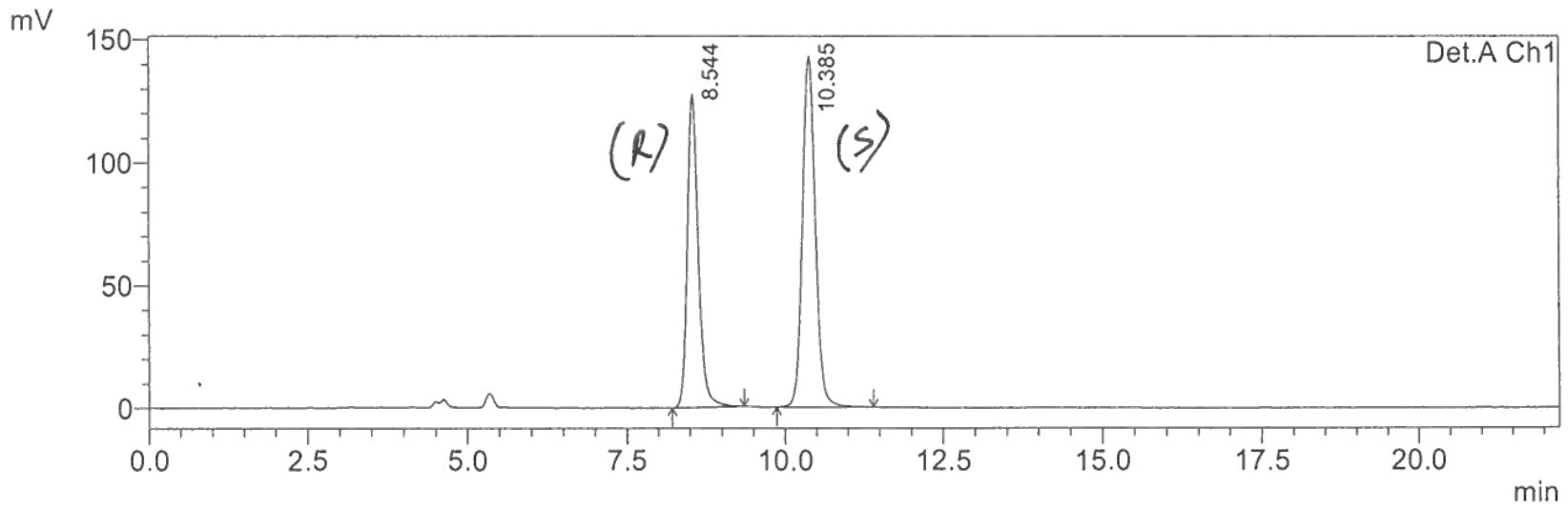

Detector A Chl 254nm

\begin{tabular}{|r|r|r|r|}
\hline Peak\# & Ret. Time & \multicolumn{1}{|c|}{ Area } & \multicolumn{1}{|c|}{ Area \% } \\
\hline 1 & 8.544 & 1538280 & 43.003 \\
\hline 2 & 10.385 & 2038875 & 56.997 \\
\hline Total & & 3577155 & 100.000 \\
\hline
\end{tabular}

$\mathrm{mV}$

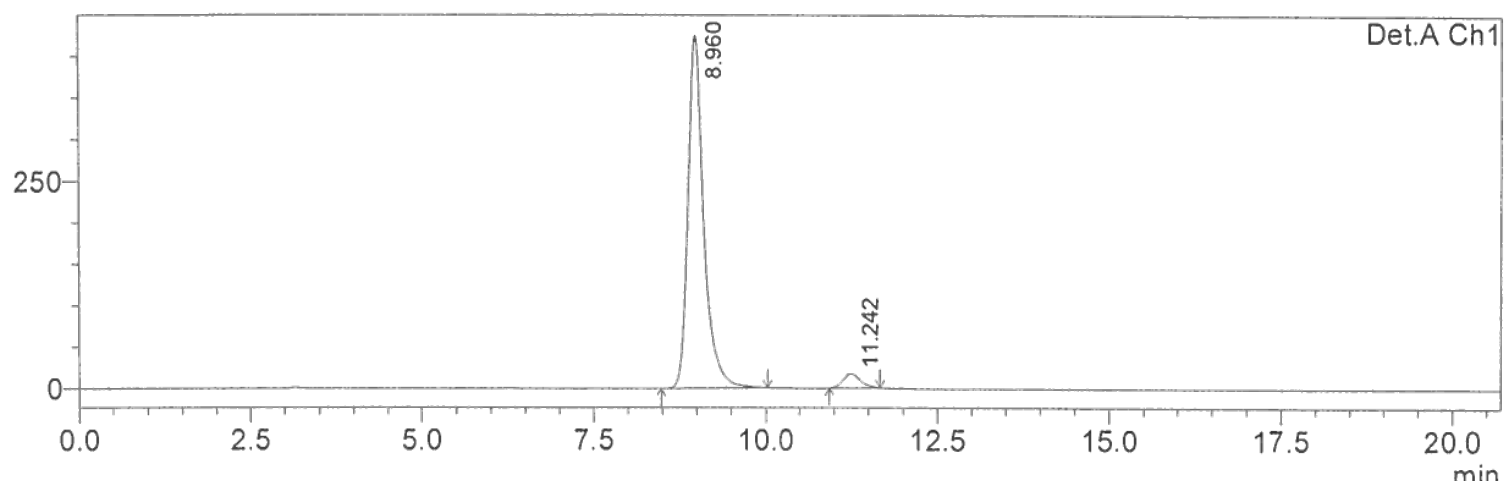

Detector A Ch1 254nm

\begin{tabular}{|r|r|r|r|}
\hline Peak\# & Ret. Time & \multicolumn{1}{|c|}{ Area } & Area \% \\
\hline 1 & 8.960 & 6748148 & 95.690 \\
\hline 2 & 11.242 & 303970 & 4.310 \\
\hline Total & & 7052118 & 100.000 \\
\hline
\end{tabular}


Figure S21

Allylic alkylation of ( \pm )-1,3-diphenylpropenyl acetate 24 with benzylamine into compound $(S)$-29

Pd-AMPP* 13i: chromatogram HPLC: Lux $5 \mu$ cellulose-1, detn 254 nm, 0.5 mL/min, Hex/i-PrOH (98:2): 95\% e.e.

$(S)$

$\mathrm{mV}$

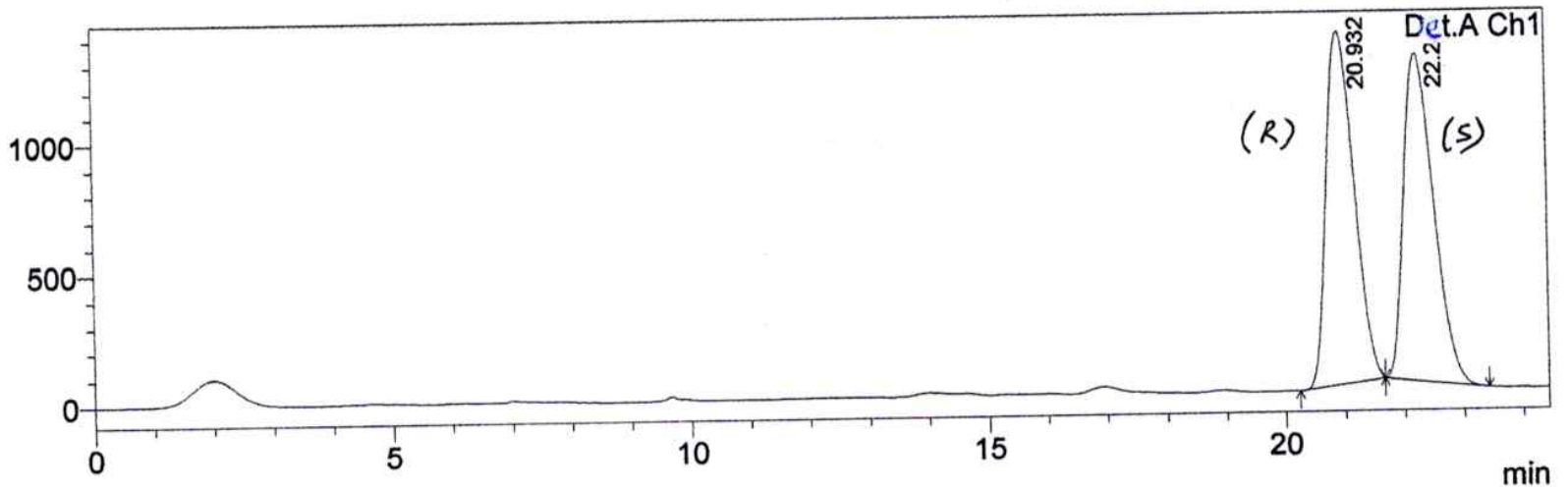

Detector A Ch1 254nm
\begin{tabular}{|r|r|r|r|}
\hline Peak\# & Ret. Time & Area & \multicolumn{1}{|c|}{ Area \% } \\
\hline 1 & 20.932 & 44213280 & 49.869 \\
\hline 2 & 22.243 & 44445317 & 50.131 \\
\hline Total & & 88658597 & 100.000 \\
\hline
\end{tabular}

$\mathrm{mV}$

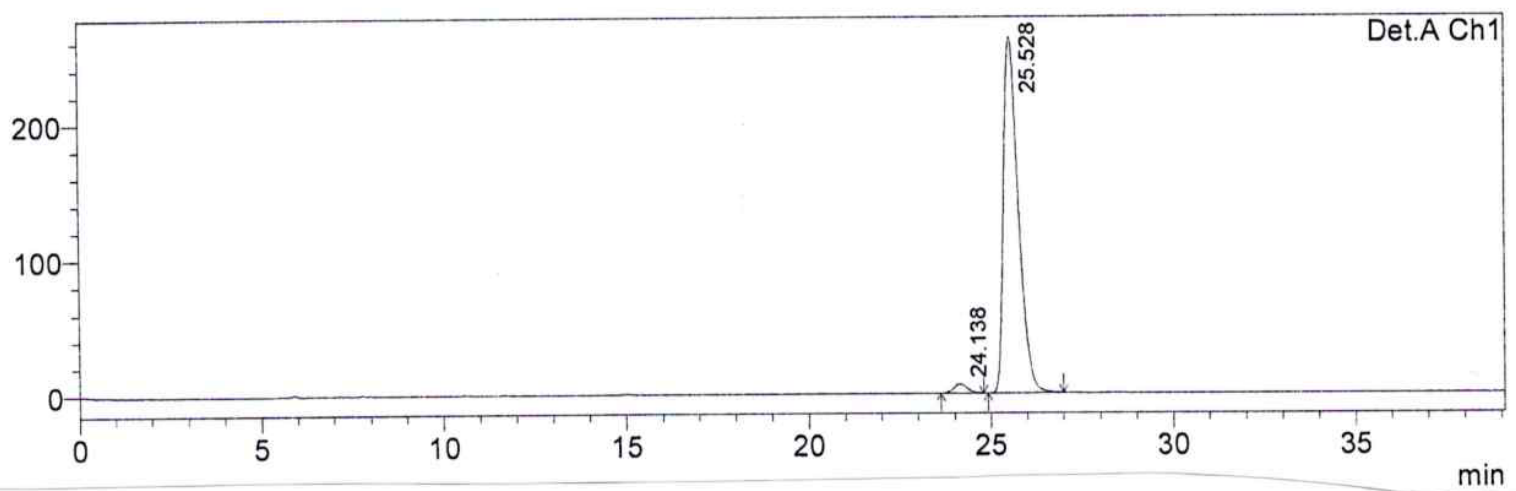

Detector A Ch1 254nm
\begin{tabular}{|r|r|r|r|}
\hline Peak\# & Ret. Time & \multicolumn{1}{|c|}{ Area } & Area $\%$ \\
\hline 1 & 24.138 & 174009 & 2.290 \\
\hline 2 & 25.528 & 7424913 & 97.710 \\
\hline Total & & 7598922 & 100.000 \\
\hline
\end{tabular}

$e x=95 \%(s$, 
Table S60. Complementary results in allylic alkylation of ( \pm )-1,3-diphenylpropenyl acetate 24 with dimethylmalonate into compound $(R)$-28, catalyzed by Pd-complexes with AMPP*a

\begin{tabular}{|c|c|c|c|c|c|}
\hline Entry & $\begin{array}{l}\text { AMPP* } \\
\text { ligand }\end{array}$ & Reaction time $^{b}$ & $\begin{array}{c}\text { Reaction } \\
\text { temperature }\end{array}$ & Yield & \%e.e. ${ }^{\mathrm{c}}($ abs. conf. $)$ \\
\hline 1 & $13 \mathbf{j}$ & $4.5 \mathrm{~h}$ & $\mathrm{RT}$ & $88 \%$ & $41 \%(S)^{\mathrm{d}}$ \\
\hline 2 & $13 \mathbf{a}$ & $5.5 \mathrm{~h}$ & RT & $90 \%$ & $62 \%(S)$ \\
\hline 3 & 131 & $16.5 \mathrm{~h}$ & RT & $86 \%$ & $55 \%(S)$ \\
\hline 4 & 131 & $16.5 \mathrm{~h}$ & $-20^{\circ} \mathrm{C}$ & $93 \%$ & $59 \%(S)$ \\
\hline 5 & $13 m$ & $13 \mathrm{~h}$ & RT & $70 \%$ & $36 \%(S)$ \\
\hline 6 & $13 n$ & $48 \mathrm{~h}$ & RT & $70 \%$ & $34 \%(S)$ \\
\hline 7 & $13 b$ & $14 \mathrm{~h}$ & RT & $87 \%$ & $44 \%(S)$ \\
\hline 8 & $13 b$ & $20 \mathrm{~h}$ & $-20^{\circ} \mathrm{C}$ & $77 \%$ & $47 \%(S)$ \\
\hline 9 & $13 c$ & $1.5 \mathrm{~h}$ & RT & $74 \%$ & $38 \%(S)$ \\
\hline 10 & $13 c$ & $16 \mathrm{~h}$ & $-20^{\circ} \mathrm{C}$ & $96 \%$ & $23 \%(S)$ \\
\hline 11 & $13 \mathrm{~h}$ & $16 \mathrm{~h}$ & $\mathrm{RT}$ & $83 \%$ & $41 \%(R)$ \\
\hline 12 & $13 \mathrm{~h}$ & $16 \mathrm{~h}$ & $-20^{\circ} \mathrm{C}$ & $83 \%$ & $56 \%(R)$ \\
\hline 13 & $13 k$ & $45 \mathrm{~h}$ & RT & $66 \%$ & $61 \%(S)$ \\
\hline 14 & $13 k$ & $47 \mathrm{~h}$ & $-30^{\circ} \mathrm{C}$ & $64 \%$ & $71 \%(S)$ \\
\hline 15 & 130 & $13 \mathrm{~h}$ & $\mathrm{RT}$ & $74 \%$ & $8 \%(S)$ \\
\hline 16 & $13 q$ & $48 \mathrm{~h}$ & $\mathrm{RT}$ & $74 \%$ & $39 \%(S)$ \\
\hline 17 & $13 f$ & $16.5 \mathrm{~h}$ & $\mathrm{RT}$ & $90 \%$ & $85 \%(S)$ \\
\hline 18 & $13 f$ & $16.5 \mathrm{~h}$ & $0^{\circ} \mathrm{C}$ & $87 \%$ & $85 \%(S)$ \\
\hline 19 & $13 f$ & $21 \mathrm{~h}$ & $-20^{\circ} \mathrm{C}$ & $88 \%$ & $85 \%(S)$ \\
\hline 20 & $13 f$ & $21 \mathrm{~h}$ & $-35^{\circ} \mathrm{C}$ & $85 \%$ & $85 \%(S)$ \\
\hline 21 & $13 \mathrm{~g}$ & $3 \mathrm{~h}$ & $\mathrm{RT}$ & $83 \%$ & $70 \%(S)$ \\
\hline 22 & $13 \mathrm{~g}$ & $5.75 \mathrm{~h}$ & $0^{\circ} \mathrm{C}$ & $90 \%{ }^{\mathrm{e}}$ & $73 \%(S)$ \\
\hline 23 & $13 \mathrm{~g}$ & $5.75 \mathrm{~h}$ & $-20^{\circ} \mathrm{C}$ & $81 \%^{\mathrm{e}}$ & $72 \%(S)$ \\
\hline 24 & $13 r$ & $17 \mathrm{~h}$ & $\mathrm{RT}$ & $71 \%$ & $43 \%(S)$ \\
\hline 25 & $16 a$ & $14 \mathrm{~h}$ & $\mathrm{RT}$ & $68 \%$ & $44 \%(S)$ \\
\hline 26 & $16 a$ & $24 \mathrm{~h}$ & $-20^{\circ} \mathrm{C}$ & $65 \%$ & $49 \%(S)$ \\
\hline 27 & $16 \mathrm{~b}$ & $15 \mathrm{~h}$ & $\mathrm{RT}$ & $68 \%$ & $4 \%(S)$ \\
\hline 28 & $16 \mathrm{~b}$ & $24 \mathrm{~h}$ & $-20^{\circ} \mathrm{C}$ & $71 \%$ & $10 \%(S)$ \\
\hline 29 & 16d & $111 \mathrm{~h}$ & $\mathrm{RT}$ & $68 \%$ & $47 \%(S)$ \\
\hline
\end{tabular}

${ }^{a}$ Reaction conditions: $0.005 \mathrm{mmol}$ of $\left[\mathrm{Pd}\left(\pi-\mathrm{C}_{3} \mathrm{H}_{5}\right) \mathrm{Cl}\right]_{2}, 0.01 \mathrm{mmol}$ of AMPP* ligand, $1 \mathrm{mmol}$ of $(E)-1,3-$ diphenylprop-2-en-1-yl acetate $\mathbf{2 4}, 3 \mathrm{mmol}$ of dimethylmalonate, $3 \mathrm{mmol}$ of BSA, and a catalytic amount of KOAc in $5 \mathrm{~mL}$ of dry dichloromethane. ${ }^{b}$ Reaction times (not optimized). ${ }^{\circ}$ Determined by chiral HPLC on Chiralcel OD-H column. ${ }^{\mathrm{d}}$ The precatalyst is obtained by decomplexation of $\mathbf{1 2} \mathbf{j}$ with DABCO and mixing the crude mixture with 0.5 equiv. of $\left[\mathrm{Pd}\left(\mathrm{C}_{3} \mathrm{H}_{5}\right) \mathrm{Cl}\right]_{2}$ in $\mathrm{CH}_{2} \mathrm{Cl}_{2}$ at room temperature for 20 minutes. ${ }^{\mathrm{e}} \mathrm{Corrected}$ yield for a conversion of $84 \%$. 


\section{Computational Details}

All density functional theory (DFT) calculations were performed with Gaussian $16^{1}$ at the Université de Sherbrooke with the Mammouth supercomputer supported by Le Réseau Québécois De Calculs Hautes Performances. The DFT ground state calculations were carried out using the B3LYP/genecp method. A 6-31g $(\mathrm{d}, \mathrm{p})$ basis set was used for $\mathrm{C}, \mathrm{H}, \mathrm{N}$ and $\mathrm{O}$ atoms. ${ }^{2} \mathrm{VDZ}$ (valence double $\zeta$ ) with SBKJC effective core potentials was used for $\mathrm{P}$ and $\mathrm{Pd}$ atoms. ${ }^{3-8}$ Seven membered ring was directly optimised from the *.cif file. Red, positive isosurface; blue, negative isosurface. Fukui index were calculated according to ref ${ }^{9}$.

\section{Number of Imaginary Frequencies and Cartesian Coordinates}

- No imaginary frequencies for structure 34M(half-chair)

\section{- Cartesian Coordinates:}

\section{Structure 33M(boat)}

110

symmetry $\mathrm{c} 1$

Pd $\quad-0.093299207$

$\begin{array}{llll}P & -1.434170894 & 0.948537815 & -0.392623162\end{array}$

$\begin{array}{llll}\text { P } & 2.062745019 & -0.015465214 & -0.203471030\end{array}$

$\begin{array}{llll}\text { C } & -1.207244042 & 1.632279446 & -2.142902354\end{array}$

$\begin{array}{llll}\text { C } & -0.785344499 & 2.945624182 & -2.370281807\end{array}$

H $\quad-0.596109773 \quad 3.605714411 \quad-1.533710034$

$\begin{array}{llll}\text { C } & -0.583564854 & 3.402448698 & -3.676046063\end{array}$

$\begin{array}{llll}\text { H } & -0.258651468 & 4.425409038 & -3.841726104\end{array}$

$\begin{array}{llll}\text { C } & -0.799877426 & 2.552914946 & -4.762092771\end{array}$

H $\quad-0.644925496 \quad 2.911627031 \quad-5.775106849$

$\begin{array}{llll}\text { C } & -1.216291396 & 1.238193439 & -4.539968610\end{array}$

H $\quad-1.384623123 \quad 0.569630262 \quad-5.379013233$

$\begin{array}{llll}\text { C } & -1.414762598 & 0.779985474 & -3.236762741\end{array}$

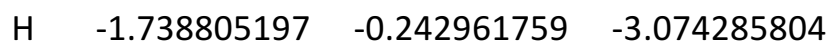

$\begin{array}{llll}\text { C } & -3.328528436 & 0.954229010 & -0.163822531\end{array}$

$\begin{array}{llll}\text { C } & -4.125841507 & 1.304160341 & -1.262569560\end{array}$

$\begin{array}{llll}\mathrm{H} & -3.660801572 & 1.545229307 & -2.210218553\end{array}$

$\begin{array}{llll}\text { C } & -5.514773815 & 1.375042909 & -1.163135843\end{array}$

$\begin{array}{llll}H & -6.104108199 & 1.655779636 & -2.030642486\end{array}$

$\begin{array}{llll}\text { C } & -6.128947937 & 1.093845035 & 0.053390904\end{array}$

$\begin{array}{llll}\text { H } & -7.209194551 & 1.141398474 & 0.151955859\end{array}$

$\begin{array}{llll}\text { C } & -5.346942235 & 0.747694173 & 1.152190949\end{array}$

H $\quad-5.827947844 \quad 0.513894802 \quad 2.096614416$

$\begin{array}{llll}\text { C } & -3.944124331 & 0.668804508 & 1.080969692\end{array}$

$\begin{array}{llll}\text { C } & -3.237629724 & 0.308414555 & 2.346514938\end{array}$

$\begin{array}{llll}\text { C } & -2.307813792 & -0.740639150 & 2.422854508\end{array}$

H $\quad-2.049698392 \quad-1.290424027 \quad 1.524849262$

$\begin{array}{llll}\text { C } & -1.742734322 & -1.110968682 & 3.644396167\end{array}$

$\begin{array}{llll}\text { H } & -1.037074947 & -1.936577447 & 3.681719367\end{array}$ 


\begin{tabular}{|c|c|c|c|}
\hline & -2.098437067 & -0.445434992 & 4.818843131 \\
\hline & -1.664425011 & -0.740094351 & 5.769429412 \\
\hline & -3.024108154 & 0.598006413 & 4.760732288 \\
\hline & -3.310308567 & 1.124619232 & 5.666231647 \\
\hline & -3.585815695 & 0.969806883 & 3.538845478 \\
\hline & -4.301464344 & 1.785855791 & 3.503844938 \\
\hline & 1.894846164 & 3.819512624 & 1.016780889 \\
\hline & 1.805852796 & 4.465719226 & -0.222638868 \\
\hline & 1.593722142 & 3.881081212 & -1.108766563 \\
\hline & 2.028183680 & 5.840458123 & -0.324163734 \\
\hline & 1.962455357 & 6.325535539 & -1.294036508 \\
\hline & 2.347863122 & 6.589609063 & 0.809082253 \\
\hline & 2.522619718 & 7.658187582 & 0.727983993 \\
\hline & 2.463087955 & 5.950516989 & 2.044683977 \\
\hline & 2.735033207 & 6.518189343 & 2.929652179 \\
\hline & 2.247331352 & 4.575626071 & 2.144609909 \\
\hline & 2.367647747 & 4.086564844 & 3.107357985 \\
\hline & 3.286474032 & -0.439900335 & 1.145117214 \\
\hline & 2.886966784 & -1.268018837 & 2.199127591 \\
\hline & 1.892954424 & -1.703514642 & 2.198216030 \\
\hline & 3.771494705 & -1.537930249 & 3.245361770 \\
\hline & 3.464354054 & -2.186839021 & 4.059658441 \\
\hline & 5.046999802 & -0.972152863 & 3.242836299 \\
\hline & 5.733896060 & -1.180174704 & 4.057702898 \\
\hline & 5.442773271 & -0.133713449 & 2.196088578 \\
\hline & 6.433121560 & 0.311158563 & 2.198984104 \\
\hline & 4.565955383 & 0.136680411 & 1.146581533 \\
\hline & 4.871286877 & 0.792473268 & 0.337374306 \\
\hline V & 1.957379547 & 1.715358581 & -0.101815801 \\
\hline & -0.838440054 & 2.229857961 & 0.668062485 \\
\hline & 3.015181764 & -0.089151967 & -1.809420895 \\
\hline 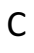 & 4.086014764 & -0.976804865 & -1.972472836 \\
\hline & 4.421131857 & -1.593390977 & -1.145551198 \\
\hline & 4.738235598 & -1.055484865 & -3.205475871 \\
\hline & 5.576063389 & -1.735470952 & -3.327544918 \\
\hline & 4.324009189 & -0.255597998 & -4.271357328 \\
\hline & 4.835933979 & -0.316401294 & -5.226945165 \\
\hline G & 3.252417825 & 0.625360633 & -4.106650841 \\
\hline & 2.930127920 & 1.252870858 & -4.931935242 \\
\hline & 2.591028599 & 0.708209570 & -2.881331322 \\
\hline 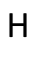 & 1.762976119 & 1.397425577 & -2.758122226 \\
\hline- & 1.642529292 & 2.330158275 & 1.163636836 \\
\hline & 2.338231207 & 1.946348308 & 1.923306819 \\
\hline C & 0.217869251 & 1.912195891 & 1.645176475 \\
\hline & 0.255559632 & 0.819506161 & 1.725751638 \\
\hline & -0.073376166 & 2.425529494 & 3.060527640 \\
\hline
\end{tabular}




$\begin{array}{lrrr}\text { H } & -0.105209722 & 3.515700292 & 3.112999004 \\ \text { H } & 0.713389781 & 2.084519409 & 3.740686424 \\ \text { H } & -1.021141738 & 2.027853855 & 3.424680689 \\ \text { C } & -1.682054615 & 3.407914390 & 0.901991379 \\ \text { H } & -2.308817843 & 3.596305544 & 0.028740700 \\ \text { H } & -1.049901950 & 4.287822096 & 1.050059719 \\ \text { H } & -2.343957810 & 3.296279348 & 1.769977505 \\ \mathrm{C} & 1.769934885 & -3.942931052 & -0.167357510 \\ \mathrm{C} & 1.630118286 & -4.414967488 & 1.151914819 \\ \mathrm{C} & 2.649071961 & -5.142798638 & 1.759050026 \\ \mathrm{C} & 3.834226430 & -5.410587017 & 1.066905060 \\ \mathrm{C} & 3.990277212 & -4.945486169 & -0.239608276 \\ \mathrm{C} & 2.968233937 & -4.220329444 & -0.850290349 \\ \mathrm{H} & 0.721345686 & -4.211779495 & 1.711372717 \\ \mathrm{H} & 2.519682059 & -5.505887395 & 2.774260306 \\ \mathrm{H} & 4.626126456 & -5.980900336 & 1.542311958 \\ \mathrm{H} & 4.903095907 & -5.157390875 & -0.788053522 \\ \mathrm{H} & 3.087965798 & -3.875502811 & -1.873505969 \\ \mathrm{C} & -0.672865076 & -3.332437003 & -0.544039266 \\ \mathrm{H} & -0.951937726 & -3.839776906 & 0.374769966 \\ \mathrm{C} & 0.697176050 & -3.230629100 & -0.883746543 \\ \mathrm{C} & -1.664255796 & -2.687123398 & -1.298582469 \\ \mathrm{C} & -3.108759082 & -2.764841200 & -1.054709869 \\ \mathrm{C} & -3.984593076 & -2.398732540 & -2.094305949 \\ \mathrm{C} & -3.664658285 & -3.247662670 & 0.146741103 \\ \mathrm{C} & -5.363974651 & -2.517153698 & -1.946277705 \\ \mathrm{H} & -3.574460070 & -2.036582094 & -3.033493117 \\ \mathrm{C} & -5.042782275 & -3.364214213 & 0.294058833 \\ \mathrm{H} & -3.022358334 & -3.537332412 & 0.972146444 \\ \mathrm{C} & -5.897992549 & -3.000865625 & -0.750921498 \\ \mathrm{H} & -6.020576035 & -2.237922384 & -2.764324051 \\ \mathrm{H} & -5.452934585 & -3.742649248 & 1.225262548 \\ \mathrm{H} & -6.972757945 & -3.098438660 & -0.633335053 \\ \mathrm{H} & -1.389515896 & -2.395122249 & -2.311903258 \\ \mathrm{H} & 0.929818884 & -2.992221509 & -1.921866340\end{array}$


Structure 33M(half-chair)

110

symmetry $\mathrm{c} 1$

$\begin{array}{llll}\text { Pd } & -0.425275376 & -1.184544285 & 0.099708544\end{array}$

$\begin{array}{lllll}P & 1.377224527 & 0.468076695 & -0.310513011\end{array}$

$\begin{array}{llll}\text { P } & -2.313575532 & 0.377792611 & 0.225624633\end{array}$

$\begin{array}{llll}\text { C } & 2.715247364 & -0.093519494 & -1.559777295\end{array}$

$\begin{array}{llll}\text { C } & 2.098479348 & -0.635457975 & -2.704212754\end{array}$

H $\quad \begin{array}{llll}\text { H } & 1.015475604 & -0.727131747 & -2.728461958\end{array}$

$\begin{array}{llll}\text { C } & 2.833801732 & -1.072371985 & -3.801412995\end{array}$

H $\quad 2.325446439-1.473375525 \quad-4.673090831$

$\begin{array}{llll}\text { C } & 4.225377281 & -0.999111798 & -3.757521288\end{array}$

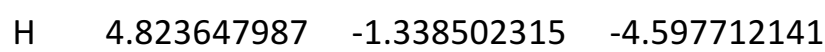

$\begin{array}{llll}\text { C } & 4.847846685 & -0.484434555 & -2.625235558\end{array}$

H $\quad 5.931076075 \quad-0.421209296 \quad-2.590407477$

$\begin{array}{llll}\text { C } & 4.125088794 & -0.007106252 & -1.512743666\end{array}$

$\begin{array}{lllll}\text { C } & 4.959938476 & 0.522801083 & -0.388374348\end{array}$

$\begin{array}{llll}\text { C } & 5.044971217 & 1.892904956 & -0.107461139\end{array}$

$\begin{array}{llll}H & 4.461933662 & 2.596185918 & -0.690583544\end{array}$

$\begin{array}{llll}\text { C } & 5.903568754 & 2.363935421 & 0.886955961\end{array}$

$\begin{array}{llll}\text { H } & 5.960188815 & 3.429776300 & 1.087385395\end{array}$

$\begin{array}{llll}\text { C } & 6.699778474 & 1.473172617 & 1.607967334\end{array}$

$\begin{array}{llll}\mathrm{H} & 7.371196133 & 1.841281869 & 2.377857550\end{array}$

$\begin{array}{llll}\text { C } & 6.641729053 & 0.108229146 & 1.319981735\end{array}$

$\begin{array}{llll}\text { H } & 7.270598427 & -0.590445216 & 1.864425367\end{array}$

$\begin{array}{lllll}\text { C } & 5.784641247 & -0.362280211 & 0.324981068\end{array}$

$\begin{array}{llll}\text { H } & 5.742699347 & -1.424144101 & 0.100290245\end{array}$

$\begin{array}{lllll}\text { C } & -1.523295508 & 4.160975473 & -0.934427563\end{array}$

$\begin{array}{llll}\text { C } & -1.103491280 & 4.689362841 & 0.293281760\end{array}$

$\begin{array}{llll}H & -0.911978308 & 4.015709323 & 1.119949121\end{array}$

$\begin{array}{lllll}\text { C } & -0.971880437 & 6.068578550 & 0.462804064\end{array}$

$\begin{array}{llll}\mathrm{H} & -0.651250624 & 6.464359475 & 1.422243217\end{array}$

$\begin{array}{llll}\text { C } & -1.259930987 & 6.939331035 & -0.589649016\end{array}$

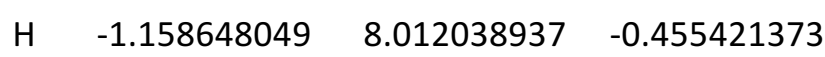

$\begin{array}{llll}\text { C } & -1.697963673 & 6.422944053 & -1.810321460\end{array}$

$\begin{array}{llll}\text { H } & -1.945524919 & 7.092250771 & -2.628887077\end{array}$

$\begin{array}{llll}\text { C } & -1.836745936 & 5.044415067 & -1.977357460\end{array}$

$\begin{array}{llll}\text { H } & -2.202951685 & 4.655277470 & -2.923787514\end{array}$

$\begin{array}{llll}\text { C } & -3.788139536 & 0.235154583 & -0.915524732\end{array}$

$\begin{array}{llll}\text { C } & -3.788751117 & -0.732115840 & -1.925423237\end{array}$

H $\quad-2.960119951-1.427574800 \quad-2.010238100$

$\begin{array}{llll}\text { C } & -4.861119118 & -0.806528517 & -2.816897133\end{array}$

H $\quad-4.864757656 \quad-1.562509336 \quad-3.595958489$

$\begin{array}{llll}\text { C } & -5.923019669 & 0.091900322 & -2.706550405\end{array}$

$\begin{array}{llll}\text { H } & -6.754967533 & 0.036112887 & -3.402127213\end{array}$

$\begin{array}{llll}\text { C } & -5.916236622 & 1.069427855 & -1.706658436\end{array}$ 


\begin{tabular}{|c|c|c|c|}
\hline & -6.738868 & & \\
\hline & -4.850818704 & 1.146277541 & -0.810630223 \\
\hline & -4.838892430 & 1.912775806 & -0.041932519 \\
\hline & -1.943323499 & 2856427 & 725128 \\
\hline & 0.764697940 & 2.017734493 & -0.937339922 \\
\hline & -2.971912253 & 0.418158843 & 1.974756125 \\
\hline & -4.270363518 & 0.004506572 & 2.292365912 \\
\hline & -4.950506807 & -0.322513250 & 1.513941697 \\
\hline 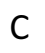 & -4.695290023 & 0.018660467 & 3.623908308 \\
\hline & -5.705780608 & -0.295193081 & 3.868001437 \\
\hline & -3.830254838 & 0.441252620 & 4.633565037 \\
\hline $\mathrm{H}$ & -4.165423568 & 0.453377580 & 5.666229107 \\
\hline & -2.531808944 & 0.848133385 & 4.315045779 \\
\hline & -1.856589660 & 1.179527584 & 3683 \\
\hline$C$ & -2.097197442 & 0.832138548 & 2.990244572 \\
\hline & -1.086900727 & 1.149743203 & 2.750666044 \\
\hline & -1.648657789 & 2.665673945 & -1.155775654 \\
\hline & -2.498206268 & 2.490928853 & -1.831746920 \\
\hline C & -0.421599906 & 1.966347985 & -1.814545702 \\
\hline H & -0.714916347 & 0.913327525 & -1.889864637 \\
\hline & -0.175205619 & 2.433723385 & -3.255916327 \\
\hline H & 0.082570882 & 3.492858668 & -3.318439058 \\
\hline $\mathrm{H}$ & -1.076858530 & 2.276411340 & -3.856439444 \\
\hline & 0.634203243 & 1.855203670 & 60054 \\
\hline & 1.752507808 & 3.067179567 & -1.212106080 \\
\hline $\mathrm{H}$ & 2.405066193 & 3.186337324 & -0.345904423 \\
\hline & 1.235028996 & 4.016837926 & -1.35 \\
\hline $\mathrm{H}$ & 2.374578250 & 2.861958555 & -2.094732678 \\
\hline C & -2.900035388 & -3.480421093 & 0.388972086 \\
\hline & -3.125328268 & -4.052882268 & -0.878237554 \\
\hline & -4.383879880 & -4.531727214 & -1.228686505 \\
\hline C & -5.448745248 & -4.444753007 & -0.326205343 \\
\hline & -5.242701683 & -3.876265408 & 0.931435589 \\
\hline 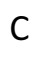 & -3.981304885 & -3.400298593 & 1.285671142 \\
\hline $\mathrm{H}$ & -2.315075602 & -4.126545873 & -1.597853863 \\
\hline$H$ & -4.536290574 & -4.978248078 & -2.206729465 \\
\hline $\mathrm{H}$ & -6.428614875 & -4.822148155 & -0.601659922 \\
\hline $\mathrm{H}$ & -6.061473869 & -3.813167556 & 1.641734499 \\
\hline $\mathrm{H}$ & -3.824035841 & -2.971376715 & 2.271035460 \\
\hline C & -0.357330862 & -3.431045236 & 0.251426975 \\
\hline $\mathrm{H}$ & -0.374140877 & -4.009902391 & -0.667704714 \\
\hline C & -1.574912161 & -3.021397178 & 0.838604559 \\
\hline C & 0.882109126 & -2.999880297 & 0.754885454 \\
\hline C & 2.180433478 & -3.444773517 & 0.226026181 \\
\hline C & 3.251741055 & -3.605361492 & 1.124461744 \\
\hline C & 2.378426474 & -3.794322295 & -1.122696578 \\
\hline
\end{tabular}




$\begin{array}{llll}\text { C } & 4.474909628 & -4.118349727 & 0.694742152 \\ \text { H } & 3.109621350 & -3.357045185 & 2.172995125 \\ \text { C } & 3.602232155 & -4.298169020 & -1.552089984 \\ \text { H } & 1.577268370 & -3.654889350 & -1.841623934 \\ \text { C } & 4.653672809 & -4.466798722 & -0.645485900 \\ \text { H } & 5.282441185 & -4.255943374 & 1.407402092 \\ \text { H } & 3.739613443 & -4.558383539 & -2.596876738 \\ \text { H } & 5.604230700 & -4.868372571 & -0.982849271 \\ \text { H } & 0.902474941 & -2.652483074 & 1.788382348 \\ \text { H } & -1.536557351 & -2.700407266 & 1.879720817 \\ \text { C } & 2.063309058 & 0.921135302 & 1.373926043 \\ \text { C } & 2.882468619 & 0.019772720 & 2.063966879 \\ \text { C } & 1.608513350 & 2.080382327 & 2.014718310 \\ \text { C } & 3.268885837 & 0.293761037 & 3.375880619 \\ \text { H } & 3.238087875 & -0.881367632 & 1.578028740 \\ \text { C } & 2.001126895 & 2.348897632 & 3.328376234 \\ \text { H } & 0.952348706 & 2.762468750 & 1.486359651 \\ \text { C } & 2.831731369 & 1.458864304 & 4.010252463 \\ \text { H } & 3.919640014 & -0.399916587 & 3.899623662 \\ \text { H } & 1.656838906 & 3.256312030 & 3.815911160 \\ \text { H } & 3.137001616 & 1.670436486 & 5.030541723\end{array}$




\begin{tabular}{|c|c|c|c|}
\hline \multicolumn{4}{|c|}{ Structure $33 \mathrm{~W}$ (boat) } \\
\hline \multicolumn{4}{|c|}{110} \\
\hline \multicolumn{4}{|c|}{ symmetry c1 } \\
\hline $\mathrm{Pd}$ & -0.053356640 & -1.185943334 & -0.405095864 \\
\hline & 1.558307272 & 0.459856648 & 0.553013012 \\
\hline b & -1.998068568 & 0.261248447 & -0.064672223 \\
\hline C & 1.200615922 & 0.315402389 & 2.405503410 \\
\hline C & 1.011179637 & 1.437863170 & 3.217285515 \\
\hline $\mathrm{H}$ & 1.079749526 & 2.432375280 & 2.795511869 \\
\hline C & 0.711989891 & 1.279724523 & 4.573511066 \\
\hline $\mathrm{H}$ & 0.569790677 & 2.157201867 & 5.197485815 \\
\hline C & 0.599892210 & 0.002773847 & 5.126363086 \\
\hline $\mathrm{H}$ & 0.370695799 & -0.116562532 & 6.180970400 \\
\hline C & 0.783463121 & -1.120871593 & 4.317576363 \\
\hline $\mathrm{H}$ & 0.695739598 & -2.117299309 & 4.740347693 \\
\hline C & 1.077947127 & -0.965602278 & 2.961925503 \\
\hline $\mathrm{H}$ & 1.224378870 & -1.843333706 & 2.339286154 \\
\hline C & 3.454503716 & 0.255589696 & 0.463379305 \\
\hline C & 4.118618200 & -0.161201008 & 1.625584878 \\
\hline $\mathrm{H}$ & 3.551754128 & -0.371296834 & 2.523541957 \\
\hline C & 5.505629738 & -0.294674869 & 1.666723366 \\
\hline $\mathrm{H}$ & 5.988967379 & -0.616112176 & 2.584037178 \\
\hline C & 6.255605983 & -0.000847061 & 0.532025202 \\
\hline $\mathrm{H}$ & 7.337394196 & -0.093548879 & 0.543753791 \\
\hline C & 5.607230871 & 0.409187965 & -0.629714211 \\
\hline $\mathrm{H}$ & 6.191005400 & 0.615069908 & -1.521262335 \\
\hline C & 4.208047954 & 0.543005413 & -0.703219122 \\
\hline C & 3.660107456 & 0.977229620 & -2.022740304 \\
\hline C & 2.642744398 & 0.270074283 & -2.682370953 \\
\hline $\mathrm{H}$ & 2.184788845 & -0.579519786 & -2.191529293 \\
\hline C & 2.237402563 & 0.624879050 & -3.970042961 \\
\hline $\mathrm{H}$ & 1.462716815 & 0.048628199 & -4.468639819 \\
\hline C & 2.840302521 & 1.698304119 & -4.627994139 \\
\hline $\mathrm{H}$ & 2.531119056 & 1.969474269 & -5.632738801 \\
\hline C & 3.849112350 & 2.417208589 & -3.983578501 \\
\hline $\mathrm{H}$ & 4.324142775 & 3.256598749 & -4.482476074 \\
\hline C & 4.254514453 & 2.059673547 & -2.697329730 \\
\hline $\mathrm{H}$ & 5.040298883 & 2.625662048 & -2.205962888 \\
\hline C & -1.198117036 & 4.176075387 & 0.452755331 \\
\hline C & -1.073842542 & 4.225603118 & 1.847089632 \\
\hline $\mathrm{H}$ & -0.970695763 & 3.301364858 & 2.401430206 \\
\hline C & -1.118896429 & 5.447719597 & 2.520441238 \\
\hline $\mathrm{H}$ & -1.027907031 & 5.469229593 & 3.602801190 \\
\hline C & -1.293115370 & 6.637902890 & 1.812255264 \\
\hline $\mathrm{H}$ & -1.330265443 & 7.587288838 & 2.337865805 \\
\hline C & -1.439536666 & 6.596805026 & 0.424840603 \\
\hline
\end{tabular}




\begin{tabular}{|c|c|c|c|}
\hline & -1.597649473 & 7.513901320 & -0.134872300 \\
\hline & -1.400895757 & 5.374366369 & -0.246948989 \\
\hline & -1.540817842 & 5.353491967 & -1.324400064 \\
\hline & -3.108598641 & 0.617474929 & -1.530764485 \\
\hline & -2.657314209 & 0.306502277 & -2.819118413 \\
\hline & -1.693340664 & -0.177351020 & -2.951668990 \\
\hline & -3.440759798 & 0.623532421 & -3.930512495 \\
\hline & -3.089712279 & 0.381184850 & -4.929062979 \\
\hline & -4.672317078 & 1.257309379 & -3.754749537 \\
\hline & -5.282517799 & 1.504786311 & -4.618124724 \\
\hline & -5.118954477 & 1.580485641 & -2.470378472 \\
\hline & -6.073454178 & 2.079946248 & -2.335581014 \\
\hline & -4.339520205 & 1.266438629 & -1.357002251 \\
\hline+ & -4.686377789 & 1.522199598 & -0.361020020 \\
\hline 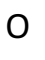 & -1.641119511 & 1.839510877 & 0.563338715 \\
\hline & 1.255922766 & 2.152020872 & 0.150800393 \\
\hline & -3.126964918 & -0.269036234 & 1.323969785 \\
\hline 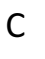 & -4.266593876 & -1.040329721 & 1.067849843 \\
\hline H & -4.534976934 & -1.314524473 & 0.053327974 \\
\hline$c$ & -5.074199999 & -1.451714120 & 2.130419910 \\
\hline & -5.962972710 & -2.041542157 & 1.928457396 \\
\hline 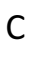 & -4.743277805 & -1.104794125 & 3.440531733 \\
\hline H & -5.376406453 & -1.423031448 & 4.263362984 \\
\hline & -3.596270264 & -0.348198387 & 3.693 \\
\hline+1 & -3.335239685 & -0.075942541 & 4.711227106 \\
\hline c & -2.781960544 & 0.067465852 & 2.640264165 \\
\hline & -1.897330619 & 0.661129824 & 81474 \\
\hline & -1.133158121 & 2.866095028 & -0.310536512 \\
\hline-1 & -1.801265692 & 2.951786356 & -1.179325184 \\
\hline & 0.262723594 & 2.472419360 & -0.888348046 \\
\hline & 0.085556750 & 1.547120042 & -1.450290342 \\
\hline C & 0.752688860 & 3.503316571 & -1.912371556 \\
\hline & 0.926389302 & 4.484527805 & -1.465732041 \\
\hline & -0.002043874 & 3.626562291 & -2.695438048 \\
\hline $\mathrm{H}$ & 1.673130554 & 3.165269529 & -2.388514228 \\
\hline 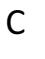 & 2.249210534 & 3.169936343 & 0.514319985 \\
\hline $\mathrm{H}$ & 2.831406337 & 2.832478080 & 1.373168896 \\
\hline $\mathrm{H}$ & 1.741938535 & 4.095672815 & 0.800507221 \\
\hline I & 2.952259562 & 3.387825928 & -0.298929483 \\
\hline & 2.556330759 & -3.383989340 & -0.282856364 \\
\hline C & 2.552308519 & -4.011808323 & 0.978724493 \\
\hline C & 3.740567634 & -4.436367416 & 1.564781041 \\
\hline C & 4.960003046 & -4.241318524 & 0.908345379 \\
\hline C & 4.981278792 & -3.615113848 & -0.338417265 \\
\hline C & 3.792134846 & -3.189881886 & -0.926697860 \\
\hline $\mathrm{H}$ & 1.616988023 & -4.181169364 & 1.504220722 \\
\hline
\end{tabular}




$\begin{array}{llll}\text { H } & 3.717380749 & -4.926313813 & 2.533496469 \\ \text { H } & 5.884601348 & -4.578845882 & 1.366302223 \\ \text { H } & 5.922710418 & -3.460705255 & -0.855945872 \\ \text { H } & 3.815420513 & -2.711393091 & -1.901831843 \\ \text { C } & 0.032940676 & -3.416728340 & -0.658165458 \\ \text { H } & -0.115945219 & -4.015830785 & 0.235001238 \\ \text { C } & 1.328769338 & -2.972963342 & -0.978699410 \\ \text { C } & -1.096166774 & -2.974778705 & -1.380917332 \\ \text { C } & -2.473594377 & -3.433352374 & -1.135903540 \\ \text { C } & -3.431548626 & -3.263114762 & -2.153233011 \\ \text { C } & -2.865571227 & -4.090970504 & 0.045903254 \\ \text { C } & -4.730531121 & -3.744437057 & -2.003458609 \\ \text { H } & -3.148613654 & -2.758211466 & -3.072461735 \\ \text { C } & -4.160326512 & -4.580646566 & 0.190386605 \\ \text { H } & -2.160406633 & -4.222157359 & 0.860511275 \\ \text { C } & -5.098090553 & -4.411439151 & -0.832652829 \\ \text { H } & -5.450843806 & -3.610243406 & -2.804584703 \\ \text { H } & -4.440202554 & -5.094143970 & 1.105022089 \\ \text { H } & -6.105826331 & -4.798959100 & -0.718482493 \\ \text { H } & -0.917156874 & -2.610912873 & -2.393048837 \\ \text { H } & 1.483516254 & -2.612354463 & -1.994998377\end{array}$




\begin{tabular}{|c|c|c|c|}
\hline \multicolumn{4}{|c|}{ Structure $33 W$ (half-chair) } \\
\hline \multicolumn{4}{|c|}{110} \\
\hline \multicolumn{4}{|c|}{ symmetry c1 } \\
\hline $\mathrm{Pd}$ & -0.392586826 & -1.174716813 & -0.493467131 \\
\hline & 1.371805820 & 0.562689478 & -0.355378409 \\
\hline & -2.290695289 & 0.272678084 & 0.067169352 \\
\hline & 1.891758404 & 0.665944432 & 1.441656736 \\
\hline C & 1.450984347 & 1.726792223 & 2.241165278 \\
\hline & 0.893235930 & 2.540731259 & 1.793533941 \\
\hline & 1.725593509 & 1.726087856 & 3.610894601 \\
\hline $\mathrm{H}$ & 1.393833739 & 2.558250386 & 4.225075608 \\
\hline ᄃ & 2.422929259 & 0.663896458 & 4.187521502 \\
\hline H & 2.635528966 & 0.665434945 & 5.252335391 \\
\hline C & 2.844661767 & -0.403029341 & 3.391013427 \\
\hline $\mathrm{H}$ & 3.388682036 & -1.231749896 & 3.833845814 \\
\hline C & 2.577274134 & -0.408184921 & 2.022180561 \\
\hline $\mathrm{H}$ & 2.915829085 & -1.239878138 & 1.414217022 \\
\hline C & 2.845422491 & 0.322265000 & -1.553513619 \\
\hline C & 2.373540639 & 0.068440708 & -2.855815210 \\
\hline $\mathrm{H}$ & 1.302201856 & -0.000814281 & -3.027326514 \\
\hline C & 3.237850988 & -0.108488919 & -3.931647638 \\
\hline $\mathrm{H}$ & 2.840891427 & -0.289800338 & -4.925967392 \\
\hline C & 4.614504009 & -0.057435424 & -3.711144204 \\
\hline $\mathrm{H}$ & 5.310874889 & -0.197838998 & -4.532327545 \\
\hline C & 5.093040149 & 0.180339122 & -2.427233794 \\
\hline $\mathrm{H}$ & 6.163573910 & 0.229625807 & -2.253493308 \\
\hline C & 4.237446872 & 0.390657657 & -1.326051801 \\
\hline C & 4.923703911 & 0.655227010 & -0.021724832 \\
\hline C & 4.964606513 & 1.937569368 & 0.541891992 \\
\hline & 4.451294019 & 2.755827391 & 0.049179717 \\
\hline C & 5.693472104 & 2.179720862 & 1.707081518 \\
\hline $\mathrm{H}$ & 5.717999258 & 3.180706571 & 2.127545027 \\
\hline C & 6.403004443 & 1.145670210 & 2.319348634 \\
\hline $\mathrm{H}$ & 6.974589745 & 1.336350585 & 3.222675201 \\
\hline C & 6.387593266 & -0.130527118 & 1.753752814 \\
\hline $\mathrm{H}$ & 6.949122560 & -0.937665641 & 2.215477326 \\
\hline C & 5.659262276 & -0.372686616 & 0.588836417 \\
\hline $\mathrm{H}$ & 5.650119999 & -1.366057468 & 0.150105108 \\
\hline C & -1.620735190 & 4.229266713 & -0.356812665 \\
\hline C & -1.218928238 & 4.541936520 & 0.948243582 \\
\hline $\mathrm{H}$ & -0.992591048 & 3.738052211 & 1.638203257 \\
\hline C & -1.149477683 & 5.870993291 & 1.369160982 \\
\hline $\mathrm{H}$ & -0.842416562 & 6.097743625 & 2.386144678 \\
\hline C & -1.482463927 & 6.906321964 & 0.494001145 \\
\hline $\mathrm{H}$ & -1.429733806 & 7.939508739 & 0.823675617 \\
\hline C & -1.902410636 & 6.603609505 & -0.802327898 \\
\hline
\end{tabular}




\begin{tabular}{|c|c|c|c|}
\hline & -2.184357275 & 7.399885002 & -1.484771849 \\
\hline & -1.978794287 & 5.274439824 & -1.220422640 \\
\hline & -2.331342172 & 5.049795424 & -2.223698576 \\
\hline & -3.746125567 & 0.313200966 & -1.113048285 \\
\hline & -3.605228621 & -0.232363154 & -2.394620801 \\
\hline & -2.677367132 & -0.723734387 & -2.674223877 \\
\hline & -4.650968560 & -0.138548967 & -3.315201457 \\
\hline & -4.539008635 & -0.562563306 & -4.308553938 \\
\hline & -5.836099290 & 0.506744074 & -2.957342183 \\
\hline & -6.650155830 & 0.580795505 & -3.672040969 \\
\hline & -5.973724957 & 1.066041078 & -1.683883848 \\
\hline & -6.892105088 & 1.576031704 & -1.409660954 \\
\hline & -4.930794732 & 0.976791800 & -0.761875118 \\
\hline & -5.037103642 & 1.420199948 & 0.222876839 \\
\hline 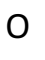 & -1.954608516 & 1.963097688 & 0.290012779 \\
\hline & 0.753120827 & 2.189336224 & -0.720133865 \\
\hline & -2.986034565 & -0.029625584 & 322688 \\
\hline 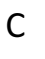 & -4.155844216 & -0.773572597 & 1.961435940 \\
\hline 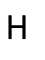 & -4.706388388 & -1.167628806 & 1.114254555 \\
\hline$c$ & -4.622073064 & -1.008120919 & 3.257168202 \\
\hline & -5.534593038 & -1.578302589 & 3.401490639 \\
\hline & -3.921870448 & -0.512814459 & 4.357116820 \\
\hline$H$ & -4.290007344 & -0.694283054 & 5.362420299 \\
\hline & -2.743521982 & 0.213220922 & 2901 \\
\hline 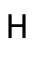 & -2.193657882 & 0.596743743 & 5.018925303 \\
\hline c & -2.268503575 & 0.451828627 & 2.876129583 \\
\hline & -1.353160064 & 1.015436773 & 2.732253391 \\
\hline & -1.679812655 & 2.796781275 & -0.851837340 \\
\hline - & -2.517254025 & 2.716442257 & -1.560120133 \\
\hline & -0.418270896 & 2.285028203 & -1.612077041 \\
\hline & -0.674324234 & 1.260889872 & -1.907475725 \\
\hline C & -0.165460153 & 3.046290026 & -2.920653520 \\
\hline & 0.056126545 & 4.102964418 & -2.758352921 \\
\hline & -1.051594698 & 2.989027798 & -3.560842773 \\
\hline $\mathrm{H}$ & 0.670862103 & 2.599485349 & -3.465374022 \\
\hline & 1.726812649 & 3.285999662 & -0.762408687 \\
\hline $\mathrm{H}$ & 2.358770085 & 3.243561996 & 0.126001960 \\
\hline $\mathrm{H}$ & 1.196035078 & 4.239310989 & -0.733672479 \\
\hline & 2.373025109 & 3.264036912 & -1.650914370 \\
\hline & 2.236889097 & -3.362051259 & -0.794855217 \\
\hline C & 2.583851188 & -3.795982927 & 0.500602722 \\
\hline C & 3.877984821 & -4.222640070 & 0.784123137 \\
\hline C & 4.857049729 & -4.221150970 & -0.215499320 \\
\hline C & 4.532701440 & -3.777119499 & -1.498640865 \\
\hline - & 3.237010581 & -3.349332479 & -1.783576572 \\
\hline & 1.841405173 & -3.802620757 & 1.292797477 \\
\hline
\end{tabular}




\begin{tabular}{|c|c|c|c|}
\hline & 4.124075722 & -4.564609881 & 1.784900742 \\
\hline & 5.862239641 & -4.566674239 & 0.005984899 \\
\hline & 5.285132988 & -3.771163334 & -2.281085510 \\
\hline & 2.990688652 & -3.011994249 & -2.785721270 \\
\hline & -0.281192423 & -3.421131105 & -0.469473066 \\
\hline & -0.153056909 & -3.909006361 & 0.492083814 \\
\hline & 0.861277407 & -3.003928678 & -1.177108778 \\
\hline & -1.585116286 & -3.089911233 & -0.894258517 \\
\hline & -2.811181945 & -3.524418508 & -0.203583870 \\
\hline & -4.013910406 & -3.581244504 & -0.932322099 \\
\hline & -2.824238722 & -3.944167088 & 1.140510049 \\
\hline & -5.184854619 & -4.058852991 & -0.346861312 \\
\hline & -4.024528676 & -3.259983187 & -1.969848551 \\
\hline & -3.992159390 & -4.428984245 & 1.721570762 \\
\hline & -1.921586771 & -3.886787706 & 1.740803175 \\
\hline & -5.176456264 & -4.491225755 & 0.980995854 \\
\hline & -6.099321155 & -4.104436694 & -0.930277601 \\
\hline & -3.980305523 & -4.755820486 & 2.756737004 \\
\hline & -6.084694114 & -4.874361402 & 1.436062396 \\
\hline & -1.715410281 & -2.872494109 & -1.954346557 \\
\hline & 0.733447996 & -2.751641228 & -2.229704526 \\
\hline
\end{tabular}




\begin{tabular}{|c|c|c|c|}
\hline \multicolumn{4}{|c|}{ Structure 34M(boat) } \\
\hline \multicolumn{4}{|c|}{110} \\
\hline \multicolumn{4}{|c|}{ symmetry c1 } \\
\hline$P d$ & -0.998760900 & -1.198229116 & 0.288832160 \\
\hline$P$ & 1.441640257 & -0.613459775 & 0.237048872 \\
\hline D & -1.702179522 & 1.137136428 & -0.010659486 \\
\hline C & 1.801151109 & 0.398009425 & 1.834449942 \\
\hline C & 2.982728329 & 1.088787328 & 2.203652474 \\
\hline C & 2.991101479 & 1.763564967 & 3.438712961 \\
\hline$H$ & 3.894625456 & 2.295382038 & 3.720674316 \\
\hline C & 1.902968341 & 1.749200651 & 4.308062729 \\
\hline $\mathrm{H}$ & 1.959259682 & 2.277354704 & 5.255123584 \\
\hline C & 0.755398856 & 1.046962338 & 3.953777513 \\
\hline $\mathrm{H}$ & -0.106356485 & 1.017407980 & 4.613034891 \\
\hline C & 0.715165118 & 0.390087058 & 2.725248719 \\
\hline $\mathrm{H}$ & -0.191604347 & -0.140962818 & 2.446430466 \\
\hline C & 2.653321804 & -2.046263529 & 0.357887818 \\
\hline C & 2.932228785 & -2.608217345 & 1.611587404 \\
\hline $\mathrm{H}$ & 2.498641474 & -2.182628124 & 2.511973735 \\
\hline C & 3.800733050 & -3.696371147 & 1.712239901 \\
\hline $\mathrm{H}$ & 4.023986592 & -4.118501951 & 2.687771265 \\
\hline C & 4.394147792 & -4.228106397 & 0.564374458 \\
\hline $\mathrm{H}$ & 5.082061562 & -5.064397435 & 0.645229889 \\
\hline C & 4.093009741 & -3.687181196 & -0.686250011 \\
\hline $\mathrm{H}$ & 4.540648248 & -4.105021684 & -1.582870987 \\
\hline C & 3.213692285 & -2.607586687 & -0.793273977 \\
\hline C & 1.004554296 & 3.189043653 & -2.154852838 \\
\hline C & 1.744052614 & 3.781366327 & -1.122667674 \\
\hline $\mathrm{H}$ & 1.677414556 & 3.371560072 & -0.121113032 \\
\hline C & 2.548665256 & 4.892774633 & -1.377462185 \\
\hline $\mathrm{H}$ & 3.108930065 & 5.349751742 & -0.566424877 \\
\hline C & 2.629050005 & 5.426561820 & -2.665278226 \\
\hline $\mathrm{H}$ & 3.253633816 & 6.293052629 & -2.860345792 \\
\hline C & 1.887841418 & 4.848960260 & -3.696773735 \\
\hline $\mathrm{H}$ & 1.928909129 & 5.266191321 & -4.698501144 \\
\hline C & 1.074075798 & 3.744493341 & -3.439583315 \\
\hline $\mathrm{H}$ & 0.477579905 & 3.322045904 & -4.243968183 \\
\hline C & -3.019845070 & 1.451042885 & -1.313588634 \\
\hline C & -3.220987115 & 0.492556942 & -2.315530504 \\
\hline C & -4.150688141 & 0.725082810 & -3.330319252 \\
\hline $\mathrm{H}$ & -4.301940413 & -0.017019701 & -4.108593630 \\
\hline C & -4.885126413 & 1.912704176 & -3.341405002 \\
\hline $\mathrm{H}$ & -5.611055182 & 2.093776957 & -4.128361512 \\
\hline C & -4.687243687 & 2.869189154 & -2.342403849 \\
\hline $\mathrm{H}$ & -5.257905300 & 3.792766810 & -2.352495905 \\
\hline C & -3.753819508 & 2.644294713 & -1.330176986 \\
\hline
\end{tabular}




\begin{tabular}{|c|c|c|c|}
\hline & & & \\
\hline & -0.363817649 & 2.102589102 & -0.546787098 \\
\hline & 1.833706924 & 0.172285691 & -1.289644454 \\
\hline & -2.211917339 & 2.199926870 & 1.439961331 \\
\hline & -3.298277892 & 1.784195381 & 2.220830928 \\
\hline & -3.839985608 & 0.875499437 & 1.976009513 \\
\hline & -3.701129250 & 2.555154821 & 3.311393418 \\
\hline & -4.548520189 & 2.237668220 & 3.911616337 \\
\hline & -3.020609318 & 3.734232716 & 3.625680078 \\
\hline & -3.335582681 & 4.332845010 & 4.475119431 \\
\hline & -1.938969889 & 4.144332314 & 2.844699138 \\
\hline & -1.411645125 & 5.062790536 & 3.084400032 \\
\hline & -1.528842286 & 3.380923795 & 1.749045840 \\
\hline & -0.693584926 & 3.696668042 & 6239 \\
\hline & 0.110241514 & 1.989246659 & -1.902472793 \\
\hline & -0.747820906 & 2.067187853 & -2.584566911 \\
\hline & 0.722889376 & 0.574973227 & 584672 \\
\hline & -0.093291438 & -0.125888065 & -1.942987948 \\
\hline & 1.052303451 & 0.377760345 & -3.651113226 \\
\hline & 1.804496393 & 1.080881050 & -4.014964813 \\
\hline & 0.147932739 & 0.522552567 & -4.250452673 \\
\hline & 1.412370813 & -0.639924850 & -3.825979864 \\
\hline & 3.199983747 & 0.570377455 & -1.656277412 \\
\hline & 3.917218758 & 0.076468355 & $-1.00<$ \\
\hline & 3.355762755 & 1.648596426 & -1.580252454 \\
\hline - & 3.417997588 & 0.255644781 & -2.681524385 \\
\hline & -4.343662679 & -1.866054254 & 12206 \\
\hline & -5.020962286 & -1.863776765 & -0.314858368 \\
\hline C & -6.368267872 & -1.524293404 & -0.382798883 \\
\hline & -7.069724384 & -1.180329854 & 0.777168441 \\
\hline & -6.412870952 & -1.176810550 & 2.009024839 \\
\hline C & -5.062552459 & -1.513782637 & 2.078406215 \\
\hline & -4.491938165 & -2.119413721 & -1.226985581 \\
\hline & -6.875035047 & -1.525776471 & -1.342775707 \\
\hline $\mathrm{H}$ & -8.121662555 & -0.918681386 & 0.719372411 \\
\hline & -6.953022808 & -0.919963635 & 2.915021317 \\
\hline & -4.558400102 & -1.526506349 & 3.041533331 \\
\hline C & -2.217686323 & -3.070775104 & 0.172263105 \\
\hline & -2.653502481 & -3.323619629 & -0.790592943 \\
\hline & -2.941706752 & -2.274578244 & 1.069392035 \\
\hline C & -0.870285459 & -3.437842607 & 0.432227810 \\
\hline & -0.104377491 & -4.272682997 & -0.511010993 \\
\hline c & 0.759610123 & -5.259958190 & -0.009090674 \\
\hline C & -0.263709828 & -4.166484949 & -1.906372374 \\
\hline C & 1.421666007 & -6.132907189 & -0.870656122 \\
\hline 7 & 0.899580220 & -5.351295405 & 1.064012477 \\
\hline
\end{tabular}




$\begin{array}{llll}\text { C } & 0.403591733 & -5.033991747 & -2.766228512 \\ \text { H } & -0.905746680 & -3.392643187 & -2.318882587 \\ \text { C } & 1.243511278 & -6.026071806 & -2.250695331 \\ \text { H } & 2.074769984 & -6.898209359 & -0.462952387 \\ \text { H } & 0.268680464 & -4.939663478 & -3.839633970 \\ \text { H } & 1.757066390 & -6.707453925 & -2.922107540 \\ \text { H } & -0.569132264 & -3.518624817 & 1.476594335 \\ \text { H } & -2.564012438 & -2.220184463 & 2.091285564 \\ \text { H } & 2.974660752 & -2.196063485 & -1.767703191 \\ \text { H } & -2.649829272 & -0.432362634 & -2.301311940 \\ \text { C } & 4.256824581 & 1.185037261 & 1.421777303 \\ \text { C } & 4.645612609 & 2.423280591 & 0.886875235 \\ \text { C } & 5.146099259 & 0.103925662 & 1.323727421 \\ \text { C } & 5.867732183 & 2.567185025 & 0.229681290 \\ \text { H } & 3.981384283 & 3.276259033 & 0.983800568 \\ \text { C } & 6.371053196 & 0.249123859 & 0.669367230 \\ \text { H } & 4.886790850 & -0.848924442 & 1.769466849 \\ \text { C } & 6.732436489 & 1.477381963 & 0.113157577 \\ \text { H } & 6.145103507 & 3.530968045 & -0.186886256 \\ \text { H } & 7.047434962 & -0.598154032 & 0.605358611 \\ \text { H } & 7.686042203 & 1.588377282 & -0.394175511\end{array}$




\begin{tabular}{|c|c|c|c|}
\hline \multicolumn{4}{|c|}{ Structure $34 \mathrm{~W}$ (boat) } \\
\hline \multicolumn{4}{|c|}{110} \\
\hline \multicolumn{4}{|c|}{ symmetry c1 } \\
\hline $\mathrm{Pd}$ & -0.888630865 & -1.233280652 & -0.420439178 \\
\hline & 1.477989733 & -0.389127886 & -0.228569663 \\
\hline & -1.893572297 & 0.966451671 & -0.037550957 \\
\hline C & 1.768946662 & -0.028584207 & 1.638518721 \\
\hline C & 2.858176356 & 0.622800441 & 2.270250674 \\
\hline C & 2.823120342 & 0.755768047 & 3.670951726 \\
\hline $\mathrm{H}$ & 3.655699981 & 1.257597294 & 4.154339887 \\
\hline C & 1.781177425 & 0.251823655 & 4.445835111 \\
\hline $\mathrm{H}$ & 1.802495653 & 0.369922463 & 5.525071075 \\
\hline C & 0.724172784 & -0.405301553 & 3.823795838 \\
\hline $\mathrm{H}$ & -0.100655504 & -0.807417265 & 4.403628293 \\
\hline C & 0.725700598 & -0.529917547 & 2.435690020 \\
\hline $\mathrm{H}$ & -0.113509635 & -1.026531308 & 1.953428355 \\
\hline C & 2.863137673 & -1.577928169 & -0.686899211 \\
\hline C & 3.372278980 & -2.478696524 & 0.256393836 \\
\hline $\mathrm{H}$ & 3.004110476 & -2.469396054 & 1.277411860 \\
\hline C & 4.377387336 & -3.376438726 & -0.107375561 \\
\hline $\mathrm{H}$ & 4.773906161 & -4.066251777 & 0.631227060 \\
\hline C & 4.873680298 & -3.385022193 & -1.412516950 \\
\hline $\mathrm{H}$ & 5.664799799 & -4.075323040 & -1.689490190 \\
\hline C & 4.344037321 & -2.509784741 & -2.362281319 \\
\hline $\mathrm{H}$ & 4.721398633 & -2.516194479 & -3.380682590 \\
\hline C & 3.333226525 & -1.615906972 & -2.004780837 \\
\hline C & 0.546954736 & 3.959075202 & -1.144240495 \\
\hline C & 1.203153078 & 4.223719946 & 0.064507132 \\
\hline $\mathrm{H}$ & 1.177029783 & 3.481991426 & 0.854447967 \\
\hline C & 1.873173164 & 5.432787008 & 0.255190868 \\
\hline $\mathrm{H}$ & 2.368254338 & 5.631169738 & 1.201769385 \\
\hline C & 1.900554108 & 6.392823601 & -0.758439096 \\
\hline $\mathrm{H}$ & 2.420029871 & 7.334117477 & -0.606445695 \\
\hline C & 1.240886226 & 6.141535635 & -1.962064015 \\
\hline $\mathrm{H}$ & 1.240678159 & 6.887837643 & -2.750888966 \\
\hline C & 0.560689072 & 4.937106303 & -2.148018752 \\
\hline $\mathrm{H}$ & 0.024573301 & 4.764649242 & -3.077668543 \\
\hline C & -3.205827372 & 1.534286356 & -1.256293433 \\
\hline C & -3.271903179 & 0.929452614 & -2.518435413 \\
\hline C & -4.194388222 & 1.379653831 & -3.464571022 \\
\hline $\mathrm{H}$ & -4.242361315 & 0.909177542 & -4.442041280 \\
\hline C & -5.051923780 & 2.436457432 & -3.151076598 \\
\hline $\mathrm{H}$ & -5.771565331 & 2.786487635 & -3.884941280 \\
\hline C & -4.982527608 & 3.046804539 & -1.896330924 \\
\hline $\mathrm{H}$ & -5.645904943 & 3.871601113 & -1.654739247 \\
\hline C & -4.059208322 & 2.602668773 & -0.949077163 \\
\hline
\end{tabular}




\begin{tabular}{|c|c|c|c|}
\hline & -4.007860582 & 3.080916109 & 0.023386261 \\
\hline & -0.709971476 & 2.233406666 & -0.096246111 \\
\hline & 1.733920944 & 0.967476836 & -1.326969320 \\
\hline & -2.596008252 & 1.363931573 & 1.645996705 \\
\hline & -3.859181677 & 0.868753856 & 1.996724490 \\
\hline & -4.449580686 & 0.300129865 & 1.286607537 \\
\hline & -4.373887712 & 1.131281854 & 3.266838366 \\
\hline & -5.356249510 & 0.753877762 & 3.533594363 \\
\hline & -3.633890766 & 1.880114652 & 4.184212689 \\
\hline & -4.039449045 & 2.085678555 & 5.170326168 \\
\hline & -2.375058525 & 2.369503731 & 3.830680159 \\
\hline & -1.800759047 & 2.959093860 & 4.538856555 \\
\hline & -1.848461984 & 36053 & 4901 \\
\hline & -0.878073774 & 2.5003 & 1041 \\
\hline & -0.198693093 & 2.658954118 & -1.375460599 \\
\hline & -1.048870954 & 2.878177695 & -2.036185629 \\
\hline & 0.586838270 & 1.50 & -2.0 \\
\hline & -0.134777493 & 0.678483985 & -2.141307467 \\
\hline & 0.940124622 & 1.868441526 & -3.520874794 \\
\hline & 1.594790852 & 719 & -3.5 \\
\hline & 0.024112032 & 2.098100547 & -4.074122596 \\
\hline & 1.428709044 & 1.025765260 & -4.018337159 \\
\hline C & 3.046811228 & & $-1.51 c$ \\
\hline & 3.818488185 & 1.001332347 & -1.035245476 \\
\hline 1 & 3.083482132 & 2.605775091 & -1.083280265 \\
\hline $\mathrm{H}$ & 3.293122425 & 1.663586149 & -2.575297318 \\
\hline & 0.472349031 & -4.410599526 & -0.778006536 \\
\hline & 0.650686405 & -4.904901804 & 0.529420591 \\
\hline C & 1.573763693 & -5.913159986 & 0.786268025 \\
\hline & 2.344548986 & -6.443990914 & -0.2 \\
\hline & 2.197461518 & -5.945811968 & -1.548612221 \\
\hline C & 1.274877630 & -4.934417734 & -1.806945060 \\
\hline & 0.066639286 & -4.4 & 64541 \\
\hline $\mathrm{H}$ & 1.692141945 & -6.289708070 & 1.797839814 \\
\hline $\mathrm{H}$ & 3.056758078 & -7.238311683 & -0.051642925 \\
\hline & 2.796147951 & -6.348700432 & -2.359530184 \\
\hline $\mathrm{H}$ & 1.153720626 & -4.560552923 & -2.819900516 \\
\hline C & -1.772378567 & -3.304839335 & -0.398433762 \\
\hline & -1.858321689 & -3.812334039 & 0.557758964 \\
\hline C & -0.583799978 & -3.452623909 & -1.130546927 \\
\hline C & -2.792167818 & -2.408718882 & -0.791015898 \\
\hline & -4.059333944 & -2.244351353 & -0.055080822 \\
\hline C & -5.176786618 & -1.724314763 & -0.734382306 \\
\hline C & -4.216507752 & -2.628024825 & 1.291004280 \\
\hline c & -6.410134736 & -1.601183784 & -0.096711944 \\
\hline & -5.075997614 & -1.420925069 & -1.772332804 \\
\hline
\end{tabular}




$\begin{array}{llll}\text { C } & -5.450892120 & -2.513088976 & 1.924261598 \\ \text { H } & -3.371466720 & -3.013508368 & 1.852966785 \\ \text { C } & -6.553403020 & -1.999594048 & 1.234210279 \\ \text { H } & -7.260764726 & -1.203813364 & -0.641870391 \\ \text { H } & -5.553627438 & -2.822888351 & 2.959792210 \\ \text { H } & -7.515714546 & -1.914092303 & 1.729487815 \\ \text { H } & -2.848686134 & -2.160695214 & -1.850616026 \\ \text { H } & -0.595952851 & -3.144751951 & -2.176412451 \\ \text { H } & 2.927925378 & -0.930703155 & -2.742295947 \\ \text { H } & -2.601724795 & 0.109648392 & -2.762843223 \\ \text { C } & 4.072308343 & 1.203809401 & 1.612172790 \\ \text { C } & 4.239373956 & 2.596995220 & 1.582936774 \\ \text { C } & 5.118353581 & 0.393295866 & 1.145691007 \\ \text { C } & 5.399345229 & 3.167641741 & 1.058279582 \\ \text { H } & 3.451737929 & 3.235134409 & 1.970916134 \\ \text { C } & 6.280820220 & 0.964339884 & 0.623927965 \\ \text { H } & 5.028484192 & -0.685147200 & 1.197624827 \\ \text { C } & 6.422125310 & 2.352090682 & 0.570871622 \\ \text { H } & 5.504538839 & 4.248217032 & 1.034145226 \\ \text { H } & 7.080555478 & 0.321180295 & 0.268592052 \\ \text { H } & 7.327373219 & 2.794251093 & 0.165824468\end{array}$




\begin{tabular}{|c|c|c|c|}
\hline \multicolumn{4}{|c|}{ Structure 34M(half-chair) } \\
\hline \multicolumn{4}{|c|}{110} \\
\hline \multicolumn{4}{|c|}{ symmetry c1 } \\
\hline $\mathrm{Pd}$ & -0.919338370 & -1.271293656 & 0.126257257 \\
\hline & 1.409599998 & -0.350319307 & -0.231003963 \\
\hline$P$ & -1.914530097 & 0.937821341 & 0.432619423 \\
\hline C & 2.072244221 & 0.274534671 & 1.462364659 \\
\hline C & 3.261516150 & 0.987648369 & 1.759653230 \\
\hline C & 3.504050640 & 1.330785545 & 3.102940456 \\
\hline $\mathrm{H}$ & 4.413565417 & 1.878139636 & 3.330632323 \\
\hline C & 2.637182507 & 0.980204940 & 4.135223078 \\
\hline $\mathrm{H}$ & 2.870670178 & 1.261813419 & 5.157629936 \\
\hline C & 1.479024143 & 0.267537294 & 3.841285135 \\
\hline$H$ & 0.782764443 & -0.013993036 & 4.625035118 \\
\hline C & 1.208712868 & -0.068573981 & 2.516304389 \\
\hline $\mathrm{H}$ & 0.291426696 & -0.605863108 & 2.290234482 \\
\hline C & 2.696709455 & -1.593208475 & -0.814425218 \\
\hline C & 3.420471660 & -2.361489484 & 0.105589021 \\
\hline $\mathrm{H}$ & 3.284561332 & -2.210054037 & 1.171984025 \\
\hline C & 4.344211732 & -3.305363750 & -0.346294224 \\
\hline H & 4.909911821 & -3.889978682 & 0.372509973 \\
\hline C & 4.542946153 & -3.494832129 & -1.715384463 \\
\hline $\mathrm{H}$ & 5.270958097 & -4.221017258 & -2.064289603 \\
\hline C & 3.798689309 & -2.752538998 & -2.634066838 \\
\hline $\mathrm{H}$ & 3.944975883 & -2.899218703 & -3.700220800 \\
\hline C & 2.869877312 & -1.811985114 & -2.186330248 \\
\hline C & 0.192920493 & 3.819569596 & -1.396915879 \\
\hline C & 1.048572556 & 4.206324720 & -0.357030665 \\
\hline $\mathrm{H}$ & 1.181411709 & 3.546404236 & 0.492872572 \\
\hline C & 1.711915468 & 5.433319572 & -0.407933499 \\
\hline $\mathrm{H}$ & 2.362584542 & 5.728413307 & 0.410701581 \\
\hline C & 1.535123345 & 6.287874100 & -1.497956692 \\
\hline $\mathrm{H}$ & 2.050405531 & 7.242979502 & -1.533568595 \\
\hline C & 0.677544098 & 5.913945126 & -2.533355227 \\
\hline $\mathrm{H}$ & 0.518566009 & 6.578275832 & -3.377518423 \\
\hline C & 0.003778494 & 4.693048714 & -2.476562109 \\
\hline $\mathrm{H}$ & -0.686489019 & 4.426546505 & -3.272681821 \\
\hline C & -3.488667383 & 1.491250159 & -0.426045195 \\
\hline C & -3.837463952 & 0.903721825 & -1.648300224 \\
\hline C & -4.966418580 & 1.349172502 & -2.337421841 \\
\hline $\mathrm{H}$ & -5.238823147 & 0.888208587 & -3.281567591 \\
\hline C & -5.745696342 & 2.378705784 & -1.807097860 \\
\hline $\mathrm{H}$ & -6.625261215 & 2.723695124 & -2.342342311 \\
\hline C & -5.398771283 & 2.964785167 & -0.586995700 \\
\hline $\mathrm{H}$ & -6.005746319 & 3.765006294 & -0.174651905 \\
\hline C & -4.270972077 & 2.525621042 & 0.105759735 \\
\hline
\end{tabular}




\begin{tabular}{|c|c|c|c|}
\hline & -4.004538359 & 2.983523698 & 1.052598706 \\
\hline & -0.790761316 & 2.193839618 & 0.029255633 \\
\hline & 1.397885104 & 0.838512197 & -1.536374844 \\
\hline & -2.157086210 & 1.398274912 & 2.229489881 \\
\hline & -2.875348216 & 0.526367018 & 3.057605243 \\
\hline & -3.278871682 & -0.400274944 & 2.660771445 \\
\hline & -3.083750795 & 0.851692716 & 4.398044441 \\
\hline & -3.646308185 & 0.177339330 & 5.036616524 \\
\hline & -2.570926309 & 2.044280220 & 4.914463164 \\
\hline & -2.731994523 & 2.296980354 & 5.958082107 \\
\hline & -1.853443066 & 2.910823989 & 4.088072724 \\
\hline & -1.455340503 & 3.838656971 & 4.487688687 \\
\hline & -1.643435598 & 2.594084618 & 2.743840261 \\
\hline & -1.085929231 & 3.261656133 & 2.097638427 \\
\hline & -0.553588561 & 2.500134289 & -1.359225245 \\
\hline & -1.522021524 & 2.651140171 & -1.856337904 \\
\hline & 0.109227780 & 1.284804281 & -2.081 \\
\hline & -0.586764085 & 0.450899058 & -1.923607588 \\
\hline & 0.156027634 & 1.502099366 & -3.601367511 \\
\hline & 0.760334916 & 2.365500844 & -3.887382639 \\
\hline & -0.857968869 & 1.668062253 & -3.978464442 \\
\hline & 0.557457010 & 0.615892498 & -4.100998894 \\
\hline$c$ & 2.628041166 & 1.456646793 & -2.049288713 \\
\hline & 3.496264570 & 0.918549748 & -1.676825206 \\
\hline & 2.721552120 & 2.503748426 & -1.752377858 \\
\hline-1 & 2.653932808 & 1.397769368 & -3.141812758 \\
\hline & -4.007546769 & -2.385989433 & -0.288965198 \\
\hline & -4.139206291 & -2.645846689 & -1.666990500 \\
\hline C & -5.377339393 & -2.544243419 & -2.295383038 \\
\hline & -6.510489655 & -2.170332564 & -1.566570332 \\
\hline & -6.394180412 & -1.897573432 & -0.202635499 \\
\hline C & -5.156268855 & -2.005359937 & 0.427526843 \\
\hline & -3.271205274 & -2.929085388 & -2.255305772 \\
\hline & -5.460701108 & -2.760795174 & -3.356320079 \\
\hline $\mathrm{H}$ & -7.475439500 & -2.095987082 & -2.058307106 \\
\hline I & -7.268504155 & -1.609302681 & 0.372699702 \\
\hline & -5.079364187 & -1.810255995 & 1.494190659 \\
\hline C & -1.682362623 & -3.387374238 & -0.025363846 \\
\hline $\mathrm{H}$ & -1.746130878 & -3.828913432 & -1.015424364 \\
\hline & -2.734538696 & -2.551752963 & 0.436646337 \\
\hline C & -0.520636259 & -3.577671848 & 0.728002493 \\
\hline C & 0.569626896 & -4.491162963 & 0.384720375 \\
\hline 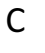 & 1.340864398 & -5.025724014 & 1.434653551 \\
\hline C & 0.813771292 & -4.941642993 & -0.927407307 \\
\hline C & 2.296346214 & -6.009071669 & 1.189771990 \\
\hline 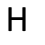 & 1.162481658 & -4.687401355 & 2.452132139 \\
\hline
\end{tabular}




$\begin{array}{llll}\text { C } & 1.773946980 & -5.916676210 & -1.170610921 \\ \text { H } & 0.256281629 & -4.522892177 & -1.759632841 \\ \text { C } & 2.511870482 & -6.460582208 & -0.113245690 \\ \text { H } & 2.866011952 & -6.426488376 & 2.014348214 \\ \text { H } & 1.950031223 & -6.256947130 & -2.186237140 \\ \text { H } & 3.252347715 & -7.230485998 & -0.307600107 \\ \text { H } & -0.562032102 & -3.292513173 & 1.779452704 \\ \text { H } & -2.812039170 & -2.430753299 & 1.516928209 \\ \text { H } & 2.297246710 & -1.229439762 & -2.900725774 \\ \text { H } & -3.241238226 & 0.092673046 & -2.053802320 \\ \text { C } & 4.314180151 & 1.442503065 & 0.794821741 \\ \text { C } & 4.456403834 & 2.814011964 & 0.531871724 \\ \text { C } & 5.253906545 & 0.555251193 & 0.249115362 \\ \text { C } & 5.480145726 & 3.282508373 & -0.291862974 \\ \text { H } & 3.753773168 & 3.514965757 & 0.970796942 \\ \text { C } & 6.280571454 & 1.024363390 & -0.572892063 \\ \text { H } & 5.189314297 & -0.502605587 & 0.472744573 \\ \text { C } & 6.392860538 & 2.387276127 & -0.852557323 \\ \text { H } & 5.565711041 & 4.346454481 & -0.492008054 \\ \text { H } & 6.999093879 & 0.322313790 & -0.985649227 \\ \text { H } & 7.192619873 & 2.750283899 & -1.491061681\end{array}$




\begin{tabular}{|c|c|c|c|}
\hline \multicolumn{4}{|c|}{ Structure $34 \mathrm{~W}$ (half-chair) } \\
\hline \multicolumn{4}{|c|}{110} \\
\hline \multicolumn{4}{|c|}{ symmetry c1 } \\
\hline $\mathrm{Pd}$ & -0.888566401 & -1.233441491 & -0.420593123 \\
\hline & 1.478039047 & -0.389108742 & -0.228517532 \\
\hline 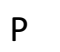 & -1.893613144 & 0.966517464 & -0.037666331 \\
\hline C & 1.768829449 & -0.028673174 & 1.638669489 \\
\hline C & 2.858101530 & 0.622438061 & 2.270601318 \\
\hline C & 2.823017285 & 0.754945023 & 3.671342682 \\
\hline $\mathrm{H}$ & 3.655628986 & 1.256502633 & 4.154940149 \\
\hline C & 1.780962240 & 0.250940325 & 4.446047359 \\
\hline $\mathrm{H}$ & 1.802299418 & 0.368746551 & 5.525307988 \\
\hline C & 0.723848160 & -0.405792250 & 3.823788091 \\
\hline $\mathrm{H}$ & -0.101105500 & -0.807880666 & 4.403448685 \\
\hline c & 0.725453906 & -0.530050418 & 2.435645182 \\
\hline $\mathrm{H}$ & -0.113790867 & -1.026447137 & 1.953196195 \\
\hline C & 2.863393345 & -1.577769908 & -0.686714380 \\
\hline C & 3.372464192 & -2.478492595 & 0.256652275 \\
\hline $\mathrm{H}$ & 3.004159722 & -2.469227945 & 1.277610952 \\
\hline C & 4.377722162 & -3.376142509 & -0.106960912 \\
\hline $\mathrm{H}$ & 4.774169887 & -4.065918470 & 0.631702700 \\
\hline C & 4.874238681 & -3.384644128 & -1.412011168 \\
\hline $\mathrm{H}$ & 5.665450316 & -4.074867974 & -1.688883250 \\
\hline C & 4.344666973 & -2.509438278 & -2.361854336 \\
\hline $\mathrm{H}$ & 4.722220589 & -2.515790722 & -3.380179041 \\
\hline C & 3.333686195 & -1.615688274 & -2.004524753 \\
\hline C & 0.546662477 & 3.959190213 & -1.144682707 \\
\hline C & 1.202964389 & 4.223969786 & 0.063989463 \\
\hline $\mathrm{H}$ & 1.177086759 & 3.482226667 & 0.853918637 \\
\hline C & 1.872774800 & 5.433155104 & 0.254592804 \\
\hline $\mathrm{H}$ & 2.367964682 & 5.631626100 & 1.201085863 \\
\hline C & 1.899822330 & 6.393203657 & -0.759044554 \\
\hline $\mathrm{H}$ & 2.419115119 & 7.334601251 & -0.607102520 \\
\hline C & 1.240064752 & 6.141785777 & -1.962585207 \\
\hline $\mathrm{H}$ & 1.239615714 & 6.888081118 & -2.751406750 \\
\hline C & 0.560089413 & 4.937212986 & -2.148462483 \\
\hline $\mathrm{H}$ & 0.023924527 & 4.764628964 & -3.078057324 \\
\hline C & -3.206067460 & 1.534285504 & -1.256168510 \\
\hline C & -3.272074961 & 0.929646827 & -2.518421739 \\
\hline C & -4.194765843 & 1.379740500 & -3.464398865 \\
\hline H & -4.242681171 & 0.909435527 & -4.441947140 \\
\hline C & -5.052596894 & 2.436231976 & -3.150641001 \\
\hline $\mathrm{H}$ & -5.772413390 & 2.786157465 & -3.884374203 \\
\hline C & -4.983271610 & 3.046383976 & -1.895803858 \\
\hline $\mathrm{H}$ & -5.646863548 & 3.870941303 & -1.654009782 \\
\hline C & -4.059732060 & 2.602362406 & -0.948704739 \\
\hline
\end{tabular}




\begin{tabular}{|c|c|c|c|}
\hline & -4.008437771 & 3.080467679 & 0.023826664 \\
\hline & -0.710099521 & 2.233540008 & -0.096498882 \\
\hline & 1.733985117 & 0.967558472 & -1.326835835 \\
\hline & -2.595829893 & 1.363838888 & 1.646038240 \\
\hline & -3.858686314 & 0.868080436 & 1.997069561 \\
\hline & -4.448956817 & 0.299079529 & 1.287146980 \\
\hline & -4.373258456 & 1.130494883 & 3.267264830 \\
\hline & -5.355374833 & 0.752638858 & 3.534260729 \\
\hline & -3.633436769 & 1.879787954 & 4.184404902 \\
\hline & -4.038890906 & 2.085267469 & 5.170571548 \\
\hline & -2.374918971 & 2.369753397 & 3.830557369 \\
\hline & -1.800756502 & 2.959685184 & 4.538552479 \\
\hline & -1.848459269 & 2.111314997 & 2.563704404 \\
\hline & -0.878328668 & 2.501132915 & 770630 \\
\hline & -0.198764227 & 2.658908530 & -1.375749905 \\
\hline & -1.048896153 & 2.877870629 & -2.036636271 \\
\hline & 0.586962785 & 1.502899752 & -2.07 \\
\hline & -0.134619321 & 0.678356485 & -2.141354453 \\
\hline & 0.940417177 & 1.868266532 & -3.520912593 \\
\hline & 1.594914071 & 2.739620761 & -3.590090437 \\
\hline & 0.024463945 & 2.097672220 & -4.074 \\
\hline & 1.429258372 & 1.025655091 & -4.018213808 \\
\hline c & 3.046791331 & 1.601778818 & -1.510266804 \\
\hline & 3.818491386 & 1.001813216 & -1.034744858 \\
\hline & 3.083146194 & 2.606098111 & -1.082698424 \\
\hline -1 & 3.293243284 & 1.664169112 & -2.574801357 \\
\hline & 0.472622692 & -4.410167601 & -0.778680586 \\
\hline & 0.650866129 & -4.904954610 & 0.528571924 \\
\hline C & 1.574058392 & -5.913179036 & 0.785136109 \\
\hline & 2.345075210 & -6.443466552 & -0.254532256 \\
\hline & 2.198088017 & -5.944791715 & -1.549667189 \\
\hline C & 1.275361817 & -4.933451832 & -1.807720744 \\
\hline & 0.066652126 & -4.499857636 & 1.349696811 \\
\hline & 1.692349377 & -6.290133370 & 1.796567349 \\
\hline $\mathrm{H}$ & 3.057381914 & -7.237752766 & -0.053089846 \\
\hline & 2.796952184 & -6.347248158 & -2.360668587 \\
\hline & 1.154258460 & -4.559226646 & -2.820549494 \\
\hline C & -1.772294259 & -3.304922247 & -0.398808685 \\
\hline 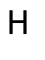 & -1.858070160 & -3.812613550 & 0.557293754 \\
\hline & -0.583654570 & -3.452208648 & -1.130972095 \\
\hline C & -2.792244702 & -2.408917731 & -0.791156797 \\
\hline C & -4.059279867 & -2.244720671 & -0.054977147 \\
\hline & -5.176897957 & -1.724675080 & -0.734006114 \\
\hline C & -4.216154680 & -2.628514737 & 1.291108348 \\
\hline C & -6.410115209 & -1.601657001 & -0.096069817 \\
\hline 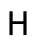 & -5.076327048 & -1.421186821 & -1.771948642 \\
\hline
\end{tabular}




$\begin{array}{llll}\text { C } & -5.450410463 & -2.513680930 & 1.924636948 \\ \text { H } & -3.370980157 & -3.014025787 & 1.852851467 \\ \text { C } & -6.553083132 & -2.000174852 & 1.234855002 \\ \text { H } & -7.260880986 & -1.204278649 & -0.641010198 \\ \text { H } & -5.552917017 & -2.823565074 & 2.960164259 \\ \text { H } & -7.515294115 & -1.914737772 & 1.730339748 \\ \text { H } & -2.848956162 & -2.160707724 & -1.850700584 \\ \text { H } & -0.595946929 & -3.144199443 & -2.176798226 \\ \text { H } & 2.928387482 & -0.930560595 & -2.742112429 \\ \text { H } & -2.601665024 & 0.110088830 & -2.763020703 \\ \text { C } & 4.072143886 & 1.203771035 & 1.612644499 \\ \text { C } & 4.238862496 & 2.597013487 & 1.583604162 \\ \text { C } & 5.118386829 & 0.393587087 & 1.146054848 \\ \text { C } & 5.398678201 & 3.168017118 & 1.059014710 \\ \text { H } & 3.451079341 & 3.234882346 & 1.971738317 \\ \text { C } & 6.280709940 & 0.964997508 & 0.624350730 \\ \text { H } & 5.028806476 & -0.684882696 & 1.197856580 \\ \text { C } & 6.421659052 & 2.352783693 & 0.571472778 \\ \text { H } & 5.503631512 & 4.248618942 & 1.035028899 \\ \text { H } & 7.080598923 & 0.322074775 & 0.268934027 \\ \text { H } & 7.326776466 & 2.795249699 & 0.166468104\end{array}$




\section{Structure 35M(boat)}

110

symmetry $\mathrm{c} 1$

Pd -0.965553509

P -2.115012983

P 1.363499436

C -2.395354500

C -1.353393236

$1.052439434 \quad 0.090083950$

H $\quad-0.438991908$

$-1.123511997$

0.176819322

0.180194727

$-0.047294344$

C -1.503431845

$-1.472541716$

1.998402812

$-2.028365643$

2.752960599

H -0.698174353

$-2.334885918$

2.255207827

$-2.193708244$

4.130777148

C -2.683056025

$-2.636229162$

4.709540803

H -2.797556456

$-1.794337904$

4.763125842

C -3.715942567

$-1.925473800$

5.834818840

H $\quad-4.636174516$

$-1.226971653$

4.014230003

C $\quad-3.574936272$

$-0.918622430$

4.501512051

H -4.386104483

$-1.062328730$

2.634537843

C $\quad-3.766817958$

$-0.629245953$

2.058007623

C -4.700221817

$-1.430105908$

$-0.674947499$

H $\quad-4.541156359$

$-2.359432736$

$-0.194452198$

C -5.844888236

$-2.857107984$

0.756314386

H $\quad-6.565262517$

$-2.646901805$

$-0.939809576$

C -6.058823009

$-3.366520474$

$-0.562500810$

H $\quad-6.948978359$

$-2.019084805$

$-2.168568276$

H

H $\quad-5.292922763$

$-2.247605402$

$-2.746859297$

$-1.099083488 \quad-2.653686482$

C $\quad-3.984032254$

$-0.606665965$

$-3.606992427$

C 1.792049070

$-0.805177152$

$-1.910063338$

C 1.825410033

$-3.751818614$

$-0.883415278$

$-4.241530946$

0.429866866

H 1.519298839

$-3.593872260$

1.243278859

C $\quad 2.264605094-5.540522310$

0.689831699

H 2.288459669

$-5.906003340$

1.712484667

C $\quad 2.677087308$

$-6.368202254$

$-0.356741227$

H 3.019580666

$-7.378108352$

$-0.152312436$

C 2.659032892

$-5.884733854$

$-1.665864500$

H 2.992154479

$-6.514924690$

$-2.485144216$

C 2.227205873

$-4.582768191$

$-1.924842624$

H 2.237109176

$-4.208451372$

$-2.945354720$

C 2.239708490

0.805580063

$-1.594001923$

C 1.616331721

1.844110300

$-2.297916717$

C $\quad 2.160021572$

2.308958567

$-3.495886372$

H 1.676689012

3.121977962

$-4.028950375$

C $\quad 3.316567295$

1.721153395

$-4.009805097$

H 3.737190187

$2.075675460 \quad-4.946106264$

C $\quad 3.925444718$

$0.668079011-3.324016412$

$\mathrm{H}$

$0.200954187-3.723304655$ 


\begin{tabular}{|c|c|c|c|}
\hline & 3.393840466 & 0.208325784 & -2.118706688 \\
\hline - & 3.874789887 & -0.608663741 & -1.597404427 \\
\hline & 1.685894849 & -1.524674708 & -0.080429788 \\
\hline & -1.051194439 & -2.467916491 & -0.320958945 \\
\hline$C$ & 2.250118983 & 0.535553814 & 1.587595672 \\
\hline & 1.360591009 & 0.835883514 & 2.633051215 \\
\hline & 0.291288056 & 0.792548274 & 2.445651829 \\
\hline$C$ & 1.814084614 & 1.179807598 & 3.904715411 \\
\hline & 1.103021773 & 1.394028550 & 4.696699344 \\
\hline & 3.186195039 & 1.245405865 & 4.141226018 \\
\hline - & 3.564530218 & 1.527660988 & 5.119143071 \\
\hline$C$ & 4.076042678 & 0.918521426 & 3.120673106 \\
\hline & 5.144217367 & 0.927238224 & 585669 \\
\hline C & 3.639977701 & 0.531122 & 9256 \\
\hline$C$ & 1.301748674 & -2.350351398 & -1.195968576 \\
\hline H & 1.825019998 & -2.000137016 & -2.096576749 \\
\hline C & -0.216016150 & -2.202924998 & -1.5 \\
\hline $\mathrm{H}$ & -0.340817988 & -1.140037356 & -1.749463668 \\
\hline C & -0.629889295 & -2.970132106 & -2.774186481 \\
\hline $\mathrm{H}$ & -0.501155292 & -4.049742622 & 5170 \\
\hline H & -0.023732431 & -2.644047391 & -3.625346405 \\
\hline $\mathrm{H}$ & -1.677747483 & -2.768760451 & -3.013986951 \\
\hline C & -1.603743198 & -3.822735531 & \\
\hline - & -1.993669417 & -3.950594364 & 0.832518878 \\
\hline $\mathrm{H}$ & -0.801442018 & -4.549795045 & -0.313727064 \\
\hline $\mathrm{H}$ & -2.408715950 & -4.046383886 & -0.892459071 \\
\hline & 0.658661672 & 4.138678595 & 0.326586969 \\
\hline C & 0.667721065 & 4.901767963 & -0.858042838 \\
\hline C & 1.740158817 & 5.735398341 & -1.158243128 \\
\hline & 2.829745361 & 5.825042555 & 28995 \\
\hline C & 2.834622259 & 5.078477958 & 0.892977839 \\
\hline C & 1.759468156 & 4.247000930 & 1.198153830 \\
\hline & -0.171713582 & 860977102 & 34914 \\
\hline $\mathrm{H}$ & 1.724713858 & 6.324032887 & -2.070405077 \\
\hline $\mathrm{H}$ & 3.663227998 & 6.479268039 & -0.522729153 \\
\hline $\mathbf{H}$ & 3.671081787 & 5.151004330 & 1.581297139 \\
\hline $\mathrm{H}$ & 1.765724687 & 3.674999138 & 2.121055134 \\
\hline C & -1.699663035 & 3.193202177 & 0.054794587 \\
\hline$\Pi$ & -1.812929007 & 3.669469575 & -0.914463266 \\
\hline C & -0.470467567 & 3.286085024 & 0.724342639 \\
\hline C & -2.755864523 & 2.388034057 & 0.541171782 \\
\hline C & -4.082948722 & 2.323054748 & -0.093141299 \\
\hline C & -5.194866568 & 1.986307958 & 0.699961046 \\
\hline C & -4.303067762 & 2.648419866 & -1.445292374 \\
\hline C & -6.482292022 & 1.981016831 & 0.166578517 \\
\hline$\pi$ & -5.045639319 & 1.752411815 & 1.750815457 \\
\hline
\end{tabular}




$\begin{array}{lrrr}\text { C } & -5.589514969 & 2.650093881 & -1.975608079 \\ \text { H } & -3.465654730 & 2.897206703 & -2.090808410 \\ \text { C } & -6.684683693 & 2.315850868 & -1.173039845 \\ \text { H } & -7.327400313 & 1.726965623 & 0.798976544 \\ \text { H } & -5.740848973 & 2.912682825 & -3.018387716 \\ \text { H } & -7.687127175 & 2.319973026 & -1.589552156 \\ \text { H } & -2.752668530 & 2.162496576 & 1.607915485 \\ \text { H } & -0.450528281 & 2.973513829 & 1.766798504 \\ \text { H } & -3.264647164 & -0.083509101 & -2.285222528 \\ \text { H } & 0.705131611 & 2.286603897 & -1.910069535 \\ \text { C } & 4.704772816 & 0.089605387 & 0.890815806 \\ \text { C } & 5.630102123 & 1.011085402 & 0.379579668 \\ \text { C } & 4.873683643 & -1.275257378 & 0.607754414 \\ \text { C } & 6.689824756 & 0.581413499 & -0.420144527 \\ \text { H } & 5.510892141 & 2.067466894 & 0.602665766 \\ \text { C } & 5.940071930 & -1.703604660 & -0.185114140 \\ \text { H } & 4.168502713 & -1.996098351 & 1.006654204 \\ \text { C } & 6.848183429 & -0.776868974 & -0.703322634 \\ \text { H } & 7.394710019 & 1.306581470 & -0.815909708 \\ \text { H } & 6.063975819 & -2.762765418 & -0.390763254 \\ \text { H } & 7.679995294 & -1.112411494 & -1.315644038\end{array}$




\section{Structure $35 \mathrm{~W}$ (boat)}

110

symmetry $\mathrm{c} 1$

$\begin{array}{llll}\text { Pd } & -0.941931656 & 1.047282512 & -0.474307912\end{array}$

$\begin{array}{llll}P & -2.143761077 & -1.070339950 & -0.117663220\end{array}$

$\begin{array}{llll}\text { P } & 1.364514843 & 0.224004788 & -0.108743636\end{array}$

$\begin{array}{llll}\text { C } & -2.633279142 & -1.274325503 & 1.676524956\end{array}$

$\begin{array}{llll}\text { C } & -1.711169735 & -1.836644408 & 2.569940134\end{array}$

H $\quad-0.767725246 \quad-2.217642375 \quad 2.193569372$

$\begin{array}{llll}\text { C } & -2.016229376 & -1.909774021 & 3.929223604\end{array}$

H $\quad-1.304214148 \quad-2.356617582 \quad 4.616594005$

$\begin{array}{llll}\text { C } & -3.231755528 & -1.411354226 & 4.404192629\end{array}$

H $\quad-3.467888112 \quad-1.471563500 \quad 5.462332720$

$\begin{array}{llll}\text { C } & -4.140408291 & -0.832341667 & 3.517074401\end{array}$

$\begin{array}{llll}\text { H } & -5.084139816 & -0.438055388 & 3.881514894\end{array}$

$\begin{array}{llll}\text { C } & -3.844752612 & -0.757040978 & 2.154117314\end{array}$

H $\quad-4.557616301 \quad-0.299904554 \quad 1.476583572$

$\begin{array}{llll}\text { C } & -3.702067132 & -1.368802078 & -1.141733248\end{array}$

$\begin{array}{llll}\text { C } & -4.764178889 & -2.165491836 & -0.695708726\end{array}$

H $\quad-4.764514932 \quad-2.565389744 \quad 0.312673593$

$\begin{array}{llll}\text { C } & -5.836114371 & -2.442846322 & -1.547428727\end{array}$

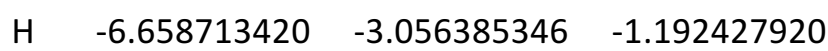

C $\quad-5.847884491 \quad-1.942283020 \quad-2.850348020$

H $\quad-6.681417399-2.163175050 \quad-3.510118499$

$\begin{array}{llll}\text { C } & -4.783009767 & -1.161306075 & -3.306226907\end{array}$

H $\quad-4.784850674 \quad-0.775199594 \quad-4.321282307$

$\begin{array}{llll}\text { C } & -3.714956249 & -0.875723438 & -2.454630639\end{array}$

$\begin{array}{llll}\text { C } & 1.775635960 & -3.789783547 & -0.520268781\end{array}$

$\begin{array}{llll}\text { C } & 1.576993526 & -4.186658049 & 0.809671381\end{array}$

$\begin{array}{llll}\text { H } & 1.128710666 & -3.489870754 & 1.508312315\end{array}$

$\begin{array}{llll}\text { C } & 1.971151396 & -5.455773357 & 1.235797589\end{array}$

$\begin{array}{llll}\text { H } & 1.815765767 & -5.748474099 & 2.270176209\end{array}$

$\begin{array}{lllll}\text { C } & 2.568661996 & -6.345873461 & 0.340745658\end{array}$

H $\quad 2.876061251 \quad-7.332259324 \quad 0.674637925$

$\begin{array}{llll}\text { C } & 2.781177161 & -5.955143533 & -0.982171898\end{array}$

H $\quad 3.258879026 \quad-6.634304749-1.682030310$

C $\quad 2.393939855 \quad-4.682727842 \quad-1.406220344$

H $\quad 2.581441373 \quad-4.380196039 \quad-2.433421336$

$\begin{array}{llll}\text { C } & 2.550983751 & 0.752116618 & -1.470154793\end{array}$

$\begin{array}{lllll}\text { C } & 2.196107211 & 0.435439596 & -2.792239241\end{array}$

$\begin{array}{llll}\text { C } & 3.013111840 & 0.824004051 & -3.853778915\end{array}$

H $\quad 2.738098873 \quad 0.560791785 \quad-4.870753943$

$\begin{array}{lllll}\text { C } & 4.179034229 & 1.552435860 & -3.606231767\end{array}$

H $\quad 4.816788886 \quad 1.854494652 \quad-4.431467192$

$\begin{array}{llll}\text { C } & 4.513271920 & 1.901866626 & -2.298000454\end{array}$

H $\quad 5.409107544 \quad 2.482114242 \quad-2.099831512$ 


\begin{tabular}{|c|c|c|c|}
\hline & 3.702868856 & 1.507306026 & -1.231539829 \\
\hline & 3.973976309 & 1.792542263 & -0.224750601 \\
\hline & 1.562621262 & -1.497918766 & 0.057668981 \\
\hline & -1.105031075 & -2.491199580 & -0.418970621 \\
\hline & 1.985852638 & 0.715155831 & 1.602879824 \\
\hline & 0.972793839 & 1.308992275 & 2.376654905 \\
\hline & -0.017515710 & 1.426482959 & 1.943604503 \\
\hline$C$ & 1.198058144 & 1.741669099 & 3.680413352 \\
\hline & 0.391571041 & 2.187713338 & 4.253992435 \\
\hline & 2.465638832 & 1.582285288 & 4.234079241 \\
\hline H & 2.669776984 & 1.906874273 & 5.250040360 \\
\hline C & 3.474608864 & 0.985989536 & 3.483534179 \\
\hline & 4.452422674 & 0.837887773 & 107251 \\
\hline & 3.277267333 & 0.527890701 & 8597 \\
\hline$C$ & 1.334453728 & -2.425467532 & -1.017303875 \\
\hline 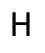 & 1.975493290 & -2.156630461 & -1.868361287 \\
\hline & -0.1360187 & -2.3 & -1.5 \\
\hline - & -0.242129399 & -1.309233574 & -1.887045969 \\
\hline$C$ & -0.390649840 & -3.243124351 & -2.737583185 \\
\hline & -0.269904056 & -4.302796 & -2.5 \\
\hline $\mathrm{H}$ & 0.316031515 & -3.001928610 & -3.538455401 \\
\hline H & -1.401083160 & -3.087099429 & -3.125543489 \\
\hline C & -1.732226698 & -3.808667835 & -0.24432 \\
\hline & -2.242413660 & -3.839551297 & 0.721185573 \\
\hline $\mathrm{H}$ & -0.955426607 & -4.574789145 & -0.224649779 \\
\hline $\mathrm{H}$ & -2.459546102 & -4.058737423 & -1.028190273 \\
\hline & -3.986956646 & 2.348385828 & -0.08 \\
\hline C & -4.056262319 & 2.711465906 & 1.278109242 \\
\hline C & -5.274022896 & 2.717208421 & 1.952477059 \\
\hline & -6.448587030 & 2.345894266 & 1.29 \\
\hline C & -6.394016896 & 1.967990514 & -0.052363347 \\
\hline C & -5.176674750 & 1.970947530 & -0.730913692 \\
\hline & -3.154892792 & 2.9853238 & 1.817 \\
\hline $\mathrm{H}$ & -5.307505459 & 3.009450097 & 2.997677248 \\
\hline $\mathrm{H}$ & -7.397546144 & 2.353465914 & 1.818318254 \\
\hline$H$ & -7.300607540 & 1.679698972 & -0.575422663 \\
\hline $\mathrm{H}$ & -5.144581399 & 1.684663252 & -1.777719257 \\
\hline C & -1.622047185 & 3.189207529 & -0.486732949 \\
\hline & -1.641945091 & 3.718990347 & 0.461389250 \\
\hline C & -2.732797750 & 2.392460895 & -0.854133038 \\
\hline C & -0.431864952 & 3.185957841 & -1.232792254 \\
\hline C & 0.754919284 & 3.993486508 & -0.917069856 \\
\hline C & 1.613889590 & 4.360929039 & -1.968257246 \\
\hline C & 1.039115645 & 4.466050751 & 0.379122076 \\
\hline C & 2.705259149 & 5.196767743 & -1.740220968 \\
\hline I & 1.414557881 & 3.995942187 & -2.971950766 \\
\hline
\end{tabular}




$\begin{array}{lrrr}\text { C } & 2.133600990 & 5.293986832 & 0.606303685 \\ \text { H } & 0.412893990 & 4.174131148 & 1.216173490 \\ \text { C } & 2.966743720 & 5.668604425 & -0.453269246 \\ \text { H } & 3.348846080 & 5.479833829 & -2.567269334 \\ \text { H } & 2.338950899 & 5.650004003 & 1.611187991 \\ \text { H } & 3.815444368 & 6.321312123 & -0.273027629 \\ \text { H } & -0.496346970 & 2.859318749 & -2.271441006 \\ \text { H } & -2.835878398 & 2.143285997 & -1.909814334 \\ \text { H } & -2.888644387 & -0.266263838 & -2.811440856 \\ \text { H } & 1.277344697 & -0.103832679 & -3.003180802 \\ \text { C } & 4.472662148 & -0.091286303 & 1.514813760 \\ \text { C } & 5.684648020 & 0.625219602 & 1.509366674 \\ \text { C } & 4.465172904 & -1.394433477 & 0.996386826 \\ \text { C } & 6.843668277 & 0.068689994 & 0.971698622 \\ \text { H } & 5.711430422 & 1.632263613 & 1.917184334 \\ \text { C } & 5.629402603 & -1.952397031 & 0.465195099 \\ \text { H } & 3.552867003 & -1.974028591 & 1.023157919 \\ \text { C } & 6.819164089 & -1.224478821 & 0.444649412 \\ \text { H } & 7.765718569 & 0.642627009 & 0.969249500 \\ \text { H } & 5.602661584 & -2.966090800 & 0.076223464 \\ \text { H } & 7.722446158 & -1.662517269 & 0.030630683\end{array}$




\begin{tabular}{|c|c|c|c|}
\hline \multicolumn{4}{|c|}{ Structure 35M(half-chair) } \\
\hline \multicolumn{4}{|c|}{110} \\
\hline \multicolumn{4}{|c|}{ symmetry c1 } \\
\hline $\mathrm{Pd}$ & 0.981176716 & -1.068120184 & 0.127624044 \\
\hline & 2.143681007 & 1.107652926 & 0.193892712 \\
\hline$P$ & -1.345480760 & -0.224637181 & 0.009082012 \\
\hline C & 2.298366999 & 1.548614073 & 2.010163650 \\
\hline C & 1.240494873 & 2.205010438 & 2.651816898 \\
\hline H & 0.383452587 & 2.528698520 & 2.070524210 \\
\hline C & 1.299569776 & 2.441036594 & 4.026476773 \\
\hline $\mathrm{H}$ & 0.482177075 & 2.960181176 & 4.517911799 \\
\hline C & 2.403267361 & 2.012678565 & 4.767032398 \\
\hline $\mathrm{H}$ & 2.447221861 & 2.198874453 & 5.835806126 \\
\hline C & 3.451398605 & 1.344040885 & 4.130744588 \\
\hline H & 4.312492223 & 1.011392562 & 4.702732758 \\
\hline C & 3.401085337 & 1.108333670 & 2.755492310 \\
\hline $\mathrm{H}$ & 4.224971730 & 0.597825048 & 2.266036598 \\
\hline C & 3.859045663 & 1.370360680 & -0.540959587 \\
\hline C & 4.769570332 & 2.297256743 & -0.014781903 \\
\hline $\mathrm{H}$ & 4.547818145 & 2.822217466 & 0.908430879 \\
\hline C & 5.971855374 & 2.547800077 & -0.678453018 \\
\hline $\mathrm{H}$ & 6.673858367 & 3.266317475 & -0.265998906 \\
\hline C & 6.266919845 & 1.884738857 & -1.871304327 \\
\hline H & 7.201928842 & 2.084287745 & -2.386082806 \\
\hline C & 5.359373772 & 0.966005892 & -2.401822866 \\
\hline $\mathrm{H}$ & 5.588216738 & 0.444364982 & -3.325912973 \\
\hline C & 4.158325073 & 0.709489047 & -1.739663269 \\
\hline C & -1.707167891 & 3.623898391 & -1.224904120 \\
\hline C & -1.730051531 & 4.259290859 & 0.024707432 \\
\hline H & -1.400555967 & 3.714494822 & 0.901858068 \\
\hline C & -2.191900770 & 5.570914494 & 0.143316879 \\
\hline $\mathrm{H}$ & -2.208633254 & 6.049284219 & 1.118328786 \\
\hline C & -2.637513364 & 6.266046491 & -0.982935500 \\
\hline $\mathrm{H}$ & -2.998144569 & 7.285796980 & -0.888197644 \\
\hline C & -2.629887619 & 5.637366457 & -2.228988989 \\
\hline $\mathrm{H}$ & -2.988749318 & 6.163878822 & -3.108346552 \\
\hline C & -2.175141864 & 4.322788658 & -2.346173068 \\
\hline $\mathrm{H}$ & -2.191684554 & 3.834833364 & -3.317465504 \\
\hline C & -2.284841848 & -1.051832865 & -1.395525212 \\
\hline C & -1.733681523 & -0.958932719 & -2.684567980 \\
\hline C & -2.365526831 & -1.571586964 & -3.766428785 \\
\hline $\mathrm{H}$ & -1.940486564 & -1.479857988 & -4.761443249 \\
\hline C & -3.537115471 & -2.304625812 & -3.566474950 \\
\hline $\mathrm{H}$ & -4.030462552 & -2.781577161 & -4.407995620 \\
\hline C & -4.062238936 & -2.434649625 & -2.280918422 \\
\hline $\mathrm{H}$ & -4.962032240 & -3.018727982 & -2.115034372 \\
\hline
\end{tabular}




\begin{tabular}{|c|c|c|c|}
\hline & -3.440149741 & -1.813358593 & -1.196764850 \\
\hline$y$ & -3.857779384 & -1.930084802 & -0.206923394 \\
\hline & -1.571245227 & 1.488607871 & -0.202567136 \\
\hline & 1.135816591 & 2.424887078 & -0.462266045 \\
\hline & -2.251747256 & -0.376063742 & 1.657693527 \\
\hline & -1.381060236 & -0.718631266 & 2.707314812 \\
\hline & -0.324768530 & -0.848743320 & 2.491894295 \\
\hline$C$ & -1.828766890 & -0.881047602 & 4.015575790 \\
\hline & -1.127659800 & -1.137820283 & 4.803500198 \\
\hline & -3.181056156 & -0.697427435 & 4.292642245 \\
\hline H & -3.559199937 & -0.816046490 & 5.303628018 \\
\hline Z & -4.050654051 & -0.337733546 & 3.267346011 \\
\hline & -5.096770600 & -0.161387599 & 3598 \\
\hline C & -3.627741060 & -0.15 & 2352 \\
\hline$C$ & -1.189811017 & 2.205872415 & -1.388326909 \\
\hline- & -1.697453080 & 1.764217410 & -2.257102779 \\
\hline & 0.33 & 2.06902 & -1.6 \\
\hline 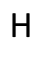 & 0.495109862 & 0.995412643 & -1.812610993 \\
\hline$C$ & 0.775336561 & 2.760338717 & -2.952220853 \\
\hline & 0.6276683 & 960 & -2.9 \\
\hline-1 & 0.194708241 & 2.374434991 & -3.796596053 \\
\hline - & 1.831685927 & 2.561014903 & -3.153561334 \\
\hline C & 1.701172847 & 3.779435692 & -0.401071851 \\
\hline - & 2.053018900 & 3.977365441 & 0.614416480 \\
\hline $\mathrm{H}$ & 0.915912212 & 4.504873072 & -0.619291674 \\
\hline $\mathrm{H}$ & 2.537685325 & 3.940264311 & -1.093793430 \\
\hline & -0.734500181 & -4.016290439 & 0.232538613 \\
\hline C & -0.868821522 & -4.515773864 & -1.077162754 \\
\hline C & -1.946879935 & -5.325621419 & -1.418865490 \\
\hline & -2.919288407 & -5.646426765 & -0.466243111 \\
\hline C & -2.811776687 & -5.140739673 & 0.830417970 \\
\hline C & -1.731104631 & -4.331569185 & 1.175416306 \\
\hline & -0.131064530 & -4.271528247 & -1.83504 \\
\hline $\mathrm{H}$ & -2.030715730 & -5.709209147 & -2.430905495 \\
\hline $\mathrm{H}$ & -3.754288417 & -6.286231799 & -0.735092494 \\
\hline H & -3.561941509 & -5.385371986 & 1.576241433 \\
\hline $\mathrm{H}$ & -1.644519184 & -3.950037701 & 2.189146006 \\
\hline C & 1.676754159 & -3.206791057 & 0.023525217 \\
\hline & 1.768314507 & -3.660681449 & -0.958575995 \\
\hline C & 0.432302329 & -3.243735646 & 0.681949414 \\
\hline C & 2.761493111 & -2.473154562 & 0.545983797 \\
\hline c & 4.100056139 & -2.428097114 & -0.061616297 \\
\hline C & 5.193554679 & -2.063151792 & 0.744708200 \\
\hline C & 4.350479868 & -2.795098005 & -1.398030591 \\
\hline C & 6.492277227 & -2.070515251 & 0.240392235 \\
\hline$H$ & 5.020203827 & -1.794063685 & 1.783593606 \\
\hline
\end{tabular}




$\begin{array}{llll}\text { C } & 5.648472458 & -2.809575633 & -1.899133769 \\ \text { H } & 3.529634709 & -3.072362943 & -2.052829222 \\ \text { C } & 6.724851421 & -2.446958071 & -1.083240513 \\ \text { H } & 7.322394296 & -1.792921022 & 0.882571119 \\ \text { H } & 5.823785388 & -3.106898172 & -2.928763616 \\ \text { H } & 7.736414215 & -2.462373153 & -1.476879281 \\ \text { H } & 2.742857796 & -2.259239946 & 1.615026537 \\ \text { H } & 0.436077751 & -3.018509511 & 1.747835416 \\ \text { H } & 3.459470680 & -0.013972772 & -2.148341836 \\ \text { H } & -0.805048308 & -0.421945978 & -2.851145051 \\ \text { C } & -4.696756182 & 0.250130012 & 0.971736311 \\ \text { C } & -5.880453763 & -0.509901945 & 0.916093217 \\ \text { C } & -4.611666957 & 1.411986609 & 0.190556233 \\ \text { C } & -6.931898899 & -0.138197976 & 0.080471881 \\ \text { H } & -5.967882311 & -1.407667803 & 1.522315890 \\ \text { C } & -5.669548847 & 1.786288616 & -0.639811463 \\ \text { H } & -3.725193760 & 2.028764807 & 0.246609899 \\ \text { C } & -6.828708383 & 1.012925674 & -0.703719835 \\ \text { H } & -7.832514497 & -0.744225030 & 0.045862894 \\ \text { H } & -5.585978160 & 2.694433516 & -1.229625355 \\ \text { H } & -7.649230358 & 1.307941657 & -1.350941642\end{array}$




\begin{tabular}{|c|c|c|c|}
\hline \multicolumn{4}{|c|}{ Structure 35W(half-chair) } \\
\hline \multicolumn{4}{|c|}{110} \\
\hline \multicolumn{4}{|c|}{ symmetry c1 } \\
\hline $\mathrm{Pd}$ & -0.942016113 & 1.047206686 & -0.474394295 \\
\hline$P$ & -2.143770888 & -1.070463127 & -0.117563347 \\
\hline$P$ & 1.364467010 & 0.224237652 & -0.108780096 \\
\hline C & -2.632970632 & -1.274110895 & 1.676762668 \\
\hline C & -1.710598870 & -1.836091630 & 2.570130876 \\
\hline $\mathrm{H}$ & -0.767138309 & -2.216945073 & 2.193669143 \\
\hline c & -2.015421036 & -1.909028701 & 3.929474101 \\
\hline $\mathrm{H}$ & -1.303201467 & -2.355586771 & 4.616815926 \\
\hline C & -3.230977008 & -1.410772009 & 4.404547501 \\
\hline $\mathrm{H}$ & -3.466935032 & -1.470848158 & 5.462731826 \\
\hline C & -4.139880124 & -0.832088197 & 3.517476878 \\
\hline $\mathrm{H}$ & -5.083628038 & -0.437924074 & 3.882001991 \\
\hline C & -3.844456865 & -0.756968348 & 2.154454495 \\
\hline $\mathrm{H}$ & -4.557499967 & -0.300051436 & 1.476957309 \\
\hline C & -3.702187080 & -1.369312284 & -1.141328512 \\
\hline C & -4.764182662 & -2.165967185 & -0.694984409 \\
\hline $\mathrm{H}$ & -4.764365471 & -2.565636129 & 0.313486745 \\
\hline C & -5.836201368 & -2.443601955 & -1.546512345 \\
\hline $\mathrm{H}$ & -6.658714267 & -3.057110398 & -1.191264090 \\
\hline C & -5.848161727 & -1.943358440 & -2.849550137 \\
\hline $\mathrm{H}$ & -6.681755385 & -2.164476048 & -3.509163921 \\
\hline C & -4.783399866 & -1.162409446 & -3.305745382 \\
\hline $\mathrm{H}$ & -4.785386847 & -0.776556019 & -4.320894029 \\
\hline C & -3.715270765 & -0.876536932 & -2.454343936 \\
\hline C & 1.776028001 & -3.789546761 & -0.519986379 \\
\hline C & 1.577047661 & -4.186615926 & 0.809850269 \\
\hline $\mathrm{H}$ & 1.128232031 & -3.490076810 & 1.508394718 \\
\hline C & 1.971542167 & -5.455611534 & 1.236015292 \\
\hline $\mathrm{H}$ & 1.815886071 & -5.748460658 & 2.270308979 \\
\hline C & 2.569734993 & -6.345402546 & 0.341107215 \\
\hline $\mathrm{H}$ & 2.877397619 & -7.331694004 & 0.675028310 \\
\hline C & 2.782578052 & -5.954481744 & -0.981698051 \\
\hline $\mathrm{H}$ & 3.260791357 & -6.633399485 & -1.681440462 \\
\hline C & 2.394994961 & -4.682179196 & -1.405782434 \\
\hline $\mathrm{H}$ & 2.582726940 & -4.379496414 & -2.432895101 \\
\hline C & 2.550859246 & 0.752157301 & -1.470318648 \\
\hline C & 2.196067517 & 0.435156315 & -2.792346262 \\
\hline C & 3.013040210 & 0.823662240 & -3.853933821 \\
\hline $\mathrm{H}$ & 2.738111669 & 0.560203562 & -4.870864885 \\
\hline C & 4.178819738 & 1.552356092 & -3.606489206 \\
\hline$H$ & 4.816550005 & 1.854360109 & -4.431759964 \\
\hline C & 4.512948624 & 1.902124827 & -2.298320670 \\
\hline $\mathrm{H}$ & 5.408662241 & 2.482590893 & -2.100251102 \\
\hline
\end{tabular}




\begin{tabular}{|c|c|c|c|}
\hline & 3.702580476 & 1.507629962 & -1.231808931 \\
\hline 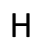 & 3.973564789 & 1.793134349 & -0.225065059 \\
\hline & 1.562441279 & -1.497714059 & 0.057903188 \\
\hline & -1.104966139 & -2.491346194 & -0.418751000 \\
\hline & 1.985995791 & 0.715548121 & 1.602723420 \\
\hline & 0.973033308 & 1.309460062 & 2.376556875 \\
\hline & -0.017311987 & 1.426947598 & 1.943601746 \\
\hline & 1.198456614 & 1.742220877 & 3.680263605 \\
\hline & 0.392044687 & 2.188311472 & 4.253909069 \\
\hline & 2.466100661 & 1.582862790 & 4.233787569 \\
\hline & 2.670362931 & 1.907520022 & 5.249699036 \\
\hline 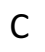 & 3.474979547 & 0.986501086 & 3.483167856 \\
\hline & 4.452845122 & 0.838427301 & 3.929635818 \\
\hline & 3.277476220 & 0.528299580 & 185807 \\
\hline & 1.334524643 & -2.425348817 & -1.017050568 \\
\hline 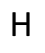 & 1.975496327 & -2.156400581 & -1.868119225 \\
\hline & -0.135974349 & -2.339018819 & -1.523917418 \\
\hline & -0.242233895 & -1.309537655 & -1.887034645 \\
\hline C & -0.390408613 & -3.243650247 & -2.737162489 \\
\hline H & -0.269519884 & -4.303248211 & -2.502105114 \\
\hline & 0.316295226 & -3.002554436 & -3.538043394 \\
\hline $\mathrm{H}$ & -1.400841723 & -3.087870070 & -3.125224025 \\
\hline C & -1.732108327 & -3.808792943 & -0.243877775 \\
\hline & -2.242219050 & -3.839561393 & 0.721677401 \\
\hline H & -0.955296980 & -4.574908655 & -0.224152068 \\
\hline $\mathrm{H}$ & -2.459476352 & -4.059004007 & -1.027657995 \\
\hline & -3.987390138 & 2.348097304 & -0.081790401 \\
\hline C & -4.056960859 & 2.711288071 & 1.277551002 \\
\hline C & -5.274833346 & 2.716935392 & 1.951718182 \\
\hline & -6.449246173 & 2.345426494 & 1.289999637 \\
\hline & -6.394413250 & 1.967422686 & -0.053241952 \\
\hline C & -5.176959432 & 1.970468207 & -0.731590341 \\
\hline$H$ & -3.155721812 & 2.985320186 & 1.817316045 \\
\hline H & -5.308522537 & 3.009264626 & 2.996887228 \\
\hline $\mathrm{H}$ & -7.398289162 & 2.352927407 & 1.817249157 \\
\hline $\mathrm{H}$ & -7.300879960 & 1.678977613 & -0.576430793 \\
\hline $\mathrm{H}$ & -5.144664564 & 1.684105658 & -1.778367705 \\
\hline C & -1.622466685 & 3.189035155 & -0.486949523 \\
\hline $\mathrm{H}$ & -1.642521783 & 3.718864530 & 0.461145143 \\
\hline 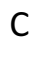 & -2.733126893 & 2.392252574 & -0.854459123 \\
\hline C & -0.432147629 & 3.185773063 & -1.232828026 \\
\hline C & 0.754533858 & 3.993418551 & -0.916967360 \\
\hline C & 1.613590213 & 4.360946439 & -1.968054867 \\
\hline C & 1.038562237 & 4.465977010 & 0.379261392 \\
\hline C & 2.704871871 & 5.196865397 & -1.739885258 \\
\hline $\mathrm{H}$ & 1.414396598 & 3.995965148 & -2.971777720 \\
\hline
\end{tabular}




$\begin{array}{lrrr}\text { C } & 2.132963490 & 5.293989108 & 0.606579079 \\ \text { H } & 0.412275619 & 4.173998743 & 1.216242510 \\ \text { C } & 2.966188936 & 5.668693485 & -0.452896797 \\ \text { H } & 3.348521843 & 5.479998509 & -2.566861873 \\ \text { H } & 2.338180772 & 5.649991673 & 1.611495454 \\ \text { H } & 3.814825691 & 6.321455583 & -0.272552288 \\ \text { H } & -0.496489340 & 2.859185016 & -2.271503332 \\ \text { H } & -2.836001295 & 2.142908096 & -1.910120643 \\ \text { H } & -2.889056754 & -0.267088780 & -2.811400559 \\ \text { H } & 1.277392730 & -0.104294713 & -3.003206335 \\ \text { C } & 4.472769370 & -0.090969232 & 1.514411945 \\ \text { C } & 5.684775605 & 0.625496309 & 1.508737509 \\ \text { C } & 4.465168226 & -1.394166401 & 0.996112490 \\ \text { C } & 6.843708653 & 0.068874366 & 0.970974687 \\ \text { H } & 5.711645850 & 1.632578786 & 1.916456228 \\ \text { C } & 5.629308939 & -1.952221556 & 0.464822850 \\ \text { H } & 3.552845577 & -1.973726626 & 1.023069790 \\ \text { C } & 6.819092650 & -1.224344574 & 0.444053895 \\ \text { H } & 7.765777890 & 0.642779931 & 0.968352111 \\ \text { H } & 5.602475790 & -2.965950986 & 0.075948949 \\ \text { H } & 7.722305873 & -1.662453948 & 0.029960048\end{array}$

\section{Investigated structures}

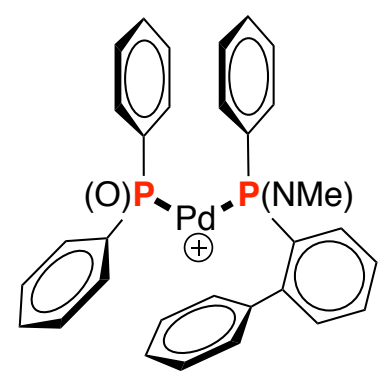

33M(boat), 33W(boat)

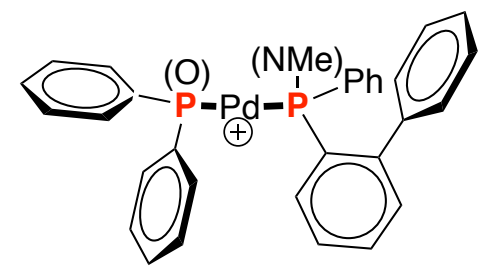

$33 \mathrm{M}$ (half-chair), 33W(half-chair)

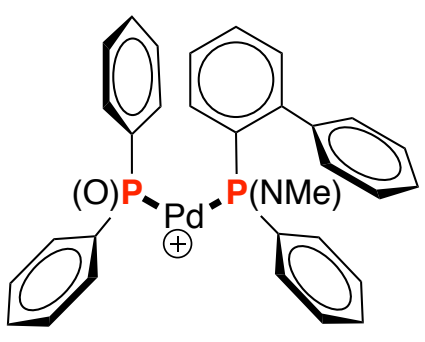

34M(boat), 34W(boat)

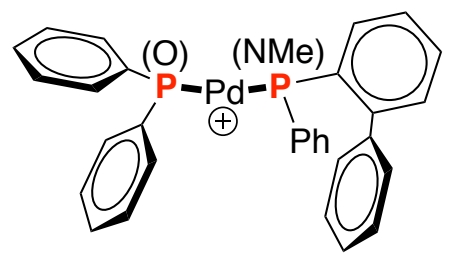

34M(half-chair), 34W(chair)

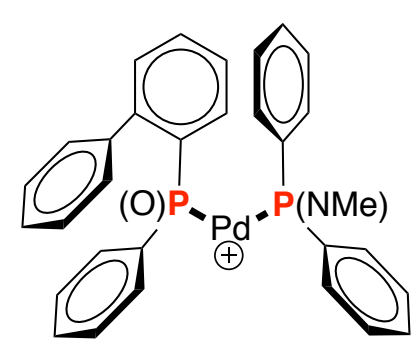

35M(boat), 35W(boat)

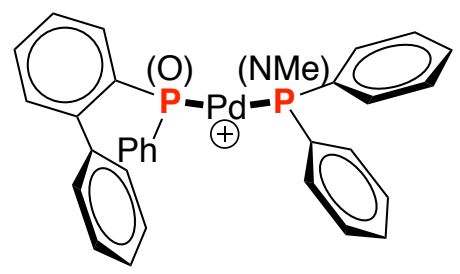

35M(half-chair), 35W(half-chair)

Figure S22. Starting configuration of investigated structures before optimisation. 
(a)

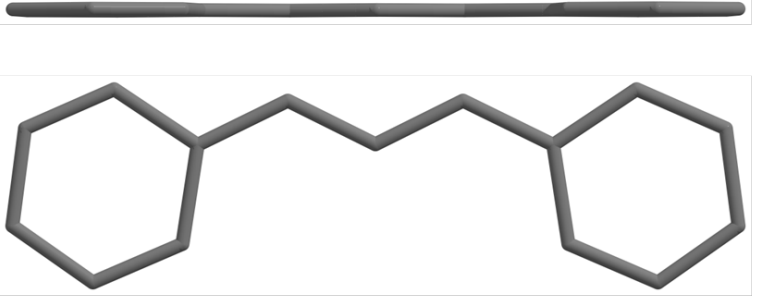

Total Energy : -15767.1903 eV

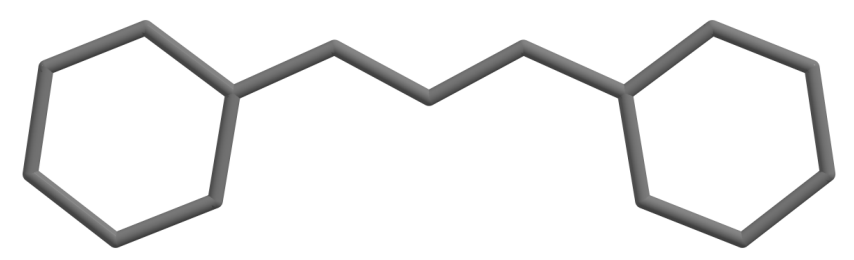

Total Energy: -15766.1582 eV

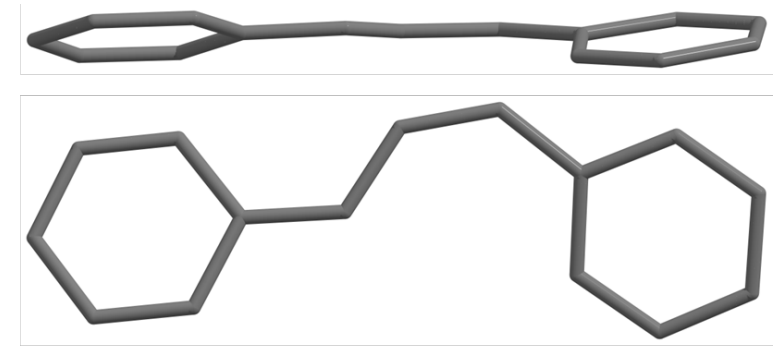

Total Energy : $-15767.0438 \mathrm{eV}$

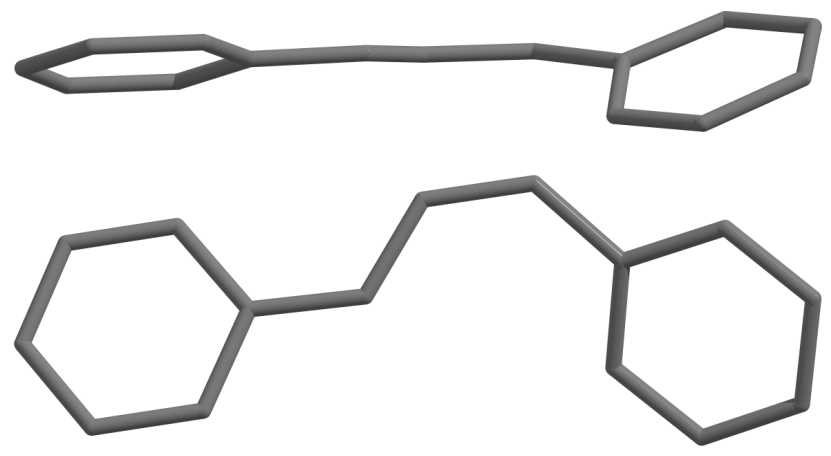

Total Energy: -15765.9902 eV

Figure S23. Optimised structure and total energies of geometrical isomers of the allyl moiety: (a) cationic form; (b) neutral form, doublet optimization

Table S61. Selected computed parameters

\begin{tabular}{|c|c|c|c|c|c|c|c|c|}
\hline \multirow{2}{*}{ Complexes } & \multirow{2}{*}{$\begin{array}{c}\text { Total energy } \\
(\mathrm{eV})\end{array}$} & \multirow{2}{*}{$\begin{array}{c}\Delta \\
\left(\mathrm{kJmol}^{-1}\right)\end{array}$} & \multicolumn{4}{|c|}{ Selected bond length $(\AA ̊)$} & \multirow{2}{*}{$\begin{array}{c}\text { HOMO } \\
(\mathrm{eV})\end{array}$} & \multirow{2}{*}{$\begin{array}{l}\text { LUMO } \\
\text { (eV) }\end{array}$} \\
\hline & & & $\mathbf{P d}-\mathbf{P}_{\mathrm{N}}$ & $\mathbf{P d}-\mathbf{P}_{0}$ & Pd-C ${ }_{3}$ & Pd-C 1 & & \\
\hline 33M(boat) & -65202.8789 & 2.5 & 2.49484 & 2.44757 & 2.36703 & 2.27063 & -7.99 & -4.74 \\
\hline $33 \mathbf{W}$ (boat) & -65202.9047 & - & 2.49481 & 2.44789 & 2.33082 & 2.28902 & -7.99 & -4.70 \\
\hline $\begin{array}{l}\text { 33M(half- } \\
\text { chair) }\end{array}$ & -65202.7369 & 16.1 & 2.47961 & 2.45406 & 2.33108 & 2.28947 & -8.16 & -4.71 \\
\hline $\begin{array}{c}\text { 33W(half- } \\
\text { chair) }\end{array}$ & -65202.7741 & 12.5 & 2.48007 & 2.45195 & 2.32068 & 2.29145 & -8.14 & -4.69 \\
\hline 34M(boat) & -65202.6722 & 2.0 & 2.51002 & 2.45732 & 2.24787 & 2.35432 & -8.04 & -4.77 \\
\hline 34W(boat) & -65202.6928 & - & 2.51998 & 2.44854 & 2.35003 & 2.26769 & -8.02 & -4.68 \\
\hline $\begin{array}{l}\text { 34M(half- } \\
\text { chair) }\end{array}$ & -65202.6132 & 7.6 & 2.52978 & 2.44222 & 2.41670 & 2.24296 & -7.98 & -4.79 \\
\hline $\begin{array}{l}\text { 34W (half- } \\
\text { chair) }\end{array}$ & -65202.6928 & - & 2.52004 & 2.44879 & 2.34958 & 2.26783 & -8.02 & -4.68 \\
\hline 35M(boat) & -65202.7893 & 3.2 & 2.46243 & 2.49082 & 2.27871 & 2.37415 & -8.16 & -4.75 \\
\hline 35W(boat) & -65202.8227 & - & 2.46088 & 2.47611 & 2.27178 & 2.32581 & -8.16 & -4.65 \\
\hline $\begin{array}{l}\text { 35M(half- } \\
\text { chair) }\end{array}$ & -65202.7659 & 5.5 & 2.46775 & 2.47767 & 2.30622 & 2.31124 & -8.14 & -4.68 \\
\hline $\begin{array}{l}\text { 35W(half- } \\
\text { chair) }\end{array}$ & -65202.8227 & 0.0 & 2.46091 & 2.47605 & 2.27193 & 2.32565 & -8.16 & -4.65 \\
\hline
\end{tabular}




\section{Fukui index calculation}

$E(N), E(N-1)$ and $E(N+1)$ are respectively the energy of the neutral, cationic and anionic systems ( $N$ being the number of electrons in the system). $I$ is the potential of the first vertical ionization and $A$ represent the electronic affinity of the molecule. $S$ stands for the global softness of the system.

Table S62. Energies of neutral $(N)$, cationic $(N-1)$, and anionic $(N+1)$ systems, ionisation potentials $I$, electron affinities $A$ and global softnesses $S$ for neutral systems.

\begin{tabular}{|c|c|c|c|c|c|c|}
\hline Complexe & $E(N)(e V)$ & $E(N-1)(e V)$ & $E(N+1)(e V)$ & $I(\mathrm{eV})$ & $A(\mathrm{eV})$ & $S\left(\mathrm{eV}^{-1}\right)$ \\
\hline $33 \mathrm{M}$ (boat) & -65202.8789 & -65193.8871 & -65206.5676 & 8.9917 & 3.6888 & 139.6262 \\
\hline $33 \mathbf{W}$ (boat) & -65202.9047 & -65193.9066 & -65206.5586 & 8.9983 & 3.6539 & 138.5527 \\
\hline $\begin{array}{l}33 M \text { (half- } \\
\text { chair) } \\
33 W \text { (half- }\end{array}$ & -65202.7369 & -65193.5888 & -65206.3933 & 9.1479 & 3.6564 & 134.8340 \\
\hline chair) & -65202.7741 & -65193.6441 & -65206.4067 & 9.1300 & 3.6327 & 134.6936 \\
\hline 34M(boat) & -65202.6722 & -65193.6233 & -65206.3648 & 9.0489 & 3.6926 & 138.2401 \\
\hline $34 \mathbf{W}$ (boat) & -65202.6928 & -65193.6645 & -65206.3071 & 9.0285 & 3.6142 & 136.7644 \\
\hline $\begin{array}{l}\text { 34M(half- } \\
\text { chair) }\end{array}$ & -65202.6132 & -65193.6167 & -65206.3319 & 12.7153 & 3.7187 & 82.3049 \\
\hline $\begin{array}{l}34 \mathbf{W} \text { (half- } \\
\text { chair) }\end{array}$ & -65202.6928 & -65193.6644 & -65206.3070 & 9.0285 & 3.6142 & 136.7592 \\
\hline $35 \mathrm{M}$ (boat) & -65202.7893 & -65193.6134 & -65206.4820 & 9.1760 & 3.6929 & 135.0449 \\
\hline $35 \mathrm{~W}$ (boat) & -65202.8227 & -65193.6900 & -65206.4100 & 9.1327 & 3.5873 & 133.5252 \\
\hline $\begin{array}{l}35 \mathrm{M} \text { (half- } \\
\text { chair) }\end{array}$ & -65202.7659 & -65193.6500 & -65206.3830 & 9.1158 & 3.6172 & 134.6591 \\
\hline $\begin{array}{l}35 W \text { (half- } \\
\text { chair) }\end{array}$ & -65202.8227 & -65193.6900 & -65206.4100 & 9.1327 & 3.5873 & 133.5249 \\
\hline
\end{tabular}


$q_{k}(N), q_{k}(N-1)$ and $q_{k}(N+1)$ are the gross electronic population of a site $k$ of the neutral, cationic and anionic systems.

Table S63. Gross electronic population for the C1 and C3 atoms of the neutral, cationic and anionic systems.

\begin{tabular}{|c|c|c|c|c|}
\hline Complexe & $\boldsymbol{k}$ & $q_{k}(N)$ & $q_{k}(N-1)$ & $q_{k}(N+1)$ \\
\hline \multirow[t]{2}{*}{ 33M(boat) } & $\mathrm{C} 1$ & 6.15511 & 6.15185 & 6.18755 \\
\hline & C3 & 6.10063 & 6.13068 & 6.17340 \\
\hline \multirow[t]{2}{*}{ 33W(boat) } & $\mathrm{C} 1$ & 6.14536 & 6.14381 & 6.18296 \\
\hline & C3 & 6.08031 & 6.14163 & 6.18057 \\
\hline \multirow[t]{2}{*}{ 33M(half-chair) } & $\mathrm{C} 1$ & 6.13962 & 6.14738 & 6.18222 \\
\hline & C3 & 6.11631 & 6.13380 & 6.17335 \\
\hline \multirow[t]{2}{*}{ 33W(half-chair) } & $\mathrm{C} 1$ & 6.14130 & 6.14434 & 6.18057 \\
\hline & $\mathrm{C} 3$ & 6.05814 & 6.13549 & 6.17131 \\
\hline \multirow[t]{2}{*}{ 34M(boat) } & $\mathrm{C} 1$ & 6.13238 & 6.11325 & 6.16020 \\
\hline & C3 & 6.10700 & 6.15709 & 6.18737 \\
\hline \multirow[t]{2}{*}{ 34W(boat) } & $\mathrm{C} 1$ & 6.07051 & 6.13839 & 6.17847 \\
\hline & C3 & 6.11065 & 6.12552 & 6.16415 \\
\hline \multirow[t]{2}{*}{ 34M(half-chair) } & $\mathrm{C} 1$ & 6.12880 & 6.15726 & 6.18886 \\
\hline & $\mathrm{C} 3$ & 6.11294 & 6.10401 & 6.14744 \\
\hline \multirow[t]{2}{*}{ 34W(half-chair) } & $\mathrm{C} 1$ & 6.07189 & 6.13832 & 6.17843 \\
\hline & C3 & 6.10318 & 6.12560 & 6.16425 \\
\hline \multirow[t]{2}{*}{ 35M(boat) } & C1 & 6.14501 & 6.12171 & 6.16053 \\
\hline & $\mathrm{C} 3$ & 6.10441 & 6.15667 & 6.18801 \\
\hline \multirow[t]{2}{*}{ 35W(boat) } & $\mathrm{C} 1$ & 6.07331 & 6.12972 & 6.16989 \\
\hline & C3 & 6.11454 & 6.14810 & 6.18300 \\
\hline \multirow[t]{2}{*}{ 35M(half-chair) } & C1 & 6.13134 & 6.13564 & 6.17333 \\
\hline & C3 & 6.09488 & 6.14706 & 6.18231 \\
\hline \multirow[t]{2}{*}{ 35W(half-chair) } & C1 & 6.06922 & 6.12978 & 6.16994 \\
\hline & C3 & 6.11444 & 6.14807 & 6.18297 \\
\hline
\end{tabular}


$f_{\mathrm{k}^{ \pm}}$is the condensed Fukui functions. The higher the value of $f_{\mathrm{k}^{ \pm}}$for a site $k$ over another, the higher is the reactivity of this site $k$ aver the other. Minus sign (-) stands for electrophilic attack and plus sign (+) for nucleophilic attack. $S_{\mathrm{k}}{ }^{ \pm}$is the condensed local softness and is obtained from the global softness $S$ and the condensed Fukui functions.

$f_{k}^{+}=\left[q_{k}(N+1)-q_{k}(N)\right] \quad$ and $f_{k}^{-}=\left[q_{k}(N)-q_{k}(N-1)\right], s_{k}^{+}=S f_{k}^{+}$and $s_{k}^{-}=S f_{k}^{-}$

Table S64. Condensed Fukui functions and condensed local softnesses for the C1 and C3 atoms.

\begin{tabular}{|c|c|c|c|c|c|c|c|c|}
\hline Complexes & $f_{1}{ }^{+}$ & $f_{1}-$ & $f_{3}{ }^{+}$ & $f_{3}$ & $S_{1_{1}}^{+}$ & $s_{1^{-}}$ & $S_{3}{ }^{+}$ & $S_{3}{ }^{-}$ \\
\hline 33M(boat) & 0.0324 & 0.0033 & 0.0728 & -0.0301 & 0.1665 & 0.0167 & 0.3734 & -0.1542 \\
\hline 33W(boat) & 0.0376 & 0.0015 & 0.1003 & -0.0613 & 0.1914 & 0.0079 & 0.5105 & -0.3122 \\
\hline 33M(half-chair & 0.0426 & -0.0078 & 0.0570 & -0.0175 & 0.2111 & -0.0385 & 0.2826 & -0.0867 \\
\hline 33w (half-chai & 0.0393 & -0.0030 & 0.1132 & -0.0774 & 0.1944 & -0.0150 & 0.5602 & -0.3829 \\
\hline 34M(boat) & 0.0278 & 0.0191 & 0.0804 & -0.0501 & 0.1413 & 0.0972 & 0.4083 & -0.2545 \\
\hline 34W(boat) & 0.1080 & -0.0679 & 0.0535 & -0.0149 & 0.5426 & -0.3412 & 0.2689 & -0.0747 \\
\hline 34M(half-chair & 0.0601 & -0.0285 & 0.0345 & 0.0089 & 0.1817 & -0.0861 & 0.1044 & 0.0270 \\
\hline 34W(half-chai & 0.1065 & -0.0664 & 0.0611 & -0.0224 & 0.5354 & -0.3339 & 0.3069 & -0.1127 \\
\hline $35 \mathrm{M}$ (boat) & 0.0155 & 0.0233 & 0.0836 & -0.0523 & 0.0770 & 0.1156 & 0.4149 & -0.2594 \\
\hline 35W(boat) & 0.0966 & -0.0564 & 0.0685 & -0.0336 & 0.4739 & -0.2768 & 0.3359 & -0.1647 \\
\hline $35 \mathrm{M}$ (half-chair & 0.0420 & -0.0043 & 0.0874 & -0.0522 & 0.2078 & -0.0213 & 0.4327 & -0.2582 \\
\hline 35W(half-chai & 0.1007 & -0.0606 & 0.0685 & -0.0336 & 0.4942 & -0.2972 & 0.3363 & -0.1650 \\
\hline
\end{tabular}




\section{Structures, representation of frontier MOs HOMO/LUMO, atomic contributions at the $C 1$ and $C 3$ positions of the complexed $\pi$-allyl group}

\section{Structure 33M(boat)}
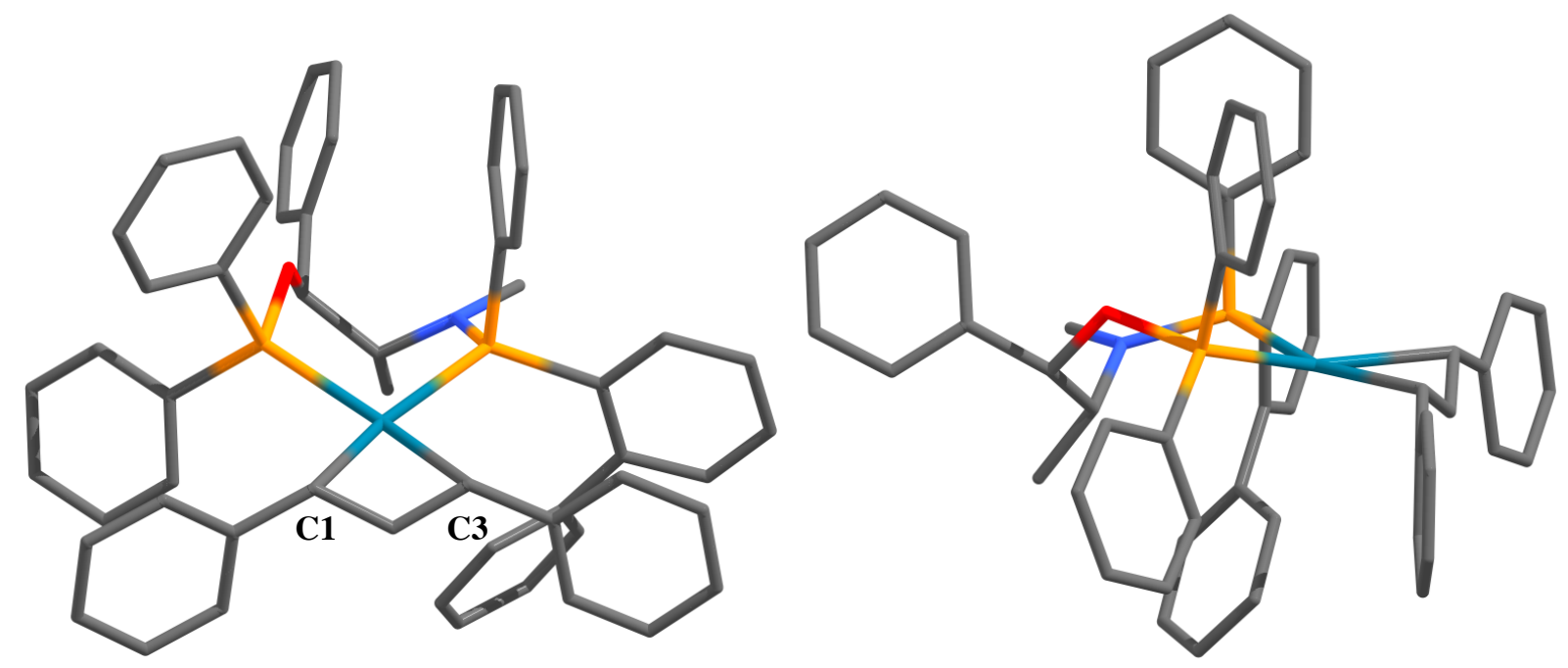

Figure S24. Optimised structure of $33 \mathbf{M}$ (boat) in the ground state.

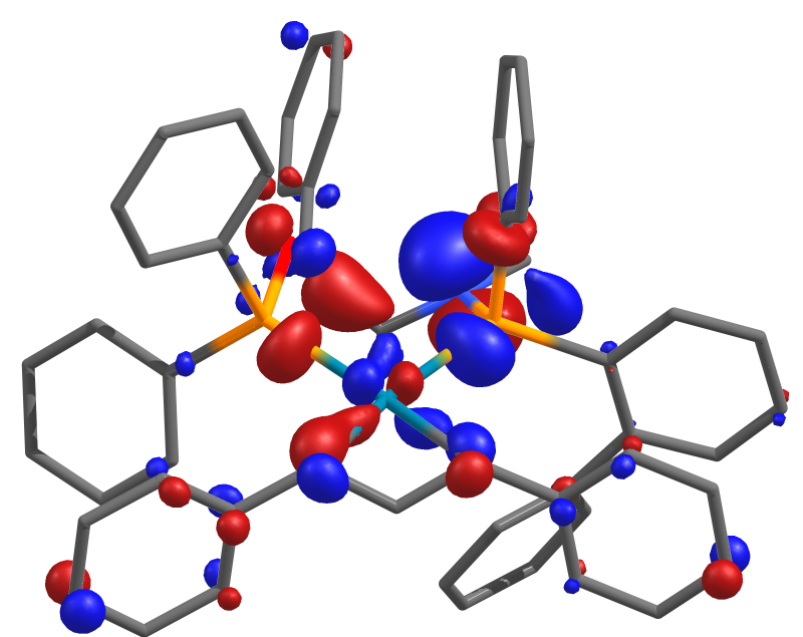

HOMO

$(-7.99 \mathrm{eV})$

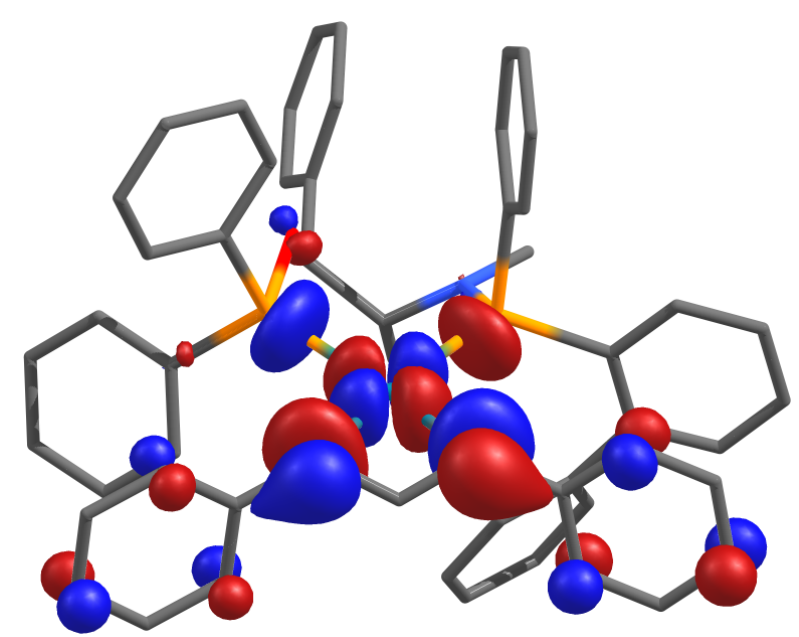

LUMO

$(-4.74 \mathrm{eV})$

Figure S25. Representations of the frontier MOs HOMO and LUMO of $33 \mathbf{M}$ (boat) in the ground state.

Table S65. Atomic contributions of HOMO and LUMO of 33M(boat) in the ground state at the C1 and C3 positions of the complexed $\pi$-allyl group.

\begin{tabular}{lcc}
\hline Fragment & HOMO & LUMO \\
\hline Energy $(\mathrm{eV})$ & -7.99 & -4.74 \\
\hline C1 $(\%)$ & 2.97 & 10.71 \\
C3 $(\%)$ & 2.29 & 13.79 \\
\hline
\end{tabular}




\section{Structure 33W(boat)}
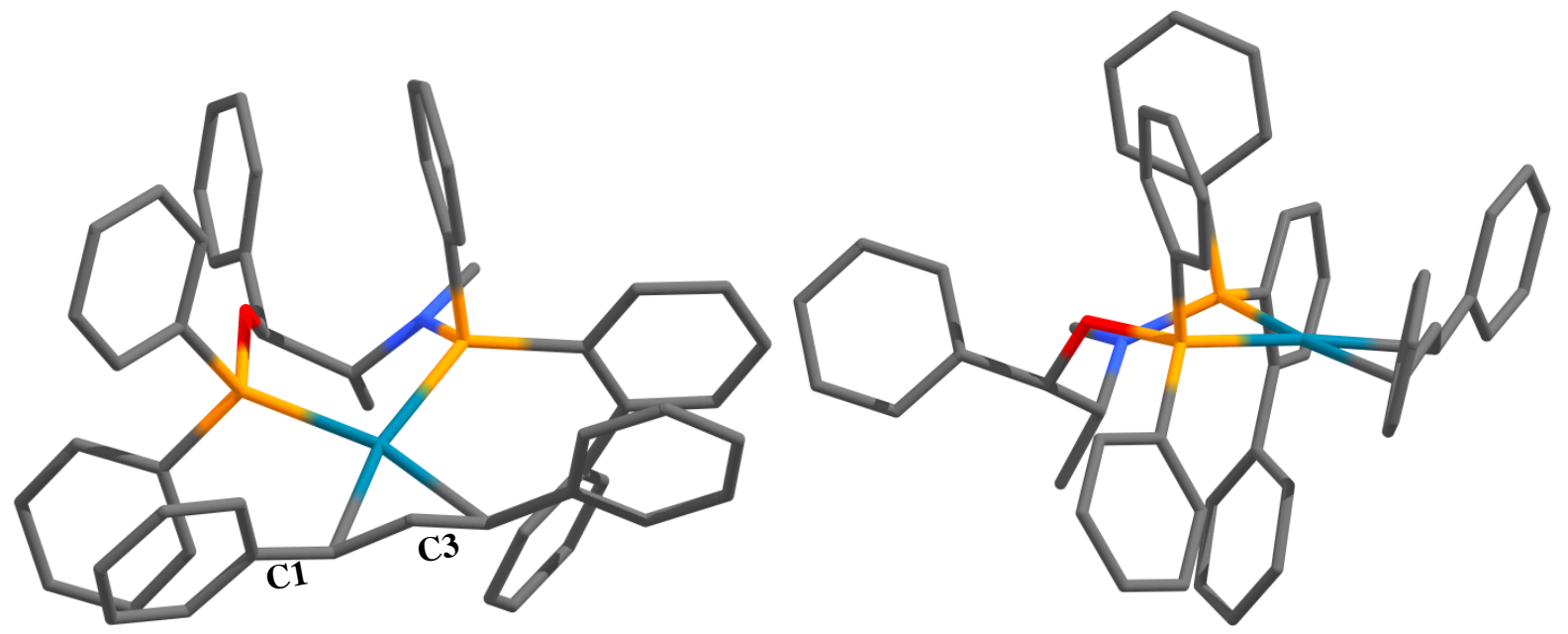

Figure S26. Optimised structure of $\mathbf{3 3} \mathbf{W}$ (boat) in the ground state.

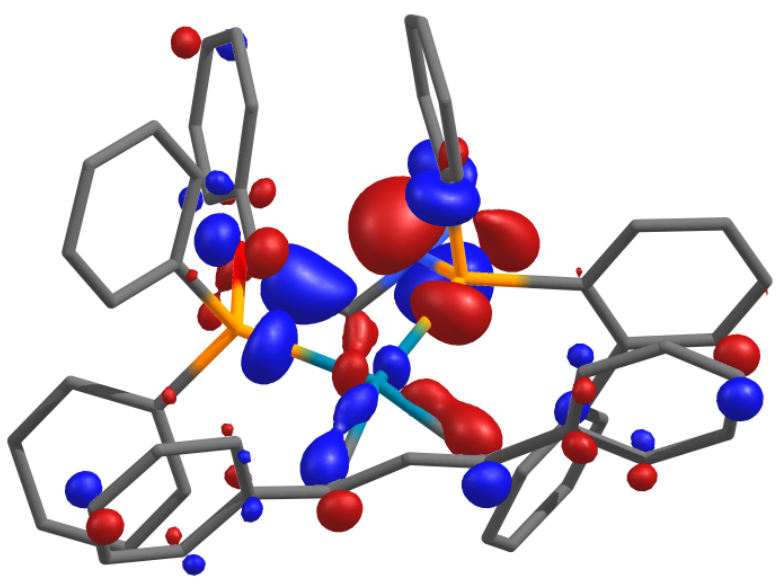

HOMO

$(-7.99 \mathrm{eV})$

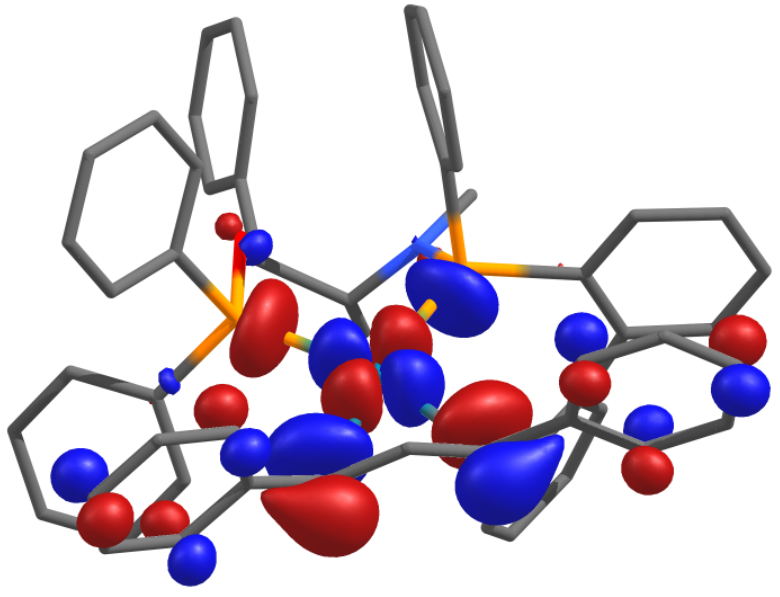

LUMO

$(-4.70 \mathrm{eV})$

Figure S27. Representations of the frontier MOs HOMO and LUMO of $\mathbf{3 3} \mathbf{W}$ (boat) in the ground state.

Table S66. Atomic contributions of HOMO and LUMO of $33 \mathbf{W}$ (boat) in the ground state at the C1 and C3 positions of the complexed $\pi$-allyl group.

\begin{tabular}{lcc}
\hline \multicolumn{1}{r}{ Fragment } & HOMO & LUMO \\
\hline Energy $(\mathrm{eV})$ & -7.99 & -4.70 \\
\hline C1 $(\%)$ & 2.56 & 11.90 \\
C3 $(\%)$ & 2.34 & 12.25 \\
\hline
\end{tabular}




\section{Structure 33M(half-chair)}

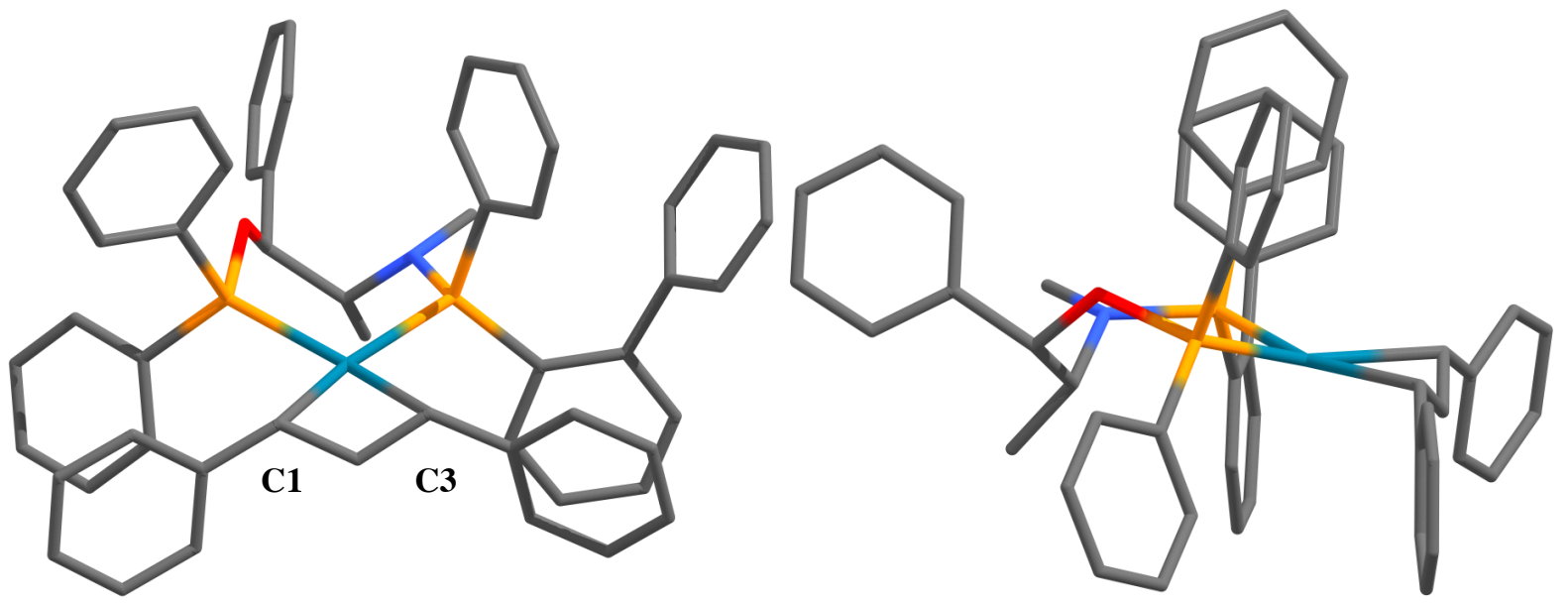

Figure S28. Optimised structure of 33M(half-chair) in the ground state.

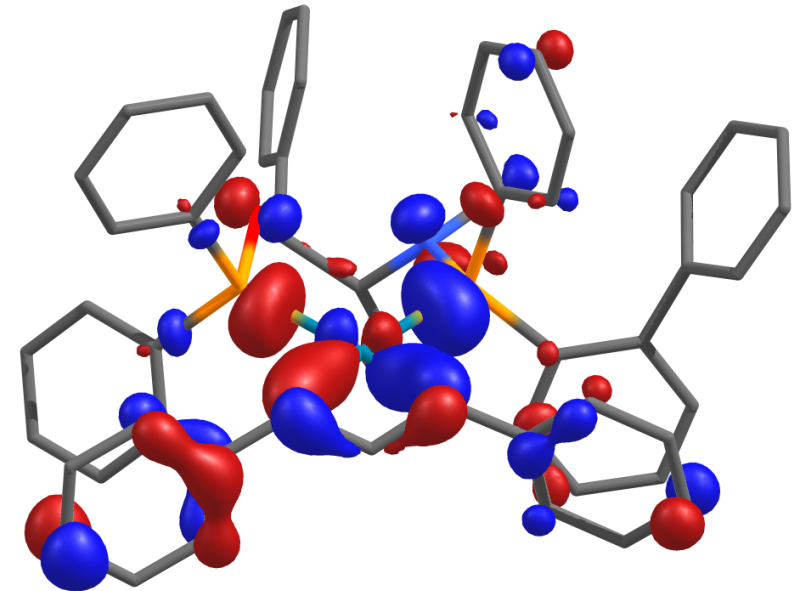

HOMO

$(-8.16 \mathrm{eV})$

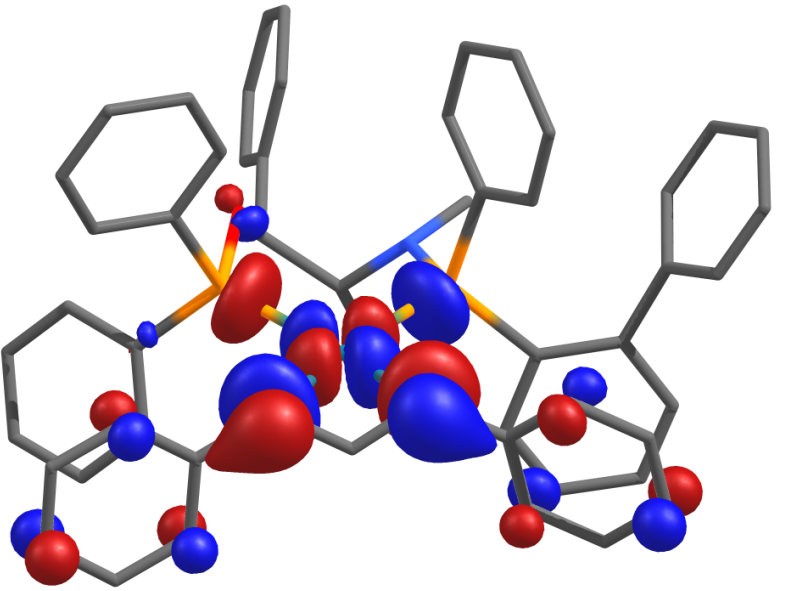

LUMO

$(-4.71 \mathrm{eV})$

Figure S29. Representations of the frontier MOs HOMO and LUMO of 33M(half-chair) in the ground state.

Table S67. Atomic contributions of HOMO and LUMO of 33M(half-chair) in the ground state at the C1 and C3 positions of the complexed $\pi$-allyl group.

\begin{tabular}{lcc}
\hline \multicolumn{1}{c}{ Fragment } & HOMO & LUMO \\
\hline Energy $(\mathrm{eV})$ & -8.16 & -4.71 \\
\hline C1 $(\%)$ & 4.78 & 11.22 \\
C3 $(\%)$ & 3.95 & 12.70 \\
\hline
\end{tabular}




\section{Structure 33W(half-chair)}

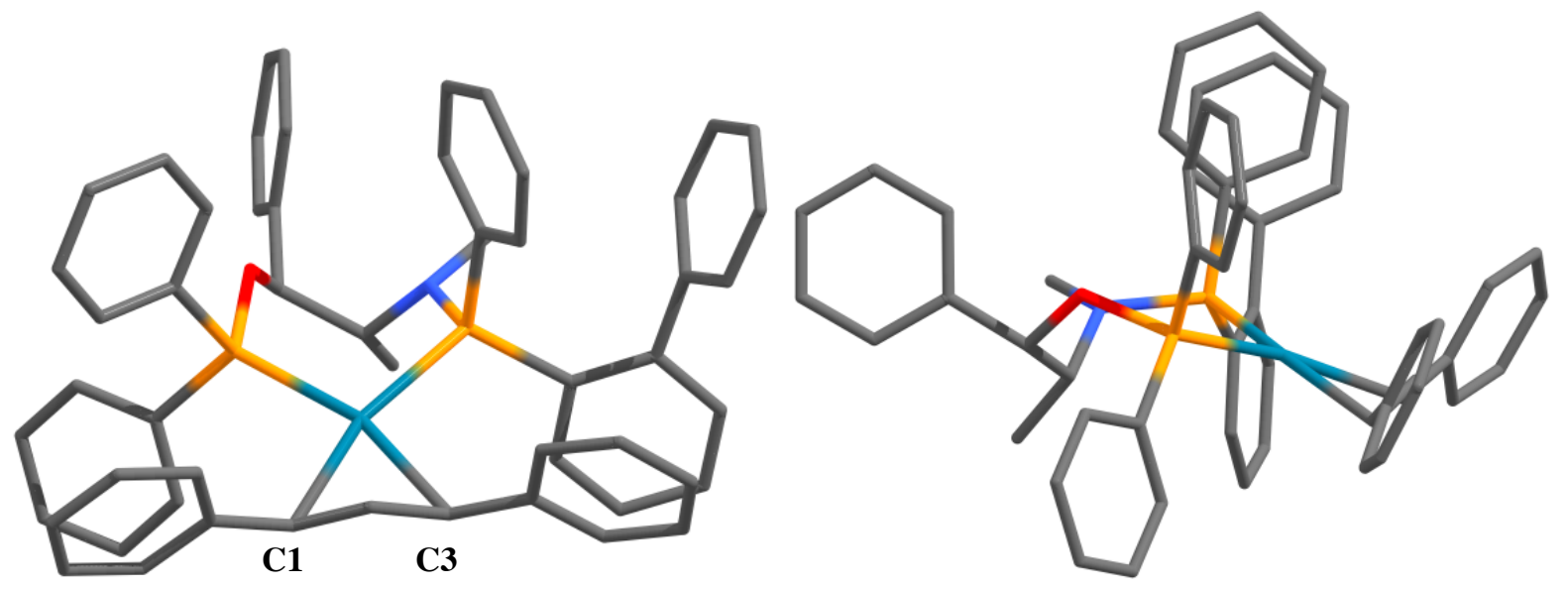

Figure S30. Optimised structure of $\mathbf{3 3 W}$ (half-chair) in the ground state.

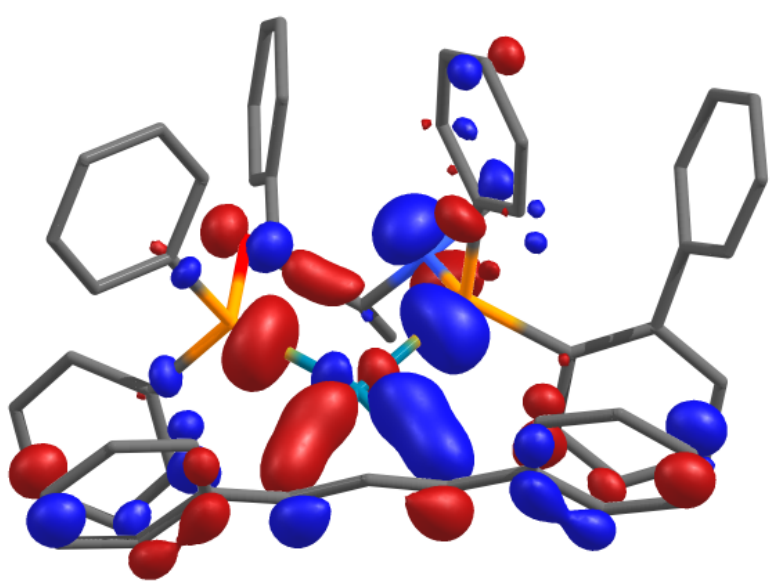

HOMO

$(-8.14 \mathrm{eV})$

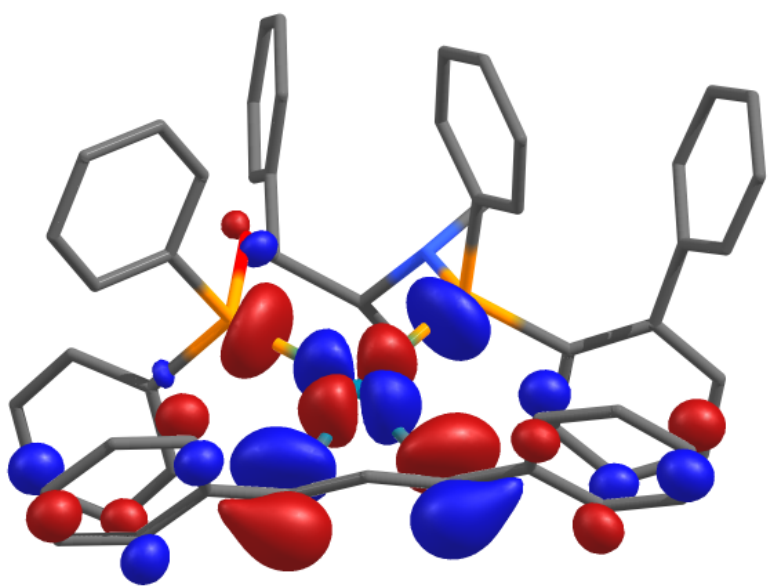

LUMO

$(-4.69 \mathrm{eV})$

Figure S31. Representations of the frontier MOs HOMO and LUMO of $\mathbf{3 3} \mathbf{W}$ (half-chair) in the ground state.

Table S68. Atomic contributions of HOMO and LUMO of 33W(half-chair) in the ground state at the C1 and C3 positions of the complexed $\pi$-allyl group.

\begin{tabular}{lcc}
\hline \multicolumn{1}{c}{ Fragment } & HOMO & LUMO \\
\hline Energy $(\mathrm{eV})$ & -8.14 & -4.69 \\
\hline C1 $(\%)$ & 3.69 & 12.07 \\
C3 $(\%)$ & 4.29 & 11.70 \\
\hline
\end{tabular}




\section{Structure 34M(boat)}
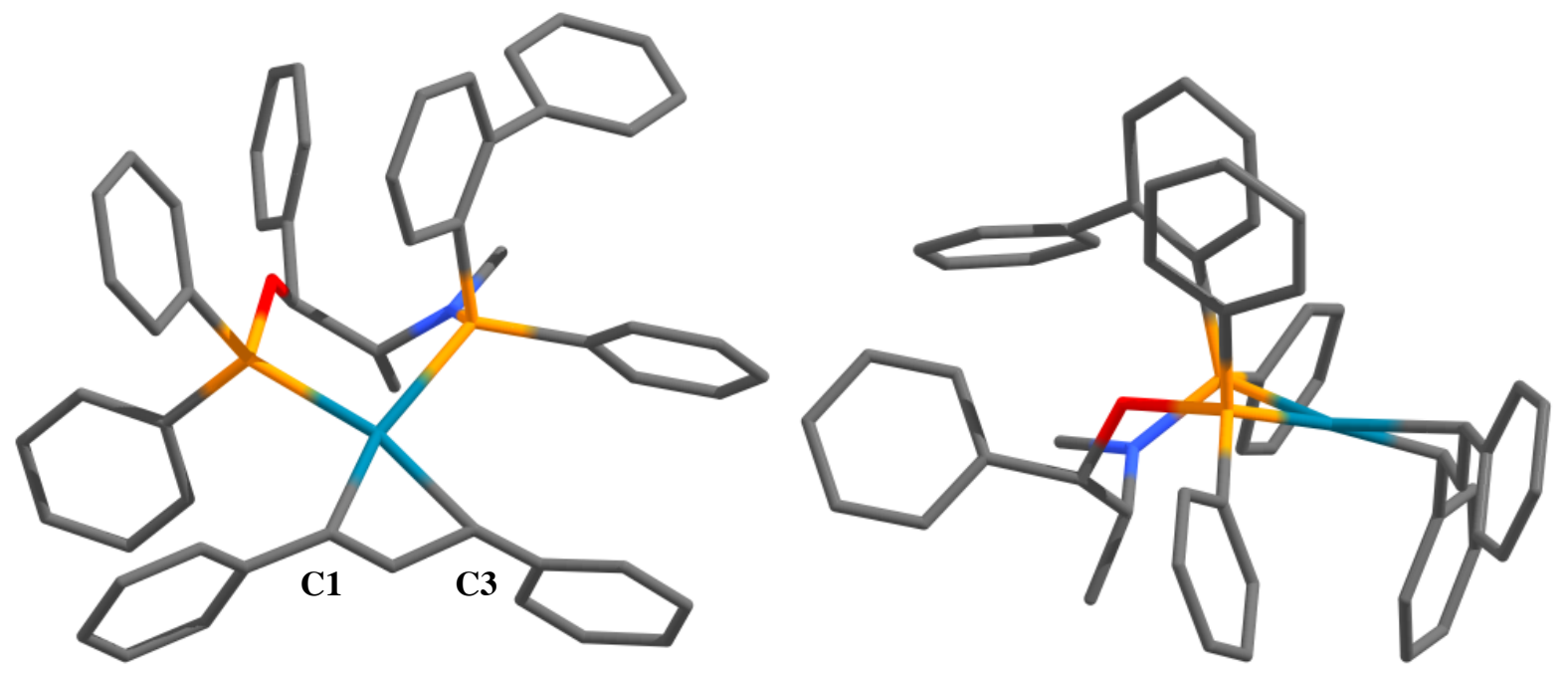

Figure S32. Optimised structure of 34M(boat) in the ground state.

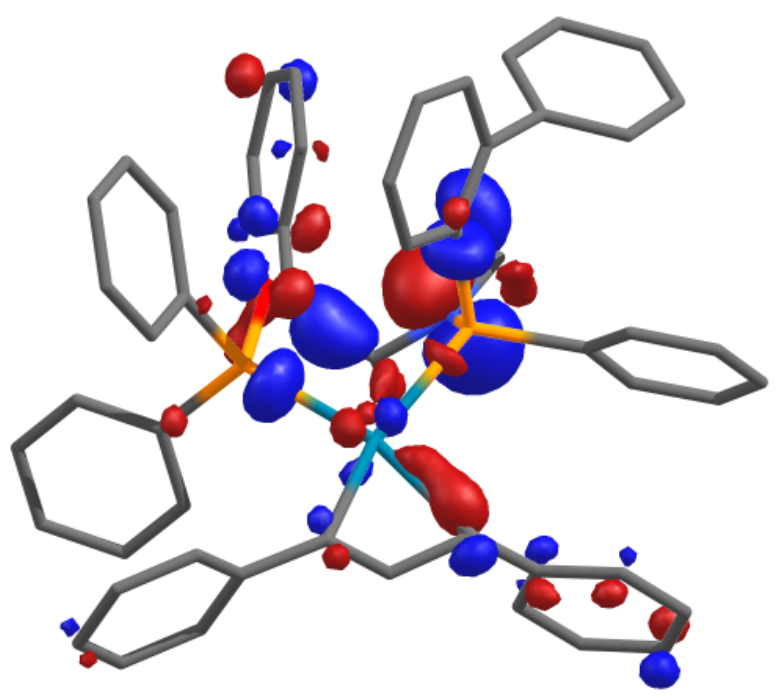

HOMO

$(-8.04 \mathrm{eV})$

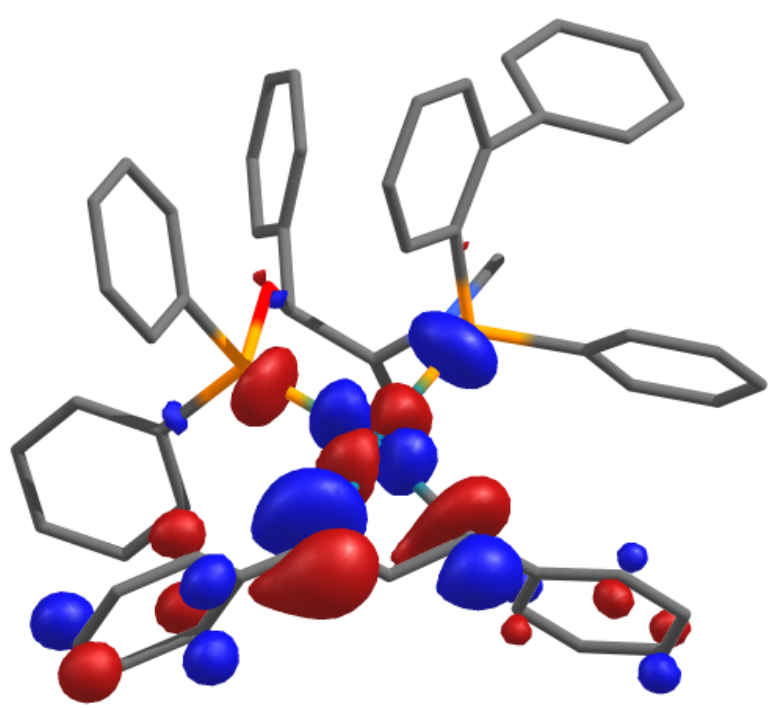

LUMO

$(-4.77 \mathrm{eV})$

Figure S33. Representations of the frontier MOs HOMO and LUMO of $34 \mathbf{M}$ (boat) in the ground state.

Table S69. Atomic contributions of HOMO and LUMO of 34M(boat) in the ground state at the C1 and C3 positions of the complexed $\pi$-allyl group.

\begin{tabular}{lcc}
\hline Fragment & HOMO & LUMO \\
\hline Energy $(\mathrm{eV})$ & -8.04 & -4.77 \\
\hline C1 $(\%)$ & 1.36 & 16.52 \\
C3 $(\%)$ & 2.40 & 9.06 \\
\hline
\end{tabular}




\section{Structure $34 \mathrm{~W}$ (boat)}
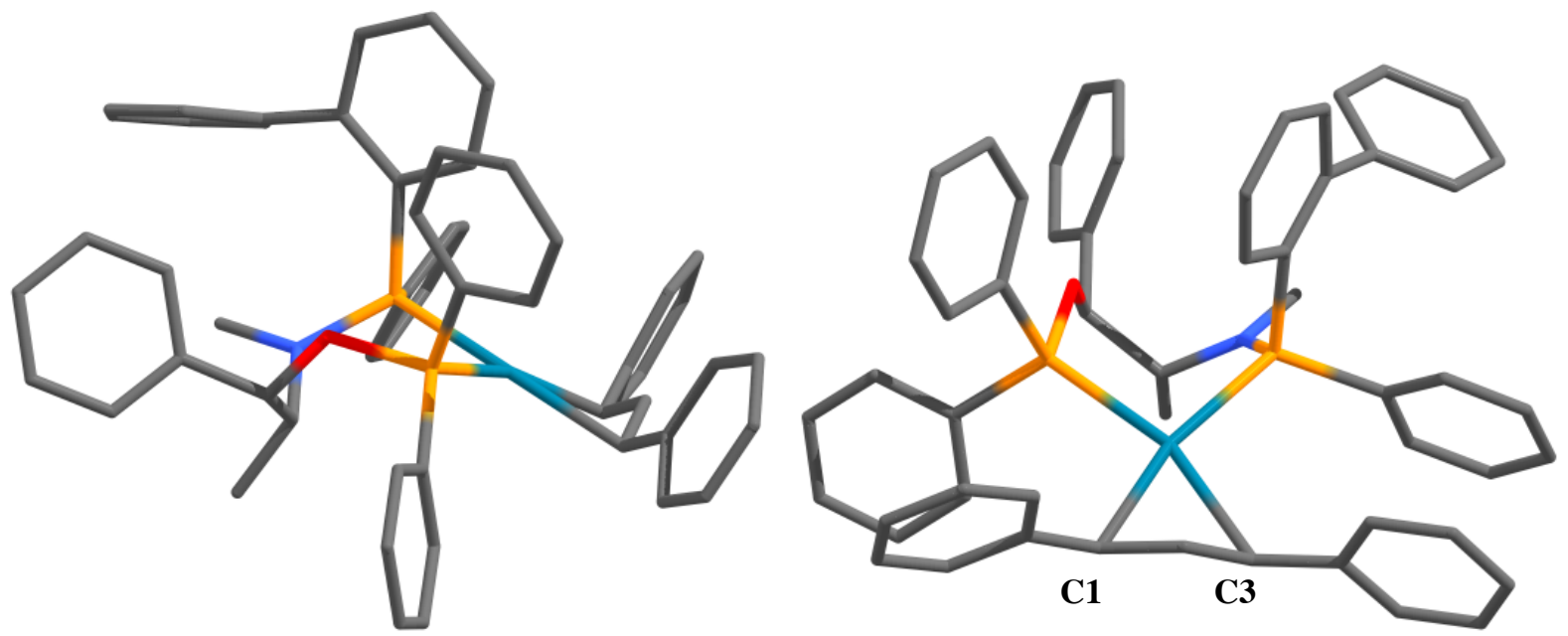

Figure S34. Optimised structure of $\mathbf{3 4 W}$ (boat) in the ground state.

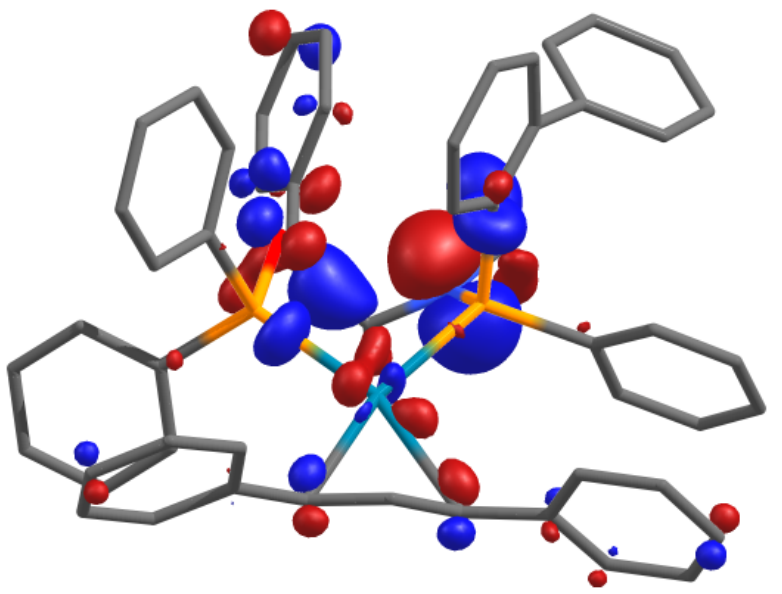

HOMO

$(-8.02 \mathrm{eV})$

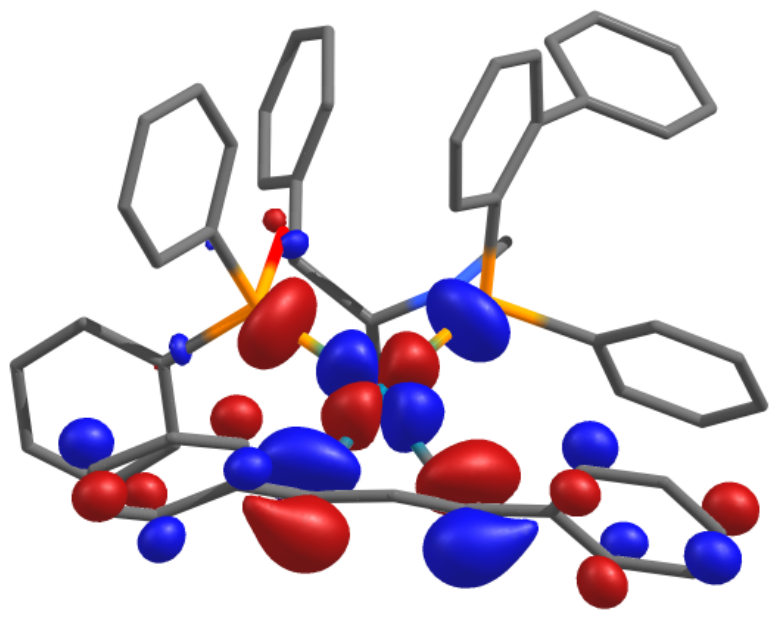

LUMO

$(-4.68 \mathrm{eV})$

Figure S35. Representations of the frontier MOs HOMO and LUMO of $\mathbf{3 4 W}$ (boat) in the ground state.

Table S70. Atomic contributions of HOMO and LUMO of $\mathbf{3 4 W}$ (boat) in the ground state at the C1 and C3 positions of the complexed $\pi$-allyl group.

\begin{tabular}{lcc}
\hline Fragment & HOMO & LUMO \\
\hline Energy $(\mathrm{eV})$ & 8.02 & 4.68 \\
\hline C1 $(\%)$ & 1.63 & 11.89 \\
C3 $(\%)$ & 1.67 & 12.73 \\
\hline
\end{tabular}




\section{Structure 34M(half-chair)}
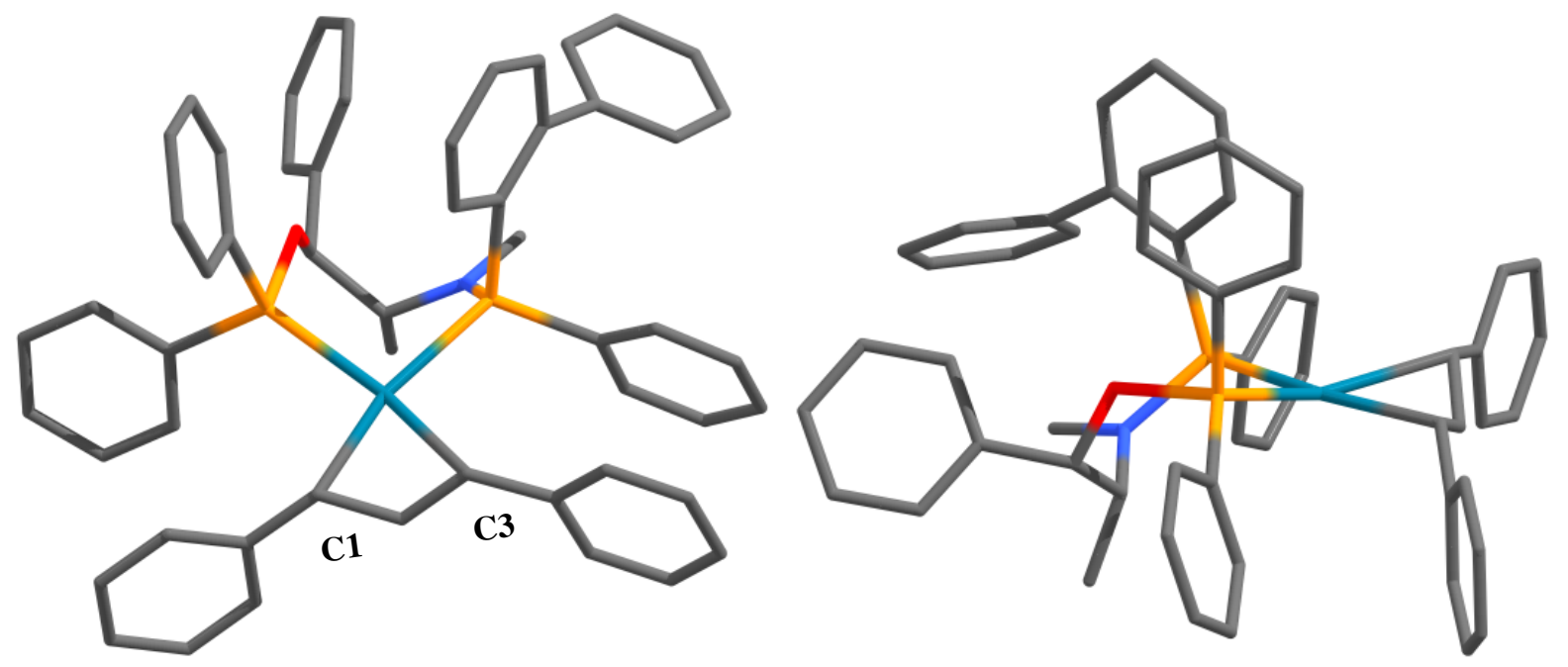

Figure S36. Optimised structure of 34M(half-chair) in the ground state.

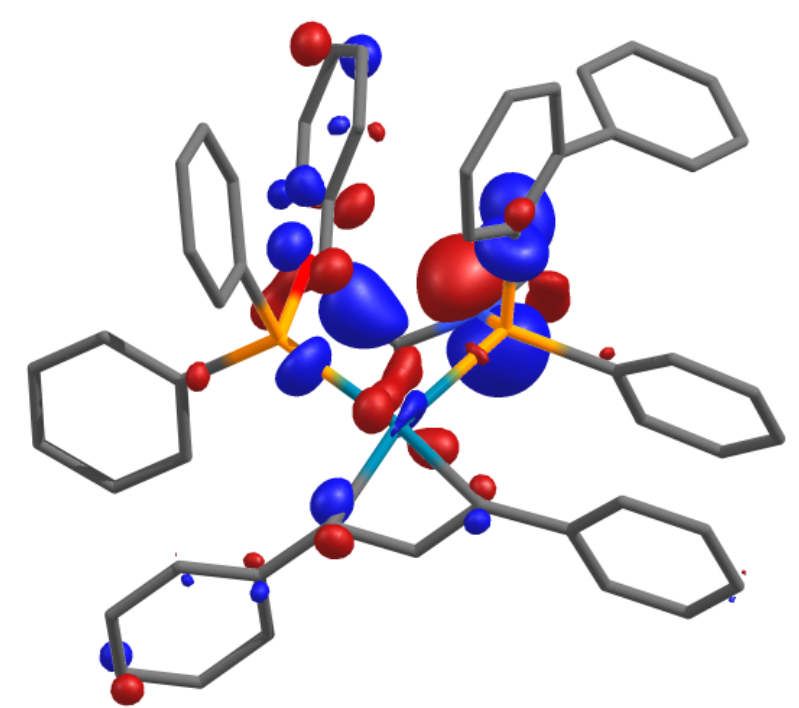

HOMO

$(-7.98 \mathrm{eV})$

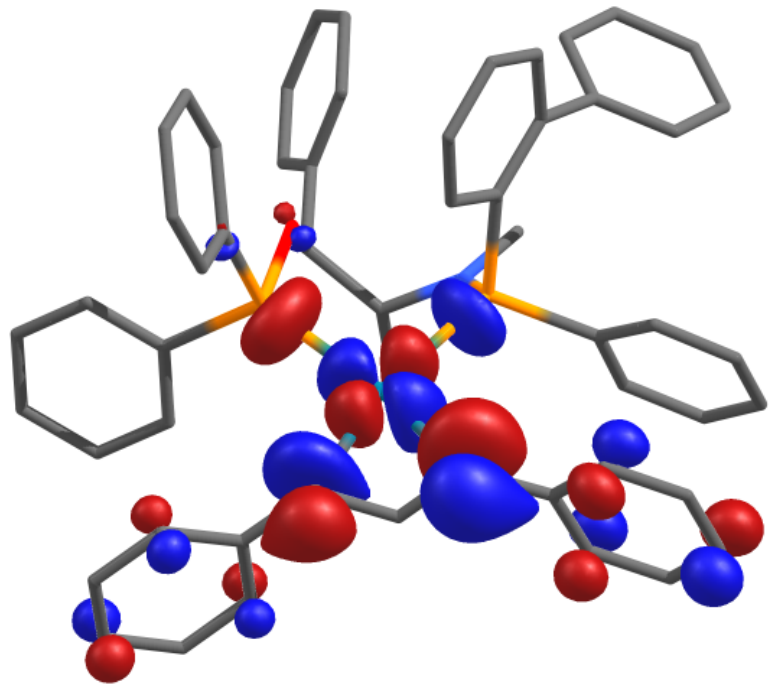

LUMO

$(-4.79 \mathrm{eV})$

Figure S37. Representations of the frontier MOs HOMO and LUMO of $34 \mathbf{M}$ (half-chair) in the ground state.

Table S71. Atomic contributions of HOMO and LUMO of $34 \mathrm{M}$ (half-chair) in the ground state at the $\mathrm{C} 1$ and $\mathrm{C} 3$ positions of the complexed $\pi$-allyl group.

\begin{tabular}{lcc}
\hline \multicolumn{1}{c}{ Fragment } & HOMO & LUMO \\
\hline Energy $(\mathrm{eV})$ & -7.98 & -4.79 \\
\hline C1 $(\%)$ & 1.90 & 9.81 \\
C3 $(\%)$ & 1.14 & 15.93 \\
\hline
\end{tabular}




\section{Structure $34 W$ (half-chair)}

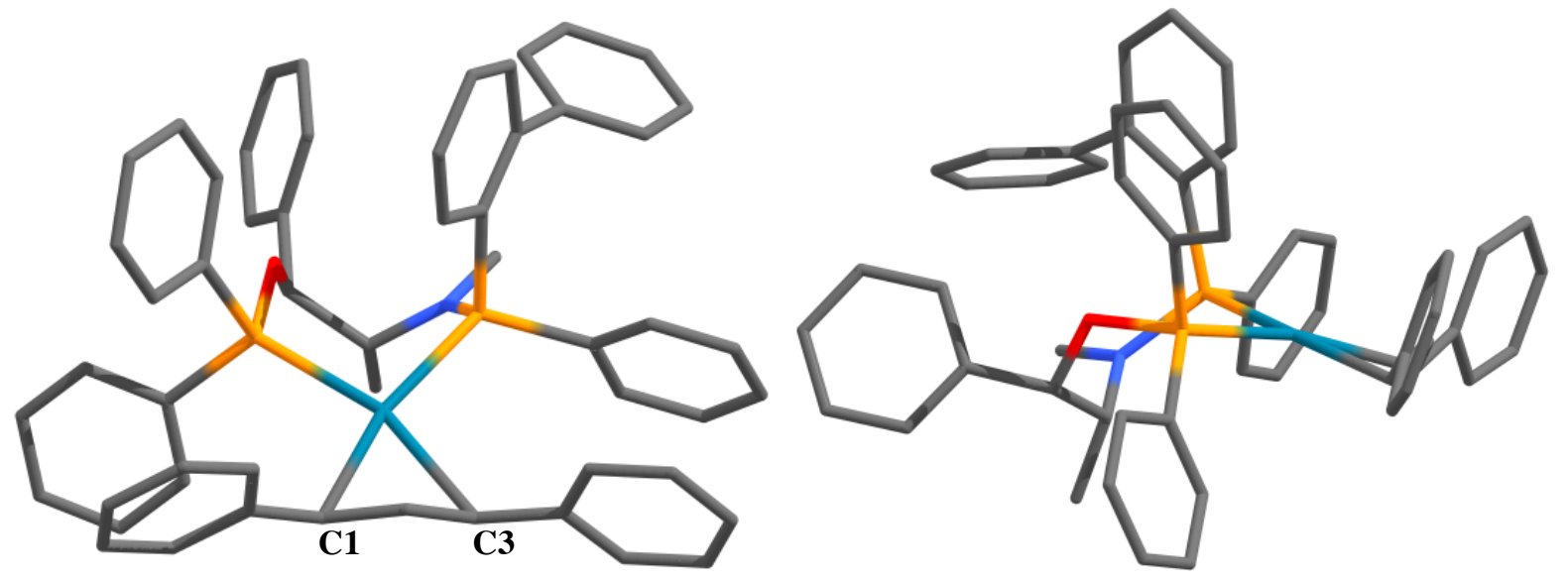

Figure S38. Optimised structure of $\mathbf{3 4 W}$ (half-chair) in the ground state.

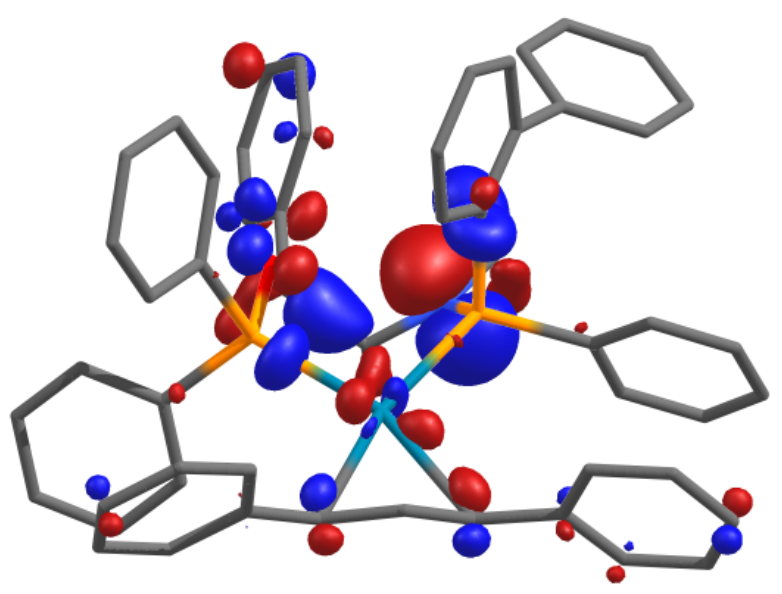

HOMO

$(-8.02 \mathrm{eV})$

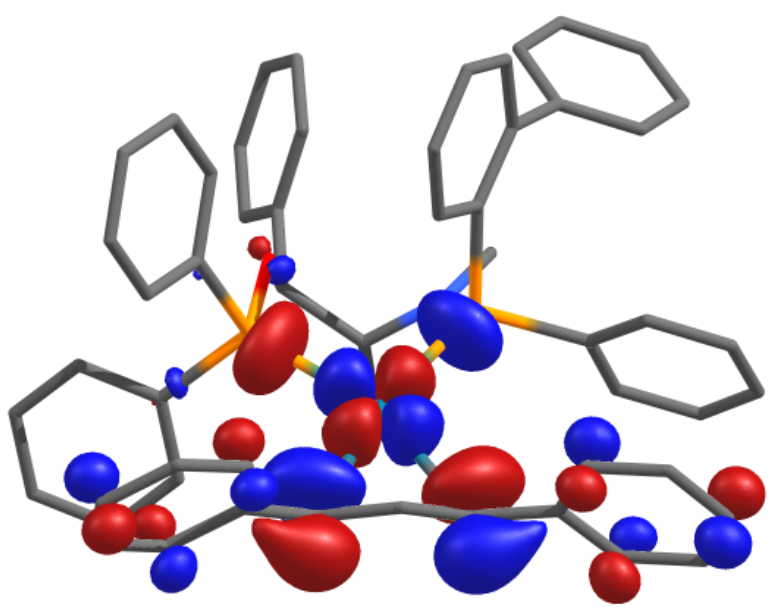

LUMO

$(-4.68 \mathrm{eV})$

Figure S39. Representations of the frontier MOs HOMO and LUMO of $34 \mathbf{W}$ (half-chair) in the ground state.

Table S72. Atomic contributions of HOMO and LUMO of $34 \mathbf{W}$ (half-chair) in the ground state at the C1 and C3 positions of the complexed $\pi$-allyl group.

\begin{tabular}{lcc}
\hline \multicolumn{1}{c}{ Fragment } & HOMO & LUMO \\
\hline Energy $(\mathrm{eV})$ & -8.02 & -4.68 \\
\hline C1 $(\%)$ & 1.66 & 12.73 \\
C3 $(\%)$ & 1.63 & 11.89 \\
\hline
\end{tabular}




\section{Structure 35M(boat)}
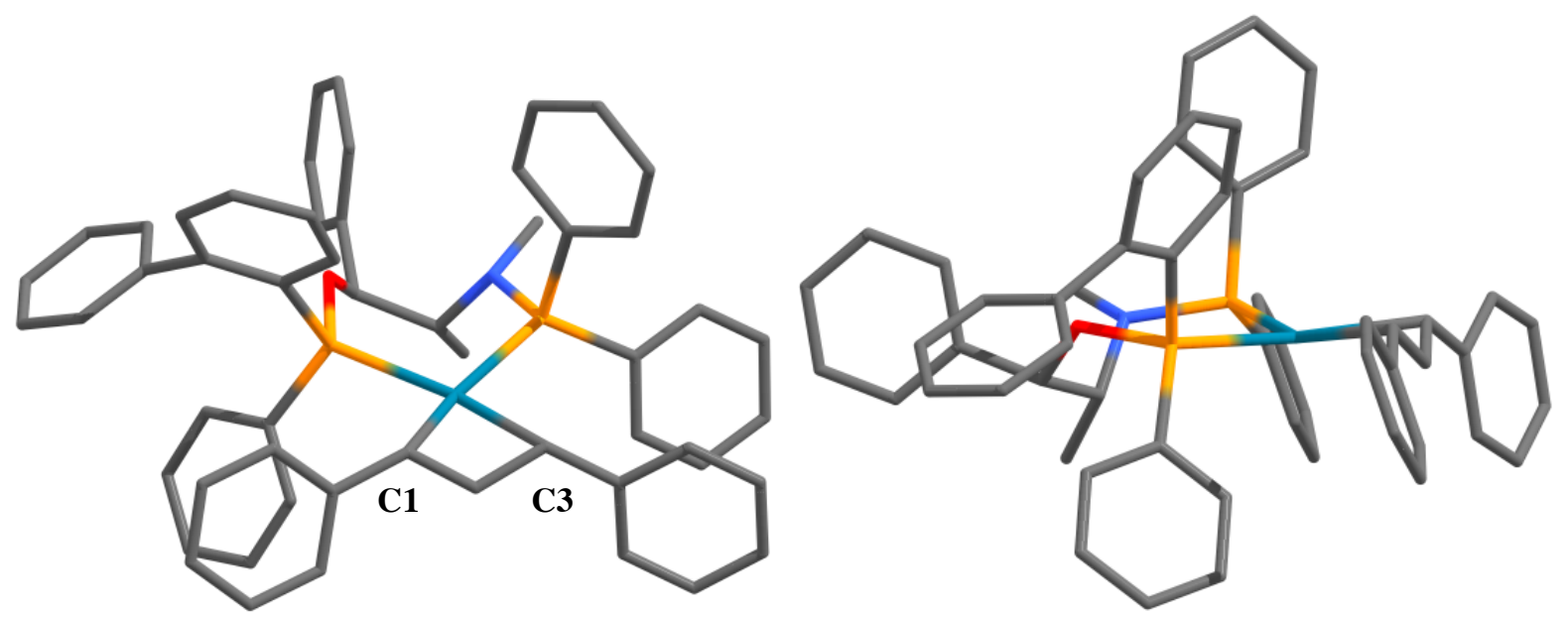

Figure S40. Optimised structure of $\mathbf{3 5 M}$ (boat) in the ground state.

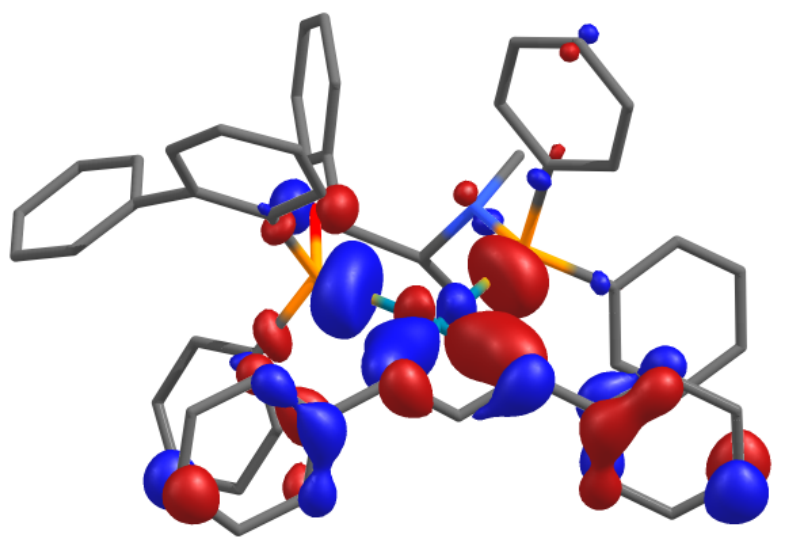

HOMO

$(-8.16 \mathrm{eV})$

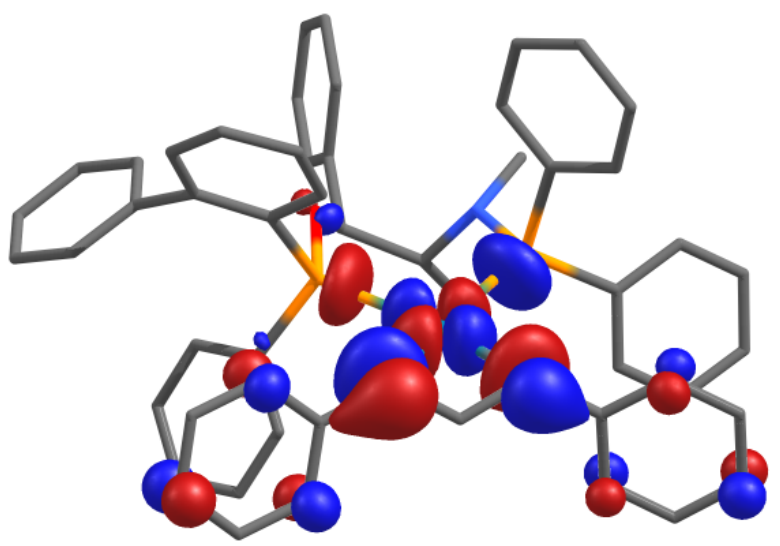

LUMO

$(-4.75 \mathrm{eV})$

Figure S41. Representations of the frontier MOs HOMO and LUMO of $\mathbf{3 5 M}$ (boat) in the ground state.

Table S73. Atomic contributions of HOMO and LUMO of 35M(boat) in the ground state at the C1 and C3 positions of the complexed $\pi$-allyl group.

\begin{tabular}{lcc}
\hline Fragment & HOMO & LUMO \\
\hline Energy $(\mathrm{eV})$ & -8.16 & -4.75 \\
\hline C1 $(\%)$ & 3.55 & 13.84 \\
C3 $(\%)$ & 4.88 & 10.94 \\
\hline
\end{tabular}




\section{Structure $35 W$ (boat)}
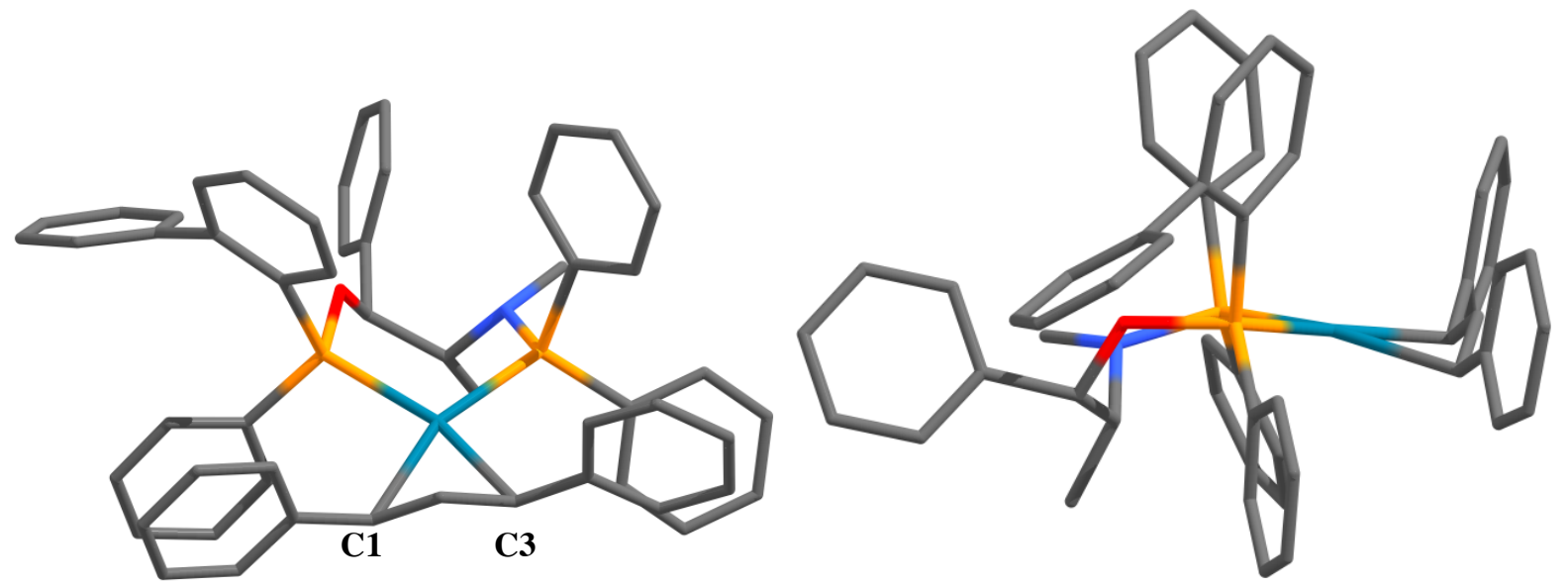

Figure S42. Optimised structure of $35 \mathbf{W}$ (boat) in the ground state.

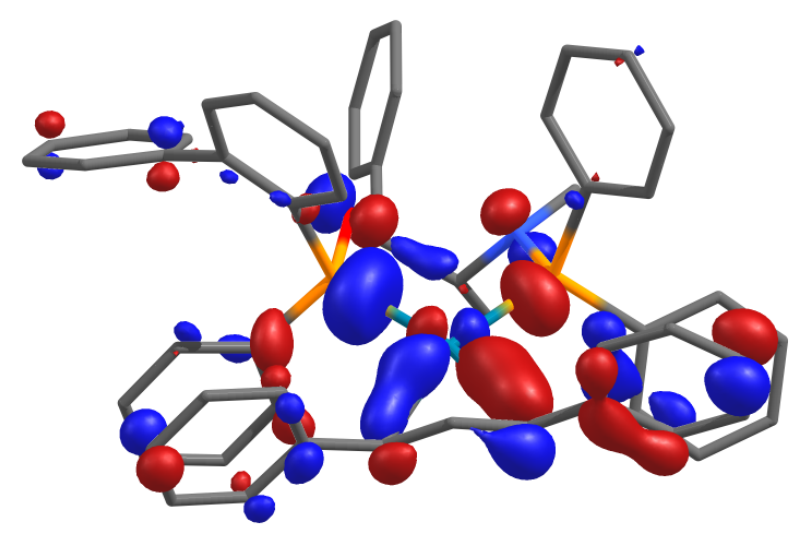

HOMO

$(-8.16 \mathrm{eV})$

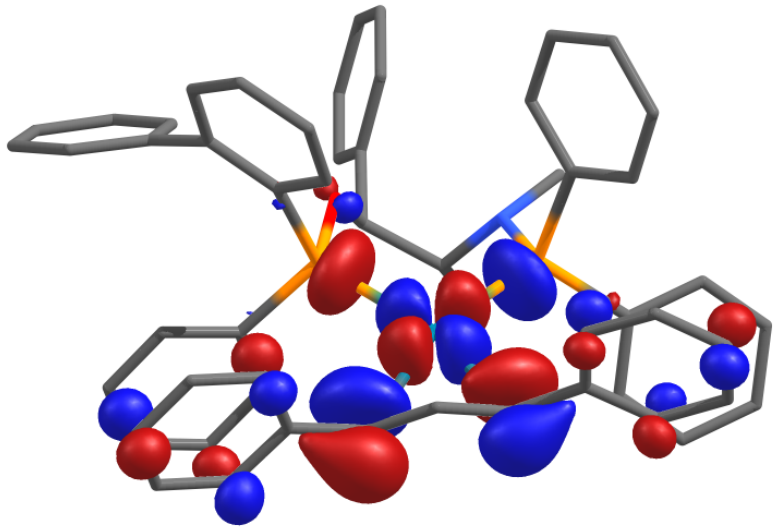

LUMO

$(-4.65 \mathrm{eV})$

Figure S43. Representations of the frontier MOs HOMO and LUMO of $\mathbf{3 5} \mathbf{W}$ (boat) in the ground state.

Table S74. Atomic contributions of HOMO and LUMO of $35 \mathbf{W}$ (boat) in the ground state at the C1 and C3 position of the complexed $\pi$-allyl group.

\begin{tabular}{lcc}
\hline \multicolumn{1}{c}{ Fragment } & HOMO & LUMO \\
\hline Energy $(\mathrm{eV})$ & -8.16 & -4.65 \\
\hline C1 $(\%)$ & 2.74 & 13.71 \\
C3 $(\%)$ & 5.06 & 10.63 \\
\hline
\end{tabular}




\section{Structure 35M(half-chair)}
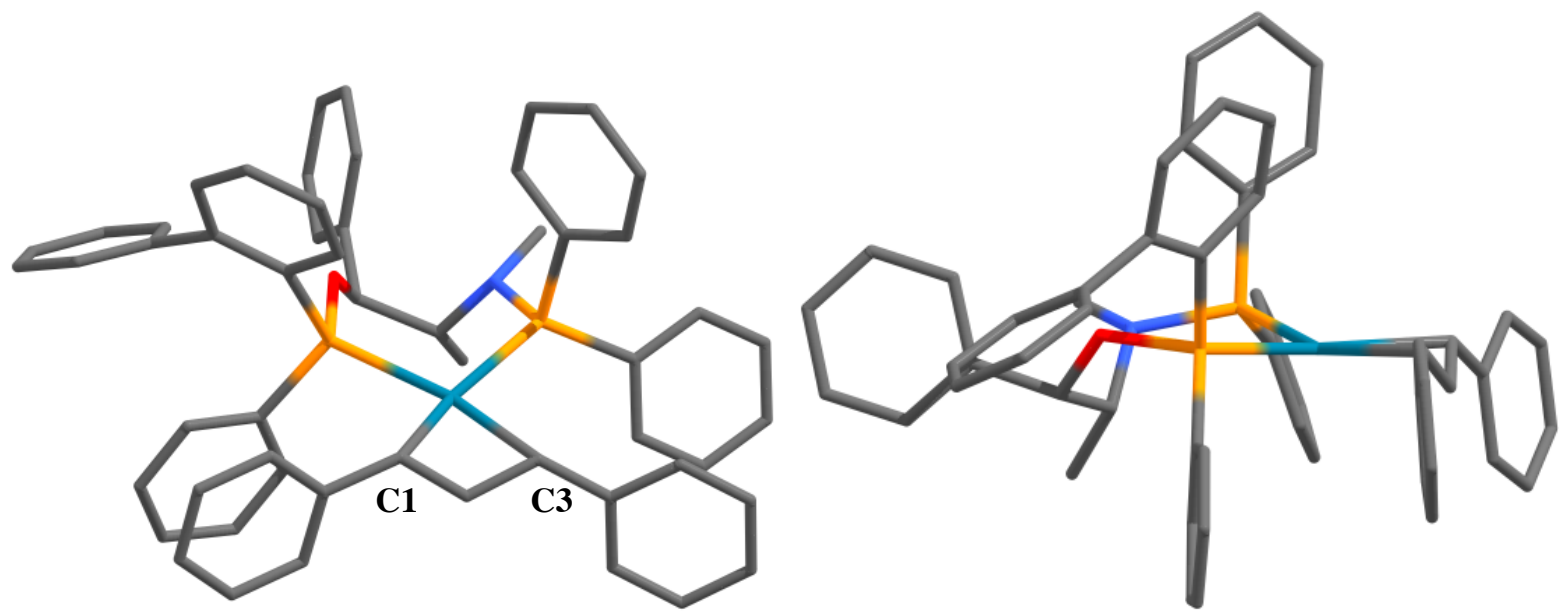

Figure S44. Optimised structure of $\mathbf{3 5 M}$ (half-chair) in the ground state.

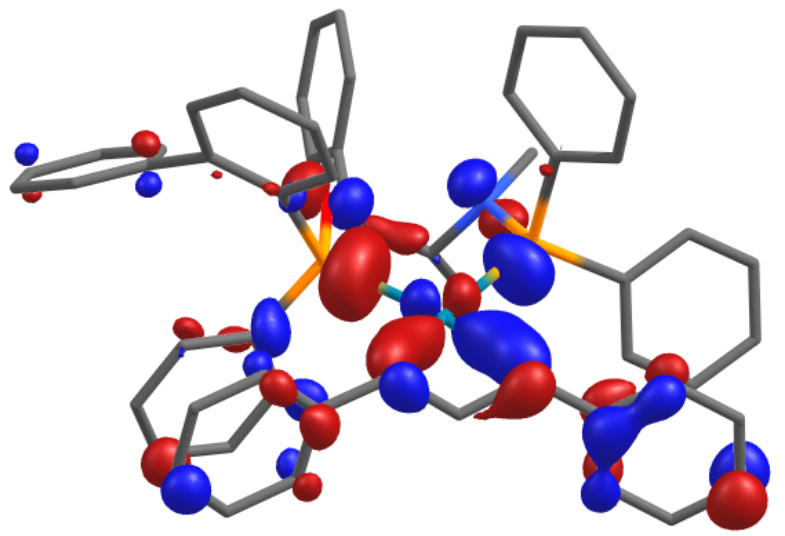

HOMO

$(-8.14 \mathrm{eV})$

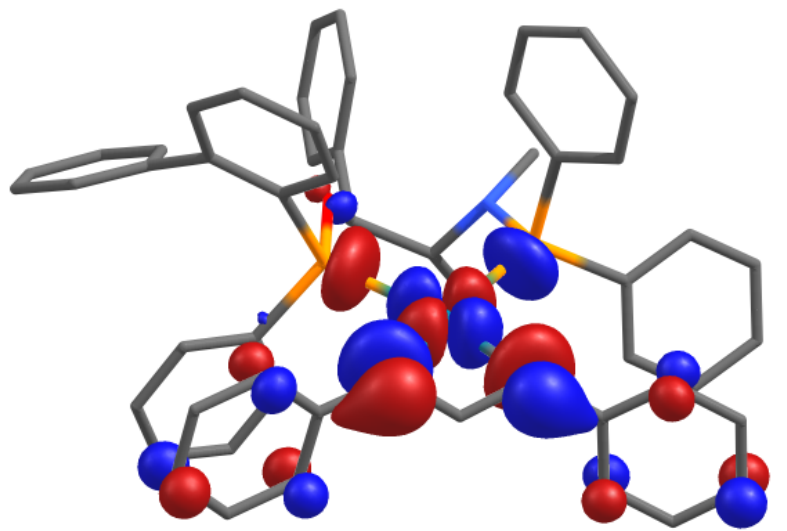

LUMO

$(-4.68 \mathrm{eV})$

Figure S45. Representations of the frontier MOs HOMO and LUMO of 35M(half-chair) in the ground state.

Table S75. Atomic contributions of HOMO and LUMO of $35 \mathrm{M}$ (half-chair) in the ground state at the $\mathrm{C} 1$ and $\mathrm{C} 3$ positions of the complexed $\pi$-allyl group.

\begin{tabular}{lcc}
\hline \multicolumn{1}{c}{ Fragment } & HOMO & LUMO \\
\hline Energy $(\mathrm{eV})$ & -8.14 & -4.68 \\
\hline C1 $(\%)$ & 4.40 & 11.81 \\
C3 $(\%)$ & 3.40 & 12.67 \\
\hline
\end{tabular}




\section{Structure 35W(half-chair)}
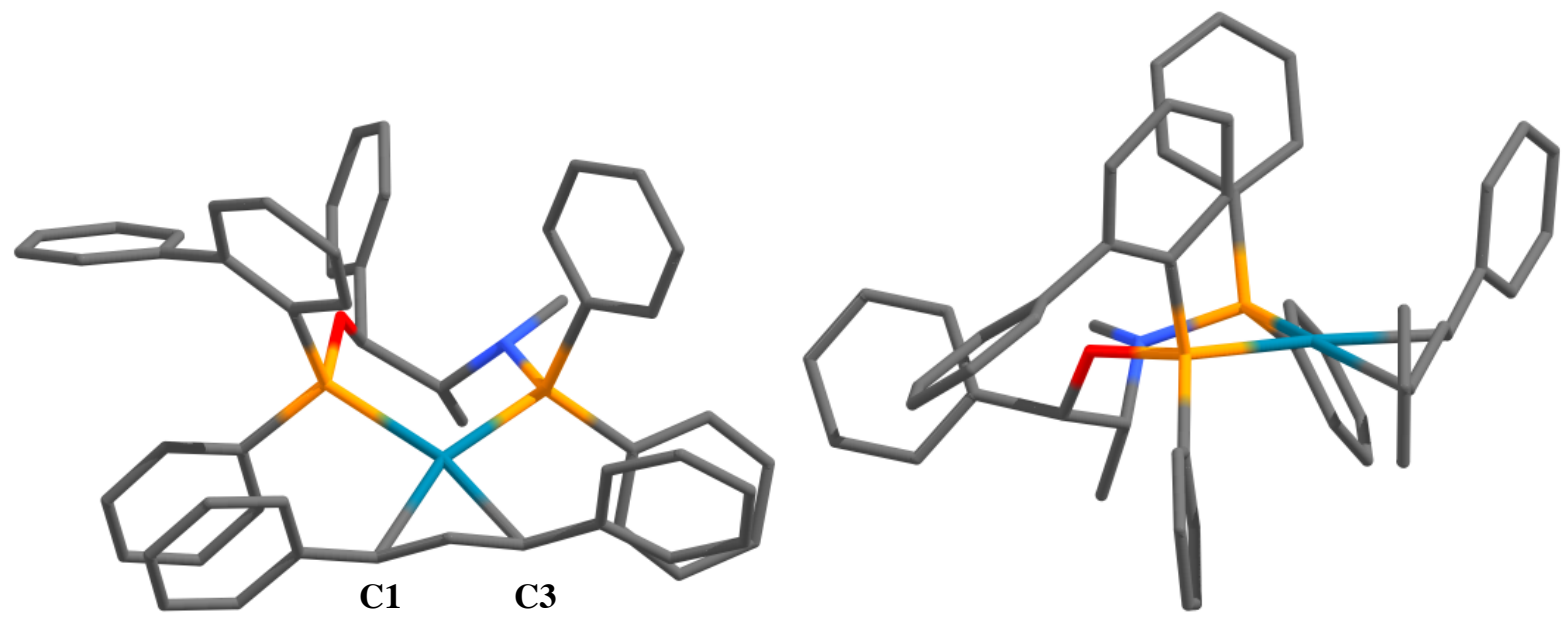

Figure S46. Optimised structure of $35 \mathrm{~W}$ (half-chair) in the ground state.

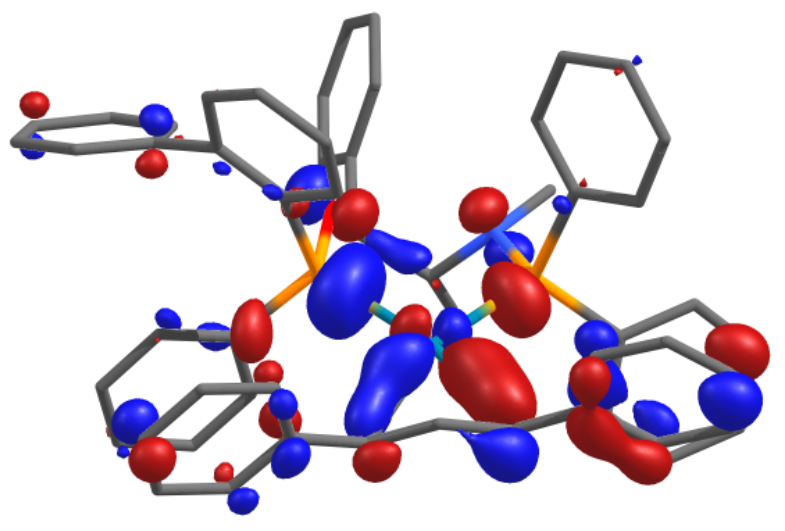

HOMO

$(-8.16 \mathrm{eV})$

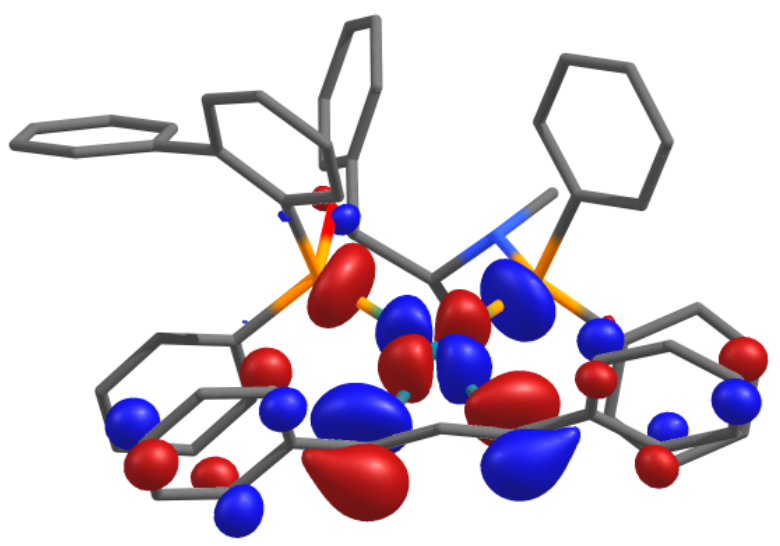

LUMO

$(-4.65 \mathrm{eV})$

Figure S47. Representations of the frontier MOs HOMO and LUMO of $\mathbf{3 5 W}$ (half-chair) in the ground state.

Table S76. Atomic contributions of HOMO and LUMO of $35 \mathbf{W}$ (half-chair) in the ground state at the C1 and C3 positions of the complexed $\pi$-allyl group.

\begin{tabular}{lcc}
\hline \multicolumn{1}{c}{ Fragment } & HOMO & LUMO \\
\hline Energy $(\mathrm{eV})$ & -8.16 & -4.65 \\
\hline C1 $(\%)$ & 2.74 & 13.71 \\
C3 $(\%)$ & 5.06 & 10.64 \\
\hline
\end{tabular}




\section{Computation references}

(1) Gaussian 16, Revision B.01, Frisch, M. J. et al. , Gaussian, Inc., W. C. Gaussian 16. Revis. A $2016,3$.

(2) Becke, A. D. Density-functional Thermochemistry. III. The Role of Exact Exchange. J. Chem. Phys. 1993, 98 (7), 5648-5652. https://doi.org/10.1063/1.464913.

(3) Binkley, J. S.; Pople, J. A.; Hehre, W. J. Self-Consistent Molecular Orbital Methods. 21. Small SplitValence Basis Sets for First-Row Elements. J. Am. Chem. Soc. 1980, 102 (3), 939-947. https://doi.org/10.1021/ja00523a008.

(4) Gordon, M. S.; Binkley, J. S.; Pople, J. A.; Pietro, W. J.; Hehre, W. J. Self-Consistent Molecular-Orbital Methods. 22. Small Split-Valence Basis Sets for Second-Row Elements. J. Am. Chem. Soc. 1982, 104 (10), 2797-2803. https://doi.org/10.1021/ja00374a017.

(5) Pietro, W. J.; Francl, M. M.; Hehre, W. J.; DeFrees, D. J.; Pople, J. A.; Binkley, J. S. Self-Consistent Molecular Orbital Methods. 24. Supplemented Small Split-Valence Basis Sets for Second-Row Elements. J. Am. Chem. Soc. 1982, 104 (19), 5039-5048. https://doi.org/10.1021/ja00383a007.

(6) Dobbs, K. D.; Hehre, W. J. Molecular Orbital Theory of the Properties of Inorganic and Organometallic Compounds 4. Extended Basis Sets for Third-and Fourth-Row, Main-Group Elements. J. Comput. Chem. 1986, 7 (3), 359-378. https://doi.org/10.1002/jcc.540070313.

(7) Dobbs, K. D.; Hehre, W. J. Molecular Orbital Theory of the Properties of Inorganic and Organometallic Compounds 5. Extended Basis Sets for First-Row Transition Metals. J. Comput. Chem. 1987, 8 (6), 861879. https://doi.org/10.1002/jcc.540080614.

(8) Dobbs, K. D.; Hehre, W. J. Molecular Orbital Theory of the Properties of Inorganic and Organometallic Compounds. 6. Extended Basis Sets for Second-Row Transition Metals. J. Comput. Chem. 1987, 8 (6), 880 893. https://doi.org/10.1002/jcc.540080615.

(9) Mekelleche, S. M.; Benhabib, R. Elucidation of the Regioselectivity in Diels-Alder Reactions by Means of Theoretical Approaches. J. Mol. Struct. THEOCHEM 2004, 709 (1-3), 31-34. https://doi.org/10.1016/j.theochem.2004.06.033. 